UNIVERSIDAD POLITÉCNICA DE MADRID

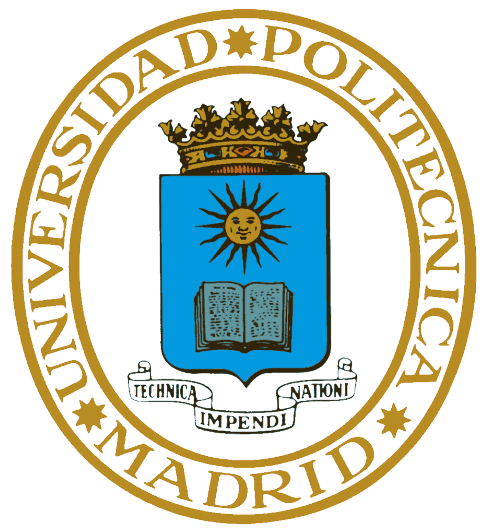

ESCUELA TÉCNICA SUPERIOR DE INGENIEROS INFORMÁTICOS

\title{
Relational logics for higher-order effectful programs PH.D THesis
}

Author: Alejandro Aguirre Galindo MSc. in Computer Science

Advisor: Gilles Barthe $\mathrm{PhD}$. in Mathematics 

Copyright $\bigcirc 2020$ by Alejandro Aguirre Galindo 
DEPARTAMENTAMENTO DE LENGUAJES Y SISTEMAS INFORMÁTICOS E INGENIERIA DE SOFTWARE

ESCUELA TÉCNICA SUPERIOR DE INGENIEROS INFORMÁTICOS

\title{
Relational logics for higher-order effectful programs
}

\author{
SUBMITTED IN PARTIAL FULFILLMENT OF THE REQUIREMENTS FOR THE DEGREE OF: \\ Doctor of Philosophy in Software, Systems and Computing
}

\author{
Author: Alejandro Aguirre Galindo \\ MSc. in Computer Science \\ Advisor: Gilles Barthe \\ PhD. in Mathematics
}

November 2020 


\section{ABSTRACT OF THE DISSERTATION ${ }^{1}$}

Relational logics express properties that relate two executions of a single program, or two executions of two different programs. Properties such as program equivalence, non-interference, differential privacy, robustness or sensitivity fall under this umbrella.

Although traditional program verification offers some capability to prove relational properties by using program transformations, ultimately these fall short because they are unable to use the program structure to guide the reasoning. On the other hand, novel logics explicitly designed for relational reasoning are often overly reliant on reasoning synchronously, that is, about programs with similar syntactic structure. We set out by developing Relational Higher-Order Logic (RHOL), a logic to prove relational properties of a pair of pure functional programs that can reason synchronously when the programs have the same syntax, but also has a wide variety of one-sided rules that allows the reasoning to progress when the programs lack this similarity. RHOL also has a companion system, Unary Higher-Order Logic (UHOL) that can be used to prove unary properties (i.e., properties of a single program). Both RHOL and UHOL are based on a standard Higher-Order Logic (HOL). RHOL is not only a logic in its own right, but can also be seen as a framework in which to embed other relational reasoning systems to prove their soundness, as well as a base on top of which to build more expressive logics.

We then showcase the versatility of RHOL by extending it to support many different extensions with the main goal of reasoning about probabilistic programs. We first focus on lifting-based properties. Liftings provide a way to erase the computational side effects from logical specifications, so that we can reason about them using standard logics. Some properties that can be proven in this manner are bounds on the probability that the program output satisfies a certain property, or differential privacy. We respectively embed reasoning about these two properties into two novel logics : Higher-Order Union Bound Logic (HO-UBL) and Higher-

\footnotetext{
${ }^{1}$ This is the version of the thesis submitted in partial fulfillment of the requirements for the degree of PhD. in Software, Systems and Computing. A corrected and updated version can be found in the author's homepage.
} 
Order Relational Probabilistic Logic (HO-RPL). We then extend these logics to support reasoning about adversarial properties that specify the behavior of a known program (an oracle) with respect to an unknown program or environment (the adversary). These properties are important concepts in fields such as security or privacy.

We then consider other related topics. First we look into proving properties of Markov chains. Many of these properties are inherently relational (e.g., stochastic dominance, recurrence, transience). We adapt the Guarded Lambda Calculus, and the Guarded HOL, a language and logic to reason about infinite streams, to our setting by extending them with probabilities and developing a relational logic on top of Guarded HOL. Second, we present a relational logic for higher-order probabilistic programs that is not lifting-based. This requires an extension of the assertion language and an axiomatization of probability theory in the base logic. Finally, we develop a predicate transformer to reason about expected sensitivity of probabilistic programs. 


\section{RESUMEN DE LA TESIS DOCTORAL}

Las lógicas relacionales expresan propiedades que relacionan dos ejecuciones de un mismo programa o dos ejecuciones de dos programas distintos. Ejemplos de estas propiedades son equivalencia, no-interferencia, privacidad diferencial, robustez o sensibilidad.

Aunque la verificación tradicional ofrece cierta capacidad para probar estas propiedades usando transformaciones de programas, no es adecuada porque no es capaz de usar la estructura de los programas para guiar el razonamiento. Por otro lado, las lógicas diseñadas para razonamiento relacional dependen demasiado de razonar de manera síncrona sobre programas con estructura sintáctica similar. Empezamos por desarrollar Relational Higher-Order Logic (RHOL), una lógica para probar propiedades relacionales de programas funcionales que puede razonar síncronamente cuando los programas tienen la misma sintaxis, y que dispone de reglas unilaterales para razonar en caso contrario. RHOL viene acompañada de UHOL, una lógica para probar propiedades de un único programa. Tanto RHOL como UHOL se basan en una lógica de orden superior estándar (HOL). RHOL no es sólo una lógica por mérito propio, sino que puede verse como un marco en el que embeber otros sistemas lógicos, así como una base para construir lógicas más expresivas.

A continuación demostramos la versatilidad de RHOL extendiéndola para dar soporte a diferentes extensiones con el objetivo de razonar sobre programas probabilísticos. Primero nos fijamos en propiedades basadas en liftings. Los liftings proporcionan una manera de borrar los efectos computacionales de las especificaciones lógicas para poder razonar acerca de ellas en lógicas estándar. Algunos ejemplos de propiedades que se pueden probar de esta forma son cotas sobre la probabilidad de que la salida de un programa satisfaga cierto predicado o privacidad diferencial. Embebemos respectivamente razonamiento sobre estas dos propiedades en dos lógicas: Higher-Order Union Bound Logic (UBL) y Higher-Order Relational Probabilistic Logic (HO-RPL). Luego extendemos estas lógicas para dar poder razonar sobre propiedades adversariales que especifican el comportamiento de un programa conocido (el oráculo) respecto a un entorno desconocido (el adversario). 
Este tipo de propiedades es habitual en los campos de seguridad o privacidad.

Luego consideramos otros temas relacionados. Primero investigamos sobre propiedades relacionales de cadenas de Markov. Para ello, extendemos el Guarded Lambda Calculus y la Guarded HOL, un lenguaje y una lógica para razonar sobre cadenas infinitas al marco probabilístico, y desarrollamos una lógica relacional sobre Guarded HOL. Segundo, presentamos una lógica para programas probabilísticos de orden superior que no se basa en liftings, sino en una axiomatización de teoría de la probabilidad. Finalmente, desarrollamos un transformador de predicados para razonar sobre sensibilidad de programas probabilísticos. 
A mi madre y a mi padre. 


\section{ACKNOWLEDGMENTS}

I would like to start by thanking my advisor, Gilles Barthe, for guiding and supporting my research for these four years. His excitement and drive for research are inspiring, and I hope we can keep collaborating in the future.

It is thanks to Gilles that I have had the opportunity to meet and work with a wonderful set of co-authors. I would like to thank them all for the constructive discussions from which this dissertation has benefited: Lars Birkedal, Aleš Bizjak, Marco Gaboardi, Deepak Garg, Justin Hsu, Benjamin Kaminski, Joost-Pieter Katoen, Shin-ya Katsumata, Christoph Matheja, Tetsuya Sato, Alexandra Silva and PierreYves Strub.

I would like to particularly thank Marco Gaboardi and Deepak Garg, with whom I have had a productive collaboration for all the years of my PhD, and discussed a big part of the material in this dissertation. Marco invited me for a fruitful visit to Buffalo and Deepak hosted me for three weeks in Saarbrücken. I want to thank Alexandra Silva as well for inviting me to visit University College London.

I am also grateful to Justin Hsu and Ugo Dal Lago for serving as external reporters for my dissertation and giving helpful feedback.

Special thanks go also to Joost-Pieter Katoen for hosting me for 3 months in Aachen. The research we started there with his students Benjamin Kaminski and Christoph Matheja has become Chapter 10 of this thesis.

Shin-ya Katsumata, and the NII Erato MMSD Project hosted me for 6 fantastic months in Tokyo, for which I am very grateful. The Erato MMSD Project is an exciting place to do research in, and Shin-ya's insight about category theory has helped me see my work in another light.

My time at IMDEA has been a delight and I was fortunate to share it with a superb group of students and postdocs that made every day nicer. The months of lockdown have made me miss our times together.

Quiero dar las gracias a mis amigos por estar ahí para mí y por ayudarme a desconectar del mundo académico.

Para acabar, me gustaría agradecer a mis padres, Ana y Luis, su cariño y apoyo incondicional y el haberme dado la oportunidad de llegar aquí. 
CONTENTS

1 Introduction 1

2 Related Work $\quad$ II

I Relational probabilistic properties of higher-order programs 16

3 Pure relational higher-order logic $\quad 17$

3.1 (A variant of) PCF ...................... 18

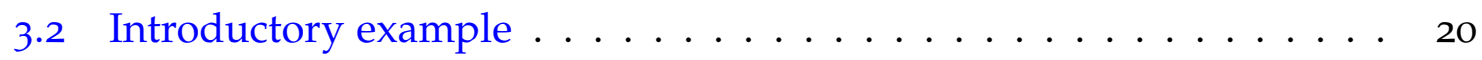

3.3 Higher-Order Logic . . . . . . . . . . . . . . . . . . 21

3.4 Unary Higher-Order Logic . . . . . . . . . . . . . . . . . . . 22

3.5 Relational Higher-Order Logic . . . . . . . . . . . . . . . . . 25

$3.5 .1 \quad$ Proof Rules . . . . . . . . . . . . . . . . . . 25

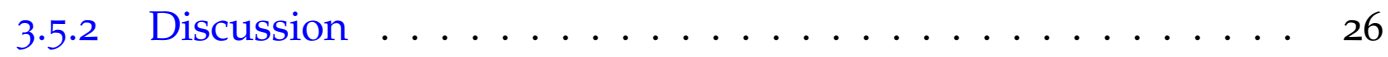

3.5.2.1 Management of the typing context ........ 26

3.5.2.2 Variants of the rules ............ 30

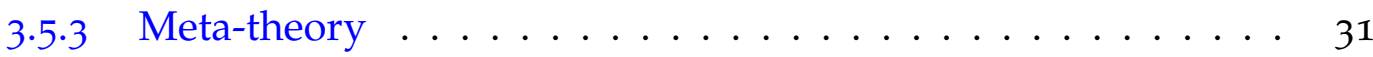

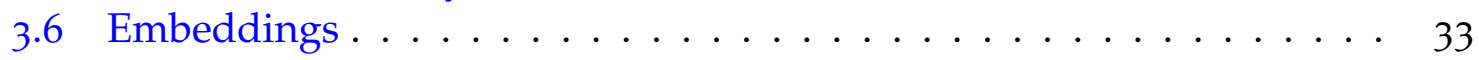

3.6.1 Refinement Types . . . . . . . . . . . . . . . 33

3.6.2 Relational Refinement Types . . . . . . . . . . . . . . 37

3.6.3 Dependency Core Calculus . . . . . . . . . . . . . . . 41

3.6 .4 Relational Cost. . . . . . . . . . . . . . . . . 45

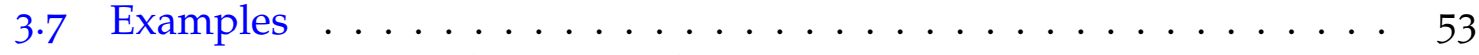

3.7.1 First Example: Factorial . . . . . . . . . . . . . 54

$3 \cdot 7.2$ Second Example: Take and Map .................. 55

$3 \cdot 7 \cdot 3$ Third example: Selection sort . . . . . . . . . . . . . 57

$3 \cdot 7.4$ Fourth Example: Insertion Sort . . . . . . . . . . . . . . 58

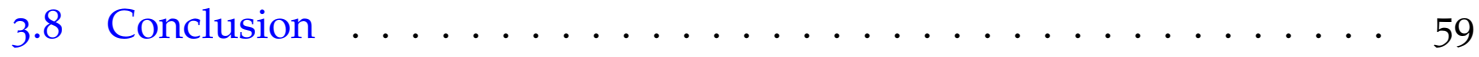


4 Unary Reasoning about probabilities: Union bounds $\quad$ 61

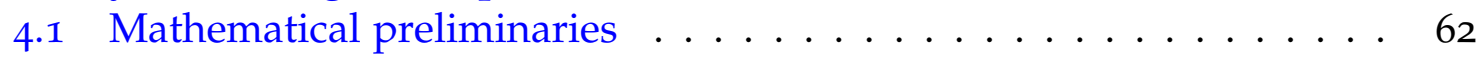

4.1.1 Measure theory . . . . . . . . . . . . . . 62

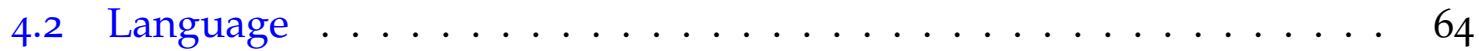

4.2.1 Syntax ............................ 64

4.3 Higher-Order Union Bound logic . . . . . . . . . . . . . . . 65

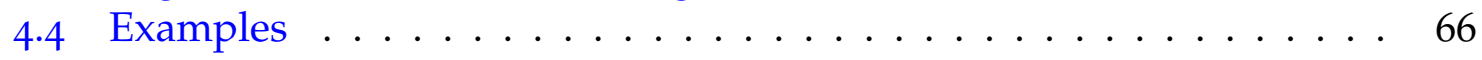

4.5 Category theory preliminaries ................ 68

4.5 .1 A Primer on Category theory . . . . . . . . . . . . . . . . . 69

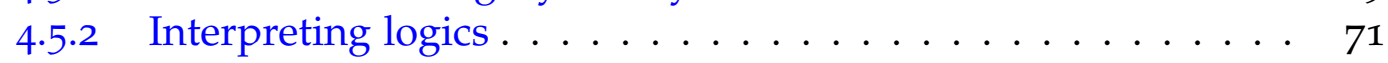

4.6 Adding effects: Graded liftings . . . . . . . . . . . . . . . . . 73

4.6.1 Monads, Graded monads and liftings . . . . . . . . . . . . . . . 73

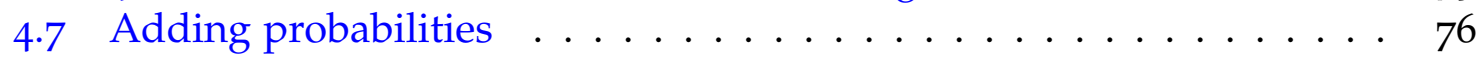

4.7.1 The category of Quasi-Borel spaces . . . . . . . . . . . . 76

4.7 .2 Predicates over Quasi-Borel spaces . . . . . . . . . . . . . . 78

$4 \cdot 7 \cdot 3$ A lifting for union bounds . . . . . . . . . . . 78

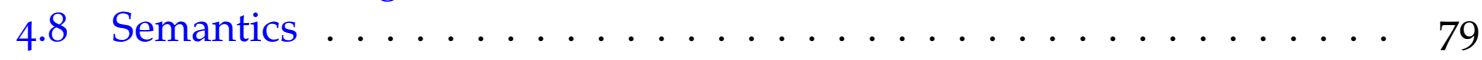

4.8.1 Semantics of the language . . . . . . . . . . . . 79

4.8 .2 Semantics of the base logics . . . . . . . . . . . . 81

4.8 .3 Semantics of HO-UBL . . . . . . . . . . . . 82

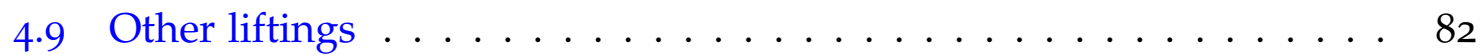

5 Relational Reasoning about probabilities: Probabilistic couplings $\quad \mathbf{8 5}$

5.1 Mathematical preliminaries ........................ 86

5.2 Higher-order probabilistic relational logic . . . . . . . . . . . . . 87

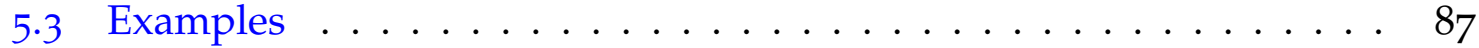

5.3.1 One-time pad/probabilistic non-interference . . . . . . . . . 88

$5 \cdot 3.2$ Adversarial Robustness in Machine Learning . . . . . . . . . 90

5.4 Semantics ................................ 92

6 Unary higher-order logic for adversarial computations 95

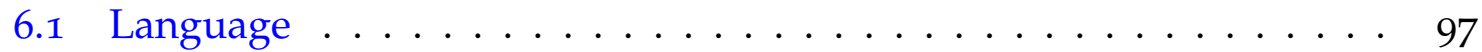

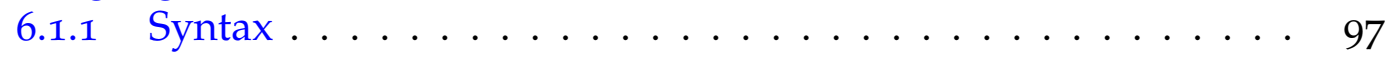

6.1 .2 Semantics .................... 100

6.2 Higher-Order Stateful Union Bound logic . . . . . . . . . . . . . . 1 IO1

6.3 Proving adversarial properties . . . . . . . . . . . 102

6.4 Example: Drifting games . . . . . . . . . . . . . . . . 104

6.5 Stateful liftings . . . . . . . . . . . . . . . . . 107

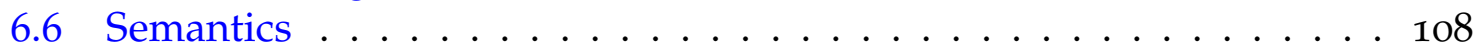

6.6.1 Semantics of HO-SUBL . . . . . . . . . . . 108

6.6.2 Soundness of the adversary rules ............. 109 
7 Relational higher-order logic for adversarial computations $\quad \mathbf{1 1 3}$

7.1 Higher-order stateful probabilistic relational logic . . . . . . . . . . 114

7.2 Relational adversarial properties .......................... 114

$7 \cdot 3$ Example: PRF/PRP Switching Lemma . . . . . . . . . . . . . 118

7.4 Relational Stateful liftings . . . . . . . . . . . . . . 120

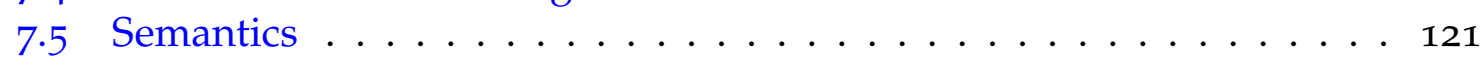

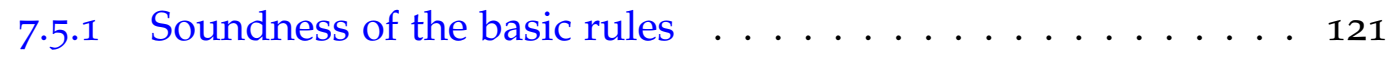

$7 \cdot 5.2$ Soundness of the adversary rules ............ 123

7.6 Related work . . . . . . . . . . . . . . 125

7.7 Conclusion ......................... 126

$\begin{array}{ll}\text { II Other topics } & 127\end{array}$

8 Probabilistic guarded relational higher-order logic $\quad 128$

8.1 Mathematical preliminaries .............................

8.1.1 Discrete probability theory .......................... 131

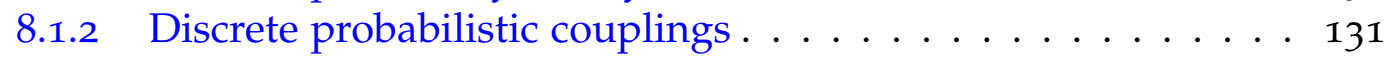

8.1.3 Probabilities in the category Set ............. 133

8.2 Overview of the system . . . . . . . . . . . . . . 134

8.2.1 Base logic: Guarded Higher-Order Logic . . . . . . . . . . . . . . . 134

8.2.2 A system for relational reasoning . . . . . . . . . . . . 135

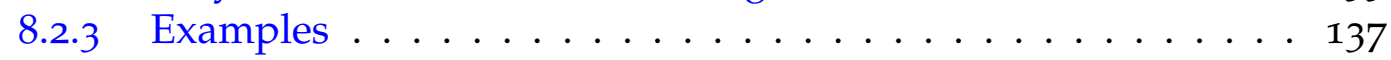

8.2.3.1 Stochastic dominance . . . . . . . . . . . . 137

8.2.3.2 Shift coupling: random walk vs lazy random walk . 139

8.2.3.3 Lumped coupling: random walks on 3 and 4 dimen-

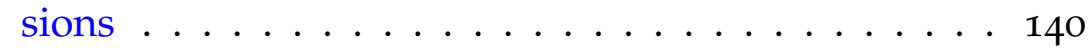

8.3 Probabilistic Guarded Lambda Calculus . . . . . . . . . . . . . . . . 141

8.4 Guarded higher-order logic . . . . . . . . . . . . . . . . 145

8.5 Relational proof system . . . . . . . . . . . . . . 148

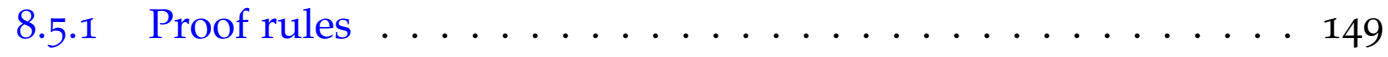

8.5 .2 Metatheory .................... 150

8.5 .3 Shift couplings revisited ....................... 152

8.6 Related Work . . . . . . . . . . . . . . . . . 153

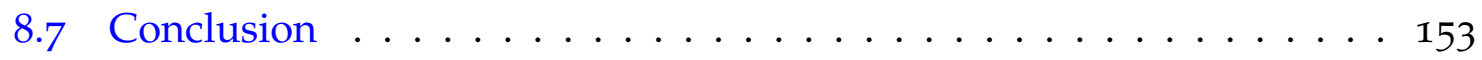

9 An assertion-based logic for higher-order probabilistic programs $\quad \mathbf{1 5 5}$

9.1 PPV by example . . . . . . . . . . . . . . . 156

9.2 HPProg: a higher-order probabilistic programming language . . . . 159

9.3 PL: A Logic for Probabilistic Programs . . . . . . . . . . . . . . . . 160

9.4 Axioms and Equations of Assertions for Statistics . . . . . . . . . . . . 162

9.4.1 Markov and Chebyshev Inequalities . . . . . . . . . . . . . 162 
9.5 Unary /Relational Logic . . . . . . . . . . . . . . . . . . . . 163

9.5.1 The Unary Logic UPL . . . . . . . . . . . . . . . . . . . 163

9.5 .2 The Relational Logic RPL . . . . . . . . . . . . . . . . . . . . . 164

9.5 .3 Special Rules . . . . . . . . . . . . . . . . . . . . 164

9.6 Semantics ............................. 166

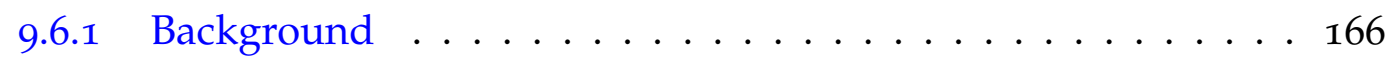

9.6 .2 Semantics for HPProg . . . . . . . . . . . . . . . . . . . . . . . 167

9.6.3 Semantics of the $\operatorname{logics} \ldots \ldots \ldots \ldots . \ldots \ldots$

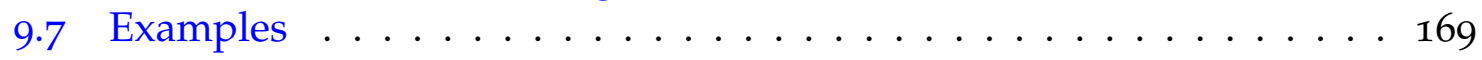

9.7.1 Slicing of Probabilistic Programs . . . . . . . . . . . . . . . . 169

9.7.2 Gaussian Mean Learning: Convergence and Stability . . . . . 171

9.8 Related work . . . . . . . . . . . . . . . . . . . 174

9.9 Conclusions . . . . . . . . . . . . . . . . . 174

10 A pre-expectation calculus for expected sensitivity $\quad \mathbf{1 7 5}$

10.1 Mathematical Preliminaries . . . . . . . . . . . . . . . 177

10.1.1 Basic probability concepts . . . . . . . . . . . . 177

10.1.2 Programming language and semantics . . . . . . . . . 178

10.1.3 Distances between probability distributions . . . . . . . . . 179

10.2 Preliminaries: Expectation-based logics . . . . . . . . . . . 180

10.3 Bounding expected sensitivity with relational pre-expectations . . . . 181

10.3.I A first unsuccessful attempt: a relational pre-expectation for

exact bounds ...................... 182

10.3.2 Compositional upper bounds by relational pre-expectation . . 183

10.3.3 Reasoning with relational pre-expectations . . . . . . . . 185

10.3.4 Embedding EPRHL . . . . . . . . . . . . . . . . . . 188

10.4 Warmup Example: Stability of SGD . . . . . . . . . . . . . . 188

10.4.1 Background . . . . . . . . . . . . . . . . . . . . 189

10.4.2 Verifying stability for stochastic gradient descent . . . . . . 190

10.5 Example: Convergence of Reinforcement Learning algorithms . . . . 192

10.5.1 Background . . . . . . . . . . . . . . . . . . 192

10.5 .2 Verifying convergence for TDo . . . . . . . . . . . . . . . 193

10.6 Example: Random Walks and Card Shuffles . . . . . . . . . . . . . 194

10.6.1 Preliminaries: Card shuffling and Markov chain mixing . . . . 195

10.6.2 Warmup: Hypercube walk . . . . . . . . . . . . . . . . 195

10.6.3 Random-to-top shuffle . . . . . . . . . . . . . . . . . . . . . 197

10.6.4 Random transpositions shuffle . . . . . . . . . . . . 200

10.6.5 Uniform riffle shuffle . . . . . . . . . . . . . . . 201

10.7 Extensions: Proving Lower Bounds and Uniformity . . . . . . . . . . 201

10.7.1 Convergence to uniform distribution . . . . . . . . . 201

10.7.2 Proving lower bounds . . . . . . . . . . . . . 202

10.8 Extensions: Rules for Asynchronous Reasoning . . . . . . . . . . . 204 
10.8.1 Asynchronous rules for bounding the Kantorovich distance . 205 10.8.2 Example: Bounding the distance between binomial distributions206 10.9 Related work . . . . . . . . . . . . . . . . . . . . . . . . . . . . . . 207 10.10Conclusion . . . . . . . . . . . . . . . . . 208

III Conclusion 209

II Conclusions \& Future Work $\quad 210$

11.1 Conclusions . . . . . . . . . . . . . . . . . . 210

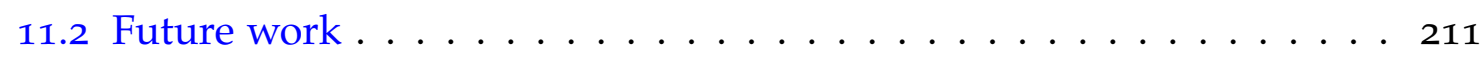

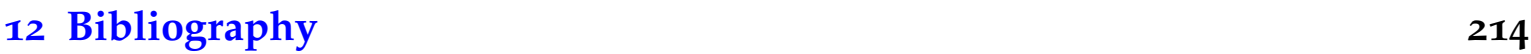

$\begin{array}{ll}\text { Index } & \mathbf{2 3 3}\end{array}$

$\begin{array}{ll}\text { Appendix } & 234\end{array}$

A Appendix of Chapter $3 \quad 234$

A.1 Example: Factorial . . . . . . . . . . . . . . . . . . . . . . 234

A.2 Example: List reversal . . . . . . . . . . . . . . . . . . . 235

A.3 Example: Proof of Theorem $3.23 \ldots \ldots \ldots \ldots . \ldots . \ldots 236$

A.4 Example: Proof of Theorem $3.24 \ldots \ldots \ldots . \ldots . \ldots 240$

A.5 Proof of Theorem $3.26 \ldots \ldots \ldots \ldots \ldots$. . . . . . . . . . . . . . .

B Appendix of Chapter 8 $\mathbf{2 4 6}$

B.o.1 Equational theory of the calculus . . . . . . . . . . . 246

B.1 Additional background . . . . . . . . . . . . . . . . . . . 247

B.2 Unary fragment . . . . . . . . . . . . . . . . . . . . 247

B.2.1 Unary fragment of GHOL . . . . . . . . . . . . . . . 247

B.2.2 Guarded UHOL . . . . . . . . . . . . . . . . . . . 248

B.2.3 Derivation rules . . . . . . . . . . . . . . . . . . . 248

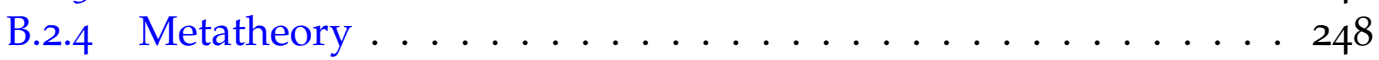

B.2.5 Probabilistic extension . . . . . . . . . . . 250

B.2.6 Unary example: Every two . . . . . . . . . . . 250

C Appendix of Chapter 10 253

C.1 Background: Real Analysis . . . . . . . . . . . . . . . . . . . . . . . . . . . . . 253

C.2 Section 2: Omitted Proofs . . . . . . . . . . . . . . . 255

C.3 Soundness and Continuity: Omitted Proofs . . . . . . . . . . . . 256

C.4 Embedding Relational Hoare Logics . . . . . . . . . . . . . . . 267 
C.5 Convergence of TD(o): Omitted details . . . . . . . . . . . . . . 271

C.6 Verifying robustness of Projected Gradient Descent (PGD) . . . . . . . 273

C.7 Random-to-top: Omitted details . . . . . . . . . . . . . . . . . . 275

C.8 Uniform riffle: Omitted details . . . . . . . . . . . . . . . . . 277

C.9 Asynchronous rules: Omitted details . . . . . . . . . . . . . . . . . 280 


\section{LIST OF FIGURES}

3.1 Selected rules for HOL . . . . . . . . . . . . . . . . . 22

3.2 Unary Higher-Order Logic rules ... . . . . . . . . . . . . . 24

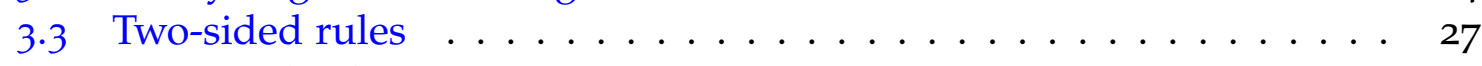

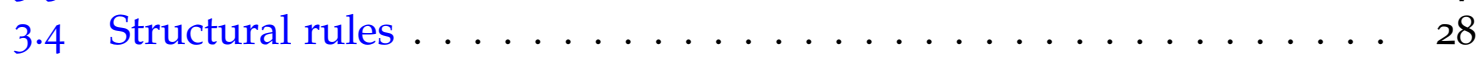

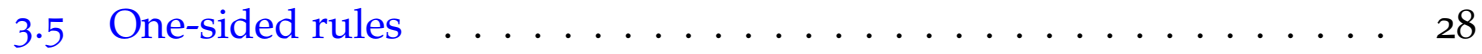

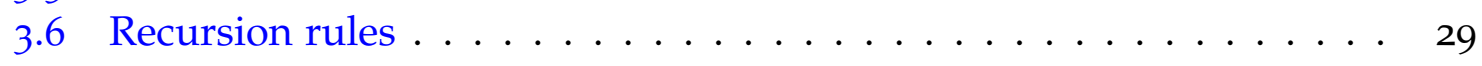

3.7 Some derived rules . . . . . . . . . . . . . . . . 29

3.8 Refinement types rules (subtyping and typing) $\ldots \ldots \ldots \ldots$

3.9 Relational Typing (Selected Rules) . . . . . . . . . . . . . 38

3.10 Selected unary RelCost rules . . . . . . . . . . . . . . . . . . 46

3.11 Selected relational RelCost rules . . . . . . . . . . . . . . . . 47

3.12 Cost-instrumented translation of expressions. . . . . . . . . 48

4.1 Selected typing rules $\ldots \ldots \ldots \ldots \ldots \ldots \ldots$

4.2 Union bound: monadic rules . . . . . . . . . . . . . 66

5.1 Relational logic: monadic rules . . . . . . . . . . . . . . . 88

5.2 Relational logic: rules for sampling . . . . . . . . . . . . . . . 89

6.1 Selected typing rules . . . . . . . . . . . . . . . . . . 99

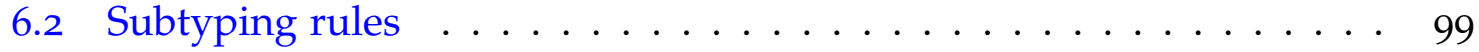

6.3 Union bound: monadic rules . . . . . . . . . . . . . 102

6.4 Union bound: structural rules . . . . . . . . . . . . . 103

7.1 Relational logic: monadic rules . . . . . . . . . . . . . . 115

7.2 Relational logic: sampling from uniform distributions . . . . . . 116

$7 \cdot 3$ Relational logic: switching rules ............... 116

8.1 Proof rules for probabilistic constructs . . . . . . . . . . . . . . 137

8.2 Semantics of types in the topos of trees . . . . . . . . . . 143 
8.3 A selection of the typing rules of the guarded lambda calculus. The rules for products, sums, and natural numbers are standard. . . . . . 145

8.4 Semantics for the Guarded $\lambda$-calculus . . . . . . . . . . . . . . 146

8.5 Selected Guarded Higher-Order Logic rules . . . . . . . . . . . . 148

8.6 Rules for probabilistic constructs . . . . . . . . . . . . . . . . . . 149

8.7 Two-sided rules for Guarded RHOL . . . . . . . . . . . . . . . 150

8.8 One-sided rules for Guarded RHOL . . . . . . . . . . . . . . 151

9.1 UPL rules for probabilistic constructs .............. 163

9.2 A selection of RPL rules. . . . . . . . . . . . . . . . . . 165

10.1 Semantics of $\mathrm{PWHILE} \mathrm{\ldots ...} \mathrm{.} \mathrm{.} \mathrm{.} \mathrm{.} \mathrm{.} \mathrm{.} \mathrm{.} \mathrm{.} \mathrm{.} \mathrm{.} \mathrm{.} \mathrm{.} \mathrm{.} 179$

10.2 Definition of the relational pre-expectation operator $\widetilde{r p e}(c, \mathcal{E}) \ldots \ldots .184$

10.3 Properties of relational pre-expectation operator $\widetilde{r p e}(c, \mathcal{E}) \ldots \ldots$. . . 186

10.4 Example programs: Stability and convergence . . . . . . . . . . . . 189

10.5 Shuffling algorithms . . . . . . . . . . . . . . . . . 198

10.6 Definition of the weakest pre-expectation operator wpe $(c, \mathcal{E}) \ldots . . .203$

10.7 Definition of the one-sided operator wpe $\langle 1\rangle(c, \varepsilon) \ldots \ldots$

B.1 Rules for the unary diamond modality . . . . . . . . . . . . . . . . 247

B.2 Guarded Unary Higher-Order Logic rules . . . . . . . . . . . . . . 249

B.3 Proof rules for probabilistic constructs - unary case . . . . . . 250

C.1 EpRHL proof pules . . . . . . . . . . . . . . . . 268

C.2 Projected Gradient Descent (PGD) . . . . . . . . . . . . . 273 


\section{LIST OF PUBLICATIONS}

This thesis is based on six papers, the first four have been published to peer-reviewed academic conferences and journals. The fourth one is an extended journal version of the first one. The fifth one has been accepted for publication and will appear in the near future. The last one is currently under preparation. The following list summarizes the aforementioned publications:

- Alejandro Aguirre, Gilles Barthe, Marco Gaboardi, Deepak Garg, Pierre-Yves Strub. A relational logic for higher-order programs. In Proceedings of ICFP, 2017

- Alejandro Aguirre, Gilles Barthe, Lars Birkedal, Aleš Bizjak, Marco Gaboardi, Deepak Garg. Relational Reasoning for Markov Chains in a Probabilistic Guarded Lambda Calculus. In Proceedings of ESOP, 2019.

- Tetsuya Sato, Alejandro Aguirre, Gilles Barthe, Marco Gaboardi, Deepak Garg, Justin Hsu. Formal verification of higher-order probabilistic programs. In Proceedings of POPL, 2019

- Alejandro Aguirre, Gilles Barthe, Marco Gaboardi, Deepak Garg, Pierre-Yves Strub. A relational logic for higher-order programs. Journal of functional programming, 2019.

- Alejandro Aguirre, Gilles Barthe, Benjamin Kaminski, Joost-Pieter Katoen, Christoph Matheja, Justin Hsu. A Pre-Expectation Calculus for Probabilistic Sensitivity. To appear in POPL 2021.

- Alejandro Aguirre, Gilles Barthe, Marco Gaboardi, Deepak Garg, Shin-ya Katsumata, Tetsuya Sato. Higher-order probabilistic adversarial computations: Categorical semantics and program logics. In preparation.

Other papers of the author not included in this thesis are the following:

- Alejandro Aguirre, Gilles Barthe, Justin Hsu, Alexandra Silva. Almost sure productivity. In Proceedings of ICALP, 2018 
- Alejandro Aguirre, Shin-ya Katsumata. Weakest preconditions in fibrations. In Proceedings of MFPS, 2020 


\section{INTRODUCTION}

Traditionally, program verification focuses on proving how a single program behaves with respect to a single input, essentially specifying the set of valid traces that are admissible behavior. This leaves behind many interesting properties that cannot be expressed in this manner. Hyperproperties [62] instead specify programs with respect to sets of sets of traces, in the sense that any $n$ parallel executions of the program should follow a valid set of $n$ traces. Relational properties are hyperproperties that specify programs with respect to sets of pairs of traces. Examples of such properties include non-interference, sensitivity or differential privacy.

Traditional verification techniques such as program logics or type systems exploit the modular nature of programs to prove specifications about them. This is best illustrated by the sequence rule of Hoare logic:

$$
\frac{\{\phi\} C_{1}\left\{\phi^{\prime}\right\} \quad\left\{\phi^{\prime}\right\} C_{2}\left\{\phi^{\prime \prime}\right\}}{\{\phi\} C_{1} ; C_{2}\left\{\phi^{\prime \prime}\right\}}
$$

To prove a specification about the composite program $C_{1} ; C_{2}$ we prove separate specifications about $C_{1}$ and $C_{2}$ and then we compose them logically. These techniques can be adapted to prove relational properties, but their syntax-directed nature often clashes against the asynchronicity of relational properties. To illustrate this, consider the usual case rule of Hoare logic:

$$
\frac{\{\psi \wedge B=\mathbf{t t}\} C_{1}\{\phi\} \quad\{\psi \wedge B=\mathbf{f f}\} C_{2}\{\phi\}}{\{\psi\} \text { if } B \text { then } C_{1} \text { else } C_{2}\{\phi\}}
$$

Standard verification considers a single trace, so it can split the proof depending on whether the conditional guard is satisfied, and analyze the corresponding branch. However, relational verification considers two traces that may not necessarily take the same branch, and in this case we also need to be able to relate an execution of $C_{1}$ with one of $\mathrm{C}_{2}$. In the imperative setting this can be solved by either using program 
transformations to construct a product program that simulates simultaneous runs of $C_{1}$ and $C_{2}$, or by designing a logic that allows us to specify two programs instead of one, and adding complex proof rules to take care of synchronization issues. In the case of functional programs, the techniques to prove relational properties are not as developed, and are very limited by program structure and the kind of properties that can be proven.

This dissertation develops semantic and logical foundations for relational reasoning about effectful higher-order programs. The program logics we present can prove relational properties of functional programs in a manner that can exploit the syntactic structure of programs, but that at the same time, can still progress through proofs in the case that the programs do not share the same structure. Our semantical models can also support reasoning about a variety of monadic effects. The tools we use for this are monadic liftings, which provide a way to specify effectful computations in a standard logic, and reason about them as if they were pure computations. We instantiate these techniques to the probabilistic setting, where we exploit the recent developments in coupling-based logics and semantics of probabilistic programming languages to develop novel relational logics for higher-order probabilistic programs. In particular, we develop reasoning principles for proving adversarial properties, which describe how a program interacts with external computations.

We then explore other topics in the realm of relational verification of probabilistic programs. We first extend the Guarded Lambda calculus-a calculus to reason about infinite streams- with a sampling operator and construct a relational logic on top of it. We use this to verify properties of Markov chains, which we model as distributions over streams. Second, we develop an assertion-based relational logic to reason about higher-order probabilistic programs. As opposed to lifting-based logics, the assertion-based logic extends the base HOL with constructs to express properties of probabilistic computations, and uses an axiomatization of probability theory to reason about them. Finally, we develop a relational weakest precondition transformer to reason about expected sensitivity of probabilistic programs. These are a family of relational properties that study how the output of a program is affected by changes to its input, and that can be applied to fields such as machine learning or numerical methods.

\section{Relational logics for imperative languages}

In principle, it is not necessary to design new logics to prove relational programs. One can simply transform the original program so that it simulates two simultaneous executions, and then state and prove the specification in a standard Hoare logic. This is the approach known as self-composition [21]. However, this kind of reasoning may be cumbersome and moreover many of the issues with synchronization in relational logics are already present when designing a self-composition 
transformation. Moreover, these transformations do not behave well under the presence of computational effects such as non-termination.

Other approaches design a new logic specifically for relational properties. Such is the case of the seminal Relational Hoare Logic by Benton [45]. Judgments of these logics are quadruples of the form:

$$
\{\psi\} C_{1} \sim C_{2}\{\phi\}
$$

where $C_{1}$ and $C_{2}$ are a pair of programs, $\psi$ is a precondition, $\phi$ is a postcondition, and both $\phi$ and $\psi$ can refer to the program variables in $C_{1}$ and $C_{2}$. The meaning of these judgments is that if we have two initial memories related by $\psi$ and we run $C_{1}$ and $C_{2}$ respectively on the first and the second of these memories, then the two final memories will be related by $\phi$.

These logics combine two-sided rules, in which the two programs have the same top-level structure, and one-sided rules, which operate on a single program. Rules for loops are further divided into synchronous, in which both programs perform the same number of iterations, and asynchronous rules, that do not have this restriction but introduce more complexity [40].

As the quintessential example of the need of these rules, consider again the case of conditional branching. Suppose we want to prove

$$
\{\psi\} \text { if } B \text { then } C_{1} \text { else } C_{2} \sim \text { if } B \text { then } C_{1} \text { else } C_{2}\{\phi\}
$$

The synchronous rule requires that $\psi$ implies that the value of $B$ is equivalent on both executions, and then the proof reduces to showing

$$
\left\{\psi \wedge B_{1}=B_{2}=\mathbf{t t}\right\} C_{1} \sim C_{1}\{\phi\}
$$

and

$$
\left\{\psi \wedge B_{1}=B_{2}=\mathbf{f f}\right\} C_{2} \sim C_{2}\{\phi\}
$$

Here $B_{1}$ refers to the evaluation of $B$ on the left-hand side, and $B_{2}$ to its evaluation on the right-hand side. However, the assumption that both executions take the same branch may be too strong, and it is not always provable. The asynchronous rule does not make this assumption, and therefore we also need to relate $C_{1}$ to $C_{2}$ and $\mathrm{C}_{2}$ to $\mathrm{C}_{1}$.

\section{Relational logics for functional programs}

As in the case of imperative programs, we also do not need specific logics to prove relational properties for functional programs. These proofs can be carried out directly in a higher-order logic. In a way, this can be seen as a higher-order version of self-composition. It is in principle possible, but hardly practical, since it does not fully exploit the structure of the program to guide the proof. 
A better alternative is to use relational refinement type systems such as $\mathrm{rF}^{*}$ [29], $\mathrm{HOARe}^{2}$ [33], or RelCost [61], or type systems to prove relational properties such as DFuzz [78]. A relational refinement type is a type characterized by a relational logical assertion. They are usually written as $\{x:: \tau \mid \phi\}$ where $\tau$ is a simple type and $\phi$ is a logical formula depending on the variables $x_{1}, x_{2}$. Morally, this denotes the pairs $\left(t_{1}, t_{2}\right)$ of inhabitants of $\tau$ that are related by $\phi$. Relational refinement type systems include rules such as:

$$
\frac{\Gamma \vdash t_{1} \sim t_{2}: \because \Pi(x:: \tau) . \sigma \quad \Gamma \vdash u_{1} \sim u_{2}:: \tau}{\Gamma \vdash t_{1} u_{1} \sim t_{2} u_{2}:: \sigma\left[u_{1} / x_{1}\right]\left[u_{2} / x_{2}\right]} \text { APP }
$$

which are reminiscent of those of dependently typed languages. Most of the logical reasoning is carried out by applying sophisticated subtyping rules, which include $\{x:: \tau \mid \phi\} \preceq\left\{x:: \tau \mid \phi^{\prime}\right\}$ whenever $\phi$ logically implies $\phi^{\prime}$.

Relational refinement types are appealing for two reasons:

- They capture many important properties of programs in a direct and intuitive manner. For instance, the type $\left\{x::\right.$ nat $\left.\mid x_{1} \leqslant x_{2}\right\} \rightarrow\left\{y::\right.$ nat $\left.\mid y_{1} \leqslant y_{2}\right\}$ captures the set of pairs of functions that preserve the natural order on natural numbers, i.e. pairs of functions $f_{1}, f_{2}$ : nat $\rightarrow$ nat such that for every $x_{1}, x_{2} \in$ nat, $x_{1} \leqslant x_{2}$ implies $f_{1}\left(x_{1}\right) \leqslant f_{2}\left(x_{2}\right)$.

- They can potentially benefit from a long and successful line of foundational [76, $162,74,118]$ and practical $[155,149]$ research on refinement types.

On the other hand, there are still many examples that cannot be verified by these systems. First of all, the two programs in a relational judgment must have the same simple type. Moreover, the rules require that they have similar or identical flow control, and these conditions must be satisfied recursively by their subprograms, e.g., in the case of applications both of the subterms in argument position must have the same type and control-flow, and so do the subterms in function position. This restriction, which can be found in more or less strict forms in the different relational type systems, limits the ability to carry fine-grained reasoning about terms that are structurally different. This raises the question whether the type-directed form of reasoning supported by refinement types can be reconciled with an expressive relational verification of higher-order programs. We provide a positive answer both for pure and for effectful higher-order programs.

Our starting point is the observation that relational refinement type systems are inherently restricted to reasoning about two structurally similar programs, because relational assertions are embedded into types. In order to provide broad support for one-sided rules (i.e., rules that contemplate only one of the two expressions), it is therefore necessary to consider relational assertions at the top-level, since one-sided rules have a natural formulation in this setting. Considering relational assertions at 
the top-level can be done in two different ways: either by supporting a rich theory of subtyping for relational refinement types, in such a way that each type admits a normal form where refinements only arise at the top-level, or simply by adapting the definitions and rules of refinement type systems so that only the top-level refinements are considered. Although both approaches are feasible, we believe that the second approach is more streamlined and leads to friendlier verification environments.

\section{Proving properties of probabilistic programs}

We then set out to extend our base language with probabilistic constructs and accordingly extend the logics to reason about them. Suppose that we want to state that a probabilistic program $C$ has probability less than $\delta$ of producing an error. One possible approach is to add to the logic predicates that explicitly mention probabilities, i.e.

$$
\{T\} C\{\underset{x \sim r}{\operatorname{Pr}}[\text { error }] \leqslant \delta\}
$$

This has the advantage of being able to precisely express the properties you want to prove, but in turn it requires to bake an axiomatization of probabilities into the logic. For instance, if we have another program $C^{\prime}$ specified by

$$
\{\top\} C^{\prime}\left\{\underset{x \sim r}{\operatorname{Pr}}[\text { error }] \leqslant \delta^{\prime}\right\}
$$

and we want to reason about $\mathrm{C} ; \mathrm{C}^{\prime}$, we need axioms to express how probabilities behave with respect to composition. Logics that follow this approach are known as assertion-based logics.

Another approach is to have a logic designed to prove only a particular subset of properties that can be proven compositionally. For instance, consider a logic with judgments of the form

$$
\{\phi\} C\left\{\phi^{\prime}\right\} \delta
$$

with the meaning that, under the assumptions in $\phi$, the program C satisfies $\phi$ with probability less than $\delta$. The property above can then simply be written as

$$
\{T\} C\{\text { error }\}_{\delta}
$$

It is not hard to prove that this logic satisfies the following sequence rule

$$
\frac{\{\phi\} C_{1}\left\{\phi^{\prime}\right\}_{\delta \delta} \quad\left\{\phi^{\prime}\right\} C_{2}\left\{\phi^{\prime \prime}\right\}_{\delta^{\prime}}}{\{\phi\} C_{1} ; C_{2}\left\{\phi^{\prime \prime}\right\}_{\delta+\delta^{\prime}}}
$$

This looks very similar to the usual sequence rule from Hoare logic, and the only changes are the probabilistic interpretation and the added parameter, but importantly the assertion language remains the same. We present a logic called 
Higher-Order Union Bound logic, based on applying these ideas to the higher-order setting. Formally, this is based on the categorical concept of monad liftings, which map predicates over base types to predicates over monadic types in a way that is coherent with the monad unit and multiplication. These logics are known as lifting-based logics. In the case of probabilistic programs, these logics also benefit from recent advances in semantics of higher-order probabilities [93].

The lifting-based approach is not as expressive as the assertion-based approach, but it is cleaner, since it does not require changing the assertion language, and it still allows us to prove many interesting properties.

In addition to the lifting-based logic to prove union bounds, which is a unary reasoning pattern, we present a lifting-based relational logic to reason about probabilistic couplings. Probabilistic couplings are a tool from Markov chain theory that has successfully been applied to verification of probabilistic programs [30], and that can be used to show for instance program equivalence or differential privacy [36]. The key idea behind couplings is that when verifying relational properties of a pair of programs, the randomness behind them can be in some sense synchronized to make the proof simpler. This means that instead of sampling from independent probability distributions on each program, we can find a particular joint distribution and sample from it. We say that two probabilistic programs are related by a $\phi$-coupling if there exists such a joint distribution where the probability of $\phi$ is 1 . This notion can also be made approximate by instead requiring that there are two joint distributions at distance $(\epsilon, \delta)$, and in this case we say that the programs are related by an $(\epsilon, \delta)$-approximate $\phi$-coupling. This could be notated as:

$$
\{\psi\} C_{1} \sim C_{2}\{\phi\}_{(\epsilon, \delta)}
$$

Existence of couplings for particular choices of $\phi$ imply a certain relation between the programs, such as equivalence, stochastic dominance or differential privacy. Couplings are also examples of monadic liftings, so they satisfy good compositionality properties, which allow us to have a sequence rule:

$$
\frac{\{\phi\} C_{1} \sim C_{2}\left\{\phi^{\prime}\right\}_{(\epsilon, \delta)} \quad\left\{\phi^{\prime}\right\} C_{1}^{\prime} \sim C_{2}^{\prime}\left\{\left\{\phi^{\prime \prime}\right\}_{\left(\epsilon^{\prime}, \delta\right)}\right.}{\{\phi\} C_{1} ; C_{1}^{\prime} \sim C_{2} ; C_{2}^{\prime}\left\{\phi^{\prime \prime}\right\}_{\left(\epsilon+\epsilon^{\prime}, \delta+\delta^{\prime}\right)}}
$$

We adapt these techniques to our higher-order setting to build sound relational logics for functional probabilistic programs.

\section{Adversarial properties}

Some program specifications, specially in fields such as security, do not only specify how a program behaves with respect to its inputs, but also how the program behaves with respect to its environment. Such is the case of pseudorandom functions, for which it can be proven that any computation with bound access to them (also known 
as an adversary) cannot distinguish them from a pseudorandom permutation except with negligible probability. It is natural to express these properties in a higherorder setting, since adversaries are functions which receive functions as arguments. Informally, this property would be stated as

$$
\forall \mathcal{A} .\{T\} \mathcal{A}(p r f) \sim \mathcal{A}(p r p)\left\{\mathbf{r}_{1}=\mathbf{r}_{2}\right\}_{\delta}
$$

Here $\mathcal{A}$ is a universally quantified higher-order term that receives a function as an argument and returns a result that we assume to be a boolean. The judgment states that no matter what $\mathcal{A}$ is, when we run it first with a pseudorandom function, and then a psueudorandom permutation, the result is the same with probability $1-\delta$.

The logic presented so far is not powerful enough to prove these properties, since the code of the adversary is not known, only its type. Proving these properties requires first an extension at type level, adding a graded state monad to restrict the capabilities of adversaries, and second new reasoning principles that exploit this additional typing information to prove properties of all inhabitants of a given type. The semantical model is also accordingly extended by generalizing the notion of liftings to state transformed monads.

\section{Markov chains}

Markov chains are ubiquitous in the analysis of probabilistic programs. Many properties regarding Markov chains are relational, such as convergence or probabilistic bisimulation. However, Markov chains (or more concretely, runs of Markov chains) are infinite objects, and cannot be modeled in our calculus.

The guarded lambda calculus (GLC) is an extension of the lambda calculus with a "later" modality and a notion of guarded recursion that can model infinite streams as a pair of an element that is given now and a stream that is given later. It has been used for instance in the analysis of reactive systems, since typing guarantees productivity. The GLC comes equipped with a logic, known as Guarded HOL (GHOL) to reason about such programs.

We start by extending the GLC with sampling instructions, and by noting that modeling probabilities over streams is very natural, since they can be modeled as a stream of probabilities over finite prefixes. We then develop a relational logic over GHOL, and coupling-based logical rules to reason about Markov chains.

\section{Predicate transformers and expected sensitivity}

A different family of techniques to prove properties of (first-order) probabilistic programs is based on predicate transformers. Analogously to Dijkstra weakest precondition transformer [71], Morgan and McIver's weakest pre-expectation transformer [121] takes as argument a program and an expectation $\mathcal{E}$ (i.e. a function from states to the non-negative reals) and return another expectation wpe $(\mathrm{c}, \varepsilon)$. We 
can prove that given an initial state $m$, the weakest pre-expectation gives us the expected value of $\mathcal{E}$ over the final distribution over states induced by $c(m)$, i.e., wpe $(\mathrm{c}, \varepsilon)(\mathrm{m})=\mathbb{E}_{\mathrm{c}(\mathrm{m})}[\varepsilon]$.

Expected sensitivity is a relational property of probabilistic programs that measures how the outputs of a program change with respect to changes in the inputs. In this thesis we will study how to develop a relational predicate transformer that can be used to prove expected sensitivity, among other properties. To do so, we use the Kantorovich metric, a canonical way of building a distance between probability distributions when the base spaces are metric spaces. The Kantorovich metric is known to over-approximate other metrics such as the statistical distance, and we will show that the Kantorovich metric itself can be over-approximated by a predicate transformer.

\section{Thesis contributions}

- We present a new logic, called Relational Higher Order Logic (RHOL, Section 3.5), for reasoning about relational properties of higher-order programs written in a variant of Plotkin's PCF (Section 3.1). The logic manipulates judgments of the form:

$$
\Gamma \mid \Psi \vdash \mathrm{t}_{1}: \sigma_{1} \sim \mathrm{t}_{2}: \sigma_{2}\{\phi\}
$$

where $\Gamma$ is a simply typed context, $\sigma_{1}$ and $\sigma_{2}$ are (possibly different) simple types, $t_{1}$ and $t_{2}$ are terms, $\Psi$ is a set of assertions, and $\phi$ is an assertion. The intended interpretation of such judgments is that assuming the assertions in $\Psi, t_{1}$ and $t_{2}$ are a pair of well-typed programs that satisfy the relation $\phi$. This logic combines two-sided rules, that reason simultaneously on a pair of terms that have the same top-level structure and one-sided rules, that operate only on either the left- or the right-hand terms. One-sided rules discharge proof obligations by using Unary Higher-Order Logic (UHOL), a program logic to prove unary properties of a single program.

- We demonstrate that RHOL can be used as a general framework, by defining sound embedding for several relational type systems: relational refinement types (Section 3.6.2), the Dependency Core Calculus (DCC) for many dependency analyses, including those for information flow security (Section 3.6.3), and the RelCost (Section 3.6.4) type system for relative cost.

- We extend our system to the probabilistic setting. We present a unary liftingbased logic HO-UBL that has judgments of the form

$$
\Gamma \mid \Psi \vdash \mathrm{t}: \mathcal{P} \sigma\{\mathrm{Q}\} \delta,
$$


with the intended interpretation that, under the assumptions in $\Gamma, t$ is a computation that satisfies $Q$ at least with probability $1-\delta$. This logic uses $\mathrm{UHOL}$ to prove premises of non-monadic programs.

- We present a relational lifting-based logic HO-RPL, that has judgments of the form

$$
\Gamma \mid \Psi \vdash \mathrm{t}_{1}: \mathcal{P} \sigma_{1} \sim \mathrm{t}_{2}: \mathcal{P} \sigma_{2}\left\{\{\mathrm{Q}\}_{(\epsilon, \delta)},\right.
$$

with the intended interpretation that, under the assumptions in $\Gamma, t_{1}$ and $t_{2}$ are computations related by an $(\epsilon, \delta)$-approximate $\mathrm{Q}-$ coupling.

- We extend our probabilistic language with a graded state monad that keeps tracks of the memory usage, as well as of the number of times that arguments are used. We use these types to develop reasoning principles to perform adversarial reasoning in the unary and in the relational setting and we add rules based on these principles to our logics.

- We apply our ideas to the Guarded Lambda Calculus and the Guarded HOL to develop a relational logic built on top of GHOL (GRHOL). We then extend the GLC with probabilities and add coupling-based reasoning rules to GHOL, and use them to prove properties of Markov chains.

- We present assertion-based unary and relational logics to reason about probabilistic programs in a general manner. This is not limited to proving liftingbased properties, but requires an axiomatization of probability theory in the base logic.

- We develop a predicate transformer that computes an over approximation of the Kantorovich distance between two executions of a probabilistic program. This can be seen as a relational version of the pre-expectation transformer by [121].

\section{Structure of the thesis}

The thesis is divided in three parts. The goal of the first part is to build a system to prove lifting-based relational properties of higher-order programs with continuous probabilities. We begin in Chapter 3 by presenting our base systems to reason about pure functional programs. This includes a base logic (HOL), a unary logic (UHOL) and a relational logic (RHOL). We then investigate probabilistic extensions. Chapter 4 presents a unary, union bound-based logic, while Chapter 5 presents a relational coupling-based logic. Chapter 4 also introduces most of the theoretical material behind our semantical models. Then, Chapters 6 and 7 extend on this by studying rules to prove adversarial properties. 
The second part of the thesis explores other topics related to relational properties and probabilities. Chapter 8 presenting rules to reason about discrete Markov chains. Chapter 9 presents an assertion-based relational logic to reason about probabilistic programs with conditioning. Chapter 10 presents a predicate transformer logic to reason about expected sensitivity of first-order programs.

Finally, the third part of the thesis presents the future work and the conclusions (Chapter 11). 


\section{RELATED WORK}

We review here some of the literature that is related to the general topics of this dissertation, and after some chapters we will present specific related work.

\section{Reasoning about higher-order programs}

The prevailing approach to reasoning about pure higher-order programs is dependent type theory, and systems such as Coq or Agda. However, several authors have explored another approach, which is crisply summarized by Jacobs [99]: "A logic is always a logic over a type theory". This alternative approach proceeds in two stages. First they introduce a typed language, and second they present a logic to reason about programs in the language. This is the approach followed by logic-enriched type theories $[3,4,44,5]$, and refinement types $[125,165,149]$, and it is also the most related to our approach. However, these works do not consider verification of relational properties.

\section{Relational reasoning for first-order programs}

Several approaches to relational reasoning in the imperative setting, including first-order probabilistic programming, have been developed in the past 15 years. We first review logics specifically designed for relational verification. Relational Hoare Logic [45] and Relational Separation Logic [164] are two such program logics, respectively based on Hoare Logic and Separation Logic, for reasoning about relational properties of (first-order) imperative programs. These logics have been used for a broad range of examples and applications, ranging from program equivalence to compiler verification and information flow analysis. Moreover, they have been extended in several directions. The line of work starting Probabilistic Relational Hoare Logic [23] and followed by approximate probabilistic Relational Hoare Logic [26] generalize Relational Hoare Logic to the probabilistic setting, 
and they have been used for applications such as probabilistic information flow, side-channel security, proofs of cryptographic strength (reductionist security) and differential privacy. Cartesian Hoare Logic [143] is also a recent generalization of Relational Hoare Logic for reasoning about bounded safety (i.e. k-safety for arbitrary but fixed k) properties of (first-order) imperative programs. This logic has been used for analyzing standard libraries. Experiments have demonstrated that such logics can be very effective in practice. Our formalism can be seen as a proposal to adapt their flexibility to pure higher-order programs.

An alternative approach is to use existing logics and instead apply transformations to the program to enable the verification of relational properties. Product programs $[21,25]$ are a general class of constructions that emulate the behavior of two programs and can be used for reducing relational verification to standard verification. While product programs naturally achieve (relative) completeness, they are often difficult to use since they require global reasoning on the obtained program. Nonetheless, there is some success in automating this approach in some settings [52]. Building product programs for (pure) higher-order languages is an intriguing possibility, and it might be possible to instrument RHOL using ideas from [40] to this effect; however, the product programs constructed in [40] appear as witnesses of a proof in a relational logic, but are not themselves used as a means of relational verification.

\section{Relational reasoning for higher-order programs}

Several type systems have been designed to support formal reasoning about relational properties for functional programs. Some of the earlier works in this direction have focused on the semantics foundations of parametricity, like the work by Abadi et al. [1] on System R, a relational version of System F. The recent work by Ghani et al. [83] has further extended this approach to give better foundations to a combination of relational parametricity and impredicative polymorphism. Interestingly, similarly to RHOL, System R also supports relations between expressions at different types, although, since System R does not support refinement types, the only relations that System R can support are the parametric ones on polymorphic terms. In RHOL, we do not support parametric polymorphism à la System F currently but the relations that we support are more general.

Some type systems have been developed to reason about specific relational properties. Such is the case of SLAM [92], Flow Caml [132] and DCC [2], which reason about information flow security. Most of these type systems consider a single expression but add information flow labels to relate different executions of the expression. In this thesis we translate DCC into RHOL, showing that this approach can also be implemented in our system.

Relational Hoare Type Theory (RHTT) [122, 146] is a formalism for relational reasoning about stateful higher-order programs. RHTT was designed to verify 
security properties like authorization and information flow policies but has been used for the verification of heterogeneous pointer data structures as well. RHTT uses a monad to separate stateful computations and relational refinements on the monadic type express relational pre- and post-conditions. RHTT supports reasoning about two different programs but the programs must have the same types at the top-level. RHTT's rules support both two- and one-sided reasoning similar to RHOL, but the focus of RHTT is on verifying properties of the program state. In particular, examples such as those in $\$ 3.7$ or embeddings such as those in $\$ 3.6$ were not considered in RHTT. RHTT is proved sound over a domain-theoretic model and continuity must be proven explicitly during the verification of recursive functions (rules are provided to prove continuity in many cases). In contrast, RHOL's settheoretic model is simpler, but admits only those recursive functions that have a unique interpretation in set-theory. RHTT does not consider the verification of probabilistic properties either.

We have already mentioned the works on relational refinement type systems for verifying cryptographic constructions [29], for differential privacy [33, 78] and for relational cost analysis [61]. This line of works is probably the most related to our work. However, RHOL improves over all of them, as also shown by some of the embeddings we give in Section 3.6. Another work in this direction is that of [10], which proposes a technique to reduce relational refinement to standard first-order refinements. Their technique is incomplete but it works well on some concrete examples. We believe that some technique of this kind can also be applied to RHOL. However, this is orthogonal to our current goal and we leave this investigation to future work.

In a recent paper, Grimm et al. [88] propose an alternative manner of proving relational properties of monadic computations in $\mathrm{F}^{\star}$. Intrinsic specification of monadic computations in this language needs to be unary, however relational properties can be proven extrinsically by using a reification operator. This operator exposes the computational content of the terms and turns them into pure expressions, about which logical lemmas can be written and proven, exploiting the automation features of $\mathrm{F}^{\star}$. The idea of exposing the pure representation of a computation generalizes the ideas behind our embeddings of DCC and RelCost. However, this work offers no specific support for relational reasoning and would correspond in our setting to embedding these frameworks directly into HOL rather that into RHOL.

Maillard et al. [115] define a framework, embedded in a relational dependent type theory, for defining and reasoning about program logics for general monadic effects. Their approach works for reasoning about discrete probabilities but it cannot handle higher order probabilistic programs. Their framework does not reason about adversaries either.

Several works have explored how to automate the verification of higher order programs by means of techniques inspired by model checking and Horn clause solving. Some of the recent works in this direction $[107,108]$ have focused on the 
use of Higher-order modal Fixed point Logics (HFL). In this approach, a program is translated to an HFL formula expressing the correctness of the program with respect to a particular property, and the verification is performed via HFL model checking. This approach is able to express several properties of higher order programs in a uniform way. The verification technique based on HFL is quite different from the one we present here but both techniques serve similar purposes. Properties that have been targeted using HFL include reachability, and trace properties, which are all non-relational. Instead, we focused on relational properties including information flow, relational cost, and program equivalence. Nevertheless, it would be interesting to investigate whether HFL model checking could be used also to support the automated verification of relational properties. Program verification based on Horn clauses has been also used for the verification of relational properties of higher order programs [153]. Their technique focuses on a principled way to encode relational properties in Horn clauses. In our approach instead, the relational nature of the properties is used in the corresponding logic RHOL and its combined use with UHOL and HOL.

\section{Logical relations}

Logical relations $[128,144,150]$ provide a fundamental tool for reasoning about programs. They have been used for a broad range of purposes, including proving unary properties (for instance strong normalization or complexity) and relational properties (for instance equivalence or information flow security). Our work can be understood as an attempt to internalize the versatility of relational logical relations in a syntactic framework. There is a large body of work on logics for logical relations, from the early work by Plotkin \& Abadi [130] to more recent work on logics for reasoning about states and concurrency $[73,72,103,111]$. In particular, the IRIS logic [103] can be seen as a powerful reasoning framework for logical relations, as shown by Krogh-Jespersen et al. [111]. Even though we also aim to internalize logical relations, our goal differs from the goal of IRIS in that we aim for syntax-driven relational verification, as opposed to IRIS.

\section{Reasoning about probabilistic programs}

A series of work develops reasoning methods for probabilistic higher-order programs for different variations of the lambda calculus. One line of work has focused on operationally-based techniques for reasoning about contextual equivalence of programs. The methods are based on probabilistic bisimulations [66] or on logical relations [50]. Most of these approaches have been developed for languages with discrete distributions, but recently there has also been work on languages with continuous distributions $[55,67]$. Another line of work has focused on denotational models, starting with the seminal work in [101]. Recent work includes support for 
relational reasoning about equivalence of programs with continuous distributions for a total programming language [145].

Closer to our work are relational refinement type systems for higher-order probabilistic programs. These were initially considered by Barthe et al. [29] for a stateful fragment of $\mathrm{F}^{*}$, and later by Barthe et al. $[33,34]$ for a pure language. Both systems are based on probabilistic couplings and the latter support approximate probabilistic couplings, which yield a natural interpretation of differential privacy [75], both in its pure and approximate forms (i.e. $\epsilon$ - and $(\epsilon, \delta)$-privacy). These systems do not support however reasoning about programs with different types or syntactic structures.

Kozen [109] introduces expectation-based reasoning for a core probabilistic programming language. Morgan, McIver and Seidel [121] define a weakest preexpectation calculus for an imperative language with both probabilistic and nondeterministic choice. These approaches support verification of arbitrary properties, but Kaminski et al [104] show how similar ideas can be used for reasoning about expected cost. All these works are cast in the setting of a core probabilistic imperative language. The author of this dissertation and Katsumata [6] show that expectationbased reasoning remains sound in a higher-order setting, but their semantics is set-theoretical (rather than based on Quasi-Borel spaces) and they do not provide proof systems or calculi.

There exist adaptations of (approximate) probabilistic relational Hoare logic in the higher-order setting $[29,33,34]$. However, these adaptations have a settheoretical (or in the topos of trees) semantics and only support distributions over discrete base types. 


\section{Part I}

\section{Relational probabilistic properties of higher-order programs}




\section{PURE RELATIONAL HIGHER-ORDER LOGIC}

We present a new logic, called Relational Higher Order Logic, for reasoning about relational properties of higher-order programs written in a variant of Plotkin's PCF. The logic manipulates judgments of the form:

$$
\Gamma \mid \Psi \vdash \mathrm{t}_{1}: \sigma_{1} \sim \mathrm{t}_{2}: \sigma_{2}\{\phi\}
$$

where $\Gamma$ is a simply typed context, $\sigma_{1}$ and $\sigma_{2}$ are (possibly different) simple types, $t_{1}$ and $t_{2}$ are terms, $\Psi$ is a set of assertions, and $\phi$ is an assertion. The set-theoretic interpretation of this judgment is that for every valuation $\rho$ (mapping variables in the context $\Gamma$ to elements in the interpretation of their types), the interpretation of $\phi$ is true whenever the interpretation of (all the assertions in) $\Psi$ is true.

In contrast with relational refinement type systems, our logic disentangles logical information from typing information. This allows us to have one-sided rules to reason about structurally different programs, and at the same time, retain the natural synchronous reasoning of these systems for programs that have the same top-level construction. In a derivation, one- and two-sided rules can be alternated freely, thus giving great expressivity to our loigic.

The system presented in this chapter is also the basis for the extensions that we will present in the ensuing chapters.

Structure of the chapter We begin by presenting our language (Section 3.1) and a motivating example of relational verification (Section 3.2). Then, we present our logics in three steps: the base logic (Section 3.3), a unary logic (Section 3.4) and a relational logic (Section 3.5), and we discuss their metatheoretical properties. Afterwards, we show how to embed several relational verification systems from the literature into our framework (Section 3.6). Finally, we verify several examples, some of which go beyond the capabilities of previous systems (Section 3.7). 


\section{1 (A variant of) PCF}

The first and simplest language we consider in this thesis is a variant of PCF [129] that has as basic types booleans, natural numbers, and lists, and allows recursive definitions over the latter two. For this, we require that all recursive calls are performed on strictly smaller elements-as a consequence, the fixpoint equation derived from the definition has a unique set-theoretical solution. The method to enforce this requirement is orthogonal to our design, and could for instance be based on a syntactic guard predicate, or on sized types [22].

Types and terms of the language are defined by the following grammar:

$$
\begin{aligned}
& \tau::=\text { bool }_{\text {nat }} \mid \text { list }_{\tau}|\tau \times \tau| \tau \rightarrow \tau \\
& \mathrm{t}::=\mathrm{x}|\langle\mathrm{t}, \mathrm{t}\rangle| \pi_{1} \mathrm{t}\left|\pi_{2} \mathrm{t}\right| \mathrm{t} \mathrm{t} \mid \lambda \mathrm{x}: \tau \text {.t }|\mathrm{c}| \mathrm{S} \mathrm{t}|\mathrm{t}:: \mathrm{t}| \text { case } \mathrm{t} \text { of } 0 \mapsto \mathrm{t} ; \mathrm{S} \mapsto \mathrm{t} \\
& \quad \text { case } \mathrm{t} \text { of } \mathrm{tt} \mapsto \mathrm{t} ; \mathrm{ff} \mapsto \mathrm{t} \mid \text { case } \mathrm{t} \text { of } \square \mapsto \mathrm{t} ;_{-}::_{-} \mapsto \mathrm{t} \mid \text { letrec } f x=\mathrm{t}
\end{aligned}
$$

where $x$ ranges over a set $\mathrm{V}$ of variables, $\mathrm{c}$ ranges over the set $\{\mathrm{tt}, \mathrm{ff}, \mathrm{O}, \square\}$ of constants, and $\lambda$-abstractions are $\grave{a} l a$ Church. Throughout the chapter we will use letters $t, u, v$ to range over terms, and letters $x, y, z$ to range over variables. Occasionally, we will also use $f, g$ for variables with arrow types.

The operational behavior of terms is captured by $\beta \iota \mu$-reduction $\rightarrow_{\beta \iota \mu}=\rightarrow_{\beta} \cup \rightarrow_{\iota}$ $\cup \rightarrow_{\mu}$, where $\beta$-reduction, t-reduction and $\mu$-reduction are defined as the contextual closure of:

$$
\begin{aligned}
& (\lambda x . t) u \rightarrow \beta t[u / x] \\
& \pi_{i}\left\langle t_{1}, t_{2}\right\rangle \rightarrow_{\beta} t_{i} \\
& \text { case } 0 \text { of } 0 \mapsto u ; S \mapsto v \rightarrow \mathfrak{u} \\
& \text { case St of } 0 \mapsto u ; S \mapsto v \rightarrow_{\iota}(v t) \\
& \text { case tt of } \mathrm{tt} \mapsto \mathrm{u} \text {;ff } \mapsto v \rightarrow_{\mathfrak{}} \mathrm{u} \\
& \text { case ff of tt } \mapsto u \text {; ff } \mapsto v \rightarrow_{\iota} v \\
& \text { case [ of [ } \mapsto \mathrm{u}_{-}:_{-} \mapsto v \rightarrow_{\mathrm{\imath}} \mathrm{u} \\
& \text { case } h:: t \text { of } \square \mapsto u ;_{-}::_{-} \mapsto v \rightarrow_{\iota}(v h t) \\
& (\text { letrec } f x=t)(C \vec{t}) \rightarrow_{\mu} t[C \vec{t} / x][\text { letrec } f x=t / f]
\end{aligned}
$$

where $t[u / x]$ denotes the usual (capture-free) notion of substitution on terms (replace $x$ by $u$ in $t$ ). As usual, we let $=_{\beta \mu}$ denote the reflexive, symmetric, and transitive closure of $\rightarrow_{\beta \mu}$. In particular, we only allow reduction of letrec when the argument has a constructor $C \in\{\mathrm{tt}, \mathrm{ff}, \mathrm{O}, \mathrm{S}, \mathrm{Z},::\}\}$ in head position.

Typing judgments are of the form $\Gamma \vdash t: \tau$, where $\Gamma$ is a set of typing declarations of the form $x: \sigma$, such that each variable is declared at most once. The typing rules are standard, except for the one for recursive functions. In this rule, we require that the domain of the recursive function be an inductive type (naturals or lists here) and that the body of the recursive definition letrec $f x=t$ satisfy a predicate $\operatorname{Def}(f, x, t)$ 
which ensures that all recursive calls are performed on smaller arguments. The typing rule for recursive definitions is thus:

$$
\frac{\Gamma, f: I \rightarrow \sigma, x: I \vdash e: \sigma \quad \mathcal{D e f}(f, x, t) \quad I \in\left\{\text { nat } \text { list }_{\tau}\right\}}{\Gamma \vdash \text { letrec } f x=t: I \rightarrow \sigma}
$$

We give set-theoretical semantics to this system. The choice of this model is motivated by simplicity, but our construction would still work in a different model. We will see later in this thesis how to interpret it in a general Cartesian closed category.

For each type $\tau$, its interpretation $\llbracket \tau \rrbracket$ is the set of its values:

$$
\llbracket \text { bool } \rrbracket \triangleq\{\mathbf{t t}, \mathbf{f f}\} \quad \llbracket \text { nat } \rrbracket \triangleq \mathbb{N} \quad \llbracket \text { list }_{\tau} \rrbracket \triangleq \text { list }_{\llbracket \tau \rrbracket} \quad \llbracket \sigma \rightarrow \tau \rrbracket \triangleq \llbracket \sigma \rrbracket \rightarrow \llbracket \tau \rrbracket
$$

where $\llbracket \sigma \rrbracket \rightarrow \llbracket \tau \rrbracket$ is the set of total functions with domain $\llbracket \sigma \rrbracket$ and codomain $\llbracket \tau \rrbracket$.

A valuation $\rho$ for a context $\Gamma$ (written $\rho \models \Gamma$ ) is a partial map such that $\rho(x) \in \llbracket \tau \rrbracket$ whenever $(x: \tau) \in \Gamma$. For every valuation $\rho$ let $\rho[v / x]$ denote its unique extension $\rho^{\prime}$ such that $\rho^{\prime}(y)=v$ if $x=y$ and $\rho^{\prime}(y)=\rho(y)$ otherwise. Given a valuation $\rho$ for a context $\Gamma$, every term $t$ for which a typing judgment $\Gamma \vdash t: \tau$ can be derived, has an interpretation $(t)_{\rho}$ :

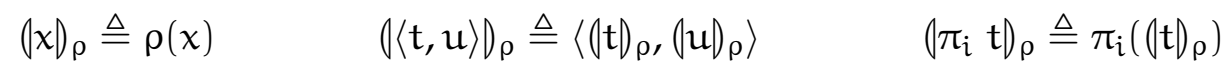

$$
\begin{aligned}
& (\lambda x: \tau \cdot \mathrm{t})_{\rho} \triangleq \lambda v: \llbracket \tau \rrbracket \cdot(\mathrm{t})_{\rho[(\nu) \rho / x]} \quad(\mathrm{c})_{\rho} \triangleq \mathrm{c} \quad(\mathrm{S} \mathrm{t})_{\rho} \triangleq \mathrm{S}(\mathrm{t})_{\rho}
\end{aligned}
$$

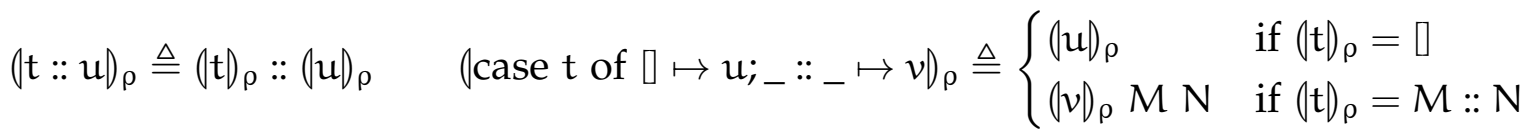

$$
\begin{aligned}
& \text { (letrec } f x=\mathrm{t})_{\rho} \triangleq \mathrm{F}
\end{aligned}
$$

In the case of letrec $f x=t$, we require that $\mathrm{F}$ be the unique solution of the fixpoint equation extracted from the recursive definition-existence and uniqueness of the solution follows from the validity of the $\operatorname{Def}(f, x, t)$ predicate.

The interpretation of well-typed terms is sound. Moreover, the interpretation equates convertible terms. (This extends to $\eta$-conversion.)

Theorem 3.1 (Soundness of set-theoretic semantics). The following hold:

- If $\Gamma \vdash \mathrm{t}: \tau$ and $\rho \models \Gamma$, then $(\mathrm{t})_{\rho} \in \llbracket \tau \rrbracket$.

- If $\Gamma \vdash \mathrm{t}: \tau$ and $\Gamma \vdash \mathrm{u}: \tau$ and $\mathrm{t}={ }_{\beta \iota \mu} \mathrm{u}$ and $\rho \models \Gamma$, then $(\mathrm{t})_{\rho}=(\mathrm{u})_{\rho}$. 


\subsection{Introductory example}

In this section we show a motivating example for our logic. We use an informal presentation style here. Later, in Section 3.7, we revisit the example and prove it formally. Consider the following pair of programs corresponding to two implementations of the factorial function:

$$
\begin{aligned}
& \text { fact }_{1} \triangleq \text { letrec } f_{1} n_{1}=\text { case } n_{1} \text { of } 0 \mapsto 1 ; S \mapsto \lambda x_{1} \cdot\left(S x_{1}\right) *\left(f_{1} x_{1}\right) \\
& \text { fact }_{2} \triangleq \text { letrec } f_{2} n_{2}=\lambda \text { acc.case } n_{2} \text { of } 0 \mapsto \operatorname{acc} ; S \mapsto \lambda x_{2} \cdot f_{2} x_{2}\left(\left(S x_{2}\right) * \text { acc }\right)
\end{aligned}
$$

We want to show a relation between the functions, namely that for any natural $n$, and any initial value $a$ of the accumulator, $\mathrm{a} *\left(\right.$ fact $\left._{1} \mathrm{n}\right)=$ fact $_{2} \mathrm{n}$ a. To avoid having to name our programs, we assume our logic has two distinguished variables $\mathbf{r}_{1}, \mathbf{r}_{2}$, that represent the pair of programs we reason about. Then, the relation we want to show is

$$
\forall \text { n. } \forall \text { a. } a *\left(\mathbf{r}_{1} \mathrm{n}\right)=\mathbf{r}_{2} \mathrm{na}
$$

This relation is not expressible in a relational refinement type system, since the two programs have different types. But they still have similar structure, and we wish to exploit this to find a proof. First we notice that both programs are recursive functions, so we apply a rule for this case, LETREC. This gives us the inductive hypothesis that the relation above holds for natural numbers strictly smaller than $n$, and the proof obligation that, for the following two programs

$$
\begin{aligned}
\text { case } n \text { of } 0 & \mapsto 1 ; S \mapsto \lambda x .(S x) *\left(f_{1} x\right) \\
\lambda \text { acc.case } n \text { of } 0 \mapsto a c c ; S & \mapsto \lambda x . f_{2} \times((S x) * a c c)
\end{aligned}
$$

the result of the first one, times $a$, is equal to the result calling the second one with argument $a$, that is,

$$
\forall \text { a. } \mathbf{a} * \mathbf{r}_{1}=\mathbf{r}_{2} a
$$

But now we have a pair of programs that have not only different types, but also different top-level term former: the first one has a case-analysis, while the second one has an abstraction. We now apply a one-sided rule ABS-R that takes care of the abstraction on the right and ignores the term on the left, leaving a pair of programs

$$
\begin{array}{r}
\text { case } n \text { of } 0 \mapsto 1 ; S \mapsto \lambda x .(S x) *\left(f_{1} x\right) \\
\text { case } n \text { of } 0 \mapsto a c c ; S \mapsto \lambda x . f_{2} \times((S x) * a)
\end{array}
$$

and a proof obligation

$$
\mathrm{a} * \mathbf{r}_{1}=\mathbf{r}_{2}
$$

Finally we have two programs with the same type and the same structure. We can do synchronous reasoning, using the fact that both programs need to take the same branch. In the 0 branch, its trivial to check that $a * 1=a$. In the successor branch we can instantiate the inductive hypothesis, since the bound variable $x$ is the predecessor of $n$, and thus strictly smaller. This concludes the proof. 


\subsection{Higher-Order Logic}

Higher-Order Logic is a predicate logic over simply-typed, higher-order terms. Its rules are written in the style of natural deduction. More specifically, its assertions are formulas over typed terms, and are defined by the following grammar:

$$
\phi::=\mathrm{P}\left(\mathrm{t}_{1}, \ldots, \mathrm{t}_{\mathrm{n}}\right)|\top| \perp|\phi \wedge \phi| \phi \vee \phi|\phi \Rightarrow \phi| \forall x: \tau . \phi \mid \exists x: \tau . \phi
$$

where $\mathrm{P}$ ranges over basic predicates, such as $=, \leqslant$, or sorted(l). As usual, we will often omit the types of bound variables, when they are clear from the context. We assume that predicates come equipped with an axiomatization. For instance, the predicate All $(l, \lambda x . \phi)$ is defined to capture lists whose elements $e$ all satisfy $\phi[e / x]$. This can be defined axiomatically for a given $\phi$ with a free variable $x$ (which we denote with and overloaded $\lambda$ symbol):

$$
\operatorname{All}(\square, \lambda x . \phi) \quad \forall h t . A l l(t, \lambda x . \phi) \Rightarrow \phi[h / x] \Rightarrow \operatorname{All}(h:: t, \lambda x . \phi)
$$

The notation $\lambda x . \phi$, used for simplicity, can be made formal by introducing a type for propositions-adding such a type is straightforward and orthogonal to our work; another alternative would be to use axiom schemes.

We define well-typed assertions using a judgment of the form $\Gamma \vdash \phi$. This judgment has the expected, straightforward rules. A HOL (inference) judgment is then of the form $\Gamma \mid \Psi \vdash \phi$, where $\Gamma$ is a simply typed context, $\Psi$ is a set of assertions, and $\phi$ is an assertion, and such that $\Gamma \vdash \psi$ for every $\psi \in \Psi$, and $\Gamma \vdash \phi$. The rules of this judgment are given in Figure 3.1, where the notation $\phi[t / x]$ denotes the (capture-free) substitution of $x$ by $t$ in $\phi$. In addition to the usual rules for equality, implication and universal quantification, there are rules for inductive types (only the rules for lists are shown; similar rules exist for booleans and natural numbers): the rule LIST models the induction principle for lists; the rules NC and CONS formalize injectivity and non-overlap of constructors. A rule for strong induction SLIST can be considered as well, and is in fact derivable from simple induction.

Higher-Order Logic inherits a set-theoretical interpretation from its underlying simply-typed $\lambda$-calculus. We assume given for each predicate $P$ of arity $\tau_{1} \times \cdots \times \tau_{n}$ an interpretation $\llbracket P \rrbracket \subseteq \llbracket \tau_{1} \rrbracket \times \cdots \times \llbracket \tau_{n} \rrbracket$ which is compatible with the axioms of $P$. The interpretation of assertions is then defined in the usual way. Specifically, an assertion in context $\Gamma \vdash \phi$ is interpreted as a subset $\llbracket \Gamma \vdash \phi \rrbracket \subseteq \llbracket \Gamma \rrbracket$. The semantics is defined inductively on the structure of $\phi$ :

$$
\begin{aligned}
& \llbracket \Gamma \vdash \mathrm{P}\left(\mathrm{t}_{1}, \ldots, \mathrm{t}_{\mathrm{n}}\right) \rrbracket \triangleq\left\{\gamma \in \llbracket \Gamma \rrbracket \mid\left(\llbracket \mathrm{t}_{1} \rrbracket \rrbracket_{\gamma}, \ldots, \llbracket \mathrm{t}_{\mathfrak{n}} \rrbracket_{\gamma}\right) \in \llbracket \mathrm{P} \rrbracket\right\} \\
& \llbracket \Gamma \vdash \top \rrbracket \triangleq \llbracket \Gamma \rrbracket \quad \llbracket \Gamma \vdash \perp \rrbracket \triangleq \emptyset \\
& \llbracket \Gamma \vdash \phi_{1} \wedge \phi_{2} \rrbracket \triangleq \llbracket \Gamma \vdash \phi_{1} \rrbracket \cap \llbracket \Gamma \vdash \phi_{2} \rrbracket \quad \llbracket \Gamma \vdash \phi_{1} \vee \phi_{2} \rrbracket \triangleq \llbracket \Gamma \vdash \phi_{1} \rrbracket \cup \llbracket \Gamma \vdash \phi_{2} \rrbracket \\
& \llbracket \Gamma \vdash \phi_{1} \Rightarrow \phi_{2} \rrbracket \triangleq\left(\llbracket \Gamma \rrbracket \backslash \llbracket \Gamma \vdash \phi_{1} \rrbracket\right) \cup \llbracket \Gamma \vdash \phi_{2} \rrbracket \\
& \llbracket \Gamma \vdash \forall x: \tau . \phi \rrbracket \triangleq\{\gamma \in \llbracket \Gamma \rrbracket \mid \forall y \in \llbracket \tau \rrbracket \cdot(\gamma, y) \in \llbracket \Gamma, x: \tau \vdash \phi \rrbracket\}
\end{aligned}
$$




$$
\begin{aligned}
& \frac{\phi \in \Psi}{\Gamma \mid \Psi \vdash \phi} A X \quad \frac{\Gamma \vdash \mathrm{t}: \tau \quad \Gamma \vdash \mathrm{t}^{\prime}: \tau \quad \mathrm{t}=\beta_{\beta \mu} \mathrm{t}^{\prime}}{\Gamma \mid \Psi \vdash \mathrm{t}=\mathrm{t}^{\prime}} \text { CONV } \\
& \frac{\Gamma|\Psi \vdash \phi[\mathrm{t} / \mathrm{x}] \quad \Gamma| \Psi \vdash \mathrm{t}=\mathrm{u}}{\Gamma \mid \Psi \vdash \phi[\mathrm{u} / \mathrm{x}]} \text { SUBST } \quad \frac{\Gamma \mid \Psi, \psi \vdash \phi}{\Gamma \mid \Psi \vdash \psi \Rightarrow \phi} \Rightarrow \text { । } \\
& \frac{\Gamma|\Psi \vdash \psi \Rightarrow \phi \quad \Gamma| \Psi \vdash \psi}{\Gamma \mid \Psi \vdash \phi} \Rightarrow_{\mathrm{E}} \quad \frac{\Gamma, \mathrm{x}: \sigma \mid \Psi \vdash \phi}{\Gamma \mid \Psi \vdash \forall \mathrm{x}: \sigma . \phi} \forall_{\mathrm{I}} \quad \frac{\Gamma \mid \Psi \vdash \forall \mathrm{x}: \sigma . \phi \quad \Gamma \vdash \mathrm{t}: \sigma}{\Gamma \mid \Psi \vdash \phi[\mathrm{t} / \mathrm{x}]} \forall_{\mathrm{E}} \\
& \overline{\Gamma \mid \Psi \vdash T} T_{1} \quad \frac{\Gamma \mid \Psi \vdash \perp \quad \Gamma \vdash \phi}{\Gamma \mid \Psi \vdash \phi} \perp_{\mathrm{E}} \\
& \frac{\Gamma\left|\Psi \vdash \phi[0 / \mathrm{l}] \quad \Gamma, \mathrm{h}: \tau, \mathrm{t}: \operatorname{list}_{\tau}\right| \Psi, \phi \vdash \phi[\mathrm{h}:: \mathrm{t} / \mathrm{t}]}{\Gamma \mid \Psi \vdash \forall \mathrm{t}: \text { list }_{\sigma} . \phi} \text { LIST } \quad \frac{\Gamma \vdash \mathrm{h}:: \mathrm{t}: \text { list }_{\tau}}{\Gamma \mid \emptyset \vdash \square \neq \mathrm{h}:: \mathrm{t}} \mathrm{NC} \\
& \frac{\Gamma \mid \Psi \vdash \mathrm{t}_{1}:: \mathrm{t}_{2}=\mathrm{t}_{1}^{\prime}:: \mathrm{t}_{2}^{\prime}}{\Gamma \mid \Psi \vdash \mathrm{t}_{\mathrm{i}}=\mathrm{t}_{\mathrm{i}}^{\prime}} \text { CONS }_{\mathrm{i}} \quad \frac{\Gamma, \mathrm{t}: \text { list }_{\tau} \mid \Psi, \forall \mathrm{u}: \text { list }_{\tau} \cdot|\mathrm{u}|<|\mathrm{t}| \Rightarrow \phi[\mathrm{u} / \mathrm{t}] \vdash \phi}{\Gamma \mid \Psi \vdash \forall \mathrm{t}: \text { list }_{\tau} . \phi} \text { SLIST }
\end{aligned}
$$

Figure 3.1: Selected rules for HOL

Higher-order logic is sound with respect to this semantics.

Theorem 3.2 (Soundness of set-theoretical semantics). If $\Gamma \mid \Psi \vdash \phi$ is a valid HOL derivation, then $\llbracket \Gamma \vdash \Lambda_{\psi \in \Psi} \psi \rrbracket \subseteq \llbracket \Gamma \vdash \phi \rrbracket$.

In particular, $\mathrm{HOL}$ is consistent, i.e. there is no derivation of $\Gamma \mid \emptyset \vdash \perp$ for any $\Gamma$.

\subsection{Unary Higher-Order Logic}

As a stepping stone towards Relational Higher-Order Logic, we define Unary HigherOrder Logic (UHOL). UHOL retains the flavor of refinement types, but dissociates typing from assertions; judgments of UHOL are of the form:

$$
\Gamma \mid \Psi \vdash t: \tau\{\phi\}
$$

where a distinguished variable $\mathbf{r}$, which does not appear in $\Gamma$, may appear free in $\phi$ as a synonym of $t$. A judgment is well-formed if $\psi$ is a valid set of assertions in the context $\Gamma$, and $\phi$ is a valid assertion in the context $\Gamma, \mathbf{r}: \tau$. The intended interpretation of the judgment above is that $t$ has type $\tau$ under the context $\Gamma$, and that $\Gamma \mid \Psi \vdash \phi[\mathrm{t} / \mathbf{r}]$ is a valid HOL judgment. In this way, UHOL combines a typing and a logical judgment. But the key difference between HOL and UHOL is that the 
logical judgment is proven following the structure of $t$ rather than on the structure of $\phi$.

Not only is UHOL a program logic in its own right, but it is also a key component for deriving one-sided rules in RHOL (Section 3.5). When reasoning about two terms with different structures, RHOL relies on UHOL to express proof obligations that affect only one of the two terms.

Figure 3.2 presents selected typing rules. The ABS rule allows proving formulas that refer to $\lambda$-abstractions, expressing that if the argument satisfies a precondition $\phi^{\prime}$, then the result satisfies a postcondition $\phi$. The APP rule, dually, proves a condition $\phi$ on an application $t u$ provided that the argument $u$ satisfies the precondition $\phi^{\prime}$ of the function $t$. The motivation behind the substitution $[\mathbf{r} x / \mathrm{r}]$ in both rules is that the type of $\mathbf{r}$ changes from the premise to the conclusion, and when $\mathbf{r}$ is bound to an arrow type, it needs to appear on the left of an application construct to recover its original type.

The VAR rule introduces a variable from the context with a formula proven in HOL. Rules for constants (e.g. NIL) work in the same way. Rule CONS proves a formula $\phi$ for a non-empty list, provided that $\phi$ on the entire list is a logical consequence (in HOL) of some conditions $\phi^{\prime}, \phi^{\prime \prime}$ on its head and its tail, respectively. Rule PAIR allows the construction of judgments about pairs in a similar manner. The rules $\mathrm{PROJ}_{i}$ for $i=1,2$ establish judgments about the projections of a pair. The rule SUB (subsumption) allows strengthening the assumed assertions $\Psi$ and weakening the concluding assertion $\phi$. It generates a HOL proof obligation. The rule CASE can be used for a case analysis over the constructor of a term. Finally, the rule LETREC supports inductive reasoning about recursive function. Recall that the domain of a recursive definition is an inductive type, for which a natural notion of size exists. If, assuming that a proposition holds for all elements smaller than the argument, we can prove that the proposition holds for the body, then the proposition must hold for the function as well. Furthermore, we require that the function we are verifying satisfies the predicate $\operatorname{Def}(f, x, t)$, as was the case in HOL. The induction is performed over the $<$ order, which varies depending on the type of the argument.

We now discuss the main meta-theoretic results of UHOL. The following result establishes that every HOL judgment can be proven in UHOL and vice versa.

Theorem 3.3 (Equivalence with HOL). For every context $\Gamma$, simple type $\sigma$, term $t$, set of assertions $\Psi$ and assertion $\phi$, the following are equivalent:

- $\Gamma \mid \Psi \vdash t: \sigma\{\phi\}$

- $\Gamma \mid \Psi \vdash \phi[\mathrm{t} / \mathbf{r}]$

The forward implication follows by induction on the derivation of $\Gamma \mid \Psi \vdash t: \sigma\{\phi\}$. The reverse implication is immediate from the rule SUB and the observation that $\Gamma \mid \Psi \vdash t: \sigma\{T\}$ whenever $\mathrm{t}$ is a term of type $\sigma$. 


$$
\begin{aligned}
& \frac{\Gamma \vdash x: \sigma \quad \Gamma \mid \Psi \vdash \phi[x / \mathbf{r}]}{\Gamma \mid \Psi \vdash x: \sigma\{\phi\}} \text { VAR } \quad \frac{\Gamma, x: \tau \mid \Psi, \phi^{\prime} \vdash t: \sigma\{\phi\}}{\Gamma \mid \Psi \vdash \lambda x: \tau . t: \tau \rightarrow \sigma\left\{\forall x . \phi^{\prime} \Rightarrow \phi[\mathbf{r} x / \mathbf{r}]\right\}} \text { ABS } \\
& \frac{\Gamma\left|\Psi \vdash \mathrm{t}: \tau \rightarrow \sigma\left\{\forall x . \phi^{\prime}[\mathrm{x} / \mathbf{r}] \Rightarrow \phi[\mathbf{r} x / \mathbf{r}]\right\} \quad \Gamma\right| \Psi \vdash u: \tau\left\{\phi^{\prime}\right\}}{\Gamma \mid \Psi \vdash \mathrm{t} u: \sigma\{\phi[\mathrm{u} / \mathrm{x}]\}} \text { APP } \quad \frac{\Gamma \mid \Psi \vdash_{\mathrm{HOL}} \phi[0 / \mathbf{r}]}{\Gamma \mid \Psi \vdash]: \operatorname{list}_{\sigma}\{\phi\}} \text { NIL } \\
& \Gamma\left|\Psi \vdash \mathrm{h}: \sigma\left\{\phi^{\prime}\right\} \quad \Gamma\right| \Psi \vdash \mathrm{t}: \operatorname{list}_{\sigma}\left\{\phi^{\prime \prime}\right\} \\
& \frac{\Gamma \mid \Psi \vdash_{\mathrm{HOL}} \forall x \mathrm{y} \cdot \phi^{\prime}[\mathrm{x} / \mathbf{r}] \Rightarrow \phi^{\prime \prime}[\mathrm{y} / \mathbf{r}] \Rightarrow \phi[\mathrm{x}:: \mathrm{y} / \mathbf{r}]}{\Gamma \mid \Psi \vdash \mathrm{h}:: \mathrm{t}: \operatorname{list}_{\sigma}\{\phi\}} \text { CONS } \\
& \Gamma\left|\Psi \vdash t: \sigma\left\{\phi^{\prime}\right\} \quad \Gamma\right| \Psi \vdash u: \tau\left\{\phi^{\prime \prime}\right\} \\
& \frac{\Gamma \mid \Psi \vdash \mathrm{t}: \sigma \times \tau\left\{\phi\left[\pi_{\mathfrak{i}}(\mathbf{r}) / \mathbf{r}\right]\right\}}{\Gamma \mid \Psi \vdash \pi_{\mathrm{i}}(\mathrm{t}): \sigma\{\phi\}} \mathrm{PROJ}_{\mathrm{i}} \quad \frac{\Gamma \mid \Psi \vdash_{\mathrm{HOL}} \forall x \mathrm{y} . \phi^{\prime}[\mathrm{x} / \mathbf{r}] \Rightarrow \phi^{\prime \prime}[\mathrm{y} / \mathbf{r}] \Rightarrow \phi[\langle x, \mathrm{y}\rangle / \mathbf{r}]}{\Gamma \mid \Psi \vdash\langle\mathrm{t}, \mathrm{u}\rangle: \sigma \times \tau\{\phi\}} \text { PAIR } \\
& \frac{\Gamma\left|\Psi \vdash \mathrm{t}: \sigma\left\{\phi^{\prime}\right\} \quad \Gamma\right| \Psi \vdash_{\mathrm{HOL}} \phi^{\prime}[\mathrm{t} / \mathbf{r}] \Rightarrow \phi[\mathrm{t} / \mathbf{r}]}{\Gamma \mid \Psi \vdash \mathrm{t}: \sigma\{\phi\}} \text { SUB } \\
& \Gamma \vdash l: \text { list }_{\tau} \\
& \Gamma \mid \Psi, l=\square \vdash u: \sigma\{\phi\} \\
& \frac{\Gamma \mid \Psi \vdash v: \tau \rightarrow \operatorname{list}_{\tau} \rightarrow \sigma\{\forall h t . l=h:: t \Rightarrow \phi[\mathbf{r} h \mathrm{t} / \mathbf{r}]\}}{\Gamma \mid \Psi \vdash \text { case } l \text { of }\left[\mapsto \mathrm{u}_{-}::_{-} \mapsto v: \sigma\{\phi\}\right.} \text { LISTCASE } \\
& \operatorname{Def}(\mathrm{f}, \mathrm{x}, \mathrm{t}) \\
& \frac{\Gamma, x: \mathrm{I}, \mathrm{f}: \mathrm{I} \rightarrow \sigma\left|\Psi, \phi^{\prime}, \forall \mathrm{m} .\right| \mathrm{m}|<| x \mid \Rightarrow \phi^{\prime}[\mathrm{m} / \mathrm{x}] \Rightarrow \phi[\mathrm{m} / \mathrm{x}][\mathrm{f} \mathrm{m} / \mathbf{r}] \vdash e: \sigma\{\phi\}}{\Gamma \mid \Psi \vdash \text { letrec } \mathrm{f} x=\mathrm{t}: \mathrm{I} \rightarrow \sigma\left\{\forall x . \phi^{\prime} \Rightarrow \phi[\mathbf{r} \mathrm{x} / \mathbf{r}]\right\}} \text { LETREC } \\
& \text { where } I \in\left\{\text { nat, } \text { list }_{\tau}\right\}
\end{aligned}
$$

Figure 3.2: Unary Higher-Order Logic rules 
We lift the HOL semantics to UHOL. Terms, types and formulas are interpreted as before. The following corollary states the soundness of UHOL.

Corollary 3.4 (Set-theoretical soundness and consistency). Let $\mathrm{t}$ be a term and $\Gamma \mid \Psi \vdash$ $t: \sigma\{\phi\}$ a valid UHOL derivation. Then,

$$
\llbracket \Gamma \vdash \bigwedge_{\psi \in \Psi} \psi \rrbracket \subseteq \llbracket \Gamma \vdash \phi[\mathrm{t} / \mathbf{r}] \rrbracket .
$$

In particular, there is no proof of the judgment $\Gamma \mid \emptyset \vdash \mathrm{t}: \sigma\{\perp\}$ for any $\Gamma, \mathrm{t}$ and $\sigma$.

Next, we prove subject conversion for UHOL. This result follows immediately from Theorem 3.3 and subject conversion of $\mathrm{HOL}$, which is itself a direct consequence of the CONV and SUBST rules.

Corollary 3.5 (Subject conversion). Assume that $\mathrm{t}={ }_{\beta \iota \mu} \mathrm{t}^{\prime}$ and $\Gamma \mid \Psi \vdash \mathrm{t}: \sigma\{\phi\}$. Then $\Gamma \mid \Psi \vdash \mathrm{t}^{\prime}: \sigma\{\phi\}$.

\subsection{Relational Higher-Order Logic}

Relational Higher-Order Logic (RHOL) extends UHOL's separation of assertions and types to a relational setting. Formally, RHOL is a relational type system which manipulates judgments of the form

$$
\Gamma \mid \Psi \vdash \mathrm{t}_{1}: \tau_{1} \sim \mathrm{t}_{2}: \tau_{2}\{\phi\}
$$

which combine a typing judgment for a pair of PCF terms and permit reasoning about the relation between them. The judgment means that $t_{1}$ and $t_{2}$ respectively have types $\tau_{1}$ and $\tau_{2}$ in $\Gamma$ and that $t_{1}, t_{2}$ are related by the assertion $\phi$. Wellformedness of the judgment requires $\Psi$ to be a valid set of assertions in $\Gamma$ and $\phi$ to be a valid assertion in $\Gamma, \mathbf{r}_{1}: \tau_{1}, \mathbf{r}_{2}: \tau_{2}$, where the special variables $\mathbf{r}_{1}$ and $\mathbf{r}_{2}$ are used as synonyms for $t_{1}$ and $t_{2}$ in $\phi$.

\subsubsection{Proof Rules}

The type system combines two-sided rules (Figure 3.3), which apply when the two terms have the same top-level constructors and one-sided rules (Figure 3.5), which analyze either one of the two terms. For instance, the APP rule applies when the two terms are applications, and requires that the functions $t_{1}$ and $t_{2}$ relate and the arguments $u_{1}$ and $u_{2}$ relate. Specifically, $t_{1}$ and $t_{2}$ must map values related by $\phi^{\prime}$ to values related by $\phi$, and $u_{1}$ and $u_{2}$ must be related by $\phi^{\prime}$. The ABS rule is dual. The PAIR rule requires that the left and right components of a pair relate independently (a stronger rule is discussed at the end of this section). The $P R O J_{i}$ rules require 
in their premise an assertion that only refers to the first or the second component of the pair. The rules for lists require that the two lists are either both empty, or both non-empty. The rule CONS requires that the two heads and the two tails relate independently. The CASE rule derives judgments about two case constructs when the terms over which the matching happens reduce to the same branch (i.e. have the same constructor) on both sides.

In contrast, one-sided typing rules only analyze one term; therefore, they come in two flavors: left rules (shown in Figure 3.5) and right rules (omitted but similar). Rule ABS-L considers the case where the left term is a $\lambda$-abstraction, and requires the body of the abstraction to be related to the right term $u_{2}$ whenever the argument on the left side satisfies a non-relational assertion $\phi^{\prime}$. Dually, rule APP-L considers the case where the left term is of the form $t_{1} \mathfrak{u}_{1}$, and $t_{1}$ is related to the right term $u_{2}$; specifically, $t_{1}$ should map every value satisfying $\phi^{\prime}$ to a value satisfying $\phi$. Moreover, $u_{1}$ should satisfy $\phi^{\prime}$ in UHOL (not RHOL, since $\phi^{\prime}$ is a non-relational assertion). One-sided rules for pairs and lists follow a similar pattern.

In addition, RHOL has structural rules (Figure 3.4). The rule SUB can be used for weakening the conclusion; the ensuing side-condition is discharged in HOL. Other structural rules assimilate rules of HOL. For instance, if we can prove two different assertions for the same terms we can prove the conjunction of the assertions $\left(\wedge_{\mathrm{I}}\right)$. Other logical connectives have similar rules. Finally, the rule UHOL-L (and a dual rule UHOL-R) allow falling back to UHOL in a RHOL proof.

Rules LETREC and LETREC-L introduce recursive function definitions (Figure 3.6). These rules allow for a style of reasoning very similar to strong induction. If, assuming that the function's specification holds for all smaller arguments, we can prove that the functions specification holds, then the specification must hold for all arguments. We require that the two functions we are relating satisfy the predicates $\operatorname{Def}\left(f_{i}, x_{i}, t_{i}\right)$, as was the case in HOL and UHOL. The induction is performed over the simultaneous order $(a, b)<(c, d)$, which holds whenever both $a \leqslant b$ and $c \leqslant d$, and at least one of the inequalities is strict.

\subsubsection{Discussion}

\subsubsection{Management of the typing context}

In a relational judgment $\Gamma \mid \Psi \vdash \mathrm{t}_{1}: \sigma_{1} \sim \mathrm{t}_{2}: \sigma_{2}\{\phi\}$, the context $\Gamma$ contains variables that can appear free in both $t_{1}$ and $t_{2}$. This forces us to add additional premises to the abstraction rules to ensure the terms in the conclusion remain well-typed. Note that we could have another sound (but less expressive) version of the ABS rule where we abstract over the same variable on both sides.

An alternative choice of presentation is to have separate contexts $\Gamma_{1}$ and $\Gamma_{2}$ for the terms on each side of the relational judgments, eliminating the need for the extra premises. However, there would be no other benefit to this alternative. For 


$$
\begin{aligned}
& \frac{\Gamma, x_{1}: \tau_{1}, x_{2}: \tau_{2} \mid \Psi, \phi^{\prime} \vdash t_{1}: \sigma_{1} \sim t_{2}: \sigma_{2}\{\phi\} \quad x_{1} \notin \mathrm{FV}\left(t_{2}\right) \quad x_{2} \notin \mathrm{FV}\left(t_{1}\right)}{\Gamma \mid \Psi \vdash \lambda x_{1}: \tau_{1} . t_{1}: \tau_{1} \rightarrow \sigma_{1} \sim \lambda x_{2}: \tau_{2} . t_{2}: \tau_{2} \rightarrow \sigma_{2}\left\{\forall x_{1}, x_{2} . \phi^{\prime} \Rightarrow \phi\left[\mathbf{r}_{1} x_{1} / \mathbf{r}_{1}\right]\left[\mathbf{r}_{2} x_{2} / \mathbf{r}_{2}\right]\right\}} \text { ABS } \\
& \Gamma \mid \Psi \vdash \mathrm{t}_{1}: \tau_{1} \rightarrow \sigma_{1} \sim \mathrm{t}_{2}: \tau_{2} \rightarrow \sigma_{2}\left\{\forall \mathrm{x}_{1}, \mathrm{x}_{2} . \phi^{\prime}\left[\mathrm{x}_{1} / \mathbf{r}_{1}\right]\left[\mathrm{x}_{2} / \mathbf{r}_{2}\right] \Rightarrow \phi\left[\mathbf{r}_{1} \mathrm{x}_{1} / \mathbf{r}_{1}\right]\left[\mathbf{r}_{2} \mathrm{x}_{2} / \mathbf{r}_{2}\right]\right\} \\
& \Gamma \mid \Psi \vdash \mathfrak{u}_{1}: \tau_{1} \sim \mathfrak{u}_{2}: \tau_{2}\left\{\phi^{\prime}\right\} \\
& \Gamma \mid \Psi \vdash \mathrm{t}_{1} \mathrm{u}_{1}: \sigma_{1} \sim \mathrm{t}_{2} \mathrm{u}_{2}: \sigma_{2}\left\{\phi\left[\mathrm{u}_{1} / \mathrm{x}_{1}\right]\left[\mathrm{u}_{2} / \mathrm{x}_{2}\right]\right\}
\end{aligned}
$$

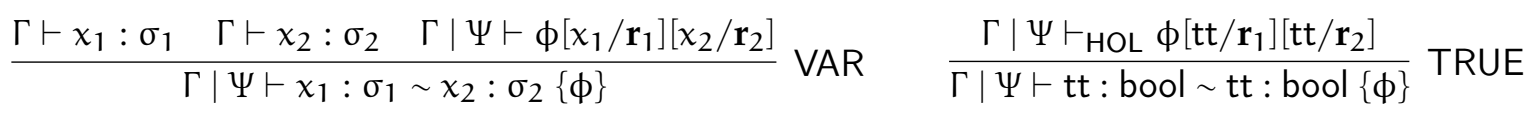

$$
\begin{aligned}
& \frac{\left.\left.\Gamma \mid \Psi \vdash_{\mathrm{HOL}} \phi[] / \mathbf{r}_{1}\right][] / \mathbf{r}_{2}\right]}{\Gamma \mid \Psi \vdash\left[: \operatorname{list}_{\sigma_{1}} \sim\right]: \text { list }_{\sigma_{2}}\{\phi\}} \mathrm{NIL} \\
& \Gamma\left|\Psi \vdash \mathrm{h}_{1}: \sigma_{1} \sim \mathrm{h}_{2}: \sigma_{2}\left\{\phi^{\prime}\right\} \quad \Gamma\right| \Psi \vdash \mathrm{t}_{1}: \operatorname{list}_{\sigma_{1}} \sim \mathrm{t}_{2}: \operatorname{list}_{\sigma_{2}}\left\{\phi^{\prime \prime}\right\} \\
& \frac{\Gamma \mid \Psi \vdash_{\mathrm{HOL}} \forall x_{1} x_{2} y_{1} y_{2} \cdot \phi^{\prime}\left[x_{1} / \mathbf{r}_{1}\right]\left[x_{2} / \mathbf{r}_{2}\right] \Rightarrow \phi^{\prime \prime}\left[y_{1} / \mathbf{r}_{1}\right]\left[y_{2} / \mathbf{r}_{2}\right] \Rightarrow \phi\left[x_{1}:: y_{1} / \mathbf{r}_{1}\right]\left[x_{2}:: y_{2} / \mathbf{r}_{2}\right]}{\Gamma \mid \Psi \vdash h_{1}:: t_{1}: \text { list }_{\sigma_{1}} \sim h_{2}:: t_{2}: \operatorname{list}_{\sigma_{2}}\{\phi\}} \text { cons } \\
& \Gamma \mid \Psi \vdash l_{1}: \text { list }_{\tau_{1}} \sim l_{2}: \text { list }_{\tau_{2}}\left\{\mathbf{r}_{1}=\square \Leftrightarrow \mathbf{r}_{2}=\square\right\} \\
& \Gamma \mid \Psi, \mathrm{l}_{1}=\square, \mathrm{l}_{2}=\square \vdash \mathfrak{u}_{1}: \sigma_{1} \sim \mathrm{u}_{2}: \sigma_{2}\{\phi\} \\
& \Gamma \mid \Psi \vdash v_{1}: \tau_{1} \rightarrow \text { list }_{\tau_{1}} \rightarrow \sigma_{1} \sim v_{2}: \tau_{2} \rightarrow \text { list }_{\tau_{2}} \rightarrow \sigma_{2}\{ \\
& \} \forall \mathrm{h}_{1} \mathrm{~h}_{2} \mathrm{t}_{1} \mathrm{t}_{2} \cdot \mathrm{l}_{1}=\mathrm{h}_{1}:: \mathrm{t}_{1} \Rightarrow \mathrm{l}_{2}=\mathrm{h}_{2}:: \mathrm{t}_{2} \Rightarrow \phi\left[\mathbf{r}_{1} \mathrm{~h}_{1} \mathrm{t}_{1} / \mathbf{r}_{1}\right]\left[\mathbf{r}_{2} \mathrm{~h}_{2} \mathrm{t}_{2} / \mathbf{r}_{2}\right] \\
& \overline{\Gamma \mid \Psi \vdash \text { case } l_{1} \text { of } \square \mapsto u_{1} i_{-}:_{-} \mapsto v_{1}: \sigma_{1} \sim \text { case } l_{2} \text { of } \square \mapsto u_{2} ;_{-}:_{-} \mapsto v_{2}: \sigma_{2}\{\phi\}} \text { LISTCASE } \\
& \Gamma\left|\Psi \vdash \mathrm{t}_{1}: \sigma_{1} \sim \mathrm{t}_{2}: \sigma_{2}\left\{\phi^{\prime}\right\} \quad \Gamma\right| \Psi \vdash \mathrm{u}_{1}: \tau_{1} \sim \mathrm{u}_{2}: \tau_{2}\left\{\phi^{\prime \prime}\right\} \\
& \frac{\Gamma \mid \Psi \vdash_{\mathrm{HOL}} \forall x_{1} x_{2} y_{1} y_{2} \cdot \phi^{\prime}\left[x_{1} / \mathbf{r}_{1}\right]\left[x_{2} / \mathbf{r}_{2}\right] \Rightarrow \phi^{\prime \prime}\left[y_{1} / \mathbf{r}_{1}\right]\left[y_{2} / \mathbf{r}_{2}\right] \Rightarrow \phi\left[\left\langle x_{1}, y_{1}\right\rangle / \mathbf{r}_{1}\right]\left[\left\langle x_{2}, y_{2}\right\rangle / \mathbf{r}_{2}\right]}{\Gamma \mid \Psi \vdash\left\langle t_{1}, u_{1}\right\rangle: \sigma_{1} \times \tau_{1} \sim\left\langle t_{2}, u_{2}\right\rangle: \sigma_{2} \times \tau_{2}\{\phi\}} \text { PAIR } \\
& \frac{\Gamma \mid \Psi \vdash \mathrm{t}_{1}: \sigma_{1} \times \tau_{1} \sim \mathrm{t}_{2}: \sigma_{2} \times \tau_{2}\left\{\phi\left[\pi_{\mathrm{i}}\left(\mathbf{r}_{1}\right) / \mathbf{r}_{1}\right]\left[\pi_{\mathfrak{i}}\left(\mathbf{r}_{2}\right) / \mathbf{r}_{2}\right]\right\}}{\Gamma \mid \Psi \vdash \pi_{\mathfrak{i}}\left(\mathrm{t}_{1}\right): \sigma_{1} \sim \pi_{\mathfrak{i}}\left(\mathrm{t}_{2}\right): \sigma_{2}\{\phi\}} \text { PROJ }_{\mathrm{i}}
\end{aligned}
$$

Figure 3.3: Two-sided rules 


$$
\begin{gathered}
\frac{\Gamma\left|\Psi \vdash \mathrm{t}_{1}: \sigma_{1} \sim \mathrm{t}_{2}: \sigma_{2}\left\{\phi^{\prime}\right\} \quad \Gamma\right| \Psi \vdash_{\mathrm{HOL}} \phi^{\prime}\left[\mathrm{t}_{1} / \mathbf{r}_{1}\right]\left[\mathrm{t}_{2} / \mathbf{r}_{2}\right] \Rightarrow \phi\left[\mathrm{t}_{1} / \mathbf{r}_{1}\right]\left[\mathrm{t}_{2} / \mathbf{r}_{2}\right]}{\Gamma \mid \Psi \vdash \mathrm{t}_{1}: \sigma_{1} \sim \mathrm{t}_{2}: \sigma_{2}\{\phi\}} \text { SUB } \\
\frac{\Gamma\left|\Psi \vdash \mathrm{t}_{1}: \sigma_{2} \sim \mathrm{t}_{2}: \sigma_{2}\{\phi\} \quad \Gamma\right| \Psi \vdash \mathrm{t}_{1}: \sigma_{2} \sim \mathrm{t}_{2}: \sigma_{2}\left\{\phi^{\prime}\right\}}{\Gamma \mid \Psi \vdash \mathrm{t}_{1}: \sigma_{2} \sim \mathrm{t}_{2}: \sigma_{2}\left\{\phi \wedge \phi^{\prime}\right\}} \wedge_{\mathrm{I}} \\
\frac{\Gamma \mid \Psi, \phi^{\prime}\left[\mathrm{t}_{1} / \mathbf{r}_{1}\right]\left[\mathrm{t}_{2} / \mathbf{r}_{2}\right] \vdash \mathrm{t}_{1}: \sigma_{2} \sim \mathrm{t}_{2}: \sigma_{2}\{\phi\}}{\Gamma \mid \Psi \vdash \mathrm{t}_{1}: \sigma_{2} \sim \mathrm{t}_{2}: \sigma_{2}\left\{\phi^{\prime} \Rightarrow \phi\right\}} \Rightarrow_{\mathrm{I}} \\
\frac{\Gamma \mid \Psi \vdash \mathrm{t}_{1}: \sigma_{1}\left\{\phi\left[\mathbf{r} / \mathbf{r}_{1}\right]\left[\mathrm{t}_{2} / \mathbf{r}_{2}\right]\right\} \quad \Gamma \vdash \mathrm{t}_{2}: \sigma_{2}}{\Gamma \mid \Psi \vdash \mathrm{t}_{1}: \sigma_{1} \sim \mathrm{t}_{2}: \sigma_{2}\{\phi\}} \mathrm{UHOL}-\mathrm{L}
\end{gathered}
$$

Figure 3.4: Structural rules

$$
\begin{aligned}
& \frac{\Gamma, x_{1}: \tau_{1} \mid \Psi, \phi^{\prime} \vdash t_{1}: \sigma_{1} \sim t_{2}: \sigma_{2}\{\phi\} \quad x_{1} \notin F V\left(t_{2}\right)}{\Gamma \mid \Psi \vdash \lambda x_{1}: \tau_{1} . t_{1}: \tau_{1} \rightarrow \sigma_{1} \sim t_{2}: \sigma_{2}\left\{\forall x_{1} . \phi^{\prime} \Rightarrow \phi\left[\mathbf{r}_{1} x_{1} / \mathbf{r}_{1}\right]\right\}} \text { ABS-L } \\
& \Gamma \mid \Psi \vdash \mathrm{t}_{1}: \tau_{1} \rightarrow \sigma_{1} \sim \mathrm{u}_{2}: \sigma_{2}\left\{\forall \mathrm{x}_{1} . \phi^{\prime}\left[\mathrm{x}_{1} / \mathbf{r}_{1}\right] \Rightarrow \phi\left[\mathbf{r}_{1} \mathrm{x}_{1} / \mathbf{r}_{1}\right]\right\} \\
& \Gamma \mid \Psi \vdash u_{1}: \sigma_{1}\left\{\phi^{\prime}\right\} \\
& \Gamma \mid \Psi \vdash \mathfrak{t}_{1} \mathfrak{u}_{1}: \sigma_{1} \sim \mathfrak{u}_{2}: \sigma_{2}\left\{\phi\left[\mathfrak{u}_{1} / \mathrm{x}_{1}\right]\right\} \\
& \frac{\phi\left[\mathrm{x}_{1} / \mathbf{r}_{1}\right] \in \Psi \quad \mathbf{r}_{2} \notin \mathrm{FV}(\phi) \quad \Gamma \vdash \mathrm{t}_{2}: \sigma_{2}}{\Gamma \mid \Psi \vdash \mathrm{x}_{1}: \sigma_{1} \sim \mathrm{t}_{2}: \sigma_{2}\{\phi\}} \mathrm{VAR}-\mathrm{L} \quad \frac{\left.\Gamma \mid \Psi \vdash \phi[] / \mathbf{r}_{1}\right]\left[\mathrm{t}_{2} / \mathbf{r}_{2}\right] \quad \Gamma \vdash \mathrm{t}_{2}: \sigma_{2}}{\Gamma \mid \Psi \vdash]: \text { list }_{\sigma_{1}} \sim \mathrm{t}_{2}: \sigma_{2}\{\phi\}} \text { NIL-L } \\
& \Gamma\left|\Psi \vdash h_{1}: \sigma_{1} \sim t_{2}: \sigma_{2}\left\{\phi^{\prime}\right\} \quad \Gamma\right| \Psi \vdash t_{1}: \text { list }_{\sigma_{1}} \sim \mathrm{t}_{2}: \sigma_{2}\left\{\phi^{\prime \prime}\right\} \\
& \frac{\Gamma \mid \Psi \vdash_{\mathrm{HOL}} \forall x_{1} x_{2} y_{1} \cdot \phi^{\prime}\left[x_{1} / \mathbf{r}_{1}\right]\left[x_{2} / \mathbf{r}_{2}\right] \Rightarrow \phi^{\prime \prime}\left[y_{1} / \mathbf{r}_{1}\right]\left[x_{2} / \mathbf{r}_{2}\right] \Rightarrow \phi\left[x_{1}:: y_{1} / \mathbf{r}_{1}\right]\left[x_{2} / \mathbf{r}_{2}\right]}{\Gamma \mid \Psi \vdash h_{1}:: t_{1}: \operatorname{list}_{\sigma_{1}} \sim t_{2}: \sigma_{2}\{\phi\}} \text { CONS-L } \\
& \Gamma \vdash \mathrm{t}_{1}: \text { list }_{\tau} \\
& \Gamma \mid \Psi, t_{1}=\square \vdash u_{1}: \sigma_{1} \sim t_{2}: \sigma_{2}\{\phi\} \\
& \frac{\Gamma \mid \Psi \vdash v_{1}: \tau \rightarrow \operatorname{list}_{\tau} \rightarrow \sigma_{1} \sim t_{2}: \sigma_{2}\left\{\forall h_{1} l_{1} \cdot t_{1}=h_{1}:: l_{1} \Rightarrow \phi\left[\mathbf{r}_{1} h_{1} l_{1} / \mathbf{r}_{1}\right]\right\}}{\left.\Gamma \mid \Psi \vdash \text { case } t_{1} \text { of }\right] \mapsto u_{1} ;_{-}:{ }_{-} \mapsto v_{1}: \sigma_{1} \sim t_{2}: \sigma_{2}\{\phi\}} \text { LISTCASE }- \text { L } \\
& \Gamma\left|\Psi \vdash \mathrm{t}_{1}: \sigma_{1} \sim \mathrm{t}_{2}: \sigma_{2}\left\{\phi^{\prime}\right\} \quad \Gamma\right| \Psi \vdash \mathrm{u}_{1}: \tau_{1} \sim \mathrm{t}_{2}: \sigma_{2}\left\{\phi^{\prime \prime}\right\} \\
& \frac{\Gamma \mid \Psi \vdash_{\text {HOL }} \forall x_{1} x_{2} y_{1} \cdot \phi^{\prime}\left[x_{1} / \mathbf{r}_{1}\right]\left[x_{2} / \mathbf{r}_{2}\right] \Rightarrow \phi^{\prime \prime}\left[y_{1} / \mathbf{r}_{1}\right]\left[x_{2} / \mathbf{r}_{2}\right] \Rightarrow \phi\left[\left\langle x_{1}, y_{1}\right\rangle / \mathbf{r}_{1}\right]\left[x_{2} / \mathbf{r}_{2}\right]}{\Gamma \mid \Psi \vdash\left\langle t_{1}, u_{1}\right\rangle: \sigma_{1} \times \tau_{1} \sim t_{2}: \sigma_{2}\{\phi\}} \text { PAIR-L } \\
& \frac{\Gamma \mid \Psi \vdash \mathrm{t}_{1}: \sigma_{1} \times \tau_{1} \sim \mathrm{t}_{2}: \sigma_{2}\left\{\phi\left[\pi_{1}\left(\mathbf{r}_{1}\right) / \mathbf{r}_{1}\right]\right\}}{\Gamma \mid \Psi \vdash \pi_{1}\left(\mathrm{t}_{1}\right): \sigma_{1} \sim \mathrm{t}_{2}: \sigma_{2}\{\phi\}} \mathrm{PROJ}_{1}-\mathrm{L}
\end{aligned}
$$

Figure 3.5: One-sided rules 


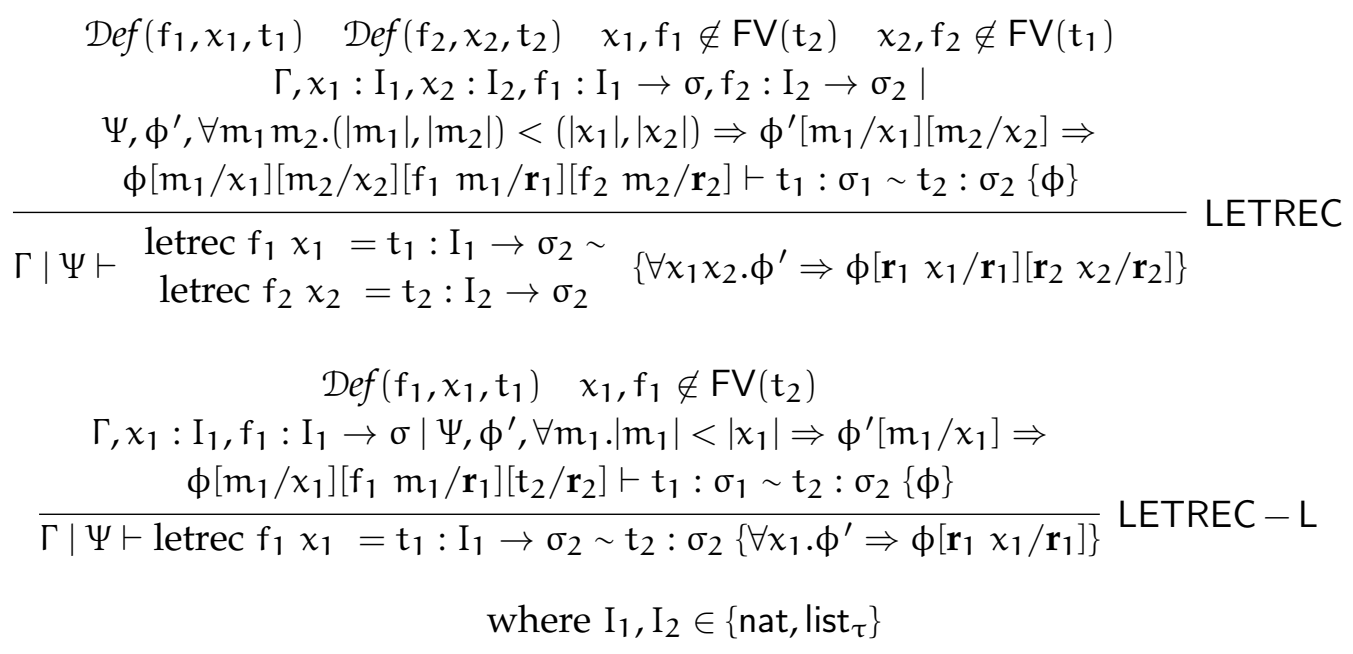

Figure 3.6: Recursion rules

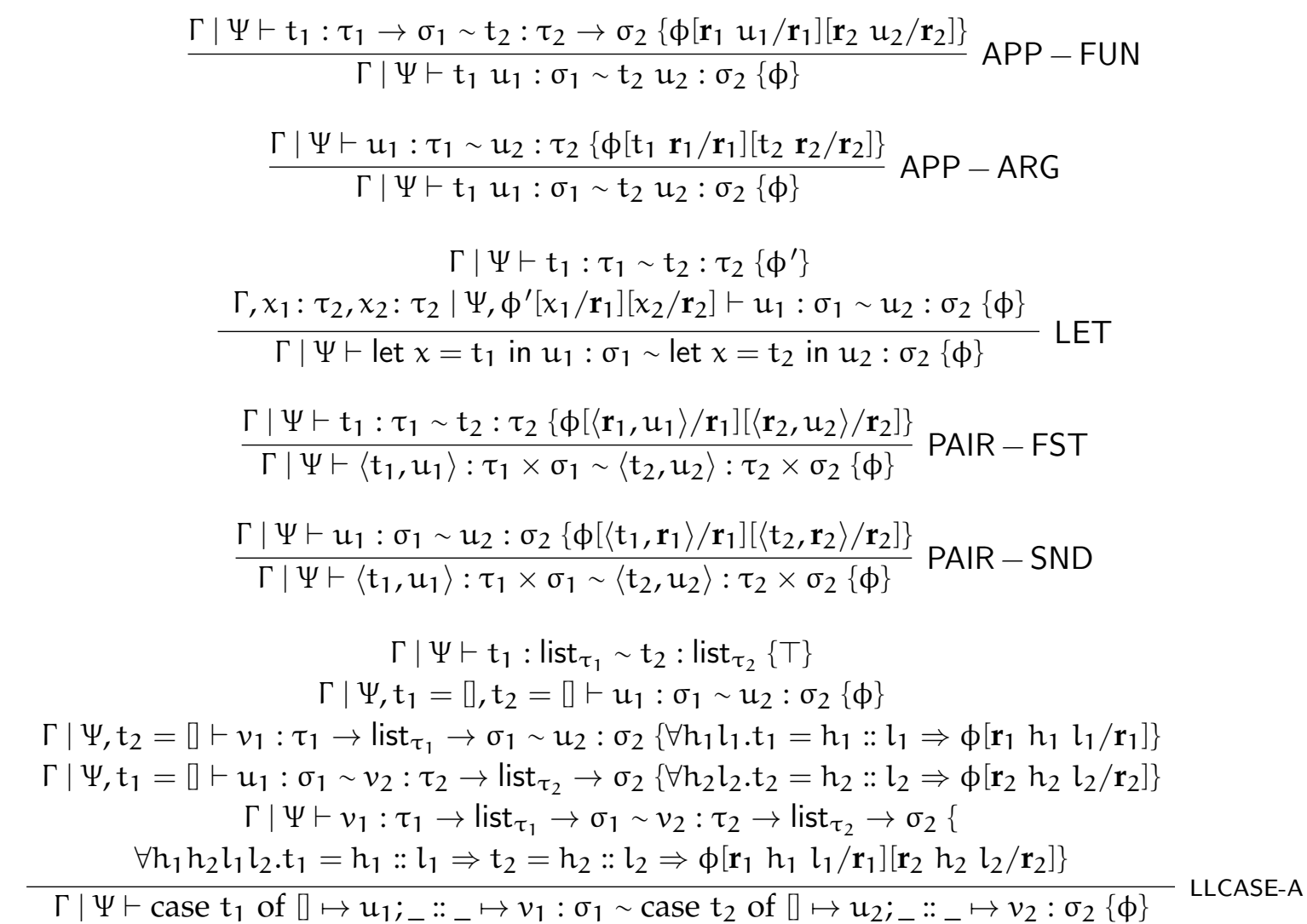

Figure 3.7: Some derived rules 
instance, UHOL premises as in APP-L would still need to use both contexts $\Gamma_{1}$ and $\Gamma_{2}$, since the logical context $\Psi$ is typed under both.

\section{3י5.2.2 Variants of the rules}

Our choice of the rules is guided by the practical considerations of being able to verify examples easily, without specifically aiming for minimality or exhaustiveness. In fact, many of our rules can be derived from others, or reduced to a more elementary form. For instance:

- The structural rules to reason about logical connectives, such as $\wedge_{\mathrm{I}}$, can be derived by induction on the length of derivations with the help of SUB.

- The VAR-L (similarly, NIL-L) rule can be weakened, without affecting the strength of the system,

$$
\frac{\phi\left[x_{1} / \mathbf{r}_{1}\right] \in \Psi \quad \mathbf{r}_{2} \notin \mathrm{FV}(\phi)}{\Gamma \mid \Psi \vdash x_{1}: \sigma_{1} \sim x_{2}: \sigma_{2}\{\phi\}} \text { VAR-L }
$$

- The premise of the VAR rule in Figure 3.3 (and similarly, NIL) can be changed to $\phi[x / r] \in \Psi$. We can recover the original rule by one application of SUB.

- The rules APP-FUN and APP-ARG in Figure 3.7 (adapted from [82]) can be derived from the rule APP. To derive APP-FUN, instantiate $\phi^{\prime}$ to $\mathbf{r}_{1}=u_{1} \wedge$ $\mathbf{r}_{2}=u_{2}$ in APP. To derive APP-ARG, we have to prove a trivial condition $\forall x_{1} x_{2} . \phi\left[t_{1} x_{1} / \mathbf{r}_{1}\right]\left[t_{2} x_{2} / \mathbf{r}_{2}\right] \Rightarrow \phi\left[t_{1} x_{1} / \mathbf{r}_{1}\right]\left[t_{2} x_{2} / r_{2}\right]$ on $t_{1}, t_{2}$.

- The PAIR-FST and PAIR-SND rules in Figure 3.7 can be derived in a similar way. These rules overcome a limitation of the original PAIR rule, namely, that the relations for the two components of the pair must be independent. PAIR-FST and PAIR-SND allow relating, for instance, pairs of integers $\left\langle m_{1}, n_{1}\right\rangle$ and $\left\langle m_{2}, n_{2}\right\rangle$ such that $m_{1}+n_{1}=m_{2}+n_{2}$.

- The LLCASE-A rule can be used to reason about case constructs when the terms over which we discriminate do not necessarily reduce to the same branch. It is equivalent to applying LISTCASE-L followed by LISTCASE-R.

- We could add a let construct to our language, defined as let $x=t$ in $u \triangleq(\lambda x . u) t$ and a derived LET rule that first proves an specification for the first argument of the let (i.e., $t$ ), and then uses this as an assumption for the second argument of the let. 


\subsubsection{Meta-theory}

RHOL retains the expressiveness of HOL, and is relatively complete with respect to it, as formalized in the following theorem.

Theorem 3.6 (Equivalence with HOL). For every context $\Gamma$, simple types $\sigma_{1}$ and $\sigma_{2}$, terms $\mathrm{t}_{1}$ and $\mathrm{t}_{2}$, set of assertions $\Psi$ and assertion $\phi$, if $\Gamma \vdash \mathrm{t}_{1}: \sigma_{1}$ and $\Gamma \vdash \mathrm{t}_{2}: \sigma_{2}$, then the following are equivalent:

- $\Gamma \mid \Psi \vdash \mathrm{t}_{1}: \sigma_{1} \sim \mathrm{t}_{2}: \sigma_{2}\{\phi\}$

- $\Gamma \mid \Psi \vdash \phi\left[\mathrm{t}_{1} / \mathbf{r}_{1}\right]\left[\mathrm{t}_{2} / \mathbf{r}_{2}\right]$

Proof. The easier direction is the reverse implication. To prove it, we can trivially apply SUB instantiating $\phi^{\prime}$ as a tautology that matches the structure of the types. For instance, for a base type nat we would use $T$, for an arrow type nat $\rightarrow$ nat we would use $\forall x . \perp \Rightarrow T$, and so on.

The forward implication follows by induction on the derivation of the judgment $\Gamma \mid \Psi \vdash t_{1}: \sigma_{1} \sim t_{2}: \sigma_{2}\{\phi\}$. We show here the cases for abstraction and application:

Case ABS. The rule is:

$$
\frac{\Gamma, x_{1}: \tau_{1}, x_{2}: \tau_{2} \mid \Psi, \phi^{\prime} \vdash t_{1}: \sigma_{1} \sim t_{2}: \sigma_{2}\{\phi\}}{\Gamma \mid \Psi \vdash \lambda x_{1} \cdot t_{1}: \tau_{1} \rightarrow \sigma_{1} \sim \lambda x_{2} \cdot t_{2}: \tau_{2} \rightarrow \sigma_{2}\left\{\forall x_{1}, x_{2} \cdot \phi^{\prime} \Rightarrow \phi\left[\mathbf{r}_{1} x_{1} / \mathbf{r}_{1}\right]\left[\mathbf{r}_{2} x_{2} / \mathbf{r}_{2}\right]\right\}}
$$

By applying the induction hypothesis on the premise:

$\Gamma, x_{1}: \tau_{1}, x_{2}: \tau_{2} \mid \Psi, \phi^{\prime} \vdash \phi\left[t_{1} / \mathbf{r}_{1}\right]\left[\mathrm{t}_{2} / \mathbf{r}_{2}\right]$

By applying $\Rightarrow_{\mathrm{I}}$ on $(\mathrm{I})$ :

$\Gamma, x_{1}: \tau_{1}, x_{2}: \tau_{2} \mid \Psi \vdash \phi^{\prime} \Rightarrow \phi\left[t_{1} / \mathbf{r}_{1}\right]\left[t_{2} / \mathbf{r}_{2}\right]$

By applying $\forall_{I}$ twice on (2):

$\Gamma \mid \Psi \vdash \forall x_{1} x_{2} . \phi^{\prime} \Rightarrow \phi\left[\mathrm{t}_{1} / \mathbf{r}_{1}\right]\left[\mathrm{t}_{2} / \mathbf{r}_{2}\right]$

Finally, by applying CONV on (3):

$\Gamma \mid \Psi \vdash \forall x_{1} x_{2} \cdot \phi^{\prime} \Rightarrow \phi\left[\left(\lambda x_{1} \cdot t_{1}\right) x_{1} / \mathbf{r}_{1}\right]\left[\left(\lambda x_{2} \cdot t_{2}\right) x_{2} / \mathbf{r}_{2}\right]$

The proof for ABS-L (and ABS-R) is analogous.

Case APP. The rule is

$$
\begin{gathered}
\Gamma \mid \Psi \vdash \mathrm{t}_{1}: \tau_{1} \rightarrow \sigma_{1} \sim \mathrm{t}_{2}: \tau_{2} \rightarrow \sigma_{2}\left\{\forall \mathrm{x}_{1}, \mathrm{x}_{2} \cdot \phi^{\prime}\left[\mathrm{x}_{1} / \mathbf{r}_{1}\right]\left[\mathrm{x}_{2} / \mathbf{r}_{2}\right] \Rightarrow \phi\left[\mathbf{r}_{1} \mathrm{x}_{1} / \mathbf{r}_{1}\right]\left[\mathbf{r}_{2} \mathrm{x}_{2} / \mathbf{r}_{2}\right]\right\} \\
\Gamma \mid \Psi \vdash \mathfrak{u}_{1}: \tau_{1} \sim \mathfrak{u}_{2}: \tau_{2}\left\{\phi^{\prime}\right\}
\end{gathered}
$$

By applying the induction hypothesis on the premises we have:

$\Gamma \mid \Psi \vdash \forall x_{1} x_{2} . \phi^{\prime}\left[x_{1} / \mathbf{r}_{1}\right]\left[x_{2} / \mathbf{r}_{2}\right] \Rightarrow \phi\left[t_{1} x_{1} / \mathbf{r}_{1}\right]\left[t_{2} x_{2} / r_{2}\right]$

and

$\Gamma \mid \Psi \vdash \phi^{\prime}\left[\mathfrak{u}_{1} / \mathbf{r}_{1}\right]\left[\mathfrak{u}_{2} / \mathbf{r}_{2}\right]$

By applying twice $\forall_{E}$ to (I) with $u_{1}, u_{2}$ :

$\Gamma \mid \Psi \vdash \phi^{\prime}\left[\mathfrak{u}_{1} / \mathbf{r}_{1}\right]\left[\mathfrak{u}_{2} / \mathbf{r}_{2}\right] \Rightarrow \phi\left[\mathfrak{t}_{1} \mathfrak{u}_{1} / \mathbf{r}_{1}\right]\left[\mathfrak{t}_{2} u_{2} / \mathbf{r}_{2}\right]$ 
and by applying $\Rightarrow_{E}$ to (3) and (2):

$\Gamma \mid \Psi \vdash \phi\left[t_{1} u_{1} / \mathbf{r}_{1}\right]\left[t_{2} u_{2} / \mathbf{r}_{2}\right]$

The proof for APP-L (and APP-R) is analogous, and it uses the UHOL embedding (Theorem 3.3) for the premise about the argument. Proofs for CONS and PAIR also follow the same structure.

This immediately entails soundness of RHOL, as formalized next.

Corollary 3.7 (Set-theoretical soundness and consistency). Let $\mathrm{t}_{1}, \mathrm{t}_{2}$ be two terms such that $\Gamma \mid \Psi \vdash \mathrm{t}_{1}: \sigma_{1} \sim \mathrm{t}_{2}: \sigma_{2}\{\phi\}$ is a valid RHOL derivation. Then,

$$
\llbracket \Gamma \vdash \bigwedge_{\psi \in \Psi} \psi \rrbracket \subseteq \llbracket \Gamma \vdash \phi\left[\mathrm{t}_{1} / \mathbf{r}_{1}\right]\left[\mathrm{t}_{2} / \mathbf{r}_{2}\right] \rrbracket .
$$

In particular, there is no proof of the judgment $\Gamma \mid \emptyset \vdash \mathrm{t}_{1}: \sigma_{1} \sim \mathrm{t}_{2}: \sigma_{2}\{\perp\}$ for any $\Gamma, \mathrm{t}_{1}, \mathrm{t}_{2}$, $\sigma_{1}$ and $\sigma_{2}$.

The equivalence also entails subject conversion (and as special cases subject reduction and subject expansion). This follows immediately from subject conversion of HOL (which, as stated earlier, is itself a direct consequence of the CONV and SUBST rules).

Corollary 3.8 (Subject conversion). Assume that $\mathrm{t}_{1}={ }_{\beta \mu \mu} \mathrm{t}_{1}^{\prime}$ and $\mathrm{t}_{2}={ }_{\beta \mu \mu} \mathrm{t}_{2}^{\prime}$ and $\Gamma \mid \Psi \vdash$ $\mathrm{t}_{1}: \sigma_{1} \sim \mathrm{t}_{2}: \sigma_{2}\{\phi\}$. Then we have $\Gamma \mid \Psi \vdash \mathrm{t}_{1}^{\prime}: \sigma_{1} \sim \mathrm{t}_{2}^{\prime}: \sigma_{2}\{\phi\}$.

Another useful consequence of the equivalence is the admissibility of the transitivity rule.

Corollary 3.9 (Admissibility of transitivity rule). Assume that:

- $\Gamma \mid \Psi \vdash \mathrm{t}_{1}: \sigma_{1} \sim \mathrm{t}_{2}: \sigma_{2}\{\phi\}$

- $\Gamma \mid \Psi \vdash \mathrm{t}_{2}: \sigma_{2} \sim \mathrm{t}_{3}: \sigma_{3}\left\{\phi^{\prime}\right\}$

Then, $\Gamma \mid \Psi \vdash \mathrm{t}_{1}: \sigma_{1} \sim \mathrm{t}_{3}: \sigma_{3}\left\{\phi\left[\mathrm{t}_{2} / \mathbf{r}_{2}\right] \wedge \phi^{\prime}\left[\mathrm{t}_{2} / \mathbf{r}_{1}\right]\right\}$.

Finally, we prove an embedding lemma for UHOL. The proof can be carried by induction on the structure of derivations, or using the equivalence between UHOL and HOL (Theorem 3.3).

Lemma 3.10 (Embedding lemma). Assume that:

- $\Gamma \mid \Psi \vdash \mathrm{t}_{1}: \sigma_{1}\{\phi\}$

- $\Gamma \mid \Psi \vdash \mathrm{t}_{2}: \sigma_{2}\left\{\phi^{\prime}\right\}$

Then $\Gamma \mid \Psi \vdash \mathfrak{t}_{1}: \sigma_{1} \sim \mathfrak{t}_{2}: \sigma_{2}\left\{\phi\left[\mathbf{r}_{1} / \mathbf{r}\right] \wedge \phi^{\prime}\left[\mathbf{r}_{2} / \mathbf{r}\right]\right\}$. 
Proof. By the embedding into HOL (Theorem 3.3), we have:

- $\Gamma \mid \Psi \vdash \phi\left[\mathrm{t}_{1} / \mathbf{r}\right]$

- $\Gamma \mid \Psi \vdash \phi^{\prime}\left[t_{2} / \mathbf{r}\right]$

and by the $\wedge_{I}$ rule,

$$
\Gamma \mid \Psi \vdash \phi\left[\mathrm{t}_{1} / \mathbf{r}\right] \wedge \phi^{\prime}\left[\mathrm{t}_{2} / \mathbf{r}\right] .
$$

Finally, by Theorem 3.6:

$$
\Gamma \mid \Psi \vdash \mathrm{t}_{1}: \sigma_{1} \sim \mathrm{t}_{2}: \sigma_{2}\left\{\phi\left[\mathbf{r}_{1} / \mathbf{r}\right] \wedge \phi^{\prime}\left[\mathbf{r}_{2} / \mathbf{r}\right]\right\} .
$$

The embedding is reminiscent of the approach of [47] to encode information flow properties in Hoare logic.

\subsection{Embeddings}

In this section, we establish the expressiveness of RHOL and UHOL by embedding several existing refinement type systems (three relational and one non-relational) from a variety of domains. All embeddings share the common idea of separating the simple typing information from the more fine-grained refinement information in the translation. We use uniform notation to represent similar ideas across the different embeddings. In particular, we use vertical bars $|\cdot|$ to denote the erasure of a type into a simple type, and floor bars $L \cdot\rfloor$ to denote the embedding of the refinement of a type in a HOL formula.

For clarity of exposition, we often present fragments or variants of systems that appear in the literature, notably excluding recursive functions that do not satisfy our well-definedness predicate. Moreover, the embeddings are given for a version of RHOL à la Curry, in which $\lambda$-abstractions do not carry the type of their bound variable.

\subsubsection{Refinement Types}

Refinement types $[76,149,155]$ are a variant of simple types where for every basic type $\tau$, there is a type $\{x: \tau \mid \phi\}$ which is inhabited by the elements $t$ of $\tau$ that satisfy the logical assertion $\phi[t / x]$. This includes dependent refinements $\Pi(x: \tau) . \sigma$, in which the variable $x$ is also bound in the refinement formulas appearing in $\sigma$. Here we present a simplified variant of these systems. (Refined) types are defined by the grammar

$$
\tau:=\text { bool } \mid \text { nat }\left|\operatorname{list}_{\tau}\right|\{x: \tau \mid \phi\} \mid \Pi(x: \tau) \cdot \tau
$$




$$
\begin{aligned}
& \frac{\Gamma \vdash \tau}{\Gamma \vdash \tau \preceq \tau} \quad \frac{\Gamma \vdash \tau_{1} \preceq \tau_{2} \quad \Gamma \vdash \tau_{2} \preceq \tau_{3}}{\Gamma \vdash \tau_{1} \preceq \tau_{3}} \quad \frac{\Gamma \vdash \tau_{1} \preceq \tau_{2}}{\Gamma \vdash \text { list }_{\tau_{1}} \preceq \text { list }_{\tau_{2}}} \\
& \frac{\Gamma \vdash\{x: \tau \mid \phi\}}{\Gamma \vdash\{x: \tau \mid \phi\} \preceq \tau} \quad \frac{\Gamma \vdash \tau \preceq \sigma \quad \Gamma, x: \tau \vdash \phi}{\Gamma \vdash \tau \preceq\{x: \sigma \mid \phi\}} \quad \frac{\Gamma \vdash \sigma_{2} \preceq \sigma_{1} \quad \Gamma, x: \sigma_{2} \vdash \tau_{1} \preceq \tau_{2}}{\Gamma \vdash \Pi\left(x: \sigma_{1}\right) \cdot \tau_{1} \preceq \Pi\left(x: \sigma_{2}\right) \cdot \tau_{2}} \\
& \overline{\Gamma, x: \tau \vdash x: \tau} \quad \frac{\Gamma, x: \tau \vdash t: \sigma}{\Gamma \vdash \lambda x . t: \Pi(x: \tau) \cdot \sigma} \quad \frac{\Gamma \vdash t_{1}: \Pi(x: \tau) \cdot \sigma \quad \Gamma \vdash t_{2}: \tau}{\Gamma \vdash t_{1} t_{2}: \sigma\left[t_{2} / x\right]} \\
& \frac{\Gamma \vdash \mathrm{t}: \text { list }_{\tau} \quad \Gamma \vdash \mathrm{t}_{1}: \sigma \quad \Gamma \vdash \mathrm{t}_{2}: \Pi(\mathrm{h}: \tau) . \Pi\left(\mathrm{l}: \text { list }_{\tau}\right) . \sigma}{\Gamma \vdash \text { case t of } \square \mapsto \mathrm{t}_{1} ;_{-}::_{-} \mapsto \mathrm{t}_{2}: \sigma} \quad \frac{\Gamma \vdash \tau \preceq \sigma \quad \Gamma \vdash \mathrm{t}: \tau}{\Gamma \vdash \mathrm{t}: \sigma} \\
& \frac{\Gamma, x: \tau, f: \Pi(\{y: \tau \mid y<x\}) \cdot \sigma[y / x] \vdash t: \sigma \quad \operatorname{Def}(f, x, t)}{\Gamma \vdash \operatorname{letrec} f x=t: \Pi(x: \tau) \cdot \sigma}
\end{aligned}
$$

Figure 3.8: Refinement types rules (subtyping and typing)

As usual, we shorten $\Pi(x: \tau) . \sigma$ to $\tau \rightarrow \sigma$ if $x \notin \mathrm{FV}(\sigma)$. We also shorten bindings of the form $x:\{x: \tau \mid \phi\}$ to $\{x: \tau \mid \phi\}$. Typing rules are presented in Figure 3.8; note that the LETREC rule requires that recursive definitions satisfy the well-definedness predicate. Judgments of the form $\Gamma \vdash \tau$ are well-formedness judgments. Judgments of the form $\Gamma \vdash \phi$ are logical judgments; we omit a formal description of the rules, but assume that the logic of assertions is consistent with HOL, i.e. $\Gamma \vdash \phi$ implies $|\Gamma| \mid\lfloor\Gamma\rfloor \vdash \phi$, where the erasure functions are defined below.

This system can be embedded in UHOL. The embedding highlights the relation between these two systems, i.e. between logical assertions embedded in the types (as in refinement types) and logical assertions at the top-level, separate from simple types (as in UHOL). The intuitive idea behind the embedding is therefore to separate refinement assertions from types. Specifically, from every refinement type we can obtain a simple type by repeatedly extracting the type $\tau$ from $\{x: \tau \mid \phi\}$. We represent this extraction using the following translation function $|\tau|$ :

$\mid$ bool $\mid \triangleq$ bool $\quad \mid$ nat $\mid \triangleq$ nat $\quad \mid$ list $_{\tau} \mid \triangleq$ list $_{|\tau|} \quad|\{x: \tau \mid \phi\}| \triangleq|\tau| \quad|\Pi(x: \tau) . \sigma| \triangleq|\tau| \rightarrow|\sigma|$

Since $|\tau|$ loses refinement information, we define a second translation that extracts the refinement as a logical predicate over a variable $x$ that names the typed expression. This second translation is written $\lfloor\tau\rfloor(x)$. 


$$
\begin{array}{cc}
\lfloor\text { bool }\rfloor(x),\lfloor\text { nat }\rfloor(x) \triangleq T & \left\lfloor\text { list }_{\tau}\right\rfloor(x) \triangleq \operatorname{All}(x, \lambda y \cdot\lfloor\tau\rfloor(y)) \\
\lfloor\{y: \tau \mid \phi\}\rfloor(x) \triangleq\lfloor\tau\rfloor(x) \wedge \phi[x / y] & \lfloor\Pi(x: \tau) \cdot \sigma\rfloor(x) \triangleq \forall y \cdot\lfloor\tau\rfloor(y) \Rightarrow\lfloor\sigma\rfloor(x y)
\end{array}
$$

The refinement of simple types is trivial. If $t$ is an expression of type $\{x: \tau \mid \phi\}$, then $t$ must satisfy both the refinement formula $\phi$ and the refinement of $\tau$. The refinement of a list uses the predicate All, which as defined in $\$ 3.3$, means that all elements of a list satisfy a given formula. Finally, if $t$ is an expression of type $\Pi(x: \tau) . \sigma$, then it must be the case that for every $x$ satisfying the refinement of $\tau,(t x)$ satisfies the refinement of $\sigma$.

The syntax of assertions and expressions is exactly the same as in HOL, and therefore there is no need for a translation. The embedding of types can be lifted to contexts in the natural way.

$$
|x: \tau, \Gamma| \triangleq x:|\tau|,|\Gamma| \quad\lfloor x: \tau, \Gamma\rfloor \triangleq\lfloor\tau\rfloor(x),\lfloor\Gamma\rfloor
$$

To encode judgments, all that remains is to put the previous definitions together. The main result about embedding typing judgments is the following:

Theorem 3.11. If $\Gamma \vdash t: \tau$ is derivable in the refinement type system, then $|\Gamma| \mid\lfloor\rfloor\rfloor t$ : $|\tau|\{\lfloor\tau\rfloor(\mathbf{r})\}$ is derivable in UHOL.

Proof. By induction on the derivation. We show the most interesting cases:

Case. $\frac{\Gamma, x: \tau \vdash t: \sigma}{\Gamma \vdash \lambda x . t: \Pi(x: \tau) \cdot \sigma}$

To prove: $|\Gamma||\lfloor\Gamma\rfloor \vdash \lambda x . t:| \Pi(x: \tau) . \sigma \mid\{\lfloor\Pi(x: \tau) . \sigma\rfloor(\mathbf{r})\}$.

Expanding the definitions:

$|\Gamma||\lfloor\Gamma\rfloor \vdash \lambda x . t:| \tau|\rightarrow| \sigma|| \forall x .\lfloor\tau\rfloor(x) \Rightarrow\lfloor\sigma\rfloor(\mathbf{r x})$

By induction hypothesis on the premise:

$|\Gamma|, x:|\tau||\lfloor\Gamma\rfloor,\lfloor\tau\rfloor(x) \vdash t:| \sigma||\lfloor\sigma\rfloor(\mathbf{r})$

Directly by ABS.

Case. $\frac{\Gamma \vdash t: \Pi(x: \tau) \cdot \sigma \quad \Gamma \vdash \mathfrak{u}: \tau}{\Gamma \vdash t \mathfrak{u}: \sigma[u / x]}$

To prove: $|\Gamma||\lfloor\Gamma\rfloor \vdash \mathrm{t} u:| \sigma[u / x] \mid\{\lfloor\sigma[u / x]\rfloor(\mathbf{r})\}$.

Expanding the definitions:

$|\Gamma|\left|\lfloor\Gamma\rfloor \vdash \mathrm{t} e_{2}:\right| \sigma||\lfloor\sigma\rfloor(\mathbf{r})[u / x]$

By induction hypothesis on the premise:

$|\Gamma||\lfloor\Gamma\rfloor \vdash \mathrm{t}:| \tau|\rightarrow| \sigma|| \forall \mathrm{x} .\lfloor\tau\rfloor(\mathrm{x}) \Rightarrow\lfloor\sigma\rfloor(\mathbf{r} \mathrm{x})$

and

$|\Gamma||\lfloor\Gamma\rfloor \vdash u:| \tau||\lfloor\tau\rfloor(\mathbf{r})$

We get the result directly by APP. 
Case. $\frac{\Gamma \vdash \tau \preceq \sigma \quad \Gamma \vdash t: \tau}{\Gamma \vdash t: \sigma}$

To prove: $|\Gamma||\lfloor\Gamma\rfloor \vdash \mathrm{t}:| \sigma \mid\{\lfloor\sigma\rfloor(\mathbf{r})\}$.

This follows from applying the induction hypothesis to the second premise, and then applying the rule SUB with Theorem 3.12 below. Note that the first premise implies $|\sigma| \equiv|\tau|$.

Case. $\frac{\Gamma, x: \tau, f: \Pi(y:\{r: \tau \mid y<x\}) \cdot \sigma[y / x] \vdash t: \sigma \quad \operatorname{Def}(f, x, t)}{\Gamma \vdash \operatorname{letrec} f x=t: \Pi(x: \tau) \cdot \sigma}$

To prove: $|\Gamma||\lfloor\Gamma\rfloor \vdash \operatorname{letrec} f \mathrm{x}=\mathrm{t}:| \Pi(\mathrm{x}: \tau) \cdot \sigma \mid\{\lfloor\Pi(\mathrm{x}: \tau) \cdot \sigma\rfloor(\mathbf{r})\}$

By induction hypothesis on the premise:

$|\Gamma|, x:|\tau|, f:|\tau| \rightarrow|\sigma||\lfloor\Gamma\rfloor,\lfloor\tau\rfloor(x), \forall y .\lfloor\tau\rfloor(y) \wedge y<x \Rightarrow\lfloor\sigma[y / x]\rfloor(f y) \vdash t:| \sigma||\lfloor\sigma\rfloor(\mathbf{r})$

Directly by LETREC.

The above proof relies on the following theorem about subtyping.

Theorem 3.12. If $\Gamma \vdash \tau \preceq \sigma$ is derivable in a refinement type system, then the judgment $|\Gamma|, x:|\tau| \mid\lfloor\Gamma\rfloor,\lfloor\tau\rfloor(x) \vdash\lfloor\sigma\rfloor(x)$ is derivable in $H O L$.

Proof. The proof is by induction on the derivation. We show here only the case of the dependent product:

Case. $\frac{\Gamma \vdash \sigma_{2} \preceq \sigma_{1} \quad \Gamma, x: \sigma_{2} \vdash \tau_{1} \preceq \tau_{2}}{\Gamma \vdash \Pi\left(x: \sigma_{1}\right) \cdot \tau_{1} \preceq \Pi\left(x: \sigma_{2}\right) \cdot \tau_{2}}$

To show: $|\Gamma|, f:\left|\Pi\left(x: \sigma_{1}\right) \cdot \tau_{1}\right| \mid\lfloor\Gamma\rfloor,\left\lfloor\Pi\left(x: \sigma_{1}\right) \cdot \tau_{1}\right\rfloor(f) \vdash\left\lfloor\Pi\left(x: \sigma_{2}\right) \cdot \tau_{2}\right\rfloor(f)$

Expanding the definitions:

$|\Gamma|, f:\left|\Pi\left(x: \sigma_{1}\right) \cdot \tau_{1}\right| \mid\lfloor\Gamma\rfloor, \forall x .\left\lfloor\sigma_{1}\right\rfloor(x) \Rightarrow\left\lfloor\tau_{1}\right\rfloor(f x) \vdash \forall x .\left\lfloor\sigma_{2}\right\rfloor(x) \Rightarrow\left\lfloor\tau_{2}\right\rfloor(f x)$

By the rules $\forall_{\mathrm{I}}$ and $\Rightarrow_{\mathrm{I}}$ it suffices to prove:

$|\Gamma|, f:\left|\Pi\left(x: \sigma_{1}\right) . \tau_{1}\right|, x:\left|\sigma_{2}\right| \mid\lfloor\Gamma\rfloor, \forall x .\left\lfloor\sigma_{1}\right\rfloor(x) \Rightarrow\left\lfloor\tau_{1}\right\rfloor(f x),\left\lfloor\sigma_{2}\right\rfloor(x) \vdash\left\lfloor\tau_{2}\right\rfloor(f x)$

On the other hand, by induction hypothesis on the premises:

$|\Gamma|, x:\left|\sigma_{2}\right| \mid\lfloor\Gamma\rfloor,\left\lfloor\sigma_{2}\right\rfloor(x) \vdash\left\lfloor\sigma_{1}\right\rfloor(x)$

and

$|\Gamma|, x:\left|\sigma_{2}\right|, y:\left|\tau_{1}\right| \mid\lfloor\Gamma\rfloor,\left\lfloor\sigma_{2}\right\rfloor(x),\left\lfloor\tau_{1}\right\rfloor(y) \vdash\left\lfloor\tau_{2}\right\rfloor(y)$

which we can weaken respectively to:

$|\Gamma|, x:\left|\sigma_{2}\right|, f:\left|\Pi\left(x: \sigma_{1}\right) \cdot \tau_{1}\right||| \Gamma \mid,\left\lfloor\sigma_{2}\right\rfloor(x), \forall x .\left\lfloor\sigma_{1}\right\rfloor(x) \Rightarrow\left\lfloor\tau_{1}\right\rfloor(f x) \vdash\left\lfloor\sigma_{1}\right\rfloor(x)$

and

$|\Gamma|, x:\left|\sigma_{2}\right|, y:\left|\tau_{1}\right|, f:\left|\Pi\left(x: \sigma_{1}\right) \cdot \tau_{1}\right||| \Gamma \mid,\left\lfloor\sigma_{2}\right\rfloor(x),\left\lfloor\tau_{1}\right\rfloor(y), \forall x .\left\lfloor\sigma_{1}\right\rfloor(x) \Rightarrow\left\lfloor\tau_{1}\right\rfloor(f x) \vdash\left\lfloor\tau_{2}\right\rfloor(y)$

(5)

From (4), by doing a cut with its own premise $\forall x .\left\lfloor\sigma_{1}\right\rfloor(x) \Rightarrow\left\lfloor\tau_{1}\right\rfloor(f x)$, we derive:

$|\Gamma|, x:\left|\sigma_{2}\right|, f:\left|\Pi\left(x: \sigma_{1}\right) \cdot \tau_{1}\right| \mid\lfloor\Gamma\rfloor,\left\lfloor\sigma_{2}\right\rfloor(x), \forall x .\left\lfloor\sigma_{1}\right\rfloor(x) \Rightarrow\left\lfloor\tau_{1}\right\rfloor(f x) \vdash\left\lfloor\tau_{1}\right\rfloor(\mathrm{fx})$

From (5), by $\Rightarrow_{\mathrm{I}}$ and $\forall_{\mathrm{I}}$ we can derive:

$|\Gamma|, x:\left|\sigma_{2}\right|, f:\left|\Pi\left(x: \sigma_{1}\right) \cdot \tau_{1}\right| \mid\lfloor\Gamma\rfloor,\left\lfloor\sigma_{2}\right\rfloor(x),, \forall x .\left\lfloor\sigma_{1}\right\rfloor(x) \Rightarrow\left\lfloor\tau_{1}\right\rfloor(f x) \vdash \forall y .\left\lfloor\tau_{1}\right\rfloor(y) \Rightarrow\left\lfloor\tau_{2}\right\rfloor(y)$

And by $\forall_{E}$

$|\Gamma|, x:\left|\sigma_{2}\right|, f:\left|\Pi\left(x: \sigma_{1}\right) \cdot \tau_{1}\right| \mid\lfloor\Gamma\rfloor,\left\lfloor\sigma_{2}\right\rfloor(x), \forall x .\left\lfloor\sigma_{1}\right\rfloor(x) \Rightarrow\left\lfloor\tau_{1}\right\rfloor(f x) \vdash\left\lfloor\tau_{1}\right\rfloor(f x) \Rightarrow\left\lfloor\tau_{2}\right\rfloor(f x)(7)$

Finally, from (6) and (7) by $\Rightarrow_{E}$ we get: 
$|\Gamma|, x:\left|\sigma_{2}\right|, f:\left|\Pi\left(x: \sigma_{1}\right) . \tau_{1}\right| \mid\lfloor\Gamma\rfloor,\left\lfloor\sigma_{2}\right\rfloor(x), \forall x .\left\lfloor\sigma_{1}\right\rfloor(x) \Rightarrow\left\lfloor\tau_{1}\right\rfloor(f x) \vdash\left\lfloor\tau_{2}\right\rfloor(f x)$ and by one last application of $\Rightarrow_{\mathrm{I}}$ we get what we wanted to prove.

Soundness of refinement types w.r.t. the set-theoretical semantics follows immediately from Theorem 3.11 and the set-theoretical soundness of UHOL (Corollary 3.4).

Corollary 3.13 (Soundness of refinement types). If $\Gamma \vdash t: \tau$, then for every valuation $\rho \models \Gamma$ we have $\llbracket t \rrbracket_{\rho} \in \llbracket \tau \rrbracket_{\rho}$.

As a final remark, note that a function with a refinement type can be interpreted in two different ways: 1) As a map whose domain is the domain type restricted to its (the type's) refinement, or 2) As a map whose domain is the entire domain type (disregarding the refinement), but whose result satisfies the co-domain's refinement only if the argument satisfies the domain's refinement. We use the second interpretation, while some prior work, for example [76], uses the first. Type systems using the first interpretation can inhabit types that this embedding would erase into uninhabited simple types. For instance, consider the type $\{x:$ nat $|x\rangle$ $1 \wedge x<1\} \rightarrow$ Emp where Emp denotes the empty type. This is inhabited (under appropriate subtyping rules), but it would be erased into the type nat $\rightarrow$ Emp, which is not inhabited.

\subsubsection{Relational Refinement Types}

Relational refinement types $[29,33]$ are a variant of refinement types that can be used to express relational properties via a syntax of the form $\{\mathbf{r}:: \tau \mid \phi\}$ where $\phi$ is a relational assertion-i.e. it may contain a left and right copy of $\mathbf{r}$, which are denoted as $\mathbf{r}_{1}$ and $\mathbf{r}_{2}$ respectively, as well as a left and a right copy of every variable in the context. In this section, we introduce a simple relational refinement type system and establish a type-preserving translation to RHOL.

The syntax of relational refinement types is given by the grammar:

$$
\begin{aligned}
\tau:: & =\text { bool } \mid \text { nat } \mid \tau \rightarrow \tau \\
\mathrm{T}, \mathrm{U} & ::=\tau\left|\operatorname{list}_{\mathrm{T}}\right| \Pi(\mathrm{x}:: \mathrm{T}) . \mathrm{U} \mid\{x:: \mathrm{T} \mid \phi\}
\end{aligned}
$$

Relational refinement types are naturally ordered by a subtyping relation $\Gamma \vdash$ $\mathrm{T} \preceq \mathrm{U}$, where $\Gamma$ is a sequence of variables declarations of the form $x:: \mathrm{U}$.

Typing judgments are of the form $\Gamma \vdash t_{1} \sim t_{2}:: T$. We present selected typing rules in Figure 3.9. Note that the form of judgments requires that $t_{1}$ and $t_{2}$ have the same simple type, and the typing rules require that $t_{1}$ and $t_{2}$ have the same structure. ${ }^{1}$ In the CASELIST rule, we require that both terms reduce to the same

\footnotetext{
${ }^{1}$ The typing rules displayed in the figure will in fact force $t_{1}$ and $t_{2}$ to be the same term modulo renaming. This is not the case in existing relational refinement type systems; however, rules that introduce different terms on the right and on the left are limited, since both terms still need to have the same type and most one-sided rules break this invariant. For instance, in [29] there is a rule similar to LLCASE-A, and a rule for reducing one of the terms of a judgment.
} 


$$
\begin{aligned}
& \text { VAR-RT } \frac{(x: T) \in \Gamma}{\Gamma \vdash x_{1} \sim x_{2}:: T} \\
& \text { ABS-RT } \frac{\Gamma, x:: T \vdash u_{1} \sim u_{2}:: U}{\Gamma \vdash \lambda x_{1} \cdot u_{1} \sim \lambda x_{2} \cdot u_{2}:: \Pi(x:: T) . u} \\
& \text { APP-RT } \frac{\Gamma \vdash \mathrm{t}_{1} \sim \mathrm{t}_{2}:: \Pi(\mathrm{x}:: \mathrm{T}) . \mathrm{U} \quad \Gamma \vdash \mathrm{u}_{1} \sim \mathrm{u}_{2}:: \mathrm{T}}{\Gamma \vdash \mathrm{t}_{1} \mathrm{u}_{1} \sim \mathrm{t}_{2} \mathrm{u}_{2}:: \mathrm{U}\left[\mathrm{u}_{1} / \mathrm{x}_{1}\right]\left[\mathrm{u}_{2} / \mathrm{x}_{2}\right]} \quad \mathrm{NIL} \frac{\Gamma \vdash \mathrm{T}}{\Gamma \vdash \square \sim \square:: \text { list }} \\
& \text { CONS } \frac{\Gamma \vdash h_{1} \sim h_{2}:: T \quad \Gamma \vdash \mathrm{t}_{1} \sim \mathrm{t}_{2}:: \text { list }_{\mathrm{T}}}{\Gamma \vdash \mathrm{h}_{1}:: \mathrm{t}_{1} \sim \mathrm{h}_{2}:: \mathrm{t}_{2}:: \text { list }_{\mathrm{T}}} \quad \text { SUB } \frac{\Gamma \vdash \mathrm{t}_{1} \sim \mathrm{t}_{2}:: \mathrm{T} \quad \Gamma \vdash \mathrm{T} \preceq \mathrm{U}}{\Gamma \vdash \mathrm{t}_{1} \sim \mathrm{t}_{2}:: \mathrm{U}} \\
& \Gamma, x:: T, f:: \Pi\left(\left\{y:: T \mid\left(y_{1}, y_{2}\right)<\left(x_{1}, x_{2}\right)\right\}\right) . U[y / x] \vdash t_{1} \sim t_{2}:: U \\
& \text { LETREC-RT } \frac{\Gamma \vdash \Pi(x:: \mathrm{T}) . \mathrm{U} \quad \operatorname{Def}(\mathrm{f}, \mathrm{x}, \mathrm{t})}{\Gamma \vdash \text { letrec } \mathrm{f}_{1} \mathrm{x}_{1}=\mathrm{t}_{1} \sim \operatorname{letrec} \mathrm{f}_{2} \mathrm{x}_{2}=\mathrm{t}_{2}:: \Pi(\mathrm{x}:: \mathrm{T}) . \mathrm{U}}
\end{aligned}
$$

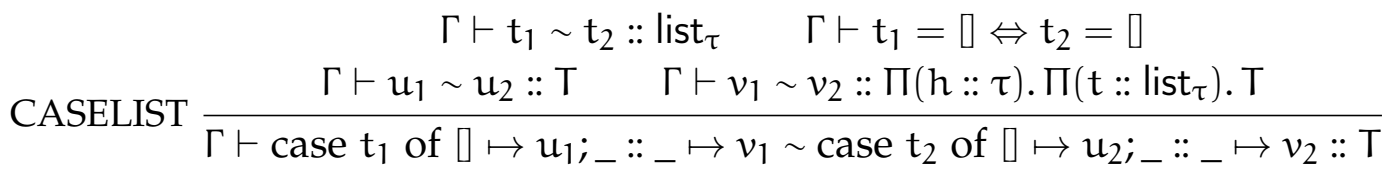

Figure 3.9: Relational Typing (Selected Rules)

branch; the case rule for natural numbers is similar. The LETREC rule uses (a straightforward adaptation of) the $\operatorname{Def}(f, x, t)$ predicate from our simply-typed language, and requires that the two recursive definitions perform exactly the same recursive calls.

Subtyping rules are the same as in the unary case, and therefore we refer to Figure 3.8 for them (allowing their instantiation for relational types $\mathrm{T}, \mathrm{U}$ as well as unary types $\sigma, \tau)$.

The embedding of refinement types into UHOL can be adapted to the relational setting. From each relational refinement type $\mathrm{T}$ we can extract a simple type $|\mathrm{T}|$. On the other hand, we can erase every relational refinement type $T$ into a relational formula $\llbracket T \Downarrow$, which is parametrized by two expressions and defined as follows: 


$$
\begin{aligned}
& \llbracket \operatorname{list}_{\tau} \rrbracket\left(x_{1}, x_{2}\right) \triangleq \bigwedge_{i \in\{1,2\}} \operatorname{All}\left(x_{i}, \lambda y \cdot\lfloor\tau\rfloor(y)\right) \\
& \|\operatorname{list} \mathrm{T}\|\left(x_{1}, x_{2}\right) \triangleq \operatorname{All2}\left(x_{1}, x_{2}, \lambda y_{1} \cdot \lambda y_{2} \cdot \| T \rrbracket\left(y_{1}, y_{2}\right)\right) \\
& \llbracket\{y: \tau \mid \phi\} \rrbracket\left(x_{1}, x_{2}\right) \triangleq \bigwedge_{i \in\{1,2\}}\lfloor\tau\rfloor\left(x_{i}\right) \wedge \phi\left[x_{i} / y\right] \\
& \llbracket\{y:: T \mid \phi\} \rrbracket\left(x_{1}, x_{2}\right) \triangleq \llbracket T \Downarrow\left(x_{1}, x_{2}\right) \wedge \phi\left[x_{1} / y_{1}\right]\left[x_{2} / y_{2}\right] \\
& \llbracket \Pi(y: \tau) \cdot \sigma \Downarrow(x) \triangleq \bigwedge_{i \in\{1,2\}} \forall y \cdot\lfloor\tau\rfloor(y) \Rightarrow\lfloor\sigma\rfloor(x y) \\
& \llbracket \Pi(y:: T) . u \Downarrow\left(x_{1}, x_{2}\right) \triangleq \forall y_{1} y_{2} \cdot \| T \Downarrow\left(y_{1}, y_{2}\right) \Rightarrow \Downarrow u \Downarrow\left(x_{1} y_{1}, x_{2} y_{2}\right)
\end{aligned}
$$

The predicate All2 relates two lists element-wise and is defined axiomatically:

$$
\begin{gathered}
\operatorname{All2}\left(\left[,\left[, \lambda x_{1} \cdot \lambda x_{2} \cdot \phi\right)\right.\right. \\
\forall h_{1} h_{2} t_{1} t_{2} \cdot \operatorname{All2}\left(t_{1}, t_{2}, \lambda x_{1} \cdot \lambda x_{2} \cdot \phi\right) \Rightarrow \phi\left(h_{1}, h_{2}\right) \Rightarrow \operatorname{All} 2\left(h_{1}:: t_{1}, h_{2}:: t_{2}, \lambda x_{1} \cdot \lambda x_{2} \cdot \phi\right)
\end{gathered}
$$

To extend the embedding to contexts, we need to duplicate every variable in them:

$$
|x:: T, \Gamma| \triangleq x_{1}, x_{2}:|T|,|\Gamma| \quad \quad \Downarrow x:: T, \Gamma \rrbracket \triangleq \llbracket T \Downarrow\left(x_{1}, x_{2}\right), \llbracket \Gamma \rrbracket
$$

Now we state the main result:

Theorem 3.14 (Soundness of embedding). If $\Gamma \vdash \mathrm{t}_{1} \sim \mathrm{t}_{2}:: \mathrm{T}$, then $|\Gamma| \mid\left\lfloor\Gamma \downarrow \vdash \mathrm{t}_{1}:|\mathrm{T}| \sim\right.$ $\mathrm{t}_{2}:|\mathrm{T}|\left\{\llbracket \mathrm{T} \rrbracket\left(\mathbf{r}_{1}, \mathbf{r}_{2}\right)\right\}$. Also, if $\Gamma \vdash \mathrm{T} \preceq \mathrm{U}$ then $|\Gamma|, \mathrm{x}_{1}, \mathrm{x}_{2}:|\mathrm{T}||| \llbracket \Gamma \rrbracket, \llbracket \mathrm{T} \Downarrow\left(\mathrm{x}_{1}, \mathrm{x}_{2}\right) \vdash \Downarrow \mathrm{U} \Downarrow\left(\mathrm{x}_{1} . \mathrm{x}_{2}\right)$.

Proof. The proof proceeds by induction on the structure of derivations. Most cases are very similar to the unary case, so we will only show the most interesting ones:

Case. $\frac{\Gamma \vdash \mathrm{T}}{\Gamma \vdash \square \sim \square:: \text { list }_{\mathrm{T}}}$

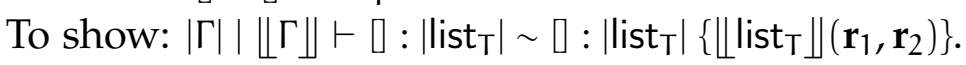

There are two options. If $\mathrm{T}$ is a unary type, we have to prove:

$|\Gamma| \mid\left\lfloor\Gamma \rrbracket \vdash \square: \|\right.$ ist $_{T}\left|\sim \square: \| \operatorname{list}_{T}\right|\left\{\bigwedge_{i \in\{1,2\}} \operatorname{All}\left(\mathbf{r}_{i}, \lambda x .\lfloor\tau \mid(x))\right\}\right.$

And by the definition of All we can directly prove:

$\emptyset \mid \emptyset \vdash \operatorname{All}(\square, \lambda x .\lfloor\tau\rfloor(x)) \wedge \operatorname{All}(\square, \lambda x .\lfloor\tau\rfloor(x))$

If $\mathrm{T}$ is a relational type, we have to prove: 
$|\Gamma| \mid \llbracket \Gamma \rrbracket \vdash \square: \|$ ist $_{T} \mid \sim \square: \|$ ist $_{T} \mid\left\{\operatorname{All} 2\left(\mathbf{r}_{1}, \mathbf{r}_{2}, \lambda x_{1} \cdot \lambda x_{2} \cdot \| T \Downarrow\left(x_{1}, x_{2}\right)\right)\right\}$

And by the definition of All2 we can directly prove:

$\emptyset \mid \emptyset \vdash \operatorname{All2}\left(\square, \square, \lambda x_{1} \cdot \lambda x_{2} \cdot \llbracket T \Downarrow\left(x_{1}, x_{2}\right)\right)$

Case. $\frac{\Gamma \vdash h_{1} \sim h_{2}:: T \quad \Gamma \vdash t_{1} \sim t_{2}:: \text { list }_{T}}{\Gamma \vdash h_{1}:: t_{1} \sim h_{2}:: t_{2}:: \text { list }_{T}}$

To show: $|\Gamma|||\left|\Gamma \rrbracket \vdash h_{1}:: \mathrm{t}_{2}:\right|$ list $_{\mathrm{T}} \mid \sim \mathrm{h}_{2}:: \mathrm{t}_{2}: \|$ list $_{\mathrm{T}} \mid\left\{\right.$ list $\left._{\top}\right\}$.

There are two options. If $\mathrm{T}$ is a unary type, we have to prove:

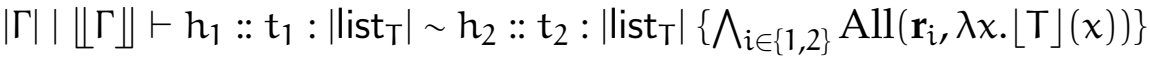

By induction hypothesis we have:

$|\Gamma| \mid\left\lfloor\Gamma \rrbracket \vdash h_{1}:|T| \sim h_{2}:: t_{2}:|T|\left\{\bigwedge_{i \in\{1,2\}} \mid T\right\rfloor\left(\mathbf{r}_{i}\right)\right\}$

and

$|\Gamma| \mid\left\lfloor\Gamma \rrbracket \vdash \mathrm{t}_{1}: \|\right.$ ist $_{\mathrm{T}} \mid \sim \mathrm{t}_{2}: \|$ ist $_{\mathrm{T}} \mid\left\{\bigwedge_{\mathrm{i} \in\{1,2\}} \operatorname{All}\left(\mathbf{r}_{i}, \lambda \mathrm{x} .[\mathrm{T}\rfloor(\mathrm{x})\right)\right\}$

And by the definition of All we can directly prove:

$\bigwedge_{i \in\{1,2\}}[T]\left(h_{i}\right) \Rightarrow \bigwedge_{i \in\{1,2\}} \operatorname{All}\left(t_{i}, \lambda x .\lfloor T\rfloor(x)\right) \Rightarrow \bigwedge_{i \in\{1,2\}} \operatorname{All}\left(h_{i}:: t_{i}, \lambda x .\lfloor T\rfloor(x)\right)$

So by the CONS rule, we prove the result. If $\mathrm{T}$ is a relational type, we have to prove:

$|\Gamma| \mid\left\|\Gamma \rrbracket \vdash \mathrm{h}_{1}:: \mathrm{t}_{1}:\right\|$ ist $_{\mathrm{T}} \mid \sim \mathrm{h}_{2}:: \mathrm{t}_{2}: \|$ ist $_{\mathrm{T}} \mid\left\{\operatorname{All} 2\left(\mathbf{r}_{1}, \mathbf{r}_{2}, \lambda \mathrm{x}_{1} . \lambda \mathrm{x}_{2} \cdot \| \mathrm{T} \Downarrow\left(\mathrm{x}_{1}, \mathrm{x}_{2}\right)\right)\right\}$

By induction hypothesis we have:

$|\Gamma|\left|\llbracket \Gamma \Downarrow \vdash \mathrm{h}_{1}:\right| \mathrm{T}\left|\sim \mathrm{h}_{2}:: \mathrm{t}_{2}:\right| \mathrm{T} \mid\left\{\left\lfloor\mathrm{T} \rrbracket\left(\mathbf{r}_{1}, \mathbf{r}_{2}\right)\right\}\right.$

and

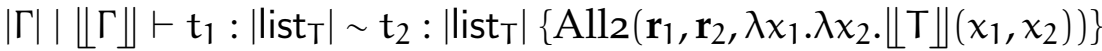

And by the definition of All2 we can directly prove:

$\| T \Downarrow\left(h_{1}, h_{2}\right) \Rightarrow \operatorname{All} 2\left(t_{1}, t_{2}, \lambda x_{1} \cdot \lambda x_{2} \cdot \| T \Downarrow\left(x_{1}, x_{2}\right)\right) \Rightarrow \operatorname{All}\left(h_{1}:: t_{1}, h_{1}:: h_{2}, \lambda x_{1} \cdot \lambda x_{2} \cdot \| T \Downarrow\left(x_{1}, x_{2}\right)\right)$

So by the CONS rule, we prove the result.

$$
\Gamma, x:: T, f:: \Pi\left(y::\left\{y:: T \mid\left(y_{1}, y_{2}\right)<\left(x_{1}, x_{2}\right)\right\}\right) . u[y / x] \vdash t_{1} \sim t_{2}:: U
$$

Case. $\frac{\Gamma \vdash \Pi(x:: \mathrm{T}) . \mathrm{U} \quad \operatorname{Def}\left(\mathrm{f}_{1}, \mathrm{x}_{1}, \mathrm{t}_{1}\right) \quad \operatorname{Def}\left(\mathrm{f}_{2}, \mathrm{x}_{2}, \mathrm{t}_{2}\right)}{\Gamma \vdash \text { letrec } \mathrm{f}_{1} \mathrm{x}_{1}=\mathrm{t}_{1} \sim \operatorname{letrec} \mathrm{f}_{2} \mathrm{x}_{2}=\mathrm{t}_{2}: \because \Pi(\mathrm{x}:: \mathrm{T}) . \mathrm{U}}$

To show:

$|\Gamma||| \mid \Gamma \Downarrow \vdash$ letrec $f_{1} \mathrm{x}_{1}=\mathrm{t}_{1}:|\Pi(\mathrm{x}:: \mathrm{T}) . \mathrm{U}| \sim \operatorname{letrec} f_{2} \mathrm{x}_{2}=\mathrm{t}_{2}:|\Pi(\mathrm{x}:: \mathrm{T}) . \mathrm{U}|\{$

$\left.\llbracket \Pi(x:: T) . u \Downarrow\left(\mathbf{r}_{1}, \mathbf{r}_{2}\right)\right\}$

Expanding the definitions:

$|\Gamma||| \llbracket \Gamma \rrbracket \vdash$ letrec $f_{1} \mathrm{x}_{1}=\mathrm{t}_{1}:|\mathrm{T}| \rightarrow|\mathrm{U}| \sim \operatorname{letrec} f_{2} \mathrm{x}_{2}=\mathrm{t}_{2}:|\mathrm{T}| \rightarrow|\mathrm{U}|\left\{\forall \mathrm{x}_{1} \mathrm{x}_{2} \cdot \| \mathrm{T} \rrbracket\left(\mathrm{x}_{1}, \mathrm{x}_{2}\right) \Rightarrow\right.$ $\left.\llbracket \mathrm{u} \Downarrow\left(\mathbf{r}_{1} x_{1}, \mathbf{r}_{2} x_{2}\right)\right\}$

By induction hypothesis on the premise:

$|\Gamma|, x_{1}, x_{2}:|T|, f_{1}, f_{2}:|T| \rightarrow|\mathrm{U}||\downarrow| \Gamma \rrbracket,\left\lfloor T \Downarrow\left(x_{1}, x_{2}\right)\right.$,

$\forall y_{1}, y_{2} \cdot\left(\llbracket T \Downarrow\left(y_{1}, y_{2}\right) \wedge\left(y_{1}, y_{2}\right)<\left(x_{1}, x_{2}\right)\right) \Rightarrow \llbracket u \rrbracket\left(f_{1} x_{1}, f_{2} x_{2}\right) \vdash$

$\mathrm{t}_{1}:|\mathrm{U}| \sim \mathrm{t}_{2}:|\mathrm{U}|\left\{\left\lfloor\mathrm{U} \rrbracket\left(\mathbf{r}_{1}, \mathbf{r}_{2}\right)\right\}\right.$

And we apply the LETREC rule to get the result.

Soundness of relational refinement types w.r.t. set-theoretical semantics follows immediately from Theorem 3.14 and the set-theoretical soundness of RHOL (Corol- 
lary 8.14).

Corollary 3.15 (Soundness of relational refinement types). If $\Gamma \vdash t_{1} \sim t_{2}:: \mathrm{T}$, then for every valuation $\theta \models \Gamma$ we have $\left(\left(\mathrm{t}_{1}\right)_{\theta},\left(\mathrm{t}_{2}\right)_{\theta}\right) \in(\mathrm{T})_{\theta}$.

\subsubsection{Dependency Core Calculus}

The Dependency Core Calculus (DCC) [2] is a higher-order calculus with a type system that tracks data dependencies. DCC was designed as a unifying framework for dependency analysis and it was shown that many other calculi for information flow analysis [92, 157], binding-time analysis [91], and program slicing, all of which track dependencies, can be embedded in DCC. Here, we show how a fragment of DCC can be embedded into RHOL. Transitively, the corresponding fragments of all the aforementioned calculi can also be embedded in RHOL. The fragment of DCC we consider excludes recursive functions. DCC admits general recursive functions, while our definition of RHOL only admits a subset of these. Extending the embedding to recursive functions admitted by RHOL is not difficult.

DCC is an extension of the simply typed lambda-calculus with a monadic type family $\mathbb{T}_{\ell}(\tau)$, indexed by labels $\ell$, which are elements of a lattice. Unlike other uses of monads, DCC's monad does not encapsulate any effects. Instead, its only goal is to track dependence. The type system forces that the result of an expression of type $\mathbb{T}_{\ell}(\tau)$ can depend on an expression of type $\mathbb{T}_{\ell^{\prime}}\left(\tau^{\prime}\right)$ only if $\ell^{\prime} \sqsubseteq \ell$ in the lattice. Dually, if $\ell^{\prime} \nsubseteq \ell$, then even if an expression e of type $\mathbb{T}_{\ell}(\tau)$ mentions a variable $x$ of type $\mathbb{T}_{\ell^{\prime}}\left(\tau^{\prime}\right)$, then $e^{\prime}$ s result must be independent of the substitution provided for $x$ during evaluation.

For simplicity and without any loss of generality, we consider here only a two point lattice $\{L, H\}$ with $L \sqsubset H$. The syntax of DCC's types and expressions is shown below. We use e to denote DCC expressions, to avoid confusion with HOL's expressions.

$$
\begin{aligned}
\tau::= & \text { bool }|\tau \rightarrow \tau| \tau \times \tau \mid \mathbb{T}_{\ell}(\tau) \\
e::= & x|\lambda x . e| e_{1} e_{2}|\mathrm{tt}| \mathrm{ff} \mid \text { case } e \text { of } \mathrm{tt} \mapsto e_{\mathrm{t}} ; \mathrm{ff} \mapsto e_{\mathrm{f}}\left|\left\langle e_{1}, e_{2}\right\rangle\right| \pi_{1}(e) \mid \pi_{2}(e) \\
& \left|\eta_{\ell}(e)\right| \operatorname{bind}\left(e_{1}, x . e_{2}\right)
\end{aligned}
$$

Here, $\eta_{\ell}(e)$ and bind $\left(e_{1}, x . e_{2}\right)$ are respectively the return and bind constructs for the monad $\mathbb{T}_{\ell}(\tau)$. Typing rules for these two constructs are shown below. Typing rules for the remaining constructs are the standard ones.

$$
\frac{\Gamma \vdash e: \tau}{\Gamma \vdash \eta_{\ell}(e): \mathbb{T}_{\ell}(\tau)} \quad \frac{\Gamma \vdash e_{1}: \mathbb{T}_{\ell}\left(\tau_{1}\right) \quad \Gamma, x: \tau_{1} \vdash e_{2}: \tau_{2} \quad \tau_{2} \searrow \ell}{\Gamma \vdash \operatorname{bind}\left(e_{1}, x . e_{2}\right): \tau_{2}}
$$

The crux of the dependency tracking is the relation $\tau_{2} \searrow \ell$ in the premise of the rule for bind. The relation, read " $\tau_{2}$ protected at level $\ell$ " and defined below, informally 
means that all primitive (boolean) values extractable from $e_{2}$ are protected by a monadic construct of the form $\mathbb{T}_{\ell^{\prime}}(\tau)$, with $\ell \sqsubseteq \ell^{\prime}$. Hence, the rule forces that the result obtained by eliminating the type $\mathbb{T}_{\ell}\left(\tau_{1}\right)$ flow only into types protected at $\ell$ in this sense.

$$
\frac{\ell \sqsubseteq \ell^{\prime}}{\mathbb{T}_{\ell^{\prime}}(\tau) \searrow \ell} \quad \frac{\tau \searrow \ell}{\mathbb{T}_{\ell^{\prime}}(\tau) \searrow \ell} \quad \frac{\tau_{1} \searrow \ell \quad \tau_{2} \searrow \ell}{\tau_{1} \times \tau_{2} \searrow \ell} \quad \frac{\tau_{2} \searrow \ell}{\tau_{1} \rightarrow \tau_{2} \searrow \ell}
$$

This fragment of DCC has a relational set-theoretical interpretation. For every type $\tau$, we define a carrier set $|\tau|$ :

$$
\text { |bool } \mid \triangleq \text { bool } \quad\left|\tau_{1} \rightarrow \tau_{2}\right| \triangleq\left|\tau_{1}\right| \rightarrow\left|\tau_{2}\right| \quad\left|\tau_{1} \times \tau_{2}\right| \triangleq\left|\tau_{1}\right| \times\left|\tau_{2}\right| \quad\left|\mathbb{T}_{\ell}(\tau)\right| \triangleq|\tau|
$$

Next, every type $\tau$ is interpreted as a lattice-indexed family of relations $\lfloor\tau\rfloor_{a} \subseteq|\tau| \times|\tau|$. The role of the lattice element $a$ is that it defines what can be observed in the system. Specifically, an expression of type $\mathbb{T}_{\ell}(\tau)$ can be observed only if $\ell \sqsubseteq a$. When $\ell \nsubseteq$ a, expressions of type $\mathbb{T}_{\ell}(\tau)$ look like "black-boxes". Technically, we force $\left\lfloor\mathbb{T}_{\ell}(\tau)\right\rfloor_{\mathrm{a}}=|\tau| \times|\tau|$ when $\ell \nsubseteq \mathrm{a}$. DCC's typing rules are sound with respect to this model. The soundness implies that if $\ell \nsubseteq \ell^{\prime}$ and $x: \mathbb{T}_{\ell}($ bool $) \vdash e: \mathbb{T}_{\ell^{\prime}}($ bool $)$, then for $e_{1}, e_{2}: \mathbb{T}_{\ell}($ bool $), e\left[e_{1} / x\right]$ and $e\left[e_{2} / x\right]$ are equal booleans in the set-theoretical model. This result, called noninterference, formalizes that DCC's dependency tracking is correct.

To translate DCC to RHOL, we actually embed this set-theoretical model in RHOL. We start by defining an erasing translation, $|\tau|$, from DCC's types into RHOL's simple types. This translation is exactly the same as the definition of carrier sets shown above, except that we treat $\times$ and $\rightarrow$ as RHOL's syntactic type constructs instead of set-theoretical constructs. Next, we define an erasure of terms:

$$
\begin{array}{cccc}
|\mathrm{tt}| & \triangleq \mathrm{tt} \quad|\mathrm{ff}| \triangleq \mathrm{ff} \quad \mid \text { case } e \text { of } \mathrm{tt} \mapsto e_{\mathrm{t}} ; \mathrm{ff} \mapsto e_{\mathrm{f}} \mid \triangleq \text { case }|e| \text { of } \mathrm{tt} \mapsto\left|e_{\mathrm{t}}\right| ; \mathrm{ff} \mapsto\left|e_{\mathrm{f}}\right| \\
|x| & \triangleq x \quad|\lambda x . e| \triangleq \lambda x .|e| & \left|e_{1} e_{2}\right| \triangleq\left|e_{1}\right|\left|e_{2}\right| & \left|\left\langle e_{1}, e_{2}\right\rangle\right| \triangleq\left\langle\left|e_{1}\right|,\left|e_{2}\right|\right\rangle \\
\left|\pi_{1}(e)\right| & \triangleq \pi_{1}(|e|) \quad\left|\pi_{2}(e)\right| \triangleq \pi_{2}(|e|) & \left|\eta_{\ell}(e)\right| \triangleq|e| & \left|\operatorname{bind}\left(e_{1}, x_{.} e_{2}\right)\right| \triangleq\left(\lambda x .\left|e_{2}\right|\right)\left|e_{1}\right|
\end{array}
$$

It is fairly easy to see that if $\vdash e: \tau$ in DCC, then $\vdash|e|:|\tau|$. Next, we define the lattice-indexed family of relations $\lfloor\tau\rfloor_{a}$ in HOL. For technical convenience, we write the relations as logical assertions, indexed by variables $x, y$ representing the two terms to be related.

$$
\begin{gathered}
\lfloor\text { bool }\rfloor_{a}(x, y) \triangleq(x=\mathrm{tt} \wedge y=\mathrm{tt}) \vee(x=\mathrm{ff} \wedge y=\mathrm{ff}) \\
\left\lfloor\tau_{1} \rightarrow \tau_{2}\right\rfloor_{\mathrm{a}}(x, y) \triangleq \forall v w \cdot\left\lfloor\tau_{1}\right\rfloor_{\mathrm{a}}(v, w) \Rightarrow\left\lfloor\tau_{2}\right\rfloor_{\mathfrak{a}}(x v, y w) \\
\left\lfloor\tau_{1} \times \tau_{2}\right\rfloor_{a}(x, y) \triangleq\left\lfloor\tau_{1}\right\rfloor_{a}\left(\pi_{1}(x), \pi_{1}(y)\right) \wedge\left\lfloor\tau_{2}\right\rfloor_{a}\left(\pi_{2}(x), \pi_{2}(y)\right) \\
\left\lfloor\mathbb{T}_{\ell}(\tau)\right\rfloor_{a}(x, y) \triangleq \begin{cases}\lfloor\tau\rfloor_{a}(x, y) & \ell \sqsubseteq a \\
T & \ell \nsubseteq \mathrm{a}\end{cases}
\end{gathered}
$$


The most important clause is the last one: When $\ell \nsubseteq a$, any two $x, y$ are in the relation $\left\lfloor\mathbb{T}_{\ell}(\tau)\right\rfloor_{\mathrm{a}}$. This generalizes to all protected types in the following sense.

Lemma 3.16. If $\ell \nsubseteq a$ and $\tau \searrow \ell$, then $\vdash \forall x y \cdot\left(\lfloor\tau\rfloor_{a}(x, y) \equiv T\right)$ in HOL.

Proof. By induction on the derivation of $\tau \searrow \ell$.

Case. $\frac{\ell \sqsubseteq \ell^{\prime}}{\mathbb{T}_{\ell^{\prime}}(\tau) \searrow \ell}$

Since $\ell \nsubseteq \mathrm{a}$ (given) and $\ell \sqsubseteq \ell^{\prime}$ (premise), it must be the case that $\ell^{\prime} \nsubseteq$ a. Hence, by definition, $\left\lfloor\mathbb{T}_{\ell^{\prime}}(\tau)\right\rfloor_{a}(x, y)=T$.

Case. $\frac{\tau \searrow \ell}{\mathbb{T}_{\ell^{\prime}}(\tau) \searrow \ell}$

We consider two cases:

If $\ell^{\prime} \nsubseteq a$, then $\left\lfloor\mathbb{T}_{\ell^{\prime}}(\tau)\right\rfloor_{a}(x, y)=\top$ by definition.

If $\ell^{\prime} \sqsubseteq a$, then $\left\lfloor\mathbb{T}_{\ell^{\prime}}(\tau)\right\rfloor_{a}(x, y)=\lfloor\tau\rfloor_{a}(x, y)$ by definition. By I.H. on the premise, we have $\lfloor\tau\rfloor_{a}(x, y) \equiv T$. Composing, $\left\lfloor\mathbb{T}_{\ell^{\prime}}(\tau)\right\rfloor_{a}(x, y) \equiv T$.

Case. $\frac{\tau_{1} \searrow \ell \quad \tau_{2} \searrow \ell}{\tau_{1} \times \tau_{2} \searrow \ell}$

By I.H. on the premises, we have $\left\lfloor\tau_{i}\right\rfloor_{a}(x, y) \equiv \top$ for $i=1,2$ and all $x, y$. Therefore, $\left\lfloor\tau_{1} \times \tau_{2}\right\rfloor_{a}(x, y) \triangleq\left\lfloor\tau_{1}\right\rfloor_{a}\left(\pi_{1}(x), \pi_{1}(y)\right) \wedge\left\lfloor\tau_{2}\right\rfloor_{a}\left(\pi_{2}(x), \pi_{2}(y)\right) \equiv \top \wedge \top \equiv \top$.

Case. $\frac{\tau_{2} \searrow \ell}{\tau_{1} \rightarrow \tau_{2} \searrow \ell}$

By I.H. on the premise, we have $\left\lfloor\tau_{2}\right\rfloor_{a}(x, y) \equiv \top$ for all $x, y$. Hence,

$\left\lfloor\tau_{1} \rightarrow \tau_{2}\right\rfloor_{a}(x, y) \triangleq\left(\forall v, w .\left\lfloor\tau_{1}\right\rfloor_{a}(v, w) \Rightarrow\left\lfloor\tau_{2}\right\rfloor_{a}(x v, y w)\right) \equiv\left(\forall v, w .\left\lfloor\tau_{1}\right\rfloor_{a}(v, w) \Rightarrow \top\right) \equiv \top$.

The translations extend to contexts as follows:

$$
\begin{gathered}
\left|x^{1}: \tau_{1}, \ldots, x^{n}: \tau_{n}\right| \triangleq x_{1}^{1}:\left|\tau_{1}\right|, x_{2}^{1}:\left|\tau_{1}\right|, \ldots, x_{1}^{n}:\left|\tau_{n}\right|, x_{2}^{n}:\left|\tau_{n}\right| \\
\left\lfloor x^{1}: \tau_{1}, \ldots, x^{n}: \tau_{n}\right\rfloor_{a} \triangleq\left\lfloor\tau_{1}\right\rfloor_{a}\left(x_{1}^{1}, x_{2}^{1}\right), \ldots,\left\lfloor\tau_{n}\right\rfloor_{a}\left(x_{1}^{n}, x_{2}^{n}\right)
\end{gathered}
$$

The following theorem states that the whole translation is sound: It preserves well-typedness. In the statement of the theorem, $|e|_{1}$ and $|e|_{2}$ replace each variable $x$ in $|e|$ with $x_{1}$ and $x_{2}$, respectively.

Theorem 3.17 (Soundness of embedding). If $\Gamma \vdash e: \tau$ in DCC, then for all $a \in\{L, H\}$, $\left.|\Gamma|\left|\lfloor\Gamma\rfloor_{\mathrm{a}} \vdash\right| e\right|_{1}:|\tau| \sim|e|_{2}:|\tau|\left\{\lfloor\tau\rfloor_{\mathrm{a}}\left(\mathbf{r}_{1}, \mathbf{r}_{2}\right)\right\}$. 
Proof. By induction on the given derivation of $\Gamma \vdash e: \tau$. We show here the cases corresponding to the monadic constructions only:

Case. $\frac{\Gamma \vdash e: \tau}{\Gamma \vdash \eta_{\ell}(e): \mathbb{T}_{\ell}(\tau)}$

To show: $\left.|\Gamma|\left|\lfloor\Gamma\rfloor_{\mathfrak{a}} \vdash\right| e\right|_{1}:|\tau| \sim|e|_{2}:|\tau|\left\{\left\lfloor\mathbb{T}_{\ell}(\tau)\right\rfloor_{\mathfrak{a}}\left(\mathbf{r}_{1}, \mathbf{r}_{2}\right)\right\}$.

By I.H. on the premise: $\left.|\Gamma|\left|\lfloor\Gamma\rfloor_{a} \vdash\right| e\right|_{1}:|\tau| \sim|e|_{2}:|\tau|\left\{\lfloor\tau\rfloor_{a}\left(\mathbf{r}_{1}, \mathbf{r}_{2}\right)\right\}$

If $\ell \sqsubseteq a$, then $\left\lfloor\mathbb{T}_{\ell}(\tau)\right\rfloor_{\mathfrak{a}}\left(\mathbf{r}_{1}, \mathbf{r}_{2}\right) \triangleq\lfloor\tau\rfloor_{\mathfrak{a}}\left(\mathbf{r}_{1}, \mathbf{r}_{2}\right)$, so the required result is the same as (1). If $\ell \nsubseteq$ a, then $\left\lfloor\mathbb{T}_{\ell}(\tau)\right\rfloor_{\mathrm{a}}\left(\mathbf{r}_{1}, \mathbf{r}_{2}\right) \triangleq T$ and the required result follows from rule SUB applied to (1).

Case. $\frac{\Gamma \vdash e: \mathbb{T}_{\ell}(\tau) \quad \Gamma, x: \tau \vdash e^{\prime}: \tau^{\prime} \quad \tau^{\prime} \searrow \ell}{\Gamma \vdash \operatorname{bind}\left(e, x . e^{\prime}\right): \tau^{\prime}}$

To show: $\left.|\Gamma|\left|[\Gamma\rfloor_{a} \vdash\left(\lambda x .\left|e^{\prime}\right|_{1}\right)\right| e\right|_{1}:\left|\tau^{\prime}\right| \sim\left(\lambda x .\left|e^{\prime}\right|_{2}\right)|e|_{2}:\left|\tau^{\prime}\right|\left\{\left\lfloor\tau^{\prime}\right\rfloor_{a}\left(\mathbf{r}_{1}, \mathbf{r}_{2}\right)\right\}$.

By I.H. on the first premise:

$|\Gamma||| \Gamma\rfloor_{\mathfrak{a}} \vdash|e|_{1}:|\tau| \sim|e|_{2}:|\tau|\left\{\left\lfloor\mathbb{T}_{\ell}(\tau)\right\rfloor_{\mathfrak{a}}\left(\mathbf{r}_{1}, \mathbf{r}_{2}\right)\right\}$

By I.H. on the second premise:

$|\Gamma|, x_{1}:|\tau|, x_{2}:\left.|\tau|\left|\lfloor\Gamma\rfloor_{a},\langle\tau\rfloor_{a}\left(x_{1}, x_{2}\right) \vdash\right| e^{\prime}\right|_{1}:\left|\tau^{\prime}\right| \sim\left|e^{\prime}\right|_{2}:\left|\tau^{\prime}\right|\left\{\left\lfloor\tau^{\prime}\right\rfloor_{a}\left(\mathbf{r}_{1}, \mathbf{r}_{2}\right)\right\}$

We consider two cases:

Subcase. $\ell \sqsubseteq$ a. Here, $\left\lfloor\mathbb{T}_{\ell}(\tau)\right\rfloor_{\mathrm{a}}\left(\mathbf{r}_{1}, \mathbf{r}_{2}\right) \triangleq\lfloor\tau\rfloor_{\mathrm{a}}\left(\mathbf{r}_{1}, \mathbf{r}_{2}\right)$, so (1) can be rewritten to:

$\left.|\Gamma|\left|\lfloor\Gamma\rfloor_{\mathfrak{a}} \vdash\right| e\right|_{1}:|\tau| \sim|e|_{2}:|\tau|\left\{\lfloor\tau\rfloor_{\mathfrak{a}}\left(\mathbf{r}_{1}, \mathbf{r}_{2}\right)\right\}$

Applying rule $\mathrm{ABS}$ to (2) yields:

$|\Gamma||| \Gamma\rfloor_{\mathrm{a}} \vdash \lambda x_{1} \cdot\left|e^{\prime}\right|_{1}:|\tau| \rightarrow\left|\tau^{\prime}\right| \sim \lambda x_{2} \cdot\left|e^{\prime}\right|_{2}:|\tau| \rightarrow\left|\tau^{\prime}\right|\{$

$\left.\forall x_{1} x_{2} \cdot\lfloor\tau\rfloor_{a}\left(x_{1}, x_{2}\right) \Rightarrow\left\lfloor\tau^{\prime}\right\rfloor_{a}\left(\mathbf{r}_{1} x_{1}, \mathbf{r}_{2} x_{2}\right)\right\}$

Applying rule APP to (4) and (3) yields:

$\left.|\Gamma|\left|\lfloor\Gamma\rfloor_{\mathrm{a}} \vdash\left(\lambda x_{1} \cdot\left|e^{\prime}\right|_{1}\right)\right| e\right|_{1}:\left|\tau^{\prime}\right| \sim\left(\lambda x_{2} \cdot\left|e^{\prime}\right|_{2}\right)|e|_{2}:\left|\tau^{\prime}\right|\left\{\left\lfloor\tau^{\prime}\right\rfloor_{\mathrm{a}}\left(\mathbf{r}_{1}, \mathbf{r}_{2}\right)\right\}$

which is what we wanted to prove.

Subcase. $\ell \nsubseteq$ a. Here, $\left\lfloor\mathbb{T}_{\ell}(\tau)\right\rfloor_{\mathrm{a}}\left(\mathbf{r}_{1}, \mathbf{r}_{2}\right) \triangleq\lfloor\tau\rfloor_{\mathrm{a}}\left(\mathbf{r}_{1}, \mathbf{r}_{2}\right)$, so (1) can be rewritten to:

$\left.|\Gamma|\left|\lfloor\Gamma\rfloor_{\mathrm{a}} \vdash\right| e\right|_{1}:|\tau| \sim|e|_{2}:|\tau|\{T\}$

Applying rule ABS to (2) yields:

$|\Gamma||| \Gamma\rfloor_{\mathrm{a}} \vdash \lambda x_{1} \cdot\left|e^{\prime}\right|_{1}:|\tau| \rightarrow\left|\tau^{\prime}\right| \sim \lambda x_{2} \cdot\left|e^{\prime}\right|_{2}:|\tau| \rightarrow\left|\tau^{\prime}\right|\{$

$\left.\forall x_{1} x_{2} \cdot\lfloor\tau\rfloor_{a}\left(x_{1}, x_{2}\right) \Rightarrow\left\lfloor\tau^{\prime}\right\rfloor_{a}\left(\mathbf{r}_{1} x_{1}, \mathbf{r}_{2} x_{2}\right)\right\}$

By Lemma 3.16 applied to the subcase assumption $\ell \nsubseteq \mathrm{a}$ and the premise $\tau^{\prime} \searrow \ell$, we have $\left\lfloor\tau^{\prime}\right\rfloor_{a}\left(\mathbf{r}_{1} x_{1}, \mathbf{r}_{2} x_{2}\right) \equiv T$. So, by rule SUB:

$\left.|\Gamma||| \Gamma\rfloor_{\mathrm{a}} \vdash \lambda x_{1} \cdot\left|e^{\prime}\right|_{1}:|\tau| \rightarrow\left|\tau^{\prime}\right| \sim \lambda x_{2} \cdot\left|e^{\prime}\right|_{2}:|\tau| \rightarrow\left|\tau^{\prime}\right|\left\{\forall x_{1} x_{2} \cdot \mid \tau\right\rfloor_{a}\left(x_{1}, x_{2}\right) \Rightarrow T\right\}$

Since $\left(\forall x_{1} x_{2} \cdot\lfloor\tau\rfloor_{a}\left(x_{1}, x_{2}\right) \Rightarrow T\right) \equiv T \equiv\left(\forall x_{1}, x_{2} \cdot T \Rightarrow T\right)$, we can use SUB again to get:

$\left.|\Gamma|\left|\lfloor\Gamma\rfloor_{\mathrm{a}} \vdash \lambda x_{1} \cdot\right| e^{\prime}\right|_{1}:|\tau| \rightarrow\left|\tau^{\prime}\right| \sim \lambda x_{2} \cdot\left|e^{\prime}\right|_{2}:|\tau| \rightarrow\left|\tau^{\prime}\right|\left\{\forall x_{1}, x_{2} . \top \Rightarrow T\right\}$

Applying rule APP to (6) and (5) yields:

$|\Gamma||| \Gamma\rfloor_{a} \vdash\left(\lambda x_{1} \cdot\left|e^{\prime}\right|_{1}\right)|e|_{1}:\left|\tau^{\prime}\right| \sim\left(\lambda x_{2} \cdot\left|e^{\prime}\right|_{2}\right)|e|_{2}:\left|\tau^{\prime}\right|\{T\}$

which is the same as our goal since $\left\lfloor\tau^{\prime}\right\rfloor_{\mathrm{a}}\left(\mathbf{r}_{1}, \mathbf{r}_{2}\right) \equiv T$. 
DCC's noninterference theorem is a corollary of this theorem and the soundness of RHOL in set theory.

\subsubsection{Relational Cost}

RelCost [61] is a relational refinement type-and-effect system designed to reason about relative cost - the difference in the execution costs of two similar programs or of two runs of the same program on two different inputs. RelCost combines reasoning about the maximum and minimum costs of a single program with relational reasoning about the relative cost of two programs. This combination of reasoning styles is motivated by the following observation: if two programs are structurally similar, then relational reasoning makes it easier to compute their relative cost. And if this approach fails, one can still establish an upper bound on the relative cost by computing the difference of the maximum cost of one program and the minimum cost of the other.

Here, we show how a fragment of RelCost can be embedded into RHOL. Similar to what we did for DCC, to just convey the main intuition, we consider a fragment of RelCost excluding recursive functions. The syntax of RelCost is based on two sorts of types:

$$
\begin{array}{rlr}
A & :=\text { nat }\left|\operatorname{list}_{A}[n]\right| A \stackrel{\operatorname{exec}(k, l)}{\longrightarrow} A \mid \forall i \stackrel{\operatorname{exec}(k, l)}{::} \text { S. A } & \text { (unary types) } \\
\tau & ::=\text { nat }_{r}\left|\operatorname{list}_{\tau}[n]^{\alpha}\right| \tau \stackrel{\operatorname{diff}(k)}{\longrightarrow} \tau \mid \forall i \stackrel{\operatorname{diff}(k)}{:} \text { S. } \tau|U A| \square \tau \quad \text { (relational types) }
\end{array}
$$

Unary types are used to type one program and they are mostly standard except for the effect annotation exec $(k, l)$ on arrow types and universally quantified types representing the min and max cost $k$ and $l$ of the body of the closure, respectively. Relational types ascribe two programs, so they are interpreted as pairs of expressions. In relational types, arrow types and universally quantified types have an effect annotation $\operatorname{diff}(k)$ representing the relative cost $k$ of the two closures. Besides, the superscript $\alpha$ refines list types with the number of elements that can differ in two lists. The type $U A$ is the weakest relation over elements of the unary type $A$, i.e. it can be used to type two arbitrary terms, while the type $\square \tau$ is the diagonal subrelation of $\tau$, i.e. it can be used to type only two terms that are equal.

There are two kinds of typing judgments, unary and relational:

$$
\Delta ; \Phi ; \Omega \vdash \vdash_{\mathrm{k}}^{\mathrm{l}} \mathrm{t}: \mathrm{A} \quad \Delta ; \Phi ; \Gamma \vdash \mathrm{t}_{1} \ominus \mathrm{t}_{2} \lesssim \mathrm{l}: \tau
$$

The unary judgment states that the execution cost of $t$ is lower bounded by $k$ and upper bounded by $l$, and the expression $t$ has the unary type $A$. The relational judgment states that the relative cost of $t_{1}$ with respect to $t_{2}$ is upper bounded by $l$ and the two expressions have the relational type $\tau$. Here $\Omega$ is a unary type environment, $\Gamma$ is a relational type environment, $\Delta$ is an environment for index 


$$
\begin{gathered}
\operatorname{vAR} \frac{\Omega(x)=A}{\Delta ; \Phi_{a} ; \Omega \vdash_{0}^{0} x: A} \quad \operatorname{CONST} \frac{\Delta ; \Phi_{a} ; \Omega \vdash_{0}^{0} \mathrm{n}: \text { int }}{\Delta ; \Phi_{a} ; x: A_{1}, \Omega \vdash_{k}^{l} t: A_{2}} \\
\operatorname{LPP} \frac{\Delta ; \Phi_{a} ; \Omega \vdash_{k_{1}}^{l_{1}} t_{1}: A_{1} \stackrel{\text { exec }(k, l)}{\Delta} A_{0}^{0} \lambda x . t: A_{1} \stackrel{\operatorname{exec}(k, l)}{\longrightarrow} A_{2}}{\Delta ; \Phi_{a} ; \Omega \vdash_{k_{1}+k_{2}+k+c_{a p p}}^{l_{1}+l_{2}+l+c_{a p p}} t_{1} t_{2}: A_{2}}
\end{gathered}
$$

Figure 3.10: Selected unary RelCost rules

variables and $\Phi$ for assumed constraints over the index terms. Figures 3.10 and 3.11 show selected rules.

To embed RelCost in RHOL, we define a monadic-style cost-instrumented translation of RelCost types. The translation is given in two-steps: First, we define an erasure of cost and size information into simple types and then we define a cost-passing style translation of simple types with a value-translation and an expression-translation. The erasure function is defined as follows:

$$
\begin{aligned}
& \mid \text { nat }|\triangleq| \text { nat }_{r} \mid \triangleq \text { nat } \quad \mid \text { list }_{A}[n] \mid \triangleq \text { list }_{|A|} \quad \mid \text { ist }_{\tau}[n]^{\alpha} \mid \triangleq \text { list }_{|\tau|} \quad|\mathrm{U} A| \triangleq|A| \\
& |\square \tau| \triangleq|\tau| \quad \mid \forall i \stackrel{\operatorname{exec}(k, l)}{::} \text { S. } A \mid \triangleq \text { nat } \rightarrow|A| \quad \mid \forall i \stackrel{\text { diff( }(k)}{::} \text { S. } \tau \mid \triangleq \text { nat } \rightarrow|\tau| \\
& |A \stackrel{\operatorname{exec}(k, l)}{\longrightarrow} B| \triangleq|A| \rightarrow|B| \quad\left|\tau_{1} \stackrel{\operatorname{diff}(k)}{\longrightarrow} \tau_{2}\right| \triangleq\left|\tau_{1}\right| \rightarrow\left|\tau_{2}\right|
\end{aligned}
$$

The cost-passing style translation of simple types is

$$
(\text { nat })_{v} \triangleq \text { nat } \quad\left(\text { list }_{A}\right)_{v} \triangleq \text { list }_{(A)_{v}} \quad(A \rightarrow B)_{v} \triangleq(A)_{v} \rightarrow(B)_{e} \quad(A)_{e} \triangleq(A)_{v} \times \text { nat }
$$

Guided by the translation of types above we can provide a cost-instrumented translation of simply-typed $\lambda$-expressions (Figure 3.12). This translation maps an expression of the simple type $\tau$ to an expression of type $\tau \times$ nat, where the second component is the number of reduction steps under an eager, call-by-value reduction strategy (which is the semantics of RelCost). It is fairly easy to see that this translation preserves typability and that it counts steps accurately.

However, this translation forgets the cost and size information in types. To recover these, we define a HOL formula for every unary type. But, first, we define axiomatically a predicate $\operatorname{list} U(n, l, P)$ that captures size information about lists:

$$
\begin{gathered}
\forall l, P . \operatorname{list} \mathrm{U}(0, l, \mathrm{P}) \equiv l=\square \\
\forall \mathrm{n}, \mathrm{l}, \mathrm{P} . \operatorname{listU}(\mathrm{n}+1, \mathrm{l}, \mathrm{P}) \equiv \exists w_{1}, w_{2} . l=w_{1}:: w_{2} \wedge \mathrm{P}\left(w_{1}\right) \wedge \operatorname{listU}\left(\mathrm{n}, w_{2}, \mathrm{P}\right)
\end{gathered}
$$




$$
\begin{aligned}
& \text { R-VAR } \frac{\Gamma(x)=\tau}{\Delta ; \Phi_{a} ; \Gamma \vdash x \ominus x \lesssim 0: \tau} \quad \text { R-CONST } \frac{}{\Delta ; \Phi_{a} ; \Gamma \vdash \mathrm{n} \ominus \mathrm{n} \lesssim 0: \text { int }_{r}} \\
& \mathrm{R}-\operatorname{CONS1} \frac{\Delta ; \Phi_{a} ; \Gamma \vdash \mathrm{t}_{1} \ominus \mathrm{t}_{1}^{\prime} \lesssim \mathrm{l}_{1}: \tau \quad \Delta ; \Phi_{a} ; \Gamma \vdash \mathrm{t}_{2} \ominus \mathrm{t}_{2}^{\prime} \lesssim \mathrm{l}_{2}: \text { list }_{\tau}[\mathrm{n}]^{\alpha}}{\Delta ; \Phi_{a} ; \Gamma \vdash \operatorname{cons}\left(\mathrm{t}_{1}, \mathrm{t}_{2}\right) \ominus \operatorname{cons}\left(\mathrm{t}_{1}^{\prime}, \mathrm{t}_{2}^{\prime}\right) \lesssim \mathrm{l}_{1}+\mathrm{l}_{2}: \text { list }_{\tau}[\mathrm{n}+1]^{\alpha+1}} \\
& \mathrm{R}-\operatorname{CoNs2} \frac{\Delta ; \Phi_{\mathrm{a}} ; \Gamma \vdash \mathrm{t}_{1} \ominus \mathrm{t}_{1}^{\prime} \lesssim \mathrm{l}_{1}: \square \tau \quad \Delta ; \Phi_{\mathrm{a}} ; \Gamma \vdash \mathrm{t}_{2} \ominus \mathrm{t}_{2}^{\prime} \lesssim \mathrm{l}_{2}: \text { list }_{\tau}[\mathrm{n}]^{\alpha}}{\Delta ; \Phi_{\mathrm{a}} ; \Gamma \vdash \operatorname{cons}\left(\mathrm{t}_{1}, \mathrm{t}_{2}\right) \ominus \operatorname{cons}\left(\mathrm{t}_{1}^{\prime}, \mathrm{t}_{2}^{\prime}\right) \lesssim \mathrm{l}_{1}+\mathrm{l}_{2}: \operatorname{list}_{\tau}[\mathrm{n}+1]^{\alpha}} \\
& \Delta ; \Phi_{a} ; \Gamma \vdash t \ominus t^{\prime} \lesssim l: \operatorname{list}_{\tau}[n]^{\alpha} \quad \Delta ; \Phi_{a} \wedge n=0 ; \Gamma \vdash t_{1} \ominus t_{1}^{\prime} \lesssim l^{\prime}: \tau^{\prime} \\
& \mathrm{i}, \Delta ; \Phi_{\mathrm{a}} \wedge \mathrm{n}=\mathrm{i}+1 ; \mathrm{h}: \square \tau, \mathrm{tl}: \text { list }_{\tau}[i]^{\alpha}, \Gamma \vdash \mathrm{t}_{2} \ominus \mathrm{t}_{2}^{\prime} \lesssim \mathrm{l}^{\prime}: \tau^{\prime}
\end{aligned}
$$

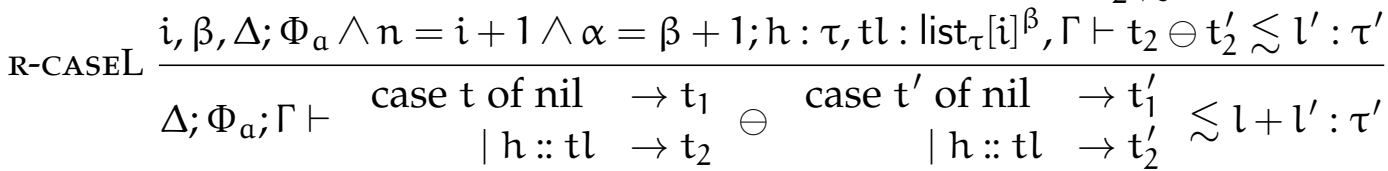

$$
\begin{aligned}
& \text { R-LAM } \frac{\Delta ; \Phi_{a} ; x: \tau_{1}, \Gamma \vdash \mathrm{t}_{1} \ominus \mathrm{t}_{2} \lesssim l: \tau_{2}}{\Delta ; \Phi_{a} ; \Gamma \vdash \lambda x . \mathrm{t}_{1} \ominus \lambda x . \mathrm{t}_{2} \lesssim 0: \tau_{1} \stackrel{\operatorname{diff(l)}}{\longrightarrow} \tau_{2}} \\
& \Delta ; \Phi_{a} ; \Gamma \vdash \mathrm{t}_{1} \ominus \mathrm{t}_{1}^{\prime} \lesssim \mathrm{l}_{1}: \tau_{1} \stackrel{\text { diff(l) }}{\longrightarrow} \tau_{2} \\
& \text { R-APP } \frac{\Delta ; \Phi_{a} ; \Gamma \vdash \mathrm{t}_{2} \ominus \mathrm{t}_{2}^{\prime} \lesssim \mathrm{l}_{2}: \tau_{1}}{\Delta ; \Phi_{a} ; \Gamma \vdash \mathrm{t}_{1} \mathrm{t}_{2} \ominus \mathrm{t}_{1}^{\prime} \mathrm{t}_{2}^{\prime} \lesssim l_{1}+\mathrm{l}_{2}+\mathrm{l}: \tau_{2}} \\
& i:: S, \Delta ; \Phi_{a} ; \Gamma \vdash t \ominus t^{\prime} \lesssim l: \tau \\
& \text { R-ILAM } \frac{i \notin \operatorname{FIV}\left(\Phi_{a} ; \Gamma\right)}{\Delta ; \Phi_{a} ; \Gamma \vdash \Lambda \mathrm{t} \ominus \Lambda \mathrm{t}^{\prime} \lesssim 0: \forall i \stackrel{\text { diff(l) }}{::} \text { S. } \tau} \\
& \Delta ; \Phi_{a} ; \Gamma \vdash t \ominus \mathrm{t}^{\prime} \lesssim l: \forall i \stackrel{\text { diff( }\left(^{\prime}\right)}{:} \text { S. } \tau \\
& \text { R-IAPP } \frac{\Delta \vdash \mathrm{J}: \mathrm{S}}{\Delta ; \Phi_{a} ; \Gamma \vdash \mathrm{t}[] \ominus \mathrm{t}^{\prime}[] \lesssim \mathrm{l}+\mathrm{l}^{\prime}[\mathrm{J} / \mathrm{i}]: \tau\{\mathrm{J} / \mathrm{i}\}} \\
& \Delta ; \Phi_{a} ; \Gamma \vdash t \ominus t \lesssim l: \tau \\
& \text { NOCHANGE } \frac{\forall x \in \operatorname{dom}(\Gamma) . \Delta ; \Phi_{a} \models \Gamma(x) \sqsubseteq \square \Gamma(x)}{\Delta ; \Phi_{a} ; \Gamma, \Gamma^{\prime} ; \Omega \vdash t \ominus t \lesssim 0: \square \tau} \\
& \operatorname{switcH} \frac{\Delta ; \Phi_{a} ; \bar{\Gamma} \vdash_{k_{1}}^{l_{1}} \mathrm{t}_{1}: A \quad \Delta ; \Phi_{a} ; \bar{\Gamma} \vdash_{\mathrm{k}_{2}}^{\mathrm{l}_{2}} \mathrm{t}_{2}: A}{\Delta ; \Phi_{a} ; \Gamma \vdash \mathrm{t}_{1} \ominus \mathrm{t}_{2} \lesssim \mathrm{l}_{1}-\mathrm{k}_{2}: \mathrm{U} A}
\end{aligned}
$$

Figure 3.11: Selected relational RelCost rules 


$$
(x) \triangleq(x, 0) \quad(\lambda x \text {.t }) \triangleq(\lambda x .(t), 0) \quad(\Lambda \text {.t }) \triangleq\left(\lambda_{-} \cdot(t), 0\right)
$$

$(\mathrm{tu}) \triangleq$ let $x=(\mathrm{t})$ in let $y=(\mathrm{u})$ in let $z=\pi_{1}(\mathrm{x}) \pi_{1}(\mathrm{y})$ in $\left(\pi_{1}(z), \pi_{2}(\mathrm{x})+\pi_{2}(\mathrm{y})+\pi_{2}(z)+\mathrm{c}_{\mathrm{app}}\right)$

$(\mathrm{t}[]) \triangleq$ let $x=(\mathrm{t})$ in let $y=\pi_{1}(\mathrm{x}) 0$ in $\left(\pi_{1}(y), \pi_{2}(\mathrm{x})+\pi_{2}(\mathrm{y})+\mathrm{c}_{\mathrm{iapp}}\right) \quad(\mathrm{nil}) \triangleq($ nil, 0$)$

$\left(\operatorname{cons}\left(t_{1}, t_{2}\right)\right) \triangleq$ let $x=\left(t_{1}\right)$ in let $y=\left(t_{2}\right)$ in $\left(\pi_{1}(x):: \pi_{1}(y), \pi_{2}(x)+\pi_{2}(y)\right)$

$$
\begin{gathered}
\left(\mid \text { case } \mathrm{t}^{\prime} \text { of nil } \rightarrow \mathrm{t}_{1}^{\prime} \mid \mathrm{h}:: \mathrm{tl} \rightarrow \mathrm{t}_{2}^{\prime}\right) \triangleq \\
\left\{\begin{array}{l}
\text { let } x=\left(\mathrm{t}^{\prime}\right) \text { in case } \pi_{1}(\mathrm{x}) \text { of } \\
\text { nil } \rightarrow \text { let } y=\left(\mathrm{t}_{1}^{\prime}\right) \text { in }\left(\pi_{1}(\mathrm{y}), \pi_{2}(\mathrm{x})+\pi_{2}(\mathrm{y})+\mathrm{c}_{\text {case }}\right) \\
\mid \mathrm{h}:: \mathrm{tl} \rightarrow \text { let } y=\left(\mathrm{t}_{2}^{\prime}\right) \text { in }\left(\pi_{1}(y), \pi_{2}(x)+\pi_{2}(y)+\mathrm{c}_{\text {case }}\right)
\end{array}\right.
\end{gathered}
$$

Figure 3.12: Cost-instrumented translation of expressions.

We can now define a HOL formula inductively on unary types.

$$
\begin{aligned}
& \lfloor\text { nat }\rfloor_{v}(x) \triangleq T \quad\left\lfloor\operatorname{list}_{A}[n]\right\rfloor_{v}(x) \triangleq \operatorname{list} U\left(n, x,\lfloor A\rfloor_{v}\right) \\
& \lfloor A \stackrel{\operatorname{exec}(k, l)}{\longrightarrow} B\rfloor_{v}(x) \triangleq \forall y \cdot\lfloor A\rfloor_{v}(y) \Rightarrow\lfloor B\rfloor_{e}^{k, l}(x y) \\
& \lfloor\forall i \stackrel{\operatorname{exc}(k, l)}{::} S . A\rfloor_{v}(x) \triangleq \forall y . \top \Rightarrow \forall i \cdot\lfloor A\rfloor_{e}^{k, l}(x y) \quad\lfloor A\rfloor_{e}^{k, l}(x) \triangleq\lfloor A\rfloor_{v}\left(\pi_{1} x\right) \wedge k \leqslant \pi_{2} x \leqslant l
\end{aligned}
$$

The type translation can also be extended to type environments:

$$
\left(\mid x_{1}: A_{1}, \ldots, x_{n}: A_{n} \|\right)=x_{1}:\left(\left|A_{1}\right| D_{v}, \ldots, x_{n}:\left(\left.\left|A_{n}\right|\right|_{v} .\right.\right.
$$

Similarly, we can associate to a type environment an HOL context that we can use to recover the cost and size information: $\left\lfloor x_{1}: A_{1}, \ldots, x_{n}: A_{n}\right\rfloor=\left\lfloor A_{1}\right\rfloor_{v}\left(x_{1}\right), \ldots,\left\lfloor A_{n}\right\rfloor_{v}\left(x_{n}\right)$. Now we can provide a cost-instrumented translation of unary judgments.

Theorem 3.18. If $\Delta ; \Phi ; \Omega \vdash_{k}^{l} t: A$, then: $(|\Omega| \mid), \Delta \mid \Phi,\lfloor\Omega\rfloor \vdash(t):\left(|A| \|_{e}\left\{\lfloor A\rfloor_{e}^{k, l}(\mathbf{r})\right\}\right.$

Proof. By induction on the derivation of $\Delta ; \Phi ; \Omega \vdash_{k}^{\mathrm{l}} \mathrm{t}$ : A. We show selected cases.

Case. $\overline{\Delta ; \Phi_{a} ; \Omega, x: A \vdash_{0}^{0} x: A}$

We can conclude by the following derivation:

$$
\begin{aligned}
& \overline{\left(|\Omega| D, x:\left(\left.|A|\right|_{v}, \Delta \mid \Phi_{a},\lfloor\Omega\rfloor,\lfloor A\rfloor_{v}(x) \vdash x:(|A|)_{v}\left\{\lfloor A\rfloor_{v}(\mathbf{r})\right\}\right.\right.} \text { VAR }
\end{aligned}
$$

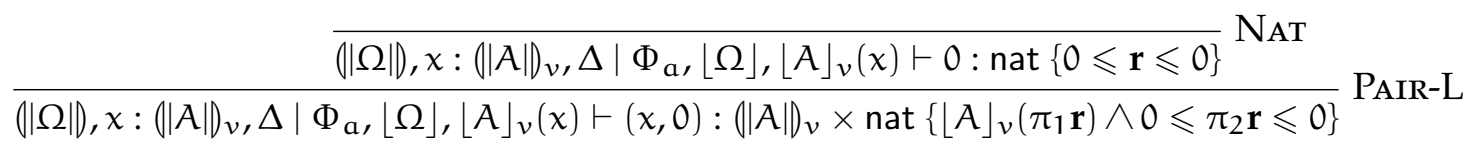


where the additional proof condition that is needed for the [PAIR-L] rule can be easily proved in HOL.

Case.

$$
\overline{\Delta ; \Phi_{a} ; \Omega \vdash_{0}^{0} \mathrm{n}: \text { int }}
$$

Then we can conclude by the following derivation:

$$
\frac{\overline{(|\Omega|), \Delta \mid \Phi_{\mathrm{a}},\lfloor\Omega\rfloor \vdash \mathrm{n}: \text { nat }\{T\}} \text { NAT } \quad \overline{(|\Omega|), \Delta \mid \Phi_{\mathrm{a}},\lfloor\Omega\rfloor \vdash 0: \text { nat }\{0 \leqslant \mathbf{r} \leqslant 0\}} \text { NAT }}{(|\Omega|), \Delta \mid \Phi_{\mathrm{a}},\lfloor\Omega\rfloor \vdash(\mathrm{n}, 0): \text { nat } \times \text { nat }\left\{0 \leqslant \pi_{2} \mathbf{r} \leqslant 0\right\}} \text { PAIR-L }
$$

where the additional proof condition that is needed for the [PAIR-L] rule can be easily proved in HOL.

Case. $\frac{\Delta ; \Phi_{a} ; x: A_{1}, \Omega \vdash_{k}^{l} t: A_{2}}{\Delta ; \Phi_{a} ; \Omega \vdash_{0}^{0} \lambda x . t: A_{1} \stackrel{\text { exec }(k, l)}{\longrightarrow} A_{2}}$

By induction hypothesis we have

$$
(|\Omega|), x:\left(|| A_{1} \mid\right)_{v}, \Delta \mid \Phi,\lfloor\Omega\rfloor,\left\lfloor A_{1}\right\rfloor_{v}(x) \vdash(t):\left(|| A_{2} \mid D_{e}\left\{\lfloor A\rfloor_{e}^{k, l}(\mathbf{r})\right\}\right.
$$

and we can conclude by the following derivation:

$$
\begin{aligned}
& \left(|\Omega| D, x:\left(\left|A_{1}\right| D_{v}, \Delta \mid \Phi,\lfloor\Omega\rfloor,\left\lfloor A_{1}\right\rfloor_{v}(x) \vdash\right.\right. \\
& (t):\left(\left|A_{2}\right|\right) e\left\{\left\lfloor A_{2}\right\rfloor_{e}^{k, l}(\mathbf{r})\right\} \\
& (|\Omega|), \Delta \mid \Phi,\lfloor\Omega\rfloor \vdash \lambda x .(t \mathrm{t}):\left(\left|A_{1}\right|\right)_{v} \rightarrow\left(\left|A_{2}\right|\right)_{e}\{\text { ABs } \\
& \frac{\left.\forall x .\left\lfloor A_{1}\right\rfloor_{v}(x) \Rightarrow\left\lfloor A_{2}\right\rfloor_{e}^{k, l}(\mathbf{r} x)\right\} \quad(|\Omega|), \Delta \mid \Phi,\lfloor\Omega\rfloor \vdash 0: \text { nat }\{0 \leqslant \mathbf{r} \leqslant 0\}}{(|| \Omega||), \Delta \mid \Phi,\lfloor\Omega\rfloor \vdash(\lambda x .(t)), 0):\left(\left(|| A_{1} \mid\right)_{v} \rightarrow\left(\left|A_{2}\right| D_{e}\right) \times \text { nat }\{\right.} \text { PAIR-L } \\
& \left.\forall x \cdot\left\lfloor A_{1}\right\rfloor_{v}(x) \Rightarrow\left\lfloor A_{2}\right\rfloor_{e}^{k, l}\left(\left(\pi_{1} \mathbf{r}\right) x\right) \wedge 0 \leqslant \pi_{2} \mathbf{r} \leqslant 0\right\}
\end{aligned}
$$

where the additional proof condition that is needed for the [PAIR-L] rule can be easily proved in HOL.

Case $\frac{\Delta ; \Phi_{a} ; \Omega \vdash_{k_{1}}^{l_{1}} t_{1}: A_{1} \stackrel{\operatorname{exec}(k, l)}{\longrightarrow} A_{2} \quad \Delta ; \Phi_{a} ; \Omega \vdash_{k_{2}}^{l_{2}} t_{2}: A_{1}}{\Delta ; \Phi_{a} ; \Omega \vdash_{k_{1}+k_{2}+k+c_{a p p}}^{l_{1}+l_{2}+l+c_{a p p}} t_{1} t_{2}: A_{2}}$

By induction hypothesis and unfolding some some definitions we have

$$
\begin{aligned}
(|\Omega|), \Delta \mid \Phi_{a},\lfloor\Omega\rfloor \vdash\left(\mathrm{t}_{1}\right):\left(\left(\left|\mathrm{A}_{1}\right|\right)_{v} \rightarrow\left(\left(|| \mathrm{A}_{2} \mid\right)_{v} \times \text { nat }\right)\right) \times \text { nat }\{\} \\
\forall \mathrm{h} .\left\lfloor A_{1}\right\rfloor_{v}(\mathrm{~h}) \Rightarrow\left(\left\lfloor\mathrm{A}_{2}\right\rfloor_{v}\left(\pi_{1}\left(\left(\pi_{1}(\mathbf{r})\right) \mathrm{h}\right)\right) \wedge \mathrm{k} \leqslant \pi_{2}\left(\left(\pi_{1}(\mathbf{r})\right) \mathrm{h}\right) \leqslant l\right) \wedge \mathrm{k}_{1} \leqslant \pi_{2}(\mathbf{r}) \leqslant l_{1}
\end{aligned}
$$

and $(|\Omega|), \Delta \mid \Phi_{a},\lfloor\Omega\rfloor \vdash\left(t_{2}\right):\left(\left|A_{1}\right|\right)_{v} \times$ nat $\left\{\left\lfloor A_{1}\right\rfloor_{v}\left(\pi_{1}(\mathbf{r})\right) \wedge k_{2} \leqslant \pi_{2}(\mathbf{r}) \leqslant l_{2}\right\}$. So, we can prove:

$$
\begin{array}{r}
(|\Omega|), \Delta \mid \Phi_{a},\lfloor\Omega\rfloor \vdash \text { let } x=\left(t_{1}\right) \text { in let } y=\left(t_{2}\right) \text { in } \pi_{1}(x) \pi_{1}(y):\left(|| A_{2} \mid\right)_{v} \times \text { nat }\{\} \\
\left.\qquad A_{2}\right\rfloor_{v}\left(\pi_{1}(\mathbf{r})\right) \wedge k \leqslant \pi_{2}(\mathbf{r}) \leqslant l \wedge k_{1} \leqslant \pi_{2}(x) \leqslant l_{1} \wedge k_{2} \leqslant \pi_{2}(y) r \leqslant l_{2}
\end{array}
$$


This combined with the definition of the cost-passing translation $\left(t_{1} t_{2}\right) \triangleq$ let $x=$ $\left(t_{1}\right)$ in let $y=\left(t_{2}\right)$ in let $z=\pi_{1}(x) \pi_{1}(y)$ in $\left(\pi_{1}(z), \pi_{2}(x)+\pi_{2}(y)+\pi_{2}(z)+c_{\text {app }}\right)$ allows us to prove as required the following:

$$
\begin{gathered}
(|\Omega|), \Delta \mid \Phi_{a},\lfloor\Omega\rfloor \vdash\left(\mathrm{t}_{1} \mathrm{t}_{2}\right):\left(|| \mathrm{A}_{2} \mid\right)_{v} \times \text { nat }\{\} \\
\left\lfloor A_{2}\right\rfloor_{v}\left(\pi_{1}(\mathbf{r})\right) \wedge \mathrm{k}+\mathrm{k}_{1}+\mathrm{k}_{2}+\mathrm{c}_{\mathrm{app}} \leqslant \pi_{2}(\mathbf{r}) \leqslant l+\mathrm{l}_{1}+\mathrm{l}_{2}+\mathrm{c}_{\mathrm{app}} .
\end{gathered}
$$

For the embedding of cost and size information in the relational case we first define a predicate list $R\left(n, l_{1}, l_{2}, a, P\right)$ in HOL axiomatically:

$$
\begin{gathered}
\forall l_{1}, l_{2}, a, P . \operatorname{listR}\left(0, l_{1}, l_{2}, a, P\right) \equiv l_{1}=l_{2}=\square \quad \forall n, l_{1}, l_{2}, a, P \cdot \operatorname{listR}\left(n+1, l_{1}, l_{2}, a, P\right) \equiv \\
\exists w_{1}, z_{1}, w_{2}, z_{2} \cdot l_{1}=w_{1}:: w_{2} \wedge l_{2}=z_{1}:: z_{2} \wedge P\left(w_{1}, z_{1}\right) \wedge \\
\left(\left(\left(w_{1}=z_{1}\right) \wedge \operatorname{listR}\left(n, w_{2}, z_{2}, a, P\right)\right) \vee\right. \\
\left.\left(a>0 \wedge \exists b . a=b+1 \wedge \operatorname{listR}\left(n, w_{2}, z_{2}, b, P\right)\right)\right)
\end{gathered}
$$

Let $\bar{\tau}$ denote RelCost's erasure of the binary type $\tau$ to a unary type. ${ }^{2}$ This erasure maps list $t_{\tau}[n]^{\alpha}$ to list $\bar{\tau}[n], \tau \stackrel{\text { diff(l) }}{\longrightarrow} \sigma$ to $\bar{\tau} \stackrel{\operatorname{exec}(0, \infty)}{\longrightarrow} \bar{\sigma}$, etc. Next, we define HOL formulas for the binary types.

$$
\begin{aligned}
& \left\lfloor\text { nat } \rrbracket_{v}(x, y) \triangleq x=y \quad \quad\left\lfloor U A \rrbracket_{v}(x, y) \triangleq\lfloor A\rfloor_{v}(x) \wedge\lfloor A\rfloor_{v}(y)\right.\right. \\
& \llbracket \square \tau \rrbracket_{v}(x, y) \triangleq(x=y) \wedge\left(\llbracket \tau \rrbracket_{v}(x, y)\right) \\
& \llbracket \tau \stackrel{\operatorname{diff}(l)}{\longrightarrow} \sigma \rrbracket_{v}(x, y) \triangleq\left\{\begin{array}{l}
\lfloor\bar{\tau} \stackrel{\operatorname{exec}(0, \infty)}{\longrightarrow} \bar{\sigma}\rfloor_{v}(x) \wedge\lfloor\bar{\tau} \stackrel{\operatorname{exec}(0, \infty)}{\longrightarrow} \bar{\sigma}\rfloor_{v}(y) \wedge \\
\left(\forall z_{1}, z_{2} \cdot \llbracket \tau \rrbracket_{v}\left(z_{1}, z_{2}\right) \Rightarrow\left\lfloor\sigma \rrbracket_{e}^{l}\left(x z_{1}, y z_{2}\right)\right)\right.
\end{array}\right.
\end{aligned}
$$

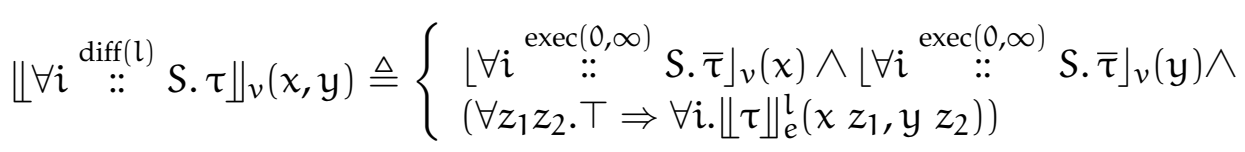

$$
\begin{aligned}
& \llbracket \operatorname{list}_{\tau}[n]^{\alpha} \Downarrow_{v}(x, y) \triangleq \operatorname{listR}\left(n, x, y, \alpha,\left\lfloor\tau \|_{v}\right)\right. \\
& \llbracket \tau \rrbracket_{e}^{l}(x, y) \triangleq \llbracket \tau \rrbracket_{v}\left(\pi_{1} x, \pi_{1} y\right) \wedge\left(\pi_{2} x-\pi_{2} y \leqslant l\right)
\end{aligned}
$$

The type translation can also be extended to relational type environments pointwise: $\left\|x^{1}: \tau_{1}, \ldots, x^{\mathfrak{n}}: \tau_{\mathfrak{n}}\right\| \triangleq x_{1}^{1}:\left(|| \tau_{1} \mid \nu_{v}, x_{2}^{1}:\left(|| \tau_{1}||_{v}, \ldots, x_{1}^{\mathfrak{n}}:\left(\left|\tau_{\mathfrak{n}}\right|\right\rangle_{v}, x_{2}^{\mathfrak{n}}:\left(|| \tau_{n} \mid \nu_{v}\right.\right.\right.$ We also need to derive from a type relational environment an HOL context that remembers the cost and size information: $\llbracket x^{1}: \tau_{1}, \ldots, x^{\mathfrak{n}}: \tau_{\mathfrak{n}} \rrbracket \triangleq \Perp \tau_{1} \rrbracket_{v}\left(x_{1}^{1}, x_{2}^{1}\right), \ldots, \llbracket \tau_{\mathfrak{n}} \rrbracket_{v}\left(x_{1}^{\mathfrak{n}}, x_{2}^{\mathfrak{n}}\right)$. Now we can provide the translation of relational judgments.

\footnotetext{
${ }^{2}$ In RelCost, this erasure is written $|\tau|$. We use a different notation to avoid confusion with our own erasure function from RelCost's types to simple types.
} 
Theorem 3.19. If $\Delta ; \Phi ; \Gamma \vdash \mathrm{t}_{1} \ominus \mathrm{t}_{2} \lesssim l: \tau$, then:

$$
\|\Gamma\|, \Delta \mid \Phi, \| \Gamma \rrbracket \vdash\left(\mathrm{t}_{1}\right)_{1}:(|\tau|)_{e} \sim\left(\mathrm{t}_{2}\right)_{2}:(|\tau|)_{e}\left\{\left\lfloor\tau \|_{e}^{\mathrm{l}}\left(\mathbf{r}_{1}, \mathbf{r}_{2}\right)\right\},\right.
$$

where $\left(t_{i}\right)_{j}$ is a copy of $t_{i}$ where each variable $x$ is replaced by a variable $x_{j}$ for $j \in\{1,2\}$.

To prove Theorem 3.19, we need three lemmas.

Lemma 3.20. Suppose $\Delta ; \Phi \vdash \tau$ wf. $^{3}$ Then, the following hold:

1. $\Delta \mid \Phi \vdash \forall x y . \llbracket \tau \rrbracket_{v}(x, y) \Rightarrow\lfloor\bar{\tau}\rfloor_{v}(x) \wedge\lfloor\bar{\tau}\rfloor_{v}(y)$

2. $\Delta \mid \Phi \vdash \forall x y \cdot \| \tau \rrbracket_{e}^{\mathrm{t}}(x, y) \Rightarrow\lfloor\bar{\tau}\rfloor_{e}^{0, \infty}(x) \wedge\lfloor\bar{\tau}\rfloor_{e}^{0, \infty}(y)$

Also, (3) $\llbracket \Gamma \rrbracket \Rightarrow\left\lfloor\bar{\Gamma}_{1}\right\rfloor \wedge\left\lfloor\bar{\Gamma}_{2}\right\rfloor$ where $\bar{\Gamma}_{1}$ and $\bar{\Gamma}_{2}$ are obtained by replacing each variable $x$ in $\bar{\Gamma}$ with $\mathrm{x}_{1}$ and $\mathrm{x}_{2}$, respectively.

Proof. (1) and (2) follow by a simultaneous induction on the given judgment. (3) follows immediately from (1).

Lemma 3.21. If $\Delta ; \Phi_{a} ; \Gamma \vdash e_{1} \ominus e_{2} \lesssim t: \tau$ in RelCost, then $\Delta ; \Phi ; \bar{\Gamma} \vdash_{0}^{\infty} e_{i}: \bar{\tau}$ for $i \in\{1,2\}$ in RelCost.

Proof. By induction on the given derivation.

Lemma 3.22. If $\Delta ; \Phi \models \tau_{1} \sqsubseteq \tau_{2}$, then $\Delta$; $\Phi \vdash \forall x y$. $\llbracket \tau_{1} \rrbracket_{v}(x, y) \Rightarrow \llbracket \tau_{2} \rrbracket_{v}(x, y)$.

Proof. By induction on the given derivation of $\Delta ; \Phi \models \tau_{1} \sqsubseteq \tau_{2}$.

Proof of Theorem 3.19. By induction on the given derivation of $\Delta ; \Phi ; \Gamma \vdash t_{1} \ominus t_{2} \lesssim k: \tau$. We show only a few representative cases here.

Case: $\quad \frac{i:: S, \Delta ; \Phi_{a} ; \Gamma \vdash e \ominus e^{\prime} \lesssim t: \tau \quad i \notin \operatorname{FIV}\left(\Phi_{a} ; \Gamma\right)}{\Delta ; \Phi_{a} ; \Gamma \vdash \wedge e \ominus \wedge e^{\prime} \lesssim 0: \forall i \stackrel{\text { diff(t) }}{::} \text { S. } \tau}$ R-ILAM

To show:

$$
\begin{gathered}
\|\Gamma\|, \Delta \mid \Phi_{a}, \| \Gamma \Downarrow \vdash\left(\lambda_{-} .(e)_{1}, 0\right):\left(\text { nat } \rightarrow(|\tau|)_{e}\right) \times \text { nat } \sim\left(\lambda_{-} \cdot\left(e e^{\prime}\right)_{2}, 0\right):\left(\text { nat } \rightarrow(|\tau|)_{e}\right) \times \text { nat }\{ \\
\left.\| \forall i \stackrel{\text { diff( }(t)}{:} \text { S. } \tau \rrbracket_{e}^{0}\left(\mathbf{r}_{1}, \mathbf{r}_{2}\right)\right\} .
\end{gathered}
$$

Expand $\llbracket \forall i \stackrel{\text { diff(t) }}{:}$ S. $\tau \rrbracket_{e}^{0}\left(\mathbf{r}_{1}, \mathbf{r}_{2}\right)$ to $\llbracket \forall i \stackrel{\text { diff(t) }}{::}$ S. $\tau \rrbracket_{v}\left(\pi_{1} \mathbf{r}_{1}, \pi_{1} \mathbf{r}_{2}\right) \wedge \pi_{2} \mathbf{r}_{1}-\pi_{2} \mathbf{r}_{2} \leqslant 0$, and apply the rule PAIR to reduce to two proof obligations:

$$
\begin{gathered}
\|\Gamma\|, \Delta \mid \Phi_{a}, \| \Gamma \rrbracket \vdash \lambda_{-} .(e)_{1}: \text { nat } \rightarrow(|| \tau \mid)_{e} \sim \lambda_{-} .\left(e^{\prime} \eta_{2}: \text { nat } \rightarrow(|\tau|)_{e}\left\{\| \forall i \stackrel{\text { diff(t) }}{::} \text { S. } \tau \|_{v}\left(\mathbf{r}_{1}, \mathbf{r}_{2}\right)\right\}\right. \\
\|\Gamma\|, \Delta \mid \Phi_{a}, \| \Gamma \rrbracket \vdash 0: \text { nat } \sim 0: \text { nat }\left\{\mathbf{r}_{1}-\mathbf{r}_{2} \leqslant 0\right\}
\end{gathered}
$$

${ }^{3}$ This judgment simply means that $\tau$ is well-formed in the context $\Delta ; \Phi$. It is defined in the original RelCost paper [61]. 
(B) follows immediately by rule ZERO. To prove (A), we start by expanding $\| \forall i \stackrel{\text { diff(t) }}{:}$ S. $\tau \|_{v}\left(\mathbf{r}_{1}, \mathbf{r}_{2}\right)$ and apply rule $\wedge_{1}$. We get three proof obligations.

$$
\begin{aligned}
& \|\Gamma\|, \Delta \mid \Phi_{a}, \| \Gamma \rrbracket \vdash \lambda_{-} .(e)_{1}: \text { nat } \rightarrow(|\tau|)_{e} \sim \lambda_{-} \cdot\left(\mid e^{\prime}\right)_{2}: \text { nat } \rightarrow\left(|\tau| \|_{e}\left\{\lfloor\forall i \stackrel{\operatorname{exe}(0, \infty)}{: !} S . \bar{\tau}\rfloor_{v}\left(\mathbf{r}_{1}\right)\right\}\right. \\
& \|\Gamma\|, \Delta \mid \Phi_{a}, \| \Gamma \rrbracket \vdash \lambda_{-} .(e)_{1}: \text { nat } \rightarrow\left(| \tau | | _ { e } \sim \lambda _ { - } \cdot ( e ^ { \prime } ) _ { 2 } : \text { nat } \rightarrow \left(|\tau| \|_{e}\left\{[\forall i \stackrel{\operatorname{exec}(0, \infty)}{:} S . \bar{\tau}\rfloor_{v}\left(\mathbf{r}_{2}\right)\right\}\right.\right. \\
& \|\Gamma\|, \Delta \mid \Phi_{a},\|\Gamma\| \vdash \lambda_{-} .(e)_{1}: \text { nat } \rightarrow(|| \tau \mid)_{e} \sim \lambda_{-} \cdot\left(e^{\prime}\right)_{2}: \text { nat } \rightarrow(|\tau|)_{e}\{ \\
& \left.\forall z_{1} z_{2} \cdot \top \Rightarrow \forall i .\|\tau\|_{e}^{\mathrm{t}}\left(\mathbf{r}_{1} z_{1}, \mathbf{r}_{2} z_{2}\right)\right\}
\end{aligned}
$$

To prove (C), apply Lemma 3.21 to the given derivation (not just the premise), obtaining a RelCost derivation for $\Delta ; \Phi_{a} ; \bar{\Gamma} \vdash_{0}^{\infty} \wedge e:(\forall i \stackrel{\operatorname{exe}(0, \infty)}{:}$ S. $\bar{\tau})$. Applying Theorem 3.18 to this yields

$$
\left.(\bar{\Gamma}), \Delta\left|\Phi_{a},\right| \bar{\Gamma}\right\rfloor \vdash\left(\lambda_{-} .(e), 0\right):\left(\text { nat } \rightarrow\left(\mid \bar{\tau} \|_{e}\right) \times \text { nat }\left\{\lfloor\forall i \stackrel{\operatorname{exec}(0, \infty)}{:} \text { S. } \bar{\tau}]_{e}^{0, \infty}(\mathbf{r})\right\}\right.
$$

in UHOL, which is the same as

$\left.(\bar{\Gamma}), \Delta\left|\Phi_{a},\right| \bar{\Gamma}\right\rfloor \vdash\left(\lambda_{-} \cdot(e), 0\right):\left(\right.$ nat $\left.\rightarrow(|\bar{\tau}|)_{e}\right) \times \operatorname{nat}\left\{[\forall i \stackrel{\operatorname{exec}(0, \infty)}{::} S . \bar{\tau}\rfloor_{v}\left(\pi_{1} \mathbf{r}\right) \wedge 0 \leqslant \pi_{2} \mathbf{r} \leqslant \infty\right\}$.

Applying rule $\mathrm{PROJ}_{1}$, we get

$$
\left.(\bar{\Gamma}), \Delta\left|\Phi_{a},\right| \bar{\Gamma}\right\rfloor \vdash \pi_{1}\left(\lambda_{-} \cdot(\mid e), 0\right): \text { nat } \rightarrow\left(\left.|\bar{\tau}|\right|_{e}\left\{[\forall i \stackrel{\operatorname{exec}(0, \infty)}{:} \text { S. } \bar{\tau}\rfloor_{v}(\mathbf{r})\right\} .\right.
$$

By subject conversion,

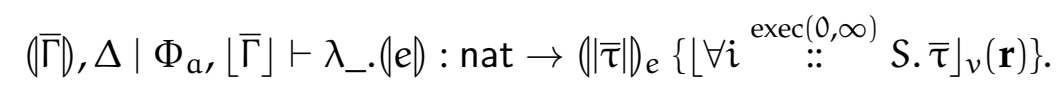

Renaming variables, we get

$$
(\bar{\Gamma})_{1}, \Delta \mid \Phi_{a},\left\lfloor\bar{\Gamma}_{1}\right\rfloor \vdash \lambda_{-} \cdot(\mid e)_{1}: \text { nat } \rightarrow\left(\left.|\bar{\tau}|\right|_{e}\left\{\lfloor\forall i \stackrel{\operatorname{exec}(0, \infty)}{:} S . \bar{\tau}\rfloor_{v}(\mathbf{r})\right\} .\right.
$$

Now note that by definition, $\|\Gamma\| \supseteq(\bar{\Gamma})_{1}$ and by Lemma 3.20(3), $\| \Gamma \rrbracket \Rightarrow\left\langle\bar{\Gamma}_{1}\right\rfloor$. Hence, we also get $\|\Gamma\|, \Delta \mid \Phi_{a}, \| \Gamma \rrbracket \vdash \lambda_{-} .(e)_{1}:$ nat $\rightarrow\|\mid \bar{\tau}\|_{e}\left\{[\forall i \stackrel{\operatorname{exec}(0, \infty)}{:} \text { S. } \bar{\tau}\rfloor_{v}(\mathbf{r})\right\}$. (C) follows immediately by rule UHOL-L.

(D) has a similar proof.

To prove $(E)$, apply the rule $A B S$, getting the obligation: $\|\Gamma\|, \Delta, z_{1}, z_{2}:$ nat $\mid \Phi_{a}, \| \Gamma \rrbracket \vdash(e)_{1}:\left(|\tau| \|_{e} \sim\left(e^{\prime}\right)_{2}:(|\tau|) e\left\{i \dot{e} .\|\tau\|_{e}^{\mathrm{t}}\left(\mathbf{r}_{1}, \mathbf{r}_{2}\right)\right\}\right.$ Since $z_{1}, z_{2}$ do not appear anywhere else, we can strengthen the context to remove them, thus reducing to: $\|\Gamma\|, \Delta \mid \Phi_{a}, \| \Gamma \Downarrow \vdash(e)_{1}:(|\tau|)_{e} \sim\left(e^{\prime}\right)_{2}:(|\tau|)_{e}\left\{\forall i .\|\tau\|_{e}^{t}\left(\mathbf{r}_{1}, \mathbf{r}_{2}\right)\right\}$ 
Next, we transpose to HOL using Theorem 3.6. We get the obligation:

$\|\Gamma\|, \Delta \mid \Phi_{a},\|\Gamma\| \vdash \forall i .\|\tau\|_{e}^{\mathrm{t}}\left((e)_{1},\left(e^{\prime}\right)_{2}\right)$

This is equivalent to:

$\|\Gamma\|, \Delta, i: S \mid \Phi_{a},\|\Gamma\| \vdash\|\tau\|_{e}^{t}\left((e)_{1},\left(e^{\prime}\right)_{2}\right)$

The last statement follows immediately from I.H. on the premise, followed by transposition to HOL using Theorem 3.6.

Case: $\frac{\Delta ; \Phi_{a} ; \Gamma \vdash e \ominus e \lesssim t: \tau \quad \forall x \in \operatorname{dom}(\Gamma) . \Delta ; \Phi_{a} \models \Gamma(x) \sqsubseteq \square \Gamma(x)}{\Delta ; \Phi_{a} ; \Gamma, \Gamma^{\prime} ; \Omega \vdash e \ominus e \lesssim 0: \square \tau}$ NOCHANGE

To show: $\|\Gamma\|, \Delta \mid \Phi_{a},\left\lfloor\Gamma \rrbracket \vdash(e)_{1}:(|\tau|)_{e} \sim(e)_{2}:(|\tau|)_{e}\left\{\|\square \tau\|_{e}^{0}\left(\mathbf{r}_{1}, \mathbf{r}_{2}\right)\right\}\right.$.

Expanding the definition of $\llbracket \square \tau \|_{e}^{0}$, this is equivalent to:

$$
\begin{gathered}
\left.\left.\|\Gamma\|, \Delta \mid \Phi_{a}, \| \Gamma \rrbracket \vdash(e)_{1}:(|\tau|)\right)_{e} \sim(e)\right\rangle_{2}:(|| \tau \mid)_{e}\{ \\
\left.\| \tau \rrbracket_{v}\left(\pi_{1} \mathbf{r}_{1}, \pi_{2} \mathbf{r}_{2}\right) \wedge\left(\pi_{1} \mathbf{r}_{1}=\pi_{1} \mathbf{r}_{2}\right) \wedge\left(\pi_{2} \mathbf{r}_{1}-\pi_{2} \mathbf{r}_{2} \leqslant 0\right)\right\} .
\end{gathered}
$$

Using rule $\wedge_{1}$, we reduce this to two obligations:

$$
\begin{gathered}
\|\Gamma\|, \Delta \mid \Phi_{a},\left\lfloor\Gamma \rrbracket \vdash(e)_{1}:(|\tau|)_{e} \sim(e)_{2}:(|\tau|)_{e}\left\{\left\lfloor\tau \|_{v}\left(\pi_{1} \mathbf{r}_{1}, \pi_{2} \mathbf{r}_{2}\right)\right\}\right.\right. \\
\|\Gamma\|, \Delta \mid \Phi_{a},\left\lfloor\Gamma \Downarrow \vdash(e)_{1}:(|\tau|)_{e} \sim(e)_{2}:(|\tau|)_{e}\left\{\left(\pi_{1} \mathbf{r}_{1}=\pi_{1} \mathbf{r}_{2}\right) \wedge\left(\pi_{2} \mathbf{r}_{1}-\pi_{2} \mathbf{r}_{2} \leqslant 0\right)\right\}\right.
\end{gathered}
$$

By I.H. on the first premise,

$\|\Gamma\|, \Delta \mid \Phi_{a}, \| \Gamma \rrbracket \vdash(e)_{1}:(|\tau|)_{e} \sim(e)_{2}:(|\tau|) e\left\{\|\tau\|_{v}\left(\pi_{1} \mathbf{r}_{1}, \pi_{2} \mathbf{r}_{2}\right) \wedge\left(\pi_{2} \mathbf{r}_{1}-\pi_{2} \mathbf{r}_{2} \leqslant t\right)\right\}$

By rule $S U B$,

$\|\Gamma\|, \Delta \mid \Phi_{a},\left\lfloor\Gamma \rrbracket \vdash(e)_{1}:(|\tau|) e_{e} \sim(e)_{2}:(|\tau|)_{e}\left\{\left\lfloor\tau \|_{v}\left(\pi_{1} \mathbf{r}_{1}, \pi_{2} \mathbf{r}_{2}\right)\right\}\right.\right.$

which is the same as (A).

To prove (B), apply Lemma 3.22 to the second premise to get for every $x \in \operatorname{dom}(\Gamma)$ that $\Delta \mid \Phi_{a} \vdash \llbracket \Gamma(x) \rrbracket_{v}\left(x_{1}, x_{2}\right) \Rightarrow \llbracket \square \Gamma(x) \rrbracket_{v}\left(x_{1}, x_{2}\right)$. Since $\llbracket \square \Gamma(x) \rrbracket_{v}\left(x_{1}, x_{2}\right) \Rightarrow x_{1}=x_{2}$ and from $\llbracket \Gamma \rrbracket$ we know that $\llbracket \Gamma(x) \rrbracket_{v}\left(x_{1}, x_{2}\right)$, it follows that $\|\Gamma\|, \Delta \mid \Phi_{a}, \| \Gamma \rrbracket \vdash x_{1}=x_{2}$. Since this holds for every $x \in \operatorname{dom}(\Gamma)$, it follows immediately that $\|\Gamma\|, \Delta \mid \Phi_{a}, \| \Gamma \Downarrow \vdash$ $(e)_{1}=(e)_{2}$. By Theorem 3.6, $\|\Gamma\|, \Delta \mid \Phi_{a},\left\lfloor\Gamma \downarrow \vdash(e)_{1}:(|\tau|)_{e} \sim(e)_{2}: \||\tau|\right)_{e}\left\{\mathbf{r}_{1}=\mathbf{r}_{2}\right\}$. Then (B) follows immediately by rule SUB.

RelCost's type-soundness theorem can be derived from Theorem 3.19 and the soundness of RHOL in set theory.

\subsection{Examples}

We present some illustrative examples to show how RHOL's rules work in practice. Our first example shows the functional equivalence of two recursive functions that perform the same number of recursive calls, and therefore we can reason about them in a synchronous manner. The second example shows the equivalence of two 
asynchronous recursive functions. The third example shows a sensitivity property of sorting. Finally, our fourth example illustrates reasoning about the relative cost of two programs, using an encoding similar to that of RelCost, but the example cannot be verified in RelCost itself.

Notational simplifications Throughout this section, we often omit types and typing contexts when they are clear. We also apply the SUB rule implicitly in some places, e.g., to change the assertion of a function from $\forall x$. $\phi$ to $\forall x$. $T \Rightarrow \phi$ so that we can apply $A B S$, to rearrange the order of quantified variables; or to pull quantifiers outwards when there is no variable capture.

\subsubsection{First Example: Factorial}

Expanding on Section 3.2, we show that the two following standard implementations of factorial, with and without an accumulator, are functionally equivalent:

$$
\begin{aligned}
& \text { fact }_{1} \triangleq \text { letrec } f_{1} n_{1}=\text { case } n_{1} \text { of } 0 \mapsto 1 ; S \mapsto \lambda x_{1} \cdot\left(S x_{1}\right) *\left(f_{1} x_{1}\right) \\
& \text { fact }_{2} \triangleq \text { letrec } f_{2} n_{2}=\lambda \text { a.case } n_{2} \text { of } 0 \mapsto a ; S \mapsto \lambda x_{2} \cdot f_{2} x_{2}\left(\left(S x_{2}\right) * a\right)
\end{aligned}
$$

Our goal is to prove that the result is the same on both implementations after scaling the result on the first one by the accumulator. In RHOL, this is expressed by the following judgment (with empty contexts):

$$
\text { fact }_{1}: \text { nat } \rightarrow \text { nat } \sim \text { fact }_{2}: \text { nat } \rightarrow \text { nat } \rightarrow \text { nat } \mid \forall n_{1} n_{2} \cdot n_{1}=n_{2} \Rightarrow \forall a .\left(\mathbf{r}_{1} n_{1}\right) * a=\mathbf{r}_{2} n_{2} a
$$

The proof starts by applying LETREC, which has the following main premise:

$$
\begin{aligned}
& \text { case } n_{1} \text { of } \quad \lambda \text { a. case } n_{2} \text { of }
\end{aligned}
$$

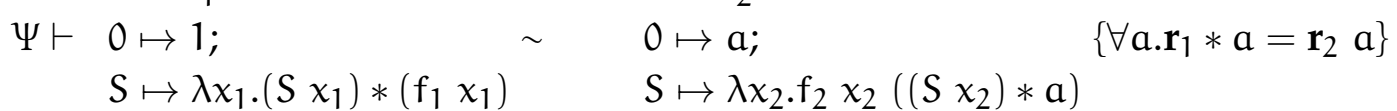

where $\Psi \triangleq n_{1}=n_{2}, \forall y_{1} y_{2} \cdot\left(y_{1}, y_{2}\right)<\left(n_{1}, n_{2}\right) \Rightarrow y_{1}=y_{2} \Rightarrow \forall a .\left(f_{1} y_{1}\right) * a=f_{2} y_{2} a$ asserts the inductive hypothesis and the equality between the arguments.

To prove this premise, we start by applying the one-sided ABS-R rule, with a trivial condition on a. Then we can apply a two-sided CASE rule, which has 3 premises. The first one asserts that the same branch is taken on both sides. The other two consider respectively the zero and the successor case. Since the branching is synchronous we do not need to consider the mixed cases (i.e., taking the zero branch on one side and the successor branch of the other). These three premises are:

1. $\Psi \vdash n_{1}=0 \Leftrightarrow n_{2}=0$

2. $\Psi, \mathrm{n}_{1}=0, \mathrm{n}_{2}=0 \vdash 1 \sim \mathrm{a}\left\{\mathbf{r}_{1} * \mathrm{a}=\mathbf{r}_{2}\right\}$ 
3. $\Psi \vdash \lambda x_{1} \cdot\left(S x_{1}\right) *\left(f_{1} x_{1}\right) \sim \lambda x_{2} \cdot f_{2} x_{2}\left(\left(S x_{2}\right) * a\right)\left\{\forall x_{1} x_{2} \cdot n_{1}=S x_{1} \Rightarrow n_{2}=S x_{2} \Rightarrow\right.$ $\left.\left(\mathbf{r}_{1} x_{1}\right) * a=\mathbf{r}_{2} x_{2}\right\}$

Premise 1 is a direct consequence of the assertion $n_{1}=n_{2}$ in $\Psi$. Premise 2 is a trivial arithmetic identity which can be proven in HOL (using rule SUB or by invoking Theorem 3.6). To prove premise 3, we first apply the (two-sided) ABS rule, which leaves the following proof obligation:

$$
\Psi, \mathrm{n}_{1}=S x_{1}, \mathrm{n}_{2}=\mathrm{S} x_{2} \vdash\left(S x_{1}\right) *\left(f_{1} x_{1}\right) \sim f_{2} x_{2}\left(\left(S x_{2}\right) * a\right)\left\{\mathbf{r}_{1} * a=\mathbf{r}_{2}\right\}
$$

This is proven in HOL by instantiating the inductive hypothesis in $\Psi$ with $y_{1} \mapsto$ $\mathrm{x}_{1}, \mathrm{y}_{2} \mapsto \mathrm{x}_{2}, \mathrm{a} \mapsto\left(\mathrm{S} \mathrm{x}_{1}\right) * \mathrm{a}$.

\subsubsection{Second Example: Take and Map}

This example establishes the equivalence of two programs that compute the same result, but using different number of recursive calls. Consider the following function take that takes a list $l$ and a natural number $n$ and returns the first $n$ elements of the list (or the whole list if its length is less than $n$ ).

$$
\begin{aligned}
\text { take } \triangleq \text { letrec } f_{1} l_{1}=\lambda n_{1} \text {.case } l_{1} \text { of }[] & \mapsto[] \\
-: *_{-} \mapsto \lambda h_{1} t_{1} . \text { case } n_{1} \text { of } 1 & \mapsto[] \\
& S \mapsto \lambda y_{1} \cdot h_{1}::\left(f_{1} t_{1} y_{1}\right)
\end{aligned}
$$

Next, consider the standard function map that applies a function $g$ to every element of a list $l$ pointwise.

$$
\begin{aligned}
\text { map } \left.\triangleq \text { letrec } f_{2} l_{2}=\lambda g_{2} . \text { case } l_{2} \text { of }\right] & \mapsto[] \\
i_{-}:_{-} & \mapsto \lambda h_{2} t_{2} \cdot\left(g_{2} h_{2}\right)::\left(f_{2} t_{2} g_{2}\right)
\end{aligned}
$$

Intuitively, it should be clear that for all $g, n, l$, map (take $l n$ ) $g=$ take (map $l g$ ) $n$ (mapping $g$ over the first $n$ elements of the list is the same as mapping $g$ over the whole list and then taking the first $n$ elements). However, the computations on the two sides of the equality are very different: For a list $l$ of length more than $n$, map (take $l n$ ) g only examines the first $n$ elements, whereas take (map $l g$ ) $n$ traverses the whole list. In the following we formalize this property in RHOL (Theorem 3.23) and outline the high-level idea of the proof. The full proof is in the appendix (Section A.3).

Theorem 3.23. $l_{1}, l_{2}$ : list $t_{\text {nat }}, n_{1}, n_{2}:$ nat, $g_{1}, g_{2}:$ nat $\rightarrow$ nat $\mid l_{1}=l_{2}, n_{1}=n_{2}, g_{1}=g_{2} \vdash$ map $\left(\right.$ take $\left.l_{1} n_{1}\right) g_{1}:$ list ${ }_{\text {nat }} \sim$ take $\left(\right.$ map $\left._{2} g_{2}\right) n_{2}$ : list $t_{\text {nat }}\left\{\mathbf{r}_{1}=\mathbf{r}_{2}\right\}$

Proof idea. Since the two sides make an unequal number of recursive calls, we need to reason asynchronously on the two sides (specifically, we use the rule LLCASE-A). However, equality cannot be established inductively with asynchronous reasoning: 
If two function applications are to be shown equal, and a recursion step is taken in only one of them, then the induction hypothesis cannot be applied. So, we strengthen the induction hypothesis, replacing the assertion $\mathbf{r}_{1}=\mathbf{r}_{2}$ in the theorem statement with $\mathbf{r}_{1} \sqsubseteq \mathbf{r}_{2} \wedge\left|\mathbf{r}_{1}\right|=\min \left(n_{1},\left|l_{1}\right|\right) \wedge\left|\mathbf{r}_{2}\right|=\min \left(n_{2},\left|l_{2}\right|\right)$ where $\sqsubseteq$ denotes the prefix ordering on lists and $|\cdot|$ is the list length function. This assertion implies $\mathbf{r}_{1}=\mathbf{r}_{2}$ and can be established inductively. The full proof is in the appendix, but at a high-level, the proof requires proving two judgments, one for the inner map-take pair and another for the outer one:

- $\Psi \vdash$ take $l_{1} n_{1} \sim \operatorname{map~}_{2} g_{2}\left\{\mathbf{r}_{1} g_{g_{2}} \mathbf{r}_{2}\right\}$

- $\Psi \vdash \operatorname{map} \sim$ take $\left\{\forall \mathrm{m}_{1} \mathrm{~m}_{2} \cdot \mathrm{m}_{1} \sqsubseteq_{g_{2}} \mathrm{~m}_{2} \Rightarrow\right.$ $\left.\left(\forall g_{1} \cdot g_{1}=g_{2} \Rightarrow \forall x_{2} \cdot x_{2} \geqslant\left|m_{1}\right| \Rightarrow\left(\mathbf{r}_{1} m_{1} g_{1}\right) \sqsubseteq\left(\mathbf{r}_{2} m_{2} x_{2}\right)\right)\right\}$

where $m_{1} \sqsubseteq_{g} m_{2}$ is an axiomatically defined predicate equivalent to (map $\left.m_{1} g\right) \sqsubseteq m_{2}$ and $\Psi$ are the assumptions in the statement of the theorem (in particular, $l_{1}=l_{2}$ ). The proof of the first premise proceeds by an analysis of map using synchronous rules. For the second premise, after applying LETREC we apply the asynchronous LLCASE-A rule, and then prove the following premises:

1. $\Psi, \Phi, x_{2} \geqslant\left|m_{1}\right|, g_{1}=g_{2}, m_{1}=[], m_{2}=[] \vdash[] \sim[]\left\{\mathbf{r}_{1} \sqsubseteq \mathbf{r}_{2}\right\}$

2. $\Psi, \Phi, x_{2} \geqslant\left|m_{1}\right|, g_{1}=g_{2}, m_{1}=[] \vdash$ [] $\sim \lambda h_{2} t_{2}$.case $x_{2}$ of $0 \mapsto[] ; S \mapsto \lambda y_{2} . h_{2}:: f_{2} t_{2} y_{2}\{$ $\left.\forall \mathrm{h}_{2} \mathrm{t}_{2} \cdot \mathrm{m}_{2}=\mathrm{h}_{2}:: \mathrm{t}_{2} \Rightarrow \mathbf{r}_{1} \sqsubseteq\left(\mathbf{r}_{2} \mathrm{~h}_{2} \mathrm{t}_{2}\right)\right\}$

3. $\Psi, \Phi, x_{2} \geqslant\left|m_{1}\right|, g_{1}=g_{2}, m_{2}=[] \vdash \lambda h_{1} t_{1} \cdot\left(g_{1} h_{1}\right)::\left(f_{1} t_{1} g_{1}\right) \sim[]\{$ $\left.\forall h_{1} t_{1} \cdot m_{1}=h_{1}:: t_{1} \Rightarrow\left(r_{1} h_{1} t_{1}\right) \sqsubseteq \mathbf{r}_{2}\right\}$

4. $\Psi, \Phi, x_{2} \geqslant\left|m_{1}\right|, g_{1}=g_{2} \vdash$ $\lambda h_{1} t_{1} \cdot\left(g_{1} h_{1}\right)::\left(f_{1} t_{1} g_{1}\right) \sim \lambda h_{2} t_{2}$.case $x_{2}$ of $0 \mapsto[] ; S \mapsto \lambda y_{2} \cdot h_{2}:: f_{2} t_{2} y_{2}\{$ $\left.\forall h_{1} t_{1} h_{2} t_{2} \cdot m_{1}=h_{1}:: t_{1} \Rightarrow m_{2}=h_{1}:: t_{1} \Rightarrow\left(r_{1} h_{1} t_{1}\right) \sqsubseteq\left(r_{2} h_{2} t_{2}\right)\right\}$

where $\Phi$ is the inductive hypothesis obtained from the LETREC application. The first two premises follow directly from the definition of $\sqsubseteq$, while the third one follows from the contradictory assumptions $m_{1} \sqsubseteq_{g} m_{2}, m_{1}=h_{1}:: t_{1}$ and $m_{2}=[]$. The last premise is proved by first applying the NATCASE-R rule and then applying the induction hypothesis.

The proof presented here is intended to show how the one-sided rules can deal with asynchronous reasoning, but we remark that a much simpler proof could be written using equational rewriting rules. However, note that our system can also be seen as a framework in which to embed and prove sound such rewriting rules, in the style of Benton [45]. 


\subsubsection{Third example: Selection sort}

This example showcases a property, namely sensitivity, that is out of reach of equational reasoning, but that is easy to prove using relational reasoning.

Given two lists of integers of the same length, we define the distance between them as the maximum of the pointwise distances:

$$
\begin{array}{ll}
d\left(l_{1}, l_{2}\right) \triangleq \max _{i}\left|l_{1}[i]-l_{2}[i]\right| & \text { if } l_{1}, l_{2} \text { have the same length } \\
d\left(l_{1}, l_{2}\right) \triangleq \infty & \text { otherwise }
\end{array}
$$

It is routine to check that this defines in fact a metric. Furthermore, it is known that sorting is 1 -Lipschitz continuous under this metric: If $\operatorname{sort}(l)$ denotes the list obtained by sorting $l$, then, for all $l_{1}, l_{2}$ we have that $d\left(\operatorname{sort}\left(l_{1}\right)\right.$, sort $\left.\left(l_{2}\right)\right) \leqslant d\left(l_{1}, l_{2}\right)$.

This result can be proved directly by showing that for any $k$ the function $\min _{k}$, which picks the $k$-th smallest element of a list, is 1-Lipschitz continuous. The above result then follows by noting that $\operatorname{sort}(l)=\left[\min _{1}(l), \min _{2}(l), \ldots, \min _{\mathfrak{n}}(l)\right]$ where $n$ is the length of $l$.

Here, we provide a different proof. We prove that a particular implementation of sorting, namely, selection sort has this property. Selection sort is a basic sorting algorithm that traverses a list, finds the least element, puts it in front, and then sorts the rest of the list recursively. The function ssort below implements selection sort.

$$
\text { ssortl } \triangleq \text { ssort }^{\prime} l(\text { length } l)
$$

$$
\begin{aligned}
& \text { ssort }^{\prime} \triangleq \text { letrec ssort }{ }^{\prime} l= \\
& \lambda \text { n.case } n \text { of } 0 \mapsto[\text {; } \\
& \mathrm{S} \mapsto \lambda \mathrm{m} \text {.case } \mathrm{l} \text { of } \\
& \text { [] } \mapsto[] \\
& { }_{-}:_{-} \mapsto \lambda \text { ht. } \quad \text { let }(\text { rest }, \text { min })=\text { restmin } t h \\
& \text { in } \min :: \text { ssort }{ }^{\prime} \text { rest } \mathrm{m}
\end{aligned}
$$

restmin $\triangleq$ letrec restmin $l=$

$\lambda$ a.case $l$ of

$$
\begin{array}{ll}
{[] \mapsto([], a)} & \\
-\because{ }_{-} \mapsto \lambda \text { ht.let } & M=\max (a, h) \\
& m=\min (a, h) \\
& (\text { rest, } \min )=\text { restmin } \mathrm{m} t \\
& \text { in }\langle M:: \text { rest, } m i n\rangle
\end{array}
$$

We then want to prove the following in RHOL:

Theorem 3.24 (1-Lipschitz continuity of ssort).

$$
\vdash \text { ssort } \sim \text { ssort }\left|\forall l_{1} l_{2} \delta .\right| l_{1}|=| l_{2} \mid \Rightarrow d\left(l_{1}, l_{2}\right) \leqslant \delta \Rightarrow d\left(r_{1} l_{1}, \mathbf{r}_{2} l_{2}\right) \leqslant \delta
$$


Note that the postcondition above is equivalent to

$$
\forall l_{1} l_{2} \cdot\left|l_{1}\right|=\left|l_{2}\right| \Rightarrow d\left(\mathbf{r}_{1} l_{1}, \mathbf{r}_{2} l_{2}\right) \leqslant d\left(l_{1}, l_{2}\right)
$$

but our version is easier to prove, because we need an explicit $\delta$ to appear in the verification of ssort' and restmin. The proof is entirely synchronous, and relies on the property of restmin that, for two lists of equal length satisfying the invariant of being closer than $\delta$ under the metric defined above, (1) the two returned minimas are spaced less than $\delta$ (under the usual metric for the integers) and (2) the two returned remaining lists satisfy the same invariant. In RHOL, this is expressed as:

$\vdash$ restmin $\sim$ restmin $\left|\forall l_{1} l_{2} \cdot d\left(l_{1}, l_{2}\right) \leqslant \delta \Rightarrow \forall h_{1} h_{2}.\right| h_{1}-h_{2} \mid \leqslant \delta \Rightarrow D\left(r_{1} l_{1} h_{1}, r_{2} l_{2} h_{2}\right) \leqslant \delta$

where we use $\mathrm{D}$ to denote the distance induced by the maximum of the componentwise distances.

The only interesting case is where we reach the $h:: t$ branch of restmin. Here, we use the following (mathematical) lemma about max and min. This lemma must be proved in HOL with sufficient axiomatization.

Lemma 3.25. Let $\delta$ a non-negative real number. For every $\mathrm{a}_{1}, \mathrm{~b}_{1}, \mathrm{a}_{2}, \mathrm{~b}_{2}$ real, if $\left|\mathrm{a}_{1}-\mathrm{a}_{2}\right| \leqslant \delta$ and $\left|\mathrm{b}_{1}-\mathrm{b}_{2}\right| \leqslant \delta$, then:

$$
\begin{gathered}
\left|\max \left(a_{1}, b_{1}\right)-\max \left(a_{2}, b_{2}\right)\right| \leqslant \delta \text { and } \\
\left|\min \left(a_{1}, b_{1}\right)-\min \left(a_{2}, b_{2}\right)\right| \leqslant \delta
\end{gathered}
$$

\subsubsection{Fourth Example: Insertion Sort}

Insertion sort is a standard sorting algorithm that sorts a list $\mathrm{h}:: \mathrm{t}$ by sorting the tail $t$ recursively and then inserting $h$ at the appropriate position in the sorted tail. Consider the following implementations of the insertion function, insert, and the insertion sort function, isort, each returning a pair, whose first element is the usual output list (inserted list for insert and sorted list for isort) and whose second element is the number of comparisons made during the execution (assuming an eager, call-by-value evaluation strategy).

$$
\begin{aligned}
& \text { insert } \triangleq \lambda x \text {. letrec insert } l=\text { case } l \text { of }[] \mapsto([x], 0) \text {; } \\
& \text { _ } \because{ }_{-} \mapsto \lambda h t \text {. case } x \leqslant h \text { of } \\
& \text { tt } \mapsto(x:: l, 1) \text {; } \\
& \mathrm{ff} \mapsto \text { let } \mathrm{s}=\text { insert } \mathrm{t} \text { in } \\
& \left(h::\left(\pi_{1} s\right), 1+\left(\pi_{2} s\right)\right)
\end{aligned}
$$

$$
\begin{aligned}
\text { isort } \triangleq \text { letrec isort } l=\text { case } l \text { of }[] & \mapsto([], 0) \text {; } \\
-\because{ }_{-} \mapsto \lambda h t . & \text { let } s=\text { isort } t \\
& \text { let } s^{\prime}=\text { insert } h\left(\pi_{1} s\right) \text { in } \\
& \left(\pi_{1} s^{\prime},\left(\pi_{2} s\right)+\left(\pi_{2} s^{\prime}\right)\right)
\end{aligned}
$$


Using this implementation, we prove the following interesting fact about insertion sort: Among all lists of the same length, insertion sort computes the fastest (with fewest comparisons) on lists that are already sorted. This is a property about the relational cost of insertion sort (on two different inputs), which cannot be established in RelCost. To state the property in RHOL, we define a list predicate sorted $(l)$ in HOL axiomatically:

$$
\operatorname{sorted}([]) \equiv T \quad \forall h t . \operatorname{sorted}(h:: t) \equiv(\operatorname{sorted}(t) \wedge h \leqslant \min (t))
$$

where the function $I \min (l)$ returns the minimum element of $l$ :

$$
\operatorname{lmin} \triangleq \text { letrec } f \mathrm{l}=\text { case } \mathrm{l} \text { of } \square \mapsto \infty ;_{-}::_{-} \mapsto \lambda \mathrm{ht} \cdot \min (\mathrm{h}, \mathrm{ft})
$$

As in the previous example, let $|\cdot|$ be the standard list length function. The property of insertion sort mentioned above is formalized in the following theorem. In words, the theorem says that if isort is executed on lists $x_{1}$ and $x_{2}$ of the same length and $x_{1}$ is sorted, then the number of comparisons made during the sorting of $x_{1}$ is no more than the number of comparisons made during the sorting of $x_{2}$.

Theorem 3.26. Let $\tau \triangleq$ list $_{\text {nat }} \rightarrow$ list $_{\text {nat }}$. Then,

- $\mid \bullet \vdash$ isort : $\tau \sim$ isort : $\tau\left\{\forall x_{1} x_{2} .\left(\operatorname{sorted}\left(x_{1}\right) \wedge\left|x_{1}\right|=\left|x_{2}\right|\right) \Rightarrow \pi_{2}\left(\mathbf{r}_{1} x_{1}\right) \leqslant \pi_{2}\left(\mathbf{r}_{2} x_{2}\right)\right\}$.

A full proof is shown in the appendix (Section A.5). The proof proceeds mostly synchronously in the two sides. Following the structure of isort, we apply the rules LETREC, LISTCASE and APP + ABS (for the let binding, which, as usual, is defined as a function application), followed by an application of the inductive hypothesis for the recursive call to isort. Eventually, we expose the call to insert on both sides. At this point, the observation is that since $x_{1}$ is already sorted, its head element must be no greater than all elements in its tail, so insert must return immediately with at most 1 comparison on the $x_{1}$ side. Formally, this last proof step can be completed by switching to either UHOL or HOL and using subject conversion; in the appendix, we switch to HOL.

\subsection{Conclusion}

We have developed Relational Higher-Order Logic (RHOL), a new formalism to reason about relational properties of (pure) higher-order programs written in a simply typed $\lambda$-calculus with inductive types and recursive definitions. The system is expressive, has solid foundations via an equivalence with Higher-Order Logic, and yet retains the (nice) "feel" of relational refinement type systems. An important direction for future work is to extend Relational Higher-Order Logic to other kinds of effects, in particular mutable state. 
For practical purposes, it will also be interesting to automate RHOL. We believe that much of the technology developed for (relational) refinement types, and in particular the automated generation of verification conditions (maybe with user hints to switch between unary and binary modes of reasoning) and the connection to SMT-solvers can be lifted without significant hurdle to Relational Higher-Order Logic. 


\section{UNARY REASONING ABOUT PROBABILITIES: UNION BOUNDS}

In the previous chapter we have developed unary and relational logics for pure, effect-free programs. However, often in practice functional programs have sideeffects, such as probabilities, global state, non-determinisim, or non-termination.

In order to reason about these programs, one could extend the base logic with constructions that e.g. explicitly mention probabilities or quantify over possible traces of executions, and there is a substantial amount of work in this direction, for instance $[58,41]$. We explore this possibility in Chapter 9.

However, an alternative is to keep the base logic intact and instead change its interpretation, so that it is only at the semantic (and not syntactic) level that the effect is taken into account. For instance, the liberal interpretation of the Hoare triple $\{P\} C\{Q\}$ states that, starting from initial conditions $P$, the terminating executions of $C$ satisfy $Q$. This does not require any explicit mention in $Q$ to termination, and therefore we can use this interpretation of Hoare logic to reason about a possibly non-terminating program $\mathrm{C}$ without changing the assertion language. This style of reasoning offers a tradeoff: on the one hand, the assertion language, and most of the logic rules remain the same, so a lot of the theory and implementations for pure programs can be reused. On the other hand, it limits the kind of properties that can be proven. We argue that a lot of interesting properties can be proven using this technique, such as bounds on the probability of some event, bounds on the expected value of the output, or differential privacy.

Formally, this interpretation is based on the notion of monadic lifting, which is a map from a predicate P over a type $\tau$ to a predicate $\dot{\mathcal{T}}$ over a type $\mathcal{T}(\tau)$, where $\mathcal{T}$ is a monad specifying a side effect. We use this notion to interpret a calculus extended with operations for sampling from probability distributions, and to develop an effectful extension of UHOL to reason about probabilistic programs.

The concepts presented in this chapter also intend to serve as a warm-up for the 
following chapters, which expand upon them by moving to the relational setting and adding state.

\section{Organization of chapter}

We start with a quick recap of measure theory (Section 4.1). We then introduce a simply typed language extended with probabilities (Section 4.2), and a unary logic to reason about it (Section 4.3). After this, we turn to the semantic foundations. We present some preliminary notions of category theory (Section 4.5 and 4.6), and the category of Quasi-Borel spaces, a model for higher-order probabilistic programs (Section 4.7). Finally, we use these categorical notions to build our semantical model (Section 4.8).

\subsection{Mathematical preliminaries}

\subsubsection{Measure theory}

We now review some of the main concepts of measure theory that we will use in this chapter and the rest of the thesis. These notions of measure theory are needed to define continuous probability distributions. For a textbook treatment of the matter, refer e.g. to [48].

Definition 4.1 (Sigma algebra). Given a set X, a $\sigma$-algebra on $X$ is a collection $\Sigma$ of subsets of $\mathrm{X}$ such that $\mathrm{X} \in \Sigma$, and $\Sigma$ is closed under the operations of complement and countable unions.

Definition 4.2 (Measurable space). A measurable space is a pair $\left(X, \Sigma_{X}\right)$ of a set $X$ and a $\sigma$-algebra over $\mathrm{X}$. Elements of $\Sigma_{X}$ are called measurable subsets (of $\mathrm{X}$ ) or events. A measurable function between $\left(X, \Sigma_{X}\right)$ and $\left(Y, \Sigma_{Y}\right)$ is a function $\mathrm{f}: \mathrm{X} \rightarrow \mathrm{Y}$ such that, for every $\mathrm{U} \in \Sigma_{Y}, \mathrm{f}^{-1}(\mathrm{U}) \in \mathrm{X}$.

Definition 4.3 (Measure, Probability measure). Given a measurable space $\left(X, \Sigma_{X}\right), a$ measure is a map $\vee: \Sigma_{X} \rightarrow[0, \infty]$ such that $v(\emptyset)=0$, and for any pairwise disjoint, countable collection $\left\{\mathrm{V}_{i}\right\}_{i \in \mathbb{N}} \subseteq \Sigma_{X}, v\left(\bigcup_{i \in \mathbb{N}} V_{i}\right)=\sum_{i \in \mathbb{N}} v\left(V_{i}\right)$. Such a triple $\left(X, \Sigma_{X}, v\right)$ is known as a measure space. If additionally, $v(X)=1$ then $v$ is called a probability measure, and $\left(\mathrm{X}, \Sigma_{\mathrm{X}}, v\right)$ is called a probability space.

Given a probability space $\left(\mathrm{X}, \Sigma_{\mathrm{X}}, \mathrm{v}\right)$, and an event $\mathrm{U} \in \Sigma_{\mathrm{X}}$, we denote the probability of $\mathrm{U}$ as $\operatorname{Pr}_{\mathrm{x} \in v}[\mathrm{x} \in \mathrm{U}]$ or $\operatorname{Pr}_{\gamma}[\mathrm{U}]$, which are equal to $v(\mathrm{U})$.

In this thesis we will also distinguish between the following types of measures:

Definition 4.4. Let $\left(X, \Sigma_{X}\right)$ be a measurable space and $v$ a measure over it.

- We say that $v$ is finite if $v(\mathrm{X})$ is finite. 
- We say that $v$ is $\sigma$-finite if $X$ can be covered with countably many sets of finite measure.

The union bound, which gives name to the logic presented on this chapter, is a bound on the probability of the union of two sets, or in general, a countable (and possibly empty) intersection of sets. We know that

$$
\operatorname{Pr}\left[E_{1} \cup E_{2}\right]=\operatorname{Pr}\left[E_{1}\right]+\operatorname{Pr}\left[E_{2}\right]-\operatorname{Pr}\left[E_{1} \cap E_{2}\right]
$$

but computing $\operatorname{Pr}\left[E_{1} \cap E_{2}\right]$ may be hard. This gets worse when unions with more than two elements, since we need to compute and add the probability of all possible intersections with the correct sign, e.g.

$$
\begin{aligned}
\operatorname{Pr}\left[E_{1} \cup E_{2} \cup E_{3}\right] & =\operatorname{Pr}\left[E_{1}\right]+\operatorname{Pr}\left[E_{2}\right]+\operatorname{Pr}\left[E_{3}\right]-\operatorname{Pr}\left[E_{1} \cap E_{2}\right] \\
& -\operatorname{Pr}\left[E_{1} \cap E_{3}\right]-\operatorname{Pr}\left[E_{2} \cap E_{3}\right]+\operatorname{Pr}\left[E_{1} \cap E_{2} \cap E_{3}\right]
\end{aligned}
$$

However we can just ignore the intersections, and get the following property (also known as subadditivity):

Lemma 4.5 (Union bound / Subadditivity of measures). Let $\left(X, \Omega_{X}, v\right)$ be a measure space, and $\left\{\mathrm{E}_{i}\right\}_{i \in \mathrm{I}}$ a countable family of sets $\mathrm{E}_{i} \in \Omega$. Then,

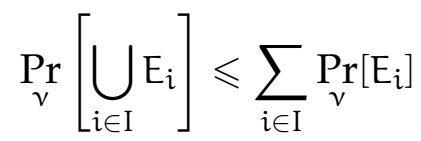

Looking at the definition of a measurable space, one could be reminded of the definition of a topological space, which is a pair $(X, T)$ of a set $X$ and a topology $T$, which is a collection of subsets of $X$ such that $X \in T, \emptyset \in T$ and $T$ is closed under countable unions and finite intersections. In fact, a $\sigma$-algebra can be constructed from a topology:

Definition 4.6 (Borel set, Borel sigma algebra). Given a topological space $(\mathrm{X}, \mathrm{T})$, the Borel $\sigma$-algebra $\Sigma_{\mathrm{T}}$ is the smallest $\sigma$-algebra containing all sets in $\mathrm{T}$. An element of $\Sigma_{\mathrm{T}}$ is called a Borel set, and $\left(\mathrm{X}, \Sigma_{\mathrm{T}}\right)$ is called a Borel space. If $\mathrm{X}$ is also a Polish space (i.e. separable and completely metrizable) then $\left(\mathrm{X}, \Sigma_{\mathrm{T}}\right)$ is called a standard Borel space.

In particular, for the real numbers with the usual topology, the Borel $\sigma$-algebra coincides with the $\sigma$-algebra generated by the open intervals $(r, q)$ the $\sigma$-algebra generated by the open intervals $(r, q)$, and the Borel sets to the sets generated from such intervals by complement or countable unions.

Finally, we recall the notions of random variables and expected value

Definition 4.7 (Random variable, Expected value). A random variable is a measurable function $\mathrm{f}$ from a space $\left(\mathrm{X}, \Sigma_{\mathrm{X}}\right)$, to the reals $\left(\mathbb{R}, \Sigma_{\mathbb{R}}\right)$. Given a measure $v$ over $\mathrm{X}$, the expected value of $\mathrm{f}$ with respect to $v$ is the value of the Lebesgue integral:

$$
\mathbb{E}_{x \sim v}[f(x)] \triangleq \int_{X} f(x) v(d x)
$$

We alternatively use the notations $\mathbb{E}_{v}[\mathrm{f}]$ or $\int \mathrm{fd} v$. 


\subsection{Language}

\subsubsection{Syntax}

We consider a simply typed lambda calculus with base types B and a unit type (that we denote by ut to distinguish it from the monad unit) extended with a monadic type former $P$ for probabilistic computations. The terms of the language are standard. The unit $(t)$ operation introduces a monadic (probabilistic) operation, and corresponds to the distribution having all its mass in $t$. The mlet $x=t$ in $u$ operation, also called bind, sequences two monadic operations by computing the expected value of $u$ with respect to an $x$ sampled from $t$. Apart from the usual monadic operations of unit and bind, we have a set of operations $\left\{\alpha_{i}\right\}_{i \in I}$ of arity $\sigma_{i, 1} \times \cdots \times \sigma_{i, \operatorname{ar}(i)} \rightarrow \mathrm{P}\left(\tau_{i}\right)$, which aim to represent probability distribution functions with parameters. The types and terms are given by the following grammar:

$$
\begin{aligned}
& \tau, \sigma::=B \mid \text { ut }|\tau \rightarrow \sigma| \tau \times \sigma \mid P \tau \\
& t, u::=c|x| \lambda x . u|t u|\langle t, u\rangle \mid \text { case } t \text { of } t t \mapsto u_{1} ; f f \mapsto u_{2}\left|\pi_{1}(t)\right| \pi_{2}(t) \\
& \quad \text { unit }(t) \mid \text { mlet } x=t \text { in } u \mid \alpha_{i}\left(t_{1}, \ldots, t_{\operatorname{ar}(i)}\right)
\end{aligned}
$$

For our examples it is enough to consider base types for booleans, reals and the $[0,1]$ interval, and the distributions $\operatorname{Bern}(\cdot):[0,1] \rightarrow \mathrm{P}($ bool $)$, Gauss $(\cdot, \cdot):$ real $\times$ real $\rightarrow$ $\mathrm{P}($ real $), \operatorname{Lap}(\cdot, \cdot):$ real $\times$ real $\rightarrow \mathrm{P}($ real $)$, and Unif $[0,1]^{n}: \mathrm{P}\left([0,1]^{\mathrm{n}}\right)$.

A typing judgment $\Gamma \vdash t: \tau$ is a relation between contexts, terms and types. Typing rules standard and presented in Figure 6.1.

$$
\begin{aligned}
& \Gamma, x: \tau, \Gamma^{\prime} \vdash x: \tau \operatorname{Var} \quad \frac{\Gamma, x: \tau \vdash t: \sigma}{\Gamma \vdash \lambda x . t: \tau \rightarrow \sigma} \text { Abs } \frac{\Gamma \vdash t_{1}: \tau \rightarrow \sigma \quad \Gamma \vdash t_{2}: \tau}{\Gamma \vdash t_{1} t_{2}: \sigma} \text { App } \\
& \frac{\Gamma \vdash \mathrm{b}: \mathbb{B} \quad \Gamma \vdash \mathrm{t}_{1}: \tau \quad \Gamma \vdash \mathrm{t}_{2}: \tau}{\Gamma \vdash \text { case } \mathrm{b} \text { of } \mathrm{tt} \mapsto \mathrm{t}_{1} ; \mathrm{ff} \mapsto \mathrm{t}_{2}: \tau} \text { Case } \\
& \frac{\Gamma \vdash \mathrm{t}: \tau_{1} \times \tau_{2}}{\Gamma \vdash \pi_{\mathfrak{i}}(\mathrm{t}): \tau_{\mathrm{i}}} \text { Proj } \quad \frac{\Gamma \vdash \mathrm{t}: \tau \quad \Gamma \vdash \mathrm{u}: \sigma}{\Gamma \vdash\langle\mathrm{t}, \mathrm{u}\rangle: \tau \times \sigma} \text { Pair } \\
& \frac{\Gamma \vdash t: \tau}{\Gamma \vdash \text { unit }(t): P \tau} \text { Unit } \quad \frac{\Gamma \vdash t_{1}: P \tau \quad \Gamma, x: \tau \vdash t_{2}: P \sigma}{\Gamma \vdash \text { mlet } x=t_{1} \text { in } t_{2}: P \sigma} \text { MLet } \\
& \frac{\Gamma \vdash \mathrm{t}_{1}: \sigma_{i, 1} \quad \ldots \quad \Gamma \vdash \mathrm{t}_{\mathrm{ar}(\mathrm{i})}: \sigma_{i, \operatorname{ar}(\mathrm{i})}}{\Gamma \vdash \alpha_{\mathfrak{i}}\left(\mathrm{t}_{1}, \ldots, \mathrm{t}_{\operatorname{ar}(\mathfrak{i})}\right): \mathrm{P}\left(\tau_{\operatorname{ar}(\mathfrak{i})}\right)} \mathrm{Op}
\end{aligned}
$$

Figure 4.1: Selected typing rules 


\subsection{Higher-Order Union Bound logic}

In this section, we present Higher-Order Union Bound Logic, or HO-UBL, a higherorder variant of the Union Bound logic [38]. This logic is used to prove an upper bound on the probability that a probabilistic program fails to satisfy an specification. It takes its name after the union bound (Lemma 4.5), which is used to compute the bound when sequencing two programs.

This logic is built on top of UHOL, by adding new judgments to reason about probabilistic computations, which are of the form:

$$
\Gamma \mid \Psi \vdash t: P \sigma\{Q\}\} \delta
$$

These judgments look similar to the ones from usual UHOL, but there is a key difference, which is indicated by the double brace and the grading $\delta$ on the postcondition. Here $t$ is a probabilistic computation over type $\sigma$, and $\Gamma, \mathbf{v}: \sigma \vdash \mathrm{Q}$ is a postcondition that can refer to the result. Note that the result actually has type $\mathrm{P}(\sigma)$, instead of $\sigma$. Therefore, we will need to use a lifting to interpret $\mathrm{Q}$. We also use the distinguished variable $\mathbf{v}$ (standing for "value") since it does not represent $t$ but rather a value sampled from $t$.

The intended interpretation of this judgment is that if $\Gamma \mid \Psi \vdash t: P \sigma\left\{\left\{Q_{\delta}\right.\right.$ is derivable, then for every $\gamma \in \Gamma$ such that $\gamma \in \Lambda \Psi, \mathrm{t}(\gamma)$ is a distribution over $\sigma$ such that $\operatorname{Pr}_{t}[\mathrm{Q}] \geqslant 1-\delta$ (or in other words, $\operatorname{Pr}_{\mathrm{t}}[\neg \mathrm{Q}] \leqslant \delta$ ).

Figure 4.2 presents the rules of the logic. Having in mind the interpretation above, the rules are not hard to understand:

- The UNIT-U states that if $t$ satisfies $\phi$, then unit( $t$ ) (with is the distribution with all its mass in $t$ ) returns with probability 1 a result satisfying $\phi$.

- The MLET-U rule is a generalization of the usual sequencing rule of Hoare logic. If $\mathrm{t}$ fails to satisfy $\mathrm{Q}$ with a probability bounded by $\delta$, and assuming $\mathrm{Q}$, $u$ fails to satisfy $R$ with probability bounded by $\delta^{\prime}$, then sequencing $t$ with $u$ fails to satisfy $R$ with probability bounded by $\delta+\delta^{\prime}$.

- The rule MCASE-U performs a case analysis for monadic computations. Note that the CASE-U rule from UHOL would not suffice, since it cannot analyze the branches, which have a monadic type.

- The CONSEQ-U rule weakens the postcondition or the bound, where the relation $\sqsubseteq$ needs to be established in HOL.

- Finally we show a rule SAMPLE-UBL for sampling from a uniform distribution. Other distributions have different sampling rules, which we omit for now. 


$$
\begin{aligned}
& \frac{\Gamma \mid \Psi \vdash t: \tau\{\phi\}}{\Gamma \mid \Psi \vdash \operatorname{unit}(\mathrm{t}): \mathrm{P}(\tau)\{\phi[\mathbf{v} / \mathbf{r}]\}_{0}} \text { UNIT-U } \\
& \frac{\Gamma\left|\Psi \vdash t: \mathcal{T}_{k}(\tau)\{\mathrm{Q}\}_{\delta} \quad \Gamma, x: \tau\right| \Psi, \mathrm{Q}[\mathrm{x} / \mathrm{v}] \vdash \mathrm{u}: \mathrm{P}(\sigma)\left\{[R\}_{\delta^{\prime}} \quad x \notin \mathrm{R}\right.}{\left.\Gamma \mid \Psi \vdash \text { mlet } x=\mathrm{t} \text { in } \mathrm{u}: \mathrm{P}(\sigma)\{R\}_{\delta}\right\}_{\delta^{\prime}}} \text { MLET-U } \\
& \frac{\Gamma \vdash \mathrm{b}: \text { bool } \quad \Gamma\left|\Psi \wedge \mathrm{b}=\mathbf{t t} \vdash \mathrm{t}_{1}: \mathrm{P}(\tau)\{\mathrm{Q}\}_{\delta} \quad \Gamma\right| \Psi \wedge \mathrm{b}=\mathbf{f f f} \vdash \mathrm{P}: \mathcal{T}_{k}(\tau)\left\{\{\mathrm{Q}\}_{\delta}\right.}{\Gamma \mid \Psi \vdash \text { case } \mathrm{b} \text { of } \mathrm{tt} \mapsto \mathrm{t}_{1} ; \mathrm{ff} \mapsto \mathrm{t}_{2}: \mathrm{P}(\tau)\{\mathrm{Q}\}_{\delta}} \text { MCASE-U } \\
& \frac{\Gamma\left|\Psi \vdash \mathrm{t}: \mathrm{P}(\tau)\left\{\mathrm{Q}^{\prime}\right\}_{\delta} \quad \Gamma\right| \Psi \vdash \mathrm{Q}^{\prime} \sqsubseteq \mathrm{Q} \quad \delta \leqslant \delta^{\prime}}{\Gamma \mid \Psi \vdash \mathrm{t}: \mathrm{P}(\tau)\left\{\left\{\mathrm{Q}_{\delta^{\prime}}\right.\right.} \text { CONSEQ } \\
& \frac{|\{x \in \sigma \mid x \in \phi\}| /|\sigma|=\delta}{\Gamma \mid \Psi \vdash \operatorname{sample}\left(\text { Unif }_{\sigma}\right): \mathrm{P}(\sigma)\left\{\{\phi\}_{1-\delta}\right.} \text { SAMPLE-UBL }
\end{aligned}
$$

Figure 4.2: Union bound: monadic rules

\subsection{Examples}

In this section we adapt the proof of the Report-Noisy-Max algorithm from [38]. Report-Noisy-Max is an algorithm that comes from the literature of differential privacy. It is applied when running a number $\mathrm{N}$ of counting queries on a database and returning the index of the query with largest result. To ensure privacy is preserved, the algorithm adds random noise to every query result and then returns the index of the largest noisy output. What we will prove about this algorithm is its accuracy, i.e., the probability that the output is actually the index of the largest non-noisy output.

To prove this example we consider a small addition to the language: an indexed type list $_{\tau}[N]$ of lists of $\tau$ of length less than $N$, plus an operator iterate $l t x . y . u$ to perform iteration over lists, where the variables $x, y$ are bound in $u$ and whose semantics is equivalent to:

$$
\text { (letrec } f z=\text { case } z \text { of } \square \mapsto t ;_{-}::_{-} \mapsto \lambda x \text {.xs.mlet } y=f x s \text { in } u \text { ) } l
$$

We will also do further simplifications that have no effect in reasoning about accuracy: instead of having a list of queries and performing those queries on a database, we will have a list of real numbers and we will pass those numbers to a 
function $\mathrm{f}$. With these considerations, the program can be written as follows:

$$
\begin{aligned}
& r n m \triangleq \lambda l . \lambda f . \quad \text { iterate } l \\
& \text { unit }(\langle 0,0\rangle) \\
& \text { x.y.mlet } z=\operatorname{Lap}(\epsilon / 2, \mathrm{f}(\mathrm{x})) \text { in } \\
& \text { case } z \geqslant \pi_{2}(\mathrm{y}) \text { of } \\
& \mathbf{t t} \mapsto \operatorname{unit}(\langle 0, z\rangle) \\
& \mathbf{f f} \mapsto \operatorname{unit}\left(\left\langle\pi_{1}(\mathrm{y})+1, \pi_{2}(\mathrm{y})\right\rangle\right)
\end{aligned}
$$

Our implementation returns a pair consistent of the maximal elements position and its corresponding output. ${ }^{1}$ When considering a new element $x$, we compare it to the maximal element and we either return the new elements position (which is o, since it is at the front) and the noisy output $z$ of $f(x)$, or the old maximal element's position, which we update by adding 1 and its noisy output. Noise is added by a Laplace distribution Lap of parameter $\epsilon / 2$ centered at $f(x)$. We can show the following result:

Lemma 4.8. Let $N \in \mathbb{N}, \in \in[0,1], l:$ list $_{\tau}[N], f: \tau \rightarrow \mathbb{R}, \delta \in[0,1]$. Then, the probability of sampling $(\mathrm{r}, \mathrm{n})$ from the output distribution of ( $r m n \mathrm{l} f$ ) such that for every $\mathrm{r}^{\prime} \in \mathrm{l}$, $\mathrm{f}(\mathrm{r})>\mathrm{f}\left(\mathrm{r}^{\prime}\right)-4 / \epsilon \cdot \log (\mathrm{N} / \delta)$ is at least $1-\delta$.

In our system, we prove the judgment:

$$
l: \operatorname{list}_{\tau}[\mathrm{N}], \mathrm{f}: \tau \rightarrow \mathbb{R} \mid \cdot \vdash r m n l f: \mathrm{P}(\mathbb{N} \times \mathbb{R})\left\{\forall \forall \mathrm{r}^{\prime} \in \operatorname{l.f}\left(\mathrm{l}\left[\pi_{1}(\mathbf{v})\right]\right)>\mathrm{f}\left(\mathrm{r}^{\prime}\right)-\frac{4}{\epsilon} \cdot \log \left(\frac{\mathrm{N}}{\delta}\right)\right\}_{\delta}
$$

We start by applying the rule ITER-U for iteration:

$$
\begin{gathered}
\Gamma, l: \operatorname{list}_{\sigma}[0] \mid \Psi \vdash t: P(\tau)\left\{\phi_{0}[[/ l]\}_{0}\right. \\
\frac{\Gamma, x: \sigma, y: \tau, l: \operatorname{list}_{\sigma}[M] \mid \Psi, \phi_{M}[y / v] \vdash u: P(\tau)\left\{\phi_{M+1}[x:: l / l]\right\}_{\delta}}{\Gamma, l: \operatorname{list}_{\sigma}[N] \mid \Psi \vdash \text { iterate } l t x . y . u: P(\tau)\left\{\phi_{N}\right\}_{N \delta}} \text { ITER-U }
\end{gathered}
$$

where $\left\{\phi_{M}\right\}_{M \in \mathbb{N}}$ is a family of indexed predicates such that, for all $M, \phi_{M} \Rightarrow \phi_{M+1}$. In our example we define it as:

$$
\phi_{N} \triangleq \forall r^{\prime} \in \operatorname{l.f}\left(l\left[\pi_{1}(\mathbf{v})\right]\right)>f\left(r^{\prime}\right)-\frac{4}{\epsilon} \cdot \log \left(\frac{N}{\delta}\right) \wedge\left|\pi_{2}(\mathbf{v})-f\left(l\left[\pi_{1}(\mathbf{v})\right]\right)\right| \leqslant \frac{2}{\epsilon} \log \frac{N}{\delta}
$$

This invariant states that the return value $\mathbf{v}$ is a pair that contains the maximal element of the list so far and a noisy output of $f$ for this element.

For the base case, we need to show:

$$
l: \operatorname{list}_{\tau}[0], f: \tau \rightarrow \mathbb{R} \mid \cdot \vdash \text { unit }(\langle 0,0\rangle): P(\mathbb{R})\left\{\left\{\phi_{0}\right\}_{0} .\right.
$$

This is proven by applying the UNIT-U rule, and some trivial reasoning in HOL, since the quantification in $\phi_{0}$ is over the elements of an empty list.

\footnotetext{
${ }^{1}$ We note that returning the output of queries can break privacy, but this does not effect reasoning about accuracy.
} 
In the inductive step, the proof uses the rule for Laplace sampling

$$
\frac{\Gamma \vdash r: \mathbb{R}}{\Gamma \mid \Psi \vdash \operatorname{Lap}(\epsilon, r): P(\mathbb{R})\left\{|\mathbf{v}-r| \leqslant \frac{1}{\epsilon} \log \frac{1}{\delta}\right\}_{\delta}} \text { LAP-U }
$$

to establish the judgment

$$
l: \operatorname{list}_{\tau}[M], f: \tau \rightarrow \mathbb{R} \mid \cdot \vdash \operatorname{Lap}(\epsilon / 2, f(z)): P(\mathbb{R})\left\{|\mathbf{v}-f(x)| \leqslant \frac{2}{\epsilon} \log \frac{M+1}{\delta}\right\}_{\delta /(M+1)} .
$$

Then, using the MLET-U rule to sequence computations and the MCASE- $U$ to do a case distinction, we show the postcondition in both branches. That is, if $z \geqslant \pi_{2}(y)$ then,

$$
\begin{aligned}
& \Gamma\left|z \geqslant \pi_{2}(y),\right| z-f(x) \mid \leqslant \frac{2}{\epsilon} \log \frac{N}{\delta}, \phi_{M}[z / v] \\
& \vdash \operatorname{unit}\left(\left\langle\pi_{1}(y)+1, \pi_{2}(y)\right\rangle\right): P(\mathbb{N} \times \mathbb{R})\left\{\left\{\phi_{M+1}\right\}_{\delta /(M+1)}\right.
\end{aligned}
$$

for $\Gamma \triangleq l: \operatorname{list}_{\tau}[M], f: \tau \rightarrow \mathbb{R}, x: \tau, y: \mathbb{N} \times \mathbb{R}, z: \mathbb{R}$, which amounts to proving in HOL

$$
\begin{aligned}
& \Gamma\left|z \geqslant \pi_{2}(y),\right| z-f(x) \mid \leqslant \frac{2}{\epsilon} \log \frac{M+1}{\delta}, \\
& \forall r^{\prime} \in \operatorname{l.f}\left(l\left[\pi_{1}(y)\right]\right)>f\left(r^{\prime}\right)-\frac{4}{\epsilon} \cdot \log \left(\frac{M}{\delta}\right),\left|\pi_{2}(y)-f\left(l\left[\pi_{1}(y)\right]\right)\right| \leqslant \frac{2}{\epsilon} \log \frac{M}{\delta}, \\
& \vdash \forall r^{\prime} \in x:: \operatorname{l.f}(x)>f\left(r^{\prime}\right)-\frac{4}{\epsilon} \cdot \log \left(\frac{M+1}{\delta}\right) \wedge|z-f(x)| \leqslant \frac{2}{\epsilon} \log \frac{M+1}{\delta}
\end{aligned}
$$

which is a routine derivation. If $z<\pi_{2}(\mathrm{y})$, the proof proceeds along similar lines. Therefore we conclude.

Remark 4.9. In this example we introduce the rule of ITER-U in an ad hoc manner. This rule considers indexed lists and families of predicates to facilitate inductive reasoning. Similar rules for natural numbers or other inductive datatypes can also be added to the system, but we do not explore this in full generality. We believe this would clutter the presentation, and moreover is an orthogonal issue.

\subsection{Category theory preliminaries}

In the previous chapter we presented set-theoretical semantics for our language PCF and our logics : HOL, UHOL and RHOL. This semantics can actually be generalized to a categorical setting, given by a Cartesian closed category that can interpret a simply-typed language, and a category of predicates to interpret the logics. In this section we will first present a recap of the category theory notions needed to understand the semantics, and then present the construction of a categorical model for our language and logics. 


\subsubsection{A Primer on Category theory}

We begin with a quick recap on the definitions and notations that will appear throughout the chapter and the rest of the thesis. For a proper textbook treatment of this topic refer for instance to [113].

\section{Categories, functors and natural transformations}

Definition 4.10 (Category). A category $\mathrm{C}$ is defined by the following data:

- A collection of objects $\mathrm{X}, \mathrm{Y}, \ldots$. If $\mathrm{X}$ is an object of $\mathbf{C}$ we will use the notation $\mathrm{X} \in \mathbf{C}$.

- For each pair of objects $\mathrm{X}, \mathrm{Y}$ a set (called the hom-set) $\mathrm{C}(\mathrm{X}, \mathrm{Y})$ of morphisms. If $f \in \mathbf{C}(X, Y)$ we call $X$ its domain and $Y$ its codomain, and often use the notation $f: X \rightarrow Y$.

Additionally, there is an associative operation o called the composition, that for every $\mathrm{X}, \mathrm{Y}, \mathrm{Z}$ sends $\mathrm{f} \in \mathrm{C}(\mathrm{X}, \mathrm{Y})$ and $\mathrm{g} \in \mathrm{C}(\mathrm{Y}, \mathrm{Z})$ to $\mathrm{g} \circ \mathrm{f} \in \mathrm{C}(\mathrm{X}, \mathrm{Z})$. We will sometimes denote $\mathrm{g} \circ \mathrm{f}$ by juxtaposition, i.e., gf. For every $\mathrm{X} \in \mathbf{C}$ there exists an identity morphism $i \mathrm{~d} \mathrm{X} \in \mathbf{C}(\mathrm{X}, \mathrm{X})$ such that for every $f \in \mathbf{C}(X, X)$, id $\circ f=f=f \circ i d$.

We will usually denote categories by bold capital letters $\mathbf{C}, \mathbf{D}, \mathbf{P}, \ldots$

Definition 4.11 (Functor). Given two categories C, D a functor F from C to D (denoted $\mathrm{F}: \mathbf{C} \rightarrow \mathbf{D})$ is a map such that:

- Sends objects of $\mathbf{C}$ to objects in $\mathbf{D}$

- For every $\mathrm{X}, \mathrm{F}\left(\mathrm{id}_{\mathrm{X}}\right)=\mathrm{id}_{\mathrm{F}}(\mathrm{X})$

- For every composable $f, g, F(f \circ g)=F(f) \circ F(g)$.

A functor is contravariant (denoted $\mathrm{F}: \mathrm{C}^{\mathrm{op}} \rightarrow \mathrm{D}$ ) if instead of the third condition we have $\mathrm{F}(\mathrm{f} \circ \mathrm{g})=\mathrm{F}(\mathrm{g}) \circ \mathrm{F}(\mathrm{f})$.

We will usually denote functors by uppercase letters $\mathrm{F}, \mathrm{G}, \mathrm{H}, \ldots$

Definition 4.12 (Natural transformation). Given two categories C, D and two functors $\mathrm{F}, \mathrm{G}: \mathbf{C} \rightarrow \mathbf{D}$ a natural transformation $\alpha$ from $\mathrm{F}$ to $\mathrm{G}$ (denoted $\alpha: \mathrm{F} \Rightarrow \mathrm{G}$ ) is defined as a collection of morphisms $\left\{\alpha_{X}: \mathrm{F}(\mathrm{X}) \rightarrow \mathrm{G}(\mathrm{X})\right\}_{\mathrm{X} \in \mathbf{C}}$ in $\mathbf{D}$ indexed by objects in $\mathbf{C}$ such that for every $X, Y \in C$ and every $f: X \rightarrow Y$, we have the equality $G(f) \circ \alpha_{X}=\alpha_{Y} \circ F(f)$.

We will usually denote natural transformations by lowercase greek letters : $\alpha, \beta, \eta, \ldots$

Example 4.13. A prototypical example of a category is the category of sets, Set, that has sets as objects and functions between sets as morphisms.

Example 4.14. Another example is the category of categories, Cat, that has as objects small categories $^{2}$ and as morphisms functors between them.

\footnotetext{
${ }^{2}$ i.e., categories whose collection of objects is a set
} 


\section{Limits and colimits}

Definition 4.15. Let $\mathbf{C}$ be a category, and $\mathrm{X}, \mathrm{Y} \in \mathbf{C}$. The product of $\mathrm{X}$ and $\mathrm{Y}$, if it exists, is an object $\mathrm{X} \times \mathrm{Y} \in \mathrm{C}$ equipped with two projections $\pi_{1}: \mathrm{X} \times \mathrm{Y} \rightarrow \mathrm{X}$ and $\pi_{2}: \mathrm{X} \times \mathrm{Y} \rightarrow \mathrm{Y}$ satisfying the following property: for every $Z \in \mathbf{C}$ and every pair of morphisms $f: Z \rightarrow X$, $\mathrm{g}: \mathrm{Z} \rightarrow \mathrm{Y}$ there exists a unique morphism $\langle\mathrm{f}, \mathrm{g}\rangle: \mathrm{Z} \rightarrow \mathrm{X} \times \mathrm{Y}$ such that $\mathrm{f}=\pi_{1} \circ\langle\mathrm{f}, \mathrm{g}\rangle$ and $g=\pi_{2} \circ\langle f, g\rangle$.

Definition 4.16. A category $\mathbf{C}$ has a terminal object (usually denoted 1 ) if for every $\mathrm{X} \in \mathbf{C}$ there exists a unique morphism from $\mathrm{X}$ to 1 (usually denoted !).

Definition 4.17. A category $\mathrm{C}$ has finite products if it has a terminal object, and for every $\mathrm{X}, \mathrm{Y}$ their Cartesian product exists.

All these definitions can be dualized

Definition 4.18. Let $\mathbf{C}$ be a category, and $X, Y \in \mathbf{C}$. The coproduct of $X$ and $Y$, if it exists, is an object $\mathrm{X}+\mathrm{Y} \in \mathrm{C}$ equipped with two injections $\operatorname{inj}_{1}: \mathrm{X} \rightarrow \mathrm{X}+\mathrm{Y}$ and $\operatorname{inj}_{2}: \mathrm{Y} \rightarrow \mathrm{X}+\mathrm{Y}$ satisfying the following property: for every $Z \in C$ and every pair of morphisms $f: X \rightarrow Z$, $\mathrm{g}: \mathrm{Y} \rightarrow \mathrm{Z}$ there exists a unique morphism $[\mathrm{f}, \mathrm{g}]: \mathrm{X}+\mathrm{Y} \rightarrow \mathrm{Z}$ such that $\mathrm{f}=[\mathrm{f}, \mathrm{g}] \circ \mathrm{inj}_{1}$ and $\mathrm{g}=[\mathrm{f}, \mathrm{g}] \circ \operatorname{inj}_{2}$.

Definition 4.19. A category $\mathbf{C}$ has an initial object (usually denoted 0 ) if for every $\mathbf{X} \in \mathbf{C}$ there exists a unique morphism from 0 to $\mathrm{X}$ (usually also denoted !).

Definition 4.20. A category $\mathbf{C}$ has finite coproducts if it has an initial object, and for every $\mathrm{X}, \mathrm{Y}$ their coproduct exists.

Definition 4.21. Let $\mathbf{C}$ be a category, $\mathrm{X}, \mathrm{Y}, \mathrm{Z}$ objects in $\mathrm{C}$, and $\mathrm{f}: \mathrm{X} \rightarrow \mathrm{Z}, \mathrm{g}: \mathrm{Y} \rightarrow \mathrm{Z}$. $A$ pullback of $\mathrm{f}$ and $\mathrm{g}$ is given by an object $\mathrm{A} \in \mathrm{C}$ and two arrows $\mathrm{h}_{1}: \mathrm{A} \rightarrow \mathrm{X}, \mathrm{h}_{2}: \mathrm{A} \rightarrow \mathrm{Y}$ such that $\mathrm{f} \circ \mathrm{h}_{1}=\mathrm{g} \circ \mathrm{h}_{2}$, and for any other $\mathrm{B} \in \mathrm{C}$ and $\mathrm{k}_{1}: \mathrm{B} \rightarrow \mathrm{X}, \mathrm{k}_{2}: \mathrm{B} \rightarrow \mathrm{Y}$ with $\mathrm{f} \circ \mathrm{k}_{1}=\mathrm{g} \circ \mathrm{k}_{2}$, then there exists a unique $\mathrm{l}: \mathrm{B} \rightarrow \mathrm{A}$ such that $\mathrm{k}_{1}=\mathrm{h}_{1} \circ \mathrm{l}$ and $\mathrm{k}_{2}=\mathrm{h}_{2} \circ \mathrm{l}$

Example 4.22. In the category Set we have all finite products, finite coproducts and pullbacks

- The product $\mathrm{X} \times \mathrm{Y}$ corresponds to the Cartesian product

- The coproduct $\mathrm{X}+\mathrm{Y}$ corresponds to the disjoint sum, i.e. all pairs of the form $(i, z)$ where either $i=1$ and $z \in X$ or $i=2$ and $z \in Y$.

- The terminal object is the singleton set $\{*\}$.

- The initial object is the empty set.

- The pullback of $\mathrm{f}: \mathrm{X} \rightarrow \mathrm{Z}$ and $\mathrm{g}: \mathrm{Y} \rightarrow \mathrm{Z}$ is given by the set $\{(\mathrm{x}, \mathrm{y}) \in \mathrm{X} \times \mathrm{Y} \mid \mathrm{f}(\mathrm{x})=$ $\mathrm{g}(\mathrm{y})\}$ and the evident projections. 
Cartesian closed categories It is well-known that Cartesian closed categories provide models for simple-typed lambda calculi. We recall the definition:

Definition 4.23 (Cartesian closed category). A Cartesian closed category (CCC) is a category $\mathbf{C}$ with finite products, and such that for every $\mathrm{Y} \in \mathbf{C}$ there exists a functor $\mathrm{Y} \Rightarrow-$ (called the exponential) such that there is a natural bijection: $\mathrm{C}(\mathrm{X} \times \mathrm{Y}, \mathrm{Z}) \cong \mathrm{C}(\mathrm{X}, \mathrm{Y} \Rightarrow \mathrm{Z})$ (in other words, $\mathrm{Y} \Rightarrow-$ is right adjoint to $-\times \mathrm{Y}$ ). We will sometimes denote the exponential $\mathrm{Y} \Rightarrow \mathrm{X}$ by $\mathrm{X}^{\mathrm{Y}}$. We denote by $\lambda: \mathrm{C}(\mathrm{X} \times \mathrm{Y}, \mathrm{Z}) \rightarrow \mathrm{C}(\mathrm{X}, \mathrm{Y} \Rightarrow \mathrm{Z})$ the map realizing the left-toright direction of the bijection, and by $\lambda^{-1}$ its inverse. These are also known as currying and uncurrying, respectively. For every $\mathrm{Y}$, there is a map eval : $(\mathrm{Y} \Rightarrow \mathrm{X}) \times \mathrm{Y} \rightarrow \mathrm{X}$ natural in $\mathrm{X}$, called the evaluation.

A bicartesian closed category (BiCCC) is a CCC with finite coproducts.

Example 4.24. The category Set is BiCCC, and $\mathrm{Y} \Rightarrow \mathrm{Z}$ is given by the set $\mathrm{C}(\mathrm{Y}, \mathrm{Z})$.

\section{Algebras}

Definition 4.25 (Algebra, Initial algebra). Let $\mathbf{C}$ be a category and $\mathrm{F}: \mathbf{C} \rightarrow \mathbf{C}$ be a functor. An algebra is a pair $(\mathrm{X}, \mathrm{f})$ of a carrier set $\mathrm{X} \in \mathrm{C}$ and a morphism $\mathrm{f}: \mathrm{F}(\mathrm{X}) \rightarrow \mathrm{X}$. Given two algebras $(\mathrm{X}, \mathrm{f})$ and $(\mathrm{Y}, \mathrm{g})$ of $\mathrm{F}$, an algebra homomorphism between them is a morphism $\mathrm{h}: \mathrm{X} \rightarrow \mathrm{Y}$ such that $\mathrm{g} \circ \mathrm{F}(\mathrm{h})=\mathrm{h} \circ \mathrm{f}$. An algebra of $\mathrm{F}$ is initial if there is exactly one algebra homomorphism to any other algebra of the same functor.

\subsubsection{Interpreting logics}

Jacobs [99] discusses how to give semantics of a logic over a simply-typed lambda calculus using the notion of fibrations. We can use this construction to give semantics of HOL, and then extend it to UHOL and RHOL.

Definition 4.26. Let $\mathbf{C}$ and $\mathbf{P}$ be two categories. A fibration is a functor $p: \mathbf{P} \rightarrow \mathbf{C}$ satisfiyng the following property (sometimes known as the Cartesian lifting property): for every object $\mathrm{Q} \in \mathbf{P}, \mathrm{X} \in \mathbf{C}$, and every morphism $\mathrm{f}: \mathrm{X} \rightarrow \mathrm{pQ}$, there exists an object $\mathbf{P} \in \mathbf{P}$ such that $\mathrm{pP}=\mathrm{X}$ and a morphism $\mathrm{s}: \mathrm{P} \rightarrow \mathrm{Q}$ that are universal, in the sense that for every $\mathrm{R} \in \mathrm{P}, \mathrm{t}: \mathrm{R} \rightarrow \mathrm{Q}$, and $\mathrm{h}: \mathrm{pR} \rightarrow \mathrm{X}$ such that $\mathrm{f} \circ \mathrm{h}=\mathrm{pt}$, there exists a unique $\mathrm{u}: \mathrm{R} \rightarrow \mathrm{P}$ such that $\mathrm{pu}=\mathrm{h}$ and $\mathrm{s} \circ \mathrm{u}=\mathrm{t}$. We usually denote $\mathrm{s} b \mathrm{y} \overline{\mathrm{f}}$ and $\mathrm{P}$ by $\mathrm{f}^{*} \mathrm{Q}$.

Given a fibration $\mathrm{p}: \mathbf{P} \rightarrow \mathbf{C}$, and $\mathbf{X} \in \mathbf{C}$, the collection of objects in $\mathbf{P}$ that are mapped to $\mathrm{X}$, and morphisms in $\mathbf{P}$ that are mapped to $i \mathrm{~d}_{\mathrm{X}}$ forms a category known as the fiber over $\mathrm{X}$ and denoted by $\mathbf{P}_{X}$. For $\mathrm{f}: \mathrm{X} \rightarrow \mathrm{Y}$ in $\mathbf{C}, \mathrm{f}^{*}$ is a functor between $\mathbf{P}_{\mathrm{Y}}$ and $\mathbf{P}_{\mathrm{X}}$ known as the reindexing functor. 3

In writing will refer to $\mathbf{C}$ as the base category (of $\mathrm{p}$ ), $\mathbf{P}$ as the total category (of $\mathrm{p}$ ), and if $\mathrm{pQ}=\mathrm{X}$ or $\mathrm{pt}=\mathrm{f}$ we respectively say that $\mathrm{Q}$ is above $\mathrm{X}$ or $\mathrm{t}$ is above $\mathrm{f}$ (in $\mathrm{p}$ ).

\footnotetext{
${ }^{3}$ Note that in general the choice of $f^{*}$ is not unique, since there may be another isomorphic object satisfying the universal property above. However, in all our uses of fibrations this choice is indeed unique and $\mathrm{f}^{*}$ is a well-defined functor
} 
These definitions are somewhat dry, however we are interested in a particular case of fibrations where the fibers have a concrete structure:

Definition 4.27. Let $\mathrm{p}: \mathbf{C} \rightarrow \mathbf{P}$ be a fibration. We say that $\mathrm{p}$ is a complete Heyting fibration if all the fibers are complete Heyting algebras and all the reindexing functors preserve the complete Heyting algebra structure.

In this setting, $\mathbf{P}_{X}$ can be seen as the set of predicates over $X$. The Cartesian lifting property implies that for every $f: X \rightarrow Y$ and every predicate $Q \in \mathbf{P}_{Y}$ the reindexing functor $f^{*} Q$ morally corresponds to the inverse image of $Q$ along $f$, that is, $f^{-1}(Q)$. For a complete Heyting fibration, each fiber has internal structure $\left(\mathbf{C}_{\mathbf{X}}, \sqsubseteq, \sqcap, \sqcup, \rightrightarrows, \top, \perp\right)$. This allows us to interpret logical implication and logical connectives. To interpret quantifiers, we need additional structure:

Definition 4.28. Let $\mathbf{C}$ be a category with finite products. A fibration $\mathrm{p}: \mathbf{P} \rightarrow \mathbf{C}$ has simple products if:

- For every projection $\pi_{1}: X \times Y \rightarrow X$, its corresponding reindexing functor $\pi_{1}^{*}: \mathbf{P}_{X} \rightarrow$ $\mathbf{P}_{X \times Y}$ has a right adjoint $\Pi_{X, Y}: \mathbf{P}_{X \times Y} \rightarrow \mathbf{P}_{X}$.

- This right adjoint satisfies the so-called Beck-Chevalley condition: for every $\mathrm{f}: \mathrm{X} \rightarrow \mathrm{Y}$ and $\mathrm{Z} \in \mathbf{C}$, the canonical natural transformation from $\mathrm{f}^{*} \circ \Pi_{\mathrm{X}, \mathrm{Z}}$ to $\Pi_{\mathrm{Y}, \mathrm{Z}} \circ(\mathrm{f} \times \mathrm{idZ})^{*}$ is an isomorphism.

The intuition is that the simple product $\Pi_{X, Y}$ takes a predicate $\phi$ over $X \times Y$ and maps it to the predicate $\forall x \in X . \phi(x)$.

Now we set up the model in which we will interpret our languages and logics. To be able to interpret product and function types in our language, as well as predicates over them, we require a pair of Cartesian closed categories $\mathbf{P}$ and $\mathbf{C}$, and a fibration $p: \mathbf{P} \rightarrow \mathbf{C}$ that preserves the Cartesian closed structure ${ }^{4}$. For convenience, we give a name to these fibrations, following [105]

Definition 4.29 (Fibration for logical relations). Let $\mathrm{p}: \mathbf{P} \rightarrow \mathbf{C}$ be a complete Heyting fibration with simple products where $\mathbf{P}$ and $\mathbf{C}$ are bicartesian closed categories, and $\mathrm{p}$ preserves the BiCCC structure. We say that $\mathrm{p}$ is a fibration for logical relations, or an $L R$ fibration for short.

Sets and predicates over them form such a fibration:

Example 4.30. Let $\operatorname{Pred}($ Set $)$ be the category that has:

- Objects: pairs of sets $(X, \phi)$ such that $\phi \subseteq X$

\footnotetext{
4In fact, it is sufficient to only require that $\mathbf{C}$ is CCC, the CCC structure of $\mathbf{P}$ can be constructed from the fiberwise structure, see Section 9.2 of [99] for details
} 
- Morphisms from $(\mathrm{X}, \phi)$ to $(\mathrm{Y}, \psi)$ : set morphisms $\mathrm{f}: \mathrm{X} \rightarrow \mathrm{Y}$ such that for all $\mathrm{x} \in \boldsymbol{\phi}$, $f(x) \in \psi$

This category is Cartesian closed:

- $(X, \phi) \times(Y, \psi) \triangleq(X \times Y,\{(x, y) \mid x \in \phi \wedge y \in \psi\})$

- $(X, \phi) \Rightarrow(Y, \psi) \triangleq(X \Rightarrow Y,\{f \mid \forall x \in \phi . f(y) \in \psi\})$

The forgetful functor $\mathrm{d}: \operatorname{Pred}($ Set $) \rightarrow$ Set mapping $(X, \phi)$ to $X$ preserves the Cartesian closed structure. Therefore, $\mathrm{d}$ is a Heyting fibration with fiberwise structure given by the usual set operations.

We will later see how to interpret HOL, UHOL and RHOL in an LR fibration $p: \mathbf{P} \rightarrow \mathbf{C}$.

\subsection{Adding effects: Graded liftings}

If we want to extend our base language with computational effects, we need to be able to reason about this computations in our logics. Concretely, to specify a property about a monadic computation of type $\mathcal{P}(\sigma)$ we need a predicate $\phi \subseteq \mathcal{P}(\sigma)$. This can be done by adding predicates that explicitly refer to effects, e.g. probabilities. However, this style of reasoning is not always convenient or intuitive. It requires adding an axiomatization of probability theory to HOL, so that the parts of the proof that deal with probabilities can be discharged to HOL. We explore this approach in Chapter 9.

Instead, we present a way to write specifications for monadic computations using predicates over pure types. The key idea is the use of monad liftings. A lifting of the monad $\mathcal{P}$ is another monad $\dot{\mathcal{P}}$ that maps a predicate $\psi \subseteq \sigma$ to a predicate $\dot{\mathcal{P}}(\psi) \subseteq \mathcal{P}(\sigma)$ and that composes along the monad $\mathcal{P}$, so that we can verify specifications in a modular way.

\subsubsection{Monads, Graded monads and liftings}

First we recall the notion of monad:

Definition 4.31 (Monad). A monad over a category $C$ is a triple $(\mathcal{T}, v, \eta)$ given by an endofunctor $\mathcal{T}: \mathbf{C} \rightarrow \mathbf{C}$, a natural transformation $\eta_{X}: X \rightarrow \mathcal{T}(X)$ called the unit, and a natural transformation $\mu_{X}: \mathcal{T} \mathcal{T}(X) \rightarrow \mathcal{T}(X)$ called the multiplication satisfying the monad coherence laws:

- For all $\mathrm{X}, i \mathrm{~d}_{\mathcal{T}(X)}=\mu_{X} \circ \eta_{\mathcal{T}(X)}=\mu_{X} \circ \mathcal{T} \eta_{X}$

- For all $\mathrm{X}, \mu_{\mathrm{X}} \circ \mu_{\mathcal{T}(X)}=\mu_{X} \circ \mathcal{T} \mu_{X}$ 
In other words, the following diagrams must commute:

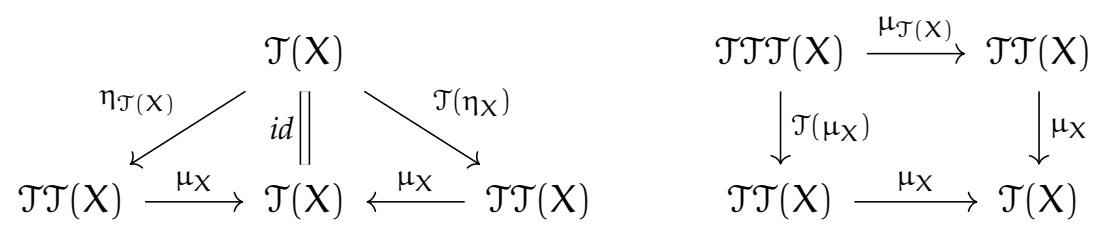

We will often denote a monad $(\mathcal{T}, \nu, \eta)$ by its first component.

A strong monad over a category $\mathbf{C}$ with finite products is a tuple $(\mathcal{T}, v, \eta, \theta)$ where $(\mathcal{T}, v, \eta)$ is a monad and and $\theta_{X, Y}: X \times \mathcal{T}(Y) \rightarrow \mathcal{T}(X \times Y)$ is a natural transformation called the strength satisfying the strength coherence laws, that is, commutativity of the diagrams below:
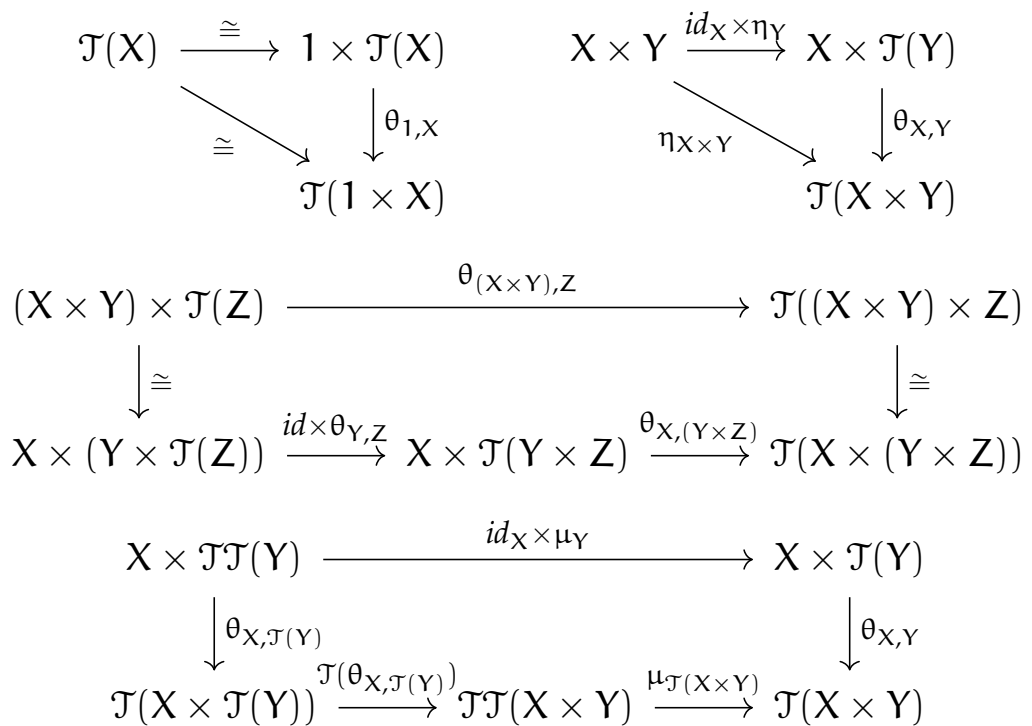

Here, we use the isomorphisms $\mathrm{X} \cong 1 \times \mathrm{X}$ and $(\mathrm{X} \times \mathrm{Y}) \times \mathrm{Z} \cong \mathrm{X} \times(\mathrm{Y} \times \mathrm{Z})$.

Alternatively, a monad can be presented as a triple $\left(\mathcal{T}, \eta,(-)^{\#}\right)$, of a functor, a unit natural transformation and an operation $(-)^{\#}: \mathrm{C}(\mathrm{X}, \mathcal{T}(\mathrm{Y})) \rightarrow \mathrm{C}(\mathcal{T}(\mathrm{X}), \mathcal{T}(\mathrm{Y}))$ called the Kleisli lifting, equivalent to $\mu_{Y} \circ \mathcal{T}(-)$.

The notion of monads can be generalized to graded monads [77], which are given by a family of functors graded by an ordered monoid:

Definition 4.32 (Graded monad). Given an ordered monoid $\mathrm{E}$, a graded monad $\mathcal{T}$ over a category $\mathbf{C}$ is given by an $\mathrm{E}$-indexed family of functors $\left\{\mathcal{T}_{e}\right\}_{e \in E}$, a natural transformation $\eta_{X}: X \rightarrow \mathcal{T}_{1_{E}}$, a family of natural transformations $\left\{\mu_{e, f, X}\right\}_{e, f \in E}: \mathcal{T}_{e} \mathcal{T}_{f} X \rightarrow \mathcal{T}_{e f} X$, a family of natural transformations $\left\{\sqsubseteq^{e \leqslant f}\right\}_{e \leqslant f, e, f \in E}: \mathcal{T}_{e} \rightarrow \mathcal{T}_{f}$ satisfying the generalization of the monad coherence laws:
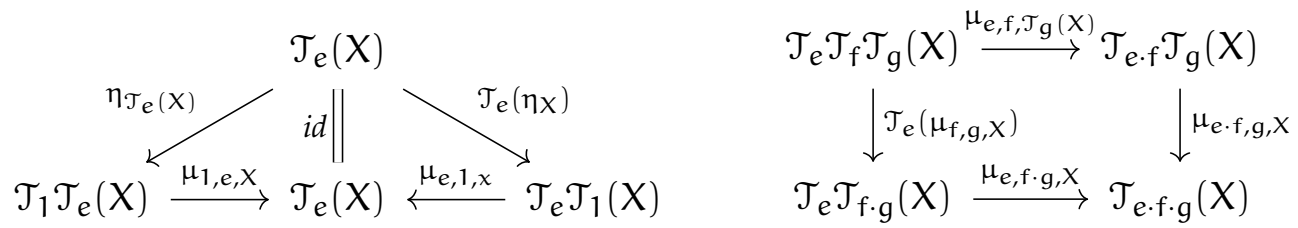
A graded monad is called strong if, in addition, there is a family of natural transformations $\left\{\theta_{e, X, Y}\right\}_{e \in E}: X \times \mathcal{T}_{e} Y \rightarrow \mathcal{T}_{e}(X \times Y)$ satisfying the generalization of the strength coherence laws.

In the theory of programming languages, monads are used to extend a type system with side-effects [120]. A monad comes with operations to introduce and compose these computations. So if we have a pure computation $f$ of type $X \rightarrow Y$, then we can turn it into a computation $\eta_{Y} \circ f$ with a trivial side effect of type $X \rightarrow \mathcal{T}(Y)$, and if we have a computation $g$ of type $Y \rightarrow \mathcal{T}(Z)$, we can extend it to a computation $\mu_{z} \circ \mathcal{T} g$ of type $\mathcal{T}(Y) \rightarrow \mathcal{T}(Z)$.

Gradings add another layer of information on top of this. At the level of monads, gradings can be seen as a minimal refinement on the monadic type that can be enforced directly by the typing system, without logical inference. This refinement is given by a monoid, whose operations are computed simultaneously as the operations of the monad: the unit of the graded monad grades the resulting type with the unit of the monoid, and the multiplication of the monad multiplies the gradings of the nested types. For instance, one could think of the identity monad graded with the monoid of natural numbers with addition to model cost. Then, the unit would introduce a computation of cost 0 , and the multiplication will add the cost of the computations.

Now look again at the rules in Figure 4.2, specially the rules UNIT-U and MLET-U, and for a moment ignore the meaning of the monad and the $\delta$ parameters. The resulting rules are rather natural, and reminiscent from the ones from UHOL or Hoare logic. We want to be able to reason in this same style once we add back the effects. This means that we need to map the predicates in the postconditions, which range over non-monadic types, to predicates over monadic types in such a way that these rules are sound. This is precisely the motivation behind the definition of liftings:

Definition 4.33 (Graded lifting of monad [105]). Given a fibration $\mathrm{p}: \mathbf{P} \rightarrow \mathbf{C}$ and $a$ monad $(\mathcal{P}, \eta, \mu)$ over $\mathbf{C}$, a graded lifting of $\mathcal{P}$ is a graded monad $\left(\left\{\dot{\mathcal{P}}_{e}\right\}_{e \in \mathrm{E}}, \dot{\eta}^{\dot{\mathcal{P}}}, \dot{\mu}^{\dot{\mathcal{P}}, e, \mathrm{f}}, \dot{\llcorner}^{e \leqslant f}\right)$

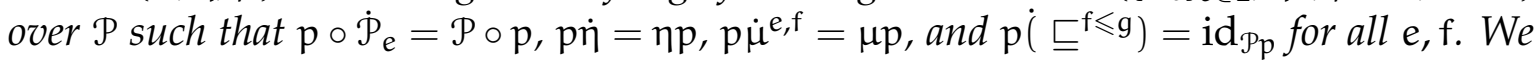
will often denote a lifting by its first component.

The following is an informal explanation of liftings, ignoring for the moment the gradings. The first condition of a lifting $p \circ \dot{\mathcal{P}}_{e}=\mathcal{P} \circ p$ states that if $\phi$ is a predicate over $X$, then $\dot{\mathcal{P}} \phi$ is a predicate over $\mathcal{P} X$. The second and third conditions specify how the unit and multiplication of the lifting interact with the unit and multiplication of the base monad: for a predicate $\phi$ over $X$ and $x \in \phi$, then $\eta(x) \in \dot{\eta}(\phi)$, and for a predicate $\psi$ over $\mathcal{P P}(X)$, if $y \in \psi$ then $\mu(y) \in \dot{\mu}(\psi)$. These properties are used in proving the soundness of the rules of HO-UBL.

At the level of liftings, gradings can be seen as some additional specification, or as adding quantitative information to the specification. For instance, we will use 
the grading on a lifting to specify the probability with which a computation may fail to satisfy the specification. This is the intuition behind the fourth condition in the definition: it allows weakenings of the grading of a lifted predicate, i.e., if $f \leqslant g$ then $\dot{\mathcal{P}}_{\mathrm{f}}(\psi) \Rightarrow \dot{\mathcal{P}}_{\mathrm{g}}(\psi)$.

We present concrete examples of liftings in Section 4.7.3, Section 4.9 and Section 5.4.

\subsection{Adding probabilities}

\subsubsection{The category of Quasi-Borel spaces}

Measurable spaces and measurable functions between them form a category denoted by Meas. This category has a monad $\mathcal{G}$ sending a measurable space to the measurable space of probability distributions over it. This is known as the Giry monad [84], and can be used to interpret first-order probabilistic languages with sampling from continuous probability distributions. However, semantics for higher-order languages is hard to accommodate in Meas, since it is well-known that it is not Cartesian closed [15].

A number of works have tried to solve the issue of defining categorical semantics for higher-order probabilistic languages with continuous probability measures. Particularly, the category of quasi-Borel spaces [93, 141] was introduced as a Cartesian closed model for higher-order probabilistic computations. Its data is given by quasi-Borel spaces and morphisms between them, defined as follows:

Definition 4.34 ([93]). A quasi-Borel space is a pair $\left(X, M_{X}\right)$ of a carrier set $X$ and a set of random elements $M_{X} \subseteq \mathbb{R} \rightarrow X$ satisfying the following closure properties:

- If $\alpha: \mathbb{R} \rightarrow X$ is constant, then $\alpha \in M_{X}$.

- If $\alpha \in M_{X}$ and $f: \mathbb{R} \rightarrow \mathbb{R}$ is (Borel) measurable, then $\alpha \circ f \in M_{X}$.

- If $\left\{S_{i}\right\}_{i \in \mathbb{N}}$ is a disjoint covering of $\mathbb{R}$ with Borel sets, and $\alpha$ is piecewise definable from $\left\{\alpha_{i}\right\}_{i} \in \mathbb{N} \subseteq M_{x}$, taking value $\alpha_{i}(x)$ for every $x \in S_{i}$, then $\alpha \in M_{x}$.

A morphism between quasi-Borel spaces $\left(\mathrm{X}, \mathrm{M}_{\mathrm{X}}\right)$ and $\left(\mathrm{Y}, \mathrm{M}_{\mathrm{Y}}\right)$ is a function $\mathrm{f}: \mathrm{X} \rightarrow \mathrm{Y}$ such that for every $\alpha \in M_{X}, f \circ \alpha \in M_{\gamma}$. Quasi-Borel spaces and morphisms between them form a category QBS.

In particular, a measurable space $\left(X, \Sigma_{X}\right)$ can be turned into a quasi-Borel space by setting $M_{X}=\operatorname{Meas}(\mathbb{R}, X)$. But, as opposed to Meas, the category QBS has a Cartesian closed structure defined by:

$$
\begin{gathered}
\left(X, M_{X}\right) \times\left(Y, M_{Y}\right) \triangleq\left(X \times Y,\left\{f: \mathbb{R} \rightarrow X \times Y \mid \pi_{1} \circ f \in M_{X} \wedge \pi_{2} \circ f \in M_{Y}\right\}\right) \\
\left(X, M_{X}\right) \Rightarrow\left(Y, M_{Y}\right) \triangleq\left(X \Rightarrow Y,\left\{f: \mathbb{R} \rightarrow(X \rightarrow Y) \mid \lambda^{-1}(f) \in Q B S(\mathbb{R} \times X, Y)\right\}\right)
\end{gathered}
$$


where we recall that $\lambda^{-1}$ : $\operatorname{Set}(\mathbb{R}, X \Rightarrow Y) \rightarrow \operatorname{Set}(\mathbb{R} \times X, Y)$ is a natural isomorphism given by the CCC structure of Set. The terminal object 1 has as carrier the singleton set $\{*\}$ and $M_{1}=\{\lambda r . *\}$. We can then define a forgetful functor $|-|:$ QBS $\rightarrow$ Set sending a QBS $\sigma$ to its carrier $|\sigma|$. This functor preserves the CCC structure and moreover it does not change functions, that is, $|f|=f$ holds for any function $f \in \mathbf{Q B S}\left(\sigma, \sigma^{\prime}\right)$. We will sometimes also denote this forgetful functor by $\mathrm{U}$.

Similarly to measurable spaces, we can define a related notion of measure over a QBS:

Definition 4.35 ([141]). Let (X, $\left.\mathrm{M}_{\mathrm{X}}\right)$ be a quasi-Borel space. A (probability) measure over $\left(\mathrm{X}, \mathrm{M}_{\mathrm{X}}\right)$ is a triple $(\Omega, \alpha, \mu)$ where $\left(\Omega, \Sigma_{\Omega}\right)$ is a standard Borel space, $\alpha \in \mathbf{Q B S}(\Omega, \mathrm{X})$, taking $\Omega$ as a QBS, and $\mu$ is a (probability) measure over $\left(\Omega, \Sigma_{\Omega}\right)$. We can define an equivalence relation between measures, in which measures are equivalent when they define the same integration operator:

$$
(\Omega, \alpha, \mu) \equiv\left(\Omega^{\prime}, \alpha^{\prime}, \mu^{\prime}\right) \Longleftrightarrow \forall f: X \rightarrow \mathbb{R}, \int_{\Omega}(f \circ \alpha) \mathrm{d} \mu=\int_{\Omega^{\prime}}\left(f \circ \alpha^{\prime}\right) \mathrm{d} \mu^{\prime}
$$

We denote the equivalence class of $(\Omega, \alpha, \mu)$ by $[\Omega, \alpha, \mu]$.

The set of probability measures over a quasi-Borel space is itself a quasi-Borel space. This allows us to define a monad $\mathcal{P}: \mathbf{Q B S} \rightarrow \mathbf{Q B S}$ of probability measures as follows:

$$
\begin{aligned}
\mathcal{P}(X) & \triangleq\{(\Omega, \alpha, \mu) \text { probability measure over } X\} / \equiv \\
M_{\mathcal{P}(X)} & \triangleq\left\{\lambda r .\left[D_{r}, \alpha(r,-), \mu_{r}\right] \mid \mu \sigma \text {-finite over } \Omega, D \subseteq \mathbb{R} \times \Omega \text { meas., } \alpha \in \mathbf{Q B S}(D, X)\right\}
\end{aligned}
$$

where $D_{r}=\{\omega \mid(r, \omega) \in D\}, \alpha(r,-):|\Omega| \rightarrow \mathcal{P}(X)$ is the partial application of $\alpha$ and $\mu_{r}$ is the measure on $D_{r}$ induced by $\mu$. Notice that in general, given $f: \Omega^{\prime} \rightarrow \mathcal{P}(X)$ and $r \in \Omega^{\prime}$ for a standard Borel space $\Omega^{\prime}$, this determines $\Omega, D \subseteq \Omega^{\prime} \times \Omega, D_{r}, \alpha \in$ QBS $(D, X)$ and $\mu$ as above such that $f(r)=\left[D_{r}, \alpha(r,-), \mu_{r}\right]$.

The unit is defined as the map sending $x \in X$ to its Dirac measure, i.e., the one assigning measure 1 to any set if it contains $x$ and o otherwise:

$$
\eta_{X}(x) \triangleq(\{*\}, \lambda y \cdot x, \delta) \quad \text { where } \delta(\{*\})=1
$$

Given a morphism $f \in \mathbf{Q B S}(X, \mathcal{P}(Y))$, we can define its Kleisli lifting $f^{\#} \in$ QBS $(\mathcal{P}(X), \mathcal{P}(Y))$ as follows:

$$
f^{\#}(\Omega, \alpha, \mu) \triangleq\left[D, \beta,\left.\left(\mu \otimes \mu^{\prime}\right)\right|_{D}\right]
$$

where $\left.f(\alpha(r))=\left[D_{r}, \beta(r,-), \mu^{\prime}\right]\right)$ are determined by $f \circ \alpha: \Omega \rightarrow \mathcal{P}(Y)$ and $\mu \otimes \mu^{\prime}$ denotes the product measure.

Moreover, this monad is strong, i.e., there exists a strength $\theta_{X, Y}: X \times \mathcal{P}(Y) \rightarrow$ $\mathcal{P}(\mathrm{X} \times \mathrm{Y})$ satisfying the strength coherence conditions. 


\subsubsection{Predicates over Quasi-Borel spaces}

To specify probabilistic programs, we need to be able to model predicates over a QBS. We present a construction of a category $\operatorname{Pred}(\mathbf{Q B S})$ of predicates over QBS, which has the following data:

An object is a pair of $X \in \mathbf{Q B S}$ and a predicate $\mathrm{P} \subseteq|\mathrm{X}|$, which is a subset of its carrier.

A morphism from $(X, P)$ to $(Y, Q)$ is a morphism $f \in \mathbf{Q B S}(X, Y)$ such that for all $x \in|X|, P(x) \Rightarrow Q(|f|(x))$ holds.

This category is a CCC, where the product and exponential are defined similarly to $\operatorname{Pred}(\operatorname{Set})$ and it comes with the forgetful functor $d: \operatorname{Pred}(\mathbf{Q B S}) \rightarrow$ QBS that preserves it.

Note that here the predicates are arbitrary subsets of the carrier (i.e., subobjects in Set) rather than measurable subsets (subobjects in QBS). This is a technical choice which comes with a trade-off. On the one hand, for a user of the logic it is easier to write specifications, since they do not have to be concerned with measurability issues. Moreover, this means that the fibration d: Pred $(\mathbf{Q B S}) \rightarrow \mathbf{Q B S}$ can be constructed as a pullback of the domain fibration Pred(Set) $\rightarrow$ Set, and the forgetful functor QBS $\rightarrow$ Set, equipping Pred (QBS) with a similar logical structure as $\operatorname{Pred}($ Set). In particular, we can the show that $d: \operatorname{Pred}(\mathbf{Q B S}) \rightarrow$ QBS is a LR fibration. On the other hand, the semantics of generalized predicates are harder to express since we cannot directly compute probabilities or measure such arbitrary predicates.

\subsubsection{A lifting for union bounds}

Inspired from the lifting for the union bound introduced in [138, Section 9.1], we construct a graded lifting of $\mathcal{P}$ along $\mathrm{d}: \operatorname{Pred}(\mathbf{Q B S}) \rightarrow$ QBS as follows:

$$
\dot{\mathcal{P}}_{\mathcal{\delta}}^{\mathrm{UB}}(X, P \subseteq|X|) \triangleq(\mathcal{P}(X),\{v \in \mathcal{P}(X) \mid \forall Q \in \mathbf{Q B S}(X,\{0,1\}) . P \subseteq \mathrm{Q} \Rightarrow \underset{v}{\operatorname{Pr}}[\mathrm{Q}] \geqslant 1-\delta\})
$$

Here we take the liberty of regarding $Q$ as a predicate, rather than a function with values in $\{0,1\}$. This construction is an instantiation of the graded TT-lifting [105]. Therefore,

Lemma 4.36. $\mathcal{P}^{\mathrm{UB}}$ is indeed $a([0, \infty],+, 0, \leqslant)$-graded lifting of $\mathcal{P}$.

The definition above is somewhat cumbersome, since we need to take into account that $\mathrm{P}$ may not be measurable, so we cannot directly compute the probability of satisfying P. However, for a measurable P, we can prove the following simplification: 
Remark 4.37. If the predicate $\mathrm{P}$ is "measurable", that is, it can be regarded as a function $\mathrm{P}: \mathrm{X} \rightarrow\{0,1\}$ in $\mathbf{Q B S}$, then every $\mu \in \mathcal{P}_{\delta}^{\mathrm{UB}}(\mathrm{X}, \mathrm{P} \subseteq|\mathrm{X}|)$ satisfies $\operatorname{Pr}_{x \sim \mu}[\mathrm{X} \in \neg \mathrm{P}] \leqslant \delta$.

That is, for measurable predicates, the lifting behaves as intended: it gives us an upper bound of the probability of a computation failing to satisfy the predicate.

\subsection{Semantics}

Now we are in shape to present the formal semantics for this chapter. In the same manner as our syntax starts from a simply-typed language with added probabilities, we build HOL over it, and then build unary and relational logics over HOL, we can give semantics to our language and logics following these steps:

- We model our language in a Cartesian closed category, QBS

- We add a monad $\mathcal{P}: \mathbf{Q B S} \rightarrow \mathbf{Q B S}$ to model probabilistic computations

- We model HOL, UHOL and RHOL in a category of predicates Pred(QBS) for which there exists a LR fibration d: $\operatorname{Pred}(\mathbf{Q B S}) \rightarrow$ QBS

- We model HO-UBL, and we interpret generalized predicates using a lifting of $\mathcal{P}, \dot{\mathcal{P}}_{\text {UB }}$.

Note however that most of the semantics is going to rely on the general properties of LR fibrations, rather than the specific properties of the categories QBS and Pred(QBS). Showing that this framework can be generalized fully (i.e., to an arbitrary CCC, monad, LR fibration and lifting) to reason about other effects is an interesting direction for future work.

\subsubsection{Semantics of the language}

A simply-typed lambda calculus has a standard interpretation in a Cartesian closed category $\mathbf{C}$, and given a strong monad $\mathcal{T}=(\mathcal{T}, \eta, \mu, \theta)$ over $\mathbf{C}$ and an interpretation $\llbracket \alpha_{i} \rrbracket$ for all the operations, we can define an interpretation for the language in Section 4.2. To accommodate probabilities we take the category QBS with the monad $\mathcal{P}$ of probability measures.

The semantics of types is defined as objects in QBS, assuming we have an object $\llbracket b_{i} \rrbracket$ for every base type $b_{i} \in B$

$$
\begin{gathered}
\llbracket u t \rrbracket \triangleq 1 \quad \llbracket \sigma \rightarrow \tau \rrbracket \triangleq \llbracket \sigma \rrbracket \Rightarrow \llbracket \tau \rrbracket \\
\llbracket \sigma \times \tau \rrbracket \triangleq \llbracket \sigma \rrbracket \times \llbracket \tau \rrbracket \quad \llbracket P(\sigma) \rrbracket \triangleq \mathcal{P} \llbracket \sigma \rrbracket
\end{gathered}
$$

Semantics of a context $\Gamma$ is given by the Cartesian product of the types in the context. Well-typed terms $\Gamma \vdash \mathrm{t}: \sigma$ are interpreted as morphisms $\llbracket \Gamma \rrbracket \rightarrow \llbracket \sigma \rrbracket$ in QBS. 
We first introduce the interpretation of non-monadic terms, which is given by the standard CCC semantics.

$$
\begin{aligned}
\llbracket \Gamma, x: \tau \vdash x: \tau \rrbracket & \triangleq \pi_{2} \\
\llbracket \Gamma \vdash \lambda x . t: \sigma \rightarrow \tau \rrbracket & \triangleq \lambda(\llbracket \Gamma, x: \sigma \vdash t: \tau \rrbracket) \\
\llbracket \Gamma \vdash t u: \tau \rrbracket & \triangleq \text { eval }\langle\llbracket \Gamma \vdash t: \sigma \rightarrow \tau \rrbracket, \llbracket \Gamma \vdash u: \tau \rrbracket\rangle \\
\llbracket \Gamma \vdash\langle t, u\rangle: \tau \times \sigma \rrbracket & \triangleq\langle\llbracket \Gamma \vdash t: \tau \rrbracket, \llbracket \Gamma \vdash u: \sigma \rrbracket\rangle \\
\llbracket \Gamma \vdash \pi_{i}(t): \tau_{i} \rrbracket & \triangleq \pi_{i} \circ \llbracket \Gamma \vdash t: \tau_{1} \times \tau_{2} \rrbracket \quad(i=1,2)
\end{aligned}
$$

The semantics of monadic terms is defined as:

$$
\begin{aligned}
\llbracket \Gamma \vdash \text { unit }(t): P \sigma \rrbracket & \triangleq \eta \circ \llbracket \Gamma \vdash t: \sigma \rrbracket \\
\llbracket \Gamma \vdash \text { mlet } x=t \text { in } u: P \tau \rrbracket & \triangleq \llbracket \Gamma, x: \sigma \vdash u: P \tau \rrbracket^{\#} \circ \theta \circ\left\langle i d_{\Gamma}, \llbracket \Gamma \vdash t: P \sigma \rrbracket\right\rangle \\
\llbracket \Gamma \vdash \alpha\left(t_{1}, \ldots, t_{k}\right): P \sigma \rrbracket & \triangleq \llbracket \alpha \rrbracket \circ\left\langle\llbracket \Gamma \vdash t_{1}: \tau_{1} \rrbracket, \ldots, \llbracket \Gamma \vdash t_{k}: \tau_{k} \rrbracket\right\rangle
\end{aligned}
$$

This semantics is sound in the following sense:

Theorem 4.38. Let $\Gamma \vdash \mathrm{t}: \sigma$ be a valid judgment. Then, $\llbracket \Gamma \vdash \mathrm{t}: \sigma \rrbracket$ is a morphism in QBS of type $\llbracket \Gamma \rrbracket \rightarrow \llbracket \sigma \rrbracket$.

Note that the set-theoretical semantics presented in Chapter 3 corresponds with the interpretation in this chapter when $\mathbf{C}$ is Set.

Inductive datatypes The language in Chapter 3 included inductive datatypes: natural numbers and lists. We can interpret them in Set, QBS, or any BiCCC with enough structure:

- Boolean numbers can be interpreted as the coproduct $1+1$, which exists in every BiCCC.

- Natural numbers can be interpreted as the carrier $N$ of the initial algebra of the functor $X \mapsto 1+X$, whenever it exists. In Set this corresponds to the set of natural numbers $\mathbb{N}$, and in QBS this corresponds to the Quasi-Borel space $\left(\mathbb{N}, M_{\mathbb{N}}\right)$, where $M_{\mathbb{N}}$ is the set of measurable functions from $\mathbb{R}$ to $\mathbb{N}$.

- Lists of a type $\tau$ can be interpreted as the carrier $L_{\tau}$ of the initial algebra of the functor $X \mapsto 1+\tau \times X$. In QBS this corresponds to the coproduct $\llbracket$ list $_{\tau} \rrbracket \triangleq \coprod_{n \in \mathbb{N}} \llbracket \tau \rrbracket^{n}$.

We can then interpret terminating using a fixed point operator fix iterating functions until termination. Since the termination criterion $\operatorname{Def}(f, x, t)$ ensures that all recursive calls are on smaller arguments, the operator fix is well-defined: for any $n \in I$ with $|n|<k$, fix $(\lambda f . \lambda x . e)(n)$ is defined within $k$ steps, so we can define:

$$
\llbracket \Gamma \vdash \text { letrec } \mathrm{fx}=\mathrm{e}: \mathrm{I} \rightarrow \sigma \rrbracket \triangleq \operatorname{fix}(\llbracket \Gamma \vdash \lambda f: \mathrm{I} \rightarrow \sigma . \lambda x: \mathrm{I} . \mathrm{e}:(\mathrm{I} \rightarrow \sigma) \rightarrow(\mathrm{I} \rightarrow \sigma) \rrbracket)
$$




\subsubsection{Semantics of the base logics}

Recall the syntax of formulas in HOL:

$$
\phi, \psi::=\top|\perp| \mathrm{P}\left(\mathrm{t}_{1}, \ldots, \mathrm{t}_{\mathrm{k}}\right)|\phi \wedge \psi| \phi \vee \psi|\phi \Rightarrow \psi| \forall x . \phi
$$

Semantics of a formula in context $\Gamma \vdash \phi$ is given by an object in $\operatorname{Pred}(\mathbf{Q B S})$ above $\llbracket \Gamma \rrbracket$, which is defined inductively by:

$$
\begin{aligned}
\llbracket \Gamma \vdash \top \rrbracket & \triangleq(\llbracket \Gamma \rrbracket, \llbracket \Gamma \rrbracket) \\
\llbracket \Gamma \vdash \mathrm{P}\left(\mathrm{t}_{1}, \ldots, \mathrm{t}_{\mathrm{k}}\right) \rrbracket & \triangleq\left(\llbracket \Gamma \vdash \mathrm{t}_{1}: \sigma_{1} \rrbracket \times \cdots \times \llbracket \Gamma \vdash \mathrm{t}_{\mathrm{k}}: \sigma_{\mathrm{k}} \rrbracket\right)^{*} \llbracket \mathrm{P} \rrbracket \\
\llbracket \Gamma \vdash \phi \wedge \psi \rrbracket & \triangleq \llbracket \Gamma \vdash \phi \rrbracket \sqcap \llbracket \Gamma \vdash \psi \rrbracket \\
\llbracket \Gamma \vdash \phi \vee \psi \rrbracket & \triangleq \llbracket \Gamma \vdash \phi \rrbracket \sqcup \llbracket \Gamma \vdash \psi \rrbracket \\
\llbracket \Gamma \vdash \phi \Rightarrow \psi \rrbracket & \triangleq \llbracket \Gamma \vdash \phi \rrbracket \Rightarrow \llbracket \Gamma \vdash \psi \rrbracket \\
\llbracket \Gamma \vdash \forall(x: \sigma) . \phi \rrbracket & \triangleq \prod_{\llbracket \Gamma \rrbracket, \llbracket \sigma \rrbracket}(\llbracket \Gamma, x: \sigma \vdash \phi \rrbracket)
\end{aligned}
$$

where the interpretation $\llbracket P \rrbracket$ for each base predicate $P: \sigma_{1} \times \cdots \times \sigma_{k}$ is a chosen object in $\mathbf{P}_{\llbracket \sigma_{1} \rrbracket \times \cdots \times \llbracket \sigma_{k} \rrbracket}$. This interpretation is well-behaved with respect to substitution. In particular, the substitution $\phi[t / x]$ of $x$ by a term $t$ of appropriate type can be interpreted by the inverse image $\llbracket \Gamma \vdash \phi[\mathrm{t} / \mathrm{x}] \rrbracket=\left\langle\mathrm{id}_{\llbracket \Gamma \rrbracket}, \llbracket \Gamma \vdash \mathrm{t}: \tau \rrbracket\right\rangle^{*} \llbracket \Gamma, x: \tau \vdash \phi \rrbracket$. We can show:

Theorem 4.39 (Soundness of HOL). Let $\Gamma \mid \Psi \vdash \phi$ be a derivable HOL judgment. Then it is valid, i.e. there is a morphism $\llbracket \Gamma \vdash \wedge \Psi \rrbracket \sqsubseteq \llbracket \Gamma \vdash \phi \rrbracket$ in $\operatorname{Pred}(\mathbf{Q B S})$ above $i \mathrm{~d}_{\llbracket \Gamma \rrbracket}$

On top of this, we build semantics for UHOL and RHOL. A judgment in UHOL can be seen a pair of a typing judgment and a logical judgment that contains an extra distinguished variable $\mathbf{r}$ referring to the typed term. With this in mind, we show:

Theorem 4.40 (Soundness of UHOL). Let $\Gamma \mid \Psi \vdash \mathrm{t}: \sigma\{\phi\}$ be a derivable UHOL judgment. Then, $\llbracket \Gamma \vdash \mathrm{t}: \sigma \rrbracket: \llbracket \Gamma \rrbracket \rightarrow \llbracket \sigma \rrbracket$ in $\mathbf{Q B S}$ and

$$
\llbracket \Gamma \vdash \bigwedge \psi \rrbracket \sqsubseteq\left\langle i \mathrm{id}_{\llbracket \Gamma \rrbracket}, \llbracket \Gamma \vdash \mathrm{t}: \sigma \rrbracket\right\rangle^{*} \llbracket \Gamma, \mathbf{r}: \sigma \vdash \phi \rrbracket
$$

in $\operatorname{Pred}(\mathbf{Q B S})$ above id $\llbracket \Gamma \rrbracket$.

Let us unpack the statement into plainer words. Semantically, $\langle i d, \llbracket \Gamma \vdash t: \sigma \rrbracket\rangle^{*}$ computes the inverse image of the predicate $\phi$ along $\langle i d, \llbracket \Gamma \vdash t: \sigma \rrbracket\rangle: \llbracket \Gamma \rrbracket \rightarrow \llbracket \Gamma, \mathbf{r}: \sigma \rrbracket$. This corresponds to the $\gamma \in \llbracket \Gamma \rrbracket$ such that $(\gamma, \llbracket \Gamma \vdash \mathrm{t}: \sigma \rrbracket(\gamma)) \in \llbracket \Gamma, \mathbf{r}: \sigma \vdash \phi \rrbracket$, which morally can be seen as the predicate resulting from substituting $\mathbf{r}$ in $\phi$ by $t$, that is, $\llbracket \Gamma \vdash \phi[t / \mathbf{r}] \rrbracket$. Therefore, what the statement is saying is that if we take a $\gamma$ in $\llbracket \Gamma \rrbracket$ satisfying $\Psi, \gamma$ also satisfies $\phi[t / \mathbf{r}]$. This corresponds to the intended interpretation. 
Even though we do not need RHOL to derive judgments in HO-UBL, we still present the semantics here for completeness. We will use them in later chapters. A judgment in RHOL can be seen as a triple formed by two typing judgments and a logical judgment with two extra distinguished variables.

Theorem 4.41 (Soundness of RHOL). Let $\Gamma \mid \Psi \vdash \mathrm{t}_{1}: \sigma_{1} \sim \mathrm{t}_{2}: \sigma_{2}\{\phi\}$ be a derivable UHOL judgment. Then, $\llbracket \Gamma \vdash \mathrm{t}_{1}: \sigma_{1} \rrbracket: \llbracket \Gamma \rrbracket \rightarrow \llbracket \sigma_{1} \rrbracket$ and $\llbracket \Gamma \vdash \mathrm{t}_{2}: \sigma_{2} \rrbracket: \llbracket \Gamma \rrbracket \rightarrow \llbracket \sigma_{2} \rrbracket$ in $\mathbf{Q B S}$ and

$$
\llbracket \Gamma \vdash \bigwedge \Psi \rrbracket \sqsubseteq\left\langle\mathrm{id}, \llbracket \Gamma \vdash \mathrm{t}_{1}: \sigma_{1} \rrbracket, \llbracket \Gamma \vdash \mathrm{t}_{1}: \sigma_{1} \rrbracket\right\rangle * \llbracket \Gamma, \mathbf{r}_{1}: \sigma_{1}, \mathbf{r}_{2}: \sigma_{2} \vdash \phi \rrbracket
$$

in $\operatorname{Pred}(\mathbf{Q B S})$ above id $\llbracket \Gamma \rrbracket$.

To conclude this section, we remark that if we instead take a model of the language in Set and model the logics in the domain fibration d : Pred(Set) $\rightarrow$ Set, we can see that it coincides with the set-theoretical interpretation we presented in Chapter 3 (Sections 3.3,3.4, and 3.5).

\subsubsection{Semantics of HO-UBL}

Finally we give semantics to the monadic extension HO-UBL. As opposed to UHOL we do not interpret the postcondition directly, since it is a predicate of the form $\Gamma, \mathbf{v}: \sigma \vdash \phi$, but the result is of type $\mathrm{P}(\sigma)$. Instead, we use the lifting $\dot{\mathcal{P}}^{U B}$. Soundness is then stated as follows:

Proposition 4.42. Let $\Gamma \mid \Psi \vdash \mathrm{t}: \mathrm{P} \tau\{\mathrm{Q}\}_{\delta}$ be a derivable HO-UBL judgment. Let also $\gamma \in \mathbf{Q B S}(1, \llbracket \Gamma \rrbracket)$ such that $\gamma: T \rightarrow \llbracket \Gamma \vdash \wedge \Psi \rrbracket$. Then $\llbracket \Gamma \vdash \mathrm{t}: \mathcal{T} \tau \rrbracket \circ \gamma$ is in

$$
\operatorname{Pred}(\mathbf{Q B S})\left(T, \dot{\mathcal{P}}_{\tau, \delta}^{\mathrm{uB}}\left(\left\langle\gamma, i \mathrm{~d}_{\llbracket \tau \rrbracket}\right\rangle^{*} \llbracket \Gamma, \mathbf{v}: \tau \vdash \mathrm{Q} \rrbracket\right)\right) .
$$

where the pull-back $\gamma^{*}$ computes the substitution of the variables in $\Gamma$ by $\gamma$.

The explanation of this theorem is similar to Theorem 4.40. First, by the isomorphism $\mathbf{Q B S}(1, \llbracket \gamma \rrbracket) \cong|\llbracket \Gamma \rrbracket|, \gamma$ can be seen as an element of the set $|\llbracket \Gamma \rrbracket|$, which corresponds to a valuation of the context $\Gamma$. Since predicates over a QBS are subsets of their carriers, then the premise of the theorem states that, in set-theoretical terms, $\gamma \in \llbracket \Gamma \vdash \Lambda \Psi \rrbracket$. The conclusion states that $\llbracket \Gamma \vdash \mathrm{t}: \mathcal{T} \tau \rrbracket \circ \gamma$, which corresponds to $t$ evaluated under $\gamma$, satisfies the lifting of $\gamma^{*} \llbracket \Gamma, \mathbf{v}: \tau \vdash \mathrm{Q} \rrbracket$, where $\left\langle\gamma, i \mathrm{id}_{\llbracket \tau}\right\rangle^{*}$ computes the reindexing of $Q$ by substituting all the variables in $\Gamma$ by their evaluation under $\gamma$.

\subsection{Other liftings}

In this section we quickly sketch how monad liftings can also be used to reason about other effects. The liftings we use are taken from [6], although they were studied before. For each effect, we will consider a simply-typed lambda calculus extended with monadic types, unit and let operators, and monadic operations. 


\section{Partiality}

As a first example, we consider liftings of the maybe monad (a.k.a. partiality monad) $\mathcal{M}$ along the domain fibration $d: \operatorname{Pred}($ Set $) \rightarrow$ Set. This monad can be used to model computations that may produce an error (e.g. division by zero) or fail to terminate. Its functor part is defined by $\mathcal{M} X \triangleq X+1$, where 1 denotes the singleton set. We will consider a simply-typed lambda calculus

$$
\begin{aligned}
\tau, \delta & :=\cdots \mid M(\tau) \\
t, u & :=\cdots \mid \text { unit }(t) \mid \text { mlet } x=t \text { in } u \mid \operatorname{div}(t, u)
\end{aligned}
$$

where div: nat $\rightarrow$ nat $\rightarrow \mathrm{P}$ (nat) computes division of natural numbers.

We can define two liftings of the partiality monad to the category Pred(Set):

$$
\begin{aligned}
& \dot{\mathcal{M}}^{\text {tot }}(\mathrm{X}, \mathrm{P} \subseteq \mathrm{X}) \triangleq(\mathrm{X}+1,\{(1, \mathrm{p}) \mid \mathrm{p} \in \mathrm{P}\}) \\
& \dot{\mathcal{M}}^{\text {par }}(\mathrm{X}, \mathrm{P} \subseteq \mathrm{X}) \triangleq(\mathrm{X}+1,\{(1, \mathrm{p}) \mid \mathrm{p} \in \mathrm{P}\} \cup\{(2, *)\})
\end{aligned}
$$

These two liftings can be used to design programs logics in which the judgment $\Gamma \mid \Psi \vdash t: M \sigma\{\{\}$ has the following interpretations:

- Under the lifting $\dot{\mathcal{M}}^{\text {tot }}, \mathrm{t}$ satisfies the judgment if it terminates correctly and the result satisfies $P$.

- Under the lifting $\dot{\mathcal{M}}^{\text {par }}$, $\mathrm{t}$ satisfies the judgment if it either does not terminate correctly, or it terminates correctly and the result satisfies P.

\section{Non-determinism}

Now we consider liftings of the non-empty powerset monad $\mathcal{N}$ along d: Pred $(\operatorname{Set}) \rightarrow$ Set. This monad can model computations with non-deterministic choices. Its functor part is defined by $\mathcal{N} X \triangleq\{\mathrm{U} \subseteq \mathrm{X} \mid \mathrm{U} \neq \emptyset\}$. We will consider a simply-typed lambda calculus

$$
\begin{aligned}
& \tau, \delta:=\cdots \mid N(\tau) \\
& t, u:=\cdots \mid \text { unit }(t) \mid \text { mlet } x=t \text { in } u \mid \operatorname{nd}(t, u)
\end{aligned}
$$

where nd: $\tau \times \tau \rightarrow P(\tau)$ performs a non-deterministic choice between its two arguments, which have the same type.

We can also define two liftings of the non-empty powerset monad to the category $\operatorname{Pred}($ Set $)$ :

$$
\begin{aligned}
& \dot{\mathcal{M}}^{\text {may }}(\mathrm{X}, \mathrm{P} \subseteq \mathrm{X}) \triangleq(\mathcal{N} \mathrm{X},\{\mathrm{U} \mid \mathrm{U} \cap \mathrm{P} \neq \emptyset\}) \\
& \dot{\mathcal{M}}^{\text {must }}(\mathrm{X}, \mathrm{P} \subseteq \mathrm{X}) \triangleq(\mathcal{N} \mathrm{X},\{\mathrm{U} \mid \mathrm{U} \subseteq \mathrm{P}\})
\end{aligned}
$$

These two liftings can be used to design program logics in which the judgment $\Gamma \mid \Psi \vdash t: N \sigma\{Q\}\}$ has the following interpretations: 
- Under the lifting $\dot{\mathcal{M}}^{\text {may }}, \mathrm{t}$ satisfies the judgment if there is at least one possible execution that satisfies P. In this case, the proof rules for non-deterministic choice are the following:

$$
\frac{\Gamma \mid \Psi \vdash \mathrm{t}_{i}: \tau\{\phi\}}{\left.\Gamma \mid \Psi \vdash \operatorname{nd}\left(\mathrm{t}_{1}, \mathrm{t}_{2}\right): \mathrm{N} \tau\{\phi\}\right\}} \mathrm{ND}_{\mathrm{i}} \quad i=1,2
$$

- Under the lifting $\dot{\mathcal{M}}^{\text {must }}$, $\mathrm{t}$ satisfies the judgment if every possible execution satisfies $P$. In this case, the proof rule for non-deterministic choice is the following:

$$
\frac{\Gamma\left|\Psi \vdash \mathrm{t}_{1}: \tau\{\phi\} \quad \Gamma\right| \Psi \vdash \mathrm{t}_{2}: \tau\{\phi\}}{\Gamma \mid \Psi \vdash \operatorname{nd}\left(\mathrm{t}_{1}, \mathrm{t}_{2}\right): \mathrm{N} \tau\{\dot{q}\}} \mathrm{ND}
$$




\section{RELATIONAL REASONING ABOUT PROBABILITIES: PROBABILISTIC COUPLINGS}

In the previous chapter we have developed a unary logic to reason about probabilistic programs. This logic could prove properties of the kind "A program P satisfies a specification $\phi$ at least with probability $1-\delta^{\prime \prime}$. The key idea was the use of liftings to keep the assertion language the same, while still being able to reason about probabilistic programs.

Now we extend these ideas to the relational setting. We will develop a relational logic to prove properties of the form

$$
\operatorname{Pr}_{x \sim P_{1}}\left[x \in \phi_{1}\right] \leqslant e^{\epsilon} \operatorname{Pr}_{y \sim P_{2}}\left[y \in \phi_{2}\right]+\delta
$$

where $P_{1}, P_{2}$ are a pair of programs and $\phi_{1}, \phi_{2}$ are a pair of assertions. This can be seen as a witness-free version of the concept of approximate probabilistic couplings [26]. Normally, approximate couplings are presented by producing either one or two witness distributions $\mu_{1}, \mu_{2}$ over the product space such that their marginals coincide with $P_{1}, P_{2}$. As observed by Sato [137], there is an equivalent presentation in the form of Equation (5.1). In the continuous setting, this presentation has an advantage, since it circumvents measurability issues when selecting witnesses. In Chapters 8 and 10 we will work instead with witness-based couplings for discrete distributions. In either of the two forms, couplings can be used to encode several properties such as equivalence, stochastic dominance or differential privacy.

We then use this logic to verify security of the one-time pad encryption scheme and certified robustness [112], a mathematical approach for (provably) protecting machine learning algorithms against adversarial examples, i.e. adversarially chosen inputs that are classified differently from another (possibly adversarially chosen) nearby input. Using our relational logic, we show that differentially private DNN computations are robust against adversarial computations. 


\section{Organization of chapter}

We begin by introducing the theory behind differential privacy and couplings (Section 5.1). We continue by presenting the logic HO-RPL (Section 5.2) and the examples (Section 5.3). After this, we show how to extend the semantics from the previous chapter to a relational setting (Section 5.4).

\subsection{Mathematical preliminaries}

We begin by reviewing the notion of differential privacy [75]. The field of differential privacy studies how to design algorithms for querying databases without compromising the privacy of the data they contain. For instance, a query to the patient database of a hospital should not reveal whether a particular patient is currently in the hospital. This is encapsulated by the following definition.

Definition 5.1 ([75, 137]). Let $f: \mathbb{R}^{k} \rightarrow \mathcal{P}(\mathbb{R})$ be a measurable function from a $k$-fold product of reals to a measure over the reals. We say that $f$ is $(\epsilon, \delta)$-differentially private $\left((\epsilon, \delta)-d p\right.$ for short) if, for every pair of vectors $\vec{x}, \vec{y} \in \mathbb{R}^{k}$ differing in at most 1 element, we have

$$
\forall \mathrm{U} \subseteq \mathbb{R} \text { measurable. } \operatorname{Pr}_{\mathrm{f}(\bar{x})}[\mathrm{U}] \leqslant e^{\epsilon} \cdot \operatorname{Pr}_{\mathrm{f}(\vec{y})}[\mathrm{U}]+\delta
$$

Informally, what the definition states is that for two vectors of data that are almost equal, the probability of observing any given output of the algorithm on the first of the vectors is close to the probability of observing the same output on the second of the vectors. The parameter $\epsilon$ represents a bound on the privacy loss, while the parameter $\delta$ is a bound on the probability that the privacy loss exceeds $\epsilon$.

There are many techniques for verification of differentially private algorithms, e.g. $[27,78,32]$. Our technique is based on probabilistic couplings, which are a tool than comes from the analysis of Markov chains [7]. We present them here in the continuous setting, and then we will present them in the discrete setting in Chapter 8.

Definition 5.2 ([137]). Let $\left(X, \Sigma_{X}, v_{X}\right)$ and $\left(Y, \Sigma_{Y}, v_{Y}\right)$ be two probability spaces, and let $\left(\mathrm{X} \times \mathrm{Y}, \Sigma_{X \times Y}\right)$ denote the product measure space. Let also $\mathrm{R} \in \Sigma_{X \times Y}$ and $\epsilon, \delta \geqslant 0$. We say that $v_{X}$ and $v_{Y}$ are related by an $(\epsilon, \delta)$-approximate $R$-coupling if

$$
\forall \mathrm{U} \in \Sigma_{\mathrm{X}} \cdot \operatorname{Pr}_{\gamma_{1}}(\mathrm{U}) \leqslant e^{\epsilon} \cdot \operatorname{Pr}_{\gamma_{2}}(\mathrm{R}(\mathrm{U}))+\delta
$$

where $\mathrm{R}(\mathrm{U})=\{\mathrm{y} \in \mathrm{Y} \mid \exists x \in \mathrm{U} .(\mathrm{x}, \mathrm{y}) \in \mathrm{R}\}$.

For a general $\mathrm{R}$ it is hard to get an intuition of what the existence of a coupling implies, but for particular choices of $R$ this existence implies interesting properties. The following lemma states the relation between differential privacy and approximate couplings: 
Lemma 5.3 ([137]). Let $\mathrm{f}: \mathbb{R}^{\mathrm{k}} \rightarrow \mathcal{P}(\mathbb{R})$ be a measurable function from a $\mathrm{k}$-fold product of reals to a measure over the reals. It is $(\epsilon, \delta)$-differentially private iff, for every pair of vectors $\vec{x}, \vec{y} \in \mathbb{R}^{k}$ differing in at most 1 element, there exists an $(\epsilon, \delta)$-approximate $(=)$-coupling of $f(\vec{x})$ and $f(\vec{y})$.

\subsection{Higher-order probabilistic relational logic}

In this section we present Higher-order probabilistic relational logic (HO-PRL), which can be seen as a higher-order version of apRHL [26]. This logic reasons about programs in the same language as Section 4.2.

Analogously to HO-UBL, we start from RHOL and we extend it with monadic judgments that have the form:

$$
\Gamma \mid \Psi \vdash \mathrm{t}_{1}: \mathcal{P} \sigma_{1} \sim \mathrm{t}_{2}: \mathcal{P} \sigma_{2}\{\mathrm{Q}\}_{(\epsilon, \delta)}
$$

where $\mathrm{Q}$ is well-formed in the context $\Gamma, \mathbf{v}_{1}: \sigma_{1}, \mathbf{v}_{2}: \sigma_{2}$. Here, the variables $\mathbf{v}_{1}, \mathbf{v}_{2}$ refer to (resp. left or right-side) result values.

The intended meaning for this judgment is that, under the conditions in $\Psi, \mathrm{t}_{1}$ and $t_{2}$ are a pair of distributions (over $\sigma_{1}$ and $\sigma_{2}$ respectively) for which there exists an $(\epsilon, \delta)$ - approximate $\mathrm{Q}-$ coupling. By Lemma 5.3, if $\mathrm{Q}$ is $\mathbf{v}_{1}=\mathbf{v}_{2}$ and the grading is $(0,0)$ then the specification corresponds to program equivalence. More generally, if $\Gamma \mid \Psi \vdash t_{1}: \mathcal{P} \tau \sim t_{2}: \mathcal{P} \tau\left\{\left\{\mathbf{v}_{1}=\mathbf{v}_{2}\right\}_{(\epsilon, \delta)}\right.$ is derivable in HO-PRL, then for every $\gamma \in \Gamma$ such that $\gamma \in \Lambda \Psi, t_{1}$ and $t_{2}$ are distributions over $\tau$ such that for every subset $Y$ of $\tau$, $\operatorname{Pr}_{t_{1}}[Y] \leqslant \exp (\epsilon) \operatorname{Pr}_{t_{2}}[Y]+\delta$, i.e. they are differentially private.

Figure 5.1 presents selected rules for the relational logic. Following the same pattern for our other relational logics, we have two-sided rules, such as UNIT, MLET, and CASE, where the two expressions have the same top-level structure, and one-sided rules, which exclusively consider the top-level construct of one expression.

Our logic also features rules for reasoning about sampling. In contrast to the other rules, whose soundness only depends on the properties of graded liftings, these rules are only valid for a specific graded lifting. We show some rules in Figure 5.2. The rule SAMPLE-ASYN reasons about sampling from uniform distributions on different sets. The rule SAMPLE-ASYN-APP proves a different, approximate conclusion for the same programs. Our examples also use other sampling rules, which we introduce when they are needed.

\subsection{Examples}

In this section we illustrate the use of our program logics with a classical example from cryptography, and an example from the machine learning literature. This latter example is new, and we develop it in some detail. 


$$
\begin{aligned}
& \frac{\Gamma \mid \Psi \vdash \mathrm{t}_{1}: \tau_{1} \sim \mathrm{t}_{2}: \tau_{2}\{\phi\}}{\Gamma \mid \Psi \vdash \operatorname{unit}\left(\mathrm{t}_{1}\right): \mathcal{P}\left(\tau_{1}\right) \sim \operatorname{unit}\left(\mathrm{t}_{2}\right): \mathcal{P}\left(\tau_{2}\right)\{\phi\}_{(0,0)}} \text { UNIT } \\
& \Gamma \mid \Psi \vdash t_{1}: \mathcal{P}\left(\tau_{1}\right) \sim t_{2}: \mathcal{P}\left(\tau_{2}\right)\left\{\{\}_{(\epsilon, \delta)}\right. \\
& \left.\Gamma, x_{1}: \tau_{1}, x_{2}: \tau_{2} \mid \Psi, Q\left[x_{1} / \mathbf{v}_{1}\right]\left[x_{2} / \mathbf{v}_{2}\right] \vdash u_{1}: \mathcal{P}\left(\sigma_{1}\right) \sim u_{2}: \mathcal{P}\left(\sigma_{2}\right)\{R\}_{(} \epsilon^{\prime}, \delta^{\prime}\right) \\
& x_{1}, x_{2} \notin \mathrm{FV}(\mathrm{R}) \quad \mathrm{x}_{1} \notin \mathrm{FV}\left(\mathrm{u}_{2}\right) \quad \mathrm{x}_{2} \notin \mathrm{FV}\left(\mathrm{u}_{1}\right) \\
& \overline{\Gamma \mid \Psi \vdash \text { mlet } x_{1}=t_{1} \text { in } u_{1}: \mathcal{P}\left(\sigma_{1}\right) \sim \text { mlet } x_{2}=t_{2} \text { in } u_{2}: \mathcal{P}\left(\sigma_{2}\right)\{R\}_{\left(\epsilon+\epsilon^{\prime}, \delta+\delta^{\prime}\right)}} \\
& \Gamma \mid \Psi \vdash \mathrm{b}_{1}: \text { bool } \sim \mathrm{b}_{2}: \text { bool }\left\{\mathbf{r}_{1} \Leftrightarrow \mathbf{r}_{2}\right\} \\
& \Gamma \mid \Psi \wedge \mathrm{b}_{1}=\mathbf{t t} \vdash \mathrm{t}_{1}: \mathcal{P}\left(\tau_{1}\right) \sim \mathrm{t}_{2}: \mathcal{P}\left(\tau_{2}\right)\{\mathbb{Q}\}_{(\epsilon, \delta)} \\
& \Gamma \mid \Psi \wedge b_{1}=\mathbf{f f} \vdash u_{1}: \mathcal{P}\left(\tau_{1}\right) \sim u_{2}: \mathcal{P}\left(\tau_{2}\right)\left\{[Q\}_{(\epsilon, \delta)}\right.
\end{aligned}
$$

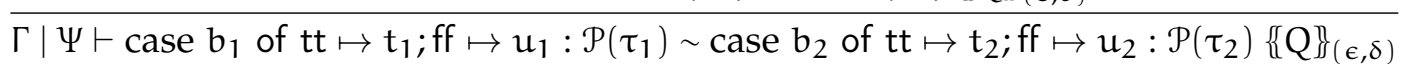

$$
\begin{aligned}
& \frac{\Gamma \mid \Psi \vdash \mathrm{t}_{1}: \tau_{1}\{\phi\}}{\Gamma \mid \Psi \vdash \operatorname{unit}\left(\mathrm{t}_{1}\right): \mathcal{P}\left(\tau_{1}\right) \sim \text { skip }: \mathcal{P}(\text { ut })\{\phi\}_{(0,0)}} \text { UNIT-L } \\
& \Gamma \mid \Psi \vdash \mathrm{t}_{1}: \mathcal{P}\left(\tau_{1}\right) \sim \text { skip : } \mathcal{P}(\text { ut })\{\mathrm{Q}\}_{(\epsilon, \delta)} \\
& \Gamma, x_{1}: \tau_{1} \mid \Psi, Q\left[x_{1} / \mathbf{v}_{1}\right]\left[* / \mathbf{v}_{2}\right] \vdash u_{1}: \mathcal{P}\left(\sigma_{1}\right) \sim u_{2}: \mathcal{P}\left(\sigma_{2}\right)\{R\}_{(\epsilon, \delta)} \\
& \frac{x_{1}, x_{2} \notin \mathrm{FV}(\mathrm{R}) \quad x_{1} \notin \mathrm{FV}\left(\mathrm{t}_{2}\right)}{\Gamma \mid \Psi \vdash \text { mlet } x_{1}=t_{1} \text { in } u_{1}: \mathcal{P}\left(\sigma_{1}\right) \sim t_{2}: \mathcal{P}\left(\sigma_{2}\right)\{R\}_{\left(\epsilon+\epsilon^{\prime}, \delta+\delta^{\prime}\right)}} \text { MLET-L }
\end{aligned}
$$

Figure 5.1: Relational logic: monadic rules

\subsubsection{One-time pad/probabilistic non-interference}

Non-interference [85] is a baseline information flow policy that is often used to model confidentiality of computations. In its simplest form, non-interference distinguishes between public (or low) and private (or high) variables and expressions, and requires that the result of a public expression not depend on the value of its private parameters. This definition naturally extends to probabilistic expressions, except that in this case the evaluation of an expression yields a distribution rather than a value. There are deep connections between probabilistic non-interference and several notions of (information-theoretic) security from cryptography. In this example, we illustrate different flavors of security properties for one-time pad encryption. Similar reasoning can be carried out for proving (passive) security of secure multiparty computation algorithms in the 3-party or multi-party setting [53, 65].

One-time pad is a perfectly secure symmetric encryption scheme. Its space of plaintexts, ciphertexts and keys is the set $\{0,1\}^{\ell}$-fixed-length bitstrings of size $\ell$. The encryption algorithm is parametrized by a key $k$-sampled uniformly over the set of bitstrings $\{0,1\}^{\ell}$-and maps every plaintext $m$ to the ciphertext $c=k \oplus m$, where the operator $\oplus$ denotes bitwise exclusive-or on bitstrings. We let otp denote the expression $\lambda$ m.mlet $\mathrm{k}=\mathcal{U}_{\{0,1\}^{e}}$ in unit $(\mathrm{k} \oplus \mathrm{m})$, where $\mathcal{U}_{X}$ is the uniform distribution over a finite set $X$. 


$$
\begin{gathered}
\frac{F: \sigma \rightarrow \sigma \text { bijection } \quad \Gamma \vdash \forall x . \phi(x, f(x))}{\Gamma \mid \Psi \vdash \text { Unif }_{\sigma}: \mathcal{T}_{\emptyset, 0}(\sigma) \sim \text { Unif }_{\sigma}: \mathcal{T}_{\emptyset, 0}(\sigma)\left\{\{\phi\}_{(0,0)}\right.} \text { SAMPLE } \\
\frac{\mathrm{B}_{1} \subseteq \mathrm{B}_{2} \text { finite }}{\Gamma \mid \Psi \vdash \text { Unif }_{\mathrm{B}_{1}}: \mathcal{P}_{\emptyset, 0}\left(\mathrm{~B}_{1}\right) \sim \text { Unif }_{\mathrm{B}_{2}}: \mathcal{P}_{\emptyset, 0}\left(\mathrm{~B}_{2}\right)\left\{\mathbf{v}_{2} \in \mathrm{B}_{1} \Rightarrow \mathbf{v}_{1}=\mathbf{v}_{2}\right\}_{(0,0)}} \text { SAMPLE-ASYN } \\
\frac{\mathrm{B}_{1} \subseteq \mathrm{B}_{2} \text { finite }}{\Gamma \mid \Psi \vdash \text { Unif }_{\mathrm{B}_{1}}: \mathcal{P}_{\emptyset, 0}\left(\mathrm{~B}_{1}\right) \sim \text { Unif }_{\mathrm{B}_{2}}: \mathcal{P}_{\emptyset, 0}\left(\mathrm{~B}_{2}\right)\left\{\mathbf{v}_{1}=\mathbf{v}_{2}\right\}_{\left(0,\left|\mathrm{~B}_{1}\right| /\left|\mathrm{B}_{2}\right|\right)}} \text { SAMPLE-ASYN-APP }
\end{gathered}
$$

Figure 5.2: Relational logic: rules for sampling

One-time pad achieves perfect security, i.e. the distributions of ciphertexts is independent of the plaintext. Perfect security can be captured as a probabilistic non-interference property:

$$
\mathrm{m}_{1}, \mathrm{~m}_{2}:\{0,1\}^{\mathrm{l}} \mid \cdot \vdash \text { otp } \mathrm{m}_{1}: \mathrm{D}\left(\{0,1\}^{\ell}\right) \sim \text { otp } \mathrm{m}_{2}: \mathrm{D}\left(\{0,1\}^{\ell}\right)\left\{\mathbf{v}_{1} \mathrm{~m}_{1}=\mathbf{v}_{2} \mathrm{~m}_{2}\right\}(0,0)
$$

The crux of the proof is to establish

$$
\mathrm{m}_{1}, \mathrm{~m}_{2}:\{0,1\}^{\ell} \mid \cdot \vdash \mathcal{U}_{\{0,1\}^{\ell}}: \mathrm{D}\left(\{0,1\}^{\ell}\right) \sim \mathcal{U}_{\{0,1\}^{\ell}}: \mathrm{D}\left(\{0,1\}^{\ell}\right)\left\{\mathbf{v}_{1} \oplus \mathrm{m}_{2}=\mathbf{v}_{2} \oplus \mathrm{m}_{1}\right\}(0,0)
$$

using the SAMPLE rule. It suffices to observe that the exclusive-or operator induces a bijection, so for any $m_{1}, m_{2}$ we can choose a bijection of $\{0,1\}^{\ell}$ onto itself satisfying the assertion. One can then conclude the proof by applying the rule for monadic sequenciation (MLET) and using algebraic properties of $\oplus$.

Interestingly, one can prove a stronger property: rather than proving that the ciphertext is independent of the plaintext, one can prove that the distribution of ciphertexts is uniform. This is captured by the following judgment

$$
\begin{gathered}
c_{1}, c_{2}, m_{1}, m_{2}:\{0,1\}^{\ell} \mid m_{1}=m_{2} \\
\vdash \text { otp } m_{1}: D\left(\{0,1\}^{\ell}\right) \sim \text { otp } m_{2}: D\left(\{0,1\}^{\ell}\right)\left\{\left\{\mathbf{v}_{1}=c_{1} \Leftrightarrow \mathbf{v}_{2}=c_{2}\right\}\right\}_{(0,0)}
\end{gathered}
$$

This style of modelling uniformity as a relational property is inspired from [39]. The proof is similar to the previous one and omitted. However, it is arguably more natural to model uniformity of the distribution of ciphertexts by the judgment

$$
\mathrm{m}:\{0,1\}^{\ell} \mid \cdot \vdash \operatorname{otp} \mathrm{m}: \mathrm{D}\left(\{0,1\}^{\ell}\right) \sim \mathcal{U}_{\{0,1\}^{\ell}}: \mathrm{D}\left(\{0,1\}^{\ell}\right)\left\{\mathbf{v}_{1}=\mathbf{v}_{2}\right\}(0,0)
$$

This judgment is closer to the simulation-based notion of security that is used pervasively in cryptography, and notably in Universal Composability [57]. Specifically, the statement captures the fact that the one-time pad algorithm can be simulated without access to the message. It is interesting to note that the judgment above (and more generally simulation-based security) could not be expressed in prior works, since the two expressions of the judgment have different types-note that in this 
specific case, the right expression is a distribution but in the general case the right expression will also be a function, and its domain will be a projection of the domain of the left expression.

The proof proceeds as follows. First, we prove

$$
m:\{0,1\}^{\ell} \vdash U_{\{0,1\}^{\ell}} \sim U_{\{0,1\}^{\ell}}\left\{\mathbf{v}_{1} \oplus m=\mathbf{v}_{2}\right\}_{(0,0)}
$$

using the [SAMPLE] rule. Then, we apply the [MLET] rule to obtain

$$
\mathrm{m}:\{0,1\}^{\ell} \vdash \begin{array}{ll}
\operatorname{mlet} k=\mathcal{U}_{\{0,1\}^{\ell}} \text { in } & \text { mlet } k=\mathcal{U}_{\{0,1\}^{\ell}} \text { in } \\
\operatorname{unit}(\mathrm{k} \oplus \mathrm{m}) & \text { unit }(k)
\end{array}\left\{\mathbf{v}_{1}=\mathbf{v}_{2}\right\}(0,0)
$$

We have mlet $k=\mathcal{U}_{\{0,1\}}$ in unit $(k) \equiv \mathcal{U}_{\{0,1\}}$; hence by equivalence, this entails

$$
m:\{0,1\}^{\ell} \vdash \text { mlet } k=\mathcal{U}_{\{0,1\}^{\ell}} \text { in unit }(k \oplus m) \mathcal{U}_{\{0,1\}^{\ell}}\left\{\mathbf{v}_{1}=\mathbf{v}_{2}\right\}(0,0)
$$

Thus we conclude.

\subsubsection{Adversarial Robustness in Machine Learning}

One main goal of machine learning is to learn classifiers, i.e., functions from a set of inputs to a finite set of outputs, called labels. Unfortunately, these classifiers can be vulnerable to misclassification attacks, where an attacker makes slight alterations to an input $x$, possibly adversarially chosen, to produce another input $y$ that is classified differently from $x$. There exist many approaches to protect against adversarial attacks. Some approaches are certified, in the sense that classifiers are provably robust against attacks. A popular example of certified approach, introduced in [112], is based on differential privacy. We review the key elements of this approach below. First, we define the notion of robustness, which formalizes the idea that small changes to the input do not alter the result of the classification:

Definition 5.4 ([112]). A classification algorithm $f: \mathbb{R}^{\mathfrak{n}} \rightarrow\{1, \ldots, k\}$ is L-robust w.r.t. $\mathrm{p}$-norm at $\vec{x}$ if $\mathrm{f}(\overrightarrow{\mathrm{x}})=\mathrm{f}(\overrightarrow{\mathrm{y}})$ for every $\vec{y} \in \mathbb{R}^{\mathfrak{n}}$ such that $|\overrightarrow{\mathrm{x}}-\overrightarrow{\mathrm{y}}|_{\mathrm{p}} \leqslant \mathrm{L}$. From now on, we set $\mathrm{L}=1$ and $\mathrm{p}=2$, although the results hold for arbitrary values.

Consider an $(\epsilon, \delta)$-differentially private probabilistic function $A: \mathbb{R}^{\mathfrak{n}} \rightarrow \mathcal{P}(\mathbf{k})$, where $\mathbf{k} \triangleq\{1, \ldots, k\}$. Given $x \in \mathbb{R}^{n}$, let $s_{1}, \ldots, s_{k}$ denote the probabilities that $A(x)=i$, where the randomness is taken over $A$. Let also $f(x)=\operatorname{argmax}\left(s_{1}, \ldots, s_{k}\right)$, i.e. $f$ returns the index of the maximal element of the tuple. The key observation of [112] is that if $\Theta(\vec{s})$, where $\Theta$ is defined as

$$
\Theta(\vec{s}) \triangleq \exists i, \forall j \neq i, s_{i}>e^{2 \epsilon} s_{j}+\left(1+e^{\epsilon}\right) \delta
$$

then $f$ is robust. This is proved by simple calculations from the following fact: 
Lemma 5.5 ([112]). If a probabilistic function $A: \mathbb{R}^{n} \rightarrow[0,1]$ is $(\epsilon, \delta)$-differentially private, then $\mathbb{E}[\mathrm{f}(\vec{x})] \leqslant e^{\epsilon} \mathbb{E}[\mathrm{f}(\vec{y})]+\delta$ for every $\vec{x}, \vec{y} \in \mathbb{R}^{n}$ such that $|\vec{x}-\vec{y}|_{2} \leqslant 1$.

Lecuyer et al [112] consider several strategies for adding noise to a Deep Neural Network (DNN) to make it differentially private.

In its simple form, we can think about a DNN as a classifier with $n$ inputs and one output, a label in $\mathbf{k}=\{1, \ldots, k\}$. This is usually computed by first computing a vector $\vec{v} \in[0,1]^{\mathrm{k}}$ of scores for each of the labels and then returning $\operatorname{argmax}(\vec{v})$. The internal structure of a DNN can be described by a series layers consisting in an affine transformation followed by an activation function. We will consider $n$ inputs, one output, 2-layer DNN, where $f_{1}, \ldots, f_{m}$ are the components of the affine transformation for the first layer and $g_{1}, \ldots, g_{k}$ are the components of the affine transformation for the second layer. We use the classical RELU as activation function, defined as $\operatorname{RELU}(x)=\max \{0, x\}$, and the Gaussian mechanism over $\mathbb{R}^{\mathrm{m}}$ as differentially private mechanism. The softmax function takes a vector in $[0, \infty)^{k}$ and returns a normalized vector in $[0,1]^{\mathrm{k}}$.

One possible strategy(for efficiency considerations) is to add Gaussian noise after the first layer of the DNN. In this paragraph, we show how our logic can be used to prove that the classifier based on the noise $\mathrm{DNN}$ is robust. We review the definition of the classifier and then sketch a proof of its robustness in our framework. The code below uses ; for the sake of readability and implicitly assumes that deterministic computations are trivial probabilistic computations:

$$
\begin{aligned}
\operatorname{DNN}(\vec{x})= \\
\\
\left(y_{1}, \ldots, y_{n}\right)=\left(f_{1}(\vec{x}), \ldots, f_{m}(\vec{x})\right) ; \\
\left(u_{1}, \ldots, u_{n}\right)=\left(\operatorname{RELU}\left(y_{1}\right), \ldots, \operatorname{RELU}\left(y_{m}\right)\right) ; \\
\left(w_{1}, \ldots, w_{m}\right)=\operatorname{Gauss}_{\sigma}\left(u_{1}, \ldots, u_{m}\right) ; \\
\left(r_{1}, \ldots, r_{k}\right)=\left(g_{1}\left(w_{1}, \ldots, w_{m}\right), \ldots, g_{k}\left(w_{1}, \ldots, w_{m}\right)\right) ; \\
\left(z_{1}, \ldots, z_{k}\right)=\left(\operatorname{RELU}\left(r_{1}\right), \ldots, \operatorname{RELU}\left(r_{k}\right)\right) ; \\
\quad \operatorname{softmax}\left(z_{1}, \ldots, z_{k}\right) ;
\end{aligned}
$$

The program is well-typed by $\mathbb{R}^{\mathfrak{n}} \rightarrow \mathcal{P}\left([0,1]^{\mathrm{k}}\right)$. Furthermore, note that the Gaussian noise we add is calibrated through $\sigma$ to the sensitivity $\Delta_{2} \vec{f}$ of the first layer, i.e. we use $\sigma=\frac{\Delta_{2} \vec{f}}{\epsilon} \sqrt{2 \ln \left(\frac{1.25}{\delta}\right)}$. The sensitivity is defined as follows:

$$
\Delta_{2} \vec{f}=\sup _{\left\{\vec{x} \mid\|\vec{x}\|_{2} \leqslant 1\right\}}\left\|\left(f_{1}(\vec{x}), f_{2}(\vec{x}), \ldots, f_{m}(\vec{x})\right)\right\|_{2}
$$

We now turn to the formalization. We will first show that DNN is $(\epsilon, \delta)$-differentially private. This is stated as:

$$
x, y: \mathbb{R}^{\mathfrak{n}} \mid\|x-y\|_{2} \leqslant 1 \vdash \operatorname{DNN}(x): \mathcal{P}\left([0,1]^{k}\right) \sim \operatorname{DNN}(y): \mathcal{P}\left([0,1]^{k}\right)\left\{\left\{\mathbf{v}_{1}=\mathbf{v}_{2}\right\}_{(\epsilon, \delta)}\right.
$$


The proof first uses the assumption on the sensitivity of the first layer to show:

$$
\begin{gathered}
x_{1}, x_{2}: \mathbb{R}^{\mathfrak{n}} \mid\left\|x_{1}-x_{2}\right\|_{2} \leqslant 1 \vdash\left(f_{1}\left(x_{1}\right), \ldots, f_{\mathfrak{m}}\left(x_{1}\right)\right): \mathcal{P}\left([0,1]^{\mathfrak{m}}\right) \sim \\
\left(f_{1}\left(x_{2}\right), \ldots, f_{m}\left(x_{2}\right)\right): \mathcal{P}\left([0,1]^{\mathfrak{m}}\right)\left\{\left\|\mathbf{v}_{1}-\mathbf{v}_{2}\right\|_{2} \leqslant \Delta_{2} \vec{f}_{\}}(0,0)\right.
\end{gathered}
$$

It is also easy to show that RELU preserves sensitivity:

$$
\begin{gathered}
y_{1}, y_{2}: \mathbb{R}^{\mathfrak{m}} \mid\left\|x_{1}-x_{2}\right\|_{2} \leqslant \Delta_{2} \vec{f} \vdash \operatorname{RELU}\left(y_{1,1}, \ldots, y_{1, m}\right): \mathcal{P}\left([0,1]^{\mathfrak{m}}\right) \sim \\
\operatorname{RELU}\left(y_{2,1}, \ldots, y_{2, m}\right): \mathcal{P}\left([0,1]^{\mathfrak{m}}\right)\left\{\left\|\mathbf{v}_{1}-\mathbf{v}_{2}\right\|_{2} \leqslant \Delta_{2} \vec{f}\right\}(0,0)
\end{gathered}
$$

We then use the rule for sampling from a Gaussian distribution:

$$
\frac{\Gamma \mid \Psi \vdash \mathfrak{u}_{1}: \mathbb{R}^{\mathfrak{n}} \sim \mathfrak{u}_{2}: \mathbb{R}^{\mathfrak{n}}\left\{\left\|\mathbf{r}_{1}-\mathbf{r}_{2}\right\|_{2} \leqslant r\right\} \quad \sigma=\frac{r}{\epsilon} \sqrt{2 \ln \left(\frac{1.25}{\delta}\right)}}{\Gamma \mid \Psi \vdash \operatorname{sample}\left(\operatorname{Gauss}_{\sigma}\left(\mathfrak{u}_{1}\right)\right): \mathcal{P}\left(\mathbb{R}^{\mathfrak{n}}\right) \sim \operatorname{sample}\left(\operatorname{Gauss}_{\sigma}\left(\mathfrak{u}_{2}\right)\right): \mathcal{P}\left(\mathbb{R}^{\mathfrak{n}}\right)\left\{\mathbf{v}_{1}=\mathbf{v}_{2}\right\}_{(\epsilon, \delta)}} \text { Gauss }
$$

The rest of the proof is post-processing: the postcomposition of an $(\epsilon, \delta)$-dp function with a deterministic function is $(\epsilon, \delta)$-dp. Once we have shown that DNN is $(\epsilon, \delta)$-dp, we can apply Lemma 5.5 to show that applying argmax to the output of DNN is robust.

\section{$5 \cdot 4$ Semantics}

In the previous chapter we have presented the construction of a category Pred (QBS) of predicates over QBS, which served as a model to interpret HOL, UHOL, RHOL and HO-UBL. However, we cannot interpret HO-RPL in Pred (QBS), because the lifting needs to map a predicate over $\tau_{1} \times \tau_{2}$ to a predicate over $\mathcal{P}\left(\tau_{1}\right) \times \mathcal{P}\left(\tau_{2}\right)$, but a lifting in $\operatorname{Pred}(\mathbf{Q B S})$ would actually map it to a predicate over $\mathcal{P}\left(\tau_{1} \times \tau_{2}\right)$.

Instead, we define a category of binary relations over Pred(QBS), that we denote by $\mathbf{B R e l}(\mathbf{Q B S})$. An explicit description of $\mathbf{B R e l}(\mathbf{Q B S})$ is:

An object is a tuple $(X, Y, P)$ where $X, Y \in Q B S$ and $P \subseteq|X \times Y|$

A morphism from $(X, Y, P)$ to $(Z, W, Q)$ is a pair $f: X \rightarrow Z, g: Y \rightarrow W$ of morphisms in QBS such that $|f \times g|(P) \subseteq Q$.

This category can also be computed by the following pullback (in the category of categories):

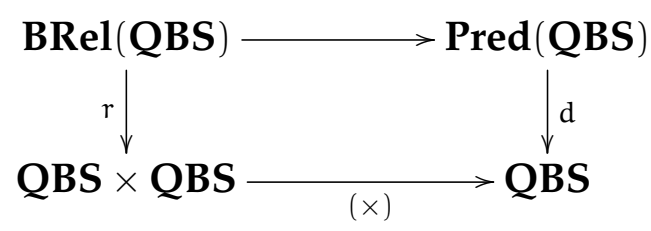

Here $(x)$ denotes the binary product functor on $\mathbf{Q B S}$, and $\mathbf{Q B S} \times \mathbf{Q B S}$ is the category that has as objects pairs $\left(X_{1}, X_{2}\right)$ of objects $X_{1}, X_{2} \in Q B S$ and as morphisms 
$(\mathbf{Q B S} \times \mathbf{Q B S})\left(\left(X_{1}, X_{2}\right),\left(Y_{1}, Y_{2}\right)\right)=\mathbf{Q B S}\left(X_{1}, Y_{1}\right) \times \mathbf{Q B S}\left(X_{2}, Y_{2}\right)$. The vertical leg of the pullback $r$ : BRel $(\mathbf{Q B S}) \rightarrow \mathbf{Q B S} \times \mathbf{Q B S}$ mapping a relation $\left(X_{1}, X_{2}, P\right)$ to its base sets $\left(\mathrm{X}_{1}, \mathrm{X}_{2}\right)$ forms a fibration. The category BRel(QBS) has a CCC structure which we denote by $(\ddot{x}, \ddot{\Rightarrow})$, and that is preserved strictly by $r$.

The category QBS $\times$ QBS can be used as a semantical model for a pair of probabilistic programs, using the monad $\mathcal{P} \times \mathcal{P}$ that maps $\left(\mathrm{X}_{1}, \mathrm{X}_{2}\right)$ to $\left(\mathcal{P} X_{1}, \mathcal{P} X_{2}\right)$.

We can then construct a lifting $\ddot{\mathcal{P}} \mathrm{dp}$ of $\mathcal{P} \times \mathcal{P}$ along $r: \mathbf{B R e l}(\mathbf{Q B S}) \rightarrow \mathbf{Q B S}$ inspired from the witness-free approximate relational coupling from Section 5.1:

$$
\ddot{\mathcal{P}}_{(\epsilon, \delta)}^{\mathrm{dp}}(X, Y, P) \triangleq\left(\mathcal{P}(X), \mathcal{P}(Y),\left\{\begin{array}{l}
\left(\mu_{1}, \mu_{2}\right) \in \mathcal{P}(X) \times \mathcal{P}(Y) \mid \forall \delta \geqslant 0, \forall \epsilon \geqslant 0, \\
\forall(f, g) \in \mathbf{R}\left((X, Y, P),\left(\mathcal{P}(\{0,1\}), \mathcal{P}(\{0,1\}), S\left(\epsilon^{\prime}, \delta^{\prime}\right)\right)\right), \\
\left.\left(f^{\sharp}\left(\mu_{1}\right), g^{\sharp}\left(\mu_{2}\right)\right) \in S\left(\epsilon+\epsilon^{\prime}, \delta+\delta^{\prime}\right)\right)
\end{array}\right\}\right)
$$

where $S(\epsilon, \delta) \triangleq\left\{\left(v_{1}, v_{2}\right) \in \mathcal{P}(\{0,1\}) \times \mathcal{P}(\{0,1\}) \mid \operatorname{Pr}_{b \sim v_{1}}[b=0] \leqslant e^{\epsilon} \operatorname{Pr}_{b \sim v_{2}}[b=0]+\delta\right\}$.

Here, $\mathbf{R}$ stands for $\mathbf{B R e l}(\mathbf{Q B S})$. This construction is an instantiation of the graded TT-lifting [105]. Therefore,

Lemma 5.6. $\ddot{\mathcal{P}} \mathrm{dp}$ is indeed $a([0, \infty],+, 0, \leqslant) \times([0, \infty],+, 0, \leqslant)$-graded lifting of $\mathcal{P}$.

A simplification for measurable predicates is the following:

Remark 5.7. Let $\chi_{S}: X \rightarrow\{0,1\}$ in $\mathbf{Q B S}$ stand for the indicator function of the subset $S$ of $X$, we have $\left(\chi_{S}, \chi_{S}\right) \in \operatorname{BRel}(Q B S)((X, Y, P),(\mathcal{P}(\{0,1\}), \mathcal{P}(\{0,1\}), S(0,0)))$. Hence $\left(\mu_{1}, \mu_{2}\right) \in$ $\ddot{\mathcal{P}}_{(\epsilon, \delta)}^{\mathrm{dp}}\left(\mathrm{X}, \mathrm{X}, \mathrm{Eq}_{\mathrm{X}}\right)$ implies $\operatorname{Pr}_{\mathrm{X} \sim \mu_{1}}[\mathrm{x} \in \mathrm{S}] \leqslant \mathrm{e}^{\epsilon} \operatorname{Pr}_{\mathrm{X} \sim \mu_{2}}[\mathrm{X} \in \mathrm{S}]+\delta$.

We can now state soundness of the HO-PRL rules in the category BRel(QBS) by using the lifting $\ddot{P} \mathrm{dp}$ :

Proposition 5.8. Let $\Gamma \mid \Psi \vdash \mathrm{t}_{1}: \mathcal{P} \tau_{1} \sim \mathrm{t}_{1}: \mathcal{P} \tau_{2}\{\mathbb{Q}\}_{(\epsilon, \delta)}$ be a derivable HO-PRL judgment, and $\gamma \in \mathbf{Q B S}(1, \llbracket \Gamma \rrbracket)$ such that $\gamma:(1, \top) \rightarrow \llbracket \Gamma \vdash \wedge \Psi \rrbracket$. Then, $\left\langle\llbracket \Gamma \vdash \mathrm{t}_{1}: \mathcal{P} \tau_{1} \rrbracket, \llbracket \Gamma \vdash \mathrm{t}_{2}\right.$ : $\left.\mathcal{P} \tau_{2} \rrbracket\right\rangle \circ \gamma$ is in

$$
\left.\left.\operatorname{BRel}(\mathbf{Q B S})\left((1,1, \top), \ddot{\mathcal{P}}_{\tau_{1}, \tau_{2},(\epsilon, \delta)}^{\mathrm{dp}}\left(\left\langle\gamma, \mathrm{id}_{\llbracket \tau_{1} \rrbracket}, \mathrm{id}_{\llbracket \tau_{2} \rrbracket}\right\rangle^{*} \llbracket \Gamma, \mathbf{v}_{1}: \tau_{1}, \mathbf{v}_{2}: \tau_{2} \vdash \mathrm{Q} \rrbracket\right)\right)\right)\right) .
$$

Here, we treat implicitly $\gamma^{*} \llbracket \Gamma, \mathbf{v}_{1}: \tau_{1}, \mathbf{v}_{2}: \tau_{2} \vdash \mathrm{Q} \rrbracket$ as a relation over $\left(\llbracket \tau_{1} \rrbracket, \llbracket \tau_{2} \rrbracket\right)$ rather than a predicate over $\llbracket \tau_{1} \rrbracket \times \llbracket \tau_{2} \rrbracket$. The proposition is stated in categorytheoretical terms, but since the fibration $\mathrm{r}: \mathbf{B R e l}(\mathbf{Q B S}) \rightarrow \mathbf{Q B S}$ has a set-theoretical description, we can restate the theorem in set-theoretical terms. First note that QBS $(1, \llbracket \Gamma \rrbracket)$ is isomorphic to $|\llbracket \Gamma \rrbracket|$ and $\operatorname{Pred}(\mathbf{Q B S})((1, T), \phi)$ is isomorphic to $\phi$. Furthermore, for $\gamma \in|\llbracket \Gamma \rrbracket|$, then $\llbracket \Gamma \vdash t: \sigma \rrbracket \circ \gamma$ corresponds to the evaluation $\llbracket \Gamma \vdash t: \sigma \rrbracket(\gamma)$ and $\langle\gamma, i d, i d\rangle^{*} \llbracket \Gamma, \Gamma^{\prime} \vdash \phi \rrbracket$ corresponds to the result of substituting in $\phi$ every $x \in \Gamma$ by the value assigned to them by $\gamma$. So the premise of the theorem just states that $\gamma$ is a valuation for $\Gamma$ satisfying the precondition $\Psi$, and the conclusion states that

$$
\left(\llbracket \Gamma \vdash \mathrm{t}_{1}: \mathcal{P} \tau_{1} \rrbracket(\gamma), \llbracket \Gamma \vdash \mathrm{t}_{2}: \mathcal{P} \tau_{2} \rrbracket(\gamma)\right)
$$


Chapter 5. Relational Reasoning about probabilities: Probabilistic couplings

are related by the lifting of

$$
\langle\gamma, \mathrm{id}, \mathrm{id}\rangle^{*} \llbracket \Gamma, \mathbf{v}_{1}: \tau_{1}, \mathbf{v}_{2}: \tau_{2} \vdash \mathrm{Q} \rrbracket
$$

Taking into account Remark 5.7, we can show:

Corollary 5.9. Let $\Gamma \mid \Psi \vdash \mathrm{t}_{1}: \mathcal{P R} \sim \mathrm{t}_{1}: \mathcal{P} \mathbb{R}\left\{\mathbf{v}_{1}=\mathbf{v}_{2}\right\}_{(\epsilon, \delta)}$ be a derivable HO-PRL judgment. Then, $\llbracket \Gamma \vdash \mathrm{t}_{1}: \mathcal{P} \mathbb{R} \rrbracket$ and $\llbracket \Gamma \vdash \mathrm{t}_{1}: \mathcal{P} \mathbb{R} \rrbracket$ are $(\epsilon, \delta)$-differentially private. 


\section{UNARY HIGHER-ORDER LOGIC FOR ADVERSARIAL COMPUTATIONS}

So far, we have seen how to write and prove specifications that model how the programs behave on a given input. However, in many other cases specifications establish how the program must interact with its environment. For instance, it is common in security and privacy to reason about the interaction between a system (sometimes called an oracle) and a malicious party (an adversary) that attacks the system and tries to gain some information. Such interactions are instances of adversarial computations, a class of computations where programs and adversaries are engaged in (sequential) interactions. Adversarial computations are also used for modelling uncertainty in classic algorithms, such as random walks [17] or multiarmed bandits [14]. In many of the aforementioned scenarios, both the programs and the adversaries are randomized, and the interactions between the two are constrained by resource policies, which limit the number of interactions between programs and adversaries, and condition the security analysis ${ }^{1}$. Unfortunately, there is a lack of a general framework for reasoning about such computations, especially for higher-order languages.

In this section, we will develop an extension of the system in Chapter 4 to reason about adversarial computations. This extension has several components: first we need to extend the base language and its semantics with state. To model this we will use the monad $\mathcal{T}(-) \triangleq M \Rightarrow \mathcal{P}(-\times M)$ where $M$ is a QBS of stores, and where $\mathcal{P}$ denotes the monad of probability measures over $\mathbf{Q B S}$

Second, we need to be able to reason about adversaries only from their type, without knowing their syntax. To allow this kind of reasoning, we track at type-level

\footnotetext{
${ }^{1}$ To illustrate the need for resource policies, consider a two-party secret sharing scheme that takes as secret input $x \in \mathbb{Z}_{\mathrm{q}}$ and returns the pair $\left(y_{1}, y_{2}\right)=(r, x-r)$, where $r$ is sampled uniformly over $\mathbb{Z}_{q}$. Now consider an oracle that takes as input $i \in\{1,2\}$ and returns $y_{i}$. An adversary that can make 2 queries will learn the secret, whereas an adversary that can make only 1 query will learn nothing about the secret.
} 
the memory usage of stateful computations, to ensure separation between oracle and adversary memories. We also need to limit the number of interactions between oracles and adversaries, as justified above. To this end, we grade the monad $\mathcal{T}$ by two parameters $\Sigma$ and $k$ : $\Sigma$ represents the memory footprint of the computation and $k$ represents the number of oracle calls. We model adversaries as higher-order, probabilistic and stateful computations. For instance, the declaration:

$$
\mathcal{A}: \forall \alpha .\left(\sigma \rightarrow \mathcal{T}_{\alpha, 1}(\tau)\right) \rightarrow \mathcal{T}_{\Sigma \cup \alpha, k}\left(\tau^{\prime}\right)
$$

defines an adversary $\mathcal{A}$ that can read and write within the memory region $\Sigma$ and can call its oracle (that is passed as argument) up to $k$ times. The memory region over which the oracle operates is abstracted away by quantifying over the effect. More general forms of adversaries can be captured by our language, e.g. adversaries with access to multiple oracles, each with a potentially different cost. However, these more general forms of adversaries do not pose any additional challenge so we focus on the simple setting of an adversary with a single oracle.

Third, we identify sound proof principles for reasoning about adversarial computations. In the exact setting, the proof principles establish that an application $\mathcal{A}(\mathcal{O})$ preserves a property $\Phi$, if the oracle $\mathcal{O}$ itself preserves the property $\Phi$, and the property $\Phi$ is "safe" with respect to the footprint $\Sigma$ of $\mathcal{A}$. In the approximate settings, the proof principles require that each oracle call preserve the property $\Phi$ with probability $1-\beta$, and ensure that the property is preserved with probability $1-k \beta$, where $k$ is an upper bound on the number of oracle calls that can be performed by the adversaries.

We integrate all of these componentts into the Higher-Order Union Bound Logic by rewriting all the existing rules to be able to reason about state, as well as adding new rules for the constructs that read and write the state, and embedding the proof principles for adversaries as a new, non-syntax directed rules.

We will apply our logic to reason about drifting games [140], a theoretical framework for analyzing online learning algorithms. Drifting games are closely related to boosting, a common technique for transforming weak learners into strong learners, see [139]. A typical use of drifting games is for proving (upper or lower) bounds on the expected loss of an algorithm. Using our unary logic, we prove an upper bound on the expected loss in a drifting game between an adversary and a so-called optimal shepherd.

\section{Organization of chapter}

We begin by introducing the language and its semantics (Section 6.1). We then present the Higher-Order Stateful Union Bound Logic (Section 6.2). After this, we present the notions of adversaries, adversarial properties and the rules to reason about them (Section 6.3). Then we use the logic to verify properties of drifting games (Section 6.4). We conclude the chapter by presenting the semantical setting. 
We first extend the notion of monad lifting to take the state into account (Section 6.5) and we use it to prove soundness of HO-SUBL (Section 6.6).

\subsection{Language}

\subsubsection{Syntax}

We extend the language from Section 4.2 with a countable set Loc of memory locations, a type Val for values stored in memory, a unit type, a monadic type for stateful probabilistic computations, graded by two indices, which represent

- An upper bound on the cost of the computation, that comes from the ordered monoid $(\mathbb{N}, 0,+, \leqslant)$

- An upper bound on the effect on memories, namely the set of memory regions the computation reads or writes, that comes from the ordered monoid $(\mathbb{P}($ Loc $), \emptyset, \cup, \subseteq)$ induced by the powerset of memory locations.

We will omit this grading when it is not relevant. The types include as well a lightweight version of effect polymorphism by allowing universal quantification over effect variables. The ordering of the monoids induces a subtyping relation, whose rules we present in Figure 6.2.

The terms of the language are standard. We have monadic unit and bind to introduce and sequence effectful programs, plus operations for reading, writing and a collection of operations $\left\{\alpha_{i}\right\}_{i \in \mathrm{I}}$ of arity $\sigma_{i, 1} \times \cdots \times \sigma_{i, \operatorname{ar}(i)} \rightarrow \mathrm{T}\left(\tau_{i}\right)$ to model sampling from a distribution. There is also a skip operation that does nothing. We introduce this as a primitive, although it can be defined as unit $(*)$, where $*$ is the single inhabitant of ut. Finally, we have terms $\wedge \alpha . t$ to introduce quantification over memory regions and $t[\Sigma]$ to eliminate it. The following grammar summarizes the terms and types:

$$
\begin{aligned}
& \tau, \sigma::=\mathrm{B} \mid \text { ut } \mid \text { Loc } \mid \text { Val }|\tau \rightarrow \sigma| \tau \times \sigma\left|\mathrm{T}_{\Sigma, \mathrm{k}} \tau\right| \forall \alpha . \tau \\
& \mathrm{t}, \mathrm{u}::=\mathrm{c}|x| \lambda x . \mathrm{u}|\mathrm{t} \mathrm{u}|\langle\mathrm{t}, \mathrm{u}\rangle \mid \text { case } \mathrm{t} \text { of } \mathrm{tt} \mapsto \mathrm{u}_{1} ; \mathrm{ff} \mapsto \mathrm{u}_{2}\left|\pi_{1}(\mathrm{t})\right| \pi_{2}(\mathrm{t}) \\
& \quad \mid \text { read } \mathrm{a}|\mathrm{a}:=\mathrm{t}| \text { skip } \mid \text { unit }(\mathrm{t}) \mid \text { mlet } x=\mathrm{t} \text { in } \mathrm{u}\left|\alpha_{\mathrm{i}}\left(\mathrm{t}_{1}, \ldots, \mathrm{t}_{\operatorname{ar}(\mathrm{i})}\right)\right| \Lambda \alpha . \mathrm{t} \mid \mathrm{t}[\Sigma]
\end{aligned}
$$

Remark 6.1. The language presented here is not a proper extension of the language from Section 4.2, since we no longer have a type $\mathrm{P}$ for stateless probabilistic computations. Alternatively, we could have separate types $\mathrm{P}$ and $\mathrm{T}$ for stateless and stateful computations, with their own operations and a coercion from $\mathrm{P}$ to $\mathrm{T}$. Out of simplicity, we choose instead a single monad for all effectful computations. Incidentally, this means that sampling from a probability distribution is a stateful operation that leaves the state unchanged. 
A grading context $\Xi$ is a collection of variables $\alpha, \beta, \ldots$ representing memory regions, which we will use to quantify over effects. Note that in our setting, all reads and writes still need to be to concrete locations (in other words, writing to some abstract $\alpha$ is not allowed). Quantification over gradings is only used to allow adversaries to call oracles independently of the regions the oracles use. For instance, a computation that uses memory region $\Sigma$ and that calls some oracle returning booleans, can be given the type $\forall \alpha . T_{\alpha, k}(B) \rightarrow T_{\alpha \cup \Sigma, k}(\tau)$. In particular, this implies that closed expressions of type $\forall \alpha . T_{\alpha, k}(\tau)$ never read nor write the memory, because they can be instantiated to $T_{\emptyset, k}(\tau)$.

A typing judgment $\Xi ; \Gamma \vdash t: \tau$ is a relation between contexts, terms and types. We present in Figure 6.1 typing rules for our language. The rules for simple types are standard. The unit and bind rules act on the grading as the unit and multiplication of the monoid from which the gradings are taken: unit introduces a computation with no memory footprint nor cost, and mlet adds the footprints and costs together. We also need to add into the footprint the effect of $\tau$, which is defined as:

$$
\begin{gathered}
\operatorname{Eff}(B) \triangleq \emptyset \quad \operatorname{Eff}\left(T_{\Sigma}(\tau)\right) \triangleq \Sigma \cup \operatorname{Eff}(\tau) \quad \operatorname{Eff}(\sigma \times \tau) \triangleq \operatorname{Eff}(\sigma) \cup \operatorname{Eff}(\tau) \\
\operatorname{Eff}(\sigma \rightarrow \tau) \triangleq \operatorname{Eff}(\tau) \quad \operatorname{Eff}(\forall \alpha . \tau) \triangleq \operatorname{Eff}(\tau) \backslash\{\alpha\}
\end{gathered}
$$

Formally, $\operatorname{Eff}(\tau)$ collects the memory footprint that a program has because of its own memory operations, rather than the ones that may come from its arguments. Therefore, we only collect the efects that appear in positive position. This will overapproximate the effect that mlet $x=t_{1}$ in $t_{2}$ has, but it will be useful in proving soundness of the adversary rules.

The rules for reading and writing have as memory footprint the memory location they are accessing, and no cost. The rules for quantifier introduction and elimination are a version of effect polymorphism. We can quantify over any grading in $\Xi$ that does not appear free in $\Gamma$, and we can instantiate a quantifier to any concrete memory region. Finally, the subtyping rule allows to change the type of a term to any larger type.

We conclude with two remarks on grading:

- when reasoning about adversaries, the subtyping rule will have the effect that the grading is an upper, rather than exact, bound on the number of calls. This is in line with many applications of the counting monad, but is occasionally cumbersome for specific examples. Such examples would be easier to formalize in a richer setting where one can specify upper bounds and exact bounds on the number or calls. For simplicity, we refrain from introducing such a system, at the cost of making our examples a bit more cumbersome;

- the grading tracks the memory footprint of the computation. However, it can be easily generalized to gradings $\left(\Sigma_{R}, \Sigma_{W}\right)$ that distinguish between memory locations that are read, and memory locations that are written. 
Chapter 6. Unary higher-order logic for adversarial computations

$$
\begin{aligned}
& \frac{\Xi ; \Gamma, x: \tau \vdash t: \sigma}{\Xi ; \Gamma \vdash \lambda x . t: \tau \rightarrow \sigma} \text { Abs } \quad \frac{\Xi ; \Gamma \vdash t_{1}: \tau \rightarrow \sigma \quad \Xi ; \Gamma \vdash t_{2}: \tau}{\Xi ; \Gamma \vdash t_{1} t_{2}: \sigma} \text { App } \\
& \Xi ; \Gamma, x: \tau, \Gamma^{\prime} \vdash x: \tau \text { Var } \quad \frac{\Xi ; \Gamma \vdash \mathrm{b}: \mathbb{B} \quad \Xi ; \Gamma \vdash \mathrm{t}_{1}: \tau \quad \Xi ; \Gamma \vdash \mathrm{t}_{2}: \tau}{\Xi ; \Gamma \vdash \text { case } \mathrm{b} \text { of } \mathrm{tt} \mapsto \mathrm{t}_{1} ; f f \mapsto \mathrm{t}_{2}: \tau} \text { Case } \\
& \frac{\Xi ; \Gamma \vdash \mathrm{t}: \tau_{1} \times \tau_{2}}{\Xi ; \Gamma \vdash \pi_{i}(\mathrm{t}): \tau_{i}} \text { Proj } \quad \frac{\Xi ; \Gamma \vdash \mathrm{t}: \tau \quad \Xi ; \Gamma \vdash \mathrm{u}: \sigma}{\Xi ; \Gamma \vdash\langle\mathrm{t}, \mathrm{u}\rangle: \tau \times \sigma} \text { Pair }
\end{aligned}
$$

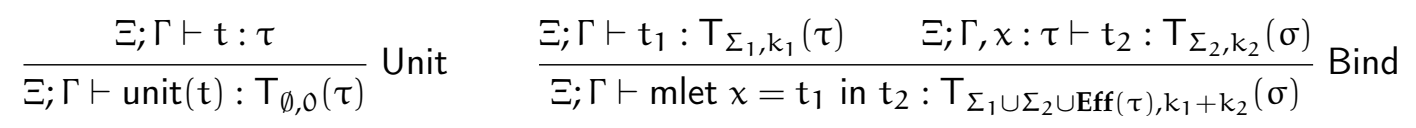

$$
\begin{aligned}
& \frac{a \in \text { Loc }}{\Xi ; \Gamma \vdash \text { read } l: \mathrm{T}_{\{\mathrm{a}\}, 0}(\mathrm{Val})} \text { Read } \quad \frac{\Xi ; \Gamma \vdash \mathrm{t}: \text { Val } \quad \mathrm{a} \in \text { Loc }}{\Xi ; \Gamma \vdash \mathrm{a}:=\mathrm{t}: \mathrm{T}_{\{\mathrm{a}\}, 0} \text { (ut) }} \text { Write }
\end{aligned}
$$

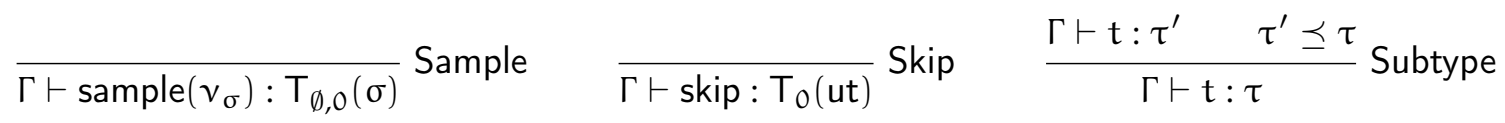

$$
\begin{aligned}
& \frac{\Xi, \alpha ; \Gamma \vdash \mathrm{t}: \tau \quad \alpha \notin \mathrm{FV}(\Gamma)}{\Xi ; \Gamma \vdash \Lambda \alpha . \mathrm{t}: \forall \alpha . \tau} \text { ForAll-I } \quad \frac{\Xi ; \Gamma \vdash \mathrm{t}: \forall \alpha . \tau \quad \Sigma \in \mathbb{P}(\text { Loc })}{\Xi ; \Gamma \vdash \mathrm{t}[\Sigma]: \tau[\Sigma / \alpha]} \text { ForAll-E }
\end{aligned}
$$

Figure 6.1: Selected typing rules

$$
\begin{gathered}
\frac{\Sigma \subseteq \Sigma^{\prime} \quad k \leqslant k^{\prime} \quad \tau \preceq \tau^{\prime}}{T_{\Sigma, k}(\tau) \preceq T_{\Sigma^{\prime}, k^{\prime}}\left(\tau^{\prime}\right)} \quad \frac{\tau \preceq \tau^{\prime} \tau^{\prime} \preceq \tau^{\prime \prime}}{\tau \preceq \tau^{\prime \prime}} \\
\frac{\tau \preceq \tau^{\prime} \quad \sigma \preceq \sigma^{\prime}}{\tau \times \sigma \preceq \tau^{\prime} \times \sigma^{\prime}} \quad \frac{\tau^{\prime} \preceq \tau \quad \sigma \preceq \sigma^{\prime}}{\tau \times \sigma \preceq \tau^{\prime} \times \sigma^{\prime}} \frac{\tau^{\prime} \preceq \tau}{\forall \alpha . \tau \preceq \forall \alpha . \tau^{\prime}}
\end{gathered}
$$

Figure 6.2: Subtyping rules

Given a typing context $\Gamma$ with no free grading variables, an instantiation of the context (denoted $\Gamma \vdash \gamma$ ) is a map from every variable $(x: \sigma) \in \Gamma$ to a closed term $\vdash \gamma(\mathrm{x}): \sigma$. Given $\Gamma \vdash \gamma$ and $\Gamma \vdash \tau$ : $\tau$, we denote by $\mathrm{t} \gamma$ the result of substituting every free variable $x \in \mathrm{FV}(\mathrm{t})$ by $\gamma(x)$ in a capture-free manner, and given $\left(y: \sigma^{\prime}\right) \notin \Gamma$ and $\vdash u$ : $\sigma^{\prime}$, we denote by $\gamma[y \mapsto u]$ the extension of $\gamma$ for which y gets mapped to $y$, and $\Gamma, y: \sigma^{\prime} \vdash \gamma[y \mapsto u]$. Similarly, given a grading context $\Xi$, an instantiation of the context (denoted $\Xi \vdash \xi$ ) is a map from the variables in $\Xi$ to memory regions in $\mathbb{P}($ Loc $)$. The instantiation of a type $\sigma$ with respect to $\xi$ is denoted by the juxtaposition $\sigma \xi$ and consists of replacing every grading variable $\alpha \in \mathrm{FV}(\sigma)$ by $\xi(\alpha)$ in a capture-free manner. The instantiation of a typing context $\Gamma$ with respect to $\xi$ is denoted by $\Gamma \xi$ and consists of replacing every $(x: \sigma)$ in $\Gamma$ by $(x: \sigma \xi)$. Given $\Xi \vdash \xi, \alpha \notin \operatorname{dom}(\Xi)$ and $\Sigma \subseteq$ Loc, we denote by $\xi[\alpha \mapsto \Sigma]$ the extension for which $\alpha$ gets mapped to $\Sigma$ and $\Xi, \alpha \vdash \xi[\alpha \mapsto \Sigma]$. 


\subsubsection{Semantics}

Here we adapt the CCC interpretation presented in Section 5.4. We will interpret our language in the category QBS of quasi-Borel spaces, and we use the monad $\mathcal{T}(-) \triangleq M \Rightarrow \mathcal{P}(-\times M)$ to interpret probabilistic stateful computations, where $M$ is an abstract set of memories of the form $\Pi_{a \in L o c} V$ al. This monad can be seen as the state monad transformer applied to $\mathcal{P}$, which is sometimes denoted by $\operatorname{St}(\mathcal{P})$ and is defined by:

$$
\begin{aligned}
& \eta_{X}^{\mathcal{T}}: X \rightarrow M \Rightarrow \mathcal{P}(X \times M) \\
& \eta^{\mathcal{T}} \triangleq \lambda . \eta_{X \times M}^{\mathcal{P}} \\
& \mu_{X}^{\mathcal{T}}:(M \Rightarrow \mathcal{P}((M \Rightarrow \mathcal{P}(X \times M)) \times M)) \rightarrow M \Rightarrow \mathcal{P}(X \times M) \\
& \mu^{\mathcal{T}} \triangleq \lambda . \mu_{X \times M}^{\mathcal{P}} \circ \mathcal{P}(\text { eval }) \circ \text { eval }
\end{aligned}
$$

where $\lambda$ denotes currying.

From the strength $\theta_{X, Y}^{\mathcal{P}}: X \times \mathcal{P}(Y) \rightarrow \mathcal{P}(X \times Y)$ we can also construct a strength $\theta_{X, Y}^{\mathcal{T}}: X \times \mathcal{T}(Y) \rightarrow \mathcal{T}(X \times Y)$ for $\mathcal{T}:$

$$
\begin{aligned}
& \theta^{\mathcal{T}}: \mathrm{X} \times(\mathrm{M} \Rightarrow \mathcal{P}(\mathrm{Y} \times \mathrm{M})) \rightarrow M \Rightarrow \mathcal{P}(\mathrm{X} \times \mathrm{Y} \times \mathrm{M}) \\
& \theta^{\mathcal{T}} \triangleq \lambda . \theta_{X, Y \times M}^{\mathcal{P}} \circ\left(\mathrm{id}_{x} \times \text { eval }\right)
\end{aligned}
$$

Recall that a type $\tau$ will get mapped to an object $\llbracket \tau \rrbracket$, and a judgment $\Gamma \vdash t: \tau$ will get mapped to a morphism $\llbracket \Gamma \vdash t: \tau \rrbracket \in \mathbf{Q B S}(\llbracket \Gamma \rrbracket$, $\llbracket \tau \rrbracket)$, where $\llbracket \Gamma \rrbracket$ is defined as the Cartesian product of the interpretations of the types in $\Gamma$. The semantics of types is defined as:

$$
\begin{gathered}
\llbracket \text { ut } \rrbracket \triangleq 1 \quad \llbracket[0, \infty] \rrbracket \triangleq[0, \infty] \quad \llbracket \sigma \rightarrow \tau \rrbracket \triangleq \llbracket \sigma \rrbracket \Rightarrow \llbracket \tau \rrbracket \quad \llbracket \sigma \times \tau \rrbracket \triangleq \llbracket \sigma \rrbracket \times \llbracket \tau \rrbracket \\
\llbracket T_{\Sigma, k}(\sigma) \rrbracket \triangleq \tau(\llbracket \sigma \rrbracket \quad \llbracket \forall \alpha . \tau \rrbracket \triangleq \llbracket \tau \rrbracket
\end{gathered}
$$

Note that the grading of the monad plays no role in the semantics of expressions. We omit here the interpretation of non-monadic terms, which is given by the standard CCC semantics (see Section 4.8). The semantics of monadic terms is defined as:

$$
\begin{aligned}
& \llbracket \Gamma \vdash \operatorname{unit}(t): T \sigma \rrbracket \triangleq \eta^{\mathcal{T}} \circ \llbracket \Gamma \vdash t: \sigma \rrbracket \\
& \llbracket \Gamma \vdash \text { mlet } x=t \text { in } u: T \tau \rrbracket \triangleq \llbracket \Gamma, x: \sigma \vdash u: T \tau \rrbracket^{\# \top} \circ \theta^{\mathcal{T}} \circ\left\langle\mathrm{id}_{\Gamma}, \llbracket \Gamma \vdash \mathrm{t}: \mathcal{T} \sigma \rrbracket\right\rangle \\
& \llbracket \Gamma \vdash \operatorname{read} a: \mathcal{T V a l} \rrbracket \triangleq \lambda . \eta^{\mathcal{P}} \circ\left\langle\pi_{a}, i \mathrm{~d}\right\rangle \circ \pi_{2} \\
& \llbracket \Gamma \vdash \mathrm{a}:=\mathrm{t}: \mathcal{T} \mathrm{ut} \rrbracket \triangleq \lambda . \eta^{\mathcal{P}} \circ \mathrm{l}_{\mathrm{a}} \circ\left\langle\llbracket \Gamma \vdash \mathrm{t}: \mathrm{Val} \rrbracket, \pi_{\neq \mathrm{a}}\right\rangle \\
& \llbracket \Gamma \vdash \alpha\left(t_{1}, \ldots, t_{k}\right): \mathcal{T} \sigma \rrbracket \triangleq \lambda .\left(\theta^{\prime}\right)^{\mathcal{T}} \circ\left\langle\llbracket \alpha \rrbracket \circ\left\langle\llbracket \Gamma \vdash \mathrm{t}_{1}: \tau_{1} \rrbracket, \ldots, \llbracket \Gamma \vdash \mathrm{t}_{k}: \tau_{k} \rrbracket\right\rangle, \mathrm{id}\right\rangle
\end{aligned}
$$

where ( $(-)^{\# \mathcal{T}}$ denotes the Kleisli lifting, $\pi_{\neq \mathrm{a}}$ is the projection $\Pi_{\mathrm{b} \in \mathrm{Loc}} \mathrm{Val} \rightarrow \Pi_{\mathrm{b} \in \mathrm{Loc}, \mathrm{b} \neq \mathrm{a}} \mathrm{Val}_{\mathrm{a}}$, $\mathrm{c}_{\mathrm{a}}$ is the injection $\mathrm{Val} \times \Pi_{\mathrm{b} \in \mathrm{Loc}, \mathrm{b} \neq \mathrm{a}} \mathrm{Val} \rightarrow \Pi_{\mathrm{b} \in \mathrm{Loc}} \mathrm{Val}$ and $\theta_{X, Y}^{\prime}: \mathcal{P}(X) \times Y \rightarrow \mathcal{P}(X \times Y)$ is the co-strength, a transformation analogous to the strength but with swapped arguments. 


\subsection{Higher-Order Stateful Union Bound logic}

In this section, we present Higher-Order Stateful Union Bound Logic, or HO-SUBL, which can be seen as an extension with state of the logic from Section 4.3.

The system is built on top of UHOL by adding a new form of judgment to reason about monadic computations of type $M \rightarrow \mathcal{P}(\sigma \times M)$. These judgments are of the form:

$$
\Gamma \mid \Psi \vdash\{\mathrm{P}\} \mathrm{t}: \mathcal{T} \sigma\{\mathrm{Q}\}_{\delta}
$$

These judgments have the flavor of an imperative Hoare logic. Here, $t$ is a monadic computation over type $\sigma, \Gamma, \mathbf{s}: M \vdash P$ is a precondition over the initial memory, and $\Gamma, \mathbf{s}: M, \mathbf{v}: \sigma \vdash \mathrm{Q}$ is a postcondition over the final memory and result. As in previous chapters, the double brace on the postcondition indicates that it should be interpreted as a lifting: even though $\mathrm{Q}$ is a predicate over $M \times \sigma$, in the interpretation of the triple it acts as a predicate over $\mathcal{P}(M \times \sigma)$. $\delta$ is a non-negative real number. The intended interpretation of this judgment is that if $\Gamma \mid \Psi \vdash\{P\} t: \mathcal{T} \tau\{Q\}_{\delta}$ is derivable, then for every $\gamma \in \Gamma$ such that $\gamma \in \Lambda \Psi$, and every $\mathrm{m} \in \mathrm{P}, \mathrm{t}(\mathrm{m})$ is a distribution over $\tau \times M$ such that $\operatorname{Pr}_{t(m)}[Q] \geqslant 1-\delta$. In particular, if $\delta \geqslant 1$, then this is trivially satisfied.

Comparing these judgments to the ones from Section 4.3 we see two main differences: there is a precondition, which can explicitly mention the initial state and the postcondition has an extra variable $\mathbf{s}$ to refer to the final state.

Figure 6.3 presents the rules of the logic. The UNIT-U rule assumes that we have a term $t$ satisfying $\phi$ and a well-formed predicate $P$, and concludes that for any initial state in $P$, unit $(t)$ returns with probability 1 a final state and result satisfying the conjunction of $\mathrm{P}$ and $\phi$. The MLET- $U$ rule is a generalization of the usual sequencing rule of Hoare logic; note how the error probabilities are added in the grading. The READ-U and WRITE-U rules propagate the effect on the heap backwards. We also include a rule MCASE- $U$ for conditional constructs of monadic type and a rule SAMPLE-UBL for sampling from a uniform distribution. Finally, we present some structural rules in Figure 6.4: a CONSEQ-U rule, where the relation $\Rightarrow$ needs to be established in HOL, a OR-PRE-U rule to perform case analysis in the preconditions, and a AND-POST-U rule to split the proof of a conjunction in the postcondition. Notice that in the latter we have a union bound $\delta+\delta^{\prime}$ on the conclusion, which comes from the sum of the union bounds on the premises.

For completeness, we add a predicate former $\mathcal{H}_{\sigma, \delta}(\mathrm{P}, \mathrm{t}, \mathrm{Q})$ that internalizes the monadic judgments into the base logic. This predicates are well formed when $\mathbf{s}: M \vdash \mathrm{P}, \vdash \mathrm{t}: \mathcal{T}(\sigma)$ and $\mathbf{s}: \mathrm{M}, \mathbf{v}: \sigma \vdash \mathrm{Q}$, and have the obvious interpretation. We add rules to switch between systems also in Figure 6.4. We do not provide here rules to reason about these predicates in HOL, but see Chapter 8 for a related logic in which we do. 


$$
\begin{aligned}
& \frac{\Gamma \mid \Psi \vdash t: \tau\{\phi\} \quad \Gamma, \mathbf{s}: M \vdash P}{\Gamma \mid \Psi \vdash\{\mathrm{P}\} \text { unit }(\mathrm{t}): \mathcal{T}_{\emptyset, 0}(\tau)\{\phi[\mathbf{v} / \mathbf{r}] \wedge \mathrm{P}\}_{0}} \text { UNIT-U } \\
& \frac{\Gamma \mid \Psi \vdash\{P\} t: \mathcal{T}_{\Sigma, k}(\tau)\left\{\{\mathrm{Q}\}_{\delta} \quad \Gamma, x: \tau \mid \Psi \vdash\{\mathrm{Q}[\mathrm{x} / \mathbf{v}]\} u: \mathcal{T}_{\Sigma^{\prime}, \mathrm{k}^{\prime}}(\sigma)\left\{\left\{R_{\}}\right\}_{\delta^{\prime}} \quad x \notin \mathrm{R}\right.\right.}{\Gamma \mid \Psi \vdash\{\mathrm{P}\} \text { mlet } x=\mathrm{t} \text { in } u: \mathcal{T}_{\Sigma \cup \Sigma^{\prime} \cup \operatorname{Eff}(\tau), k+k^{\prime}}(\sigma)\left\{R_{\}}\right\}_{\delta+\delta^{\prime}}} \text { MLET-U } \\
& \frac{\Xi ; \Gamma \vdash \mathrm{a}: \text { Loc }}{\Gamma \mid \Psi \vdash\{\mathrm{P}[\mathbf{s}[\mathbf{a}] / \mathbf{v}]\} \text { read } \mathrm{a}: \mathcal{T}_{\{\mathbf{a}\}, 0}(\mathrm{Val})\left\{[\mathrm{P}\}_{0}\right.} \text { READ-U } \\
& \frac{\Xi ; \Gamma \vdash \mathrm{a}: \text { Loc } \quad \Xi ; \Gamma \vdash \mathrm{t}: \text { Val }}{\Gamma \mid \Psi \vdash\{\mathrm{P}[\mathbf{s}[\mathrm{a} \mapsto \mathrm{t}] / \mathbf{s}]\} \mathrm{a}:=\mathrm{t}: \mathcal{T}_{\{\mathrm{a}\}, 0}(\text { ut })\{\mathrm{P}\}_{\}_{0}}} \text { WRITE-U } \\
& \Gamma \vdash \mathrm{b} \text { : bool } \\
& \frac{\Gamma\left|\Psi \wedge \mathrm{b}=\mathbf{t t} \vdash\{\mathrm{P}\} \mathrm{t}_{1}: \mathcal{T}_{\Sigma, \mathrm{k}}(\tau)\{\mathrm{Q}\}_{\delta} \quad \Gamma\right| \Psi \wedge \mathrm{b}=\mathbf{f f} \vdash\{\mathrm{P}\} \mathrm{t}_{2}: \mathcal{T}_{\Sigma, \mathrm{k}}(\tau)\left\{\{\mathrm{Q}\}_{\delta}\right.}{\Gamma \mid \Psi \vdash\{\mathrm{P}\} \text { case } \mathrm{b} \text { of } \mathrm{tt} \mapsto \mathrm{t}_{1} ; \mathrm{ff} \mapsto \mathrm{t}_{2}: \mathcal{T}_{\Sigma, \mathrm{k}}(\tau)\left\{\{\mathrm{Q}\}_{\delta}\right.} \text { MCASE-U } \\
& \frac{|\{x \in \sigma \mid x \in \phi\}| /|\sigma|=\delta}{\Gamma \mid \Psi \vdash\{P\} \text { Unif }_{\sigma}: \mathcal{T}_{\emptyset, 0}(\sigma)\{\mathrm{P} \wedge \phi\}_{1-\delta}} \text { SAMPLE-UBL }
\end{aligned}
$$

Figure 6.3: Union bound: monadic rules

\subsection{Proving adversarial properties}

Security properties are often expressed in an adversarial setting. Instead of specifying how a program behaves with respect to its input, we specify how this program must behave when interacting with another unknown and arbitrary program. We now fix some terminology that we borrow from the field of security. Let $\mathcal{O}: \sigma \rightarrow \mathcal{T}(\tau)$ be a probabilistic program (called the oracle). An adversarial property of $\mathcal{O}$ is a property stating that for every $\mathcal{A}:(\sigma \rightarrow \mathcal{T}(\tau)) \rightarrow \mathcal{T}\left(\tau^{\prime}\right)$ (called an adversary), $\mathcal{A}(\mathcal{O})$ preserves $\phi$ (called the invariant). In HO-UBL, these properties can be expressed (omitting gradings) as:

$$
\left.\mathcal{A}:(\sigma \rightarrow \mathcal{T}(\tau)) \rightarrow \mathcal{T}\left(\tau^{\prime}\right) \vdash\{\phi\} \mathcal{A}(\mathcal{O}): \mathcal{T}\left(\tau^{\prime}\right)\{\phi\}\right\}
$$

Here $\mathcal{A}$ is a variable, but $\mathcal{O}$ stands for a concrete term with a known syntax, from which we assume:

$$
\vdash\{\phi\} \mathcal{O}: \sigma \rightarrow \mathcal{T}(\tau)\{\phi\}
$$

In general, it is not possible to prove (6.1) with any further assumption. If there is no memory separation between $\mathcal{O}$ and $\mathcal{A}$, then $\mathcal{A}$ could conceivably break the invariant. This memory separation can be enforced by restricting the syntax of $\mathcal{A}$ to not access any location that $\mathcal{O}$ accesses, but a more principled alternative is to restrict it by properly annotating the type of $\mathcal{A}$. Assume the adversary has type:

$$
\mathcal{A}: \forall \alpha .\left(\sigma \rightarrow \mathcal{T}_{\alpha, 1}(\tau)\right) \rightarrow \mathcal{T}_{\Sigma \cup \alpha, k}\left(\tau^{\prime}\right)
$$




$$
\begin{gathered}
\frac{\Gamma\left|\Psi \vdash \mathrm{P} \Rightarrow \mathrm{P}^{\prime} \quad \Gamma\right| \Psi \vdash\left\{\mathrm{P}^{\prime}\right\} \mathrm{t}: \mathcal{T}_{\Sigma, \mathrm{k}}(\tau)\left\{\left\{\mathrm{Q}^{\prime}\right\}_{\delta^{\prime}} \quad \Gamma \mid \Psi \vdash \mathrm{Q}^{\prime} \Rightarrow \mathrm{Q} \quad \delta^{\prime} \leqslant \delta\right.}{\Gamma \mid \Psi \vdash\{\mathrm{P}\} \mathrm{t}: \mathcal{T}_{\Sigma, \mathrm{k}}(\tau)\left\{\{\mathrm{Q}\}_{\delta}\right.} \text { CONSEQ-U } \\
\frac{\Gamma \mid \Psi \vdash\{\mathrm{P}\} \mathrm{t}: \mathcal{T}_{\Sigma, \mathrm{k}}(\tau)\left\{\mathrm{Q}_{\delta} \quad \Gamma \mid \Psi \vdash\left\{\mathrm{P}^{\prime}\right\} \mathrm{t}: \mathcal{T}_{\Sigma, \mathrm{k}}(\tau)\{\mathrm{Q}\}_{\delta}\right.}{\Gamma \mid \Psi \vdash\left\{\mathrm{P} \vee \mathrm{P}^{\prime}\right\} \mathrm{t}: \mathcal{T}_{\Sigma, \mathrm{k}}(\tau)\{\mathrm{Q}\}_{\delta}} \text { OR-PRE-U } \\
\frac{\Gamma\left|\Psi \vdash\{\mathrm{P}\} \mathrm{t}: \mathcal{T}_{\Sigma, \mathrm{k}}(\tau)\{\mathrm{Q}\}_{\delta} \quad \Gamma\right| \Psi \vdash\{\mathrm{P}\} \mathrm{t}: \mathcal{T}_{\Sigma, \mathrm{k}}(\tau)\left\{\left\{\mathrm{Q}^{\prime}\right\}_{\delta^{\prime}}\right.}{\Gamma \mid \Psi \vdash\{\mathrm{P}\} \mathrm{t}: \mathcal{T}_{\Sigma, \mathrm{k}}(\tau)\left\{\mathrm{Q} \wedge \mathrm{Q}^{\prime}\right\}_{\delta+\delta^{\prime}}} \text { AND-POST-U } \\
\frac{\Gamma \mid \Psi \vdash\{\mathrm{P}\} \mathrm{t}: \mathcal{T}_{\Sigma, \mathrm{k}}(\tau)\{\mathrm{Q}\}_{\delta^{\prime}}}{\Gamma \mid \Psi \vdash \mathrm{t}: \mathcal{T}_{\Sigma, \mathrm{k}}(\tau)\left\{\mathcal{H}_{\tau, \delta}(\mathrm{P}, \mathbf{r}, \mathrm{Q})\right\}} \operatorname{SWITCH}-\mathrm{U} \frac{\Gamma \mid \Psi \vdash \mathrm{t}: \mathcal{T}_{\Sigma, \mathrm{k}}(\tau)\left\{\mathcal{H}_{\tau, \delta}(\mathrm{P}, \mathbf{r}, \mathrm{Q})\right\}}{\Gamma \mid \Psi \vdash\{\mathrm{P}\} \mathrm{t}: \mathcal{T}_{\Sigma, \mathrm{k}}(\tau)\{\mathrm{Q}\}_{\delta^{\prime}}} \text { UNSWITCH-U }
\end{gathered}
$$

Figure 6.4: Union bound: structural rules

This states that the oracle has a private memory region that is abstracted by $\alpha$ and that the memory footprint of the whole computation is $\alpha \cup \Sigma$. Since the memory footprint of the adversary cannot depend on $\alpha$, then it must be that the memory region that the adversary accesses directly is restricted to $\Sigma$. In addition, the type states that the adversary can call the oracle up to $k$ times.

If we set an invariant $\phi$ that does not depend on $\Sigma$ then the adversary should not be able to break $\phi$. This is captured by the following notion of safety:

Definition 6.2. Let $\phi$ be a predicate on memories and $\Sigma \subseteq$ Loc. We say that $\phi \in \operatorname{Safe}(\Sigma)$ if and only if,

$$
\forall \mathrm{m} . \forall \mathrm{l} \in \Sigma . \forall v \in \operatorname{Val} . \phi(\mathrm{m}) \Rightarrow \phi(\mathrm{m}[\mathrm{l} \mapsto v])
$$

As an example, let $\phi \equiv \mathbf{s}[\mathrm{a}]=\mathbf{s}[\mathrm{b}]$ for some $\mathrm{a}, \mathrm{b} \in$ Loc. Then, for all $\Sigma$ such that $\mathrm{a}, \mathrm{b} \notin \Sigma$ we have that $\phi \in \operatorname{Safe}(\Sigma)$.

Below we present the adversary rule:

$$
\begin{gathered}
\cdot \vdash \mathcal{A}: \forall \alpha .\left(\sigma \rightarrow \mathcal{T}_{\alpha, 1}(\tau)\right) \rightarrow \mathcal{T}_{\Sigma \cup \alpha, k}\left(\tau^{\prime}\right) \quad \chi: \sigma \mid \Psi \vdash\{\phi\} \mathrm{t}: \mathcal{T}_{\Sigma^{\prime}, 1}(\tau)\{\phi\}_{\delta} \\
\mathbf{s}: M \vdash \phi \quad \phi \in \operatorname{Safe}(\Sigma) \quad \sigma, \tau, \tau^{\prime} \text { non-monadic types }
\end{gathered}
$$

What this rule states is that if

1. $\phi$ is an invariant safe for $\Sigma$,

2. $\mathcal{O}$ is an oracle that preserves $\phi$ at least with probability $1-\delta$, and

3. $\mathcal{A}$ is an arbitrary adversary that only writes to $\Sigma$ and that can call $\mathcal{O}$ up to $k$ times, 
then $\mathcal{A}(\mathcal{O})$ will preserve $\phi$ at least with probability $1-\delta$

For simplicity we present the rule in an empty context and assuming $\sigma, \tau$ and $\tau^{\prime}$ are non-monadic. These restrictions can be lifted by adding extra logical premises.

In the following section we will see how this rule is applied in practice.

\subsection{Example: Drifting games}

Drifting games [140] are a theoretical framework for reasoning about boosting and online learning algorithms. One goal of this framework is to measure how well algorithms perform w.r.t. a perfect scenario. A common way to achieve this goal rigorously is to define a loss function, which can be thought as some kind of quality metric, and to estimate the loss of the algorithm. For probabilistic programs, the loss of an algorithm is often defined as the expected value of the loss function on its output distribution. In this section, we use our logic from Section 6.2 to verify an upper bound for the expected loss of drifting games [140, Theorem 2].

A drifting game has two players: an adversary and a shepherd, that interact for $T$ rounds. The game is played on a n-dimensional continuous and infinite board, modelled as $\mathbb{R}^{n}$, using $m$ tokens. At each round the tokens are moved to new positions; we let $p_{i}^{t}$ denote the position of token $i$ at time t. $p_{i}^{0}=(0, \ldots, 0)$ for all $i$. For each round, the shepherd and the adversary play in turn, according to the following rules:

shepherd chooses a weight vector $w_{i}^{t} \in \mathbb{R}^{n}$ for every token;

adversary chooses a drift vector $z_{i}^{t}$ for every token; the drift vectors must be admissible w.r.t. the $w_{i}^{t}$, as defined below, else $z_{i}^{t}$ is set to a default admissible vector w.r.t. the $w_{i}^{t}$;

updates tokens are moved to their new positions $p_{i}^{t}=p_{i}^{t-1}+z_{i}^{t}$;

At the end of the game, one computes the loss w.r.t. a loss function $L: \mathbb{R}^{n} \rightarrow[0, \lambda]$. We assume the adversary to be probabilistic, therefore we define the expected loss as $\ell=\frac{1}{m} \sum_{i=1}^{m} E\left[L\left(p_{i}^{\top}\right)\right]$. Our goal is to define potential functions $\phi_{t}: \mathbb{R}^{\mathfrak{n}} \rightarrow \mathbb{R}^{+}$such that $\phi_{\mathrm{T}}=\mathrm{L}$ and for every admissible adversary choice $\left(z_{\mathfrak{i}}^{\mathrm{t}}\right)_{\mathrm{i}}$,

$$
\sum_{i=1}^{m} \phi_{t}\left(p_{i}^{t}+z_{i}^{t}\right) \leqslant \sum_{i=1}^{m} \phi_{t-1}\left(p_{i}^{t}\right)
$$

Assuming the existence of such potential functions, one can prove that $\ell \leqslant \phi_{0}(\overrightarrow{0})$ holds with probability 1 , where the randomness is taken from the adversary. First, note that $\phi_{t}=L$ and $\ell=\frac{1}{m} \sum_{i=1}^{m} E\left[L\left(p_{i}^{t}\right)\right]$, so by basic properties of expectation, it suffices to prove that $\frac{1}{m} \sum_{i=1}^{m} \phi_{t}\left(p_{i}^{t}\right) \leqslant \phi_{0}(\overrightarrow{0})$ holds with probability I for every 
$1 \leqslant t \leqslant T$. The proof is by induction on $t$. The base case follows immediately from the initialization of the game. For the induction step, we have:

$$
\begin{aligned}
\frac{1}{\mathfrak{m}} \sum_{i=1}^{\mathfrak{m}} \phi_{\mathrm{t}}\left(p_{i}^{\mathrm{t}}\right) & =\frac{1}{\mathfrak{m}} \sum_{i=1}^{\mathfrak{m}} \phi_{\mathrm{t}}\left(p_{i}^{\mathrm{t}-1}+z_{i}^{\mathrm{t}}\right) & & \text { by def. of } p_{i}^{\mathrm{t}} \\
& \leqslant \frac{1}{\mathfrak{m}} \sum_{i=1}^{\mathfrak{m}} \phi_{\mathrm{t}-1}\left(p_{i}^{\mathrm{t}}\right) & & \text { by prop. of potential function } \\
& \leqslant \phi_{0}(\overrightarrow{0}) & & \text { by I.H. }
\end{aligned}
$$

We are left to prove the existence of potential functions. For simplicity, we only consider the 2-norm, although the result extends to p-norms. We define $\phi_{\mathrm{T}}=\mathrm{L}$ and $\phi_{\mathrm{t}-1}(\mathrm{x})=\min _{w \in \mathbb{R}^{n}} \max _{z \in \mathrm{B}} \phi_{\mathrm{t}}(\mathrm{x}+z)+w \cdot z-\delta|w|_{2}$ where $\delta \in \mathbb{R}$ and $\mathrm{B} \subseteq \mathbb{R}^{\mathrm{n}}$ are two parameters of the game. Then we say that $\left(z_{i}^{\mathfrak{t}}\right)_{i}$ is admissible w.r.t. $\left(w_{i}^{\mathrm{t}}\right)_{i}$ iff $z_{i}^{\mathrm{t}} \in \mathrm{B}$ and $\sum_{i=1}^{\mathfrak{m}} w_{i}^{\mathrm{t}} \cdot z_{i}^{\mathrm{t}} \geqslant \delta \sum_{i=1}^{\mathfrak{m}}\left|w_{i}^{\mathrm{t}}\right|_{2}$. Under mild assumptions on $\delta$ and $\mathrm{B}$, one can show that for every round $t$ and token $i$ there exists a weight vector $\tilde{w}_{i}^{t}$ such that $\Psi\left(\tilde{w}_{i}^{t}, p_{i}^{t}, t\right)$ is valid, where: $\Psi(w, p, t) \triangleq \forall z \in$ B. $\phi_{t}(p+z) \leqslant \phi_{t-1}(p)-w \cdot z+\delta|w|_{2}$. Moreover only knowledge of $p_{i}^{t-1}$ is required to defined $\tilde{w}_{i}^{t}$. The vector $\tilde{w}_{i}^{t}$ has the nice property that if $\left(z_{i}^{t}\right)_{i}$ is admissible w.r.t. $\left(\tilde{w}_{i}^{t}\right)_{i}$, then

$$
\sum_{i=1}^{\mathfrak{m}} \phi_{t}\left(p_{i}^{t}+z_{i}^{t}\right) \leqslant \sum_{i=1}^{\mathfrak{m}} \phi_{t-1}\left(p_{i}^{t}\right)
$$

as required for our potential functions. The optimal shepherd is then the one that on round t picks $\tilde{w}_{i}^{t}$ satisfying the quantified inequality above.

We now turn to the formalization of this example in our logic. We model the drifting game using an adversary with oracle access to a shepherd oracle. For simplicity, we write the code for a single token. The shepherd has type $S: \mathbb{R}^{n} \rightarrow$ $\mathcal{T}_{\{t, p, q\}, 1}\left(\mathbb{R}^{\mathfrak{n}}\right)$ and uses memory locations $t, p$, and $w$ to store the round number, the position and the weight vector. The shepherd oracle receives as input the drift value $z$ of the adversary, and checks its admissibility before updating the position of the tokens. If the drift value is not admissible, the shepherd uses a default value to update the position of the token. Then it updates the round number, generates the next optimal weight value, which it returns to the adversary. The code is given below in a convenient syntax; for instance, we use some syntactic sugar, e.g. we write $t:=! t+1$ instead of mlet $x=$ read $t$ in $t:=x+1$ and we use semicolon instead of let:

$$
\begin{aligned}
& \lambda z . \text { (case } \operatorname{adm}(! w, z) \text { of } \\
& \quad \mathbf{t t} \mapsto p:=! p+z \cdot t w \\
& \quad \mathbf{f f} \mapsto p:=! p+z \cdot \operatorname{defz}(! w)) ; \\
& \mathrm{t}:=! \mathrm{t}+1 ; \\
& w:=\operatorname{optw}(! p) ; \\
& \text { unit }(! w)
\end{aligned}
$$

where $\operatorname{defz}(w)$ computes an admissible drift vector and optw(p) chooses an optimal weight. In the following, we let $\operatorname{defz}(w)=\overrightarrow{1}$, which we can easily check to be admissible. 
We establish the following judgment (we omit the typing of $\mathcal{A}$ and assumptions on $\mathrm{B}$ and $\delta$ to guarantee the existence of an optimal shepherd):

$$
\cdot \mid \cdot \vdash\left\{p=\overrightarrow{0} \wedge t=0 \wedge w=\tilde{w}_{0}\right\} \mathcal{A}[\{p, w, t\}] S: \mathcal{T}_{\{t, p, q\}, T}\left(\mathbb{R}^{\mathfrak{n}}\right)\left\{\left\{\phi_{t}(p) \leqslant \phi_{0}(\overrightarrow{0})\right\}_{0}\right.
$$

We use as invariant: $\Phi \triangleq \phi_{\mathrm{t}}(\mathrm{p}) \leqslant \phi_{0}(\overrightarrow{0}) \wedge \Psi(w, p, t)$, and we apply the CONSEQ rule to show that $p=\overrightarrow{0} \wedge t=0 \wedge w=\tilde{w}_{0} \Rightarrow \Phi$. This is trivial, since $\phi_{0}(\overrightarrow{0}) \leqslant \phi_{0}(\overrightarrow{0})$, and by assumption, $\Psi\left(\tilde{w}_{0}, \overrightarrow{0}, 0\right)$. By application of the ADV rule, it suffices to show:

$$
z: B \mid \cdot \vdash\{\Phi\} S(z): \mathcal{T}_{\{t, p, q\}, 1}\left(\mathbb{R}^{\mathfrak{n}}\right)\left\{\{\Phi\}_{0}\right.
$$

There are two cases, depending on whether $z$ is admissible. If it is, then we need to show (using CONSEQ again):

$\phi_{\mathrm{t}}(\mathrm{p}) \leqslant \phi_{0}(\overrightarrow{0}) \wedge \Psi(w, p, t) \Rightarrow \phi_{\mathrm{t}+1}(\mathrm{p}+z \cdot w) \leqslant \phi_{0}(\overrightarrow{0}) \wedge \Psi(\mathrm{optw}(\mathrm{p}+z \cdot w), \mathrm{p}+z \cdot w, \mathrm{t}+1)$

Otherwise we need to show:

$$
\phi_{\mathrm{t}}(\mathrm{p}) \leqslant \phi_{0}(\overrightarrow{0}) \wedge \Psi(w, p, t) \Rightarrow \phi_{\mathrm{t}+1}(\mathrm{p}+w) \leqslant \phi_{0}(\overrightarrow{0}) \wedge \Psi(\mathrm{optw}(\mathrm{p}+(w) \cdot w), \mathrm{p}+\cdot w, \mathrm{t}+1)
$$

In both cases, the second conjunct to the right of the implication is established from properties of optw, whereas the first conjunct follows from (6.2), assuming optimality of $w$.

As mentioned in Section 6.1, our type system does not enforce that an adversary makes exactly $\mathrm{T}$ calls, so we cannot apply the rule of consequence to establish $\ell \leqslant \phi_{0}(\overrightarrow{0})$ as a valid postcondition. This is a minor issue, since our goal with this example is to illustrate the use of our logic. However, for the sake of completeness, we also mention three possible workarounds:

1. the most elegant solution would be to work in a system that tracks the exact number of calls;

2. the most practical solution would be to model the interaction between the adversary and the shepherd using a loop. In this case, the adversary does not have access to the shepherd as a procedure, and we can complete the proof fully within the logic. However, this style of formalization is a poorer illustration of the use of the adversary rule;

3. one can complete the proof above completely within our system and without changing the formalization by considering potentials $\left(\phi_{i, j}\right)_{0 \leqslant i \leqslant j \leqslant T}$, such that $\phi_{i, i}=\mathrm{L}, \phi_{0}=\min \left(\phi_{0, j}\right)$, and $\phi_{i, j}$ is defined recursively from $\phi_{i+1, j}$ as previously. However, this is a tedious exercise that does not illustrate our logic, so we do not develop this workaround further. 


\subsection{Stateful liftings}

In this section we present an abstract setting in which we can give semantics to our logic. Let $\mathcal{P}$ be the monad of measures over QBS, and let $\mathcal{T} \triangleq \operatorname{St}(\mathcal{P}) \triangleq M \Rightarrow \mathcal{P}(-\times M)$ as in Section 6.1. We need to adapt the material from Section 4.6 to be able to model predicates over $\mathcal{T}$.

Recall from Section 4.7 the definition of the category Pred (QBS) of predicates over QBS. In this section, we will use for it the shorthand notation P.

The same approach of finding a lifting of the $\mathcal{T}$ monad does not work directly because it would not allow us to include the specification about states. Such a lifting would map a predicate over $X$ to a predicate over over $M \Rightarrow \mathcal{P}(X \times M)$, but this does not match the way we write specifications in HO-SUBL. We actually need to lift a pair of predicates over $M$ (the precondition) and over $X \times M$ (the postcondition) into a predicate over $M \Rightarrow \mathcal{P}(X \times M)$.

Therefore, we need to find a different construction. Assume there exists a graded lifting of $\mathcal{P}$ along $\mathrm{d}: \mathbf{P} \rightarrow \mathbf{Q B S}$, denoted $\left(\left\{\dot{\mathcal{P}}_{\mathrm{k}}\right\}, \dot{\eta}^{\mathcal{P}}, \dot{\mu}^{\dot{\mathcal{P}}, k, l}\right)$. We define a QBS-indexed family of functors $\dot{\mathrm{St}}(\dot{\mathcal{P}})_{\sigma, k}:\left(\mathbf{P}_{M}\right)^{\text {op }} \times \mathbf{P}_{\sigma \times M} \rightarrow \mathbf{P}_{\mathcal{T}(\sigma)}$ defined as:

$$
\dot{\mathrm{S} t}(\dot{\mathcal{P}})_{\sigma, k}(\mathrm{P}, \mathrm{Q}) \triangleq \mathrm{P} \Rightarrow \dot{\mathcal{P}}_{\mathrm{k}}(\mathrm{Q})=\left\{\mathrm{f} \in|\mathcal{T}(\sigma)| \mid f: \mathrm{P} \rightarrow \dot{\mathcal{P}}_{k}(\mathrm{Q}) \text { in } \mathbf{P}\right\}
$$

We call $\dot{S t}(\dot{\mathcal{P}})$ a stateful lifting. This can be seen as a transformer that takes a graded lifting $\dot{\mathcal{P}}_{k}$ of some monad $\mathcal{P}$ and returns a generalized lifting of the state transformed monad $\mathrm{St}(\mathcal{P})$. In plain words, $\dot{\mathrm{St}}(\dot{\mathcal{P}})_{\sigma, k}$ maps a precondition $\mathrm{P}$ over $\mathrm{M}$ and a postcondition $\mathrm{Q}$ over $\sigma \times M$ to the computations in $M \rightarrow \mathcal{P}(\sigma \times M)$ that send initial memories in $\mathrm{P}$ to distributions over $\sigma \times M$ satisfying the lifting $\dot{\mathcal{P}}_{k}(\mathrm{Q})$. In a way, this can be seen as the set of computations $f: \mathcal{T}(\sigma)$ satisfying the generalized Hoare triple $\{\mathrm{P}\} \mathrm{f}\{\mathrm{Q}\}$.

This operator is not a graded monad, because it is not an endofunctor. However, the compositionality properties of graded liftings can be extended to $\dot{\mathcal{T}}$ as stated in the following lemma:

Lemma 6.3. Let $\dot{\mathcal{P}}_{\mathrm{k}}$ be a graded lifting of $\mathcal{P}$. Then:

- For $\mathrm{X} \in \mathbf{Q B S}, \phi \in \mathbf{P}_{X^{\prime}}$ and $\mathrm{Q} \in \mathbf{P}_{\mathrm{M}^{\prime}} \eta^{\mathcal{T}}: \phi \rightarrow \dot{\mathrm{St}}(\dot{\mathcal{P}})_{\mathrm{X}, 0}(\mathrm{Q}, \phi \dot{\times} \mathrm{Q})$ in $\mathbf{P}$.

- For $\mathrm{X}, \mathrm{Y} \in \mathbf{Q B S}, \mathrm{P} \in \mathbf{P}_{\mathrm{M}^{\prime}}, \mathrm{Q} \in \mathbf{P}_{\mathrm{X} \times \mathrm{M}^{\prime}}, \mathrm{R} \in \mathbf{P}_{\mathrm{Y} \times \mathrm{M}^{\prime}}$ and $\mathrm{f}: \mathrm{X} \rightarrow \mathcal{T}(\mathrm{Y})$, if $\lambda^{-1}(\mathrm{f}): \mathrm{Q} \rightarrow$ $\dot{\mathcal{P}}_{\mathrm{k}}(\mathrm{R})$ in $\mathbf{P}$, then $\mathrm{f}^{\#}: \dot{\mathrm{St}}(\dot{\mathcal{P}})_{X, \mathrm{k}^{\prime}}(\mathrm{P}, \mathrm{Q}) \rightarrow \dot{\mathrm{S}} \mathrm{t}(\dot{\mathcal{P}})_{\mathrm{Y}, \mathrm{k}^{\prime}+\mathrm{k}}(\mathrm{P}, \mathrm{R})$ in $\mathbf{P}$ for all $\mathrm{k}, \mathrm{k}^{\prime}$.

- For $X, Y \in$ QBS, $\phi \in \mathbf{P}_{X^{\prime}}, \mathrm{P} \in \mathbf{P}_{M}$ and $\mathrm{Q} \in \mathbf{P}_{Y \times M^{\prime}} \theta^{\mathcal{T}}: \phi \dot{\times} \dot{\mathrm{St}}(\dot{\mathcal{P}})_{\mathrm{Y}, \mathrm{k}}(\mathrm{P}, \mathrm{Q}) \rightarrow$ $\dot{\mathrm{St}}(\dot{\mathcal{P}})_{X \times Y, k}(\mathrm{P}, \phi \dot{\times} \mathrm{Q})$ in $\mathbf{P}$ for all $\mathrm{k}$.

These conditions can be explained informally by using the language of Hoare logic: 
- The first condition states that if $x \in \phi$, then $\{Q\} \eta(x)\{\phi \wedge Q\}_{0}$ is a valid Hoare triple for any $\mathrm{Q}$.

- The second condition gives us a way to sequence computations as in Hoare logic. It states that if $f$ satisfies $\{Q(x,-)\} f(x)\{R\}_{j k}$ for every argument $x$, and $t$ satisfies $\{P\} t\{Q\}_{k^{\prime}}$, then $\{P\}$ mlet $x=t$ in $f\left\{[R\}_{k+k^{\prime}}\right.$ is a valid Hoare triple. Here $\mathrm{Q}(\mathrm{x},-)$ is the set of memories $\mathrm{m}$ such that $(x, \mathrm{~m}) \in \mathrm{Q}$.

- The third condition states that if $x \in \phi$ and $t$ is such that $\{P\} t\{Q\}_{k}$ then $\{P\} \theta(x, t)\{\phi \wedge Q\}_{k}$ is a valid Hoare triple.

This is also related to the notion of parametrized monad introduced by Atkey [12], which are functors of type $\mathbf{D}^{o p} \times \mathbf{C} \times \mathbf{D} \rightarrow \mathbf{C}$ that generalize monads and satisfy similar coherence conditions.

\subsection{Semantics}

\subsubsection{Semantics of HO-SUBL}

We interpret HO-SUBL in the fibration $d: \operatorname{Pred}($ QBS $) \rightarrow$ QBS with the lifting $\dot{P}^{\text {UB }}$, and construct $\dot{\mathcal{S}}\left(\dot{\mathcal{P}}^{\mathrm{UB}}\right)$ from $\dot{\mathcal{P}}^{\mathrm{UB}}$ as in Section 6.5. The soundness result is stated as:

Proposition 6.4. Let $\Gamma \mid \Psi \vdash\{\mathrm{P}\} \mathrm{t}: \mathcal{T} \tau\{\mathbb{Q}\}_{\delta}$ be a derivable HO-UBL judgment without the adversary rule. If $\gamma \in \mathbf{Q B S}(1, \llbracket \Gamma \rrbracket)$ such that $\gamma: T \rightarrow \llbracket \Gamma \vdash \Lambda \Psi \rrbracket$, then $\llbracket \Gamma \vdash \mathrm{t}: \mathcal{T} \tau \rrbracket \circ \gamma$ is in

$\operatorname{Pred}(\mathbf{Q B S})\left(T, \dot{\mathrm{St}}\left(\dot{\mathcal{P}}^{\mathrm{UB}}\right)_{\tau, \delta}\left(\left\langle\gamma, \mathrm{id}_{\llbracket M \rrbracket}\right\rangle^{*} \llbracket \Gamma, \mathbf{s}: \mathbf{M} \vdash \mathrm{P} \rrbracket,\left\langle\gamma, \mathrm{id}_{\llbracket M \rrbracket \times} \times \llbracket \tau\right\rangle^{*} \llbracket \Gamma, \mathbf{s}: \mathbf{M}, \mathbf{v}: \tau \vdash \mathrm{Q} \rrbracket\right)\right.$.

Here recall again that $\left\langle\gamma, \mathrm{id}_{\llbracket M \rrbracket}\right\rangle^{*}$ is the reindexing that computes the substitution in P of every variable in $\Gamma$ by the value assigned to it by $\gamma$. Therefore, we can state this in set-theoretical terms, by saying that for every $\gamma \in \llbracket \Gamma \rrbracket$, if $\gamma$ satisfies $\llbracket \Gamma \vdash \wedge \Psi \rrbracket$, then $\llbracket \Gamma \vdash t: \mathcal{T} \tau \rrbracket(\gamma)$ is a map from $M$ to $\mathcal{P}(\tau \times M)$ sending memories in $\llbracket \Gamma, \mathbf{s}: M \vdash \mathrm{P} \rrbracket(\gamma)$ to distributions over memories in $\dot{\mathcal{P}}_{\delta}(\llbracket \Gamma, \mathbf{s}: M \vdash \mathrm{Q} \rrbracket(\gamma))$.

Proof. To simplify the proof, we will use set theory notation as explained above. We also use the shorthand $\dot{\mathcal{T}}_{\sigma, \delta} \triangleq \dot{S}_{\mathbf{t}}(\dot{\mathcal{P}} U B)_{\sigma, \delta}$. We only show the most interesting cases:

- Unit. By soundness of UHOL, we have that

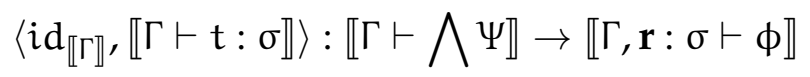

so for all $\gamma \in \llbracket \Gamma \vdash \Lambda \Psi \rrbracket, \llbracket \Gamma \vdash t: \sigma \rrbracket(\gamma) \in \llbracket \Gamma, \mathbf{r}: \sigma \vdash \phi \rrbracket(\gamma)$. Consider an arbitrary $\Gamma, \mathbf{s}: M \vdash$ P. By definition, $\llbracket \Gamma \vdash$ unit $(\mathrm{t}): \mathcal{T} \sigma \rrbracket(\gamma)=\eta^{\mathcal{T}} \circ \llbracket \Gamma \vdash \mathrm{t}: \sigma \rrbracket(\gamma)$. By Lemma 6.3 we can conclude that

$$
\eta^{\mathcal{T}}(\llbracket \Gamma \vdash t: \sigma \rrbracket(\gamma)) \in \dot{\mathcal{T}}_{\sigma, 0}(\llbracket \Gamma, \mathbf{s}: M \vdash \mathrm{P} \rrbracket(\gamma), \llbracket \Gamma, \mathbf{r}: \sigma \vdash \phi \rrbracket(\gamma) \dot{x} \llbracket \Gamma, \mathbf{s}: M \vdash \mathrm{P} \rrbracket(\gamma))
$$


- Bind. By applying I.H. to the first premise we have that, for all $\gamma \in \llbracket \Gamma \vdash \Psi \rrbracket$

$$
\llbracket \Gamma \vdash \mathrm{t}: \mathcal{T} \tau \rrbracket(\gamma) \in \dot{\mathcal{T}}_{\tau, \delta}(\llbracket \Gamma, \mathbf{s}: M \vdash \mathrm{P} \rrbracket(\gamma), \llbracket \Gamma, \mathbf{s}: M, \mathbf{v}: \tau \vdash \mathrm{Q} \rrbracket(\gamma))
$$

and for all $e: \tau,(\gamma, e) \in \llbracket \Gamma, x: \tau \vdash \Psi \rrbracket$, so by applying I.H to the second premise, $\llbracket \Gamma, x: \tau \vdash u: \mathcal{T} \sigma \rrbracket(\gamma, e)$ is in

$$
\dot{\mathcal{T}}_{\mathcal{\delta}^{\prime}}(\llbracket \Gamma, x: \tau, \mathbf{s}: M \vdash \mathrm{Q} \rrbracket(\gamma, e), \llbracket \Gamma, x: \tau, \mathbf{s}: M, \mathbf{v}: \sigma \vdash R \rrbracket(\gamma, e))
$$

Since $x \notin F V(R)$, then also $\llbracket \Gamma, x: \tau \vdash u: \mathcal{T} \sigma \rrbracket(\gamma, e)$ is in

$$
\dot{\mathcal{T}}_{\delta^{\prime}}(\llbracket \Gamma, x: \tau, \mathrm{t}: M \vdash \mathrm{Q} \rrbracket(\gamma, e), \llbracket \Gamma, \mathbf{s}: M, \mathbf{v}: \sigma \vdash R \rrbracket(\gamma))
$$

Note that $\lambda^{-1}(\llbracket \Gamma, x: \tau \vdash u: \mathcal{T} \sigma \rrbracket(\gamma))$ is a morphism $\tau \times M \rightarrow \mathcal{P}(\sigma \times M)$ and that we can derive

$$
\begin{gathered}
\lambda^{-1}(\llbracket \Gamma, x: \tau \vdash u: \mathcal{T} \sigma \rrbracket(\gamma)): \\
\llbracket \Gamma, x: \tau, \mathbf{s}: M \vdash \mathrm{Q} \rrbracket(\gamma) \rightarrow \dot{\mathcal{P}}_{\mathcal{\prime}^{\prime}}(\llbracket \Gamma, \mathbf{s}: M, \mathbf{v}: \sigma \vdash R \rrbracket(\gamma))
\end{gathered}
$$

By Lemma 6.3,

$$
\begin{gathered}
(\llbracket \Gamma, x: \tau \vdash u: \mathcal{T} \sigma \rrbracket(\gamma))^{\#}: \dot{\mathcal{T}}_{\mathcal{\delta}}(\llbracket \Gamma, \mathbf{s}: M \vdash P \rrbracket(\gamma), \llbracket \Gamma, \mathbf{s}: M, \mathbf{v}: \tau \vdash \mathrm{Q} \rrbracket(\gamma)) \rightarrow \\
\dot{\mathcal{T}}_{\delta+\delta^{\prime}}(\llbracket \Gamma, \mathbf{s}: M \vdash P \rrbracket(\gamma), \llbracket \Gamma, \mathbf{s}: M, \mathbf{v}: \sigma \vdash R(\gamma) \rrbracket)
\end{gathered}
$$

The Bind operator is defined as:

$$
\llbracket \Gamma \vdash \text { mlet } x=\mathrm{t} \text { in } u: \mathcal{T} \sigma \rrbracket \triangleq \llbracket \Gamma, x: \sigma \vdash u: \mathcal{T} \sigma \rrbracket^{\#} \circ \theta \circ\left\langle\mathrm{id}_{\Gamma}, \llbracket \Gamma \vdash \mathrm{t}: \mathcal{T} \tau \rrbracket\right\rangle
$$

so we conclude.

\subsubsection{Soundness of the adversary rules}

To prove soundness of the adversary rules we will use logical relations. Logical predicates and relations [128] are a technique used in programming language theory to prove properties such as strong normalization or contextual equivalence. The idea of logical relations (or predicates) is that they allow us to prove that all inhabitants of a certain type satisfy a particular property that is defined inductively on the structure of types, rather than terms.

We now define an indexed logical predicate $\mathcal{L}_{\phi, \delta}$ mapping types to predicates (more concretely, it maps a type $\tau$ to a set of closed terms of type $\tau$ ). The logical predicate is indexed by an invariant $\phi$, which is a predicate over memories and a 
real $\delta \in[0,1]$ and it is defined as follows:

$$
\begin{aligned}
\mathcal{L}_{\phi, \delta}(\mathrm{B}) & \triangleq\{\mathrm{b}: \mathrm{B}\} \\
\mathcal{L}_{\phi, \delta}(\sigma \rightarrow \tau) & \triangleq\left\{\begin{array}{l}
\left\{\mathrm{t}: \sigma \rightarrow \tau \mid \forall x: \sigma . x \in \mathcal{L}_{\phi, \delta}(\sigma) \Rightarrow(\mathrm{t} x) \in \mathcal{L}_{\phi, \delta}(\tau)\right\} \quad \text { if } \operatorname{Eff}(\sigma) \subseteq \operatorname{Eff}(\tau) \\
\left\{\mathrm{t}: \sigma \rightarrow \tau \mid \forall x: \sigma .(\mathrm{t} x) \in \mathcal{L}_{\phi, \delta}(\tau)\right\} \quad \text { otherwise }
\end{array}\right. \\
\mathcal{L}_{\phi, \delta}(\sigma \times \tau) & \triangleq\left\{\mathrm{t}: \sigma \times \tau \mid \pi_{1}(\mathrm{t}) \in \mathcal{L}_{\phi, \delta}(\sigma) \wedge \pi_{2}(\mathrm{t}) \in \mathcal{L}_{\phi, \delta}(\tau)\right\} \\
\mathcal{L}_{\phi, \delta}\left(\mathcal{T}_{\Sigma, k}(\sigma)\right) & \triangleq\left\{\mathrm{t}: \mathcal{T}_{\Sigma, \mathrm{k}}(\sigma) \mid \mathcal{H}_{\sigma, k \delta}\left(\phi, \mathrm{t}, \mathbf{v} \in \mathcal{L}_{\phi, \delta}(\sigma) \wedge \phi\right)\right\} \\
\mathcal{L}_{\phi, \delta}(\forall \alpha . \tau) & \triangleq\left\{\mathrm{t}: \forall \alpha . \tau \mid \forall \Sigma . \mathrm{t}[\Sigma] \in \mathcal{L}_{\phi, \delta}(\tau[\Sigma / \alpha])\right\}
\end{aligned}
$$

The definition of the logical predicate involves two subtleties. First note that there are two different definitions of the logical predicate for arrow types, depending on whether the effect of the argument is contained in the effect of the result. The idea is that if it is not, then the argument is ignored, so we do not need to require that it satisfies the logical predicate. Otherwise, we get the usual definition: a function satisfies the logical predicate for $\sigma \rightarrow \tau$ if arguments that satisfy the predicate for $\sigma$ get mapped to results satisfying the predicate the predicate for $\tau$. The second subtlety is that the definition ignores the first grading of the monad. A different definition, without indexing the predicate by $\phi$ and defining instead:

$$
\mathcal{L}_{\delta}\left(\mathcal{T}_{\Sigma, k}(\sigma)\right) \triangleq\left\{\mathrm{t}: \mathcal{T}_{\Sigma, k}(\sigma) \mid \forall \phi \in \operatorname{Safe}(\Sigma) . \mathcal{H}_{\sigma, k \delta}\left(\phi, \mathrm{t}, \mathbf{v} \in \mathcal{L}_{\phi, \delta}(\sigma) \wedge \phi\right)\right\}
$$

would impose overly strong conditions on monadic types that appear in argument position. Namely, it would force us to prove that they preserve all the invariants that are safe for a given region $\Sigma$, but we only know that the oracle preserves a particular invariant $\phi$. Note however that the grading $k$ is used to scale the grading of the lifting. The grading $\Sigma$ of the monad is used in the premise of the Basic Lemma, which we now state, using the notion of safety that we defined in Section 6.3.

Lemma 6.5 (Basic lemma). Let $\dot{\mathcal{P}}$ be as above, and assume that for every $v \in \Delta$, sample $\left(v_{\sigma}\right) \in \dot{\mathcal{P}}_{0}\left(\mathcal{L}_{\phi, \delta}(\sigma)\right)$. Let $\vdash \mathrm{t}: \sigma$ be a closed term, and $\mathbf{s}: M \vdash \phi$ such that $\phi \in$ $\operatorname{Safe}(\operatorname{Eff}(\sigma))$. Then, for all $\delta \geqslant 0, \mathrm{t} \in \mathcal{L}_{\phi, \delta}(\sigma)$.

Proof. We actually prove a generalization of the Basic Lemma, which makes the cases of abstraction and application easier to handle.

Lemma 6.6. Let $\Xi ; \Gamma, \Gamma^{\prime} \vdash t: \sigma$ be a well-typed term and $\phi$ a predicate over memories such that $\phi \in \operatorname{Safe}(\operatorname{Eff}(\sigma) \backslash \Xi)$. Assume further that for every $(\mathrm{x}: \tau) \in \Gamma^{\prime}, \operatorname{Eff}(\tau) \nsubseteq \mathbb{E f f}(\sigma)$ and that $\Xi \vdash \xi$ is an instantiation of the context $\Xi$. Let also $\Gamma \xi \vdash \gamma, \Gamma^{\prime} \xi \vdash \gamma^{\prime}$ be instantiations of the typing contexts. If $\gamma$ is such that $\gamma\left(x_{i}\right) \in \mathcal{P}_{\phi, \delta}\left(\sigma_{i}\right)$ for every $\left(x_{i}: \sigma_{i}\right) \in \Gamma$, then $\mathrm{t} \gamma \gamma^{\prime} \in \mathcal{P}_{\phi, \delta}(\sigma \xi)$.

This can be proven by induction on the typing derivation. We show a few cases, omitting the $\Xi$ context when irrelevant. 
- Variable. We have $\Gamma, \Gamma^{\prime} \vdash x: \sigma$, and by assumption, $x$ cannot be in $\Gamma^{\prime}$. Therefore, also by assumption, $\llbracket x \gamma \rrbracket \in \mathcal{P}_{\phi, \delta}(\sigma)$.

- Abstraction. Assume $\phi \in \operatorname{Safe}(\operatorname{Eff}(\sigma \rightarrow \tau))$. If $\operatorname{Eff}(\sigma) \subseteq \operatorname{Eff}(\tau)$, then also $\phi \in \operatorname{Safe}(\operatorname{Eff}(\sigma))$. We apply I.H., and we have that

$$
\mathrm{t} \gamma[\mathrm{x} \mapsto \mathrm{u}] \gamma^{\prime} \in \mathcal{P}_{\phi, \delta}(\tau)
$$

for all $u \in \mathcal{P}_{\phi, \delta}(\sigma)$. Therefore, $(\lambda x . t) \gamma \gamma^{\prime} \in \mathcal{P}_{\phi, \delta}(\sigma, \tau)$.

If $\operatorname{Eff}(\sigma) \not \subset \operatorname{Eff}(\tau)$, then by I.H., for all $u: \sigma, t \gamma\left(\gamma^{\prime}[x \mapsto u]\right) \in \mathcal{P}_{\phi, \delta}(\tau)$. Therefore,

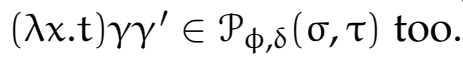

- Application. By I.H., $t \gamma \gamma^{\prime} \in \mathcal{L}_{\phi, \delta}(\sigma \rightarrow \tau)$. If $\operatorname{Eff}(\sigma) \subseteq \operatorname{Eff}(\tau)$, then by I.H. we also have $u \gamma \gamma^{\prime} \in \mathcal{L}_{\phi, \delta}(\sigma)$, and therefore by definition $(\mathrm{t} u) \gamma \gamma^{\prime} \in \mathcal{L}_{\phi, \delta}(\sigma)$. Otherwise, by definition of the logical predicate we have again $(\mathrm{t} u) \gamma \gamma^{\prime} \in \mathcal{L}_{\phi, \delta}(\sigma)$.

- Unit. WLOG we can assume that $\Gamma^{\prime}=\emptyset$. We have that $\phi \in \operatorname{safe}\left(\operatorname{Eff}\left(\mathcal{T}_{\Sigma}(\sigma)\right)\right)$, so also $\phi \in \operatorname{safe}(\operatorname{Eff}(\sigma)))$. By I.H., $\mathrm{t} \gamma \in \mathcal{L}_{\phi, \delta}(\sigma)$. By the properties of $\dot{\mathcal{P}}$ :

$$
(\text { unit }(\mathrm{t})) \gamma \in \mathcal{H}_{\sigma, 0}\left(\phi,-, \mathbf{v} \in \mathcal{L}_{\phi, \delta}(\sigma) \wedge \phi\right)
$$

- Bind. WLOG we can assume that $\Gamma^{\prime}=\emptyset$. Our premises are $\Gamma \vdash t: \mathcal{T}_{\Sigma}(\tau)$ and $\Gamma, x: \tau \vdash u: \mathcal{T}_{\Sigma^{\prime}}(\sigma)$, and by assumption, $\Phi \in \operatorname{Safe}\left(\Sigma \cup \Sigma^{\prime} \cup \operatorname{Eff}(\tau) \cup \operatorname{Eff}(\sigma)\right)$. Therefore, $\Phi \in \operatorname{Safe}(\Sigma \cup \operatorname{Eff}(\tau))$ and $\Phi \in \operatorname{Safe}\left(\Sigma^{\prime} \cup \operatorname{Eff}(\sigma)\right)$. So we can apply I.H. to both premises. For the first one, we have that

$$
\mathrm{t} \gamma \in \mathcal{H}_{\sigma, k \delta}\left(\phi,-, \mathbf{v} \in \mathcal{L}_{\phi, \delta}(\sigma) \wedge \phi\right)
$$

and for the second one, we have that for all $e \in \mathcal{L}_{\phi, \delta}(\sigma)$,

$$
\mathrm{u} \gamma[\mathrm{x} \mapsto e] \in \mathcal{H}_{\tau, \mathrm{k}^{\prime} \delta}\left(\phi,-, \mathbf{v} \in \mathcal{L}_{\phi, \delta}(\tau) \wedge \phi\right)
$$

By properties of the lifting, we get

$$
(\text { mlet } x=\mathrm{t} \text { in } u) \gamma \in \mathcal{H}_{\tau,\left(k+k^{\prime}\right) \delta}\left(\phi,-, \mathbf{v} \in \mathcal{L}_{\phi, \delta}(\tau) \wedge \phi\right)
$$

- Forall introduction. By assumption, $\Xi, \alpha ; \Gamma \vdash t: \tau$, and $\phi \in \operatorname{Safe}(\operatorname{Eff}(\forall \alpha . \tau) \backslash \Xi)$, so $\phi \in \operatorname{Safe}(\operatorname{Eff}(\tau) \backslash(\Xi \cup \alpha))$. For all $\Xi \vdash \xi$ and $\Sigma$, by I.H., $t \gamma \in \mathcal{L}_{\phi, \delta}(\tau \xi[\alpha \mapsto \Sigma])$, and therefore $(\wedge \alpha . t) \gamma \in \mathcal{L}_{\phi, \delta}(\forall \alpha . \tau)$.

- Forall elimination. By assumption, $\Xi ; \Gamma \vdash \mathrm{t}: \forall \alpha . \tau$ and $\phi \in \operatorname{Safe}(\operatorname{Eff}(\tau[\Sigma / \alpha]) \backslash \Xi)$. Recall that $\operatorname{Eff}(\forall \alpha \cdot \tau)=\operatorname{Eff}(\tau[\emptyset / \alpha])$. Since $\operatorname{Eff}(\tau[\emptyset / \alpha]) \subseteq \operatorname{Eff}(\tau[\Sigma / \alpha]) \backslash \Xi$, then also $\phi \in \operatorname{Safe}(\operatorname{Eff}(\forall \alpha . \tau) \backslash \Xi)$ (in other words, if the effect is smaller, then the predicate is still safe). Therefore, we can apply I.H., and we get that for $\Xi \vdash \xi$ $\mathrm{t} \gamma \in \mathcal{P}_{\phi, \delta}(\forall \alpha . \tau \xi)$. From this, we can conclude that $\mathrm{t}[\Sigma] \gamma \in \mathcal{P}_{\phi, \delta}(\tau[\Sigma / \alpha] \xi)$. 
And from this, we can conclude:

Corollary 6.7. The ADV-U rule is sound.

Proof. By the premise on $\mathcal{A}$, safety of $\phi$ and the Basic Lemma, we can prove that

$$
\mathcal{A} \in \mathcal{L}_{\phi, \delta}\left(\forall \alpha .\left(\sigma \rightarrow \mathcal{T}_{\alpha, 1}(\tau)\right) \rightarrow \mathcal{T}_{\Sigma \cup \alpha, k}\left(\tau^{\prime}\right)\right)
$$

On the other hand, by the assumption on the oracle we have that:

$$
\lambda x . t \in \mathcal{L}_{\phi, \delta}\left(\sigma \rightarrow \mathcal{T}_{\Sigma^{\prime}, 1}(\tau)\right)
$$

From this, we can derive:

$$
\mathcal{A}\left[\Sigma^{\prime}\right](\lambda x . \mathrm{t}) \in \mathcal{L}_{\phi, \delta}\left(\mathcal{T}_{\Sigma \cup \Sigma^{\prime}, \mathrm{k}}\left(\tau^{\prime}\right)\right)
$$

and the conclusion follows directly. 


\section{RELATIONAL HIGHER-ORDER LOGIC FOR ADVERSARIAL COMPUTATIONS}

As the next and final step of our progression, we present a logic to reason about relational adversarial properties of stateful programs from the language in Section 6.1. This builds on RHOL and aims to prove properties of the form

$$
\Gamma \mid \Psi \vdash\{\psi\} \mathrm{t}_{1} \sim \mathrm{t}_{2}\{\phi\}_{(\epsilon, \delta)}
$$

where $\psi$ is a relational precondition on the initial states and $\phi$ is a relational postcondition on the final states and return values. As in Chapter 5 , the postcondition will be interpreted using $(\epsilon, \delta)$-approximate couplings.

For the particular case of adversarial properties, we will look into proving judgments of the form

$$
\left.\{\phi\} \mathcal{A}\left(\mathcal{O}_{1}\right) \sim \mathcal{A}\left(\mathcal{O}_{2}\right)\left\{\phi \wedge \mathbf{v}_{1}=\mathbf{v}_{2}\right\}\right\}_{(\epsilon, \delta)}
$$

stating that an adversary $\mathcal{A}$ preserves a relational invariant $\phi$ and is $(\epsilon, \delta)$-differentially private for oracles $\mathcal{O}_{1}$ and $\mathcal{O}_{2}$.

We call this logic Higher-Order Stateful Relational Probabilistic Logic (HO-SRPL), which in a sense generalizes both the HO-RPL from Chapter 5 and the HO-SUBL form Chapter 7 .

Organization of the chapter. We start by presenting the logic HO-SUBL in Section 7.1. After this, we motivate and present the relational adversary rule (Section 7.2) and we verify the PRF-PRP examples (Section 7.3). Then we present the semantical setting (Section 7.4) and the semantics of the logic and the adversary rule (Section 7.5). 


\subsection{Higher-order stateful probabilistic relational logic}

Higher-order stateful probabilistic relational logic (HO-SPRL) is a stateful version of the HO-RPL from Chapter 5. As it is the case of HO-RPL, we build it on top of RHOL and we extend it with monadic judgments of the form:

$$
\Gamma \mid \Psi \vdash\{P\} t_{1}: \mathcal{T} \sigma_{1} \sim t_{2}: \mathcal{T} \sigma_{2}\{Q Q\}_{(\epsilon, \delta)}
$$

where $P$ is well-formed in the context $\Gamma, \mathbf{s}_{1}: M, \mathbf{s}_{2}: M$, and $Q$ is well-formed in the context $\Gamma, \mathbf{s}_{1}: M, \mathbf{s}_{2}: M, \mathbf{v}_{1}: \sigma_{1}, \mathbf{v}_{2}: \sigma_{2}$. Here, the variables $\mathbf{s}_{1}, \mathbf{s}_{2}$ are refer to (resp. left or right-side) memories, and $\mathbf{v}_{1}, \mathbf{v}_{2}$ refer to (resp. left or right-side) result values.

The intended interpretation of such a judgment is that for every $\gamma \in \Gamma$ such that $\gamma$ satisfies $\Lambda \Psi$, and for every pair $m_{1}, m_{2} \in M$, such that $\left(m_{1}, m_{2}\right) \in P, t_{1}\left(m_{1}\right)$ and $t_{2}\left(m_{2}\right)$ are distributions over $\sigma_{1} \times M$ and $\sigma_{2} \times M$ respectively for which there exists an $(\epsilon, \delta)$-approximate $\mathrm{Q}$-coupling. Recall that by Lemma 5.3 if $\mathrm{Q}$ is of the form $\mathbf{s}_{1}=\mathbf{s}_{2} \wedge \mathbf{v}_{1}=\mathbf{v}_{2}$ then we can say that $t_{1}$ and $t_{2}$ are $(\epsilon, \delta)$-differentially private.

Figure 7.1 presents selected rules for the relational logic. Following our pattern for relational logics, we have 2-sided rules, where the two expressions have the same top-level structure, and one-sided rules, which exclusively consider the top-level construct of one expression:

- The UNIT, MLET, READ, and WRITE rules follow the same intuition as their unary versions of HO-SUBL

- The MCASE rule requires synchronization between the guards, either they are both false, or both true. We also include a one-sided MCASE-L rule for asynchronous reasoning.

- Some one-sided rules, such as MLET-L assume as a premise a relational judgment in which one of the sides is an empty (skip) instruction. These can eventually be discharged by the UNIT-L rule or by a one-sided sampling rule.

Our logic also features rules for reasoning about sampling. We show some rules in Figure 7.2. The rules SAMPLE-ASYN and SAMPLE-ASYN-APP are generalizations of their stateless counterparts.

Finally, we add a predicate former $\mathcal{H}_{\sigma,(\epsilon, \delta)}\left(\mathrm{P}, \mathrm{t}_{1}, \mathrm{t}_{2}, \mathrm{Q}\right)$ to internalize the monadic judgments. This is well formed when $\mathbf{s}_{1}: M, \mathbf{s}_{2}: M \vdash P, \vdash t_{1}: \mathcal{T}_{\sigma}, \vdash t_{2}: \mathcal{T}_{\sigma}$ and $\mathbf{v}_{1}: \sigma, \mathbf{s}_{1}: M, \mathbf{v}_{2}: \sigma, \mathbf{s}_{2}: M \vdash Q$. We show rules to switch between the systems in Figure $7 \cdot 3$.

\subsection{Relational adversarial properties}

We have seen in the previous chapter the notion of adversarial property, in which we prove for an oracle $\mathcal{O}$ that any adversary $\mathcal{A}$ interacting with $\mathcal{O}$ preserves some invariant $\phi$ (up to some probability) 


$$
\begin{aligned}
& \frac{\Gamma \mid \Psi \vdash \mathrm{t}_{1}: \tau_{1} \sim \mathrm{t}_{2}: \tau_{2}\{\phi\} \quad \Gamma, \mathbf{s}_{1}: M, \mathbf{s}_{2}: M \vdash \mathrm{P}}{\Gamma \mid \Psi \vdash\{\mathrm{P}\} \text { unit }\left(\mathrm{t}_{1}\right): \mathcal{T}_{\emptyset, 0}\left(\tau_{1}\right) \sim \operatorname{unit}\left(\mathrm{t}_{2}\right): \mathcal{T}_{\emptyset, 0}\left(\tau_{2}\right)\{\mathrm{P} \wedge \phi\}_{(0,0)}} \text { UNIT } \\
& \Gamma \mid \Psi \vdash\{P\} t_{1}: \mathcal{T}_{\Sigma_{1}, k_{1}}\left(\tau_{1}\right) \sim t_{2}: \mathcal{T}_{\Sigma_{2}, k_{2}}\left(\tau_{2}\right)\{Q \mathrm{Q}\}_{(\epsilon, \delta)} \\
& \Gamma, x_{1}: \tau_{1}, x_{2}: \tau_{2} \mid \Psi \vdash\left\{Q\left[x_{1} / v_{1}\right]\left[x_{2} / v_{2}\right]\right\} u_{1}: \mathcal{T}_{\Sigma_{1}^{\prime}, k_{1}^{\prime}}\left(\sigma_{1}\right) \sim u_{2}: \mathcal{T}_{\Sigma_{2}^{\prime}, k_{2}^{\prime}}\left(\sigma_{2}\right)\{R\}_{\left(\epsilon^{\prime}, \delta^{\prime}\right)} \\
& x_{1}, x_{2} \notin \mathrm{FV}(\mathrm{R}) \quad \mathrm{x}_{1} \notin \mathrm{FV}\left(\mathrm{u}_{2}\right) \quad \mathrm{x}_{2} \notin \mathrm{FV}\left(\mathrm{u}_{1}\right) \\
& \Gamma \mid \Psi \vdash\{P\} \begin{array}{c}
\text { mlet } x_{1}=t_{1} \text { in } u_{1}: \mathcal{T}_{\Sigma_{1} \cup \Sigma_{1}^{\prime} \cup \operatorname{Eff}\left(\tau_{1}\right), k_{1}+k_{1}^{\prime}}\left(\sigma_{1}\right) \sim \\
\text { mlet } x_{2}=t_{2} \text { in } u_{2}: \mathcal{T}_{\Sigma_{2} \cup \Sigma_{2}^{\prime} \operatorname{Eff}\left(\tau_{2}\right), k_{2}+k_{2}^{\prime}}\left(\sigma_{2}\right)
\end{array}\{R\}_{\left(\epsilon+\epsilon^{\prime}, \delta+\delta^{\prime}\right)}
\end{aligned}
$$

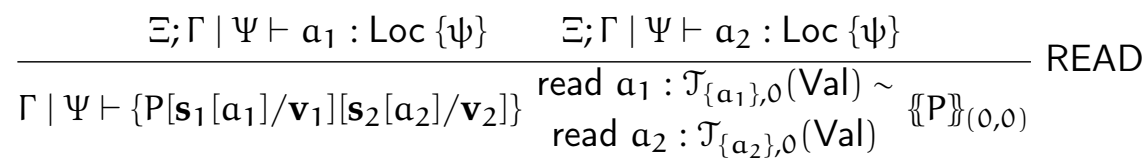

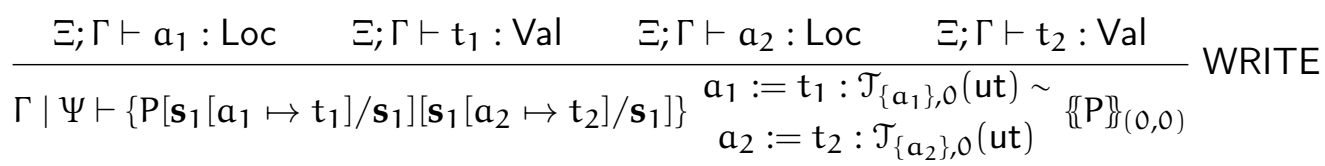

$$
\begin{aligned}
& \Gamma \mid \Psi \vdash \mathrm{b}_{1}: \text { bool } \sim \mathrm{b}_{2}: \text { bool }\left\{\mathbf{r}_{1} \Leftrightarrow \mathbf{r}_{2}\right\} \\
& \Gamma \mid \Psi \wedge b_{1}=\mathbf{t t} \vdash\{P\} t_{1}: \mathcal{T}_{\Sigma_{1}, k_{1}}\left(\tau_{1}\right) \sim t_{2}: \mathcal{T}_{\Sigma_{2,}, k_{2}}\left(\tau_{2}\right)\left\{Q Q_{(\epsilon, \delta)}\right.
\end{aligned}
$$

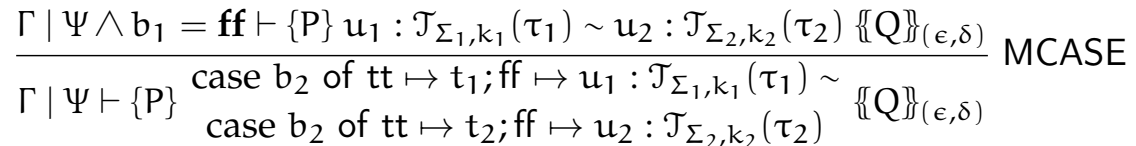

$$
\begin{aligned}
& \frac{\Gamma \mid \Psi \vdash \mathrm{t}_{1}: \tau_{1}\{\phi\} \quad \Gamma, \mathbf{s}_{1}: M, \mathbf{s}_{2}: M \vdash \mathrm{P}}{\Gamma \mid \Psi \vdash\{\mathrm{P}\} \text { unit }\left(\mathrm{t}_{1}\right): \mathcal{T}_{\emptyset, 0}\left(\tau_{1}\right) \sim \text { skip }: \mathcal{T}_{\emptyset, 0}(\text { ut })\{\mathrm{P} \wedge \phi\}_{(0,0)}} \text { UNIT-L } \\
& \Gamma \mid \Psi \vdash\{\mathrm{P}\} \mathrm{t}_{1}: \mathcal{T}_{\Sigma_{1}, \mathrm{k}_{1}}\left(\tau_{1}\right) \sim \text { skip : } \mathcal{T}_{\emptyset, 0}(\text { ut })\left\{\{\mathrm{Q}\}_{(\epsilon, \delta)}\right. \\
& \Gamma, x_{1}: \tau_{1} \mid \Psi \vdash\left\{Q\left[x_{1} / \mathbf{v}_{1}\right]\left[\star / \mathbf{v}_{2}\right]\right\} u_{1}: \mathcal{T}_{\Sigma_{1}^{\prime}, k_{1}^{\prime}}\left(\sigma_{1}\right) \sim u_{2}: \mathcal{T}_{\Sigma_{2}, k_{2}}\left(\sigma_{2}\right)\{R\}_{(\epsilon, \delta)} \\
& \mathrm{x}_{1} \notin \mathrm{FV}(\mathrm{R}) \quad \mathrm{x}_{1} \notin \mathrm{FV}\left(\mathrm{u}_{2}\right) \\
& \overline{\Gamma \mid \Psi \vdash\{\text { P }\} \text { mlet } x_{1}=\mathrm{t}_{1} \text { in } u_{1}: \mathcal{T}_{\Sigma_{1} \cup \Sigma_{1}^{\prime} \cup \operatorname{Eff}(\tau), k_{1}+k_{1}^{\prime}}\left(\sigma_{1}\right) \sim \mathrm{t}_{2}: \mathcal{T}_{\Sigma_{2}, k_{2}}\left(\sigma_{2}\right)\{R\}_{\left(\epsilon+\epsilon^{\prime}, \delta+\delta^{\prime}\right)}} \\
& \Gamma \mid \Psi \vdash \mathrm{b}_{1}: \text { bool }\{T\} \\
& \Gamma \mid \Psi \wedge b_{1}=\mathbf{t t} \vdash\{P\} t_{1}: \mathcal{T}_{\Sigma_{1}, k_{1}}\left(\tau_{1}\right) \sim t_{2}: \mathcal{T}_{\Sigma_{2}, k_{2}}\left(\tau_{2}\right)\left\{\{Q\}_{(\epsilon, \delta)}\right. \\
& \Gamma \mid \Psi \wedge b_{1}=\mathbf{f f} \vdash\{P\} u_{1}: \mathcal{T}_{\Sigma_{1}, k_{1}}\left(\tau_{1}\right) \sim t_{2}: \mathcal{T}_{\Sigma_{2}, k_{2}}\left(\tau_{2}\right)\left\{\{\mathrm{Q}\}_{(\epsilon, \delta)}\right. \\
& \overline{\Gamma \mid \Psi \vdash\{\mathrm{P}\} \text { case } \mathrm{b}_{2} \text { of } \mathrm{tt} \mapsto \mathrm{t}_{1} ; \mathrm{ff} \mapsto \mathrm{u}_{1}: \mathcal{T}_{\Sigma_{1}, \mathrm{k}_{1}}\left(\tau_{1}\right) \sim \mathrm{t}_{2}: \mathcal{T}_{\Sigma_{2}, \mathrm{k}_{2}}\left(\tau_{2}\right)\left\{\{\mathrm{Q}\}_{(\epsilon, \delta)}\right.}
\end{aligned}
$$

Figure 7.1: Relational logic: monadic rules

Relational adversarial properties are the natural generalization of these. In this case, we have two oracles $\mathcal{O}_{1}, \mathcal{O}_{2}$ and a single adversary $\mathcal{A}$ interacting with them. We will consider the setting in which the adversary is given access to either one of the oracles, which is often the situation in which adversarial properties are stated in 


$$
\begin{aligned}
& \frac{\mathrm{B}_{2} \subseteq \mathrm{B}_{1} \text { finite }}{\Gamma \mid \Psi \vdash\{\mathrm{P}\} \operatorname{Unif}_{\mathrm{B}_{1}}: \mathcal{T}_{\emptyset, 0}\left(\mathrm{~B}_{1}\right) \sim \mathrm{Unif}_{\mathrm{B}_{2}}: \mathcal{T}_{\emptyset, 0}\left(\mathrm{~B}_{2}\right)\left\{\mathrm{P} \wedge\left(\mathbf{v}_{1}=\mathbf{v}_{2} \vee \mathbf{v}_{1} \notin \mathrm{B}_{2}\right)\right\}_{(0,0)}} \\
& \frac{\mathrm{B}_{1} \subseteq \mathrm{B}_{2} \text { finite }}{\Gamma \mid \Psi \vdash\{\mathrm{P}\} \operatorname{Unif}_{\mathrm{B}_{1}}: \mathcal{T}_{\emptyset, 0}\left(\mathrm{~B}_{1}\right) \sim \operatorname{Unif}_{\mathrm{B}_{2}}: \mathcal{T}_{\emptyset, 0}\left(\mathrm{~B}_{2}\right)\left\{\mathrm{P} \wedge \mathbf{v}_{1}=\mathbf{v}_{2}\right\}_{\left(0,\left|\mathrm{~B}_{1}\right| /\left|\mathrm{B}_{2}\right|\right)}} \mathrm{SAM}
\end{aligned}
$$

Figure 7.2: Relational logic: sampling from uniform distributions

$$
\begin{aligned}
& \frac{\Gamma \mid \Psi \vdash \mathrm{t}_{1}: \tau_{1} \sim \mathrm{t}_{2}: \tau_{2}\left\{\mathcal{H}_{\sigma,(\epsilon, \delta)}\left(\mathrm{P}, \mathbf{r}_{1}, \mathbf{r}_{2}, \mathrm{Q}\right)\right\}}{\Gamma \mid \Psi \vdash\{\mathrm{P}\} \operatorname{unit}\left(\mathrm{t}_{1}\right): \mathcal{T}_{\emptyset, 0}\left(\tau_{1}\right) \sim \operatorname{unit}\left(\mathrm{t}_{2}\right): \mathcal{T}_{\emptyset, 0}\left(\tau_{2}\right)\{\mathrm{Q}\}_{(\epsilon, \delta)}} \text { UNSWITCH } \\
& \frac{\Gamma \mid \Psi \vdash\{\mathrm{P}\} \operatorname{unit}\left(\mathrm{t}_{1}\right): \mathcal{T}_{\emptyset, 0}\left(\tau_{1}\right) \sim \operatorname{unit}\left(\mathrm{t}_{2}\right): \mathcal{T}_{\emptyset, 0}\left(\tau_{2}\right)\{\mathrm{Q} \wedge \phi\}_{(\epsilon, \delta)}}{\Gamma \mid \Psi \vdash \mathrm{t}_{1}: \tau_{1} \sim \mathrm{t}_{2}: \tau_{2}\left\{\mathcal{H}_{\sigma,(\epsilon, \delta)}\left(\mathrm{P}, \mathbf{r}_{1}, \mathbf{r}_{2}, \mathrm{Q}\right)\right\}} \text { SWITCH }
\end{aligned}
$$

Figure 7.3: Relational logic: switching rules

cryptography or security. Other properties that require that the adversary be able to query both of the oracles could be considered by extending the type of adversaries to support multiple oracles and then comparing $\mathcal{A}\left(\mathcal{O}_{1}, \mathcal{O}_{2}\right)$ and $\mathcal{A}\left(\mathcal{O}_{2}, \mathcal{O}_{1}\right)$.

We also generalize the notion of safety for a region $\Sigma$. In the unary case we only required that writing to $\Sigma$ preserves the invariant. In the relational case, we also need to require that $\phi$ is also "safe for reading in $\Sigma$ ", meaning that an adversary reading from two different memories related by $\phi$ at the same location in $\Sigma$ sees the same value. This prevents the two executions from diverging due to a read operation:

Definition 7.1. Let $\phi$ be a predicate and $\Sigma \subseteq$ Loc. We say that $\phi \in \operatorname{RSafe}(\Sigma)$ if and only if,

$$
\forall \mathrm{m}_{1}, \mathrm{~m}_{2}, \forall \mathrm{l} \in \Sigma . \forall v \in \text { Val. } \phi\left(\mathrm{m}_{1}, \mathrm{~m}_{2}\right) \Rightarrow \phi\left(\mathrm{m}_{1}[\mathrm{l} \mapsto v], \mathrm{m}_{2}[\mathrm{l} \mapsto v]\right)
$$

and

$$
\forall \mathrm{m}_{1}, \mathrm{~m}_{2} . \forall \mathrm{l} \in \Sigma . \phi\left(\mathrm{m}_{1}, \mathrm{~m}_{2}\right) \Rightarrow \mathrm{m}_{1}[\mathrm{l}]=\mathrm{m}_{2}[\mathrm{l}]
$$

A more precise access control would distinguish between the locations that are safe for reading and the locations that are safe for writing, but it is an orthogonal matter.

We present now the main relational adversary rule, which can be used to reason 
about differential privacy:

$$
\begin{aligned}
& \cdot \vdash \mathcal{A}: \forall \alpha .\left(\sigma \rightarrow \mathcal{T}_{\alpha, 1}(\tau)\right) \rightarrow \mathcal{T}_{\Sigma \cup \alpha, k}\left(\tau^{\prime}\right) \\
& x_{1}: \sigma, x_{2}: \sigma \mid \mathrm{Eq}_{\sigma}\left(x_{1}, x_{2}\right) \vdash\{\phi\} \mathrm{t}_{1}: \mathcal{T}_{\Sigma^{\prime}, 1}(\tau) \sim \mathrm{t}_{2}: \mathcal{T}_{\Sigma^{\prime}, 1}(\tau)\left\{\phi \wedge \mathrm{Eq}_{\tau}\left(\mathbf{v}_{1}, \mathbf{v}_{2}\right)\right\}_{(\epsilon, \delta)} \\
& \mathbf{s}_{1}: M, \mathbf{s}_{2}: M \vdash \phi \\
& \frac{\operatorname{RSafe}(\Sigma) \quad \mathrm{x}_{1} \notin \mathrm{FV}\left(\mathrm{t}_{2}\right), \mathrm{x}_{2} \notin \mathrm{FV}\left(\mathrm{t}_{1}\right) \quad \sigma, \tau, \tau^{\prime} \text { non-monadic types }}{\cdot \mid \cdot \vdash\{\phi\}} \mathcal{A}\left[\Sigma^{\prime}\right]\left(\lambda x_{1} \cdot \mathrm{t}_{1}\right): \mathcal{T}_{\Sigma \cup \Sigma^{\prime}, \mathrm{k}}(\tau) \sim \Sigma^{\prime}(\phi)-D P \\
& \mathcal{A}\left[\Sigma^{\prime}\right]\left(\lambda x_{2} . t_{2}\right): \mathcal{T}_{\Sigma \cup \Sigma^{\prime}, k}(\tau) \quad\left\{\phi \wedge E q_{\tau^{\prime}}\left(\mathbf{v}_{1}, \mathbf{v}_{2}\right)\right\}_{(k \in, k \delta)}
\end{aligned}
$$

Here, $\mathrm{Eq}_{\tau}$ denotes extensional equality at type $\tau$, which is defined as:

$$
\begin{aligned}
\mathrm{Eq}_{\mathrm{b}}\left(x_{1}, x_{2}\right) & \triangleq x_{1}=x_{2} \\
\mathrm{Eq}_{\tau_{1} \rightarrow \tau_{2}}\left(x_{1}, x_{2}\right) & \triangleq \forall y_{1}, y_{2} \in \tau_{1} . \mathrm{Eq}_{\tau_{1}}\left(y_{1}, y_{2}\right) \Rightarrow \mathrm{Eq}_{\tau_{1}}\left(x_{1} y_{1}, x_{2} y_{2}\right) \\
\mathrm{Eq}_{\tau_{1} \times \tau_{2}}\left(x_{1}, x_{2}\right) & \triangleq \mathrm{Eq}_{\tau_{1}}\left(\pi_{1}\left(x_{1}\right), \pi_{1}\left(x_{2}\right)\right) \wedge \mathrm{Eq}_{\tau_{2}}\left(\pi_{2}\left(x_{1}\right), \pi_{2}\left(x_{2}\right)\right)
\end{aligned}
$$

In the simplified case where $\sigma$ is a base type, $\mathrm{Eq}_{\sigma}\left(\mathrm{x}_{1}, \mathrm{x}_{2}\right)$ can be replaced by $\mathrm{x}_{1}=\mathrm{x}_{2}$ (similarly for $\tau, \tau^{\prime}$ ). The premises of the rule state:

- $\phi$ is a relational invariant safe for the memory region $\Sigma$

- We have two oracles $\mathcal{O}_{1}, \mathcal{O}_{2}$ such for extensionally equal inputs and initial memories related by $\phi$, the final memories are related by an $(\epsilon, \delta)$-approximate $\phi$-coupling, and the outputs are $(\epsilon, \delta)$-approximately equal

- $\mathcal{A}$ is an arbitrary adversary that only writes to and reads from $\Sigma$ and that can call its argument (either $\mathcal{O}_{1}$ or $\mathcal{O}_{2}$ ) up to $k$ times

From them, we conclude that executing the adversary with these oracles and initial memories related by $\phi$ should yield output memories related by an $(k \epsilon, k \delta)-$ approximate $\phi$-coupling and output results $(k \epsilon, k \delta)$-approximately equal.

We also provide another rule for adversaries, that performs up-to-bad reasoning. This is a reasoning pattern in which we define a bad event $\chi$ that captures the conditions under which the invariant $\phi$ is broken. The rule can be stated as:

$$
\begin{aligned}
& \cdot \vdash \mathcal{A}: \forall \alpha .\left(\sigma \rightarrow \mathcal{T}_{\alpha, 1}(\tau)\right) \rightarrow \mathcal{T}_{\Sigma \cup \alpha, k}\left(\tau^{\prime}\right) \\
& x_{1}: \sigma, x_{2}: \sigma \mid \mathrm{Eq}_{\sigma}\left(x_{1}, x_{2}\right) \vdash\{\phi\} \begin{array}{c}
\mathrm{t}_{1}: \mathcal{T}_{\Sigma^{\prime}, 1}(\tau) \sim \\
\mathrm{t}_{2}: \mathcal{T}_{\Sigma^{\prime}, 1}(\tau)
\end{array}\left\{\left(\phi \wedge \mathrm{Eq}_{\tau}\left(\mathbf{v}_{1}, \mathbf{v}_{2}\right)\right) \vee \chi\right\}_{(\epsilon, \delta)} \\
& x_{1}: \sigma \mid \cdot \vdash\{\chi\} t_{1}: \mathcal{T}_{\Sigma^{\prime}, 1}(\tau)\{\chi \chi\}_{0} \\
& \mathbf{s}_{1}: M, \mathbf{s}_{2}: M \vdash \phi \quad \mathbf{s}_{1}: M \vdash \chi \quad \phi \in \operatorname{RSafe}(\Sigma)
\end{aligned}
$$

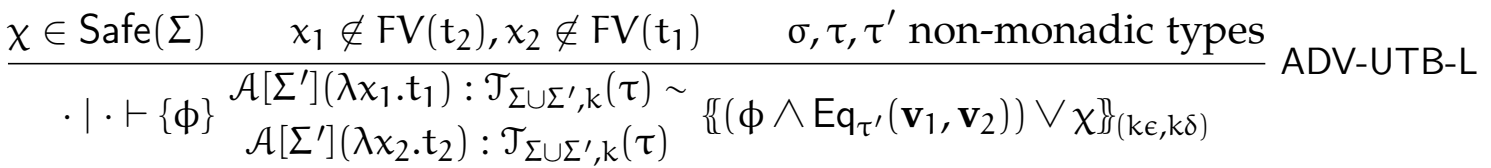

The premises of this rule state: 
- $\phi$ and $\chi$ are relational properties safe for the memory region $\Sigma$

- The oracles $\mathcal{O}_{1}, \mathcal{O}_{2}$ such for extensionally equal inputs and initial memories related by $\phi$, either return final memories are related by an $(\epsilon, \delta)$-approximate $\phi$-coupling, and the outputs are $(\epsilon, \delta)$-approximately equal, or return final memories related by an $(\epsilon, \delta)$-approximate $x$-coupling (i.e., the bad event occurs).

- The oracles $\mathcal{O}_{1}, \mathcal{O}_{2}$ preserve $\chi$ under an $(\epsilon, \delta)$-approximate coupling

- $\mathcal{A}$ is an arbitrary adversary that only writes to and reads from $\Sigma$ and that can call its argument (either $\mathcal{O}_{1}$ or $\mathcal{O}_{2}$ ) up to $k$ times

Therefore, we can conclude that after executing the adversary with initial condition $\phi$, either we reach $\chi$ at some point, and then have $\chi$ at the end, or we never reach $\chi$ and then have at $\phi$ and equal outputs at the end. The postcondition is graded by $(k \in, k \delta)$ since the oracles may be called up to $k$ times.

\subsection{Example: PRF/PRP Switching Lemma}

In this section we illustrate the use of our program logic with a classic example in computer-aided cryptography. We also briefly discuss how examples from the literature can be verified in our setting.

The PRF/PRP Switching Lemma [98] is a classic tool for upper bounding the probability of an adversary with bounded oracle access to distinguish between a pseudo-random function (PRF) and a pseudo-random permutation (PRP). The PRF/PRP Switching Lemma is a popular benchmark for computer-aided cryptography, so multiple formalizations are available, e.g. [24]. One common trait of these formalizations is that the PRF and the PRP are modeled as stateful procedures, which sample a uniform output for every fresh input. This style of modeling has the advantage to sample from base types, which is necessary for first-order imperative languages. In our setting, we can formalize the proof of the PRF/PRP Switching Lemma exactly as in other approaches, but we can also sample from function spaces. Since our purpose is to illustrate the use of the adversary rule in a relational setting, we follow the proof of [24] and use the exact fragment of the relational logic. Moreover, we only present the first part of the proof, where we prove that the two expressions are equivalent up to a failure event. The remaining part of the proof, namely bounding the probability of a collision, can be carried in the Union Bound logic.

We define pseudo-random functions and pseudo-random permutations as follows, where $\{0,1\}^{l}$ denotes the set of bitstrings of length $l$ and we consider for our state two set of locations $L, L^{\prime}$ for the oracle and the adversary stores. These are indexed by $\{0,1\}^{\mathrm{l}}$ and contain values also in $\{0,1\}^{\mathrm{l}}$. It is convenient to view $\mathrm{L}$ as a 
finite map, and we use dom $\mathrm{L}$ and im $\mathrm{L}$ to denote its domain and image respectively. We also use if $b$ then $t$ to denote a case distinction with an empty else clause, and semicolon (;) to denote sequencing:

$$
\begin{aligned}
\operatorname{PRF}\left(\mathrm{x}_{1}:\{0,1\}^{\mathrm{l}}\right) \triangleq & \text { if } \mathrm{x}_{1} \notin \operatorname{dom} \mathrm{L}_{1} \text { then }\left\{z_{1}=\operatorname{Unif}_{\{0,1\}} ; \mathrm{L}_{1}\left[\mathrm{x}_{1}\right]:=z_{1}\right\} ; \\
& \mathrm{L}_{1}\left[\mathrm{x}_{1}\right] \\
\operatorname{PRP}\left(\mathrm{x}_{2}:\{0,1\}^{\mathrm{l}}\right) \triangleq \quad & \text { if } \mathrm{x}_{2} \notin \operatorname{dom} \mathrm{L}_{2} \text { then }\left\{z_{2}=\operatorname{Unif}_{\{0,1\}} \backslash\left(\text { im } \mathrm{L}_{2}\right) ; \mathrm{L}_{2}\left[\mathrm{x}_{2}\right]:=z_{2}\right\} ; \\
& \mathrm{L}\left[\mathrm{x}_{2}\right]
\end{aligned}
$$

We will prove the example using up-to-bad reasoning. In our example, the bad event $\chi$ corresponds to having a collision when sampling in the first program:

$$
x \equiv \exists x, x^{\prime} . x \neq x^{\prime} \wedge x^{\prime} \in \operatorname{dom} \mathrm{L}_{1} \wedge \mathrm{L}_{1}[\mathrm{x}]=\mathrm{L}_{1}\left[\mathrm{x}^{\prime}\right]
$$

We consider an adversary $\mathcal{A}$ of type $\forall \alpha .\left(\{0,1\}^{l} \rightarrow \mathcal{T}_{\alpha, 1}\left(\{0,1\}^{l}\right)\right) \rightarrow \mathcal{T}_{L^{\prime} \cup \alpha, N}(\{0,1\})$. Such an adversary can call the oracle $\mathrm{N}$ times and returns a bit $\mathrm{b}$. We want to prove:

$\vdash\left\{\mathbf{s}_{1}=\mathbf{s}_{2}\right\} \mathcal{A}[\mathrm{L}](P R F): \mathcal{T}_{\mathrm{LUL}^{\prime}, \mathrm{N}}(\mathbb{B}) \sim \mathcal{A}[\mathrm{L}](P R P): \mathcal{T}_{\mathrm{LUL}^{\prime}, \mathrm{N}}(\mathbb{B})\left\{\left\{\mathbf{s}_{1}=\mathbf{s}_{2} \wedge \mathbf{v}_{1}=\mathbf{v}_{2}\right) \vee \chi\right\}_{(0,0)}$

By the applying the ADV-UTB rule, there are two premises to show:

$\Gamma \mid \Psi \vdash\left\{\mathbf{s}_{1}=\mathbf{s}_{2}\right\} \operatorname{PRF}\left(\mathrm{x}_{1}\right): \mathcal{T}_{\mathrm{L}, 1}\left(\{0,1\}^{\mathrm{l}}\right) \sim P R P\left(\mathrm{x}_{2}\right): \mathcal{T}_{\mathrm{L}, 1}\left(\{0,1\}^{\mathrm{l}}\right)\left\{\left\{\left(\mathbf{s}_{1}=\mathbf{s}_{2} \wedge \mathbf{v}_{1}=\mathbf{v}_{2}\right) \vee \chi\right\}_{(0,0)}\right.$ and

$$
\left.x_{1}:\{0,1\}^{l} \mid \cdot \vdash\{\chi\} \operatorname{PRF}\left(x_{1}\right): \mathcal{T}_{L, 1}\left(\{0,1\}^{l}\right)\left\{\mathcal{T}_{L, 1}\left(\{0,1\}^{l}\right)\right\}\right\}_{\chi} 0,0
$$

where $\Gamma \triangleq x_{1}:\{0,1\}^{l}, x_{2}:\{0,1\}^{l}, \Psi \triangleq x_{1}=x_{2}$. The second premise can be proven by simple unary reasoning, noting that memory cells are not rewritten, so once there is a collision between two memory cells, it will be preserved. To prove the first premise, we apply the MCASE rule. In the empty else branch, we need to prove:

$$
\begin{aligned}
& \left\{\mathbf{s}_{1}=\mathbf{s}_{2}\right\} \\
\Gamma \mid \Psi, x_{1} \in \operatorname{dom} \mathrm{L}_{1} \vdash & \mathrm{L}\left[\mathrm{x}_{1}\right]: \mathcal{T}_{\mathrm{L}, 0}\left(\{0,1\}^{\mathrm{l}}\right) \sim \mathrm{L}\left[\mathrm{x}_{2}\right]: \mathcal{T}_{\mathrm{L}, 1}\left(\{0,1\}^{\mathrm{l}}\right) \\
& \left\{\left[\mathbf{s}_{1}=\mathbf{s}_{2} \wedge \mathbf{v}_{1}=\mathbf{v}_{2}\right) \vee \chi^{\sharp}\right\}_{(0,0)}
\end{aligned}
$$

which is a simple derivation. In the then branch, we first apply the synchronous WRITE rule, and then we are left to prove:

$$
\begin{aligned}
& \left\{\mathbf{s}_{1}=\mathbf{s}_{2}\right\}
\end{aligned}
$$

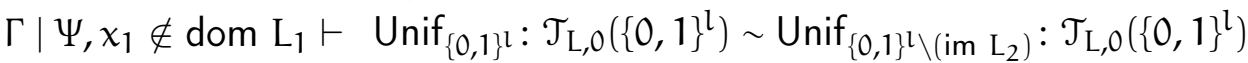

$$
\begin{aligned}
& \left.\left\{\mathbf{s}_{1}\left[\mathrm{x}_{1} \mapsto \mathbf{v}_{1}\right]=\mathbf{s}_{2}\left[\mathrm{x}_{2} \mapsto \mathbf{v}_{2}\right] \wedge \mathbf{v}_{1}=\mathbf{v}_{2}\right) \vee \mathbf{v}_{1} \in \operatorname{im~} L_{1}\right\}(0,0)
\end{aligned}
$$

which we prove by first strengthening the postcondition to

$$
\left\{\mathbf{s}_{1}=\mathbf{s}_{2} \wedge\left(\mathbf{v}_{1}=\mathbf{v}_{2} \vee \mathbf{v}_{1} \in \mathrm{im} L_{1}\right)\right\}
$$

and then using the SAMPLE-ASYN rule.

From the relational judgment, one concludes that the advantage of an adversary $\mathcal{A}$ to distinguish between a PRF and a PRP is upper bounded by the probability of the bad event in the first game. Using the union bound logic, we can prove that this probability is upper bounded by $\frac{\mathrm{N}(\mathrm{N}-1)}{2^{\mathrm{l}}}$. The proof is standard and omitted. 


\subsection{Relational Stateful liftings}

To interpret relational logics, recall the category BRel(QBS) defined by the following pullback (in the category of categories):

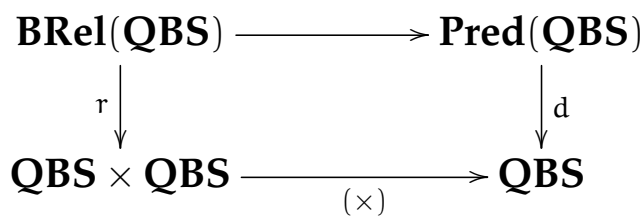

An analogous construction to $\dot{\mathcal{T}}$ can be implemented using a graded lifting $\ddot{\mathcal{P}}_{\mathrm{k}}$ of $\mathcal{P} \times \mathcal{P}$ along $r$. That is, we can define

$$
\begin{aligned}
& \ddot{\operatorname{St}}(\ddot{\mathcal{P}})_{\sigma_{1}, \sigma_{2}, k}:\left(\mathbf{B R e l}(\mathbf{Q B S})_{M, M}\right)^{o p} \times\left(\operatorname{BRel}(\mathbf{Q B S})_{\sigma_{1} \times M, \sigma_{2} \times M}\right) \rightarrow \operatorname{BRel}(\mathbf{Q B S})_{\mathcal{T}\left(\sigma_{1}\right), \mathcal{T}\left(\sigma_{2}\right)} \\
& \ddot{\mathrm{St}}(\ddot{\mathcal{P}})_{\sigma_{1}, \sigma_{2}, k}(P, Q)=\left\{\left(f_{1}, f_{2}\right): P \rightarrow \ddot{\mathcal{P}}_{k} Q \operatorname{in} \operatorname{BRel}(\mathbf{Q B S})\right\}
\end{aligned}
$$

In other words, this construction takes a relation over $(M, M)$ and a relation over $\left(\sigma_{1} \times M, \sigma_{2} \times M\right)$ and returns a relation over $\left(M \Rightarrow \mathcal{P}\left(\sigma_{1} \times M\right), M \Rightarrow \mathcal{P}\left(\sigma_{2} \times M\right)\right.$. Lemma 6.3 can then be generalized accordingly.

Lemma 7.2. Let $\ddot{\mathcal{P}}_{\mathrm{k}}$ be a graded lifting of $\mathcal{P} \times \mathcal{P}$. Then:

- For $\mathrm{X}_{1}, \mathrm{X}_{2} \in \mathbf{Q B S}, \phi \in \operatorname{BRel}(\mathbf{Q B S})_{\mathrm{X}_{1}, \mathrm{X}_{2}}$, and $\mathrm{Q} \in \operatorname{BRel}(\mathbf{Q B S})_{\mathrm{M}, \mathrm{M}}$, then

$$
\left(\eta^{\mathcal{T}}, \eta^{\mathcal{T}}\right): \phi \rightarrow \ddot{\mathrm{S}} \mathrm{t}(\ddot{\mathcal{P}})_{X_{1}, X_{2}, 0}(Q, \phi \ddot{\times} Q)
$$

in $\operatorname{BRel}(\mathbf{Q B S})$.

- For $X_{1}, X_{2}, Y_{1}, Y_{2} \in \mathbf{Q B S}, P \in \operatorname{BRel}(Q B S)_{M, M}, Q \in \operatorname{BRel}(Q B S)_{X_{1} \times M, X_{2} \times M}, R \in$ $\operatorname{BRel}(Q B S)_{Y_{1} \times M, Y_{2} \times M}, f_{1}: X_{1} \rightarrow \mathcal{T}\left(Y_{1}\right)$ and $f_{2}: X_{2} \rightarrow \mathcal{T}\left(Y_{2}\right)$, if $\left(\lambda^{-1}\left(f_{1}\right), \lambda^{-1}\left(f_{2}\right)\right):$ $\mathrm{Q} \rightarrow \ddot{\mathcal{P}}_{\mathrm{k}}(\mathrm{R})$ in $\mathbf{B R e l}(\mathbf{Q B S})$, then

$$
\left(f_{1}^{\#}, f_{2}^{\#}\right): \ddot{S} t(\ddot{\mathcal{P}})_{X_{1}, X_{2}, k^{\prime}}(P, Q) \rightarrow \ddot{S} t(\ddot{\mathcal{P}})_{Y_{1}, Y_{2}, k^{\prime}+k}(P, R)
$$

in $\operatorname{BRel}(\mathbf{Q B S})$ for all $k, k^{\prime}$

- For $\mathrm{X}_{1}, \mathrm{X}_{2}, \mathrm{Y}_{1}, \mathrm{Y}_{2} \in \mathbf{Q B S}, \phi \in \mathbf{B R e l}(\mathbf{Q B S})_{\mathrm{X}_{1}, \mathrm{X}_{2}}, \mathrm{P} \in \operatorname{BRel}(\mathbf{Q B S})_{\mathrm{M}, \mathrm{M}}$ and $\mathrm{Q} \in$ $\operatorname{BRel}(\mathbf{Q B S})_{Y_{1} \times M, Y_{2} \times M}$, then

$$
\left(\theta^{\mathcal{T}}, \theta^{\mathcal{T}}\right): \phi \ddot{\times} \ddot{\mathrm{S}} \mathrm{t}(\ddot{\mathcal{P}})_{Y_{1}, Y_{2}, k}(P, Q) \rightarrow \ddot{S} t(\ddot{\mathcal{P}})_{X_{1} \times Y_{1}, X_{2} \times Y_{2}, k}(P, \phi \ddot{\times} Q)
$$

in $\mathbf{B R e l}(\mathbf{Q B S})$ for all $\mathrm{k}$. 


\subsection{Semantics}

\subsubsection{Soundness of the basic rules}

The semantics of the relational logics are a generalization of the semantics of the unary logics. We interpret the monadic rules in the category of relations over QBS, with the lifting $\ddot{\mathcal{P}} d p$, which induces $\ddot{\mathrm{St}}(\ddot{\mathcal{P}} d p)$ as in the previous section. We can show:

Proposition 7.3. Let $\Gamma \mid \Psi \vdash\{\mathrm{P}\} \mathrm{t}_{1}: \mathcal{T}_{\tau_{1}} \sim \mathrm{t}_{1}: \mathcal{T}_{\tau_{2}}\{\mathrm{Q}\}_{(\epsilon, \delta)}$ be a derivable HO-SPRL judgment without the ADV rule, and $\gamma \in \mathbf{Q B S}(1, \llbracket \Gamma \rrbracket)$ such that $\gamma: \top \rightarrow \llbracket \Gamma \vdash \wedge \Psi \rrbracket$. Then $\left(\llbracket \Gamma \vdash \mathrm{t}_{1}: \mathcal{T}_{\tau_{1} \rrbracket \circ \gamma}, \llbracket \Gamma \vdash \mathrm{t}_{2}: \mathcal{T}_{\tau_{2}} \rrbracket \circ \gamma\right)$ is a morphism in $\mathbf{B R e l}(\mathbf{Q B S})$ from $(1,1, \top)$ to

$$
\begin{aligned}
\ddot{\mathrm{St}}\left(\mathcal{P}^{\mathrm{dp}}\right)_{(\epsilon, \delta)}\left(\left\langle\gamma, \mathrm{id} \rrbracket_{\llbracket M \rrbracket \times \llbracket M \rrbracket}\right\rangle^{*} \llbracket \Gamma, \mathbf{s}_{1}: M, \mathbf{s}_{2}: M \vdash P \rrbracket,\right. \\
\left.\left.\left\langle\gamma, i d_{\llbracket M \rrbracket \times \llbracket \tau_{1} \rrbracket \times \llbracket M \rrbracket \times \llbracket \tau_{2} \rrbracket}\right\rangle^{*} \llbracket \Gamma, \mathbf{s}_{1}: M, \mathbf{v}_{1}: \tau_{1}, \mathbf{s}_{2}: M, \mathbf{v}_{2}: \tau_{2} \vdash \mathrm{Q} \rrbracket\right)\right) .
\end{aligned}
$$

The proof is by induction on the derivation and is largely independent of the specific lifting. Here, we are implicitly interpreting

$$
\left\langle\gamma, \mathrm{id}_{\llbracket M \rrbracket \times \llbracket M \rrbracket}\right\rangle^{*} \llbracket \Gamma, \mathbf{s}_{1}: M, \mathbf{s}_{2}: M \vdash P \rrbracket
$$

as a relation over $(M, M)$ (as opposed to a predicate over $M \times M)$, and

$$
\left\langle\gamma, \mathrm{id}_{\llbracket M \rrbracket \times \llbracket \tau_{1} \rrbracket \times \llbracket M \rrbracket \times \llbracket \tau_{2} \rrbracket}\right\rangle^{*} \llbracket \Gamma, \mathbf{s}_{1}: M, \mathbf{v}_{1}: \tau_{1}, \mathbf{s}_{2}: M, \mathbf{v}_{2}: \tau_{2} \vdash \mathrm{Q} \rrbracket
$$

as a relation over $\left(M \times \tau_{1}, M \times \tau_{2}\right)$.

Proof. To simplify the proof, we will use set theory notation, i.e. instead of $\gamma \in$ QBS $(1, \llbracket \Gamma \rrbracket)$ and $\left\langle\gamma^{*}, i d\right\rangle \llbracket \Gamma, \mathbf{s}: M \vdash P \rrbracket$, we write $\gamma \in \llbracket \Gamma \rrbracket$ and $\llbracket \Gamma, \mathbf{s}: M \vdash P \rrbracket(\gamma)$. We also use the shorthand $\ddot{\mathfrak{T}}_{\sigma_{1}, \sigma_{2},(\epsilon, \delta)} \triangleq \ddot{\mathrm{St}}(\ddot{\mathrm{P}} d p)_{\sigma_{1}, \sigma_{2},(\epsilon, \delta)}$, and omit $\sigma_{1}, \sigma_{2}$ when they are clear from the context. We only show a few interesting cases:

- UNIT-L. By soundness of UHOL,

$$
\left\langle\mathrm{id}_{\llbracket \Gamma \rrbracket,}, \llbracket \Gamma \vdash \mathrm{t}: \tau_{1} \rrbracket\right\rangle: \llbracket \Gamma \vdash \bigwedge \Psi \rrbracket \rightarrow \llbracket \Gamma, \mathbf{r}: \tau_{1} \vdash \phi \rrbracket
$$

so for all $\gamma \in \llbracket \Gamma \vdash \Lambda \Psi \rrbracket, \llbracket \Gamma \vdash t: \tau_{1} \rrbracket(\gamma) \in \llbracket \Gamma, \mathbf{r}: \tau_{1} \vdash \phi \rrbracket(\gamma)$. Consider an arbitrary predicate $\Gamma, \mathbf{s}_{1}: M, \mathbf{s}_{2}: M \vdash P$. By definition,

$$
\llbracket \Gamma \vdash \operatorname{unit}(\mathrm{t}): \mathcal{T} \tau_{1} \rrbracket(\gamma)=\eta^{\mathcal{T}}\left(\llbracket \Gamma \vdash \mathrm{t}: \tau_{1} \rrbracket(\gamma)\right)
$$

and

$$
\llbracket \Gamma \vdash \text { skip: } \mathcal{T} u t \rrbracket(\gamma)=\eta(*)
$$

Where $*$ is the only element of the singleton set. By Lemma 7.2 we can conclude that

$$
\begin{aligned}
& \left(\eta^{\mathcal{T}}\left(\llbracket \Gamma \vdash \mathrm{t}: \tau_{1} \rrbracket(\gamma)\right), \eta^{\mathcal{T}}(*)\right) \in \\
& \ddot{\mathfrak{T}}_{\tau_{1}, \mathrm{ut},(0,0)}\left(\llbracket \Gamma, \mathbf{s}_{1}: M, \mathbf{s}_{2} \vdash \mathrm{P} \rrbracket(\gamma), \llbracket \Gamma \vdash \phi \rrbracket(\gamma) \ddot{x} \llbracket \Gamma, \mathbf{s}_{1}: M, \mathbf{s}_{2}: M \vdash P \rrbracket(\gamma)\right)
\end{aligned}
$$


- BIND. By applying I.H. to the first premise we have that, for all $\gamma \in \llbracket \Gamma \vdash \Psi \rrbracket$

$$
\begin{aligned}
& \left(\llbracket \Gamma \vdash \mathrm{t}_{1}: \mathcal{T} \tau_{1} \rrbracket(\gamma), \llbracket \Gamma \vdash \mathrm{t}_{2}: \mathcal{T} \tau_{2} \rrbracket(\gamma)\right) \in \\
& \ddot{\mathcal{T}}_{(\epsilon, \delta)}\left(\llbracket \Gamma, \mathbf{s}_{1}: M, \mathbf{s}_{2}: M \vdash P \rrbracket(\gamma), \llbracket \Gamma, \mathbf{s}_{1}: M, \mathbf{v}_{1}: \tau_{1}, \mathbf{s}_{2}: M, \mathbf{v}_{2}: \tau_{2} \vdash Q \rrbracket(\gamma)\right)
\end{aligned}
$$

and for all $e_{1}: \tau_{2}, e_{2}: \tau_{2}$, we have $\left(\gamma, e_{1}, e_{2}\right) \in \llbracket \Gamma, x_{1}: \tau_{1}, x_{2}: \tau_{2} \vdash \Psi \rrbracket$, so by applying I.H. to the second premise,

$$
\begin{gathered}
\left(\llbracket \Gamma, x_{1}: \tau_{1}, x_{2}: \tau_{2}, \vdash u_{1}: \mathcal{T} \sigma_{1} \rrbracket\left(\gamma, e_{1}, e_{2}\right), \llbracket \Gamma, x_{1}: \tau_{1}, x_{2}: \tau_{2} \vdash u_{2}: \mathcal{T} \sigma_{2} \rrbracket\left(\gamma, e_{1}, e_{2}\right)\right) \in \\
\ddot{\mathcal{T}}_{\left(\epsilon^{\prime}, \delta^{\prime}\right)}\left(\llbracket \Gamma, x_{1}: \tau_{1}, x_{2}: \tau_{2}, \mathbf{s}_{1}: M, \mathbf{s}_{2}: M \vdash Q \mathbb{Q} \rrbracket\left(\gamma, e_{1}, e_{2}\right),\right. \\
\left.\llbracket \Gamma, x_{1}: \tau_{1}, x_{2}: \tau_{2}, \mathbf{s}_{1}: M, \mathbf{v}_{1}: \sigma_{1}, \mathbf{s}_{2}: M, \mathbf{v}_{2}: \sigma_{2} \vdash R \rrbracket\left(\gamma, e_{1}, e_{2}\right)\right)
\end{gathered}
$$

Since $x_{1}, x_{2} \notin F V(R)$, then also

$$
\begin{gathered}
\left(\llbracket \Gamma, x_{1}: \tau_{1}, x_{2}: \tau_{2}, \vdash u_{1}: \mathcal{T} \sigma_{1} \rrbracket\left(\gamma, e_{1}, e_{2}\right), \llbracket \Gamma, x_{1}: \tau_{1}, x_{2}: \tau_{2} \vdash u_{2}: \mathcal{T} \sigma_{2} \rrbracket\left(\gamma, e_{1}, e_{2}\right)\right) \in \\
\ddot{\mathcal{T}}_{\left(\epsilon^{\prime}, \delta^{\prime}\right)} \llbracket \Gamma, x_{1}: \tau_{1}, x_{2}: \tau_{2}, \mathbf{s}_{1}: M, \mathbf{s}_{2}: M \vdash Q \rrbracket\left(\gamma, e_{1}, e_{2}\right), \\
\left.\llbracket \Gamma, \mathbf{s}_{1}: M, \mathbf{v}_{1}: \sigma_{1}, \mathbf{s}_{2}: M, \mathbf{v}_{2}: \sigma_{2} \vdash R \rrbracket(\gamma)\right)
\end{gathered}
$$

Also, since $x_{1} \notin F V\left(u_{2}\right)$ and $x_{2} \notin F V\left(u_{1}\right)$, we have morphisms

$$
\lambda^{-1}\left(\llbracket \Gamma, \chi_{1}: \tau_{1} \vdash \mathfrak{u}_{1}: \mathcal{T} \sigma_{1} \rrbracket(\gamma)\right): \tau_{1} \times M \rightarrow \mathcal{P}\left(\sigma_{1} \times M\right)
$$

and

$$
\lambda^{-1}\left(\llbracket \Gamma, \chi_{2}: \tau_{2} \vdash \mathfrak{u}_{2}: \mathcal{T} \sigma_{2} \rrbracket(\gamma)\right): \tau_{2} \times M \rightarrow \mathcal{P}\left(\sigma_{2} \times M\right)
$$

so,

$$
\begin{gathered}
\left(\lambda^{-1}\left(\llbracket \Gamma, x_{1}: \tau_{1} \vdash \mathfrak{u}_{1}: \mathcal{T} \sigma_{1} \rrbracket(\gamma)\right), \lambda^{-1}\left(\llbracket \Gamma, x_{2}: \tau_{2} \vdash \mathfrak{u}_{2}: \mathcal{T} \sigma_{2} \rrbracket(\gamma)\right)\right): \\
\llbracket \Gamma, x_{1}: \tau_{1}, \mathbf{s}_{1}: M, x_{2}: \tau_{2}, \mathbf{s}_{1}: M \vdash \mathrm{Q} \rrbracket(\gamma) \rightarrow \\
\ddot{P}_{\left(\epsilon^{\prime}, \delta^{\prime}\right)}^{d p}\left(\llbracket \Gamma, \mathbf{s}_{1}: M, \mathbf{v}_{1}: \sigma_{1}, \mathbf{s}_{2}: M, \mathbf{v}_{1}: \sigma_{2} \vdash R \rrbracket(\gamma)\right)
\end{gathered}
$$

By Lemma 7.2, we get

$$
\begin{aligned}
& \left(\left(\llbracket \Gamma, x_{1}: \tau_{1} \vdash u_{1}: \mathcal{T} \sigma_{1} \rrbracket(\gamma)\right)^{\#},\left(\llbracket \Gamma, x_{2}: \tau_{2} \vdash u_{2}: \mathcal{T} \sigma_{2} \rrbracket(\gamma)\right)^{\#}\right): \\
& \ddot{\mathcal{T}}_{(\epsilon, \delta)}\left(\llbracket \Gamma, \mathbf{s}_{1}: M, \mathbf{s}_{2}: M \vdash P \rrbracket(\gamma), \llbracket \Gamma, \mathbf{s}_{1}: M, \mathbf{v}_{1}: \tau_{1}, \mathbf{s}_{2}: M, \mathbf{v}_{2}: \tau_{2} \vdash \mathrm{Q} \rrbracket(\gamma)\right) \rightarrow \\
& \ddot{\mathfrak{T}}_{\left(\epsilon+\epsilon^{\prime}, \delta+\delta^{\prime}\right)}\left(\llbracket \Gamma, \mathbf{s}_{1}: M, \mathbf{s}_{2}: M \vdash P \rrbracket(\gamma), \llbracket \Gamma, \mathbf{s}_{1}: M, \mathbf{v}_{1}: \sigma_{1}, \mathbf{s}_{2}: M, \mathbf{v}_{2}: \sigma_{2} \vdash R(\gamma) \rrbracket\right)
\end{aligned}
$$

and from this we can conclude. 


\subsubsection{Soundness of the adversary rules}

\section{Soundness of the ADV-DP rule}

We generalize the logical predicates to the relational case. For an invariant $\mathbf{s}_{1}: M, \mathbf{s}_{2}: M \vdash$ $\phi$ and $(\epsilon, \delta) \in[0, \infty] \times[0, \infty]$ we define a logical relation, i.e., a map from a type $\sigma$ to pairs of closed terms of type $\sigma$ as follows:

$$
\begin{aligned}
& \mathcal{R}_{\phi,(\epsilon, \delta)}(\mathrm{B}) \triangleq\left\{\mathrm{b}_{1}, \mathrm{~b}_{2}: \mathrm{B} \mid \mathrm{b}_{1}=\mathrm{b}_{2}\right\} \\
& \mathcal{R}_{\phi,(\epsilon, \delta)}(\sigma \rightarrow \tau) \triangleq\left\{\begin{array}{l}
\left\{t_{1}, t_{2}: \sigma \rightarrow \tau \mid \begin{array}{l}
\forall u_{1}, u_{2}: \sigma \cdot\left(u_{1}, u_{2}\right) \in \mathcal{R}_{\phi,(\epsilon, \delta)}(\sigma) \\
\Rightarrow\left(t_{1} u_{1}, t_{2} u_{2}\right) \in \mathcal{R}_{\phi,(\epsilon, \delta)}(\tau)
\end{array}\right\} \text { if } \operatorname{Eff}(\sigma) \subseteq \operatorname{Eff}(\tau) \\
\left\{t_{1}, t_{2}: \sigma \rightarrow \tau \mid \forall u_{1}, u_{2}: \sigma \cdot\left(t_{1} u_{1}, t_{2} u_{2}\right) \in \mathcal{R}_{\phi,(\epsilon, \delta)}(\tau)\right\} \quad \text { otherwise }
\end{array}\right. \\
& \mathcal{R}_{\phi,(\epsilon, \delta)}(\sigma \times \tau) \triangleq\left\{\begin{array}{l|l}
t_{1}, t_{2}: \sigma \times \tau \mid & \begin{array}{l}
\left(\pi_{1}\left(t_{1}\right), \pi_{1}\left(t_{2}\right)\right) \in \mathcal{R}_{\phi,(\epsilon, \delta)}(\sigma) \\
\wedge\left(\pi_{2}\left(t_{1}\right), \pi_{2}\left(t_{2}\right)\right) \in \mathcal{R}_{\phi,(\epsilon, \delta)}(\tau)
\end{array}
\end{array}\right\} \\
& \mathcal{R}_{\phi,(\epsilon, \delta)}\left(\mathcal{T}_{\Sigma, k}(\sigma)\right) \triangleq\left\{\mathrm{t}_{1}, \mathrm{t}_{2}: \mathcal{T}_{\Sigma, \mathrm{k}}(\sigma) \mid \mathcal{H}_{\sigma,(\mathrm{k \epsilon}, \mathrm{k} \delta)}\left(\phi, \mathrm{t}_{1}, \mathrm{t}_{2},\left(\mathbf{v}_{1}, \mathbf{v}_{2}\right) \in \mathcal{R}_{\phi,(\epsilon, \delta)}(\sigma) \wedge \phi\right)\right\} \\
& \mathcal{R}_{\phi,(\epsilon, \delta)}(\forall \alpha . \tau) \triangleq\left\{\mathrm{t}_{1}, \mathrm{t}_{2}: \forall \alpha . \tau \mid \forall \Sigma .\left(\mathrm{t}_{1}[\Sigma], \mathrm{t}_{2}[\Sigma]\right) \in \mathcal{R}_{\phi,(\epsilon, \delta)}(\tau[\Sigma / \alpha])\right\}
\end{aligned}
$$

The definition is analogous to the unary case. Note that the relation at the base types changes, we now require equality to ensure that the computations have the same control flow. We state now the Basic Lemma:

Lemma 7.4 (Basic lemma). Let $\ddot{\mathcal{P}}$ be as above, and assume that for every $v \in \mathcal{D}$, $(\operatorname{sample}(v)$, sample $(v)) \in \ddot{\mathcal{P}}_{(0,0)}^{d p}\left(\mathcal{R}_{\phi,(\epsilon, \delta)}(\mathrm{B})\right)$. Let $\vdash \mathrm{t}: \sigma$ be a closed term and $\mathbf{s}_{1}: M, \mathbf{s}_{2}: M \vdash$ $\phi$ such that $\phi \in \operatorname{RSafe}(\operatorname{Eff}(\sigma))$. Then, for all $\epsilon, \delta \geqslant 0,(\mathrm{t}, \mathrm{t}) \in \mathcal{R}_{\phi,(\epsilon, \delta)}(\sigma)$

Proof. The proof is very similar to the unary case, by first stating a generalization and then proving it by induction on the typing judgment.

By instantiating the Basic Lemma at the type of adversaries, we get the following:

Corollary 7.5. The ADV-DP rule is sound.

\section{Soundness of the ADV-UTB rule}

Now we define the logical relation that we use to prove soundness of the upto-bad adversary rule. We use $\mathcal{B}$ to denote it, and we index it by an invariant 
$\mathbf{s}_{1}: M, \mathbf{s}_{2}: M \vdash \phi$ and a bad event $\mathbf{s}: M \vdash \chi:$

$$
\begin{aligned}
& \mathcal{B}_{\phi, \xi,(\epsilon, \delta)}(B) \triangleq\left\{b_{1}, b_{2}: B \mid b_{1}=b_{2}\right\} \\
& \mathcal{B}_{\phi, \xi,(\epsilon, \delta)}(\sigma \rightarrow \tau) \triangleq\left\{\begin{array}{l}
\left\{t_{1}, t_{2}: \sigma \rightarrow \tau \mid \begin{array}{c}
\forall u_{1}, u_{2}: \sigma \cdot\left(u_{1}, u_{2}\right) \in \mathcal{B}_{\phi, \xi,(\epsilon, \delta)}(\sigma) \\
\Rightarrow\left(t_{1} u_{1}, t_{2} u_{2}\right) \in \mathcal{B}_{\phi, \xi,(\epsilon, \delta)}(\tau)
\end{array}\right\} \operatorname{Eff}(\sigma) \subseteq \operatorname{Eff}(\tau) \\
\left\{t_{1}, t_{2}: \sigma \rightarrow \tau \mid \forall u_{1}, u_{2}: \sigma \cdot\left(t_{1} u_{1}, t_{2} u_{2}\right) \in \mathcal{B}_{\phi, \xi,(\epsilon, \delta)}(\tau)\right\} \text { otherwise }
\end{array}\right. \\
& \mathcal{B}_{\phi, \xi,(\epsilon, \delta)}(\sigma \times \tau) \triangleq\left\{\begin{array}{l|l}
t_{1}, t_{2}: \sigma \times \tau \mid \begin{array}{l}
\left(\pi_{1}\left(t_{1}\right), \pi_{1}\left(t_{2}\right)\right) \in \mathcal{B}_{\phi, \xi,(\epsilon, \delta)}(\sigma) \\
\wedge\left(\pi_{2}\left(t_{1}\right), \pi_{2}\left(t_{2}\right)\right) \in \mathcal{B}_{\phi, \xi,(\epsilon, \delta)}(\tau)
\end{array}
\end{array}\right\} \\
& \mathcal{B}_{\phi, \xi,(\epsilon, \delta)}\left(\mathcal{T}_{\Sigma, k}(\sigma)\right) \triangleq \\
& \left\{\begin{array}{l|l}
\mathrm{t}_{1}, \mathrm{t}_{2}: \mathcal{T}_{\Sigma, \mathrm{k}}(\sigma) \mid \begin{array}{l}
\mathcal{H}_{\sigma,(\mathrm{ke}, \mathrm{k} \delta)}\left(\phi, \mathrm{t}_{1}, \mathrm{t}_{2},\left(\left(\mathbf{v}_{1}, \mathbf{v}_{2}\right) \in \mathcal{B}_{\phi, \xi,(\epsilon, \delta)}(\sigma) \wedge \phi\right) \vee \xi\left[\mathbf{s}_{1} / \mathbf{s}\right]\right) \\
\wedge \mathrm{t}_{1} \in \mathcal{L}_{\xi, 0}\left(\mathcal{T}_{\Sigma, k}(\sigma)\right)
\end{array}
\end{array}\right\} \\
& \mathcal{B}_{\phi, \xi,(\epsilon, \delta)}(\forall \alpha . \tau) \triangleq\left\{t_{1}, t_{2}: \forall \alpha . \tau \mid \forall \Sigma .\left(t_{1}[\Sigma], t_{2}[\Sigma]\right) \in \mathcal{B}_{\phi, \xi,(\epsilon, \delta)}(\tau[\Sigma / \alpha])\right\}
\end{aligned}
$$

The only change to this definition with respect to the previous section is for computations of type $\mathcal{T}_{\Sigma, k}(\sigma)$. A pair of computations $\left(t_{1}, t_{2}\right)$ is in the relation if for initial memories are related by $\phi$, then the final memories and return values are related by the lifting of $\left(\mathcal{B}_{\phi, \chi,(\epsilon, \delta)}(\sigma) \wedge \phi\right) \vee \chi$, and furthermore $t_{1}$ is in the unary logical predicate $\mathcal{L}_{\chi, 0}(\delta)$, that is, it preserves the invariant $\chi$ with probability 1 . We can now show:

Lemma 7.6 (Basic lemma). Let $\ddot{\mathcal{P}}$ be as above, and assume that for every $v \in \mathcal{D}$, $(\operatorname{sample}(v)$, sample $(v)) \in \ddot{\mathcal{P}}_{(0,0)}^{d p}\left(\mathcal{R}_{\phi,(\epsilon, \delta)}(\mathrm{B})\right)$. Let $\vdash \mathrm{t}: \sigma$ be a closed term, $\mathbf{s}_{1}: \mathrm{M}, \mathbf{s}_{2}: M \vdash \phi$ and $\mathbf{s}: M \vdash \chi$ such that $\phi \in \operatorname{RSafe}(\operatorname{Eff}(\sigma))$ and $\chi \in \operatorname{Safe}(\operatorname{Eff}(\sigma))$. Then, for all $\epsilon, \delta \geqslant 0$, $(t, t) \in \mathcal{B}_{\phi, \chi,(\epsilon, \delta)}(\sigma)$

Proof. First we strengthen the lemma, and then we prove it by induction on the derivation. The only new case that is interesting is the case of binding, which we quickly sketch. Suppose we have $\Gamma \vdash t: \mathcal{T}_{\Sigma}(\tau)$ and $\Gamma, x: \tau \vdash u: \mathcal{T}_{\Sigma^{\prime}}(\sigma)$, and two substitutions $\Gamma \vdash \gamma_{1}, \Gamma \vdash \gamma_{2}$ such that for every $\left(x: \tau^{\prime}\right) \in \Gamma,\left(\gamma_{1}(x), \gamma_{2}(x)\right) \in \mathcal{B}_{\phi, \xi,(\epsilon, \delta)}\left(\tau^{\prime}\right)$. By I.H., for $\left(t \gamma_{1}, t \gamma_{2}\right)$ we can show that under the precondition $\phi$, the final memories and return values are related by the lifting of $\left(\mathcal{R}_{\phi, \chi(\epsilon, \delta)}(\tau) \wedge \phi\right) \vee \chi$. Then we have two cases for $\Gamma, x: \tau \vdash u: \mathcal{T}(\sigma)$, depending on which clause is established in the postcondition. If $\left(\mathcal{R}_{\phi, x,(\epsilon, \delta)}(\tau) \wedge \phi\right)$, then we can apply the I.H. to show that for all $\left(e_{1}, e_{2}\right) \in \mathcal{B}_{\phi, \chi,(\epsilon, \delta)}(\tau),\left(u \gamma_{1}\left[x \mapsto e_{1}\right], u \gamma_{2}\left[x \mapsto e_{2}\right]\right) \in \mathcal{B}_{\phi, \chi,(\epsilon, \delta)}(\sigma)$, and therefore $\left((\right.$ mlet $x=t$ in $u) \gamma_{1}$, (mlet $x=t$ in $\left.\left.u\right) \gamma_{2}\right) \in \mathcal{B}_{\phi, \chi,(\epsilon, \delta)}(\sigma)$. Otherwise, we have the precondition $\chi$ and by the Basic Lemma of $\mathcal{L}, \chi$, for all $e: \sigma, u \gamma_{1}[\chi \mapsto e] \in \mathcal{L}_{\xi, 0}(\sigma)$. So $\chi$ is preserved with probability 1 by the computation on the left, and it is also preserved by the computation on the right since it only depends on $\mathbf{s}_{1}$. Therefore $\Gamma \vdash$ mlet $x=t$ in $u: \mathcal{T}(\sigma)$ satisfies the relation.

And from this, we deduce: 
Corollary 7.7. The ADV-UTB rule is sound.

\subsection{Related work}

We present some work related to Chapters 4 to 7 .

\section{Reasoning about adversaries}

Garg et al. [79] develop a program logic to reason about safety properties of firstorder concurrent and stateful programs in the presence of adversaries. Jia et al. [10o] generalize the program logic to higher-order programs. Both logics contain rules - called RES and INV, respectively - to reason about adversary code based only on invariant properties of state-affecting functions available to the adversary. In spirit, this is very similar to our rules for adversaries. Devriese et al. [7o] develop sound proof principles for reasoning about adversary code. Their principles are cast in terms of parametricity properties of side-effects, an idea they call "effect parametricity". They use effect parametricity to verify programs that use object capabilities-no program logic is given, therefore reasoning must be carried directly in the model of programs. In all these works, the underlying programming language is non-probabilistic, and they only consider the unary, non-quantitative, setting.

Barthe, Grégoire and Zanella-Béguelin [24] define a probabilistic relational Hoare logic (pRHL) for reasoning about the security of cryptographic constructions. Their logic considers a probabilistic imperative language with adversarial calls and features a proof rule for adversaries. Our rule for the relational setting closely matches their rule. Barthe et al. [37] define an adversary rule for reasoning about differential privacy in a quantitative variant of pRHL; their rule uses bounds on the maximal numbers of oracle queries to derive privacy bounds of adversarial computations from privacy bounds of oracles. Barthe et al. [38] define a Union Bound logic to reason about accuracy of adversarial computations, for a similar language. However their proof rule is restricted to adversaries (called external procedures) without oracles.

\section{Other works}

Several works have studied equivalence for languages with states and modules, starting from the work of Pitts and Stark [127] to the more recent series of work on Iris [103]. Some of the techniques developed in this setting share similarities with our work. However, the addition of probabilities and the different flavours of reasoning make our problem essentially different. We will comment only on the most related works. Benton et al. [46] study a notion of proof-relevant logical relation based on effects and regions and abstract locations. They use this notion of logical relations to prove the equivalence of stateful programs. Our treatment 
of states and regions is similar to theirs. Bizjak and Birkedal [50] study logical relations and program equivalence for a higher order probabilistic programming language, and they show how to extend them to accommodate states. They do not study program logics and their model does not support all the flavours of reasoning we consider here. Atkey [12] studies the notion of parameterised monads, which are a generalized version of monads whose underlying functor is graded by a precondition and a postcondition and that compose in a very similar way to our stateful liftings. Maillard et al. [114] consider a semantics framework for reasoning about general effectful computations using the Dijkstra monad. They consider unary reasoning and focus on stateful computations, but they do not consider relational reasoning or probabilities.

\subsection{Conclusion}

Throughout the previous 4 chapters we have introduced proof principles for reasoning about adversarial computations, and embedded these principles into several program logics. There are several interesting directions for future work. First, we would like to generalize our approach to a setting where instead of relations we consider metrics. This would allow us to prove metric preservation, to explore applications to differential privacy, using ideas from [19] or to study expected sensitivity, as in [42]. Second, we would like to extend our approach to account for the cost of (adversarial) computations, and to explore applications to provable security. It would also be interesting to support our approaches and these potential extensions with an implementation that could be used to mechanize examples. 


\section{Part II}

\section{Other topics}




\section{PROBABILISTIC GUARDED RELATIONAL HIGHER-ORDER LOGIC}

Stochastic processes are often used in mathematics, physics, biology or finance to model evolution of systems with uncertainty. In particular, Markov chains are "memoryless" stochastic processes, in the sense that the evolution of the system depends only on the current state and not on its history. Perhaps the most emblematic example of a (discrete time) Markov chain is the simple random walk over the integers, that starts at $\mathrm{o}$, and that on each step moves one position either left or right with uniform probability. Let $p_{i}$ be the position at time $i$. Then, this process is described by:

$$
p_{0}=0 \quad p_{i+1}=\left\{\begin{array}{l}
p_{i}+1 \text { with probability } 1 / 2 \\
p_{i}-1 \text { with probability } 1 / 2
\end{array}\right.
$$

The goal of this chapter is to develop a programming and reasoning framework for probabilistic computations over infinite objects, such as Markov chains. Although programming and reasoning frameworks for infinite objects and probabilistic computations are well-understood in isolation, their combination is challenging. In particular, one must develop a proof system that is powerful enough for proving interesting properties of probabilistic computations over infinite objects, and practical enough to support effective verification of these properties.

A first challenge is to model probabilistic infinite objects. We focus on the case of Markov chains, due to its importance. A (discrete-time) Markov chain is a sequence of random variables $\left\{X_{i}\right\}$ over some fixed type $T$ satisfying some independence property. Thus, the straightforward way of modelling a Markov chain is as a stream of distributions over T. Going back to the simple example outlined above, it is natural to think about this kind of discrete-time Markov chain as characterized by the sequence of positions $\left\{p_{i}\right\}_{i \in \text { nat }}$, which in turn can be described as an infinite set indexed by the natural numbers. This suggests that a natural way to model such a 
Markov chain is to use streams in which each element is produced probabilistically from the previous one. However, there are some downsides to this representation. First of all, it requires explicit reasoning about probabilistic dependency, since $X_{i+1}$ depends on $X_{i}$. Also, we might be interested in global properties of the executions of the Markov chain, such as "The probability of passing through the initial state infinitely many times is $1^{\prime \prime}$. These properties are naturally expressed as properties of the whole stream. For these reasons, we want to represent Markov chains as distributions over streams. Seemingly, one downside of this representation is that the set of streams is not countable, which suggests the need for introducing heavy measure-theoretic machinery in the semantics of the programming language, even when the underlying type is discrete or finite.

Fortunately, measure-theoretic machinery can be avoided (for discrete distributions) by developing a probabilistic extension of the simply-typed guarded $\lambda$-calculus and giving a semantic interpretation in the topos of trees [63]. Informally, the simplytyped guarded $\lambda$-calculus [63] extends the simply-typed lambda calculus with a later modality, denoted by $\triangleright$. The type $\triangleright A$ ascribes expressions that are available one unit of logical time in the future. The $\triangleright$ modality allows one to model infinite types by using "finite" approximations. For example, a stream of natural numbers is represented by the sequence of its (increasing) prefixes in the topos of trees. The prefix containing the first $i$ elements has the type $S_{i} \triangleq$ nat $\times \triangleright$ nat $\times \ldots \times \triangleright^{(i-1)}$ nat, representing that the first element is available now, the second element a unit time in the future, and so on. This is the key to representing probability distributions over infinite objects without measure-theoretic semantics: We model probability distributions over non-discrete sets as discrete distributions over their (the sets') approximations. For example, a distribution over streams of natural numbers (which a priori would be non-discrete since the set of streams is uncountable) would be modelled by a sequence of distributions over the finite approximations $S_{1}, S_{2}, \ldots$ of streams. Importantly, since each $S_{i}$ is countable, each of these distributions can be discrete.

The topos of trees has a rich internal logic known as Guarded HOL, which is a higher-order logic equipped with two modalities: the later modality, introduced above, describes assertions that will be valid one step into the future; and the constant modality (denoted by $\square$ ) describes assertions that are valid at every point in time. This logic is in principle enough to prove relational properties. But as we have argued before in this thesis, this kind of proof would not exploit the program structure or structural similarities to guide the proof. To address this issue, we define a relational proof system that supports syntax-directed proofs, with necessary provisions for escaping the syntax-directed discipline when the expressions do not have the same structure. The proof system manipulates judgments of the form:

$$
\Delta|\Sigma| \Gamma \mid \Psi \vdash \mathrm{t}_{1}: A_{1} \sim \mathrm{t}_{2}: A_{2}\{\phi\}
$$

This logic is presented in a dual context style. Here $\Delta$ and $\Gamma$ are two typing 
contexts, respectively containing constant and non-constant types. Similarly $\Sigma$ and $\Psi$ respectively denote sets of constant and non-constant assertions over variables in $\Delta$ and $\Gamma$. Then, $t_{1}$ and $t_{2}$ are well-typed expressions of type $A_{1}$ and $A_{2}$, and $\phi$ is an assertion that may contain the special variables $\mathbf{r}_{1}$ and $\mathbf{r}_{2}$ that respectively correspond to the values of $t_{1}$ and $t_{2}$. The context $\Delta$ and $\Gamma$, the terms $t_{1}$ and $t_{2}$ and the types $A_{1}$ and $A_{2}$ provide a type specification, while $\Sigma, \Psi$, and $\phi$ are a logical specification.

Our relational logic comes with typing rules that allow one to reason about relational properties by exploiting as much as possible the syntactic similarities between $t_{1}$ and $t_{2}$, and to fall back on pure logical reasoning when these are not available. In order to apply relational reasoning to guarded computations the logic provides relational rules for the later and constant modalities. These rules allow the relational verification of general relational properties that go beyond the traditional notion of program equivalence and, moreover, they allow the verification of properties of guarded computations over different types.

Importantly, our approach for reasoning formally about probabilistic computations is based on probabilistic couplings. The couplings we consider in this chapter are simpler than the ones we have seen in previous chapters in two ways: they are discrete and they are exact. In this setting, $R$-couplings for some relation $R$ are usually presented by the existence of a witness joint distribution whose support is contained in R. We use a classical result by Strassen [147] that shows existence of a coupling via a universal quantification, rather than a existential one to develop a more expressive proof rule. We also develop proof rules for shift-couplings, which relate states of two Markov chains at possibly different times.

We show the flexibility of our formalism by verifying several examples of relational properties of probabilistic computations, and Markov chains in particular. These examples could not be verified with existing approaches. In particular we focus on examples about random walks that exploit the capabilities of our system involving shift relations in an infinite computation. This style of reasoning is motivated by "shift" couplings in Markov chains. In contrast to a standard coupling, which relates the states of two Markov chains at the same time $t$, a shift coupling relates the states of two Markov chains at possibly different times. Our specific example relates a standard random walk (described earlier) to a variant called a lazy random walk; the verification requires relating the state of standard random walk at time $t$ to the state of the lazy random walk at time $2 t$. We note that this kind of reasoning is impossible with conventional relational proof rules even in a non-probabilistic setting. Therefore, we provide a novel family of proof rules for reasoning about shift relations. At a high level, the rules combine a careful treatment of the later and constant modalities with a refined treatment of fixpoint operators, allowing us to relate different iterates of function bodies. 
Outline of the chapter We begin by reviewing some concepts in discrete probability theory (Section 8.1). We then present an informal overview of the system (Section 8.2) in which we discuss some examples. After that, we introduce the Guarded Lambda calculus and our probabilisic extension (Section 8.3). Then we present the logics: the base logic on the topos of trees (Section 8.4) and the relational logic we build on top (Section 8.5), and we revisit the examples from the earlier secion in fuller detail. Finally, we present related work (Section 8.6) and conclusions (Section 8.7).

\subsection{Mathematical preliminaries}

\subsubsection{Discrete probability theory}

This section reviews the definition of discrete probability distributions and introduces probabilistic couplings.

Definition 8.1 (Discrete probability distribution). Let C be a set. A (total) discrete probability distribution over $\mathrm{C}$ is a function $\mu: \mathrm{C} \rightarrow[0,1]$ such that its support, supp $\mu \triangleq$ $\{x \in C \mid \mu(x)>0\}$ is countable and $\sum_{x \in \text { supp } \mu} \mu(x)=1$. We denote the set of discrete distributions over $\mathrm{C}$ as $\mathrm{D}(\mathrm{C})$. Given a subset $\mathrm{E} \subseteq \mathrm{C}$ (called an event), the probability of sampling from $\mu$ a point in $\mathrm{E}$ is denoted $\operatorname{Pr}_{x \leftarrow \mu}[x \in \mathrm{E}]$, or $\operatorname{Pr}_{\mu}[\mathrm{E}]$, and is equal to $\sum_{x \in E} \mu(x)$.

From a distribution over a product set, we can define distributions over its components, called the marginals.

Definition 8.2 (Marginals). Let $\mu$ be a distribution over a product space $C_{1} \times C_{2}$. The first (resp. second) marginal of $\mu$ is another distribution $\mathrm{D}\left(\pi_{1}\right)(\mu)\left(\mathrm{D}\left(\pi_{2}\right)(\mu)\right)$ over $\mathrm{C}_{1}\left(\mathrm{C}_{2}\right)$ defined as:

$$
D\left(\pi_{1}\right)(\mu)(x)=\sum_{y \in C_{2}} \mu(x, y) \quad\left(D\left(\pi_{2}\right)(\mu)(y)=\sum_{x \in C_{1}} \mu(x, y)\right)
$$

\subsubsection{Discrete probabilistic couplings}

Probabilistic couplings [7] are a fundamental tool in the analysis of Markov chains. We have presented approximate couplings in Section 5.1, but in this case we use exact discrete couplings, which are much simpler to understand.

When analyzing a relation between two Markov chains it is sometimes useful to consider that the transitions are sampled from a joint distribution that "couples" the randomness in a convenient manner.

Consider for instance the case of the following Markov chain, which counts the total amount of tails observed when tossing repeatedly a biased coin with 
probability of tails $\mathrm{p}$ :

$$
n_{0}=0 \quad n_{i+1}=\left\{\begin{array}{l}
n_{i}+1 \text { with probability } p \\
n_{i} \text { with probability }(1-p)
\end{array}\right.
$$

If we have two biased coins with probabilities of tails $p$ and $q$ with $p \leqslant q$ and we respectively observe $\left\{n_{i}\right\}$ and $\left\{m_{i}\right\}$ we would expect that, in some sense, $n_{i} \leqslant m_{i}$ should hold for all $i$ (this property is known as stochastic dominance). A formal proof of this fact using elementary tools from probability theory would require to compute the cumulative distribution functions for $n_{i}$ and $m_{i}$ and then to compare them. The coupling method reduces this proof to showing a way to pair the coin flips so that if the first coin shows tails, so does the second coin.

We now review the definition of couplings and state relevant properties.

Definition 8.3 (Couplings). Let $\mu_{1} \in \mathrm{D}\left(\mathrm{C}_{1}\right)$ and $\mu_{2} \in \mathrm{D}\left(\mathrm{C}_{2}\right)$, and $\mathrm{R} \subseteq \mathrm{C}_{1} \times \mathrm{C}_{2}$.

- A distribution $\mu \in \mathrm{D}\left(\mathrm{C}_{1} \times \mathrm{C}_{2}\right)$ is a coupling for $\mu_{1}$ and $\mu_{2}$ iff its first and second marginals coincide with $\mu_{1}$ and $\mu_{2}$ respectively, i.e. $\mathrm{D}\left(\pi_{1}\right)(\mu)=\mu_{1}$ and $\mathrm{D}\left(\pi_{2}\right)(\mu)=$ $\mu_{2}$.

- A distribution $\mu \in \mathrm{D}\left(\mathrm{C}_{1} \times \mathrm{C}_{2}\right)$ is a R-coupling for $\mu_{1}$ and $\mu_{2}$ if it is a coupling for $\mu_{1}$ and $\mu_{2}$ and, moreover, $\operatorname{Pr}_{\left(x_{1}, x_{2}\right) \leftarrow \mu}\left[R x_{1} x_{2}\right]=1$, i.e., if the support of the distribution $\mu$ is included in $\mathrm{R}$.

Moreover, we write $\diamond_{\mu_{1}, \mu_{2}} . \mathrm{R}$ iff there exists a R-coupling for $\mu_{1}$ and $\mu_{2}$.

Couplings always exist. For instance, the product distribution of two distributions is always a coupling. Going back to the example about the two coins, it can be proven by computation that the following is a coupling that lifts the less-or-equal relation ( 0 indicating heads and 1 indicating tails):

$$
\begin{cases}(0,0) \mathrm{w} / \text { prob }(1-\mathrm{q}) & (0,1) \mathrm{w} / \operatorname{prob}(\mathrm{q}-\mathrm{p}) \\ (1,0) \mathrm{w} / \text { prob } 0 & (1,1) \mathrm{w} / \operatorname{prob} \mathrm{p}\end{cases}
$$

The following theorem by Strassen [147] gives a necessary and sufficient condition for the existence of R-couplings between two distributions. The theorem is remarkable in the sense that it proves an equivalence between an existentially quantified property (namely the existence of a particular coupling) and a universally quantified property (checking, for each event, an inequality between probabilities).

Theorem 8.4 (Strassen's theorem $\left.{ }^{1}\right)$. Consider $\mu_{1} \in \mathrm{D}\left(\mathrm{C}_{1}\right)$ and $\mu_{2} \in \mathrm{D}\left(\mathrm{C}_{2}\right)$, and $\mathrm{R} \subseteq$ $\mathrm{C}_{1} \times \mathrm{C}_{2}$. Then $\diamond_{\mu_{1}, \mu_{2}} . \mathrm{R}$ iff for every $\mathrm{X} \subseteq \mathrm{C}_{1}, \operatorname{Pr}_{\mathrm{x}_{1} \leftarrow \mu_{1}}\left[\mathrm{x}_{1} \in X\right] \leqslant \operatorname{Pr}_{\mathrm{x}_{2} \leftarrow \mu_{2}}\left[\mathrm{x}_{2} \in \mathrm{R}(\mathrm{X})\right]$, where $R(X)$ is the image of $X$ under $R$, i.e. $R(X)=\left\{y \in C_{2} \mid \exists x \in X . R \times y\right\}$.

\footnotetext{
${ }^{1}$ Compare this theorem with the Definition 5.2 of continuous couplings, which can be seen as an approximate version of Strassen's theorem
} 
An important property of couplings is closure under sequential composition.

Lemma 8.5 (Sequential composition couplings). Let $\mu_{1} \in D\left(C_{1}\right), \mu_{2} \in D\left(C_{2}\right), M_{1}$ : $\mathrm{C}_{1} \rightarrow \mathrm{D}\left(\mathrm{D}_{1}\right)$ and $\mathrm{M}_{2}: \mathrm{C}_{2} \rightarrow \mathrm{D}\left(\mathrm{D}_{2}\right)$. Moreover, let $\mathrm{R} \subseteq \mathrm{C}_{1} \times \mathrm{C}_{2}$ and $\mathrm{S} \subseteq \mathrm{D}_{1} \times \mathrm{D}_{2}$. Assume: (1) $\diamond_{\mu_{1}, \mu_{2}}$.R; and (2) for every $x_{1} \in C_{1}$ and $x_{2} \in C_{2}$ such that $R x_{1} x_{2}$, we have $\diamond_{M_{1}\left(x_{1}\right), M_{2}\left(x_{2}\right)}$.S. Then $\left.\diamond_{\left(\text {bind } \mu_{1}\right.} M_{1}\right),\left(\right.$ bind $\left.\mu_{2} M_{2}\right) . S$, where bind $\mu M$ is defined as

$$
\text { (bind } \mu M)(y)=\sum_{x} \mu(x) \cdot M(x)(y)
$$

We conclude this section with the following lemma, which follows from Strassen's theorem:

Lemma 8.6 (Fundamental lemma of couplings). Let $\mathrm{R} \subseteq \mathrm{C}_{1} \times \mathrm{C}_{2}, \mathrm{E}_{1} \subseteq \mathrm{C}_{1}$ and $\mathrm{E}_{2} \subseteq \mathrm{C}_{2}$ such that for every $\mathrm{x}_{1} \in \mathrm{E}_{1}$ and $\mathrm{x}_{2} \in \mathrm{C}_{2}, \mathrm{R} \mathrm{x}_{1} \mathrm{x}_{2}$ implies $\mathrm{x}_{2} \in \mathrm{E}_{2}$, i.e. $\mathrm{R}\left(\mathrm{E}_{1}\right) \subseteq \mathrm{E}_{2}$. Moreover, let $\mu_{1} \in \mathrm{D}\left(\mathrm{C}_{1}\right)$ and $\mu_{2} \in \mathrm{D}\left(\mathrm{C}_{2}\right)$ such that $\diamond_{\mu_{1}, \mu_{2}}$.R. Then

$$
\operatorname{Pr}_{1} \leftarrow \mu_{1}\left[x_{1} \in E_{1}\right] \leqslant \operatorname{Pr}_{x_{2} \leftarrow \mu_{2}}\left[x_{2} \in E_{2}\right]
$$

This lemma can be used to prove probabilistic inequalities from the existence of suitable couplings:

Corollary 8.7. Let $\mu_{1}, \mu_{2} \in \mathrm{D}(\mathrm{C})$ :

1. If $\diamond_{\mu_{1}, \mu_{2}} \cdot(=)$, then for all $x \in C, \mu_{1}(x)=\mu_{2}(x)$.

2. If $C=$ nat and $\diamond_{\mu_{1}, \mu_{2}} .(\geqslant)$, then for all $n \in$ nat, $\operatorname{Pr}_{x \leftarrow \mu_{1}}[x \geqslant n] \geqslant \operatorname{Pr}_{x \leftarrow \mu_{2}}[x \geqslant n]$

In the example at the beginning of the section, the property we want to prove is precisely that, for every $k$ and $i$, the following holds:

$$
\operatorname{Pr}_{1} \leftarrow n_{i}\left[x_{1} \geqslant k\right] \leqslant \operatorname{Pr}_{x_{2} \leftarrow m_{i}}\left[x_{2} \geqslant k\right]
$$

Since we have a $\leqslant$-coupling, this proof is immediate. This example is formalized in subsection 8.2.3.

\subsubsection{Probabilities in the category Set}

To interpret probabilistic programs in Set we need to build a monad of probability distributions in Set. The map $\mathcal{D}$ sending a set to the set of discrete distributions over it can be turned into a functor by specifying its behaviour on morphisms, namely:

$$
\mathcal{D}(f: X \rightarrow Y) \triangleq \lambda(v: \mathcal{D}(X))(y: Y)=\sum_{x \in X}[f(x)=y] \cdot v(x)
$$

where $[f(x)=y]$ has value 1 if the equality holds and o otherwise. 
Moreover, $\mathcal{D}$ can in fact be turned into a monad by setting:

$$
\begin{gathered}
\eta_{X}(x: X) \triangleq \lambda\left(x^{\prime}: X\right)\left[x=x^{\prime}\right] \\
\mu_{X}(F: \mathcal{D D}(X)) \triangleq \lambda(x: X) \sum_{v \in \mathcal{D}(X)} F(v) v(x)
\end{gathered}
$$

As every monad in Set, $\mathcal{D}$ is also strong. We can therefore use it to give semantics to the unit and bind operations of a higher-order language. This approach is enough for our uses, as we will later see, however, it has its limitions. Primarily, we cannot model continuous probability distributions, such as the Gaussian or the Laplace distributions, which are extensively used in real-life applications to privacy or machine learning.

\subsection{Overview of the system}

In this section we give a high-level overview of our system, with the details on sections 8.3, 8.4 and 8.5. We start by presenting the base logic, and then we show how to extend it with probabilities and how to build a relational reasoning system on top of it.

\subsubsection{Base logic: Guarded Higher-Order Logic}

Our starting point is the Guarded Higher-Order Logic [63] (Guarded HOL) inspired by the topos of trees. In addition to the usual constructs of HOL to reason about lambda terms, this logic features the $\triangleright$ and $\square$ modalities to reason about infinite terms, in particular streams. The $\triangleright$ modality is used to reason about objects that will be available in the future, such as tails of streams. For instance, suppose we want to define an $\operatorname{All}(s, \phi)$ predicate, expressing that all elements of a stream $s \equiv n:: x s$ satisfy a property $\phi$. This can be axiomatized as follows:

$$
\forall\left(x s: \triangleright \operatorname{Str}_{n a t}\right)(n: \text { nat }) . \phi n \Rightarrow \triangleright[s \leftarrow x s] . \operatorname{All}(s, x . \phi) \Rightarrow \operatorname{All}(n:: x s, x . \phi)
$$

We use $x . \phi$ to denote that the formula $\phi$ depends on a free variable $x$, which will get replaced by the first argument of All. We have two antecedents. The first one states that the head $n$ satisfies $\phi$. The second one, $\triangleright[s \leftarrow x s]$. All $(s, x . \phi)$, states that all elements of xs satisfy $\phi$. Formally, $x s$ is the tail of the stream and will be available in the future, so it has type $\triangleright \operatorname{Str}_{n a t}$. The delayed substitution $\triangleright[s \leftarrow x s]$ replaces $s$ of type $\operatorname{Str}_{n a t}$ with $x$ s of type $\triangleright \operatorname{Str}_{n a t}$ inside All and shifts the whole formula one step into the future. In other words, $\triangleright[s \leftarrow x s]$. All $(s, x . \phi)$ states that $\operatorname{All}(-, x . \phi)$ will be satisfied by $x \mathrm{~s}$ in the future, once it is available.

On the other hand, the constant modality $\square$ is used to denote that a formula holds at every step. 


\subsubsection{A system for relational reasoning}

As we have argued before in this thesis, when proving relational properties it is often convenient to build proofs guided by the syntactic structure of the two expressions to be related. This is particularly convenient when both expressions have the same structure and control flow. But, at the same time a strict adherence to the syntax-directed discipline is detrimental to the expressiveness of the system; for instance, it makes it difficult or even impossible to reason about structurally dissimilar terms. To achieve the best of both worlds, we present a relational proof system built on top of Guarded HOL, which we call Guarded RHOL. Judgments have the shape:

$$
\Delta|\Sigma| \Gamma \mid \Psi \vdash \mathrm{t}_{1}: \mathrm{A}_{1} \sim \mathrm{t}_{2}: \mathrm{A}_{2}\{\phi\}
$$

where $\phi$ is a logical formula that may contain two distinguished variables $\mathbf{r}_{1}$ and $\mathbf{r}_{2}$ that respectively represent the expressions $t_{1}$ and $t_{2}$. This judgment subsumes two typing judgments on $t_{1}$ and $t_{2}$ and a relation $\phi$ on these two expressions. As in Chapter 3 the proof system combines rules of two different flavors: two-sided rules, which relate expressions with the same top-level constructs, and one-sided rules, which operate on a single expression.

We then extend Guarded HOL with a modality $\diamond$ that lifts assertions over types $A_{1}$ and $A_{2}$ to assertions over $D\left(A_{1}\right)$ and $D\left(A_{2}\right)$. Concretely, we define for every assertion $\phi$, variables $x_{1}$ and $x_{2}$ of type $A_{1}$ and $A_{2}$ respectively, and expressions $t_{1}$ and $t_{2}$ of type $D\left(A_{1}\right)$ and $D\left(A_{2}\right)$ respectively, the modal assertion $\diamond\left[x_{1} \leftarrow t_{1}, x_{2} \leftarrow t_{2}\right] \phi$ which holds iff the interpretations of $t_{1}$ and $t_{2}$ are related by the probabilistic lifting of the interpretation of $\phi$. We call this new logic Probabilistic Guarded HOL.

We accordingly extend the relational proof system to support reasoning about probabilistic expressions by adding judgments of the form:

$$
\Delta|\Sigma| \Gamma \mid \Psi \vdash \mathrm{t}_{1}: \mathrm{D}\left(A_{1}\right) \sim \mathrm{t}_{2}: \mathrm{D}\left(A_{2}\right)\left\{\diamond_{\left[x_{1} \leftarrow \mathbf{r}_{1}, x_{2} \leftarrow \mathbf{r}_{2}\right]} \phi\right\}
$$

expressing that $t_{1}$ and $t_{2}$ are distributions related by a $\phi$-coupling. We call this proof system Probabilistic Guarded RHOL. These judgments can be built by using the following rule, that lifts relational judgments over types $A_{1}$ and $A_{2}$ to judgments over distribution types $D\left(A_{1}\right)$ and $D\left(A_{2}\right)$ when the premises of Strassen's theorem are satisfied.

$$
\frac{\Delta|\Sigma| \Gamma \mid \Psi \vdash \forall X_{1} \subseteq C_{1} \cdot P_{y_{1} \leftarrow t_{1}}\left[y_{1} \in X_{1}\right] \leqslant \operatorname{Pr}_{y_{2} \leftarrow t_{2}}\left[\exists y_{1} \in X_{1} \cdot \phi\right]}{\Delta|\Sigma| \Gamma \mid \Psi \vdash t_{1}: D\left(C_{1}\right) \sim t_{2}: D\left(C_{2}\right)\left\{\diamond_{\left[y_{1} \leftarrow r_{1}, y_{2} \leftarrow r_{2}\right]} \phi\right\}} \text { COUPLING }
$$

Note that this differs with the presentation from Chapter 5 , in the sense that we internalize the lifting into our logic by using the $\diamond$ modality, rather than defining another kind of judgment for monadic types. The presentation with the $\diamond$ modality makes it simpler to deal with functions returning distribution types, which will be useful for reasoning about Markov chains. 
Recall that (discrete time) Markov chains are "memoryless" probabilistic processes, whose specification is given by a (discrete) set $C$ of states, an initial state $s_{0}$ and a probabilistic transition function step : $C \rightarrow D(C)$, where $D(S)$ represents the set of discrete distributions over C. As explained in the introduction, a convenient modeling of Markov chains is by means of probabilistic streams, i.e. to model a Markov chain as an element of $D\left(\operatorname{Str}_{C}\right)$, where $C$ is its underlying state space. To model Markov chains, we introduce a markov operator with type $\mathrm{C} \rightarrow(\mathrm{C} \rightarrow \mathrm{D}(\mathrm{C})) \rightarrow \mathrm{D}\left(\mathrm{Str}_{\mathrm{C}}\right)$ that, given an initial state and a transition function, returns a distribution over streams. We can reason about Markov chains by the Markov rule (the context, omitted, does not change):

$$
\begin{aligned}
& \vdash \mathrm{t}_{1}: \mathrm{C}_{1} \sim \mathrm{t}_{2}: \mathrm{C}_{2}\{\phi\} \\
& \vdash \mathrm{h}_{1}: \mathrm{C}_{1} \rightarrow \mathrm{D}\left(\mathrm{C}_{1}\right) \sim \mathrm{h}_{2}: \mathrm{C}_{2} \rightarrow \mathrm{D}\left(\mathrm{C}_{2}\right)\left\{\psi_{3}\right\} \\
& \vdash \psi_{4}
\end{aligned}
$$

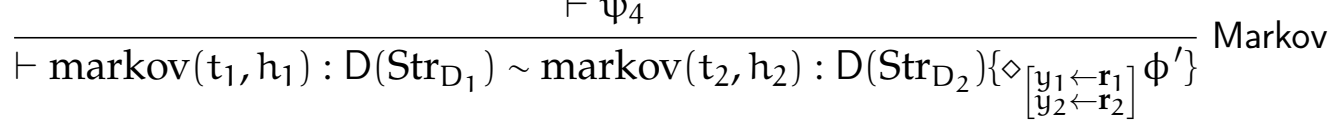

$$
\begin{aligned}
& \text { where }\left\{\begin{array}{c}
\psi_{3} \equiv \forall x_{1} x_{2} \cdot \phi\left[x_{1} / \mathbf{r}_{1}\right]\left[x_{2} / \mathbf{r}_{2}\right] \Rightarrow \diamond_{\left[y_{1} \leftarrow \mathbf{r}_{1} x_{1}, y_{2} \leftarrow \mathbf{r}_{2} x_{2}\right]} \phi\left[y_{1} / \mathbf{r}_{1}\right]\left[y_{2} / \mathbf{r}_{2}\right] \\
\psi_{4} \equiv \forall x_{1} x_{2} x s_{1} x s_{2} \cdot \phi\left[x_{1} / \mathbf{r}_{1}\right]\left[x_{2} / \mathbf{r}_{2}\right] \Rightarrow \triangleright\left[y_{1} \leftarrow x_{1}, y_{2} \leftarrow x s_{2}\right] . \phi^{\prime} \Rightarrow \\
\phi^{\prime}\left[x_{1}:: x s_{1} / y_{1}\right]\left[x_{2}:: x s_{2} / y_{2}\right]
\end{array}\right.
\end{aligned}
$$

Informally, the rule stipulates the existence of an invariant $\phi$ over states. The first premise insists that the invariant hold on the initial states, the condition $\psi_{3}$ states that the transition functions preserve the invariant, and $\psi_{4}$ states that the invariant $\phi$ over pairs of states can be lifted to a stream property $\phi^{\prime}$.

Other rules of the logic are given in Figure 8.1. The language construct unit creates a point distribution whose entire mass is at its argument. Accordingly, the [UNIT] rule creates a straightforward coupling. The [MLET] rule internalizes sequential composition of couplings (Lemma 8.5) into the proof system. The construct mlet $\mathrm{x}=\mathrm{t}$ in $\mathrm{t}^{\prime}$ composes a distribution $\mathrm{t}$ with a probabilistic computation $t^{\prime}$ with one free variable $x$ by sampling $x$ from $t$ and running $t^{\prime}$. The [MLET-L] rule supports one-sided reasoning about mlet $x=t$ in $t^{\prime}$ and relies on the fact that couplings are closed under convex combinations. Note that one premise of the rule uses a unary judgment, with a non-relational modality $\diamond_{[x \leftarrow r]} \phi$ whose informal meaning is that $\phi$ holds with probability 1 in the distribution $\mathbf{r}$.

The following table summarizes the different base logics we consider,the relational systems we build on top of them, including the ones presented in Chapter 3 , and the equivalences between both sides: 


$$
\begin{aligned}
& \frac{\Delta|\Sigma| \Gamma \mid \Psi \vdash \mathrm{t}_{1}: \mathrm{C}_{1} \sim \mathrm{t}_{2}: \mathrm{C}_{2}\left\{\phi\left[\mathbf{r}_{1} / \mathrm{x}_{1}, \mathbf{r}_{2} / \mathrm{x}_{2}\right]\right\}}{\Delta|\Sigma| \Gamma \mid \Psi \vdash \operatorname{unit}\left(\mathrm{t}_{1}\right): \mathrm{D}\left(\mathrm{C}_{1}\right) \sim \operatorname{unit}\left(\mathrm{t}_{2}\right): \mathrm{D}\left(\mathrm{C}_{2}\right)\left\{{ }_{\left[\mathrm{x}_{1} \leftarrow \mathbf{r}_{1}, \mathrm{x}_{2} \leftarrow \mathbf{r}_{2}\right]} \phi\right\}} \text { UNIT } \\
& \Delta|\Sigma| \Gamma \mid \Psi \vdash \mathrm{t}_{1}: \mathrm{D}\left(\mathrm{C}_{1}\right) \sim \mathrm{t}_{2}: \mathrm{D}\left(\mathrm{C}_{2}\right)\left\{\diamond_{\left[x_{1} \leftarrow \mathbf{r}_{1}, x_{2} \leftarrow \mathbf{r}_{2}\right]} \phi\right\}
\end{aligned}
$$

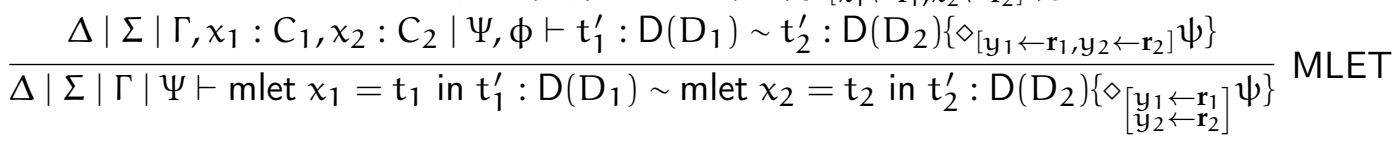

$$
\begin{aligned}
& \Delta|\Sigma| \Gamma \mid \Psi \vdash \mathrm{t}_{1}: \mathrm{D}\left(\mathrm{C}_{1}\right)\left\{\diamond_{[\mathrm{x} \leftarrow \mathbf{r}]} \phi\right\} \\
& \frac{\Delta|\Sigma| \Gamma, x_{1}: C_{1} \mid \Psi, \phi \vdash \mathrm{t}_{1}^{\prime}: \mathrm{D}\left(\mathrm{D}_{1}\right) \sim \mathrm{t}_{2}^{\prime}: \mathrm{D}\left(\mathrm{D}_{2}\right)\left\{\diamond_{\left[y_{1} \leftarrow \mathbf{r}_{1}, y_{2} \leftarrow \mathbf{r}_{2}\right]} \psi\right\}}{\Delta|\Sigma| \Gamma \mid \Psi \vdash \text { mlet } x_{1}=\mathrm{t}_{1} \text { in } \mathrm{t}_{1}^{\prime}: \mathrm{D}\left(\mathrm{D}_{1}\right) \sim \mathrm{t}_{2}^{\prime}: \mathrm{D}\left(\mathrm{D}_{2}\right)\left\{\diamond_{\left[\mathrm{y}_{1} \leftarrow \mathbf{r}_{1}, \mathrm{y}_{2} \leftarrow \mathbf{r}_{2}\right]} \psi\right\}} \text { MLET }-\mathrm{L}
\end{aligned}
$$

\begin{tabular}{|c|c|c|}
\hline Relational logic & & Base logic \\
\hline $\begin{array}{l}\text { RHOL } \$ 3.5 \\
\Gamma\left|\Psi \vdash \mathrm{t}_{1} \sim \mathrm{t}_{2}\right| \phi\end{array}$ & $\stackrel{\operatorname{Thm} 3.6}{\Longleftrightarrow}$ & $\begin{array}{l}\text { HOL } \S 3 \cdot 3 \\
\Gamma \mid \Psi \vdash \phi\left[\mathrm{t}_{1} / \mathbf{r}_{1}\right]\left[\mathrm{t}_{2} / \mathbf{r}_{2}\right]\end{array}$ \\
\hline $\begin{array}{l}\text { Guarded RHOL } \S 8.5 \\
\Delta|\Sigma| \Gamma\left|\Psi \vdash \mathrm{t}_{1} \sim \mathrm{t}_{2}\right| \phi\end{array}$ & $\stackrel{\operatorname{Thm} 8.13}{\Longleftrightarrow}$ & $\begin{array}{l}\text { Guarded HOL }\left[6_{3}\right] \\
\Delta|\Sigma| \Gamma \mid \Psi \vdash \phi\left[\mathrm{t}_{1} / \mathbf{r}_{1}\right]\left[\mathrm{t}_{2} / \mathbf{r}_{2}\right]\end{array}$ \\
\hline $\begin{array}{l}\text { Probabilistic Guarded RHOL } \S 8.5 \\
\Delta|\Sigma| \Gamma\left|\Psi \vdash \mathrm{t}_{1} \sim \mathrm{t}_{2}\right| \diamond_{\left[y_{1} \leftarrow \mathbf{r}_{1}, y_{2} \leftarrow \mathbf{r}_{2}\right]} . \phi\end{array}$ & $\operatorname{Thm} 8.13$ & $\begin{array}{l}\text { Probabilistic Guarded HOL } \S 8.4 \\
\Delta|\Sigma| \Gamma \mid \Psi \vdash \diamond_{\left[y_{1} \leftarrow t_{1}, y_{2} \leftarrow t_{2}\right]} . \phi\end{array}$ \\
\hline
\end{tabular}

Figure 8.1: Proof rules for probabilistic constructs

\subsubsection{Examples}

We formalize elementary examples from the literature on security and Markov chains. None of these examples can be verified in prior systems. Lumping of random walks cannot even be stated in prior systems because the two related expressions in these examples have different types. The random walk vs lazy random walk (shift coupling) cannot be proved in prior systems because it requires either asynchronous reasoning or code rewriting. Finally, the biased coin example (stochastic dominance) cannot be proved in prior work because it requires Strassen's formulation of the existence of coupling (rather than a bijection-based formulation) or code rewriting. We give additional details below.

\subsubsection{Stochastic dominance}

Stochastic dominance defines a partial order between random variables whose underlying set is itself a partial order; it has many different applications in statistical biology (e.g. in the analysis of the birth-and-death processes), statistical physics (e.g. in percolation theory), and economics. First-order stochastic dominance, which we define below, is also an important application of probabilistic couplings. We 
demonstrate how to use our proof system for proving (first-order) stochastic dominance for a simple Markov process which samples biased coins. While the example is elementary, the proof method extends to more complex examples of stochastic dominance, and illustrates the benefits of Strassen's formulation of the coupling rule over alternative formulations stipulating the existence of bijections (explained later).

We start by recalling the definition of (first-order) stochastic dominance for the $\mathbb{N}$-valued case. The definition extends to arbitrary partial orders.

Definition 8.8 (Stochastic dominance). Let $\mu_{1}, \mu_{2} \in \mathrm{D}(\mathbb{N})$. We say that $\mu_{2}$ stochastically dominates $\mu_{1}$, written $\mu_{1} \leqslant S D \mu_{2}$, iff for every $n \in \mathbb{N}$,

$$
\operatorname{Pr}_{x \leftarrow \mu_{1}}[x \geqslant n] \leqslant \operatorname{Pr}_{x \leftarrow \mu_{2}}[x \geqslant n]
$$

The following result, equivalent to 8.7, characterizes stochastic dominance using probabilistic couplings.

Proposition 8.9. Let $\mu_{1}, \mu_{2} \in \mathrm{D}(\mathbb{N})$. Then $\mu_{1} \leqslant S D \mu_{2}$ iff $\diamond_{\mu_{1}, \mu_{2}}$. $(\leqslant)$.

We now turn to the definition of the Markov chain. For $p \in[0,1]$, we consider the parametric $\mathbb{N}$-valued Markov chain coins $\triangleq \operatorname{markov}(0, h)$, with initial state 0 and (parametric) step function:

$$
h \triangleq \lambda x \text {.mlet } b=\operatorname{Bern}(p) \text { in unit }(x+b)
$$

where, for $p \in[0,1]$, Bern( $p)$ is the Bernoulli distribution on $\{0,1\}$ with probability $p$ for 1 and $1-p$ for 0 . Our goal is to establish that coins is monotonic, i.e. for every $p_{1}, p_{2} \in[0,1], p_{1} \leqslant p_{2}$ implies coins $p_{1} \leqslant S D$ coins $p_{2}$. We formalize this statement as

$$
\vdash \text { coins : }[0,1] \rightarrow \mathrm{D}\left(\operatorname{Str}_{\mathbb{N}}\right) \sim \text { coins }:[0,1] \rightarrow \mathrm{D}\left(\operatorname{Str}_{\mathbb{N}}\right)\{\psi\}
$$

where $\psi \triangleq \forall p_{1}, p_{2} \cdot p_{1} \leqslant p_{2} \Rightarrow \diamond_{\left[y_{1} \leftarrow \mathbf{r}_{1}, y_{2} \leftarrow \mathbf{r}_{2}\right]} \operatorname{All}\left(y_{1}, y_{2}, z_{1} \cdot z_{2} \cdot z_{1} \leqslant z_{2}\right)$. The crux of the proof is to establish stochastic dominance for the Bernoulli distribution:

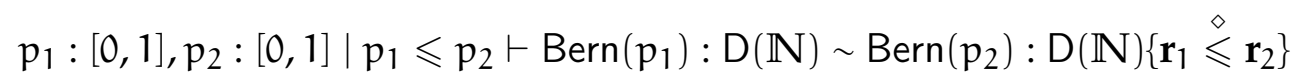

where we use $e_{1} \stackrel{\gtrless}{\leqslant} e_{2}$ as shorthand for $\diamond_{\left[y_{1} \leftarrow e_{1}, y_{2} \leftarrow e_{2}\right]} y_{1} \leqslant y_{2}$. This is proved directly by the COUPLING rule and checking by simple calculations that the premise of the rule is valid.

We briefly explain how to conclude the proof. Let $h_{1}$ and $h_{2}$ be the step functions for $p_{1}$ and $p_{2}$ respectively. It is clear from the above that (context omitted):

$$
x_{1} \leqslant x_{2} \vdash h_{1} x_{1}: D(\text { bool }) \sim h_{2} x_{2}: D(\text { bool })\left\{\diamond_{\left[y_{1} \leftarrow r_{1}, y_{2} \leftarrow \mathbf{r}_{2}\right]} \cdot y_{1} \leqslant y_{2}\right\}
$$


and by the definition of All:

$$
x_{1} \leqslant x_{2} \Rightarrow \operatorname{All}\left(x_{1}, x s_{2}, z_{1} \cdot z_{2} . z_{1} \leqslant z_{2}\right) \Rightarrow \operatorname{All}\left(x_{1}:: \triangleright x_{1}, x_{2}:: \triangleright x_{2}, z_{1} \cdot z_{2} . z_{1} \leqslant z_{2}\right)
$$

So, we can conclude by applying the Markov rule.

It is instructive to compare our proof with prior formalizations, and in particular with the proof in [31]. Their proof is carried out in the pRHL logic, whose [COUPLING] rule is based on the existence of a bijection that satisfies some property, rather than on our formalization based on Strassen's Theorem. Their rule is motivated by applications in cryptography, and works well for many examples, but is inconvenient for our example at hand, which involves non-uniform probabilities. Indeed, their proof is based on code rewriting, and is done in two steps. First, they prove equivalence between sampling and returning $x_{1}$ from $\operatorname{Bern}\left(\mathfrak{p}_{1}\right)$; and sampling $z_{1}$ from $\operatorname{Bern}\left(\mathrm{p}_{2}\right), z_{2}$ from $\operatorname{Bern}\left({ }^{\mathfrak{p}_{1}} / \mathrm{p}_{2}\right)$ and returning $z=z_{1} \wedge z_{2}$. Then, they find a coupling between $z$ and $\operatorname{Bern}\left(\mathrm{p}_{2}\right)$.

\subsubsection{Shift coupling: random walk vs lazy random walk}

The previous example is an instance of a lockstep coupling, in that it relates the $k$-th element of the first chain with the k-th element of the second chain. Many examples from the literature follow this lockstep pattern; however, it is not always possible to establish lockstep couplings. Shift couplings are a relaxation of lockstep couplings where we relate elements of the first and second chains without the requirement that their positions coincide.

We consider a simple example that motivates the use of shift couplings. Consider the random walk and lazy random walk (which, at each time step, either chooses to move or stay put), both defined as Markov chains over $\mathbb{Z}$. For simplicity, assume that both walks start at position o. It is not immediate to find a coupling between the two walks, since the two walks necessarily get desynchronized whenever the lazy walk stays put. Instead, the trick is to consider a lazy random walk that moves two steps instead of one. The random walk and the lazy random walk of step 2 are defined by the step functions:

$$
\begin{aligned}
& \text { step } \triangleq \lambda x \text {.mlet } z=U_{\{-1,1\}} \text { in unit }(z+x) \\
& \text { 1step2 } \triangleq \lambda x \text {.mlet } z=U_{\{-1,1\}} \text { in mlet } b=\mathcal{U}_{\{0,1\}} \text { in unit }(x+2 * z * b)
\end{aligned}
$$

After 2 iterations of step, the position has either changed two steps to the left or to the right, or has returned to the initial position, which is the same behavior lstep2 has on every iteration. Therefore, the coupling we want to find should equate the elements at position $2 i$ in step with the elements at position $i$ in lstep2. The details on how to prove the existence of this coupling are in section 8.5. 


\subsubsection{Lumped coupling: random walks on 3 and 4 dimensions}

A Markov chain is recurrent if it has probability 1 of returning to its initial state, and transient otherwise. A classic result by Pólya [131] states that the random walk over $\mathbb{Z}$ is recurrent as well as the random walk over $\mathbb{Z}^{2}$. However, the random walk over $\mathbb{Z}^{3}$ is transient.

For higher dimensions, we can use a coupling argument to prove transience. Specifically, we can define a coupling between a lazy random walk in $n$ dimensions and a random walk in $n+m$ dimensions, and derive transience of the latter from transience of the former. We define the (lazy) random walks below, and sketch the coupling arguments.

Specifically, we show here the particular case of the transience of the 4-dimensional random walk from the transience of the 3-dimensional lazy random walk. We start by defining the stepping functions:

$$
\begin{aligned}
\operatorname{step}_{4} & : \mathbb{Z}^{4} \rightarrow \mathrm{D}\left(\mathbb{Z}^{4}\right) \triangleq \lambda z_{1} \cdot \text { mlet } x_{1}=\text { Unif }_{\mathrm{U}_{4}} \text { in unit }\left(z_{1}+{ }_{4} x_{1}\right) \\
\text { lstep }_{3} & : \mathbb{Z}^{3} \rightarrow \mathrm{D}\left(\mathbb{Z}^{3}\right) \triangleq \lambda z_{2} \cdot \text { mlet } x_{2}=\text { Unif }_{\mathrm{U}_{3}} \text { in mlet } \mathrm{b}_{2}=\operatorname{Bern}(3 / 4) \text { in unit }\left(z_{2}+{ }_{3} \mathrm{~b}_{2} * x_{2}\right)
\end{aligned}
$$

where $U_{i}=\{( \pm 1,0, \ldots 0), \ldots,(0, \ldots, 0, \pm 1)\}$ are the vectors of the basis of $\mathbb{Z}^{i}$ and their opposites. The random walk of dimension 4 is modeled by rwalk $4 \triangleq \operatorname{markov}\left(0, \operatorname{step}_{4}\right)$, and the lazy walk of dimension 3 is modeled by lwalk $3 \triangleq \operatorname{markov}\left(0, \operatorname{step}_{3}\right)$. We want to prove:

$$
\vdash \operatorname{rwalk}_{4}: \mathrm{D}\left(\operatorname{Str}_{\mathbb{Z}^{4}}\right) \sim \operatorname{lwalk}_{3}: \mathrm{D}\left(\operatorname{Str}_{\mathbb{Z}^{3}}\right)\left\{\diamond\left[\begin{array}{l}
y_{1} \leftarrow \mathbf{r}_{1} \\
y_{2} \leftarrow \mathbf{r}_{2}
\end{array}\right] \text { All }\left(y_{1}, y_{2}, z_{1} \cdot z_{2} \cdot \operatorname{pr}_{3}^{4}\left(z_{1}\right)=z_{2}\right)\right\}
$$

where $\operatorname{pr}_{n_{1}}^{n_{2}}$ denotes the standard projection from $\mathbb{Z}^{n_{2}}$ to $\mathbb{Z}^{n_{1}}$.

We apply the Markov rule. The only interesting premise requires proving that the transition function preserves the coupling:

$$
p_{2}=\operatorname{pr}_{3}^{4}\left(p_{1}\right) \vdash \operatorname{step}_{4} \sim \operatorname{lstep}_{3} \mid \forall x_{1} x_{2} \cdot x_{2}=\operatorname{pr}_{3}^{4}\left(x_{1}\right) \Rightarrow \diamond_{\left[\begin{array}{lll}
y_{1} \leftarrow \mathbf{r}_{1} & x_{1} \\
y_{2} \leftarrow \mathbf{r}_{2} & x_{2}
\end{array}\right]} \operatorname{pr}_{3}^{4}\left(y_{1}\right)=y_{2}
$$

To prove this, we need to find the appropriate coupling, i.e., one that preserves the equality. The idea is that the step in $\mathbb{Z}^{3}$ must be the projection of the step in $\mathbb{Z}^{4}$. This corresponds to the following judgment:

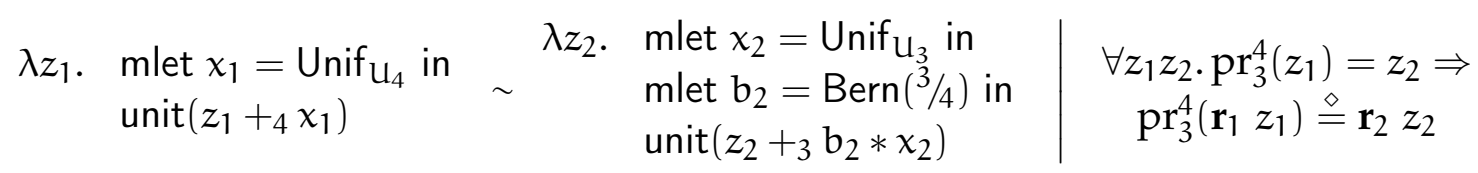

which by simple equational reasoning is the same as

$$
\begin{aligned}
& \lambda z_{1} \text {. mlet } x_{1}=\text { Unif }_{\mathrm{U}_{4}} \text { in } \sim \lambda z_{2} \text {. mlet } \mathrm{p}_{2}=\text { Unif }_{\mathrm{U}_{3}} \times \operatorname{Bern}(3 / 4) \text { in } \quad \forall z_{1} z_{2} \cdot \operatorname{pr}_{3}^{4}\left(z_{1}\right)=z_{2} \Rightarrow \\
& \operatorname{unit}\left(z_{1}+{ }_{4} x_{1}\right) \sim \operatorname{unit}\left(z_{2}+{ }_{3} \pi_{1}\left(\mathrm{p}_{2}\right) * \pi_{2}\left(\mathrm{p}_{2}\right)\right) \quad \operatorname{pr}_{3}^{4}\left(\mathbf{r}_{1} z_{1}\right) \stackrel{\ominus}{=} \mathbf{r}_{2} z_{2}
\end{aligned}
$$


We want to build a coupling such that if we sample $(0,0,0,1)$ or $(0,0,0,-1)$ from Unif $_{\mathrm{U}_{3}}$, then we sample 0 from Bern $(3 / 4)$, and otherwise if we sample $\left(x_{1}, x_{2}, x_{3}, 0\right)$ from $U_{n i f} u_{4}$, we sample $\left(x_{1}, x_{2}, x_{3}\right)$ from $U_{3}$. Formally, we prove this with the Coupling rule. Given $X: U_{4} \rightarrow$ bool, by simple computation we show that:

$$
\operatorname{Pr}_{z_{1} \sim \operatorname{Unif}_{\mathrm{u}_{4}}}\left[z_{1} \in X\right] \leqslant \underset{z_{2} \sim \operatorname{Unif}_{\mathrm{u}_{3}} \times \operatorname{Pern}(3 / 4)}{\operatorname{Pr}}\left[z_{2} \in\left\{y \mid \exists x \in X \cdot \operatorname{pr}_{3}^{4}(x)=\pi_{1}(y) * \pi_{2}(y)\right\}\right]
$$

This concludes the proof. From the previous example, it follows that the lazy walk in 3 dimensions is transient, since the random walk in 3 dimensions is transient. By simple reasoning, we now conclude that the random walk in 4 dimensions is also transient.

\subsection{Probabilistic Guarded Lambda Calculus}

To ensure that a function on infinite datatypes is well-defined, one must check that it is productive. This means that any finite prefix of the output can be computed in finite time. For instance, consider the following function on streams:

$$
\text { letrec bad }(\mathrm{x}: \mathrm{xs})=\mathrm{x}: \operatorname{tail}(\operatorname{bad} \mathrm{xs})
$$

This function is not productive since only the first element can be computed. We can argue this as follows: Suppose that the tail of a stream is available one unit of time after its head, and that that $\mathrm{x}$ : $\mathrm{xs}$ is available at time o. How much time does it take for bad to start outputting its tail? Assume it takes $k$ units of time. This means that tail (bad xs) will be available at time $k+1$, since $x s$ is only available at time 1 . But tail (bad xs) is exactly the tail of $\operatorname{bad}(x: x s)$, and this is a contradiction, since $\mathrm{x}$ : $\mathrm{xs}$ is available at time $\mathrm{o}$ and therefore the tail of $\operatorname{bad}(\mathrm{x}: \mathrm{xs})$ should be available at time $k$. Therefore, the tail of bad will never be available.

The guarded lambda calculus solves the productivity problem by distinguishing at type level between data that is available now and data that will be available in the future, and restricting when fixpoints can be defined. Specifically, the guarded lambda calculus extends the usual simply typed lambda calculus with two modalities: $\triangleright$ (pronounced later) and $\square$ (constant). The later modality represents data that will be available one step in the future, and is introduced and removed by the term formers $\triangleright$ and prev respectively. This modality is used to guard recursive occurrences, so for the calculus to remain productive, we must restrict when it can be eliminated. This is achieved via the constant modality, which expresses that all the data is available at all times. In the remainder of this section we present a probabilistic extension of this calculus.

Syntax Types of the calculus are defined by the grammar

$$
A, B::=b \mid \text { nat }|A \times B| A+B|A \rightarrow B| \operatorname{Str}_{A}|\square A| \triangleright A \mid D(A)
$$


where $b$ ranges over a collection of base types. $\operatorname{Str}_{A}$ is the type of guarded streams of elements of type A. Formally, the type $\operatorname{Str}_{A}$ is isomorphic to $A \times \triangleright \operatorname{Str}_{A}$. This isomorphism gives a way to introduce streams with the function (::) :A $\rightarrow \triangleright \operatorname{Str}_{A} \rightarrow$ $\operatorname{Str}_{A}$ and to eliminate them with the functions hd : $\operatorname{Str}_{A} \rightarrow A$ and $t \mathrm{t}: \operatorname{Str}_{A} \rightarrow \triangleright \operatorname{Str}_{A}$. $D(A)$ is the type of distributions over $A$.

We also need to distinguish between arbitrary types $A, B$ and constant types $S, T$, which are defined by the following grammar

$$
\mathrm{S}, \mathrm{T}::=\mathrm{b}_{\mathrm{C}} \mid \text { nat }|\mathrm{S} \times \mathrm{T}| \mathrm{S}+\mathrm{T}|\mathrm{S} \rightarrow \mathrm{T}| \square \mathrm{A}
$$

where $b_{C}$ is a collection of constant base types. Note in particular that for any type A the type $\square A$ is constant.

The terms of the language $t$ are defined by the following grammar

$$
\begin{aligned}
& \mathrm{t}::=x|\mathrm{c}| 0 \mid \text { St } \mid \text { case } \mathrm{t} \text { of } 0 \mapsto t ; S \mapsto t|\mu| \text { unit }(\mathrm{t}) \mid \text { mlet } x=t \text { in } \mathrm{t} \\
& |\langle t, t\rangle| \pi_{1} t\left|\pi_{2} t\right| \operatorname{inj}_{1} t\left|\operatorname{inj}_{2} t\right| \text { case } t \text { of inj }_{1} x . t ; \text { inj }_{2} \text { y.t }|\lambda x . t| t t \mid \text { fix } x . t \\
& \mid t:: \text { ts } \mid \text { hd } t|t l t| \text { box } t \mid \text { letb } x \leftarrow t \text { in } t \mid \text { letc } x \leftarrow t \text { in } t \mid \triangleright \xi \text {.t } \mid \text { prev } t
\end{aligned}
$$

where $\xi$ is a delayed substitution, a sequence of bindings $\left[x_{1} \leftarrow t_{1}, \ldots, x_{n} \leftarrow t_{n}\right]$. The terms $\mathrm{c}$ are constants corresponding to the base types used and unit $(t)$ and mlet $x=$ $t$ in $t$ are the introduction and sequencing construct for probability distributions. The meta-variable $\mu$ stands for base distributions like Unif $C_{C}$ and Bern $(p)$.

Delayed substitutions were introduced in [51] in a dependent type theory to be able to work with types dependent on terms of type $\triangleright A$. In the setting of a simple type theory, such as the one considered in this chapter, delayed substitutions are equivalent to having the applicative structure [116] $\circledast$ for the $\triangleright$ modality. However, delayed substitutions extend uniformly to the level of propositions, and thus we choose to use them in this chapter in place of the applicative structure.

Denotational semantics The meaning of terms is given by a denotational model in the category $\mathcal{S}$ of presheaves over $\omega$, the first infinite ordinal. This category $\delta$ is also known as the topos of trees [49]. In previous work [63], it was shown to model most of the constructions of the guarded lambda calculus and its internal logic, with the notable exception of the probabilistic features. Below we give an elementary presentation of the semantics.

Informally, the idea behind the topos of trees is to represent (infinite) objects from their finite approximations, which we observe incrementally as time passes. Given an object $x$, we can consider a sequence $\left\{x_{i}\right\}$ of its finite approximations observable at time $i$. These are trivial for finite objects, such as a natural number, since for any number $n, n_{i}=n$ at every $i$. But for infinite objects such as streams, the $i$ th approximation is the prefix of length $i+1$.

Concretely, the category $\mathcal{S}$ consists of: 
- Objects $X$ : families of sets $\left\{X_{i}\right\}_{i \in \text { nat }}$ together with restriction functions $r_{n}^{X}: X_{n+1} \rightarrow$ $X_{n}$. We will write simply $r_{n}$ if $X$ is clear from the context.

- Morphisms $X \rightarrow Y$ : families of functions $\alpha_{n}: X_{n} \rightarrow Y_{n}$ commuting with restriction functions in the sense of $r_{n}^{Y} \circ \alpha_{n+1}=\alpha_{n} \circ r_{n}^{X}$. Moreover if $x \in X_{i}$ and $j \leqslant i$ we will write $x \uparrow_{j}$ for the element $r_{j}\left(\cdots\left(r_{i-1}(x)\right) \cdots\right) \in X_{j}$

The topos of trees is a CCC, and as such can interpret product and arrow types via products and exponentials. The full interpretation of types of the calculus can be found in Figure 8.2.

$$
\begin{aligned}
& \llbracket \mathrm{b} \rrbracket \triangleq \text { chosen object of } \delta \\
& \llbracket \text { nat } \rrbracket \triangleq \text { nat } \stackrel{\text { id }}{\longleftarrow} \text { nat } \stackrel{\text { id }}{\longleftarrow} \text { nat } \stackrel{\text { id }}{\longleftarrow} \ldots \\
& \llbracket A \times B \rrbracket \triangleq \llbracket A \rrbracket_{0} \times \llbracket B \rrbracket_{0} \stackrel{r_{0} \times r_{0}}{\longleftarrow} \llbracket A \rrbracket_{1} \times \llbracket B \rrbracket_{1} \stackrel{r_{1} \times r_{1}}{\longleftarrow} \llbracket A \rrbracket_{2} \times \llbracket B \rrbracket_{2} \stackrel{r_{2} \times r_{2}}{\longleftarrow} \ldots \\
& \llbracket A \rightarrow B \rrbracket \triangleq\left(\llbracket B \rrbracket^{\llbracket A \rrbracket}\right)_{0} \leftarrow\left(\llbracket B \rrbracket^{\llbracket A} \rrbracket\right)_{1} \leftarrow\left(\llbracket B \rrbracket^{\llbracket A \rrbracket}\right)_{2} \leftarrow \cdots \\
& \llbracket \operatorname{Str}_{A} \rrbracket \triangleq \llbracket A \rrbracket_{0} \times 1 \stackrel{r_{0} \times !}{\longleftarrow} \llbracket A \rrbracket_{1} \times\left(\llbracket A \rrbracket_{0} \times 1\right) \stackrel{r_{1} \times r_{0} \times !}{\longleftarrow} \llbracket A \rrbracket_{2} \times\left(\llbracket A \rrbracket_{1} \times\left(\llbracket A \rrbracket_{0} \times 1\right)\right) \leftarrow \cdots \\
& \llbracket \triangleright A \rrbracket \triangleq 1 \stackrel{!}{\leftarrow} \llbracket A \rrbracket_{0} \stackrel{\mathrm{r}_{0}}{\leftarrow} \llbracket A \rrbracket_{1} \stackrel{\mathrm{r}_{1}}{\leftarrow} \ldots \\
& \llbracket \square A \rrbracket \triangleq G(\llbracket A \rrbracket) \stackrel{\text { id }}{\longleftarrow} G(\llbracket A \rrbracket) \stackrel{i \mathrm{~d}}{\longleftarrow} \ldots \\
& \llbracket \mathrm{D}(\mathrm{C}) \rrbracket \triangleq \mathrm{D}\left(\llbracket \mathrm{C} \rrbracket_{0}\right) \stackrel{\mathrm{D}\left(\mathrm{r}_{0}\right)}{\longleftarrow} \mathrm{D}\left(\llbracket \mathrm{C} \rrbracket_{1}\right) \stackrel{\mathrm{D}\left(\mathrm{r}_{1}\right)}{\longleftarrow} \mathrm{D}\left(\llbracket \mathrm{C} \rrbracket_{2}\right) \stackrel{\mathrm{D}\left(\mathrm{r}_{2}\right)}{\longleftarrow} \ldots
\end{aligned}
$$

Figure 8.2: Semantics of types in the topos of trees

Here we detail some key points of the semantics:

- Base types such as nat are interpreted as a constant sequence with the identity as the restriction function: $\llbracket$ nat $\rrbracket \triangleq$ nat $\stackrel{\text { id }}{\longleftarrow}$ nat $\stackrel{\text { id }}{\longleftarrow}$ nat $\stackrel{\text { id }}{\longleftarrow}$...

- Products are computed pointwise. The exponential $X \Rightarrow Y$ at time $n$ is given by the set of tuples $\left(f_{0}, f_{1}, \ldots, f_{n}\right)$ such that for every $i, f_{i}: X_{i} \rightarrow Y_{i}$ and $f_{i} \circ r_{i}^{X}=r_{i}^{Y} \circ f_{i+1}$.

- The later modality shifts the sequence by 1 to the future and inserts at $o$ the terminal object (i.e., singleton set), plus the unique morphism to it:

$$
\llbracket \triangleright A \rrbracket \triangleq 1 \stackrel{!}{\leftarrow} \llbracket A \rrbracket_{0} \stackrel{r_{0}}{\leftarrow} \llbracket A \rrbracket_{1} \stackrel{r_{1}}{\leftarrow} \cdots
$$

- The constant modality flattens whole sequences into each of the steps:

$$
\llbracket \square A \rrbracket \triangleq G(\llbracket A \rrbracket) \stackrel{\text { id }}{\longleftarrow} G(\llbracket A \rrbracket) \stackrel{\text { id }}{\longleftarrow} \cdots,
$$

where $G(\llbracket A \rrbracket)$ is a set of sequences $\left\{x_{n}\right\}_{n} \in$ nat such that $x_{i} \in \llbracket A \rrbracket_{i}$ and $r_{i}\left(x_{i+1}\right)=x_{i}$ for all $i$. 
- An object $X$ of $S$ is constant if all its restriction functions are bijections. Constant types are interpreted as constant objects of $\delta$ and for a constant type $A$ the objects $\llbracket \square A \rrbracket$ and $\llbracket A \rrbracket$ are isomorphic in $S$.

- Streams over a type $A$ are interpreted as sequences of finite prefixes of elements of $A$ with the restriction functions of $A$ :

$$
\llbracket \operatorname{Str}_{A} \rrbracket \triangleq \llbracket A \rrbracket_{0} \times 1 \stackrel{r_{0} \times !}{\longleftarrow} \llbracket A \rrbracket_{1} \times \llbracket \operatorname{Str}_{A} \rrbracket_{0} \stackrel{r_{1} \times r_{0} \times !}{\longleftarrow} \llbracket A \rrbracket_{2} \times \llbracket \operatorname{Str}_{A} \rrbracket_{1} \leftarrow \cdots
$$

- To interpret distributions we use the following observation:

Proposition 8.10. Let $(\mathcal{T}, \eta, \mu)$ be a monad on Set. Then, $(\mathcal{T} \circ-, \eta \circ-, \mu \circ-)$ is a monad on $\mathcal{S}$.

This allows us to define distributions over an object $A$ as a sequence of distributions over each $\llbracket A \rrbracket_{i}$ :

$$
\llbracket D(A) \rrbracket \triangleq(\mathcal{D} \circ-) \llbracket A \rrbracket=\mathcal{D}\left(\llbracket A \rrbracket_{0}\right) \stackrel{\mathcal{D}\left(r_{0}\right)}{\longleftarrow} \mathcal{D}\left(\llbracket A \rrbracket_{1}\right) \stackrel{\mathcal{D}\left(r_{1}\right)}{\longleftarrow} \mathcal{D}\left(\llbracket A \rrbracket_{2}\right) \stackrel{\mathcal{D}\left(r_{2}\right)}{\longleftarrow} \ldots,
$$

Combining these last two definitions, distributions over streams are defined as a sequence of distributions over finite prefixes:

$$
\llbracket \mathrm{D}\left(\mathrm{Str}_{\mathrm{A}}\right) \rrbracket \triangleq \mathcal{D}\left(\llbracket A \rrbracket_{0} \times 1\right) \stackrel{\mathcal{D}\left(\mathrm{r}_{0} \times !\right)}{\longleftarrow} \mathcal{D}\left(\llbracket A \rrbracket_{1} \times \llbracket \operatorname{Str}_{A} \rrbracket_{0}\right) \stackrel{\mathcal{D}\left(\mathrm{r}_{1} \times \mathrm{r}_{0} \times !\right)}{\longleftarrow} \cdots
$$

Typing rules Terms are typed under a dual context $\Delta \mid \Gamma$, where $\Gamma$ is a usual context that binds variables to a type, and $\Delta$ is a constant context containing variables bound to types that are constant. The term letc $x \leftarrow u$ in $t$ allows us to shift variables between constant and non-constant contexts. The typing rules can be found in Figure 8.3.

The semantics of such a dual context $\Delta \mid \Gamma$ is given as the product of types in $\Delta$ and $\Gamma$, except that we implicitly add $\square$ in front of every type in $\Delta$. In the particular case when both contexts are empty, the semantics of the dual context correspond to the terminal object 1 , which is the singleton set $\{*\}$ at each time.

The interpretation of the well-typed term $\Delta \mid \Gamma \vdash \mathrm{t}: \mathrm{A}$ is defined by induction on the typing derivation, and can be found in Figure 8.4.

Applicative structure of the later modality As in previous work we can define the operator $\circledast$ satisfying the typing rule

$$
\frac{\Delta|\Gamma \vdash \mathrm{t}: \triangleright(\mathrm{A} \rightarrow \mathrm{B}) \quad \Delta| \Gamma \vdash \mathrm{u}: \triangleright \mathrm{A}}{\Delta \mid \Gamma \vdash \mathrm{t} \circledast \mathrm{u}: \triangleright \mathrm{B}}
$$

and the equation $(\triangleright t) \circledast(\triangleright u) \equiv \triangleright(t) u$ as the term $t \circledast u \triangleq \triangleright[f \leftarrow t, x \leftarrow u] . f x$. 


$$
\begin{aligned}
& \frac{x: A \in \Gamma}{\Delta \mid \Gamma \vdash x: A} \quad \frac{x: A \in \Delta}{\Delta \mid \Gamma \vdash x: A} \quad \frac{\Delta \mid \Gamma, x: A \vdash t: B}{\Delta \mid \Gamma \vdash \lambda x \cdot t: A \rightarrow B} \\
& \frac{\Delta|\Gamma \vdash \mathrm{t}: \mathrm{A} \rightarrow \mathrm{B} \quad \Delta| \Gamma \vdash \mathrm{u}: \mathrm{A}}{\Delta \mid \Gamma \vdash \mathrm{tu}: \mathrm{B}} \quad \frac{\Delta \mid \Gamma, \mathrm{f}: \Delta \mathrm{A} \vdash \mathrm{t}: \mathrm{A}}{\Delta \mid \Gamma \vdash \text { fix f.t:A }} \quad \frac{\Delta \mid \cdot \vdash \mathrm{t}: \Delta \mathrm{A}}{\Delta \mid \Gamma \vdash \operatorname{prevt}: \mathrm{A}} \\
& \frac{\Delta \mid \cdot \vdash \mathrm{t}: \mathrm{A}}{\Delta \mid \Gamma \vdash \text { box } \mathrm{t}: \square \mathrm{A}} \quad \frac{\Delta|\Gamma \vdash \mathrm{u}: \square \mathrm{B} \quad \Delta, \mathrm{x}: \mathrm{B}| \Gamma \vdash \mathrm{t}: \mathrm{A}}{\Delta \mid \Gamma \vdash \text { letb } \mathrm{x} \leftarrow \mathrm{u} \text { in } \mathrm{t}: \mathrm{A}} \\
& \frac{\Delta|\Gamma \vdash \mathrm{u}: \mathrm{B} \quad \Delta, \mathrm{x}: \mathrm{B}| \Gamma \vdash \mathrm{t}: \mathrm{A} \quad \mathrm{B} \text { constant }}{\Delta \mid \Gamma \vdash \text { letc } x \leftarrow u \text { in } \mathrm{t}: \mathrm{A}} \\
& \frac{\Delta\left|\Gamma, x_{1}: A_{1}, \cdots x_{n}: A_{n} \vdash t: A \quad \Delta\right| \Gamma \vdash t_{i}: \triangleright A_{i}}{\Delta \mid \Gamma \vdash \triangleright\left[x_{1} \leftarrow t_{1}, \ldots, x_{n} \leftarrow t_{n}\right] . t: \triangleright A} \quad \frac{\Delta \mid \Gamma \vdash t: A \quad \text { A discrete }}{\Delta \mid \Gamma \vdash \text { unit }(\mathrm{t}): D(A)} \\
& \frac{\Delta|\Gamma \vdash \mathrm{t}: \mathrm{D}(\mathrm{A}) \quad \Delta| \Gamma, \mathrm{x}: \mathrm{A} \vdash \mathrm{u}: \mathrm{D}(\mathrm{B})}{\Delta \mid \Gamma \vdash \operatorname{mlet} \mathrm{x}=\mathrm{t} \text { in } \mathrm{u}: \mathrm{D}(\mathrm{B})} \quad \frac{\mu \text { primitive distribution on type } A}{\Delta \mid \Gamma \vdash \mu: \mathrm{D}(\mathrm{A})}
\end{aligned}
$$

Figure 8.3: A selection of the typing rules of the guarded lambda calculus. The rules for products, sums, and natural numbers are standard.

Example: Modeling Markov chains As an application of $\circledast$ and an example of how to use guardedness and probabilities together, we now give the precise definition of the markov construct that we used to model Markov chains earlier:

$$
\begin{aligned}
\text { markov }: & C \rightarrow(C \rightarrow D(C)) \rightarrow D\left(\operatorname{Str}_{C}\right) \\
\text { markov } \triangleq & \text { fix } f . \lambda x . \lambda h . \\
& \quad \text { mlet } z=h x \text { in mlet } t=\operatorname{swap}_{\triangleright D} \operatorname{Str}_{C}(f \circledast \triangleright z \circledast \triangleright h) \text { in unit }(x:: t)
\end{aligned}
$$

The guardedness condition gives $f$ the type $\triangleright\left(C \rightarrow(C \rightarrow D(C)) \rightarrow D\left(S \operatorname{tr}_{C}\right)\right)$ in the body of the fixpoint. Therefore, it needs to be applied functorially (via $\circledast$ ) to $\triangleright z$ and $\triangleright h$, which gives us a term of type $\triangleright D\left(\operatorname{Str}_{C}\right)$. To complete the definition we need to build a term of type $D\left(\triangleright \operatorname{Str}_{C}\right)$ and then sequence it with :: to build a term of type $D\left(\operatorname{Str}_{C}\right)$. To achieve this, we use the primitive operator swap $\operatorname{sD}^{C}: \triangleright D(C) \rightarrow D(\triangleright C)$, which witnesses the isomorphism between $\triangleright D(C)$ and $D(\triangleright C)$. For this isomorphism to exist, it is crucial that distributions be total (i.e., we cannot use subdistributions). Indeed, the denotation for $\triangleright D(C)$ is the sequence $\{*\} \leftarrow D\left(C_{1}\right) \leftarrow D\left(C_{2}\right) \leftarrow \ldots$, while the denotation for $D(\triangleright C)$ is the sequence $D(\{*\}) \leftarrow D\left(C_{1}\right) \leftarrow D\left(C_{2}\right) \leftarrow \ldots$, and $\{*\}$ is isomorphic to $D(\{*\})$ in Set only if $D$ considers only total distributions.

\subsection{Guarded higher-order logic}

We now introduce Guarded HOL (GHOL), which is a higher-order logic to reason about terms of the guarded lambda calculus. The logic is essentially that of [63], but 


$$
\begin{aligned}
& \llbracket \Delta \mid \Gamma \vdash \lambda x: A . t: A \rightarrow B \rrbracket_{i}(\delta, \gamma) \triangleq\left(f_{0}, \ldots, f_{i}\right) \\
& \text { where } f_{i}(x)=\llbracket \Delta \mid \Gamma, x: A \vdash t: B \rrbracket\left(\delta,\left(\gamma \uparrow_{i}, x\right)\right) \\
& \llbracket \Delta \mid \Gamma \vdash \mathrm{t}_{1} \mathrm{t}_{2}: \mathrm{B} \rrbracket_{i}(\delta, \gamma) \triangleq \mathrm{f}_{\mathrm{i}}\left(\llbracket \Delta \mid \Gamma \vdash \mathrm{t}_{2}: A \rrbracket(\delta, \gamma)\right) \\
& \text { where } \llbracket \Delta \mid \Gamma \vdash \mathrm{t}_{1}: A \rightarrow B \rrbracket_{i}(\delta, \gamma)=\left(f_{0}, \ldots, f_{i}\right) \\
& \llbracket \Delta \mid \Gamma \vdash \triangleright\left[x_{1} \leftarrow t_{1}, \ldots, x_{n} \leftarrow t_{n}\right] . t: \triangleright A \rrbracket_{0}(\delta, \gamma) \triangleq * \\
& \llbracket \Delta \mid \Gamma \vdash \triangleright\left[x_{1} \leftarrow t_{1}, \ldots, x_{n} \leftarrow t_{n}\right] . t: \triangleright A \rrbracket_{i+1}(\delta, \gamma) \triangleq \\
& \llbracket \Delta \mid \Gamma,\left\{x_{k}: A_{k}\right\}_{k=1}^{n} \vdash \mathrm{t}: A \rrbracket_{i}\left(\delta,\left(\gamma \uparrow_{i},\left\{\llbracket \Delta \mid \Gamma \vdash t_{k}: \triangleright A_{k} \rrbracket_{i+1}\right\}_{k=1}^{n}(\delta, \gamma)\right)\right) \\
& \llbracket \Delta\left|\Gamma \vdash \operatorname{prev} \mathrm{t}: A \rrbracket_{\mathfrak{i}}(\delta, \gamma) \triangleq \llbracket \Delta\right| \cdot \vdash \mathrm{t}: \triangleright A \rrbracket_{\mathfrak{i}+1}(\delta) \\
& \llbracket \Delta \mid \Gamma \vdash \text { box } \mathrm{t}: \square A \rrbracket_{i}(\delta, \gamma) \triangleq\left\{\llbracket \Delta \mid \cdot \vdash \mathrm{t}: A \rrbracket_{j}(\delta)\right\}_{j=0}^{\infty} \\
& \llbracket \Delta \mid \Gamma \vdash \text { letb } x \leftarrow u \text { in } t: A \rrbracket_{i}(\delta, \gamma) \triangleq \\
& \llbracket \Delta, \mathrm{x}: \mathrm{B} \mid \Gamma \vdash \mathrm{t}: \mathrm{A} \rrbracket_{\mathfrak{i}}\left(\left(\delta, \llbracket \Delta \mid \Gamma \vdash \mathfrak{u}: \square \mathrm{B} \rrbracket_{\mathfrak{i}}(\delta, \gamma)\right), \gamma\right) \\
& \llbracket \Delta \mid \Gamma \vdash \text { letc } x \leftarrow u \text { in } \mathrm{t}: A \rrbracket_{\mathfrak{i}}(\delta, \gamma) \triangleq \\
& \llbracket \Delta, \mathrm{x}: \mathrm{B} \mid \Gamma \vdash \mathrm{t}: \mathrm{A} \rrbracket_{\mathfrak{i}}\left(\left(\delta, \varepsilon_{\mathfrak{i}}^{-1}\left(\llbracket \Delta \mid \Gamma \vdash \mathfrak{u}: \square \mathrm{B} \rrbracket_{\mathfrak{i}}(\delta, \gamma)\right)\right), \gamma\right) \\
& \llbracket \Delta \mid \Gamma \vdash \mathrm{hd} t: A \rrbracket_{i}(\delta, \gamma) \triangleq \pi_{0}\left(\llbracket \Delta \mid \Gamma \vdash \mathrm{t}: \operatorname{Str}_{A} \rrbracket_{i}(\delta, \gamma)\right) \\
& \llbracket \Delta \mid \Gamma \vdash \mathrm{tl} \mathrm{t}: \triangleright \operatorname{Str}_{A} \rrbracket_{i}(\delta, \gamma) \triangleq \pi_{1}\left(\llbracket \Delta \mid \Gamma \vdash \mathrm{t}: \operatorname{Str}_{A} \rrbracket_{i}(\delta, \gamma)\right) \\
& \llbracket \Delta \mid \Gamma \vdash \mathrm{t}:: \mathrm{u}: \operatorname{Str}_{A} \rrbracket_{\mathfrak{i}}(\delta, \gamma) \triangleq\left(\llbracket \Delta\left|\Gamma \vdash \mathrm{t}: A \rrbracket_{\mathfrak{i}}(\delta, \gamma), \llbracket \Delta\right| \Gamma \vdash \mathrm{u}: \operatorname{Str}_{\mathrm{A}} \rrbracket_{\mathfrak{i}}(\delta, \gamma)\right) \\
& \llbracket \Delta \mid \Gamma \vdash \text { unit }(\mathrm{t}): \mathrm{D}(\mathrm{C}) \rrbracket_{\mathfrak{i}}(\delta, \gamma) \triangleq \operatorname{unit}\left(\llbracket \Delta \mid \Gamma \vdash \mathrm{t}: \mathrm{C} \rrbracket_{\mathfrak{i}}(\delta, \gamma)\right) \\
& \llbracket \Delta \mid \Gamma \vdash \text { mlet } x=\mathrm{t} \text { in } \mathrm{u}: \mathrm{D}(\mathrm{C}) \rrbracket_{i}(\delta, \gamma) \triangleq \text { mlet } v=\llbracket \Delta \mid \Gamma \vdash \mathrm{t}: \mathrm{D}(\mathrm{D}) \rrbracket_{i}(\delta, \gamma) \text { in } \\
& \llbracket \Delta \mid \Gamma, x: \mathrm{D} \vdash \mathrm{u}: \mathrm{D}(\mathrm{C}) \rrbracket_{\mathfrak{i}}(\delta, \gamma[\mathrm{x}:=v])
\end{aligned}
$$

Figure 8.4: Semantics for the Guarded $\lambda$-calculus

presented with the dual context formulation analogous to the dual-context typing judgment of the guarded lambda calculus. Compared to standard intuitionistic higher-order logic, the logic GHOL has two additional constructs, corresponding to additional constructs in the guarded lambda calculus. These are the later modality $(\triangleright)$ on propositions, with delayed substitutions, which expresses that a proposition holds one time unit into the future, and the "always" modality $\square$, which expresses that a proposition holds at all times. Formulas are defined by the grammar:

$$
\phi, \psi::=\top|\phi \wedge \psi| \phi \vee \psi|\neg \psi| \forall x . \phi|\exists x . \phi| \triangleright\left[x_{1} \leftarrow t_{1} \ldots x_{n} \leftarrow t_{n}\right] . \phi \mid \square \phi
$$

The basic judgment of the logic is $\Delta|\Sigma| \Gamma \mid \Psi \vdash \phi$ where $\Sigma$ is a logical context for $\Delta$ (that is, a list of formulas well-formed in $\Delta$ ) and $\Psi$ is another logical context for the dual context $\Delta \mid \Gamma$. The formulas in context $\Sigma$ must be constant propositions. We say that a proposition $\phi$ is constant if it is well-typed in context $\Delta \mid \cdot$ and moreover if 
every occurrence of the later modality in $\phi$ is under the $\square$ modality. Selected rules are displayed in Figure 8.5 on page 22. We highlight Loeb induction, which is the key to reasoning about fixpoints: to prove that $\phi$ holds now, one can assume that it holds in the future.

Denotational semantics. Formally, the guarded HOL has a model in the internal logic of the topos of trees. To make more explicit how the distribution monad interacts with the model, we instead define the category $\operatorname{Pred}(\mathcal{S})$ of predicates over the topos of trees:

- Given an object $\left\{X_{i}, r_{i}\right\}_{i \in \omega}$ in $\mathcal{S}$, a predicate over it is a sequence of predicates $\left\{\phi_{i}\right\}_{i \in \omega}$ such that for all $\mathrm{i}, \phi_{i} \subseteq X_{i}$ and $r_{i}\left(\phi_{i+1}\right) \subseteq \phi_{i}$

- Given objects $\left\{X_{i}, r_{i}\right\}_{i \in \omega},\left\{Y_{i}, s_{i}\right\}_{i \in \omega}$ in $\mathcal{S}$ and predicates $\left\{\phi_{i}\right\}_{i \in \omega},\left\{\psi_{i}\right\}_{i \in \omega}$ over them, a morphism between the predicates is given by a morphism $\left(f_{i}\right)_{i \in \omega}$ between the objects such that for all $i, f_{i}\left(\phi_{i}\right) \subseteq \psi_{i}$

The forgetful fibration $\operatorname{Pred}(\mathcal{S}) \rightarrow \mathcal{S}$ is a LR fibration, so we can use it as a semantical model for our logic. The interpretation of the formula $\Delta \mid \Gamma \vdash \phi$ is a predicate over the interpretation $\llbracket \Delta \mid \Gamma \rrbracket$. The interpretation of formulas is defined by induction on the typing derivation. In the interpretation of the context $\Delta|\Sigma| \Gamma \mid \Psi$ the formulas in $\Sigma$ are interpreted with the added $\square$ modality. Moreover all formulas $\phi$ in $\Sigma$ are typeable in the context $\Delta \mid \cdot \vdash \phi$ and thus their interpretations are subsets of $\llbracket \square \Delta \rrbracket$. We treat these subsets of $\llbracket \Delta \mid \Gamma \rrbracket$ in the obvious way.

The cases for the semantics of the judgment $\Delta \mid \Gamma \vdash \phi$ is defined inductively:

$$
\begin{gathered}
\llbracket \Delta\left|\Gamma \vdash \top \rrbracket_{i} \triangleq \llbracket \Delta\right| \Gamma \rrbracket_{i} \\
\llbracket \Delta\left|\Gamma \vdash \phi \wedge \psi \rrbracket_{i} \triangleq \llbracket \Delta\right| \Gamma \vdash \phi \rrbracket_{i} \cap \llbracket \Delta \mid \Gamma \vdash \psi \rrbracket_{i} \\
\llbracket \Delta\left|\Gamma \vdash \phi \vee \psi \rrbracket_{i} \triangleq \llbracket \Delta\right| \Gamma \vdash \phi \rrbracket_{i} \cup \llbracket \Delta \mid \Gamma \vdash \psi \rrbracket_{i} \\
\llbracket \Delta \mid \Gamma \vdash \phi \Rightarrow \psi \rrbracket_{i} \triangleq\left\{x\left|\forall j \leqslant i, x \Upsilon_{j} \in \llbracket \Delta\right| \Gamma \vdash \phi \rrbracket_{j} \Rightarrow x \uparrow_{j} \in \llbracket \Delta \mid \Gamma \vdash \psi \rrbracket_{j}\right\} \\
\llbracket \Delta \mid \Gamma \vdash \forall x: A . \phi \rrbracket_{i} \triangleq \\
\left\{(\delta, \gamma) \mid \forall j \leqslant i, \forall x \in \llbracket A \rrbracket_{j},\left(\delta,\left(\gamma\left\lceil_{j}\right), x\right) \in \llbracket \Delta \mid \Gamma, x: A \vdash \phi \rrbracket\right\}\right. \\
\llbracket \Delta \mid \Gamma \vdash \triangleright\left[x_{1} \leftarrow t_{1}, \ldots, x_{n} \leftarrow t_{n}\right] . \phi \rrbracket_{i} \triangleq \\
\left\{(\delta, \gamma) \mid i>0 \Rightarrow\left(\delta, \gamma\left\lceil_{i-1},\left\{\llbracket t_{k} \rrbracket_{i}(\delta, \gamma)\right\}_{k=1}^{n}\right) \in \llbracket \phi \rrbracket_{i-1}\right\}\right. \\
\llbracket \square \phi \rrbracket_{i} \triangleq\left\{x \mid \forall j, x \in \llbracket \phi \rrbracket_{j}\right\}
\end{gathered}
$$

It can be shown that this logic is sound with respect to its model in the topos of trees.

Theorem 8.11 (Soundness of the semantics). The semantics of guarded higher-order logic is sound: if $\Delta|\Sigma| \Gamma \mid \Psi \vdash \phi$ is derivable then, $\llbracket \Delta|\Gamma \vdash \Lambda \square \Sigma \rrbracket \cap \llbracket \Delta| \Gamma \vdash \Lambda \Psi \rrbracket \subseteq \llbracket \Delta \mid \Gamma \vdash \phi \rrbracket$. 


$$
\begin{aligned}
& \frac{\phi \in \Psi}{\Delta|\Sigma| \Gamma \mid \Psi \vdash \phi} \mathrm{AX}_{\mathrm{U}} \quad \frac{\phi \in \Sigma}{\Delta|\Sigma| \Gamma \mid \Psi \vdash \phi} \mathrm{AX}_{\mathrm{G}} \quad \frac{\Gamma \vdash \mathrm{t}: \tau \quad \Gamma \vdash \mathrm{t}^{\prime}: \tau \quad \mathrm{t} \equiv \mathrm{t}^{\prime}}{\Delta|\Sigma| \Gamma \mid \Psi \vdash \mathrm{t}=\mathrm{t}^{\prime}} \text { CONV } \\
& \frac{\Delta|\Sigma| \Gamma|\Psi \vdash \phi[\mathrm{t} / \mathrm{x}] \quad \Delta| \Sigma|\Gamma| \Psi \vdash \mathrm{t}=\mathrm{u}}{\Delta|\Sigma| \Gamma \mid \Psi \vdash \phi[\mathrm{u} / \mathrm{x}]} \text { SUBST } \quad \frac{\Delta|\Sigma| \Gamma \mid \Psi, \triangleright \phi \vdash \phi}{\Delta|\Sigma| \Gamma \mid \Psi \vdash \phi} \text { Loeb } \\
& \frac{\Delta|\Sigma| \Gamma, x_{1}: A_{1}, \ldots, x_{n}: A_{n}|\Psi \vdash \phi \quad \Delta| \Gamma \vdash t_{1}: \triangleright A_{1} \quad \ldots \quad \Delta \mid \Gamma \vdash t_{n}: \triangleright A_{n}}{\Delta|\Sigma| \Gamma \mid \Psi \vdash \triangleright\left[x_{1} \leftarrow t_{1}, \ldots, x_{n} \leftarrow t_{n}\right] . \phi} \triangleright_{1} \\
& \frac{\Delta|\Sigma| \cdot\left|\cdot \vdash \triangleright\left[x_{1} \leftarrow t_{1} \ldots x_{n} \leftarrow t_{n}\right] . \phi \quad \Delta\right| \bullet \vdash t_{1}: \triangleright A_{1} \quad \ldots \quad \Delta \mid \bullet \vdash t_{n}: \triangleright A_{n}}{\Delta|\Sigma| \Gamma \mid \Psi \vdash \phi\left[\text { prev } t_{1} / x_{1}\right] \ldots\left[\text { prev } t_{n} / x_{n}\right]} \triangleright_{E} \\
& \Delta|\Sigma| \Gamma\left|\Psi \vdash \triangleright\left[\mathrm{x}_{1} \leftarrow \mathrm{t}_{1}, \ldots, \mathrm{x}_{\mathrm{n}} \leftarrow \mathrm{t}_{\mathrm{n}}\right] . \psi \quad \Delta\right| \Gamma \vdash \mathrm{t}_{1}: \triangleright \mathrm{A}_{1} \ldots \Delta \mid \Gamma \vdash \mathrm{t}_{\mathrm{n}}: \triangleright \mathrm{A}_{\mathrm{n}} \\
& \Delta|\Sigma| \Gamma, x_{1}: A_{1}, \ldots, x_{n}: A_{n} \mid \Psi, \psi \vdash \phi \\
& \Delta|\Sigma| \Gamma \mid \Psi \vdash \triangleright\left[\mathrm{x}_{1} \leftarrow \mathrm{t}_{1}, \ldots, \mathrm{x}_{\mathrm{n}} \leftarrow \mathrm{t}_{\mathrm{n}}\right] . \phi \quad \triangleright_{\mathrm{App}} \\
& \frac{\Delta|\Sigma| \cdot \mid \cdot \vdash \phi}{\Delta|\Sigma| \Gamma \mid \Psi \vdash \square \phi} \square_{l} \quad \frac{\Delta|\Sigma| \Gamma|\Psi \vdash \square \psi \quad \Delta| \Sigma, \psi|\Gamma| \Psi \vdash \phi}{\Delta|\Sigma| \Gamma \mid \Psi \vdash \phi} \square_{E}
\end{aligned}
$$

Figure 8.5: Selected Guarded Higher-Order Logic rules

To interpret predicates over probabilistic computations we will first define a lifting of the $\mathcal{D} \circ-$ monad, and the internalize it into our logic. It is simple to check that the lifting $\dot{\mathcal{D}}^{\text {Coup }}=(\dot{\mathcal{D}}$ Coup $, \dot{\eta}, \dot{\mu})$ over Pred(Set) can be extended to a lifting $\dot{\mathcal{D}}^{\text {Coup }} \circ-$ over Pred $(\mathcal{S})$ by postcomposition: we take $(\dot{\eta} \circ-)$ as the unit and $(\dot{\mu} \circ-)$ as the multiplication.

For convenience, we will internalize the lifting $\dot{\mathcal{D}}^{\text {Coup }} \circ-$ into our logic as the $\diamond$ modality. For every $\tau_{1}, \tau_{2}$ we have:

$$
\llbracket x_{1}: \mathcal{D}\left(\tau_{1}\right), x_{2}: \mathcal{D}\left(\tau_{2}\right) \vdash \diamond_{y_{1} \leftarrow x_{1}, y_{2} \leftarrow x_{2}} \phi \rrbracket=\left(\dot{\mathcal{D}}^{\text {Coup }} \circ-\right) \llbracket y_{1}: \tau_{1}, y_{2}: \tau_{2} \vdash \phi \rrbracket
$$

Furthermore, we can prove from this definition additional rules to reason about couplings, which can be seen in Figure 8.6. These rules will be the key to proving the soundness of the probabilistic fragment of the relational proof system.

Proposition 8.12. The additional rules are sound.

\subsection{Relational proof system}

We complete the formal description of the system by describing the proof rules for the non-probabilistic fragment of the relational proof system (the rules of the probabilistic fragment were described in Section 8.2.2). 
Chapter 8. Probabilistic guarded relational higher-order logic

$$
\begin{aligned}
& \frac{\Delta|\Sigma| \Gamma\left|\Psi \vdash \diamond_{\left[x_{1} \leftarrow \mathrm{t}_{1}, \mathrm{x}_{2} \leftarrow \mathrm{t}_{2}\right]} \phi \quad \Delta\right| \Sigma\left|\Gamma, \mathrm{x}_{1}: A_{1}, \mathrm{x}_{2}: A_{2}\right| \Psi, \phi \vdash \psi}{\Delta|\Sigma| \Gamma \mid \Psi \vdash \diamond_{\left[\mathrm{x}_{1} \leftarrow \mathrm{t}_{1}, \mathrm{x}_{2} \leftarrow \mathrm{t}_{2}\right]} \psi} \text { MONO2 } \\
& \frac{\Delta|\Sigma| \Gamma \mid \Psi \vdash \phi\left[\mathrm{t}_{1} / \mathrm{x}_{1}\right]\left[\mathrm{t}_{2} / \mathrm{x}_{2}\right]}{\Delta|\Sigma| \Gamma \mid \Psi \vdash \diamond\left[\mathrm{x}_{1} \leftarrow \operatorname{unit}\left(\mathrm{t}_{1}\right), \mathrm{x}_{2} \leftarrow \operatorname{unit}\left(\mathrm{t}_{2}\right)\right]} \text { UNIT2 }
\end{aligned}
$$

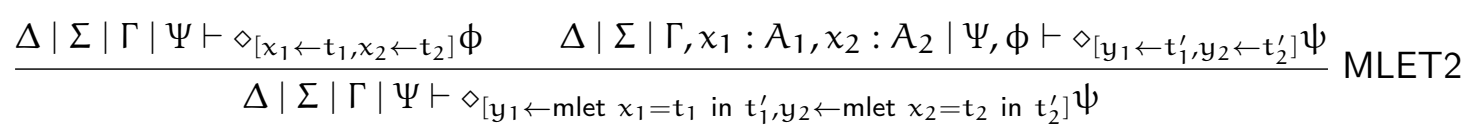

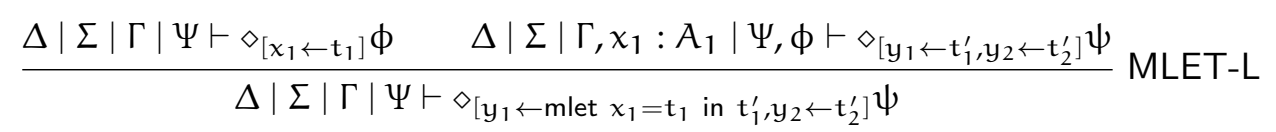

Figure 8.6: Rules for probabilistic constructs

\subsubsection{Proof rules}

The rules for core $\lambda$-calculus constructs are identical to those of RHOL, and the extended context is ignored.

We briefly comment on the two-sided rules for the new constructs (Figure 8.7). The notation $\Omega$ abbreviates a context $\Delta|\Sigma| \Gamma \mid \Psi$. The rule Next relates two terms that have a $\triangleright$ term constructor at the top level. We require that both have one term in the delayed substitutions and that they are related pairwise. Then this relation is used to prove another relation between the main terms. This rule can be generalized to terms with more than one term in the delayed substitution. The rule Prev proves a relation between terms from the same delayed relation by applying prev to both terms. The rule Box proves a relation between two boxed terms if the same relation can be proven in a constant context. Dually, LetBox uses a relation between two boxed terms to prove a relation between their unboxings. LetConst is similar to LetBox, but it requires instead a relation between two constant terms, rather than explicitly $\square$-ed terms. The rule Fix relates two fixpoints following the Loeb rule from Guarded HOL. Notice that in the premise, the fixpoints need to appear in the delayed substitution so that the inductive hypothesis is well-formed. The rule Cons proves relations on streams from relations between their heads and tails, while Head and Tail behave as converses of Cons.

Figure 8.8 contains the one-sided versions of the rules. We only present the left-sided versions as the right-sided versions are completely symmetric. The rule Next- $L$ relates at $\phi$ a term that has a $\triangleright$ with a term that does not have a $\triangleright$. First, a unary property $\phi^{\prime}$ is proven on the term $u$ in the delayed substitution, and it is then used as a premise to prove $\phi$ on the terms with delays removed. Rules for proving unary judgments can be found in the appendix. Similarly, LetBox-L proves a unary property on the term that gets unboxed and then uses it as a precondition. The rule Fix-L builds a fixpoint just on the left, and relates it with an arbitrary term $t_{2}$ at a 
property $\phi$. Since $\phi$ may contain the variable $\mathbf{r}_{2}$ which is not in the context, it has to be replaced when adding $\triangleright \phi$ to the logical context in the premise of the rule. The remaining rules are similar to their two-sided counterparts.

$$
\begin{aligned}
& \Delta|\Sigma| \Gamma, x_{1}: A_{1}, x_{2}: A_{2} \mid \Psi, \phi^{\prime}\left[x_{1} / \mathbf{r}_{1}\right]\left[x_{2} / \mathbf{r}_{2}\right] \vdash t_{1}: A_{1} \sim t_{2}: A_{2}\{\phi\} \\
& \Omega \vdash u_{1}: \triangleright A_{1} \sim u_{2}: \triangleright A_{2}\left\{\triangleright\left[\mathbf{r}_{1}, \mathbf{r}_{2} \leftarrow \mathbf{r}_{1}, \mathbf{r}_{2}\right] . \phi^{\prime}\right\}
\end{aligned}
$$

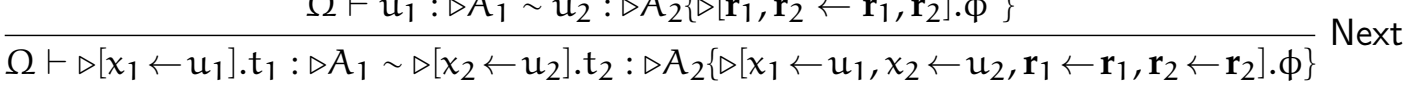

$$
\begin{aligned}
& \frac{\Delta|\Sigma| \cdot \mid \cdot \vdash \mathrm{t}_{1}: \triangleright A_{1} \sim \mathrm{t}_{2}: \triangleright A_{2}\left\{\triangleright\left[\mathbf{r}_{1}, \mathbf{r}_{2} \leftarrow \mathbf{r}_{1}, \mathbf{r}_{2}\right] . \phi\right\}}{\Omega \vdash \text { prev } \mathrm{t}_{1}: A_{1} \sim \text { prev } \mathrm{t}_{2}: A_{2}\{\phi\}} \text { Prev } \\
& \Delta|\Sigma| \cdot \mid \cdot \vdash \mathrm{t}_{1}: \mathrm{A}_{1} \sim \mathrm{t}_{2}: \mathrm{A}_{2}\{\phi\}
\end{aligned}
$$

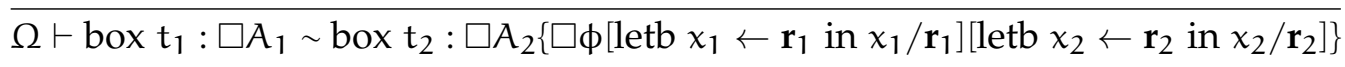

$$
\begin{aligned}
& \Omega \vdash \mathrm{u}_{1}: \square \mathrm{B}_{1} \sim \mathrm{u}_{2}: \square \mathrm{B}_{2}\left\{\square \phi\left[\text { letb } x_{1} \leftarrow \mathbf{r}_{1} \text { in } x_{1} / \mathbf{r}_{1}\right]\left[\text { letb } x_{2} \leftarrow \mathbf{r}_{2} \text { in } x_{2} / \mathbf{r}_{2}\right]\right\} \\
& \frac{\Delta, x_{1}: B_{1}, x_{2}: B_{2}\left|\Sigma, \phi\left[x_{1} / r_{1}\right]\left[x_{2} / r_{2}\right]\right| \Gamma \mid \Psi \vdash t_{1}: A_{1} \sim t_{2}: A_{2}\left\{\phi^{\prime}\right\}}{\Omega \vdash \text { letb } x_{1} \leftarrow u_{1} \text { in } t_{1}: A_{1} \sim \text { letb } x_{2} \leftarrow u_{2} \text { in } t_{2}: A_{2}\left\{\phi^{\prime}\right\}} \text { LetBox } \\
& \mathrm{B}_{1}, \mathrm{~B}_{2}, \phi \text { constant } \quad \mathrm{FV}(\phi) \cap \mathrm{FV}(\Gamma)=\emptyset \quad \Omega \vdash \mathrm{u}_{1}: \mathrm{B}_{1} \sim \mathrm{u}_{2}: \mathrm{B}_{2}\{\phi\} \\
& \frac{\Delta, x_{1}: B_{1}, x_{2}: B_{2}\left|\Sigma, \phi\left[x_{1} / r_{1}\right]\left[x_{2} / r_{2}\right]\right| \Gamma \mid \Psi \vdash t_{1}: A_{1} \sim t_{2}: A_{2}\left\{\phi^{\prime}\right\}}{\Omega \vdash \text { letc } x_{1} \leftarrow u_{1} \text { in } t_{1}: A_{1} \sim \text { letc } x_{2} \leftarrow u_{2} \text { in } t_{2}: A_{2}\left\{\phi^{\prime}\right\}} \text { LetConst } \\
& \frac{\Delta|\Sigma| \Gamma, \mathrm{f}_{1}: \triangleright A_{1}, \mathrm{f}_{2}: \triangleright A_{2} \mid \Psi, \triangleright\left[\mathbf{r}_{1}, \mathbf{r}_{2} \leftarrow \mathrm{f}_{1}, \mathrm{f}_{2}\right] . \phi \vdash \mathrm{t}_{1}: A_{1} \sim \mathrm{t}_{2}: A_{2}\{\phi\}}{\Omega \vdash \text { fix } \mathrm{f}_{1} . \mathrm{t}_{1}: A_{1} \sim \text { fix } \mathrm{f}_{2} . \mathrm{t}_{2}: A_{2}\{\phi\}} \text { Fix } \\
& \Omega \vdash x_{1}: A_{1} \sim x_{2}: A_{2}\left\{\phi_{h}\right\} \quad \Omega \vdash x_{1}: \triangleright \operatorname{Str}_{A_{1}} \sim x_{2}: \triangleright \operatorname{Str}_{A_{2}}\left\{\phi_{t}\right\} \\
& \frac{\Omega \vdash \forall x_{1}, x_{2}, s_{1}, s_{2} \cdot \phi_{h}\left[x_{1} / \mathbf{r}_{1}\right]\left[x_{2} / \mathbf{r}_{2}\right] \Rightarrow \phi_{t}\left[s_{1} / \mathbf{r}_{1}\right]\left[s_{2} / \mathbf{r}_{2}\right] \Rightarrow \phi\left[x_{1}:: s_{1} / \mathbf{r}_{1}\right]\left[x_{2}:: s_{2} / \mathbf{r}_{2}\right]}{\Omega \vdash x_{1}:: s_{1}: \operatorname{Str}_{A_{1}} \sim x_{2}:: s_{2}: \operatorname{Str}_{A_{2}}\{\phi\}} \text { Cons } \\
& \frac{\Omega \vdash t_{1}: \operatorname{Str}_{A_{1}} \sim t_{1}: \operatorname{Str}_{A_{1}}\left\{\phi\left[h d \mathbf{r}_{1} / \mathbf{r}_{1}\right]\left[h d \mathbf{r}_{2} / \mathbf{r}_{2}\right]\right\}}{\Omega \vdash h d t_{1}: A_{1} \sim h d t_{2}: A_{2}\{\phi\}} \text { Head } \\
& \frac{\Omega \vdash \mathrm{t}_{1}: \operatorname{Str}_{\mathrm{A}_{1}} \sim \mathrm{t}_{2}: \operatorname{Str}_{\mathrm{A}_{2}}\left\{\phi\left[\mathrm{tl} \mathbf{r}_{1} / \mathbf{r}_{1}\right]\left[\mathrm{tl} \mathbf{r}_{2} / \mathbf{r}_{2}\right]\right\}}{\Omega \vdash \operatorname{tl} \mathrm{t}_{1}: \triangleright \operatorname{Str}_{\mathrm{A}_{1}} \sim \mathrm{tl} \mathrm{t}_{2}: \triangleright \operatorname{Str}_{\mathrm{A}_{2}}\{\phi\}} \operatorname{Tail}
\end{aligned}
$$

Figure 8.7: Two-sided rules for Guarded RHOL

\subsubsection{Metatheory}

We review some of the most interesting metatheoretical properties of our relational proof system, highlighting the equivalence with Guarded HOL.

Theorem 8.13 (Equivalence with Guarded HOL). For all contexts $\Delta, \Gamma$; types $\sigma_{1}, \sigma_{2}$; 


$$
\begin{aligned}
& \frac{\Delta|\Sigma| \Gamma, x_{1}: B_{1} \mid \Psi, \phi^{\prime}\left[x_{1} / \mathbf{r}\right] \vdash t_{1}: A_{1} \sim t_{2}: A_{2}\{\phi\} \quad \Omega \vdash u_{1}: \triangleright B_{1}\left\{\triangleright[\mathbf{r} \leftarrow \mathbf{r}] . \phi^{\prime}\right\}}{\Omega \vdash \triangleright\left[x_{1} \leftarrow u_{1}\right] . t_{1}: \triangleright A_{1} \sim t_{2}: A_{2}\left\{\triangleright\left[x_{1} \leftarrow u_{1}, \mathbf{r}_{1} \leftarrow \mathbf{r}_{1}\right] . \phi\right\}} \text { Next-L } \\
& \frac{\Delta|\Sigma| \cdot \mid \cdot \vdash \mathrm{t}_{1}: \triangleright A_{1} \sim \mathrm{t}_{2}: A_{2}\left\{\triangleright\left[\mathbf{r}_{1} \leftarrow \mathbf{r}_{1}\right] . \phi\right\}}{\Delta|\Sigma| \Gamma_{1} ; \Gamma_{2} \mid \Psi_{1} ; \Psi_{2} \vdash \text { prev } t_{1}: A_{1} \sim t_{2}: A_{2}\{\phi\}} \text { Prev-L } \\
& \frac{\Delta|\Sigma| \Gamma_{2} \mid \Psi_{2} \vdash \mathrm{t}_{1}: \mathrm{A}_{1} \sim \mathrm{t}_{2}: \mathrm{A}_{2}\{\phi\} \quad \mathrm{FV}\left(\mathrm{t}_{1}\right) \not \subset \mathrm{FV}\left(\Gamma_{2}\right) \quad \mathrm{FV}\left(\Psi_{2}\right) \subseteq \mathrm{FV}\left(\Gamma_{2}\right)}{\Delta|\Sigma| \Gamma_{1} ; \Gamma_{2} \mid \Psi_{1} ; \Psi_{2} \vdash \text { box } \mathrm{t}_{1}: \square \mathrm{A}_{1} \sim \mathrm{t}_{2}: \mathrm{A}_{2}\left\{\square \phi\left[\text { letb } x_{1} \leftarrow \mathbf{r}_{1} \text { in } x_{1} / \mathbf{r}_{1}\right]\right\}} \text { Box-L } \\
& \Omega \vdash \mathfrak{u}_{1}: \square \mathrm{B}_{1}\left\{\square \phi\left[\text { letb } x_{1} \leftarrow \mathbf{r}_{1} \text { in } x_{1} / \mathbf{r}\right]\right\} \\
& \frac{\Delta, x_{1}: B_{1}\left|\Sigma, \phi\left[x_{1} / \mathbf{r}\right]\right| \Gamma \mid \Psi \vdash t_{1}: A_{1} \sim t_{2}: A_{2}\left\{\phi^{\prime}\right\}}{\Omega \vdash \text { letb } x_{1} \leftarrow u_{1} \text { in } t_{1}: A_{1} \sim t_{2}: A_{2}\left\{\phi^{\prime}\right\}} \text { LetBox-L } \\
& \mathrm{B}_{1}, \phi \text { constant } \quad \mathrm{FV}(\phi) \cap \mathrm{FV}(\Gamma)=\emptyset \quad \Omega \vdash \mathfrak{u}_{1}: \mathrm{B}_{1}\{\phi\} \\
& \frac{\Delta, x_{1}: B_{1}\left|\Sigma, \phi\left[x_{1} / \mathbf{r}\right]\right| \Gamma \mid \Psi \vdash t_{1}: A_{1} \sim t_{2}: A_{2}\left\{\phi^{\prime}\right\}}{\Omega \vdash \text { letc } x_{1} \leftarrow u_{1} \text { in } t_{1}: A_{1} \sim t_{2}: A_{2}\left\{\phi^{\prime}\right\}} \text { LetConst-L } \\
& \frac{\Delta|\Sigma| \Gamma, f_{1}: \triangleright A_{1} \mid \Psi, \triangleright\left[\mathbf{r}_{1} \leftarrow f_{1}\right] .\left(\phi\left[t_{2} / \mathbf{r}_{2}\right]\right) \vdash t_{1}: A_{1} \sim t_{2}: A_{2}\{\phi\}}{\Delta|\Sigma| \Gamma \mid \Psi \vdash \text { fix } f_{1} . t_{1}: A_{1} \sim t_{2}: A_{2}\{\phi\}} \text { Fix-L } \\
& \Omega \vdash x_{1}: A_{1} \sim t_{2}: A_{2}\left\{\phi_{h}\right\} \quad \Omega \vdash x_{1}: \triangleright \operatorname{Str}_{A_{1}} \sim t_{2}: A_{2}\left\{\phi_{t}\right\} \\
& \frac{\Omega \vdash \forall x_{1}, x_{2}, x s_{1} . \phi_{h}\left[x_{1} / \mathbf{r}_{1}\right]\left[x_{2} / \mathbf{r}_{2}\right] \Rightarrow \phi_{t}\left[x_{1} / \mathbf{r}_{1}\right]\left[x_{2} / \mathbf{r}_{2}\right] \Rightarrow \phi\left[x_{1}:: x_{s_{1}} / \mathbf{r}_{1}\right]\left[x_{2} / \mathbf{r}_{2}\right]}{\Omega \vdash x_{1}:: x_{1}: \operatorname{Str}_{A_{1}} \sim t_{2}: A_{2}\{\phi\}} \text { Cons-L } \\
& \frac{\Omega \vdash \mathrm{t}_{1}: \operatorname{Str}_{\mathrm{A}_{1}} \sim \mathrm{t}_{1}: \mathrm{A}_{2}\left\{\phi\left[h d \mathbf{r}_{1} / \mathbf{r}_{1}\right]\right\}}{\Omega \vdash h d \mathrm{t}_{1}: \mathrm{A}_{1} \sim \mathrm{t}_{2}: \mathrm{A}_{2}\{\phi\}} \text { Head-L } \\
& \frac{\Omega \vdash \mathrm{t}_{1}: \operatorname{Str}_{\mathrm{A}_{1}} \sim \mathrm{t}_{2}: \mathrm{A}_{2}\left\{\phi\left[\mathrm{tl} \mathbf{r}_{1} / \mathbf{r}_{1}\right]\right\}}{\Omega \vdash \mathrm{tl} \mathrm{t}_{1}: \triangleright \operatorname{Str}_{\mathrm{A}_{1}} \sim \mathrm{t}_{2}: \mathrm{A}_{2}\{\phi\}} \text { Tail-L }
\end{aligned}
$$

Figure 8.8: One-sided rules for Guarded RHOL

terms $\mathrm{t}_{1}, \mathrm{t}_{2}$; sets of assertions $\Sigma, \Psi$; and assertions $\phi$ :

$$
\Delta|\Sigma| \Gamma\left|\Psi \vdash \mathrm{t}_{1}: \sigma_{1} \sim \mathrm{t}_{2}: \sigma_{2}\{\phi\} \quad \Longleftrightarrow \quad \Delta\right| \Sigma|\Gamma| \Psi \vdash \phi\left[\mathrm{t}_{1} / \mathbf{r}_{1}\right]\left[\mathrm{t}_{2} / \mathbf{r}_{2}\right]
$$

The forward implication follows by induction on the given derivation. The reverse implication is immediate from the rule SUB which allows to fall back on Guarded HOL in relational proofs. The consequence of this theorem is that the syntax-directed, relational proof system we have built on top of Guarded HOL does not lose expressiveness.

The intended semantics of a judgment $\Delta|\Sigma| \Gamma \mid \Psi \vdash t_{1}: A_{1} \sim t_{2}: A_{2}\{\phi\}$ is is that there is a morphism

$$
\left\langle\mathrm{id}, \llbracket \Delta\left|\Gamma \vdash \mathrm{t}_{1} \rrbracket, \llbracket \Delta\right| \Gamma \vdash \mathrm{t}_{2} \rrbracket\right\rangle: \llbracket \Delta|\Gamma \vdash(\square \Sigma) \wedge \Psi \rrbracket \rightarrow \llbracket \Delta| \Gamma, \mathbf{r}_{1}, \mathbf{r}_{2} \vdash \phi \rrbracket
$$


In set-theoretic terms, this means that, for every valuation $\delta \models \Delta, \gamma \models \Gamma$,

$$
\llbracket \square \Sigma \rrbracket(\delta) \cap \llbracket \Psi \rrbracket(\delta, \gamma) \subseteq \llbracket \phi \rrbracket\left(\delta, \gamma\left[\mathbf{r}_{1} \leftarrow \llbracket \mathrm{t}_{1} \rrbracket(\delta, \gamma), \mathbf{r}_{2} \leftarrow \llbracket \mathrm{t}_{2} \rrbracket(\delta, \gamma)\right]\right)
$$

Since Guarded HOL is sound with respect to its semantics in the topos of trees, and our relational proof system is equivalent to Guarded HOL, we obtain that our relational proof system is also sound in the topos of trees.

Corollary 8.14 (Soundness and consistency). If $\Delta|\Sigma| \Gamma \mid \Psi \vdash \mathrm{t}_{1}: \sigma_{2} \sim \mathrm{t}_{2}: \sigma_{2}\{\phi\}$, then for every valuation $\delta \models \Delta, \gamma \models \Gamma$ :

$$
\begin{gathered}
\llbracket \Delta \vdash \square \Sigma \rrbracket(\delta) \wedge \llbracket \Delta \mid \Gamma \vdash \Psi \rrbracket(\delta, \gamma) \Rightarrow \\
\llbracket \Delta \mid \Gamma, \mathbf{r}_{1}: \sigma_{1}, \mathbf{r}_{1}: \sigma_{2} \vdash \phi \rrbracket\left(\delta, \gamma\left[\mathbf{r}_{1} \leftarrow \llbracket \Delta \mid \Gamma \vdash \mathrm{t}_{1} \rrbracket(\delta, \gamma)\right]\left[\mathbf{r}_{2} \leftarrow \llbracket \Delta \mid \Gamma \vdash \mathrm{t}_{2} \rrbracket(\delta, \gamma)\right]\right)
\end{gathered}
$$

In particular, there is no proof of $\Delta|\emptyset| \Gamma \mid \emptyset \vdash \mathrm{t}_{1}: \sigma_{1} \sim \mathrm{t}_{2}: \sigma_{2}\{\perp\}$.

\subsubsection{Shift couplings revisited}

We give further details on how to prove the example with shift couplings from Section 8.2.3. (Additional examples of relational reasoning on non-probabilistic streams can be found in the appendix) Recall the step functions:

$$
\begin{aligned}
& \text { step } \triangleq \lambda x \text {.mlet } z=U_{\{-1,1\}} \text { in unit }(z+x) \\
& \text { 1step } 2 \triangleq \lambda x \text {.mlet } z=\mathcal{U}_{\{-1,1\}} \text { in mlet } \mathrm{b}=\mathfrak{U}_{\{0,1\}} \text { in unit }(x+2 * z * \mathrm{~b})
\end{aligned}
$$

We axiomatize the predicate $\mathrm{All}_{2,1}$, which relates the element at position $2 \mathrm{i}$ in one stream to the element at position $i$ in another stream, as follows.

$$
\begin{aligned}
& \forall x_{1} x_{2} x s_{1} x s_{2} y_{1} \cdot \phi\left[z_{1} / x_{1}\right]\left[z_{2} / x_{2}\right] \Rightarrow \\
& \quad \triangleright\left[y s_{1} \leftarrow x s_{1}\right] . \triangleright\left[z s_{1} \leftarrow y s_{1}, y s_{2} \leftarrow x s_{2}\right] . A_{2,1}\left(z s_{1}, y s_{2}, z_{1} . z_{2} \cdot \phi\right) \Rightarrow \\
& \quad \quad \quad A l l_{2,1}\left(x_{1}:: y_{1}: x s_{1}, x_{2}: x s_{2}, z_{1} . z_{2} . \phi\right)
\end{aligned}
$$

In fact, we can assume that, in general, we have a family of $\mathrm{All}_{\mathfrak{m}_{1}, \mathrm{~m}_{2}}$ predicates relating two streams at positions $m_{1} \cdot i$ and $m_{2} \cdot i$ for every $i$.

We can now express the existence of a shift coupling by the statement:

$$
\mathrm{p}_{1}=\mathrm{p}_{2} \vdash \operatorname{markov}\left(\mathrm{p}_{1}, \text { step }\right) \sim \operatorname{markov}\left(\mathrm{p}_{2}, \text { lstep } 2\right) \mid \diamond_{\left[\begin{array}{l}
y_{1} \leftarrow \mathbf{r}_{1} \\
y_{2} \leftarrow \mathbf{r}_{2}
\end{array}\right]} \operatorname{All}_{2,1}\left(y_{1}, y_{2}, z_{1} . z_{2} \cdot z_{1}=z_{2}\right)
$$

For the proof, we need to introduce an asynchronous rule for Markov chains:

$$
\begin{aligned}
& \Omega \vdash \mathrm{t}_{1}: \mathrm{C}_{1} \sim \mathrm{t}_{2}: \mathrm{C}_{2}\{\phi\} \\
& \Omega \vdash\left(\lambda x_{1} \text {. mlet } x_{1}^{\prime}=h_{1} x_{1} \text { in } h_{1} x_{1}^{\prime}\right): C_{1} \rightarrow D\left(C_{1}\right) \sim h_{2}: C_{2} \rightarrow D\left(C_{2}\right)\{
\end{aligned}
$$

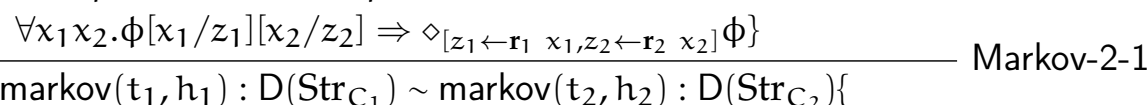

$$
\begin{aligned}
& \left.\diamond_{\left[y_{1} \leftarrow \mathbf{r}_{1}, y_{2} \leftarrow \mathbf{r}_{2}\right]} \operatorname{All}_{2,1}\left(y_{1}, y_{2}, z_{1} \cdot z_{2} . \phi\right)\right\}
\end{aligned}
$$


This asynchronous rule for Markov chains shares the motivations of the rule for loops proposed in [40]. Note that one can define a rule Markov-m-n for arbitrary $m$ and $n$ to prove a judgment of the form $\mathrm{All}_{m, n}$ on two Markov chains.

We show the proof of the shift coupling. By equational reasoning, we get:

$\lambda x_{1}$. mlet $x_{1}^{\prime}=h_{1} x_{1}$ in $h_{1} x_{1}^{\prime} \equiv \lambda x_{1}$. mlet $z_{1}=$ Unif $_{\{-1,1\}}$ in $h_{1}\left(z_{1}+x_{1}\right) \equiv$ $\equiv \lambda x_{1}$.mlet $z_{1}=\operatorname{Unif}_{\{-1,1\}}$ in mlet $z_{1}^{\prime}=\operatorname{Unif}_{\{-1,1\}}$ in unit $\left(z_{1}^{\prime}+z_{1}+x_{1}^{\prime}\right)$

and the only interesting premise of Markov-2-1 is:

$$
\begin{aligned}
& \lambda x_{1} \text {. mlet } z_{1}=\operatorname{Unif}_{\{-1,1\}} \text { in } \lambda x_{2} \text {. mlet } z_{2}=\operatorname{Unif}_{\{-1,1\}} \text { in } \\
& \text { mlet } z_{1}^{\prime}=\operatorname{Unif}_{\{-1,1\}} \text { in } \sim \quad \text { mlet } \mathrm{b}_{2}=\operatorname{Unif}_{\{1,0\}} \text { in } \\
& \text { unit }\left(z_{1}^{\prime}+z_{1}+x_{1}^{\prime}\right) \quad \text { unit }\left(x_{2}+2 * b_{2} * z_{2}\right) \\
& \forall x_{1} x_{2} \cdot x_{1}=x_{2} \Rightarrow \\
& \mathbf{r}_{1} x_{1} \stackrel{\ominus}{=} \mathbf{r}_{2} x_{2}
\end{aligned}
$$

Couplings between $z_{1}$ and $z_{2}$ and between $z_{1}^{\prime}$ and $b_{2}$ can be found by simple computations. This completes the proof.

\subsection{Related Work}

\section{Guarded Lambda Calculus}

Our probabilistic guarded $\lambda$-calculus and the associated logic Guarded HOL build on top of the guarded $\lambda$-calculus and its internal logic [63]. The guarded $\lambda$-calculus has been extended to guarded dependent type theory [51], which can be understood as a theory of guarded refinement types and as a foundation for proof assistants based on guarded type theory. These systems do not reason about probabilities, and do not support syntax-directed (relational) reasoning, both of which we support.

\section{Logical relations}

Relational models for higher-order programming languages are often defined using logical relations. [130] showed how to use second-order logic to define and reason about logical relations for the second-order lambda calculus. Recent work has extended this approach to logical relations for higher-order programming languages with computational effects such as nontermination, general references, and concurrency $[73,152,110,111]$. The logics used in loc. cit. are related to our work in two ways: (1) the logics in loc. cit. make use of the later modality for reasoning about recursion, and (2) the models of the logics in loc. cit. can in fact be defined using guarded type theory.

\subsection{Conclusion}

We have developed a probabilistic extension of the (simply typed) guarded $\lambda$ calculus, and proposed a syntax-directed proof system for relational verification. 
Moreover, we have verified a series of examples that are beyond the reach of prior work. Finally, we have proved the soundness of the proof system with respect to the topos of trees.

There are several natural directions for future work. One first direction is to enhance the expressiveness of the underlying simply typed language. For instance, it would be interesting to introduce clock variables and some type dependency as in [51], and extend the proof system accordingly. This would allow us, for example, to type the function taking the n-th element of a guarded stream, which cannot be done in the current system. Another exciting direction is to consider approximate couplings, as in [33, 34], and to develop differential privacy for infinite streamspreliminary work in this direction, such as [106], considers very large lists, but not arbitrary streams. A final direction would be to extend our approach to continuous distributions to support other application domains. 


\section{AN ASSERTION-BASED LOGIC FOR HIGHER-ORDER PROBABILISTIC PROGRAMS}

On this chapter we present unary and relational logics to reason about general properties of probabilistic programs. As opposed to the logics presented on Chapters 4 or 5 , this logic is not limited to reason about lifting-based properties. Instead, it aims to prove general properties of probabilistic programs, such as equivalence, approximation, convergence, or expected values of their output.

The probabilistic programming language we consider in this chapter differs in some ways from the language from the previous chapters. Before, we have used a higher-order language extended with sampling commands. Now we consider a higher-order language for inference, in the spirit of Anglican [161] Church [86] or Hakaru [123]. These languages are often used to build statistical models or program inference algorithms and feature, along sampling commands, a conditioning operator, that allows us to model conditional probabilities by re-scaling a measure according to a likelihood function that models observations. The higher-order setting is ideal for these languages, treating parametric models, densities or likelihood functions as first-class citizens

We introduce a framework, called the Probabilistic Programming Verification framework (PPV), for proving (unary and relational) properties of probabilistic higher-order programs with discrete and continuous distributions. Following the same style as the rest of this thesis, PPV's design is based on three different logics: PL, UPL and RPL. The base logic PL is a higher-order logic featuring specific constructs to handle measures and write specifications about them, such as expected values or probabilities. The three proof systems are equi-expressive, but the UPL and RPL are closer to the syntax-directed style of reasoning generally favored in unary and relational program verification, respectively. We define an interpretation of assertions in the category of QBS predicates and prove that the logics are sound with respect to the interpretation. 
To further ease program verification, we define carefully crafted axiomatizations of fundamental probabilistic definitions and results, including expectations as well as concentration bounds. Following Ścibior et al. [141], we validate the soundness of these axiomatizations using synthetic measure theory for the QBS framework. This ensures that a derivation based on our proof system and axioms is valid in quasiBorel spaces. A consequence of this approach is that, in order to verify programs, a user of PPV can focus on higher-level reasoning about probabilistic programs, rather than the specific details of QBS.

We validate our design through a series of examples from statistics, Bayesian inference and machine learning.

Structure of the chapter We begin by presenting two warm up examples (Section 9.1). We then present the probabilistic language we consider in this chapter (Section 9.2). After this, we present our base logic, its extensions with probabilistic expressions (Section 9.3) and the axioms to reason about them (Section 9.4). Then, we introduce the unary and relational program logics (Section 9.5). Afterwards, we discuss the semantics of the programming language and the logics (Section 9.6). Finally, we use our logics to verify other examples (Section 9.7).

\subsection{PPV by example}

In this section we introduce the general ideas behind PPV through two examples.

Continuous Observations: Two Uniform Samples This warm-up example serves as an introduction to Bayesian conditioning and how we can reason about it in our system. Let us consider the following program twoUs:

$$
\begin{aligned}
\text { twoUs } \equiv & \text { let } u_{1}=\operatorname{Uniform}(0,1) \text { in let } u_{2}=\operatorname{Uniform}(0,1) \text { in } \\
& \text { let } y=u_{1} \otimes u_{2} \text { in } \\
& \text { query } \left.y \Rightarrow \lambda x \text {. (if } \pi_{1}(x)<1 / 2 \vee \pi_{2}(x)>1 / 2 \text { then } 1 \text { else } 0\right)
\end{aligned}
$$

The first line defines two uniform distributions $\mathfrak{u}_{1}$ and $\mathfrak{u}_{2}$. The second line pairs the two distributions together using the product measure of $\mathfrak{u}_{1}$ and $u_{2}$ which we denote $\mathfrak{u}_{1} \otimes \mathfrak{u}_{2}$ (this is defined formally in Section 9.2). Then, the third line performs Bayesian conditioning on this product measure using the construction query. The prior $y$ gets conditioned by the likelihood function corresponding to the observation $\pi_{1}(x)<1 / 2 \vee \pi_{2}(x)>1 / 2$, and a posterior is computed. In this simple example, this is morally equivalent to giving score 1 to the traces that do satisfy the assertion, and score 0 to the ones that do not satisfy it, and rescaling the distribution. In general, we can use the conditioning construct with an arbitrary likelihood function to perform more general inference. After the observation, the posterior is a uniform distribution over the set $\left\{\left(x_{1}, x_{2}\right) \mid x_{1}<1 / 2 \vee x_{2}>1 / 2\right\}$. 
The simple property we will show is that $\operatorname{Pr}_{\left(x_{1}, x_{2}\right) \sim \mu}\left[x_{1}>1 / 2\right]=1 / 3$, where $\mu$ is the posterior after the observation and the pair $\left(x_{1}, x_{2}\right)$ is distributed by $\mu$. This is expressed in the unary logic UPL—since this is a unary property-through the following judgment:

$$
\vdash_{\text {UPL }} \text { twoUs : } M[\text { real } \times \text { real }]\left\{\operatorname{Pr}_{z \sim \mathbf{r}}\left[\pi_{1}(z)>1 / 2\right]=1 / 3\right\}
$$

where the distinguished variable $\mathbf{r}$ in the logical assertion represents the given term twoUs and the variable $z$ is bound by $\operatorname{Pr}_{z \sim \mathbf{r}}[\ldots]$ and it is used to represent the value sampled from the probability distribution $\mathbf{r}$. We show informally how to derive this assertion. The system UPL allows us to reason in a syntax-directed manner. Since the program starts with three let bindings, the first step will be to apply the rule for let bindings three times. This rule, which we will present formally in Section 9.5, moves $u_{1}, u_{2}$ and $y$ plus the logical assertions about them into the context. The resulting judgment is:

$$
\begin{aligned}
& \operatorname{Pr}_{z \sim u_{1}}[z>1 / 2]=1 / 2, \operatorname{Pr}_{z \sim u_{2}}[z<1 / 2]=1 / 2, \operatorname{Pr}_{z \sim u_{1}}[T]=1, \operatorname{Pr}_{z \sim u_{2}}[T]=1, y=u_{1} \otimes u_{2} \\
& \left.\vdash \text { UPL query } \Rightarrow \lambda x \text {. (if } \pi_{1}(x)<1 / 2 \vee \pi_{2}(x)>1 / 2 \text { then } 1 \text { else } 0\right): M[\text { real } \times \text { real }] \\
& \left\{\operatorname{Pr}_{z \sim \mathbf{r}}\left[\pi_{1}(z)>1 / 2\right]=1 / 3\right\}
\end{aligned}
$$

where for simplicity we omitted the typing context. The logical assertions on $u_{1}$ and $u_{2}$ can be easily discharged using the assumption that they are distributed uniformly as Uniform $(0,1)$, i.e. uniformly between 0 and 1 . To finish the proof, we want to use the fact that query corresponds to conditioning. In UPL we can do this using the following special rule that internalizes the Bayesian properties of query: ${ }^{1}$

$$
\frac{\Gamma, x: \tau \vdash e^{\prime}: \text { bool } \quad \Gamma, x: \tau \vdash e^{\prime \prime}: \text { bool } \quad \Gamma \vdash e: M[\tau]}{\left.\Gamma \mid \Psi \vdash_{\text {UPL }} \text { query } e \Rightarrow \lambda x \text {. (if } e^{\prime} \text { then } 1 \text { else } 0\right): M[\tau]\left\{\operatorname{Pr}_{y \sim r}\left[e^{\prime \prime}[y / x]\right]=\frac{\operatorname{Pr}_{x \sim e}\left[e^{\prime} \wedge e^{\prime \prime}\right]}{\operatorname{Pr}_{x \sim e}\left[e^{\prime}\right]}\right\}} \text { [Bayes] }
$$

This rule corresponds to a natural reasoning principle (derived by Bayes' theorem) for query when we have a boolean condition as the likelihood function: the probability of an event $e^{\prime \prime}$ under the posterior distribution is equal to the probability of the intersection of the event $e^{\prime \prime}$ and the observation $e^{\prime}$, under the prior distribution $e$, divided by the probability of $e^{\prime}$ under the prior distribution $e$.

To apply this rule we need to rewrite the postcondition into the appropriate shape: a fraction that has the probability of a conjunction of events in the numerator and the probability of the observed event in the denominator. This can be done in UPL through a SUB rule which generates the following proof obligations in the logic PL:

$$
\begin{aligned}
& \operatorname{Pr}_{z \sim u_{1}}[z>1 / 2]=1 / 2, \operatorname{Pr}_{z \sim u_{2}}[z<1 / 2]=1 / 2, \operatorname{Pr}_{z \sim u_{1}}[T]=1, \operatorname{Pr}_{z \sim u_{2}}[T]=1, y=u_{1} \otimes u_{2} \\
& \vdash_{\mathrm{PL}} \frac{\operatorname{Pr}_{z \sim y}\left[\left(\pi_{1}(z)<1 / 2 \vee \pi_{2}(z)>1 / 2\right) \wedge\left(\pi_{1}(z)>1 / 2\right)\right]}{\operatorname{Pr}_{z \sim y}\left[\pi_{1}(z)<1 / 2 \vee \pi_{2}(z)>1 / 2\right]}=\frac{1 / 4}{3 / 4}=1 / 3
\end{aligned}
$$

${ }^{1}$ We introduce the rule here to give some intuition, but this is also discussed in Section 9.5 after introducing PPV. 
Chapter 9. An assertion-based logic for higher-order probabilistic programs

Using this equivalence and SUB we can rewrite the judgment we need to prove as follows:

$$
\begin{aligned}
& \operatorname{Pr}_{z \sim u_{1}}[z>1 / 2]=1 / 2, \operatorname{Pr}_{z \sim u_{2}}[z<1 / 2]=1 / 2, \operatorname{Pr}_{z \sim u_{1}}[T]=1, \operatorname{Pr}_{z \sim u_{2}}[T]=1, y=u_{1} \otimes u_{2} \\
& \vdash_{\text {UPL query } y \Rightarrow \lambda x .\left(\text { if } \pi_{1}(x)<1 / 2 \vee \pi_{2}(x)>1 / 2 \text { then } 1 \text { else } 0\right): M[\text { real } \times \text { real }]\{}^{\left.\operatorname{Pr}_{z \sim \mathbf{r}}\left[\pi_{2}(z)>1 / 2\right]=\frac{\operatorname{Pr}_{z \sim y}\left[\left(\pi_{1}(z)<1 / 2 \vee \pi_{2}(z)>1 / 2\right) \wedge\left(\pi_{1}(z)>1 / 2\right)\right]}{\operatorname{Pr}_{z \sim y}\left[\pi_{1}(z)<1 / 2 \vee \pi_{2}(z)>1 / 2\right]}\right\}}
\end{aligned}
$$

and this can be proved by applying the [Bayes] rule above, concluding the proof. We saw different components of PPV at work here: unary rules, subtyping, and a special rule for query. All these components can be assembled in more complex examples, as we show in Section 9.7.

Monte Carlo Approximation As a second example, we show how to use PPV to reason about other classical applications that do not use observations. We consider reasoning about expected value and variance of distributions. Concretely, we show convergence in probability of an implementation of the naive Monte Carlo approximation. This algorithm considers a distribution $\mathrm{d}$ and a real-valued function $h$, and tries to approximate the expected value of $h(x)$ where $x$ is sampled from $d$ by sampling a number $i$ of values from $d$ and computing their mean.

Consider the following implementation of Monte Carlo approximation:

$$
\begin{aligned}
\text { MonteCarlo } \equiv & \text { letrec } f(i: \text { nat })=\text { if }(i \leqslant 0) \text { then unit }(0) \\
& \text { else } m \text { let } m=f(i-1) \text { in } \operatorname{mlet} x=\operatorname{din} \text { unit }((1 / i) *(h(x)+m *(i-1)))
\end{aligned}
$$

Our goal is to prove the convergence in probability of this algorithm, that is, the result can be made as accurate as desired by increasing the sample size (denoted by $i$ above and $n$ below). This is formalized in the following UPL judgment (we omit the typing context for simplicity):

$$
\begin{aligned}
& \left(\mathbb{E}_{x \sim d}[1]=1\right),\left(\sigma^{2}=\operatorname{Var}_{x \sim d}[h(x)]\right),\left(\mu=\mathbb{E}_{x \sim d}[h(x)]\right),(\varepsilon>0) \vdash \text { UPL } \\
& \text { MonteCarlo: nat } \rightarrow M[\text { real }] \mid \forall n,(n>0) \Rightarrow \operatorname{Pr}_{y \sim \mathbf{m}}[|y-\mu| \geqslant \varepsilon] \leqslant \sigma^{2} / n \varepsilon^{2}
\end{aligned}
$$

Formally, we are showing that the probability that the computed mean $y$ differs from the actual mean $\mu$ by more than $\varepsilon$ is upper bounded by a value that depends inversely on $n-$ more samples lead to better estimates. To derive (9.1) in UPL we need to perform two steps:

- Calculating the mass, mean, and variance of MonteCarlo in UPL:

$$
\begin{aligned}
& \left(\mathbb{E}_{x \sim d}[1]=1\right),\left(\sigma^{2}=\operatorname{Var}_{x \sim d}[h(x)]\right),\left(\mu=\mathbb{E}_{x \sim d}[h(x)]\right),(\varepsilon>0) \vdash \mathrm{UPL} \\
& \text { MonteCarlo: nat } \rightarrow M[\text { real] }\{ \\
& \left.\forall n: \text { nat. }(n>0) \Rightarrow\left(\mathbb{E}_{y \sim \mathbf{m n}}[1]=1\right) \wedge\left(\mathbb{E}_{y \sim \mathbf{m n}}[y]=\mu\right) \wedge\left(\operatorname{Var}_{y \sim \mathbf{m n}}[y]=\sigma^{2} / n\right)\right\}
\end{aligned}
$$

- Applying the Chebyshev inequality (formula 9.17 in Section 9.4.1) to (9.2) using subtyping. 
We focus on the proof of (9.2), which is carried out by induction on $n$. In our system, the rule for letrec lets us prove inductive properties of (terminating) recursive functions by introducing an inductive hypothesis into the set of assertions that can only be instantiated for smaller arguments. After applying this rule, the new goal is:

$$
\begin{gathered}
\phi_{I H} \equiv \forall n: \text { nat. }(n<i) \Rightarrow(n>0) \Rightarrow \\
\left(\mathbb{E}_{y \sim f(n)}[1]=1\right) \wedge\left(\mathbb{E}_{y \sim f(n)}[y]=\mu\right) \wedge\left(\operatorname{Var}_{y \sim f(n)}[y]=\sigma^{2} / n\right)
\end{gathered}
$$

On this, we can apply a rule for case distinction according to the two branches of the if-then-else, which gives us the following two premises:

$$
\begin{aligned}
& \Psi,(i \leqslant 0) \vdash \operatorname{unit}(0) \mid \psi \\
& \Psi,(i>0) \vdash \operatorname{mlet} m=f(i-1) \text { in }\left(m l e t x=\operatorname{din} \operatorname{unit}\left(\frac{1}{\mathfrak{i}}(h(x)+m *(i-1))\right)\right) \mid \psi
\end{aligned}
$$

where $\Psi=\left(\mathbb{E}_{x \sim d}[1]=1\right),\left(\mu=\mathbb{E}_{x \sim d}[h(x)]\right),\left(\sigma^{2}=\operatorname{Var}_{x \sim d}[h(x)]\right),(i>0), \phi_{I H}$ and $\psi=$ $\left(\mathbb{E}_{y \wedge \text { ri }}[1]=1\right) \wedge\left(\mathbb{E}_{y \wedge \text { ri }}[y]=\mu\right) \wedge\left(\operatorname{Var}_{y \sim r i}[y]=\sigma^{2} / i\right)$. The first premise is obvious since the assumptions $(i>0)$ and $(i \leqslant 0)$ are contradictory. The second premise follows from subtyping applied to a PL-judgment that is proved by instantiating the induction hypothesis with $i-1$ and applying axioms on expected values. This concludes the proof.

Again, we have seen here several different components of PPV: unary rules (including the rule for inductive reasoning), subtyping, and the use of equations and axioms. We further illustrate these components of PPV as well as others in verifying more involved examples (including relational examples) in Section 9.7.

Remark: In this chapter, we assume that query is always defined: we don't consider programs "observing" events with zero probability. We make this simplification to focus here on program verification without the need to reason about whether a query statement is defined or not. This approach was used, for example, in Barthe et al. [35] to reason about differential privacy for Bayesian processes. We believe that the problem of identifying ways to reason about when a query statement is defined is an important one, but it is orthogonal to the formal reasoning we consider here. Other work has focused on this problem [54, 141, 142, 93]. In a similar way, we consider only programs that terminate, without stipulating a specific method to prove termination.

\subsection{HPProg: a higher-order probabilistic programming language}

We present the probabilistic language HPProg we use in this section. It is a simplytyped lambda calculus extended with with products, coproducts, natural numbers, 
Chapter 9. An assertion-based logic for higher-order probabilistic programs

lists, (terminating) recursion and a monadic type for probabilistic computations. The types of HPProg are defined by the following grammar.

$$
\begin{array}{lll}
\mathrm{B}::= & \text { ut } \mid \text { bool } \mid \text { nat } \mid \text { real }|\mathrm{pReal}| \mathrm{B} \times \mathrm{B} \mid \operatorname{list}(\tilde{\tau}) & \text { (Basic Types) } \\
\tau::=\mathrm{B}|\mathrm{M}(\tau)| \tau \rightarrow \tau|\tau \times \tau| \operatorname{list}(\tau) & \text { (Types) }
\end{array}
$$

We distinguish two sorts of types: Basic Types and Types. The former, as the name suggests, include standard basic types (where pReal is the type of positive real numbers), and products and lists of them. The latter include a monadic type $M[\tau]$ for general measures on $\tau$, as well as function and product types. The language of HPProg expressions is defined by the following grammar.

$$
\begin{aligned}
t, u::= & x|c| f|t u| \lambda x . t|\langle t, u\rangle| \pi_{i}(t) \mid \text { case } t \text { with }\left[d_{i} \overline{x_{i}} \Rightarrow u_{i}\right]_{i} \mid \text { letrec } f x=t \\
& \mid \text { unit } t \mid \text { bind } t u \mid \text { query } t \Rightarrow u|\operatorname{Uniform}(t, u)| \operatorname{Bern}(t) \mid \operatorname{Gauss}(t, u)
\end{aligned}
$$

Most of the constructs are standard. We use $c$ to range over a set of basic constants and $f$ to range over a set of primitive functions. We have monadic constructions unit $t$ and bind $t_{1} t_{2}$ for the monadic type $M(\tau)$, a conditioning construction query $t_{1} \Rightarrow t_{2}$ for computing the posterior distribution given a prior distribution $t_{1}$, and a likelihood function $t_{2}$, and primitives representing basic probability distributions.

HPProg expressions are simply-typed, using rules that are mostly standard and that have appeared in other chapters. We only show rules for the new constructs:

$$
\begin{gathered}
\frac{\Gamma \vdash \mathrm{t}: \mathrm{M}(\tau) \quad \Gamma \vdash \mathrm{t}^{\prime}: \tau \rightarrow \text { pReal }}{\Gamma \vdash \text { query } \mathrm{t} \Rightarrow \mathrm{t}^{\prime}: \mathrm{M}(\tau)} \quad \frac{\Gamma \vdash \mathrm{t}: \mathrm{M}(\tau) \quad \Gamma \vdash \mathrm{t}^{\prime}: \tau \rightarrow \mathrm{M}\left(\tau^{\prime}\right)}{\Gamma \vdash \operatorname{bind} \mathrm{t} \mathrm{t}^{\prime}: \mathrm{M}\left(\tau^{\prime}\right)} \\
\frac{\Gamma, \mathrm{f}: \mathrm{I} \rightarrow \sigma, x: I \vdash \mathrm{t}: \sigma \quad \mathrm{I} \in\{\text { nat, list }(\tau)\} \quad \operatorname{Def}(\mathrm{f}, \mathrm{x}, \mathrm{t})}{\Gamma \vdash \text { letrec } \mathrm{fx}=\mathrm{t}: \mathrm{I} \rightarrow \sigma}
\end{gathered}
$$

In the last rule, $\operatorname{Def}(f, x, t)$ is any termination criterion which ensures that all recursive calls are on smaller arguments. We also consider a basic equational theory for expressions based on $\beta$-reduction, extensionality and monadic rules. These are also standard and we omit them here. We enrich this equational theory with axioms and equations reflecting common reasoning principles for probabilistic programming in Section 9.4.

Also note that we use bind as opposed to mlet as in the previous chapter. For convenience, we use some syntactic sugar: (let $x=e_{1}$ in $\left.e_{2}\right) \equiv\left(\lambda x . e_{2}\right) e_{1},(\operatorname{mlet} x=$ $e_{1}$ in $\left.e_{2}\right) \equiv$ bind $e_{1}\left(\lambda x . e_{2}\right)$, and $e_{1} \otimes e_{2} \equiv$ bind $e_{1}\left(\lambda x\right.$. bind $e_{2}(\lambda y$. unit $\langle x, y\rangle)$ ). Thanks to the commutativity of $M$ (the Fubini-Tonelli equality; see Section 9.4), the semantics of $e_{1} \otimes e_{2}$ is exactly the product measure of $e_{1}$ and $e_{2}$.

\subsection{PL: A Logic for Probabilistic Programs}

In this section we introduce a logic, named PL, for reasoning about higher-order probabilistic programs. This logic forms the basis of PPV. PL contains basic predi- 
cates over expressions of HPProg. To support more natural verification in PPV, we enrich PL with a set of axioms encompassing a wide variety of reasoning principles over probabilistic programs.

PL follows the style of HOL, as we have presented in other chapters, but extends it with assertions about probabilistic constructions. Terms and formulas of PL are defined by the following grammar:

$$
\begin{array}{rlr}
e::= & t\left|\mathbb{E}_{x \sim e}\left[e^{\prime}(x)\right]\right| \operatorname{scale}\left(e, e^{\prime}\right) \mid \operatorname{normalize}(t) & \text { (enriched expressions) } \\
\phi::= & \left(e=e^{\prime}\right)\left|\left(e<e^{\prime}\right)\right| \top|\perp| \phi \wedge \phi|\phi \Rightarrow \phi| \neg \phi \mid & \\
& |\forall x: \tau . \phi| \exists x: \tau . \phi
\end{array}
$$

Enriched expressions enrich HPProg terms $t$ with constructions to explicitly deal with measures: expectations $\mathbb{E}_{x \sim e}\left[e^{\prime}(x)\right]$, rescaling of measures $\operatorname{scale}\left(e, e^{\prime}\right)$, and normalization normalize $(e)$. These have the following typing rules:

$$
\begin{gathered}
\frac{\Gamma \vdash e_{1}: M(\tau) \quad \Gamma \vdash e_{2}: \tau \rightarrow \text { pReal }}{\Gamma \vdash \mathbb{E}_{\chi \sim e_{1}}\left[e_{2}(x)\right]:} \frac{\Gamma \vdash e_{1}: M(\tau) \quad \Gamma \vdash e_{2}: \tau \rightarrow \text { pReal }}{\Gamma \vdash \operatorname{scale}\left(e_{1}, e_{2}\right): M(\tau)} \\
\frac{\Gamma \vdash e: M(\tau)}{\Gamma \vdash \operatorname{normalize}(e): M(\tau)}
\end{gathered}
$$

Intuitively, $\mathbb{E}_{x \sim e_{1}}\left[e_{2}(x)\right]$ is the expected value of the function $e_{2}$ over the distribution $e_{1}, \operatorname{scale}\left(e_{1}, e_{2}\right)$ is a distribution obtained from an underlying measure $e_{1}$ by rescaling its components by means of the density function $e_{2}$, and normalize(e) is the normalization of a measure $e$ to a probability distribution (a measure with mass 1). Expectations of real-valued functions are defined by the difference of positive and negative parts. Precisely, for given $\Gamma \vdash e_{1}: M(\tau)$ and $\Gamma \vdash e_{2}: \tau \rightarrow$ real, we define the expectation as the following syntactic sugar: ${ }^{2}$

$$
\left.\mathbb{E}_{x \sim e_{1}}\left[e_{2}(x)\right] \equiv \mathbb{E}_{x \sim e_{1}}\left[\text { if } e_{2}(x)>0 \text { then }\left|e_{2}(x)\right| \text { else } 0\right]-\mathbb{E}_{x \sim e_{1}} \text { [if } e_{2}(x)<0 \text { then }\left|e_{2}(x)\right| \text { else } 0\right]
$$

We can also define variance and probability in terms of expectation:

$$
\underset{x \sim e}{\operatorname{Pr}}\left[e^{\prime}\right] \equiv \mathbb{E}_{x \sim e}\left[\text { if } e^{\prime} \text { then } 1 \text { else } 0\right] \quad \operatorname{Var}_{x \sim e_{1}}\left[e_{2}\right] \equiv \mathbb{E}_{x \sim e_{1}}\left[\left(e_{2}\right)^{2}\right]-\left(\mathbb{E}_{x \sim e_{1}}\left[e_{2}\right]\right)^{2}
$$

A PL judgment has the form $\Gamma \mid \Psi \vdash_{\mathrm{PL}} \phi$ where $\Gamma$ is a context assigning types to variables, $\Psi$ is a set of formulas well-formed in the context $\Gamma$, and $\phi$ is a formula also well-formed in $\Gamma$. Rules to derive well-formedness judgments $\Gamma \vdash \phi$ wf are standard and we omit them here. The proof rules of PL are also standard, and equivalent to those from Chapter 3.

We extend equational rules and axioms for standard expressions to enriched expressions. We also introduce some axioms specific to enriched expressions in Section 9.4.

\footnotetext{
${ }^{2}$ We use absolute values $|-|:$ real $\rightarrow$ pReal to adjust the typing. The right-hand side is undefined if both expectations are infinity. We could avoid this kind of undefinedness by stipulating $\infty-\infty=-\infty$, but we leave it undefined since this actually never shows up in our concrete examples.
} 


\subsection{Axioms and Equations of Assertions for Statistics}

In this section, we introduce axioms and equations for the logic PL. First, we have the standard equational theory for (enriched) expressions covering $\alpha$-conversion, $\beta$-reduction, extensionality, and the monadic rules of the monadic type $M$. We omit these rules, which we have presented before. The monadic type $M$ also has commutativity (the Fubini-Tonelli equality), represented by the following equation:

(bind $e_{1} \lambda x .\left(\right.$ bind $\left.\left.e_{2} \lambda y . e(x, y)\right)\right)=\left(\right.$ bind $e_{2} \lambda y .\left(\right.$ bind $\left.e_{1} \lambda x . e(x, y)\right) \quad\left(x, y \notin F V\left(e_{1}, e_{2}\right)\right)$

We introduce some equalities pertaining to expectation. We have the monotonicity and linearity of expectation (axioms 9.4, 9.5), and we also have the CauchySchwarz inequality (axiom 9.6). Finally, we add a rule that expresses the interaction between the monad bind and the expected value (axiom 9.7).

$$
\begin{gathered}
\left(\forall x: \tau . e^{\prime} \geqslant 0\right) \Longrightarrow \\
\mathbb{E}_{x \sim e}\left[e_{1} * e_{2}\right]=e_{1} * \mathbb{E}_{x \sim e}\left[e_{2}\right] \quad\left(x \notin \mathrm{FV}\left(e_{1}\right)\right), \quad \mathbb{E}_{x \sim e}\left[e_{1}+e_{2}\right]=\mathbb{E}_{x \sim e}\left[e_{1}\right]+\mathbb{E}_{x \sim e}\left[e_{2}\right] \\
\left(\mathbb{E}_{x \sim e}\left[e_{1} * e_{2}\right]\right)^{2} \leqslant \mathbb{E}_{x \sim e}\left[e_{1}^{2}\right] * \mathbb{E}_{x \sim e}\left[e_{2}^{2}\right] \\
\mathbb{E}_{x \sim \text { bind } e \lambda y \text {.unit }\left(e^{\prime}\right)}\left[e^{\prime \prime}\right]=\mathbb{E}_{y \sim e}\left[e^{\prime \prime}\left[e^{\prime} / x\right]\right]
\end{gathered}
$$

We also introduce some basic equalities pertaining to observation, rescaling, and normalization.

$$
\begin{gathered}
\mathbb{E}_{x \sim d^{\prime}}[h(x) \cdot g(x)]=\mathbb{E}_{x \sim \operatorname{scale}\left(d^{\prime}, g\right)}[h(x)] . \\
\left(\operatorname{scale}\left(\operatorname{scale}\left(e_{1}, e_{2}\right), e_{3}\right)=\left(\operatorname{scale}\left(e_{1}, \lambda x .\left(e_{2}(x) * e_{3}(x)\right)\right), \quad e=\operatorname{scale}\left(e, \lambda \_.1\right)\right.\right. \\
\left(\operatorname{mlet} x=\operatorname{scale}\left(e_{1}, e_{2}\right) \text { in } e_{3}(x)\right)=\left(\operatorname{mlet} x=e_{1} \operatorname{inscale}\left(e_{3}(x), \lambda u . e_{2}(x)\right)\right) \\
\operatorname{scale}\left(e_{1}, e_{2}\right) \otimes \operatorname{scale}\left(e_{3}, e_{4}\right)=\operatorname{scale}\left(e_{1} \otimes e_{2}, \lambda w . e_{2}\left(\pi_{1}(w)\right) * e_{4}\left(\pi_{2}(w)\right)\right) \\
\mathbb{E}_{y \sim e}[1]<\infty \Longrightarrow\left(\operatorname{bind} e^{\prime} \lambda x . e\right)=\operatorname{scale}\left(e, \mathbb{E}_{y \sim e^{\prime}}[1]\right) \quad(x \notin \mathrm{FV}(e)) \\
\text { (query } \left.e_{1} \Rightarrow e_{2}\right)=\operatorname{normalize}\left(\operatorname{scale}\left(e_{1}, e_{2}\right)\right) \\
\text { normalize }(e)=\operatorname{scale}\left(e, \lambda u .1 / \mathbb{E}_{x \sim e}[1]\right) \quad\left(u \notin \operatorname{FV}\left(\mathbb{E}_{x \sim e}[1]\right)\right) \\
0<\alpha<\infty \Longrightarrow \text { normalize }\left(\operatorname{scale}\left(e_{1}, e_{2}\right)\right)=\operatorname{normalize}\left(\operatorname{scale}\left(e_{1}, \alpha * e_{2}\right)\right)
\end{gathered}
$$

We can also introduce some axioms for particular distributions. For instance, for the Bernoulli distribution we have $\mathbb{E}_{x \sim \operatorname{Bern}(e)}$ [if $x$ then 1 else 0$]=e(0 \leqslant e \leqslant 1)$, and for the Gaussian we have $\mathbb{E}_{\chi \sim \operatorname{Gauss}\left(e_{1}, e_{2}\right)}[x]=e_{1}$.

\subsubsection{Markov and Chebyshev Inequalities}

The axioms we introduced above are not only very basic but also very expressive. For instance, we can prove the Markov inequality (9.16) and the Chebyshev inequality 


$$
\begin{aligned}
& \frac{\Gamma \mid \Psi \vdash_{\text {UPL }} e: \tau\{\phi[\text { unit }(\mathbf{r}) / \mathbf{r}]\}}{\Gamma \mid \Psi \vdash_{\text {UPL }} \text { unit }(e): M[\tau]\{\phi\}} \text { RET-U } \\
& \Gamma \mid \Psi \vdash_{\mathrm{UPL}} e: M\left[\tau_{1}\right]\left\{\phi_{1}\right\} \\
& \frac{\Gamma \mid \Psi \vdash_{\mathrm{UPL}} e^{\prime}: \tau_{1} \rightarrow M\left[\tau_{2}\right]\left\{\forall s: M\left[\tau_{1}\right] \cdot\left(\phi_{1}[\mathrm{~s} / \mathbf{r}] \Longrightarrow \phi_{2}[\text { bind } \mathrm{s} \mathbf{r} / \mathbf{r}]\right)\right\}}{\Gamma \mid \Psi \vdash_{\mathrm{UPL}} \text { bind e } e^{\prime}: M\left[\tau_{2}\right]\left\{\phi_{2}\right\}} \text { BIND-U } \\
& \Gamma \mid \Psi \vdash_{\text {UPL }} e: M[\tau]\left\{\phi_{1}\right\} \\
& \frac{\Gamma \mid \Psi \vdash_{\text {UPL }} e^{\prime}: \tau \rightarrow \text { pReal }\left\{\forall s: M[\tau] .\left(\phi_{1}[\mathrm{~s} / \mathbf{r}] \Longrightarrow \phi_{2}[\text { query } s \Rightarrow \mathbf{r} / \mathbf{r}]\right)\right\}}{\Gamma \mid \Psi \vdash_{\text {UPL }} \text { query } e \Rightarrow e^{\prime}: M[\tau]\left\{\phi_{2}\right\}} \text { QRY-U }
\end{aligned}
$$

Figure 9.1: UPL rules for probabilistic constructs

(9.17) in PL using these axioms.

$$
\begin{aligned}
& \mathrm{d}: M[\text { real }] \text {, a: real } \vdash_{\mathrm{PL}}(\mathrm{a}>0) \Longrightarrow \operatorname{Pr}_{x \sim \mathrm{d}}[|x| \geqslant a] \leqslant \mathbb{E}_{x \sim \mathrm{d}}[|x|] / \mathrm{a} . \\
& \mathrm{d}: M[\text { real], b: real, } \mu \text { : real } \vdash_{\mathrm{PL}} \mathbb{E}_{x \sim \mathrm{d}}[1]=1 \wedge \mu=\mathbb{E}_{x \sim \mathrm{d}}[x] \wedge b^{2}>0 \\
& \Longrightarrow \operatorname{Pr}_{x \sim d}[|x-\mu| \geqslant b] \leqslant \operatorname{Var}_{x \sim \mathrm{d}}[x] / b^{2} .
\end{aligned}
$$

\subsection{Unary/Relational Logic}

As we did for HOL, we present two proof systems built on top of PL to reason about probabilistic programs: a unary logic UPL and a relational logic RPL.

We will show that the logics UPL and RPL are sound and complete with respect to the underlying logic PL, and recover soundness of UPL and RPL from this fact.

\subsubsection{The Unary Logic UPL}

Judgments in the unary logic UPL have the shape $\Gamma \mid \Psi \vdash_{\mathrm{UPL}} e: \tau\{\phi\}$ where $\Gamma$ is a context, $\Psi$ is a set of assertions on the context variables, $e$ is a HPProg expression, $\tau$ a type, and $\phi$ is an assertion (possibly) containing a distinguished variable $\mathbf{r}$ of type $\tau$ which is used to refer to the expression $e$ in the formula $\phi$.

We give in Figure 9.1 a selection of proof rules in UPL. We present only the rules for probabilistic constructs, the rules for pure constructs are as in UHOL (Section 3.4). In the case of monadic computations, we have rules for monadic return, bind and query. It is worth noting the difference between the BIND-U rule here and the MLET-U rule from Section 4.3. In MLET-U the predicates are lifted, so the specification of the first premise (here, $\phi_{1}$ ) can be used unlifted in the context of the second premise. Meanwhile, in the second premise of the rule u-BIND (and also $u-Q R Y)$, we have an assertion that quantifies over elements in $M\left[\tau_{1}\right]$, while the 
input type of the function is just $\tau_{1}$. This follows the spirit of the interpretation (see Section 9.6), where the Kleisli lifting (-) is used to lift a function $\tau_{1} \rightarrow M\left[\tau_{2}\right]$ to a function $M\left[\tau_{1}\right] \rightarrow M\left[\tau_{2}\right]$. The quantification over distributions, rather than over elements, is essential to establish a connection with the assertion on the first premise.

Same as for UHOL, we can prove that, despite being syntax directed, UPL does not lose expressiveness relative to PL, as stated by the following theorem:

Theorem 9.1 (Equi-derivability of PL and UPL). The judgment $\Gamma \mid \Psi \vdash_{\mathrm{PL}} \phi[e / \mathbf{r}]$ is derivable if and only if the judgment $\Gamma \mid \Psi \vdash_{\mathrm{UPL}} e: \tau\{\phi\}$ is derivable.

\subsubsection{The Relational Logic RPL}

Judgments in the relational logic RPL have the shape

$$
\Gamma \mid \Psi \vdash_{\mathrm{UPL}} e_{1}: \tau_{1} \sim e_{2}: \tau_{2}\{\phi\},
$$

where $\Gamma$ is a context, $\Psi$ is a set of assertions on the context, $e_{1}$ and $e_{2}$ are HPProg expressions, $\tau_{1}$ and $\tau_{2}$ are types, and $\phi$ is an assertion (possibly) containing two distinguished variables $\mathbf{r}_{1}$ of type $\tau_{1}$ and $\mathbf{r}_{2}$ of type $\tau_{2}$ which are used to refer to the expressions $e_{1}$ and $e_{2}$ in the formula $\phi$. We give in Figure 9.2 a selection of proof rules in RPL. We present two groups of rules for probabilistic constructs, the rules for pure constructs are as in RHOL (Section 3.5). The first group consists of two-sided relational rules for probabilistic computations, The second group consists of one-sided relational rules for probabilistic computations, As usual, we show just the left-sided rules that have the constructor on the left; right-sided rules are symmetrical. As in the unary case, we use an approach that is mostly syntax-directed except for the [SUB] rule.

For the probabilistic constructions, we have relational rules for the monadic return and bind, and for query. These rules are the natural generalization of the unary rules to the relational case. In particular, in all the rules for bind and query we use assertions quantifying over distributions, similarly to what we have in UPL, to establish a connection between the different assertions.

The equi-derivability result for UPL can be lifted to the relational setting: RPL is also sound and complete with respect to the logic PL.

Theorem 9.2 (Equi-derivability of PL and RPL). The judgment $\Gamma \mid \Psi \vdash_{\mathrm{PL}} \phi\left[e_{1} / \mathbf{r}_{1}, e_{2} / \mathbf{r}_{2}\right]$ is derivable if and only if $\Gamma \mid \Psi \vdash_{\mathrm{RPL}} e_{1}: \tau_{1} \sim e_{2}: \tau_{2}\{\phi\}$ is derivable.

\subsubsection{Special Rules}

As already discussed in the introduction, we enrich PPV with rules to deal explicitly with some constructs appearing in probabilistic programs. One example is the 


\section{Relational rules for probabilistic constructions - two-sided}

$$
\begin{aligned}
& \frac{\Gamma \mid \Psi \vdash_{\mathrm{RPL}} e_{1}: \tau_{1} \sim e_{2}: \tau_{2}\left\{\phi\left[\operatorname{unit}\left(\mathbf{r}_{1}\right) / \mathbf{r}_{1}, \operatorname{unit}\left(\mathbf{r}_{2}\right) / \mathbf{r}_{2}\right]\right\}}{\Gamma \mid \Psi \vdash_{\mathrm{RPL}} \operatorname{unit}\left(e_{1}\right): M\left[\tau_{1}\right] \sim \operatorname{unit}\left(e_{2}\right): M\left[\tau_{2}\right]\{\phi\}} \operatorname{RET} \\
& \phi=\forall s_{1}: M\left[\tau_{1}\right] \cdot \forall s_{2}: M\left[\tau_{2}\right] .\left(\phi_{1}\left[s_{1} / \mathbf{r}_{1}, s_{2} / \mathbf{r}_{2}\right] \Longrightarrow \phi_{2}\left[\text { bind } s_{1} \mathbf{r}_{1} / \mathbf{r}_{1}, \text { bind } s_{2} \mathbf{r}_{2} / \mathbf{r}_{2}\right]\right) \\
& \frac{\Gamma\left|\Psi \vdash_{\mathrm{RPL}} e_{1}: M\left[\tau_{1}\right] \sim e_{2}: M\left[\tau_{2}\right]\left\{\phi_{1}\right\} \quad \Gamma\right| \Psi \vdash_{\mathrm{RPL}} e_{1}^{\prime}: \tau_{1} \rightarrow M\left[\tau_{3}\right] \sim e_{2}^{\prime}: \tau_{2} \rightarrow M\left[\tau_{4}\right]\{\phi\}}{\Gamma \mid \Psi \vdash_{\mathrm{RPL}} \text { bind } e_{1} e_{1}^{\prime}: M\left[\tau_{3}\right] \sim \text { bind } e_{2} e_{2}^{\prime}: M\left[\tau_{4}\right]\left\{\phi_{2}\right\}} \text { BIND } \\
& \left.\phi=\forall s_{1}: M\left[\tau_{1}\right], s_{2}: M\left[\tau_{2}\right] . \phi_{1}\left[s_{1} / \mathbf{r}_{1}, s_{2} / \mathbf{r}_{2}\right] \Longrightarrow \phi_{2} \text { [query } s_{1} \Rightarrow \mathbf{r}_{1} / \mathbf{r}_{1} \text {, query } s_{2} \Rightarrow \mathbf{r}_{2} / \mathbf{r}_{2}\right] \\
& \frac{\Gamma\left|\Psi \vdash_{\mathrm{RPL}} e_{1}: M\left[\tau_{1}\right] \sim e_{2}: M\left[\tau_{2}\right]\left\{\phi_{1}\right\} \quad \Gamma\right| \Psi \vdash_{\mathrm{RPL}} e_{1}^{\prime}: \tau_{1} \rightarrow \text { pReal } \sim e_{2}^{\prime}: \tau_{2} \rightarrow \text { pReal }\{\phi\}}{\Gamma \mid \Psi \vdash_{\mathrm{RPL}} \text { query } e_{1} \Rightarrow e_{1}^{\prime}: M\left[\tau_{1}\right] \sim \text { query } e_{2} \Rightarrow e_{2}^{\prime}: M\left[\tau_{2}\right]\left\{\phi_{2}\right\}} \text { QRY }
\end{aligned}
$$

Relational rules for probabilistic constructions - one-sided

$$
\begin{gathered}
\frac{\Gamma \mid \Psi \vdash_{\mathrm{RPL}} e_{1}: \tau_{1} \sim e_{2}: \tau_{2}\left\{\phi\left[\text { unit }\left(\mathbf{r}_{1}\right) / \mathbf{r}_{1}\right]\right\}}{\Gamma \mid \Psi \vdash_{\mathrm{RPL}} \operatorname{unit}\left(e_{1}\right): M\left[\tau_{1}\right] \sim e_{2}: \tau_{2}\{\phi\}} \text { RET-L } \\
\frac{\Gamma \mid \Psi \vdash_{\mathrm{UPL}} e_{1}: M\left[\tau_{1}\right]\left\{\phi_{1}\right\}}{\Gamma \mid \Psi \vdash_{\mathrm{RPL}} e_{1}^{\prime}: \tau_{1} \rightarrow M\left[\tau_{3}\right] \sim e_{2}: M\left[\tau_{2}\right]\left\{\forall s_{1}: M\left[\tau_{1}\right] . \phi_{1}\left[s_{1} / \mathbf{r}\right] \Longrightarrow \phi_{2}\left[\text { bind } s_{1} \text { in } \mathbf{r}_{1} / \mathbf{r}_{1}\right]\right\}} \\
\Gamma \mid \Psi \vdash_{\mathrm{RPL}} \operatorname{bind} e_{1} e_{1}^{\prime}: M\left[\tau_{3}\right] \sim e_{2}: M\left[\tau_{2}\right]\left\{\phi_{2}\right\} \\
\Gamma \mid \Psi \vdash_{\mathrm{UPL}} e_{1}: M\left[\tau_{1}\right]\left\{\phi_{1}\right\} \\
\frac{\Gamma \mid \Psi \vdash_{\mathrm{RPL}} e_{1}^{\prime}: \tau_{1} \rightarrow \operatorname{pReal} \sim e_{2}: M\left[\tau_{2}\right]\left\{\forall s_{1}: M\left[\tau_{1}\right] . \phi_{1}\left[s_{1} / \mathbf{r}_{1}\right] \Longrightarrow \phi_{2}\left[\text { query } s_{1} \Rightarrow \mathbf{r}_{1} / \mathbf{r}_{1}\right]\right\}}{\Gamma \mid \Psi \vdash_{\mathrm{RPL}} \text { query } e_{1} \Rightarrow e_{1}^{\prime}: M\left[\tau_{1}\right] \sim e_{2}\left\{\phi_{2}\right\}} \text { QRY-L }
\end{gathered}
$$

Figure 9.2: A selection of RPL rules. 
following rule, which internalizes Bayes law into our system:

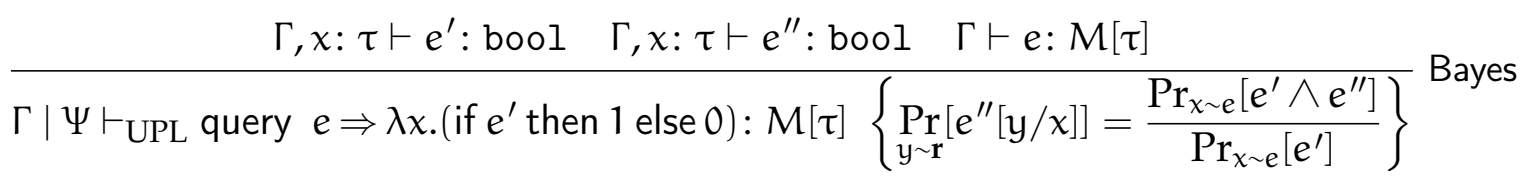

This rule can be derived by first using [u-QRY], and then reasoning in PL through the [u-SUB] rule, which is why the premises are just simply typed assumptions. In particular, in PL we use the characterization of query given in Section 9.4.

Notice that Theorem 9.1 can be used to convert UPL derivation trees into PL ones and vice versa. Similarly, Theorem 9.2 is used to convert RPL proofs to PL proofs. These conversions are useful to switch between the different levels of our system and to reason in whichever one is more convenient. To this end, we introduce the following admissible rules:

$$
\frac{\Gamma \mid \Psi \vdash_{\mathrm{UPL}} e: \tau\{\phi\}}{\Gamma \mid \Psi \vdash_{\mathrm{PL}} \phi[e / \mathbf{r}]} \text { conv-UPL } \frac{\Gamma \mid \Psi \vdash_{\mathrm{RPL}} e_{1}: \tau_{1} \sim e_{2}: \tau_{2}\{\phi\}}{\Gamma \mid \Psi \vdash_{\mathrm{PL}} \phi\left[e_{1} / \mathbf{r}_{1}, e_{2} / \mathbf{r}_{2}\right]} \text { conv-RPL }
$$

\subsection{Semantics}

\subsubsection{Background}

In this section we present the semantic foundation of PPV, which we model in the category of quasi-Borel spaces. The main difference with the interpretations we presented before is that we now consider a monad of arbitrary $\sigma$-finite measures instead of probability measures, to accomodate for conditioning, scaling and normalizing.

Definition 9.3 (Ścibior et al. [141]). The monad of $\sigma$-finite measures $\mathcal{M}$ is defined as follows.

- For any X in QBS, $\mathcal{M} X$ is the set of equivalence classes of $\sigma$-finite measures over $\mathrm{X}$ equipped with the following structure:

$$
M_{\mathcal{M} X}=\left\{\begin{array}{l|l}
\lambda r .\left[D_{r}, \alpha(r,-), \mu_{r}\right] & \begin{array}{l}
\mathrm{D} \subseteq \text { measurable } \mathbb{R} \times \Omega, \mu: \sigma \text {-finite measure on } \Omega, \\
\alpha: D \rightarrow X, D_{r}=\{\omega \mid(r, \omega) \in D\}, \mu_{r}=\left.\mu\right|_{D_{r}}
\end{array}
\end{array}\right\}
$$

Recall that two measures are equivalent if they define the same integration operator.

- The unit $\eta_{X}: X \rightarrow \mathcal{M} X$ is defined by $\eta_{X}(x)=\left[1, \lambda * . x, \mathbf{d}_{*}\right]$.

- The Kleisli lifting is defined for any $\mathrm{f}: \mathrm{X} \rightarrow \mathcal{M} Y$ and $[\Omega, \alpha, v] \in \mathcal{M} X$ as

$$
f^{\sharp}[\Omega, \alpha, v]=\left[D, \beta,\left.\left(v \otimes v^{\prime}\right)\right|_{D}\right]
$$

where $\mathrm{D}=\left\{(\mathrm{r}, \omega) \mid \omega \in \mathrm{D}_{\mathrm{r}}\right\}$ and $\beta(-)=\lambda \mathrm{r} . \beta(\mathrm{r},-)$ are defined for every $\gamma: \Omega \rightarrow \mathbb{R}$ and $\gamma^{*}: \mathbb{R} \rightarrow \Omega$ satisfying $\gamma^{*} \circ \gamma=\mathrm{id}_{\Omega}$ through $(f \circ \alpha)\left(\gamma^{*}(r)\right)=\left[D_{r}, \beta(r,-), v^{\prime}\right]$. 
Let us unpack this definition. The set of functions $M_{\mathcal{M} X}$ can be seen as a set of (uncountable) families of measures, indexed by $r$, supporting infinite measures. The Kleisli lifting uses the fact that each $(f \circ \alpha)\left(\gamma^{*}(-)\right)$ is a function in $M_{\mathcal{M} Y}$, that $D$ built as a product measure starting from $r$ and $D_{r}$ is measurable, and $\beta$ is a morphism from $D$ to $Y$.

Thanks to the Fubini-Tonelli theorem, the monad $\mathcal{M}$ on QBS is strong. We can also use the structure of QBS to define the product measure of $[\Omega, \alpha, v]$ and $\left[\Omega^{\prime}, \alpha^{\prime}, \nu^{\prime}\right]$ as $\left[\left(\Omega \times \Omega^{\prime}\right),\left(\alpha \times \alpha^{\prime}\right),\left(\nu \otimes v^{\prime}\right)\right]$. Using the isomorphism $\mathcal{M} 1 \cong[0, \infty]$, usual Lebesgue integration $\int f d \mu$ for $f: \mathbb{R} \rightarrow[0, \infty]$ and $\mu \in \mathcal{M}(\mathbb{R})$ corresponds to $f^{\sharp}(\mu)$. We can define the mass $|\mu|$ of measure $\mu=[\Omega, \alpha, \nu]$ by $\int_{X} 1 \mathrm{~d} \mu$ which is the same as the mass $|v|$ of base measure $v$. We also define a null measure as $\mathbf{o}=[\Omega, \alpha, 0]$.

\subsubsection{Semantics for HPProg}

We begin by giving a model in QBS of expressions in HPProg and to enriched expressions in PL. This is the usual CCC semantics, and we interpret the type $M(\tau)$ with the measure monad:

$$
\begin{aligned}
& \llbracket \text { unit } \rrbracket \triangleq 1, \quad \llbracket \text { bool } \rrbracket \triangleq 1+1, \quad \llbracket \text { nat } \rrbracket \triangleq \mathbb{N}, \quad \llbracket \text { real } \rrbracket \triangleq \mathbb{R}, \\
& \llbracket \text { pReal } \rrbracket \triangleq[0, \infty], \quad \llbracket \tau_{1} \rightarrow \tau_{2} \rrbracket \triangleq \llbracket \tau_{1} \rrbracket \Rightarrow \llbracket \tau_{2} \rrbracket, \quad \llbracket \tau_{1} \times \tau_{2} \rrbracket \triangleq \llbracket \tau_{1} \rrbracket \times \llbracket \tau_{2} \rrbracket, \\
& \llbracket \operatorname{list}(\tau) \rrbracket \triangleq \coprod_{n \in \mathbb{N}} \llbracket \tau \rrbracket^{n}, \quad \llbracket M(\tau) \rrbracket \triangleq \mathcal{M}(\llbracket \tau \rrbracket)
\end{aligned}
$$

Here 1 is the terminal object in QBS and $\bigsqcup_{n \in \mathbb{N}} \llbracket \tau \rrbracket^{n}$ is the coproduct of the countable family $\llbracket \tau \rrbracket^{\mathfrak{n}}=\llbracket \tau \rrbracket \times \cdots \times \llbracket \tau \rrbracket$ (n times). As usual, we interpret each term $\Gamma \vdash e: \tau$ as a morphism $\llbracket \Gamma \rrbracket \rightarrow \llbracket \tau \rrbracket$ in QBS, and in particular the interpretation $\llbracket \Gamma \rrbracket$ of a context $\Gamma$ is the product of the interpretations of its components.

We interpret return and bind using the structure of the monad $\mathcal{M}$ of measures on QBS.

$$
\begin{aligned}
& \llbracket \Gamma \vdash \text { return } e: M[\tau] \rrbracket \triangleq \eta_{\llbracket \tau \rrbracket} \circ \llbracket \Gamma \vdash e: \tau \rrbracket \\
& \llbracket \Gamma \vdash \text { bind } e_{1} e_{2}: M\left[\tau_{2}\right] \rrbracket \triangleq \llbracket \Gamma \vdash e_{2}: \tau_{1} \rightarrow M\left[\tau_{2}\right] \rrbracket \\
& \|
\end{aligned}
$$

where $\eta,(-)^{\sharp}$, and $\theta$ are the unit, the Kleisli lifting, and the strength of the commutative monad $\mathcal{M}$. To interpret the other constructions we first introduce two semantics constructions for scaling and normalizing:

$$
\operatorname{scale}(v, f) \triangleq\left(\mathcal{M}\left(\pi_{2}\right) \circ \mathrm{dst}_{1, X} \circ\left\langle f, \eta_{X}\right\rangle\right)^{\sharp}(v) \quad \operatorname{normalize}(v) \triangleq \begin{cases}\mathbf{o} & |v|=0, \infty \\ v /|v| & \text { (otherwise) }\end{cases}
$$

where dst is the double strength of the commutative monad $\mathcal{M}$, and $|v|$ is the mass of $v$. In the definition of scale $(v, f)$, the construction $\mathcal{M}\left(\pi_{2}\right) \circ$ dst $_{1, X} \circ\left\langle f, \eta_{X}\right\rangle$ corresponds to a function mapping an element $x \in X$ to a Dirac distribution centered at $x$ and scaled by $f(x)$, whose domain is then lifted to measures using the Kleisli lifting. 
To achieve this, we use the equivalence $\llbracket p R e a l \rrbracket=[0, \infty] \cong \mathcal{M} 1$, and pairing and projection constructions to manage the duplication of $x$. The definition of scale $(v)$ is more straightforward and reflects the semantics we described before. tions.

Using these constructions we can interpret the corresponding syntactic construc-

$$
\begin{aligned}
\llbracket \Gamma \vdash \operatorname{scale}\left(\mathrm{t}, \mathrm{t}^{\prime}\right): M[\tau] \rrbracket & \triangleq \operatorname{scale}\left(\llbracket \Gamma \vdash \mathrm{t}: M[\tau] \rrbracket, \llbracket \Gamma \vdash \mathrm{t}^{\prime}: \tau \rightarrow \mathrm{pReal} \rrbracket\right) \\
\llbracket \Gamma \vdash \operatorname{normalize}(\mathrm{t}): M[\tau] \rrbracket & \triangleq \text { normalize }(\llbracket \Gamma \vdash \mathrm{t}: M[\tau] \rrbracket)
\end{aligned}
$$

We can now interpret query as follows:

$$
\llbracket \Gamma \vdash \text { query } e \Rightarrow e^{\prime}: M[\tau] \rrbracket \triangleq \text { normalize }\left(\text { scale }\left(\llbracket \Gamma \vdash e: M[\tau] \rrbracket, \llbracket \Gamma \vdash e^{\prime}: \tau \rightarrow \text { pReal } \rrbracket\right)\right)
$$

Using the equivalence $\llbracket p R e a l \rrbracket=[0, \infty] \cong \mathcal{M} 1$ again, we interpret expectation as:

$$
\llbracket \Gamma \vdash \mathbb{E}_{x \sim \mathrm{t}}\left[\mathrm{t}^{\prime}(\mathrm{x})\right]: \mathrm{pRea} \rrbracket \triangleq \lambda \gamma \in \llbracket \Gamma \rrbracket .\left(\llbracket \Gamma \vdash \mathrm{t}^{\prime}: \tau \rightarrow \mathrm{pReal} \rrbracket(\gamma)\right)^{\sharp}(\llbracket \Gamma \vdash \mathrm{t}: M[\tau] \rrbracket(\gamma)) .
$$

The primitives of basic probability distributions Uniform, Bern, Gauss are interpreted by rescaling a measure (given as a constant) with density functions (cf. Section 9.7.2), and the usual operations on real numbers are given by embedding measurable real functions in QBS.

\subsubsection{Semantics of the logics}

To interpret formulas in PL we use the category Pred(QBS) of predicates over quasi-Borel spaces.

We interpret a typed formula $\Gamma \vdash \phi$ as an object $\llbracket \Gamma \vdash \phi \rrbracket \triangleq(\llbracket \Gamma \rrbracket,(\Gamma \vdash \phi 0)$ in $\operatorname{Pred}(\mathbf{Q B S})$ where the predicate part $(\Gamma \vdash \phi)$ is interpreted inductively. This is equivalent to the presentation in Section 4.8. We can show:

Theorem 9.4 (PL Soundness). If a judgment $\Gamma \mid \Psi \vdash_{\mathrm{PL}} \phi$ is derivable then we have the inclusion $\left(\bigcap_{\psi \in \Psi}(\Gamma \vdash \psi)\right) \subseteq(\Gamma \vdash \phi)$ of predicates, which is equivalent to having a morphism $\operatorname{id}_{\llbracket \Gamma \rrbracket}: \llbracket \Gamma \vdash \Lambda_{\psi \in \Psi} \psi \rrbracket \rightarrow \llbracket \Gamma \vdash \phi \rrbracket$ in the category $\operatorname{Pred}(\mathbf{Q B S})$.

Here, the soundness of PL axioms introduced in Section 9.4 is proved from the basic facts discussed by Ścibior et al. [141], in particular, the isomorphism $\mathcal{N} 1 \cong[0, \infty]$, the commutativity of the monad $\mathcal{M}$, the correspondence between $\mathrm{f}^{\sharp}(\mu)$ and usual integration $\int \mathrm{fd} \mu$ for any $f: \mathbb{R} \rightarrow[0, \infty]$ and $\mathcal{N}(\mathbb{R})$, and that measurable functions between standard Borel spaces are exactly morphisms in QBS.

Using Theorem 9.1 and Theorem 9.4, we can prove the soundness of UPL.

Corollary 9.5 (UPL Soundness). If $\Gamma\left|\Psi \vdash_{\mathrm{UPL}} e: \tau\right| \phi$ then

$$
\left\langle\operatorname{id}_{\llbracket \Gamma \rrbracket}, \llbracket \Gamma \vdash e: \tau \rrbracket\right\rangle: \llbracket \Gamma \vdash \Lambda_{\psi \in \Psi} \psi \rrbracket \rightarrow \llbracket \Gamma, \mathbf{r}: \tau \vdash \phi \rrbracket
$$

in $\operatorname{Pred}(\mathbf{Q B S})$. 
Chapter 9. An assertion-based logic for higher-order probabilistic programs

Similarly, using Theorem 9.2 and Theorem 9.4, we can prove the soundness of RPL.

Corollary 9.6 (RPL Soundness). If $\Gamma\left|\Psi \vdash_{\mathrm{RPL}} e_{1}: \tau_{1} \sim e_{2}: \tau_{2}\right| \phi$ then

$$
\left\langle\mathrm{id}_{\llbracket \Gamma \rrbracket}, \llbracket \Gamma \vdash \mathrm{e}_{1}: \tau_{1} \rrbracket, \llbracket \Gamma \vdash \mathrm{e}_{2}: \tau_{2} \rrbracket\right\rangle: \llbracket \Gamma \vdash \bigwedge_{\psi \in \psi} \psi \rrbracket \rightarrow \llbracket \Gamma, \mathbf{r}_{1}: \tau_{1}, \mathbf{r}_{2}: \tau_{2} \vdash \phi \rrbracket
$$

in $\operatorname{Pred}(\mathbf{Q B S})$.

\subsection{Examples}

In Section 9.1 we showed two examples of how to use PPV to reason about probabilistic inference and Monte Carlo approximation. In this section, we demonstrate further how PPV can be used to verify a wide range of properties of probabilistic programs. We will start by showing how to reason formally about probabilistic program slicing for continuous random variables as a relational property. We will then consider an example of the use of PPV to reason about the convergence of probabilistic inference.

\subsubsection{Slicing of Probabilistic Programs}

In this example, we show how PPV can be used to reason about relational properties of probabilistic programs with continuous random variables. Specifically, we show that a combination of relational reasoning in RPL, and equational reasoning in PL allow us to reason about slicing of probabilistic programs [9]. Slicing is a program analysis technique that can be used to speed up probabilistic inference tasks. Previous work has shown how to slice probabilistic programs with discrete random variables in an efficient way. Here, we consider the problem of checking the correctness of a slice, when the program contains continuous random variables. We look at an example adapted from Amtoft and Banerjee [9]. Consider the following two programs left and right:

$$
\begin{aligned}
\text { left } \equiv & \text { let } x=\operatorname{Uniform}(0,1) \text { in let } y=\operatorname{Uniform}(0,1) \text { in let } z=x \otimes y \text { in } \\
& \text { mlet } v=\left(\text { query } z \Rightarrow \lambda w . \text { if } \pi_{2}(w)>0.5 \text { then } 1 \text { else } 0\right) \text { in unit }\left(\pi_{1}(v)\right)
\end{aligned}
$$

Intuitively, even if query in left is applied to the product measure $z$, and not just to the measure of $y$, the conditioning concerns only $y$ and it does not affect the distribution of $x$. Indeed, right is a correct slice of left. We can show this in RPL by proving the following judgment.

$$
\vdash_{\text {RPL }} \text { left: } M[\text { real }] \sim \text { right: } M[\text { real }]\left\{\mathbf{r}_{1}=\mathbf{r}_{2}\right\}
$$


To prove this judgment, we first apply the relational LET rule, which allows us to introduce an assumption about $x$ on both sides. Then we apply a sequence of asynchronous LET-L rules on the program on the left, which introduce preconditions about $y$ and $z$ into the context:

$$
\begin{aligned}
& x=\operatorname{Uniform}(0,1), y=\operatorname{Uniform}(0,1), z=x \otimes y \vdash_{\mathrm{RPL}} \\
& \text { mlet } v=\left(\text { query } z \Rightarrow \lambda w \text {. if } \pi_{2}(w)>\frac{1}{2} \text { then } 1 \text { else } 0\right) \text { in unit }\left(\pi_{1}(v)\right) \sim x \mid \mathbf{r}_{1}=\mathbf{r}_{2}
\end{aligned}
$$

To prove this judgment we rely on the equalities on monadic bind, rescaling, and conditioning in Section 9.4. Starting from the HPProg term on the left, by applying the equations (9.13), (9.14), and (9.9), we reduce it to mlet $v=(x \otimes X)$ in unit $\left(\pi_{1}(v)\right)$ where $X$ is a normalized distribution defined by the term query $y \Rightarrow \lambda w_{2}$. if $w_{2}>$ 0.5 then 1 else 0 . We then conclude this is equal to $x$ by applying the equality (9.9) and the equality

$$
\text { mlet } w=e_{1} \otimes e_{2} \text { in unit } \pi_{1}(w)=\operatorname{scale}\left(e_{1}, \mathbb{E}_{x \sim e_{2}}[1]\right)
$$

proved from the equalities (9.12), (9.7) and monadic laws.

Using RPL we can also reason about situations where we cannot slice a program. Adapting again from Amtoft and Banerjee [9], let us consider the following two programs left and right:

$$
\begin{aligned}
& \text { left } \equiv \text { let } x=\text { Uniform }(0,1) \text { in let } y=\operatorname{Uniform}(0,1) \text { in let } z=x \otimes y \text { in } \\
& \text { mlet } v=\text { (query } z \Rightarrow \lambda w \text {. if } \pi_{1}(w)+\pi_{2}(w)>0.5 \text { then } 1 \text { else } 0 \text { ) in unit }\left(\pi_{1}(v)\right) \\
& \text { right } \equiv \text { let } x=\operatorname{Uniform}(0,1) \text { in } x
\end{aligned}
$$

Now we prove that it is not correct to slice left into right by means of the judgment below:

$$
\vdash_{\text {RPL }} \text { left: } M[\text { real }] \sim \text { right: } M[\text { real }]\left\{\mathbf{r}_{1} \neq \mathbf{r}_{2}\right\}
$$

The proof for this judgment follows the structure of the proof of the previous example. The main difference is that now we need to see the first coordinate of the variable $w$ in the conditioning. To prove that left and right are different, we use the probabilistic inference in the first example to prove $\vdash_{\mathrm{UPL}}$ left $\mid \operatorname{Pr}_{y \sim \mathrm{r}}[y\rangle$ $.5]>1 / 2$ using the [Bayes] rule and the following calculation: $\frac{\operatorname{Pr}_{w}\left[\pi_{1}(w)>5\right]}{\operatorname{Pr}_{w}\left[\pi_{1}(w)+\pi_{2}(w)>.5\right]} \geqslant$ $\frac{\operatorname{Pr}_{x}[x>.5]}{1-\operatorname{Pr}_{x}[x>.25] * \operatorname{Pr}_{y}[y>.25]}=\frac{8}{15}>\frac{1}{2}$.

Similarly, we can look at the following two programs:

$$
\begin{aligned}
\text { left } \equiv & \text { mlet } x=\operatorname{Uniform}(0,1) \text { in } \\
& \text { mlet }-=(\text { if } x>.5 \text { then }(\text { let } y=\operatorname{Uniform}(0,1) \text { in let } z=\text { unit }(x) \otimes y \text { in } \\
& \text { query } \left.\left.z \Rightarrow \lambda w . \text { if } \pi_{2}(w)>.5 \text { then } 1 \text { else } 0\right) \text { else unit }(x \otimes x)\right) \text { in unit }(x) \\
\text { right } \equiv & \text { mlet } x=\operatorname{Uniform}(0,1) \text { in unit }(x)
\end{aligned}
$$

and show that we can slice left into right. 
Chapter 9. An assertion-based logic for higher-order probabilistic programs

A key point in deriving the slicing property of the above examples is the equation mlet $w=e_{1} \otimes e_{2}$ in unit $\pi_{1}(w)=\operatorname{scale}\left(e_{1}, \mathbb{E}_{\text {} e_{2}}[1]\right)$ of splitting product measure, which is obtained by applying the axioms in Section 9.4. When $e_{2} \equiv$ query $e_{3} \Rightarrow e_{4}$, we have mlet $w=e_{1} \otimes e_{2}$ in unit $\pi_{1}(w)=e_{1}$ since our conditioning construction is normalized, and hence $\mathbb{E}_{x \sim e_{2}}[1]=1$. On the other hand, when $e_{2}$ consists of unnormalized conditioning, we may have the non-slicing mlet $w=e_{1} \otimes e_{2}$ in unit $\pi_{1}(w) \neq e_{1}$ because $\mathbb{E}_{x \sim e_{2}}[1]<1$. This is an advantage of our conditioning operator. Since we renormalize in conditioning construction, we can slice the algorithm left into right in the third example.

Putting the first and the third example together we can consider the following two programs left and right:

$$
\begin{aligned}
& \text { left } \equiv \text { mlet } x=\text { Uniform }(0,1) \text { in } \\
& \text { mlet }{ }_{-}=(\text {if } x>.5 \text { then (let } y=\operatorname{Uniform}(0,1) \text { in let } z=\operatorname{unit}(x) \otimes y \text { in } \\
& \text { query } \left.\left.z \Rightarrow \lambda w \text {. if } \pi_{2}(w)>.5 \text { then } 1 \text { else } 0\right) \text { else unit }(x \otimes x)\right) \text { in } \\
& \text { let } u=\operatorname{Uniform}(0,1) \text { in let } k=\operatorname{unit}(x) \otimes u \text { in } \\
& \text { mlet } v=\text { (query } k \Rightarrow \lambda w \text {. if } \pi_{2}(w)>.5 \text { then } 1 \text { else } 0 \text { ) in unit }\left(\pi_{1}(v)\right. \text { ) } \\
& \text { right } \equiv \text { mlet } x=\operatorname{Uniform}(0,1) \text { in } \\
& \text { mlet }_{-}=(\text {if } x>.5 \text { then (let } y=\operatorname{Uniform}(0,1) \text { in let } z=\operatorname{unit}(x) \otimes y \text { in } \\
& \text { query } \left.\left.z \Rightarrow \lambda w \text {. if } \pi_{2}(w)>.5 \text { then } 1 \text { else } 0\right) \text { else unit }(x \otimes x)\right) \\
& \text { in unit }(x)
\end{aligned}
$$

Again, we want to show that right is a correct slice of left by proving that $\vdash_{\text {RPL }}$ left: $M\left[\right.$ real] $\sim$ right: $M\left[\right.$ real] $\left\{\mathbf{r}_{1}=\mathbf{r}_{2}\right\}$. The proof of this judgment can be carried out mostly in RPL, by using the similarity between the two programs left and right. The proof starts by using relational reasoning, and afterwards reuses the proof of the first example. This shows that reasoning relationally about slicing can be better than reasoning directly about equivalence by computing the two distributions.

\subsubsection{Gaussian Mean Learning: Convergence and Stability}

Probabilistic programs are often used as models for probabilistic inference tasks in data analysis. We now show how PPV can be used to reason about such processes. Taking the example of the closed-form Bayesian update, we show how to use PPV to reason about two quite common properties, convergence and stability under changes of priors. These two properties allow us to illustrate two different aspects of PPV: 1) The support it offers for reasoning about iterative probabilistic tasks and for reasoning about densities of random variables, and 2) The support it offers for relational reasoning about measures of divergence of one distribution with respect to another. To show this, we first prove the convergence of the iterative closed-form learning of the mean of a Gaussian distribution (with fixed variance). We then 
prove this process stable for a precise notion of stability formulated in terms of Kullback-Leibler (KL) divergence.

Let us start by considering the following implementation GaussLearn of an algorithm for Bayesian learning of the mean of a Gaussian distribution with known variance $\sigma^{2}$ from a sample list L:

$$
\text { GaussLearn } \equiv \lambda \text {. letrec } f(L)=\text { case } L \text { of } \square \Rightarrow p, y:: \text { ls } \Rightarrow \text { query } f(l s) \Rightarrow \operatorname{GPDF}\left(y, \sigma^{2}\right)
$$

where $\operatorname{GPDF}\left(y, \sigma^{2}\right)$ is a shorthand for the density function $\lambda \mathrm{r} \cdot \frac{1}{\sqrt{2 \pi \sigma^{2}}} \exp \left(\frac{(\mathrm{r}-\mathrm{y})^{2}}{2 \sigma^{2}}\right)$ of a Gaussian distribution Gauss $\left(y, \sigma^{2}\right)$ with mean $y$ and variance $\sigma^{2}$. This algorithm starts by assuming a prior $p$ on the unknown mean. Then, on each iteration, a sample $y$ is read from the list and the prior gets updated by observing it as a Gaussian with mean $y$ and variance $\sigma^{2}$.

We now want to show two properties of this algorithm. The first property we show is convergence: the mean of the posterior should roughly converge to the mean of the data, but we need to take into account that the posterior also depends on the prior. More precisely, when the prior is also a Gaussian, we can show that:

$$
\begin{aligned}
(\sigma>0),(\xi>0) \vdash_{\mathrm{RPL}} \text { GaussLearn } \sim \text { Total }\left\{\forall \mathrm{L}^{\prime}: \text { list }(\text { real }) . \forall n: \text { nat. }\left(\mathrm{n}=\left|\mathrm{L}^{\prime}\right|\right)\right. \\
\\
\left.\Longrightarrow \mathbf{r}_{1}\left(\operatorname{Gauss}\left(\delta, \xi^{2}\right)\right)\left(\mathrm{L}^{\prime}\right)=\operatorname{Gauss}\left(\frac{\mathbf{r}_{2}\left(\mathrm{~L}^{\prime}\right) * \xi^{2}+\delta * \sigma^{2}}{n * \xi^{2}+\sigma^{2}}, \frac{\xi^{2} * \sigma^{2}}{n * \xi^{2}+\sigma^{2}}\right)\right\}
\end{aligned}
$$

where Total is an algorithm summing all the elements of a list L.

$$
\text { Total } \equiv \operatorname{letrec} f(\mathrm{~L}: \text { list }(\text { real }))=\text { case } \mathrm{L} \text { with } \square \Rightarrow 0, y:: \mathrm{ls} \Rightarrow \mathrm{y}+\mathrm{f}(\mathrm{ls}) .
$$

This judgment states that, if the prior on the mean is a Gaussian of mean $\delta$ and variance $\xi^{2}$, then the posterior is a Gaussian with mean close to the mean of Total $(\mathrm{L})$ and variance close to 0 , but that they are still influenced by the parameters $\delta, \xi^{2}$ of the prior.

The proof of this judgment proceeds relationally by first applying the onesided [ABS-L] rule to introduce the prior in the context. Then the proof continues synchronously by applying the [r-LETREC] and [r-LISTCASE] rules. To conclude the proof we need to show the following two premises corresponding to the base case and to the inductive step:

$$
\begin{aligned}
& (\sigma>0),(\xi>0), \phi_{\text {ind.hyp }}(\mathrm{L}=\mathrm{L}), \mathrm{d}_{\text {prior }}=\operatorname{Gauss}\left(\delta, \xi^{2}\right),(\mathrm{n}=|\mathrm{L}|) \\
& \quad \vdash_{\mathrm{RPL}} \mathrm{d}_{\text {prior }} \sim 0\left\{\mathbf{r}_{1}=\operatorname{Gauss}\left(\frac{\mathbf{r}_{2} * \xi^{2}+\delta * \sigma^{2}}{n * \xi^{2}+\sigma^{2}}, \frac{\xi^{2} * \sigma^{2}}{n * \xi^{2}+\sigma^{2}}\right)\right\} \\
& (\sigma>0),(\xi>0), \phi_{\text {ind.hyp }}(\mathrm{L}=\mathrm{y}:: l s), \mathrm{d}_{\text {prior }}=\operatorname{Gauss}\left(\delta, \xi^{2}\right),(\mathrm{n}=|\mathrm{L}|) \\
& \quad \mathrm{RPL}_{\mathrm{RPL}} \text { query } \mathrm{f}_{1}(\mathrm{ls}) \Rightarrow \operatorname{Gauss}\left(\mathrm{y}, \sigma^{2}\right) \sim \mathrm{y}+\mathrm{f}_{2}(\mathrm{ls})\left\{\mathbf{r}_{1}=\operatorname{Gauss}\left(\frac{\mathbf{r}_{2} * \xi^{2}+\delta * \sigma^{2}}{n * \xi^{2}+\sigma^{2}}, \frac{\xi^{2} * \sigma^{2}}{n * \xi^{2}+\sigma^{2}}\right)\right\}
\end{aligned}
$$

The first premise is obvious. The second premise requires a little more work, and can be proved by applying [r-QRY-L] and [r-SUB] rules and several equations in PL. We first show in PL that Gaussian distributions are conjugate prior with respect to the 
Gaussian likelihood function by applying the equations on rescaling, normalization, and conditioning.

$\vdash_{\mathrm{PL}}(\sigma>0) \wedge(\xi>0) \Longrightarrow$ query Gauss $\left(\delta, \xi^{2}\right) \Rightarrow \operatorname{GPDF}\left(z, \sigma^{2}\right)=\operatorname{Gauss}\left(\frac{z \xi^{2}+\delta \sigma^{2}}{\xi^{2}+\sigma^{2}}, \frac{\xi^{2} \sigma^{2}}{\xi^{2}+\sigma^{2}}\right)$.

Then, we apply [r-QRY-L] and [r-SUB] to the premise (9.7.2) to introduce the observations in the precondition, and apply the above fact and the induction hypothesis.

The second property we show is stability. If we run GaussLearn twice with different prior Gaussian distributions, we can show that the posteriors will be close if the list of samples is long enough and not diverging. This closeness is defined in terms of the Kullback-Leibler (KL) divergence. The KL divergence of two distributions with known density functions, can be defined by expectations: $\left(d_{1}=\right.$ scale $\left.\left.\left(d_{2}, f\right)\right) \Longrightarrow\left(K L\left(d_{1} \| d_{2}\right)=\mathbb{E}_{x \sim d_{1}} \log f(x)\right]\right)$. In particular, the KL divergence of two Gaussian distributions can be calculated as follows:

$$
\operatorname{KL}\left(\operatorname{Gauss}\left(\mu_{1}, \sigma_{1}^{2}\right) \| \operatorname{Gauss}\left(\mu_{2}, \sigma_{2}^{2}\right)\right)=\left(\log \left|\sigma_{2}\right|-\log \left|\sigma_{1}\right|\right)+\frac{\left.\sigma_{1}^{2}+\left(\mu_{1}-\mu_{2}\right)^{2}\right)}{\sigma_{2}^{2}}-\frac{1}{2}
$$

Formally, we want to prove the following judgment.

$$
\begin{aligned}
& \sigma: \text { real, } \delta: \text { real, } \xi \text { : real, } \delta_{2}: \text { real, } \xi_{2}: \text { real } \mid(\sigma>0),(\xi>0),\left(\xi_{2}>0\right) \\
& \vdash_{\mathrm{RPL}} \text { GaussLearn } \sim \text { GaussLearn }\left\{\forall \mathrm{L}^{\prime}: \text { list(real). } \forall \varepsilon: \text { real. } \forall \mathrm{C}:\right. \text { real. } \\
& (\varepsilon>0) \Longrightarrow \exists \mathrm{N}: \text { nat. }\left(\left|\mathrm{L}^{\prime}\right|>\mathrm{N}\right) \wedge\left|\operatorname{Total}\left(\mathrm{L}^{\prime}\right)\right|<\mathrm{C} *\left|\mathrm{~L}^{\prime}\right| \\
& \left.\Longrightarrow \operatorname{KL}\left(\mathbf{r}_{1}\left(\operatorname{Gauss}\left(\delta, \xi^{2}\right)\right)\left(\mathrm{L}^{\prime}\right) \| \mathbf{r}_{1}\left(\operatorname{Gauss}\left(\delta_{2}, \xi_{2}^{2}\right)\right)\left(\mathrm{L}^{\prime}\right)\right)<\varepsilon\right\}
\end{aligned}
$$

Intuitively, this states that if the algorithm is run twice with different Gaussian priors, and the mean of the data is bounded by some $\mathrm{C}$, then the KL divergence of the posteriors can be made as small as desired by increasing the size of the data. In other words, the effect of the prior on the posterior can be minimized by having enough samples.

By simple calculations, we can prove in PL the following assertion in a similar way as proofs of convergence of sequences using the epsilon-delta definition of limit.

$$
\begin{aligned}
& \vdash_{\mathrm{PL}} \forall \mathrm{L}^{\prime} \text { : list(real). } \forall \varepsilon \text { : real. } \forall \mathrm{C} \text { : real. } \\
& (\varepsilon>0) \Longrightarrow \exists \mathrm{N} \text { : nat. }\left(\left|\mathrm{L}^{\prime}\right|>\mathrm{N}\right) \wedge\left|\operatorname{Total}\left(\mathrm{L}^{\prime}\right)\right|<\mathrm{C} *\left|\mathrm{~L}^{\prime}\right| \Longrightarrow \\
& \left|\frac{\text { Total }\left(\mathrm{L}^{\prime}\right) * \xi^{2}+\delta_{2} * \sigma^{2}}{\left|\mathrm{~L}^{\prime}\right| * \xi^{2}+\sigma^{2}}-\frac{\text { Total }\left(\mathrm{L}^{\prime}\right) * \xi_{2}^{2}+\delta_{2} * \sigma^{2}}{\left|\mathrm{~L}^{\prime}\right| * \xi_{2}^{2}+\sigma^{2}}\right|<\varepsilon \wedge\left|\log \frac{n * \xi^{2} * \xi_{2}^{2}+\xi^{2} * \sigma^{2}}{n * \xi^{2} * \xi_{2}^{2}+\xi_{2}^{2} * \sigma^{2}}\right|<\varepsilon
\end{aligned}
$$

To prove (9.20), we want to combine (9.18) with (9.19) and (9.21). To do this, we apply the relational [r-SUB] rule to the judgment (9.20), which has the following PL premise:

$$
\begin{aligned}
& \vdash_{\mathrm{PL}}^{\top} \Longrightarrow \forall \mathrm{L}^{\prime}: \text { list }(\text { real }) . \forall \varepsilon \text { : real. } \forall \mathrm{C}: \text { real. } \\
& \quad(\varepsilon>0) \Longrightarrow \exists \mathrm{N}: \text { nat. }\left(\left|\mathrm{L}^{\prime}\right|>\mathrm{N}\right) \wedge \mid \text {Total}^{\prime}\left(\mathrm{L}^{\prime}\right)|<\mathrm{C} *| \mathrm{L}^{\prime} \mid \\
& \quad \Longrightarrow \mathrm{KL}\left(\text { GaussLearn }\left(\operatorname{Gauss}\left(\delta, \xi^{2}\right)\right)\left(\mathrm{L}^{\prime}\right) \| \text { GaussLearn }\left(\operatorname{Gauss}\left(\delta_{2}, \xi_{2}^{2}\right)\right)\left(\mathrm{L}^{\prime}\right)\right)<\varepsilon .
\end{aligned}
$$


We prove this in PLby first applying the rule [conv-RPL] to (9.18) and then using (9.19) and (9.21).

\subsection{Related work}

Assertion-based logics Other papers focus on program logics where the precondition and post-condition are probabilistic assertions about the input and output distributions. A non-exhausive list is [133, 69, 58, 134]. Barthe et al. [41] propose an assertion-based logic, named ELLORA, using expectation for verifying properties of imperative probabilistic programs with discrete random variables. Our assertion logic PL is similar in spirit to the one of ELLORA, but it further supports continuous distributions and the verification of higher-order programs. On the other hand, ELLORA has powerful rules for probabilistic while loops that PPV does not support. It would be interesting to explore if similar rules can also be added to PPV. Formalizations of measure and integration theory in general purpose interactive theorem provers have been considered in many papers [13, 97, 136, 64, 95]. Avigad et al. [16] recently completed a proof of the Central Limit theorem, which is the principle underlying concentration bounds. Hölzl [94] formalized discrete-time Markov chains and Markov decision processes. These and other existing formalizations have been used to verify several case studies, but they are scattered and not easily accessible for our purposes.

\subsection{Conclusions}

In this chapter we have introduced a framework PPV supporting the (unary and relational) verification of probabilistic programs including constructions for higher-order computations, continuous distributions and conditioning. PPV is more expressive than the logics presented in Chapters 4 and 5 since it supports conditioning and it allows to reason directly about probabilistic constructs, but to support this kind of reasoning it needs to combine axiomatizations of basic probabilistic constructions with rules of three different logics in order to ease the verification of examples from probabilistic inference, statistics, and machine learning. The soundness of the approach still relies on quasi-Borel spaces, using a monad of general $\sigma$-finite measures instead of a monad of probability measures. 


\section{A PRE-EXPECTATION CALCULUS FOR EXPECTED SENSITIVITY}

Sensitivity properties are a particular case of relational properties describing how changes in program inputs affect program outputs, for particular notions of distance. By varying these distances, we can apply sensitivity properties to many areas, including:

1. numerical computations, where distances are taken between real numbers,

2. numerical queries, where program inputs are databases, and the distance between them is the number of differing entries, and

3. learning algorithms, where the distance between two training sets is the number of differing examples, and the distance between outputs measures the difference in errors labeling unseen examples.

This chapter is concerned with sensitivity properties of first-order probabilistic programs. As such programs return distributions over their output space, the corresponding notions of sensitivity use distances over distributions. The Total Variation (TV) distance (a.k.a. statistical distance), for example, is a widely used notion of distance that measures the maximal difference of probabilities for two distributions. One key benefit of the TV distance is that it is defined for distributions over arbitrary spaces. However, it is sometimes desirable to consider distances inherited from the underlying space. It is common to consider classes of distances on distributions that are obtained by lifting a distance on an underlying space. This lifting is defined by the so-called Kantorovich metric, which yields a family of probabilistic metrics obtained by lifting a distance $\mathcal{E}$ on a ground set $X$ to a distance $\varepsilon^{\#}$ on distributions over $X$. The class of Kantorovich metrics cover many notions of distance, including the TV distance which can be obtained by applying the Kantorovich lifting to the discrete distance. 
We develop a relational pre-expectation calculus for reasoning about sensitivity of probabilistic computations under the Kantorovich metric. Semantically, this is given by an operator $\widetilde{r p e}(\mathrm{c}, \cdot)$ that given a program $\mathrm{c}$, maps relational expectations (which are maps of type State $\times$ State $\rightarrow[0, \infty]$ ) over the final states to relational expectations over the initial states.

Relational pre-expectations generalize pre-expectations as introduced by Morgan et al. [121], but they also present some special characteristics. As opposed to the weakest pre-expectation operator, the weakest relational pre-expectation operator composes laxly. That is, we have an inequality $\widetilde{r p e}\left(\mathrm{c} ; \mathrm{c}^{\prime}, \varepsilon\right) \leqslant \widetilde{\mathrm{rpe}}\left(\mathrm{c}, \widetilde{\mathrm{rpe}}\left(\mathrm{c}^{\prime}, \mathcal{E}\right)\right)$ instead of a strict equality. In any case, this inequality is still enough to study sensitivity properties, because we are interested in establishing upper-bounds. Our metatheory is also more involved: it uses results from optimal transportation theory, and soundness and continuity cannot be proven in full generality. In particular, we show that soundness holds for discrete distributions, and continuity for finitely-supported distributions. In addition, we cannot support fully asynchronous reasoning as we have seen in other logics of this thesis. Nonetheless, we provide rules for asynchronous programs that work well for non-trivial examples.

We demonstrate our techniques on several applications. First, we formalize an algorithmic stability property of machine-learning algorithms. Informally, algorithmic stability describes how much the output parameters from a learning algorithm are affected when one input training example is changed; this notion of probabilistic sensitivity is known to imply generalization and prevent overfitting [56]. We use our calculus for proving algorithmic stability of a commonly-used learning algorithm: stochastic gradient descent (SGD). We use these examples to contrast our approach with prior work.

Then, we consider a pair of applications showing convergence properties. We first formalize convergence of a reinforcement learning algorithm [148], following a recent analysis by Amortila et al. [8]. Then, we show in our setting classic results about convergence and rapid mixing of several card shuffling algorithms [7]. We show that the TV distance between the outputs of two probabilistic loops decreases to $o$ as the number of loop iterations increases-that is, the output distributions from any two inputs converge to the same distribution. Moreover, our technique is precise enough to describe the rate of this convergence. Upper bounds on convergence speed are key properties in algorithms that generate samples form complex distributions, such as Markov Chain Monte Carlo.

We also show how to formalize other properties complementing our bounds on convergence rate. First, we prove with our system that some card shuffling examples converge to the uniform distribution. Second, we study lower bounds-a task already challenging in the non-relational wpe calculus [90]. To conclude, we present some proof rules that allow us to use our techniques to reason about the expected distance between program executions that have different control-flow. 


\section{Outline of the chapter.}

We begin by introducing some preliminaries on probability theory and the Kantorovich distance (Section 10.1) and on pre-expectation techniques (Section 10.2). We then present our relational pre-expectation calculus for finding upper-bounds on the Kantorovich distance (Section 10.3) and look into several case studies. We first apply our calculus to studying stability of the stochastic gradient descent [89] (Section 10.4). Second, we look into the algorithm TD(o) from Reinforcement Learning [148] (Section 10.5). Third, we prove in our setting classic results in convergence of random walks and card shuffling algorithms by Aldous [7]. We then study other applications of our calculus: showing convergence to the uniform distribution and proving lower bounds (Section 10.7) and extended rules to reason about programs with asynchronous control flow (Section 10.8). Finally, we present related work (Section 10.9) and conclude (Section 10.10).

\subsection{Mathematical Preliminaries}

We briefly recap the foundations required for relational reasoning about sensitivity properties:

1. discrete probability theory,

2. probabilistic imperative programming languages, and

3. distances on probability distributions.

A comprehensive treatment of these topics is found, e.g., in the textbooks [11, 117, 156].

\subsubsection{Basic probability concepts}

Instead of full distributions, we will use sub-distributions to model the probabilistic behavior of programs that may not terminate with probability 1 .

Definition 10.1 (Probability sub-distribution). A sub-distribution over a countable set $\mathrm{A}$ is a function $\mu: \mathrm{A} \rightarrow[0,1]$ assigning a probability to each element of $\mathrm{A}$. Probabilistic events are subsets $B \subseteq A$; the probability of $B$ is denoted $\mu(B)$ and defined by $\mu(B)=$ $\sum_{b \in B} \mu(b)$. The support of $\mu$ is the set of all events $a \in A$ with $\mu(a)>0$. Moreover, we let $|\mu|=\mu(A)$. As usual, the probabilities in any sub-distribution must sum up to at most 1: $|\mu| \leqslant 1$. We call $\mu$ a distribution if $|\mu|=1$. We let $\operatorname{SD}(A)$ denote the set of sub-distributions over A.

Marginals can also be defined for subdistributions: 
Definition 10.2 (Marginals). Given a sub-distribution $\mu \in \mathbf{S D}\left(A_{1} \times A_{2}\right)$ over a product, its left and right marginals, $\pi_{1}(\mu)$ and $\pi_{2}(\mu)$, are sub-distributions over $A_{1}$ and $A_{2}$, respectively, which are given by $\pi_{1}(\mu)\left(x_{1}\right)=\sum_{x_{2} \in X} \mu\left(x_{1}, x_{2}\right)$, and $\pi_{2}(\mu)\left(x_{2}\right)=\sum_{x_{1} \in X} \mu\left(x_{1}, x_{2}\right)$.

Definition 10.3 (Dirac distribution). The Dirac distribution $\delta(a) \in \mathbf{S D}(A)$ is the point distribution at $a \in A, \delta(a)\left(a^{\prime}\right)=\left[a=a^{\prime}\right]$, where the right-hand-side is an Iverson-bracket which evaluates to 1 if the formula inside (in this case, $\mathrm{a}=\mathrm{a}^{\prime}$ ) evaluates to true, and to 0 otherwise.

Definition 10.4 (Expected value). If $\mathrm{f}: \mathrm{A} \rightarrow \mathbb{R}_{\geqslant 0}^{\infty}$ is a function mapping into the extended reals, we can take its expected value $\mathbb{E}_{\mu}[\mathrm{f}]$ with respect to some sub-distribution $\mu \in \mathbf{S D}(A)$ : $\mathbb{E}_{\mu}[f]=\sum_{a \in A} f(a) \cdot \mu(a)$. If the sum diverges, the expected value is $\infty$. We assume that addition and multiplication are extended in the natural way, with the convention $0 \cdot \infty=\infty \cdot 0=0$.

\subsubsection{Programming language and semantics}

We work with a standard probabilistic imperative language PWHILE. This language has commands defined by the following grammar:

$$
\mathrm{c}:=\operatorname{skip}|x \leftarrow e| x \leftarrow \mathrm{s}|\mathrm{c} ; \mathrm{c}| \text { if } e \text { then } \mathrm{c} \text { else } \mathrm{c} \mid \text { while } e \text { do } \mathrm{c} .
$$

Variables $x$ are drawn from an arbitrary but finite set Var of variable names. Expressions $e$ are largely standard, formed from variables and basic operations (e.g., integer addition, boolean conjunction). To handle programs with (static) arrays, we assume expressions include basic array operations for accessing and updating. For instance, when $a$ is an array variable we have syntactic sugar:

$$
\begin{array}{cc}
\mathrm{a}[\mathrm{e}] & \triangleq \operatorname{Lookup}(\mathrm{a}, e) \\
\mathrm{a}[e] \leftarrow \mathrm{e}^{\prime} \triangleq \mathrm{a} \leftarrow \operatorname{Update}\left(\mathrm{a}, e, \mathrm{e}^{\prime}\right) & \text { (expression) }
\end{array}
$$

The random sampling command $x \leqslant d$ takes a sample from some primitive distribution $d$ and stores it in $x$. For simplicity, we assume that primitive distributions do not have free program variables, and we interpret them as full distributions $\llbracket d \rrbracket: ~ S D(D)$ over some countable set $D$, possibly different for different distributions. We will often use the uniform distribution Unif $S$ when $S$ is a finite, non-empty set; for instance, for a positive integer $N$ we will write $[N]$ for the set of integers $\{0, \ldots, N-1\}$, so that $x \notin U_{n i f} f_{[N]}$ samples each number in $[N]$ with probability $1 / N$ and stores it in $x$. The distributions can also be parameterized by some more complex expression, for instance in $x \leqslant[s]$ for a program variable $y$.

PWHILE programs transform states, which are finite maps $s$ : Var $\rightarrow D$; we write State for the set of all states. The semantics of a program $c$ is a map $\llbracket c \rrbracket:$ State $\rightarrow$ SD (State) assigning a sub-distribution over possible outputs to each input. The semantics of the remaining language constructs is standard and presented 


$$
\begin{aligned}
&(\llbracket \text { skip } \rrbracket s)\left(s^{\prime}\right) \triangleq\left\{\begin{array}{l}
1 \text { if } s=s^{\prime} \\
0 \text { otherwise }
\end{array}\right. \\
&(\llbracket x \leftarrow e \rrbracket s)\left(s^{\prime}\right) \triangleq\left\{\begin{array}{l}
1 \text { if } s^{\prime}(x)=s(e) \text { and } s(y)=s^{\prime}(y) \text { for all } y \neq x \\
0 \text { otherwise }
\end{array}\right. \\
&(\llbracket x \leqslant e \rrbracket s)\left(s^{\prime}\right) \triangleq \begin{cases}d\left(s^{\prime}(x)\right): s(y)=s^{\prime}(y) \text { for all } y \neq x \\
0 \quad: \text { otherwise }\end{cases} \\
&\left(\llbracket c_{1} ; c_{2} \rrbracket s\right)\left(s^{\prime}\right) \triangleq \sum_{s^{\prime \prime} \in M e m}\left(\llbracket c_{1} \rrbracket s\right)\left(s^{\prime \prime}\right) \cdot\left(\llbracket c_{2} \rrbracket s\right)\left(s^{\prime}\right) \\
&\left(\llbracket \text { if } e \text { then } c_{1} \text { else } c_{2} \rrbracket s\right)\left(s^{\prime}\right) \triangleq[s(e)] \cdot\left(\llbracket c_{1} \rrbracket s\right)\left(s^{\prime}\right)+[\neg s(e)] \cdot\left(\llbracket c_{2} \rrbracket s\right)\left(s^{\prime}\right) \\
&(\llbracket \text { while } e \text { do } c \rrbracket s)\left(s^{\prime}\right) \triangleq\left(\lim _{n \rightarrow \infty}\left(\llbracket c_{n} \rrbracket s\right)\right)\left(s^{\prime}\right) \\
& \text { where } c_{0} \triangleq \text { abort and } c_{i+1} \triangleq \text { if } e \text { then } c_{;} ; c_{i}
\end{aligned}
$$

Figure 10.1: Semantics of PWHILE

in Figure 10.1. Here, we abuse the notation $s(e)$ to denote the natural generalization of $s$ to a map from expressions to values in D. We also use a dummy instruction abort that has as a denotation the null distribution over states. As we only work with discrete primitive distributions and states have finitely many variables, output distributions programs always have countable support.

\subsubsection{Distances between probability distributions}

Various notions of distances between distributions allow us to specify sensitivity properties of probabilistic programs. A popular example is the following:

Definition 10.5 (Total Variation distance). The Total Variation (TV) distance between $\mu_{1}, \mu_{2} \in \mathbf{S D}(X)$ is defined as: $T V\left(\mu_{1}, \mu_{2}\right) \triangleq \frac{1}{2} \sum_{x \in X}\left|\mu_{1}(x)-\mu_{2}(x)\right|$.

The term distance (or metric) is justified as $T V\left(\mu_{1}, \mu_{2}\right)$ is symmetric, satisfies the triangle inequality, and maps to zero if and only if $\mu_{1}=\mu_{2}$. The normalization factor of $\frac{1}{2}$ ensures that the TV distance is within $[0,1]$. Roughly speaking, the TV distance measures the largest difference in probabilities of any event between two given distributions.

Note that the TV distance does not require a metric space, i.e., the underlying set $X$ is not necessarily equipped with any metric. If $X$ is a metric space, we can define:

Definition 10.6 (Kantorovich distance). Let $\mathrm{X}$ be a (extended) metric space with a distance $\mathcal{E}: \mathrm{X} \times \mathrm{X} \rightarrow \mathbb{R}_{\geqslant 0}^{\infty}$. The Kantorovich distance is a canonical lifting of $\mathcal{E}$ to a 
function $\mathcal{E}^{\#}: \mathbf{S D}(X) \times \mathbf{S D}(X) \rightarrow \mathbb{R}_{\geqslant 0}^{\infty}$ that defines a metric on $\mathbf{S D}(X)$. This distance is defined as

$$
\mathcal{E}^{\#}\left(\mu_{1}, \mu_{2}\right)=\inf _{\mu \in \Gamma\left(\mu_{1}, \mu_{2}\right)} \mathbb{E}_{\mu}[\varepsilon],
$$

where $\Gamma\left(\mu_{1}, \mu_{2}\right)$ is the set of probabilistic couplings of $\mu_{1}, \mu_{2}$, given by

$$
\Gamma\left(\mu_{1}, \mu_{2}\right)=\left\{\mu \in \mathbf{S D}(X \times X) \mid \pi_{i}(\mu)=\mu_{i}, \text { for } i=1,2\right\} .
$$

The set $\Gamma\left(\mu_{1}, \mu_{2}\right)$ is non-empty provided $\left|\mu_{1}\right|=\left|\mu_{2}\right|$. Otherwise, $\Gamma\left(\mu_{1}, \mu_{2}\right)=\emptyset$ and $\varepsilon^{\#}\left(\mu_{1}, \mu_{2}\right)=\infty$.

The coupling-based definition of the Kantorovich distance is more abstract than other distances between distributions, but its generality turns out to be a strength. First, we can recover the TV distance as a lifting of the discrete metric:

Theorem 10.7 (Total variation and Kantorovich distance). Let $\mu_{1}, \mu_{2} \in \mathbf{S D}(X)$ such that $\left|\mu_{1}\right|=\left|\mu_{2}\right|=1$. If the discrete metric $\mathcal{E}: X \times X \rightarrow\{0,1\}$ is given by $\mathcal{E}\left(x_{1}, x_{2}\right)=\left[x_{1} \neq x_{2}\right]$, then $T V\left(\mu_{1}, \mu_{2}\right)=\mathcal{E}^{\#}\left(\mu_{1}, \mu_{2}\right)$.

Another advantage of the Kantorovich distance is that it is defined as an infimum. For our goal of proving continuity, it suffices to compute an upper bound of the distance, which corresponds to determining $\mathbb{E}_{\mu}[\mathcal{E}]$ for some particular coupling $\mu$.

Traditionally, the definition of $\mathcal{E}^{\#}$ is restricted to functions $\mathcal{E}$ defining a metric on X. However, the definition of $\varepsilon^{\#}$ extends mutatis mutandis to arbitrary functions $\varepsilon$. We abuse terminology and use the term Kantorovich distance also in the more general case. For instance, we can use this more general notion to bound the difference between the expected values of two functions on the outputs of two program runs:

Theorem 10.8 (Absolute expected difference). Let $\mu_{1}, \mu_{2} \in \mathbf{S D}(X)$ such that $\left|\mu_{1}\right|=$ $\left|\mu_{2}\right|=1$, and let $\mathrm{f}_{1}, \mathrm{f}_{2}: X \rightarrow \mathbb{R}_{\geqslant 0}^{\infty}$. Let $\mathcal{\varepsilon}: \mathrm{X} \times \mathrm{X} \rightarrow \mathbb{R}_{\geqslant 0}^{\infty}$ be defined by $\mathcal{\varepsilon}\left(\mathrm{x}_{1}, \mathrm{x}_{2}\right)=\mid \mathrm{f}_{1}\left(\mathrm{x}_{1}\right)-$ $f_{2}\left(x_{2}\right) \mid$. Then $\left|\mathbb{E}_{\mu_{1}}\left[f_{1}\right]-\mathbb{E}_{\mu_{2}}\left[f_{2}\right]\right| \leqslant \mathcal{E}^{\#}\left(\mu_{1}, \mu_{2}\right)$.

We can also obtain bounds on the TV distance when lifting other base distances that assign a minimum, non-zero distance to all pairs of distinct elements.

Theorem 10.9 (Scaled TV distance). Let $\mu_{1}, \mu_{2} \in \mathbf{S D}(X)$ with $\left|\mu_{1}\right|=\left|\mu_{2}\right|=1$, let $\varepsilon_{\rho}: X \times X \rightarrow[0,1]$, and let $\rho \in \mathbb{R}_{>0}$ be a strictly positive constant with $\varepsilon_{\rho}\left(x_{1}, x_{2}\right) \geqslant \rho \cdot\left[x_{1} \neq\right.$ $\left.x_{2}\right]$. Then, $T V\left(\mu_{1}, \mu_{2}\right) \leqslant \frac{1}{\rho} \cdot \varepsilon_{\rho}^{\#}\left(\mu_{1}, \mu_{2}\right)$.

\subsection{Preliminaries: Expectation-based logics}

In this thesis we have seen different unary and binary logics to reason about probabilistic programs. These had in common that they where presented in Hoare 
style (i.e., as a triple or quadruple of a precondition, a postcondition and one or two programs) and that they were Boolean, meaning that predicates over a base set $X$ are subsets of $X$.

Expectation-based logics, on the other hand, consider non-negative, real-valued predicates, also known as expectations. Expectations are then maps of type State $\rightarrow$ $\mathbb{R}_{\geqslant 0}^{\infty}$. They generalize Boolean predicates, which can be modeled by expectations by taking an appropriate $\{0,1\}$-valued function. An early example of such a logic is the work by Kozen [109] on probabilistic dynamic logic.

The weakest-preexpectation transformer, introduced by McIver and Morgan [117] is a predicate transformer wpe $(\mathrm{c}, \cdot)$ that given a program $\mathrm{c}$ maps post-expectations to pre-expectations. This is defined inductively on the structure of $c$ and, importantly, satisfies the compositionality property $w p e\left(c^{\prime} ; c^{\prime}, \mathcal{E}\right)=w p e\left(c, w p e\left(c^{\prime}, \varepsilon\right)\right)$. The calculus computes the expected value of $\mathcal{E}$ over the final distribution of a program $c$, that is, $\mathbb{E}_{\llbracket \mathfrak{c} \rrbracket \mathfrak{m}}[\varepsilon]=\operatorname{wpe}(\mathrm{c}, \mathcal{\varepsilon})(\mathrm{m})$.

This calculus enjoys strong theoretical properties: it is both sound and complete (in an extensional sense) w.r.t. common program semantics (cf. Gretz et al. [87]). Therefore, weakest pre-expectations can-in principle-be applied to reason about bounds on the Total Variation distance by using techniques similar to selfcomposition. However, it is often not practical. In fact, mathematicians have long observed that reasoning about the TV distance or the Kantorovich metric directly from their definition is inappropriate. Rather, they rely on probabilistic couplings

To express properties about pairs of states we use relational expectations, which are maps of type State $\times$ State $\rightarrow \mathbb{R}_{\geqslant 0}^{\infty}$; we write Exp for the set of all relational expectations. This set is equipped with the pointwise order inherited from the order on $\mathbb{R}_{\geqslant 0}^{\infty}$, i.e., $\mathcal{E} \leqslant \mathcal{E}^{\prime}$ if and only if $\mathcal{\varepsilon}\left(s_{1}, s_{2}\right) \leqslant \mathcal{E}^{\prime}\left(s_{1}, s_{2}\right)$ for all pairs $\left(s_{1}, s_{2}\right)$ of states. Since $\mathbb{R}_{\geqslant 0}^{\infty}$ is a complete lattice and Exp has the pointwise order, Exp is also a complete lattice; the top and bottom elements are the constant relational expectations $\infty$ and 0 , which send all pairs of states to $\infty$ and 0 respectively.

For denoting specific relational expectations, we borrow notation from relational Hoare logic [45]: We tag variables with $\langle 1\rangle$ or $\langle 2\rangle$ to refer to their value in the first or the second state, respectively. For instance, $[x\langle 1\rangle=x\langle 2\rangle]$ is a relational expectation encoding the predicate $\lambda\left\langle s_{1}, s_{2}\right\rangle$. [s $\left.s_{1}(x)=s_{2}(x)\right]$.

Relational pre-expectations naturally connect with probabilistic couplings, and capture well-established proof principles used by mathematicians for reasoning about the TV distance.

\subsection{Bounding expected sensitivity with relational pre- expectations}

As we have seen, the Kantorovich distance encompasses many specific distances on distributions. To reason about probabilistic and expected sensitivity, we would like 
to bound the Kantorovich distance between two output distributions in terms of the distance between two program inputs. In this section, we develop a relational pre-expectation operation to prove these bounds.

\subsubsection{A first unsuccessful attempt: a relational pre-expectation for exact bounds}

Since we want to reason about the Kantorovich distance lifting of a relational expectation $\varepsilon$ : State $\times$ State $\rightarrow \mathbb{R}_{\geqslant 0}^{\infty}$ between output distributions of a program c, an initial idea is to define a relational pre-expectation operator $r p e(c, \varepsilon)$ coinciding exactly with the Kantorovich distance:

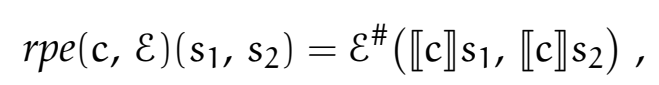

and then prove bounds of the form rpe $\left(\mathrm{c}, \mathcal{E}_{\text {out }}\right) \leqslant \mathcal{E}_{\text {in }}$ in order to bound the Kantorovich distance between outputs by some distance between inputs. While this definition is appealing, it turns out to be inconvenient for formal reasoning because it does not behave well under sequential composition: the expected sequence rule $\operatorname{rpe}\left(c ; c^{\prime}, \varepsilon\right)=\operatorname{rpe}\left(c, \operatorname{rpe}\left(c^{\prime}, \varepsilon\right)\right)$ does not hold. Roughly, this is because choosing local infima on each step does not necessarily amount to a global infimum. In fact, in some cases no local choice amounts to a global infimum.

Example 10.10. The Bernoulli distribution $\mathrm{B}(\mathrm{p})$ with bias $\mathrm{p}$ returns 1 with probability $\mathrm{p}$ and 0 with probability $1-p$. Consider the following programs:

$$
\begin{aligned}
c & =\text { if } b \text { then } x \leqslant B(1 / 2) \text { else } y \leqslant B(1 / 2) \\
c^{\prime} & =\text { if } b \text { then } y \leqslant B(1 / 2) \text { else } x \leqslant B(1 / 2) .
\end{aligned}
$$

Moreover, consider the relational expectation $\mathcal{E}=[x\langle 1\rangle \neq x\langle 2\rangle \vee y\langle 1\rangle \neq y\langle 2\rangle]$. If we fix $\mathrm{b}\langle 1\rangle=$ true and $\mathrm{b}\langle 2\rangle=$ false throughout, then

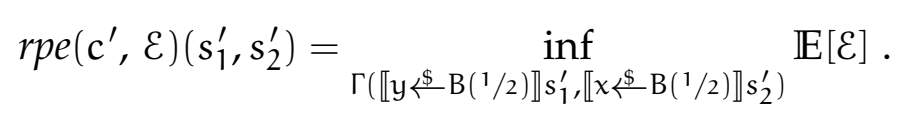

To compute the above relational pre-expectation, we first need to understand the possible couplings. Hence, we compute the marginals of the involved distributions:

$$
\begin{aligned}
& \mu_{1} \triangleq \llbracket y \ll B(1 / 2) \rrbracket s_{1}^{\prime}=\left\{\begin{array}{l}
\frac{1}{2}: x \mapsto s_{1}^{\prime}(x), y \mapsto 0 \\
\frac{1}{2}: x \mapsto s_{1}^{\prime}(x), y \mapsto 1
\end{array}\right. \\
& \mu_{2} \triangleq \llbracket x \notin B(1 / 2) \rrbracket s_{2}^{\prime}=\left\{\begin{array}{l}
\frac{1}{2}: x \mapsto 0, y \mapsto s_{2}^{\prime}(y) \\
\frac{1}{2}: x \mapsto 1, y \mapsto s_{2}^{\prime}(y) .
\end{array}\right.
\end{aligned}
$$


The marginal conditions for couplings (Def. 10.6) then yield that any coupling in $\Gamma\left(\mu_{1}, \mu_{2}\right)$ is of the form

$$
\begin{aligned}
\mu_{\rho}\left(s_{1}, s_{2}\right) & =\rho \cdot\left[s_{1}(x)=s_{1}^{\prime}(x) \wedge s_{1}(y)=1\right] \cdot\left[s_{2}(x)=1 \wedge s_{2}(y)=s_{2}^{\prime}(y)\right] \\
& +\left(\frac{1}{2}-\rho\right) \cdot\left[s_{1}(x)=s_{1}^{\prime}(x) \wedge s_{1}(y)=1\right] \cdot\left[s_{2}(x)=0 \wedge s_{2}(y)=s_{2}^{\prime}(y)\right] \\
& +\left(\frac{1}{2}-\rho\right) \cdot\left[s_{1}(x)=s_{1}^{\prime}(x) \wedge s_{1}(y)=0\right] \cdot\left[s_{2}(x)=1 \wedge s_{2}(y)=s_{2}^{\prime}(y)\right] \\
& +\rho \cdot\left[s_{1}(x)=s_{1}^{\prime}(x) \wedge s_{1}(y)=0\right] \cdot\left[s_{2}(x)=0 \wedge s_{2}(y)=s_{2}^{\prime}(y)\right]
\end{aligned}
$$

for some $0 \leqslant \rho \leqslant \frac{1}{2}$ and the previously fixed states $s_{1}^{\prime}$ and $s_{2}^{\prime}$. Hence,

$$
\begin{aligned}
\mathbb{E}_{\mu_{\rho}}[\mathcal{E}] & =\rho \cdot\left[s_{1}^{\prime}(x) \neq 1 \vee s_{2}^{\prime}(y) \neq 1\right]+\left(\frac{1}{2}-\rho\right)\left[s_{1}^{\prime}(x) \neq 0 \vee s_{2}^{\prime}(y) \neq 1\right] \\
& +\left(\frac{1}{2}-\rho\right)\left[s_{1}^{\prime}(x) \neq 1 \vee s_{2}^{\prime}(y) \neq 0\right]+\rho \cdot\left[s_{1}^{\prime}(x) \neq 0 \vee s_{2}^{\prime}(y) \neq 0\right] .
\end{aligned}
$$

Since rpe $\left(c^{\prime}, \mathcal{E}\right)$ takes the minimum over all couplings, i.e., the minimum over all $\rho \in\left[0, \frac{1}{2}\right]$, by simple computation we get that rpe $\left(\mathrm{c}^{\prime}, \mathcal{E}\right)\left(\mathrm{s}_{1}^{\prime}, \mathrm{s}_{2}^{\prime}\right)=1 / 2$, setting $\rho=1 / 2$ if $\mathrm{s}_{1}^{\prime}(\mathrm{x})=\mathrm{s}_{2}^{\prime}(\mathrm{y})$ and $\rho=0$ otherwise. Since $s_{1}^{\prime}(x), s_{2}^{\prime}(y)$ are sampled from $\llbracket c \rrbracket s_{1}$ and $\llbracket c \rrbracket s_{2}$, for any way to couple them rpe $\left(\mathrm{c}, \operatorname{rpe}\left(\mathrm{c}^{\prime}, \mathcal{E}\right)\right)\left(\mathrm{s}_{1}, \mathrm{~s}_{2}\right)=\frac{1}{2}>0$. However, $\llbracket \mathrm{c} ; \mathrm{c}^{\prime} \rrbracket \mathrm{s}_{1}$ and $\llbracket \mathrm{c} ; \mathrm{c}^{\prime} \rrbracket \mathrm{s}_{2}$ have the same marginal distributions for $(x, y)$ and thus distance 0 . Therefore,

$$
0=\operatorname{rpe}\left(\mathrm{c} ; \mathrm{c}^{\prime}, \mathcal{E}\right)\left(\mathrm{s}_{1}, \mathrm{~s}_{2}\right)<\operatorname{rpe}\left(\mathrm{c}, \operatorname{rpe}\left(\mathrm{c}^{\prime}, \mathcal{E}\right)\right)\left(\mathrm{s}_{1}, \mathrm{~s}_{2}\right)=\frac{1}{2} \text {. }
$$

Fortunately, we generally do not need to compute the exact Kantorovich distance to prove sensitivity properties: an upper bound suffices. Since the Kantorovich distance is an infimum over all couplings, we can establish upper bounds by exhibiting a specific coupling - of course, the tightness of these upper bounds will depend on the particular coupling we chose. Crucially, couplings can be constructed compositionally: a coupling for a sequential composition $c ; c^{\prime}$ can be obtained by combining a coupling for $c$ with a coupling for $c^{\prime}$. We leverage this observation into our compositional relational pre-expectation calculus, which provides upper bounds on the Kantorovich distance.

\subsubsection{Compositional upper bounds by relational pre-expectation}

To facilitate compositional reasoning, we define an upper bound $\widetilde{r p e}(c, \mathcal{E})$ of the Kantorovich distance $\varepsilon$ with respect to program $c$. Technically, $\widetilde{r p e}(c, \varepsilon)$ is a relational pre-expectation calculus defined by induction on the structure of $c$, similarly to the calculus by McIver and Morgan [121]. The rules of our calculus are shown in Figure 10.2. We take the indicator expectation $[\phi]$ be 1 if $\phi$ is true, otherwise 0 , and we define addition and multiplication on expectations pointwise. The cases of skipping, assignments and sequential composition are straightforward and apply the backwards semantics of commands. The relational pre-expectation of sampling is expressed directly in terms of the Kantorovich distance, i.e., an infimum is taken over the set of all couplings, which is not always possible in practice. We give 


$$
\begin{aligned}
& \widetilde{r p e}(\text { skip }, \varepsilon) \triangleq \varepsilon \\
& \widetilde{r p e}(x \leftarrow e, \varepsilon) \triangleq \varepsilon\{e\langle 1\rangle, e\langle 2\rangle / x\langle 1\rangle, x\langle 2\rangle\} \\
& \triangleq \lambda s_{1} s_{2} \cdot \mathcal{E}\left(s_{1}[x \mapsto e\langle 1\rangle], s_{2}[x \mapsto e\langle 2\rangle]\right) \\
& \widetilde{\operatorname{rpe}}(x \leftarrow d, \mathcal{E}) \triangleq \lambda s_{1} s_{2} . \mathcal{E}^{\#}\left(\llbracket x \leqslant d \rrbracket s_{1}, \llbracket x \leqslant d \rrbracket s_{2}\right), \text { where } \mathcal{E}^{\#}\left(\mu_{1}, \mu_{2}\right) \triangleq \inf _{\mu \in \Gamma\left(\mu_{1}, \mu_{2}\right)} \mathbb{E}_{\mu}[\mathcal{E}] \\
& \widetilde{r p e}\left(\mathrm{c} ; \mathrm{c}^{\prime}, \varepsilon\right) \triangleq \widetilde{r p e}\left(\mathrm{c}, \widetilde{\mathrm{rpe}}\left(\mathrm{c}^{\prime}, \varepsilon\right)\right) \\
& \widetilde{r p e}\left(\text { if } e \text { then } c \text { else } c^{\prime}, \mathcal{E}\right) \triangleq[e\langle 1\rangle \wedge e\langle 2\rangle] \cdot \widetilde{r p e}(c, \mathcal{E})+[\neg e\langle 1\rangle \wedge \neg e\langle 2\rangle] \cdot \widetilde{r p e}\left(c^{\prime}, \varepsilon\right)+[e\langle 1\rangle \neq e\langle 2\rangle] \cdot \infty \\
& \widetilde{\operatorname{rpe}}(\text { while e do } c, \mathcal{E}) \triangleq \operatorname{IfpX} . \Phi_{\varepsilon, \mathrm{c}}(X), \\
& \text { where } \Phi_{\varepsilon, c}(X) \triangleq[e\langle 1\rangle \wedge e\langle 2\rangle] \cdot \widetilde{r p e}(c, X)+[\neg e\langle 1\rangle \wedge \neg e\langle 2\rangle] \cdot \varepsilon+[e\langle 1\rangle \neq e\langle 2\rangle] \cdot \infty
\end{aligned}
$$

Figure 10.2: Definition of the relational pre-expectation operator $\widetilde{p e}(c, \varepsilon)$.

more details on this problem in Section 10.3.3. The relational pre-expectation for conditionals assumes the two runs are synchronized. If not, $[e\langle 1\rangle \neq e\langle 2\rangle]=1$ and the distance is (trivially) upper bounded by $\infty$, since the branches may not terminate with the same probability, so the set of couplings may be empty. Finally, in the case of while loops, we take the least fixed point of the characteristic functional $\Phi_{\varepsilon, \mathrm{c}}$ of the loop. It is not hard to show that $\Phi_{\mathcal{\varepsilon}, \mathrm{c}}(-): \operatorname{Exp} \rightarrow \operatorname{Exp}$ is monotonic (see Lemma C.3 in the appendix), so by the Knaster-Tarski theorem the least fixed point is well-defined. As in the previous case, the relational pre-expectation returns $\infty$ when runs are not synchronized, i.e., only one loop guard is true. Computing the least fixed point is usually not possible. We present an invariant-based rule in Section 10.3.3.

Remark (Synchronous vs. asynchronous control flow). In contrast to the Kantorovich distance operator rpe $(c, \varepsilon)$, cour compositional relational pre-expectation operator $\widetilde{r p e}(\mathrm{c}, \mathcal{E})$ only gives useful (i.e., finite) bounds when the control flows in the two executions of $\mathrm{c}$ can be synchronized. For deterministic guards, this means that pairs of related executions always take the same branches; for randomized guards, this means that we can relate the random samplings so that pairs of related executions always take the same branches. In Section 10.8, we describe extensions of our calculus that can give more useful bounds when reasoning asynchronously.

Remark (Tightness of bounds). It is also complicated to estimate the exact loss between $\widetilde{r p e}(\mathrm{c}, \mathcal{E})$ and $r p e(\mathrm{c}, \mathcal{E})$, since lower bounds on $r p e(\mathrm{c}, \mathcal{E})$ are not given by a witness coupling. Nonetheless, in our setting this limitation is not exclusive to our technique-in the statistical literature, lower bounds for stochastic processes such as the ones we analyze in Section 10.6 are in general hard to compute and so the exact distance is often not known. We will return to this topic in Section 10.7. 
We now study the metatheory of our calculus. Our first result is that our calculus is sound: it correctly upper bounds the Kantorovich distance.

Theorem 10.11 (Soundness of $\widetilde{r p e}$ ). Let $\mathrm{c}$ be a PWHILE program and $\mathcal{E} \in \operatorname{Exp}$ be $a$ relational expectation. Then $r p e(c, \mathcal{E}) \leqslant \widetilde{r p e}(c, \mathcal{E})$, i.e., if $\widetilde{\text { rpe }}(\mathrm{c}, \mathcal{E})\left(\mathrm{s}_{1}, \mathrm{~s}_{2}\right)<\infty$ for $\mathrm{s}_{1}, \mathrm{~s}_{2} \in$ State then

$$
\mathbb{E}_{\mu_{s_{1}, s_{2}}}[\mathcal{E}] \leqslant \widetilde{r p e}(c, \mathcal{E})\left(s_{1}, s_{2}\right) \quad \text { for some coupling } \quad \mu_{s_{1}, s_{2}} \in \Gamma\left(\llbracket c \rrbracket s_{1}, \llbracket c \rrbracket s_{2}\right) .
$$

Proof Sketch. By induction on c. The most challenging cases are for sampling and loops. The case for sampling requires first showing that there exists a coupling realizing the infimum defining the Kantorovich distance; such existence results belong to the theory of optimal transport [156].

The case for loops is challenging for another reason: it is not clear how to show that the pre-expectation operator is continuous in its second argument (but see Thm. 10.12). Instead, our proof relies on extracting a convergent sequence of couplings. We defer the details to Appendix C.3.

While it is not clear whether our relational pre-expectation operator is continuous for all programs, continuity does hold for programs that sample from finite distributions. Note that such programs can still produce distributions with infinite support by sampling in a loop.

Theorem 10.12 (Continuity of $\widetilde{r p e}$ ). Let c be a PWHILE program where all primitive distributions have finite support, and let $\mathcal{E}_{n} \in \operatorname{Exp}$ for $n \in \mathbb{N}$ be a monotonically increasing chain of relational expectations converging pointwise to $\varepsilon \in$ Exp. Then,

$$
\widetilde{\operatorname{rpe}}(\mathrm{c}, \varepsilon)=\sup _{\mathrm{n} \in \mathbb{N}} \widetilde{\operatorname{rpe}}\left(\mathrm{c}, \mathcal{E}_{\mathrm{n}}\right) \text {. }
$$

Proof Sketch. By induction on the structure of c. The most challenging case is for sampling instructions, where the proof depends on a continuity property for the Kantorovich distance. We establish this property for distributions with finite support, and complete the proof of continuity for relational pre-expectations. We defer details to Appendix C.3.

\subsubsection{Reasoning with relational pre-expectations}

The definition of $\widetilde{r p e}$ in Fig. 10.2 is sufficient to prove relational properties of probabilistic programs in theory, but there are some practical obstacles:

- Comparing different relational pre-expectations for the same program is difficult-using the definition to compute each relational pre-expectation separately is tedious. 
Chapter 10. A pre-expectation calculus for expected sensitivity

$$
\begin{aligned}
& \frac{\mathcal{E} \leqslant \mathcal{E}^{\prime}}{\widetilde{r p e}(\mathrm{c}, \mathcal{E}) \leqslant \widetilde{r p e}\left(\mathrm{c}, \mathcal{E}^{\prime}\right)} \text { Mono } \quad \frac{\operatorname{FV}\left(\mathcal{E}^{\prime}\right) \cap \mathrm{MV}(\mathrm{c})=\emptyset}{\widetilde{r p e}\left(\mathrm{c}, \mathcal{E}+\mathcal{E}^{\prime}\right) \leqslant \widetilde{r p e}(\mathrm{c}, \mathcal{E})+\mathcal{E}^{\prime}} \text { Const } \\
& \widetilde{\widetilde{r p e}}(\mathrm{c}, \mathcal{E})+\widetilde{r p e}\left(\mathrm{c}, \mathcal{E}^{\prime}\right) \leqslant \widetilde{r p e}\left(\mathrm{c}, \mathcal{E}+\mathcal{E}^{\prime}\right) \text { SupAdD } \\
& \frac{f: \mathbb{R}_{\geqslant 0} \rightarrow \mathbb{R}_{\geqslant 0} \text { linear, with } f(\infty) \triangleq \infty}{\widetilde{\operatorname{rpe}}(c, f \circ \mathcal{E})=\mathrm{f} \circ \widetilde{\mathrm{rpe}}(\mathrm{c}, \mathcal{E})} \text { CALE } \\
& \frac{M: \text { State } \times \text { State } \rightarrow \Gamma(\llbracket d \rrbracket, \llbracket d \rrbracket)}{\widetilde{r p e}(x \leftrightarrow d, \mathcal{E}) \leqslant \mathbb{E}_{\left(v_{1}, v_{2}\right) \sim M(-,-)}\left[\mathcal{E}\left\{v_{1}, v_{2} / x\langle 1\rangle, x\langle 2\rangle\right\}\right]} \text { SAMP } \\
& \frac{f: \text { State } \times \text { State } \rightarrow(\mathrm{D} \rightarrow \mathrm{D}) \text { bijection }}{\widetilde{r p e}\left(x \leqslant \text { Unif }_{\mathrm{D}}, \mathcal{E}\right) \leqslant \frac{1}{|\mathrm{D}|} \sum_{v \in \mathrm{D}} \mathcal{E}\{v, \mathrm{f}(-,-)(v) / x\langle 1\rangle, x\langle 2\rangle\}} \mathrm{UNIF}_{\mathrm{N}} \\
& \frac{[e\langle 1\rangle \wedge e\langle 2\rangle] \cdot \widetilde{\operatorname{rpe}}(\mathrm{c}, \mathcal{J})+[\neg e\langle 1\rangle \wedge \neg e\langle 2\rangle] \cdot \mathcal{E}+[e\langle 1\rangle \neq e\langle 2\rangle] \cdot \infty \leqslant \mathcal{J}}{\widetilde{r p e}(\text { while e do } c, \mathcal{E}) \leqslant \mathcal{J}} \operatorname{INV}
\end{aligned}
$$

Figure 10.3: Properties of relational pre-expectation operator $\widetilde{r p e}(\mathrm{c}, \varepsilon)$.

- Computing the relational pre-expectation for random sampling is difficult: it requires computing a minimum over all couplings.

- Computing the relational pre-expectation for loops is also difficult: in general, it is not possible to compute the least fixed point in closed form.

To make our operator easier to use, we introduce a collection of auxiliary properties in Fig. 10.3. We briefly describe the rules below.

Basic properties. The first four rules are basic properties satisfied by relational pre-expectations. Rule Mono states that the $\widetilde{r p e}$ transformer is monotone, and ConsT intuitively states that the relational pre-expectation of $\varepsilon$ is $\varepsilon$ if $c$ doesn't modify $\varepsilon$; the rule is carefully stated to behave correctly when $\widetilde{r p e}(c, \varepsilon)$ is infinite.

We also encode linearity-like properties of relational pre-expectations. SuPAdD states that the property is super-additive: the relational pre-expectation of a sum can be greater than the sum of the relational pre-expectations. Intuitively, this is because $\widetilde{r p e}(c, \varepsilon)$ involves an infimum for random sampling, and the infimum of a sum is always less than the sum of the infima. SCALE states that the relational pre-expectation is preserved by scaling. The requirement that the scaling function satisfies $f(\infty)=\infty$ is needed for correctly handle scaling by $0: \widetilde{r p e}(c, \varepsilon)$ may be infinite, even if $\varepsilon$ is identically zero.

Bounding the pre-expectation for sampling. Using the Kantorovich distance for defining the relational pre-expectation of a sampling command $x \leqslant d$ is theoretically 
clean, but inconvenient in practice for two reasons. First, the set of couplings $\Gamma\left(\llbracket x \leqslant d \rrbracket s_{1}, \llbracket x \leqslant d \rrbracket s_{2}\right)$ over which the infimum is computed is a set of distributions over pairs of states. Given denotations of primitive distributions $\llbracket d \rrbracket \in \mathbf{S D}(D)$, it would be more convenient to reason about the set $\Gamma(\llbracket d \rrbracket$, $\llbracket d \rrbracket)$-this is a set of distributions over pairs of sampled values $\mathrm{D} \times \mathrm{D}$, rather than pairs of memories. Second, computing the infimum is often difficult, and moreover unnecessary for establishing upper bounds.

Corresponding to the SAMP rule, the following result states that we can actually upper bound this Kantorovich distance by picking any coupling of the primitive distribution with itself; we call such a function $M$ : State $\times$ State $\rightarrow \Gamma(\llbracket d \rrbracket, \llbracket d \rrbracket)$ a coupling function (on d).

Proposition 10.13. Let $\mathrm{d}$ be a primitive distribution, and let $\mathrm{M}$ be a coupling function on d. For any relational expectation $\mathcal{E} \in \mathbf{E x p}$, we have:

$$
\widetilde{r p e}(x \leqslant \mathrm{~d}, \varepsilon) \leqslant \mathbb{E}_{\left(v_{1}, v_{2}\right) \sim M(-,-)}\left[\mathcal{E}\left\{v_{1}, v_{2} / x\langle 1\rangle, x\langle 2\rangle\right\}\right] .
$$

We can reuse common couplings of primitive distributions across different proofs. For example, let $\mathrm{D}$ be a finite, non-empty set and let $\mathrm{f}$ : State $\times$ State $\rightarrow(\mathrm{D} \rightarrow \mathrm{D})$ map pairs of program states to bijections on D. Then the bijection coupling $M_{f}$, the coupling function on $U_{n i f}$ is defined by

$$
f\left(s_{1}, s_{2}\right)\left(x_{1}, x_{2}\right)=\left\{\begin{array}{ll}
1 /|D| & : f\left(s_{1}, s_{2}\right)\left(x_{1}\right)=x_{2} \\
0 & : \text { otherwise }
\end{array},\right.
$$

where $x_{1}$ and $x_{2}$ are elements in D. Specialized to this case, Proposition 10.13 gives UNIF:

$$
\begin{aligned}
\widetilde{r p e}\left(x \leqslant U^{\prime} \text { Unf }_{\mathrm{D}}, \mathcal{E}\right) & \leqslant \widetilde{r p e}(x \leqslant \mathrm{~d}, \mathcal{E}) \leqslant \mathbb{E}_{\left(v_{1}, v_{2}\right) \sim M_{\mathrm{f}}(-,-)}\left[\mathcal{E}\left\{v_{1}, v_{2} / x\langle 1\rangle, x\langle 2\rangle\right\}\right] \\
& \leqslant \mathbb{E}_{v \sim \llbracket U_{n i f} \rrbracket}[\mathcal{E}\{v, \mathrm{f}(-,-)(v) / x\langle 1\rangle, x\langle 2\rangle\}] \\
& =\frac{1}{|\mathrm{D}|} \sum_{v \in \mathrm{D}} \mathcal{E}\{v, \mathrm{f}(-,-)(v) / x\langle 1\rangle, x\langle 2\rangle\} .
\end{aligned}
$$

Different coupling functions can give upper bounds of different strengths-selecting appropriate couplings to show the target property is the key part of reasoning by couplings. This technique is well-known to probability theory, where it is called the coupling method [7].

Bounding the pre-expectation for loops. As in the case of sampling, it may not always be desirable or possible to compute the fixed point for loops. Instead, we can upper bound the relational pre-expectation by a relational expectation $\mathrm{J}$, called an invariant-intuitively, if the relational pre-expectation of $\mathcal{J}$ with respect to the loop body is at most $\mathcal{J}$, then the relational pre-expectation of the loop is also at most J. Formally, this reasoning is captured by INV and the following theorem: 
Theorem 10.14. Let $\mathcal{J} \in \operatorname{Exp}$ be a relational expectation. If

$$
[e\langle 1\rangle \wedge e\langle 2\rangle] \cdot \widetilde{r p e}(c, \mathcal{J})+[\neg e\langle 1\rangle \wedge \neg e\langle 2\rangle] \cdot \mathcal{E}+[e\langle 1\rangle \neq e\langle 2\rangle] \cdot \infty \leqslant \mathcal{J},
$$

then $\widetilde{\text { rpe }}($ while e do $c, \mathcal{E}) \leqslant \mathcal{J}$.

Proof. Let $\Phi$ be the characteristic functional of the loop, as defined for the relational pre-expectation. The hypothesis implies $\Phi(\mathcal{J}) \leqslant \mathcal{J}$, so $\mathcal{J}$ is a prefixed point of $\Phi$. By the Knaster-Tarski fixed point theorem, the least fixed point $\widetilde{r p e}($ while e do $c, \mathcal{E})$ is less than $\mathrm{J}$.

\subsubsection{Embedding EpRHL}

Expectation Probabilistic Relational Hoare Logic (EPRHL) is a quantitative extension of pRHL [42]. Judgments of ErRHL are of the form: $\{P ; \varepsilon\} c_{1} \sim_{f} c_{2}\left\{Q ; \mathcal{E}^{\prime}\right\}$ where P, Q are boolean-valued assertions, $\mathcal{E}, \mathcal{E}^{\prime}$ are relational expectations, $f$ is an affine function of the form $a x+b$, where $a, b \in \mathbb{R}_{\geqslant 0}$, and $c_{1}$ and $c_{2}$ are PWHILE programs. This judgment states that for every pair of input states $s_{1}, s_{2}$ satisfying the pre-condition $P$, there is a coupling $\mu$ of $\llbracket c_{1} \rrbracket\left(s_{1}\right), \llbracket c_{2} \rrbracket\left(s_{2}\right)$ whose support lies within the post-condition $\mathrm{Q}$, and moreover $\mathbb{E}_{\mu}\left[\mathcal{E}^{\prime}\right] \leqslant f\left(\mathcal{E}\left(s_{1}, s_{2}\right)\right)$. We can embed the core inference rules of EPRHL in our proof system (see Appendix C.4 for details).

Theorem 10.15 (Embedding EPRHL). Let $\vdash\{P ; \mathcal{E}\} \mathfrak{c} \sim_{f} c\left\{Q ; \mathcal{E}^{\prime}\right\}$ be a valid $\mathbb{E P R H L}$ judgment derived using the rules of Figure C.I in Appendix C.4, with finite $\mathcal{E}$ and $\mathcal{E}^{\prime}$. Then:

$$
\widetilde{\mathrm{rpe}}\left(\mathrm{c}, \mathcal{E}^{\prime}+[\neg \mathrm{Q}] \cdot \infty\right) \leqslant \mathrm{f}(\mathcal{E})+[\neg \mathrm{P}] \cdot \infty .
$$

Furthermore, this inequality can be derived using just the definition of $\widetilde{r p e}(c, \varepsilon)$ for skip, assignment, sequence, and conditionals in Figure 10.2, and the auxiliary proof rules in Figure 10.3.

Intuitively, the bound on the relational pre-expectation captures the validity of the original EPRHL judgment. For any pair of states $\left(s_{1}, s_{2}\right)$, if $\left(s_{1}, s_{2}\right)$ does not satisfy $\mathrm{P}$, then the right-hand side is infinite and the bound trivially holds. If $\left(s_{1}, s_{2}\right)$ satisfies $P$, then the right-hand side is finite (since $\mathcal{E}$ is finite) and the relational pre-expectation is finite. This implies that $\mathrm{Q}$ must be satisfied almost surely in the coupling and $\widetilde{p e}\left(c, \mathcal{E}^{\prime}\right) \leqslant f(\mathcal{E})$. This last inequality recovers the EpRHL judgment's bound on the output distance in terms of the input distance. Furthermore, the embedding shows that the bound is derivable in our calculus without computing infimums over couplings for sampling, or computing least fixed points for loops.

\subsection{Warmup Example: Stability of SGD}

To demonstrate our relational pre-expectation operator, we analyze the stability of Stochastic Gradient Descent (SGD) as our warmup example. SGD is a core tool in 


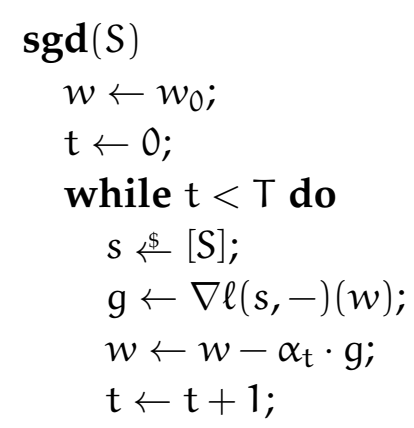

(a) Stochastic Gradient Descent (SGD)

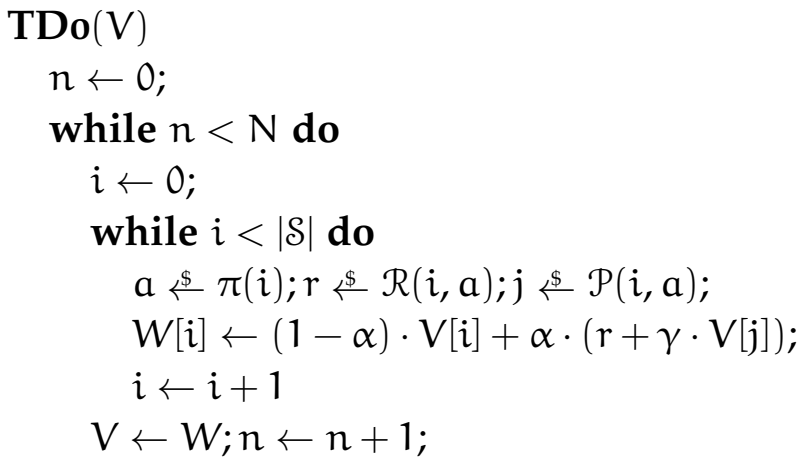

(b) $\mathrm{TD}(\mathrm{o})$ learning algorithm

Figure 10.4: Example programs: Stability and convergence

modern machine learning; SGD is the most common learning algorithm used in practice for training neural networks. Its stability was first established in Hardt et al. [89], and it was later formalized in a relational program logic EpRHL [42]. The corresponding proof in EPRHL involves complex proof rules-our calculus can establish the same property with significantly cleaner reasoning.

\subsubsection{Background}

Let $Z$ be a space of labeled examples, e.g., images annotated with the main subject. A learning algorithm $A: S \rightarrow \mathbb{R}^{\mathrm{d}}$ takes a set $S \in Z^{\mathrm{n}}$ of examples as input and produces ("learns") parameters $w \in \mathbb{R}^{\mathrm{d}}$. The algorithm is tailored to a given loss function $\ell: Z \rightarrow \mathbb{R}^{\mathrm{d}} \rightarrow[0,1]$, which describes how well an example is labeled by some parameters. The goal is to find parameters that have low loss on examples.

In machine learning, uniform stability is a useful property for learning algorithms. In a nutshell, a randomized learning algorithm $A$ is $\epsilon$-uniformly stable if for all pairs $S, S^{\prime}$ of training sets differing in exactly one example, and for all examples $z \in Z$, the expected losses of $z$ are close:

$$
\left|\mathbb{E}_{A(S)}[\ell(z)]-\mathbb{E}_{A\left(S^{\prime}\right)}[\ell(z)]\right| \leqslant \epsilon .
$$

Stable learning algorithms generalize: their performance on new, unseen examples is similar to their performance on the training set [56]. In particular, stability controls how much a learning algorithm can overfit the training set. 


\subsubsection{Verifying stability for stochastic gradient descent}

We consider the program sgd in Figure 10.4a. The gradient $\nabla$ is a higher-order function ${ }^{1}$ with type $\nabla:\left(\mathbb{R}^{\mathrm{d}} \rightarrow[0,1]\right) \rightarrow\left(\mathbb{R}^{\mathrm{d}} \rightarrow \mathbb{R}^{\mathrm{d}}\right)$; we assume that it is well-defined and given. In SGD, the true gradient of a function is approximated by a gradient $\mathrm{g}$ at a single sample $s$. The step sizes $\alpha_{t}$ (with $t \in \mathbb{N}$ ) are a sequence of real numbers that control (together with the local gradient $\mathrm{g}$ ) how to adjust the parameters in each iteration of SGD. Following Hardt et al. [89], we make the following assumptions:

1. The loss function $\ell$ is convex and L-Lipschitz in its second argument, i.e., $\left|\ell(z, w)-\ell\left(z, w^{\prime}\right)\right| \leqslant \mathrm{L} \cdot\left\|w-w^{\prime}\right\|$ for all parameters $w, w^{\prime} \in \mathbb{R}^{\mathrm{d}}$.

2. The gradient $\nabla \ell(z,-): \mathbb{R}^{\mathrm{d}} \rightarrow \mathbb{R}^{\mathrm{d}}$ is $\beta$-Lipschitz for every $z \in Z$.

3. The step sizes satisfy $0 \leqslant \alpha_{t} \leqslant 2 / \beta$.

To show uniform stability, for any two training sets $S\langle 1\rangle, S\langle 2\rangle$ differing in one element and every example $z \in Z$, our proof obligation is

$$
\left|\mathbb{E}_{\mathbf{s g d}(S\langle 1\rangle)}[\ell(z)]-\mathbb{E}_{\mathbf{s g d}(S\langle 2\rangle)}[\ell(z)]\right| \leqslant \gamma \mathrm{L} \quad \text { where } \gamma \triangleq \frac{2 \mathrm{~L}}{\mathrm{n}} \sum_{\mathrm{t}=0}^{\mathrm{T}-1} \alpha_{\mathrm{t}} .
$$

Rather than working with the loss function directly, we will first bound the preexpectation of the distance $\|w\langle 1\rangle-w\langle 2\rangle\|$ and then use the L-Lipschitz property of $\ell$ to conclude uniform stability. As usual, the main part of the proof is bounding the pre-expectation of the loop. We use the following loop invariant:

$$
\mathcal{J} \triangleq[t\langle 1\rangle \neq t\langle 2\rangle] \cdot \infty+[t\langle 1\rangle=t\langle 2\rangle] \cdot\left(\|w\langle 1\rangle-w\langle 2\rangle\|+\frac{2 L}{n} \sum_{j=t\langle 1\rangle}^{T-1} \alpha_{j}\right) .
$$

By the loop rule (Theorem 10.14), it suffices to show the following invariant condition:

$$
[e\langle 1\rangle \wedge e\langle 2\rangle] \cdot \widetilde{r p e}(b d, \mathcal{J})+[\neg e\langle 1\rangle \wedge \neg e\langle 2\rangle] \cdot\|w\langle 1\rangle-w\langle 2\rangle\|+[e\langle 1\rangle \neq e\langle 2\rangle] \cdot \infty \leqslant \quad \mathcal{J} .
$$

The main case corresponds to the first term, where both loop guards $e\langle 1\rangle$ and $e\langle 2\rangle$ are true. To bound the pre-expectation $\widetilde{r p e}(b d, \mathcal{J})$, we consider $\widetilde{r p e}(b d, J)=\widetilde{\mathrm{rpe}}(\mathrm{s} \leqslant$ Unif $_{S}, J^{\prime}$ ) where

$$
\begin{aligned}
& \mathcal{J}^{\prime} \triangleq[\mathrm{t}\langle 1\rangle+1 \neq \mathrm{t}\langle 2\rangle+1] \cdot \infty+[\mathrm{t}\langle 1\rangle+1=\mathrm{t}\langle 2\rangle+1] \cdot \mathrm{P}, \text { with } \\
& \mathrm{P} \triangleq \frac{2 \mathrm{~L}}{\mathrm{n}} \sum_{j=\mathrm{t}\langle 1\rangle+1}^{\mathrm{T}-1} \alpha_{j}+\left\|\begin{array}{c}
\left(w\langle 1\rangle-\alpha_{\mathrm{t}\langle 1\rangle} \nabla \ell(s\langle 1\rangle,-)(w\langle 1\rangle)\right) \\
-\left(w\langle 2\rangle-\alpha_{\mathrm{t}\langle 2\rangle} \nabla \ell(s\langle 2\rangle,-)(w\langle 2\rangle)\right)
\end{array}\right\| .
\end{aligned}
$$

\footnotetext{
${ }^{1}$ This makes our states non-discrete, but the distributions over them will still have discrete support, since they are generated by a composition of discrete samplings.
} 
To handle the random sampling command, we apply the sampling rule (Proposition 10.13) with the coupling function $M$ for the two uniform distributions $[S\langle 1\rangle]$ and $[S\langle 2\rangle]$ induced by the bijection $f: S\langle 1\rangle \rightarrow S\langle 2\rangle$ mapping the differing example in $S\langle 1\rangle$ to its counterpart in $S\langle 2\rangle$, and fixing all other examples. We then have $\widetilde{\text { rpe }}\left(s \leqslant\right.$ Unif $\left._{S}, J^{\prime}\right) \leqslant J^{\prime \prime}$, where

$$
\begin{aligned}
& \mathrm{J}^{\prime \prime} \triangleq[\mathrm{t}\langle 1\rangle+1 \neq \mathrm{t}\langle 2\rangle+1] \cdot \infty+[\mathrm{t}\langle 1\rangle+1=\mathrm{t}\langle 2\rangle+1] \cdot \mathrm{P}^{\prime}, \text { with } \\
& \mathrm{P}^{\prime}=\frac{2 \mathrm{~L}}{\mathrm{n}} \sum_{j=\mathrm{t}\langle 1\rangle+1}^{\mathrm{T}-1} \alpha_{j}+\frac{1}{n} \sum_{\mathrm{s} \in S\langle 1\rangle}^{\mathrm{n}-1}\left\|\begin{array}{c}
\left(w\langle 1\rangle-\alpha_{\mathrm{t}\langle 1\rangle} \nabla \ell(\mathrm{s},-)(w\langle 1\rangle)\right) \\
-\left(w\langle 2\rangle-\alpha_{\mathrm{t}\langle 2\rangle} \nabla \ell(\mathrm{f}(\mathrm{s}),-)(w\langle 2\rangle)\right)
\end{array}\right\|
\end{aligned}
$$

We focus on the terms of the last sum. Using the L-Lipschitz property of $\ell$, when $s$ is the differing example, we can bound the absolute difference by $\|w\langle 1\rangle-w\langle 2\rangle\|+$ $2 \alpha_{t\langle 1\rangle} \mathrm{L}$. When $s$ is not the differing example, we have $s\langle 1\rangle=s\langle 2\rangle$. By the $\beta$-Lipschitz property of $\nabla \ell$, convexity, and $0 \leqslant \alpha_{t} \leqslant 2 / \beta$, we can bound each of the terms by $\|w\langle 1\rangle-w\langle 2\rangle\|$. Combining the two cases gives

$$
\widetilde{r p e}(b d, \mathcal{J}) \leqslant\left(\|w\langle 1\rangle-w\langle 2\rangle\|+\frac{2 L}{n} \sum_{j=t\langle 1\rangle}^{\mathrm{T}-1} \alpha_{j}\right)
$$

for all input states with $t\langle 1\rangle=t\langle 2\rangle$ and $e\langle 1\rangle \wedge e\langle 2\rangle$. This establishes (10.1). Theorem 10.14 gives

$$
\widetilde{r p e}(\text { while } e \text { do } b d,\|w\langle 1\rangle-w\langle 2\rangle\|) \leqslant \mathcal{J} .
$$

Finally, taking the pre-expectations of both sides with respect to the initial assignments yields

$$
\widetilde{r p e}(\operatorname{sgd}(S),\|w\langle 1\rangle-w\langle 2\rangle\|) \leqslant \frac{2 L}{n} \sum_{j=0}^{T-1} \alpha_{j}=\gamma,
$$

when $S\langle 1\rangle$ and $S\langle 2\rangle$ differ in exactly one training example. Since $\ell$ is L-Lipschitz, we conclude

$$
\widetilde{r p e}(\operatorname{sgd}(S),|\ell(z, w)\langle 1\rangle-\ell(z, w)\langle 2\rangle|) \leqslant \gamma \mathrm{L},
$$

for any example $z \in Z$. By Theorem 10.8, the expected losses are at most $\gamma \mathrm{L}$ apart:

$$
\left|\mathbb{E}_{\text {sgd }(S\langle\langle\rangle)}[\ell(z)]-\mathbb{E}_{\text {sgd }(S\langle 2\rangle)}[\ell(z)]\right| \leqslant \gamma \mathrm{L},
$$

and so SGD satisfies $\gamma \mathrm{L}$-uniform stability.

Remark 10.16. This stability bound for SGD was previously verified in the program logic EPRHL [42], using a complex rule for sequential composition (SEQCASE) that required bounding the probability of selecting two differing examples. Our proof using r re is much simpler, involving just compositional reasoning for sequencing and a loop invariant.

Remark 10.17. While our calculus was designed for probabilistic programs, it is also a useful tool for proving relational properties of deterministic programs. In the Appendix C.6, we show how to prove a sensitivity bound for projected gradient descent, a deterministic version of SGD. 


\subsection{Example: Convergence of Reinforcement Learn- ing algorithms}

In the previous section, the stability guarantee weakens as the program progresses: starting from two initially-equal parameter settings, the learned parameters may drift apart as SGD runs for more iterations. In the following two sections, we will apply our technique to prove a different style of guarantee: probabilistic convergence of two outputs, starting from two different inputs. Our first example shows convergence for a classical algorithm from Reinforcement Learning (RL) [148], guided by a novel analysis by Amortila et al. [8].

\subsubsection{Background}

In the standard reinforcement learning setting, an agent (the learning algorithm) repeatedly interacts with the environment, a Markov Decision Process (MDP) with state space $\mathcal{S}$. At each step, the agent chooses an action from a set $\mathcal{A}$. The MDP draws a numeric reward according to a function $\mathcal{R}: \mathcal{S} \times \mathcal{A} \rightarrow \mathbf{S D}([0, \mathrm{R}])$, and transitions to a new random state drawn from a transition function $\mathcal{P}: \mathcal{S} \times \mathcal{A} \rightarrow \mathbf{S D}(\mathcal{S})$. The current state of the process is known to the learner-imagine the current position of a chessboard-but the exact reward and transition functions $(\mathcal{R}, \mathcal{P})$ are not known. Given black-box access to $\mathcal{R}$ and $\mathcal{S}$, the goal of the learner is to find a policy map $\pi: \mathcal{S} \rightarrow \mathcal{A}$ that maximizes the learner's expected reward when interacting with the unknown MDP over an infinite time horizon; estimated rewards in the future are reduced by a discount factor $\gamma \in[0,1)$ for each step into the future.

For many approaches to learning the optimal policy, an important requirement is estimating the value function $\mathrm{V}: \mathcal{S} \rightarrow[0, \mathrm{R}]$ of the MDP, i.e., the expected reward at each state if the agent were to repeatedly act according to some given policy $\pi$. Temporal difference (TD) learning is one approach to estimating the value function [148]. In brief, a TD learner maintains an estimate of $V$ and loops through states in $\mathcal{S}$. At each state $s$, the learner selects an action $a \sim \pi(s)$, draws a reward $r \sim \mathcal{R}(s, a)$, and draws a transition $s^{\prime} \sim \mathcal{R}(s, a)$. Then, the estimate $V(s)$ is updated by incorporating the observed reward $r$ and the estimated value $V\left(s^{\prime}\right)$ of the new state.

Figure 10.4b shows one simple approach, known as $\mathrm{TD}(\mathrm{o})$. We assume that the program takes only one argument $V$, the initial estimate of the value function. All other parameters are assumed to be fixed: the current policy $\pi$, the reward and transition functions $\mathcal{R}$ and $\mathcal{P}$, the discount factor $\gamma$, the step size $\alpha \in(0,1)$-higher $\alpha$ allows $\mathrm{V}$ to evolve faster-and the number of iterations $\mathrm{N}$. 


\subsubsection{Verifying convergence for TDo}

Since the true value function is not known, the initial estimate $V$ chosen with little information. A natural question is: does the algorithm converge to the same distribution no matter how $V$ is initialized? If so, how fast does convergence happen, as a function of the number of iterations N? To answer these questions, we will verify that TDo is contractive on V. More specifically, we will show the bound

$$
\widetilde{\operatorname{rpe}}\left(\operatorname{TDo}(\mathrm{V}),\|\mathrm{V}\langle 1\rangle-\mathrm{V}\langle 2\rangle\|_{\infty}\right) \leqslant \mathrm{k}^{\mathrm{N}} \cdot\|\mathrm{V}\langle 1\rangle-\mathrm{V}\langle 2\rangle\|_{\infty},
$$

where $k \triangleq(1-\alpha+\alpha \gamma)<1$. Before we describe the verification, we unpack the guarantee. First, the $\infty$-norms are defined by $\|\mathrm{V}\langle 1\rangle-\mathrm{V}\langle 2\rangle\|_{\infty} \triangleq \max _{i<|\delta|} \mid \mathrm{V}\langle 1\rangle[\mathrm{i}]-$ $V\langle 2\rangle[i]$. By Theorem 10.11, Eq. (10.2) implies that for any inputs $V_{1}$ and $V_{2}$, there exists a coupling $\mu$ of the output distributions $\mu_{1}$ and $\mu_{2}$ from TDo $(V\langle 1\rangle)$ and $\operatorname{TDo}(\mathrm{V}\langle 2\rangle)$, such that:

$$
\begin{aligned}
k^{\mathrm{N}} \cdot\left\|\mathrm{V}_{1}-\mathrm{V}_{2}\right\|_{\infty} & \geqslant \mathbb{E}_{\left(s_{1}, s_{2}\right) \sim \mu}\left[\left\|s_{1}(\mathrm{~V})-s_{2}(\mathrm{~V})\right\|_{\infty}\right] \\
& \geqslant \max _{i<|\delta|} \mathbb{E}_{\left(s_{1}, s_{2}\right) \sim \mu}\left[\left|s_{1}(\mathrm{~V}[i])-s_{2}(\mathrm{~V}[i])\right|\right] \\
& \geqslant \max _{i<|\delta|}\left|\mathbb{E}_{\left(s_{1}, s_{2}\right) \sim \mu}\left[s_{1}(\mathrm{~V}[i])-s_{2}(\mathrm{~V}[i])\right]\right| \\
& =\max _{i<|\delta|}\left|\mathbb{E}_{s_{1} \sim \mu_{1}}\left[s_{1}(\mathrm{~V}[i])\right]-\mathbb{E}_{s_{2} \sim \mu_{2}}\left[s_{2}(\mathrm{~V}[i])\right]\right| \quad \text { (by Theorem 10.8) }
\end{aligned}
$$

In words, the right-hand side of the final line is the maximum difference between the average estimates of $\mathrm{V}[i]$ in the two outputs, taking the maximum over all indices $i$. Since $k<1$, both sides tend to zero exponentially quickly from any pair of starting states $V_{1}$ and $V_{2}$.

Inner loop. We start by analyzing the inner loop $w_{\text {in }}$. We first show that

$$
\widetilde{r p e}\left(w_{\text {in }},\|W\langle 1\rangle-W\langle 2\rangle\|_{\infty}\right) \leqslant \mathcal{J}_{\text {in }}
$$

for the invariant $\mathcal{J}_{\text {in }}$ :

$$
\begin{aligned}
& \mathcal{J}_{\text {in }} \triangleq[i\langle 1\rangle \neq i\langle 2\rangle] \cdot \infty \\
& \quad+[i\langle 1\rangle=i\langle 2\rangle] \cdot \max _{l<|S|}\left([l<i\langle 1\rangle] \cdot|W\langle 1\rangle[l]-W\langle 2\rangle[l]|+[i\langle 1\rangle \leqslant l] \cdot k \cdot\|V\langle 1\rangle-V\langle 2\rangle\|_{\infty}\right) .
\end{aligned}
$$

Let $c_{i n}$ be the body, and $c_{s a m p}$ be the three sampling statements. Applying INv, it suffices to show:

$$
\begin{array}{r}
{[i\langle 1\rangle<|\mathcal{S}| \wedge \mathfrak{i}\langle 2\rangle<|\mathcal{S}|] \cdot \widetilde{\text { rpe }}\left(c_{\text {in }}, \mathcal{J}_{\text {in }}\right)} \\
+[i\langle 1\rangle \geqslant|\mathcal{S}| \wedge \mathfrak{i}\langle 2\rangle \geqslant|\mathcal{S}|] \cdot\|W\langle 1\rangle-W\langle 2\rangle\|_{\infty}+[i\langle 1\rangle \neq \mathfrak{i}\langle 2\rangle] \cdot \infty \leqslant \mathcal{J}_{\mathfrak{i n}}
\end{array}
$$


The main case is bounding $\widetilde{r p e}\left(c_{i n}, \mathcal{J}_{\text {in }}\right)$; the other cases are simpler. We describe the overall idea here, deferring details to Appendix C.5. To bound the relational pre-expectation for the three sampling instructions, we apply the sampling rule SAMP. Since the relational pre-expectation is computed in reverse order, we must choose a coupling for sampling $j$ first, then choose a coupling for sampling $r$, and then finally choose a coupling for sampling $a$. We aim to take the identity coupling in each case, ensuring $j\langle 1\rangle=j\langle 2\rangle, r\langle 1\rangle=r\langle 2\rangle$, and $a\langle 1\rangle=a\langle 2\rangle$, but there is a small problem: we can only take the identity coupling when samples are taken from the same distributions, e.g., $\mathcal{R}(i\langle 1\rangle, a\langle 1\rangle)=\mathcal{R}(i\langle 2\rangle, a\langle 2\rangle)$. The invariant assumes $i\langle 1\rangle=i\langle 2\rangle$, but we can only ensure $a\langle 1\rangle=a\langle 2\rangle$ after we have specified the couplings for $j$ and $r$. Accordingly, our coupling functions for SAMP will be of the following form: if $a\langle 1\rangle=a\langle 2\rangle$ then we take the identity coupling, otherwise we take the trivial (independent) coupling.

Outer loop. We now turn to the analysis of the outer loop. Consider the invariant:

$$
\mathcal{J}_{\text {out }} \triangleq[n\langle 1\rangle \neq n\langle 2\rangle] \cdot \infty+[n\langle 1\rangle=n\langle 2\rangle] \cdot k^{(\mathrm{N} \ominus n\langle 1\rangle)}\|\mathrm{V}\langle 1\rangle-\mathrm{V}\langle 2\rangle\|_{\infty},
$$

where $\mathrm{N} \ominus n$ denotes $\max (\mathrm{N}-\mathrm{n}, 0)$. We compute:

$$
\begin{aligned}
& \widetilde{r p e}\left(\mathrm{i} \leftarrow 0 ; w_{\text {in }} ; \mathrm{V} \leftarrow \mathrm{W} ; \mathrm{n} \leftarrow \mathrm{n}+1, \mathcal{J}_{\text {out }}\right) \\
& =\widetilde{r p e}\left(\mathrm{i} \leftarrow 0 ; w_{\text {in }},[n\langle 1\rangle \neq \mathrm{n}\langle 2\rangle] \cdot \infty+[\mathrm{n}\langle 1\rangle=\mathrm{n}\langle 2\rangle] \cdot k^{(\mathrm{N} \ominus(\mathrm{n}\langle 1\rangle+1))}\|\mathrm{W}\langle 1\rangle-\mathrm{W}\langle 2\rangle\|_{\infty}\right) \\
& \leqslant \widetilde{r p e}\left(\mathrm{i} \leftarrow 0,[\mathrm{n}\langle 1\rangle \neq \mathrm{n}\langle 2\rangle] \cdot \infty+[\mathrm{n}\langle 1\rangle=\mathrm{n}\langle 2\rangle] \cdot \mathrm{k}^{(\mathrm{N} \ominus(\mathrm{n}\langle 1\rangle+1))} \cdot \mathcal{J}_{\text {in }}\right) \\
& \leqslant[\mathrm{n}\langle 1\rangle \neq \mathrm{n}\langle 2\rangle] \cdot \infty+[\mathrm{n}\langle 1\rangle=\mathrm{n}\langle 2\rangle] \cdot \mathrm{k} \cdot \mathrm{k}^{(\mathrm{N} \ominus(\mathrm{n}\langle 1\rangle+1))}\|\mathrm{V}\langle 1\rangle-\mathrm{V}\langle 2\rangle\|_{\infty}=J_{\text {out }}
\end{aligned}
$$

where the last step holds because $\mathcal{J}_{i_{n}}=k \cdot\|V\langle 1\rangle-V\langle 2\rangle\|_{\infty}$ when $i=0$. This establishes the outer invariant. Computing the pre-expectation of the first initialization, we conclude:

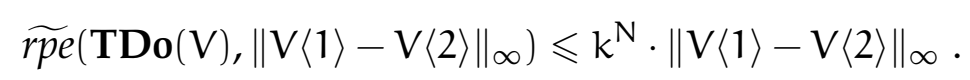

\subsection{Example: Random Walks and Card Shuffles}

In this section, we verify more challenging examples of probabilistic convergence from the theory of Markov chains, formalizing arguments by Aldous [7] in his seminal work introducing the coupling method. Our use of relational pre-expectations is similar in spirit to the previous section, but there are two key differences: (1) we aim to prove convergence under Total Variation (TV) distance, which is the standard notion of distance in this field, and (2) our arguments will require selecting more complex couplings, instead of just the identity coupling. 


\subsubsection{Preliminaries: Card shuffling and Markov chain mixing}

Distributions that are easy to describe can be surprisingly difficult to sample from. For instance, consider the uniform distribution over all permutations of a deck of playing cards. It is not clear how to sample from this distribution-i.e., perform a perfect shuffle - but we can implement a card shuffle algorithm that executes a sequence of simple randomized steps (e.g. swapping pairs of cards) and hope that after a small number steps, we will produce a shuffle that is close to uniform.

Abstracting a bit, card shuffling algorithms are a representative example of random walks for approximating complex distributions. This is a technique with a long history, combining elements of probability theory with statistical physics; and it is the basis of many heuristic algorithms used today, e.g., Markov Chain Monte Carlo (MCMC). From a theoretical perspective, the central question is: how fast do these processes converge to their target distribution? How many steps do we need to get within $\epsilon$ distance of the uniform distribution on shuffles?

Random walks and card shuffling algorithms are classical examples of Markov chains. A finite, discrete-time Markov chain is defined by a finite state space $\Sigma$ and a transition function P: $\Sigma \rightarrow \mathbf{S D}(\Sigma)$. Given an initial state $\sigma$, the associated Markov process $\left\{X_{k}^{\sigma}\right\}_{k \in \mathbb{N}}$ is a sequence of distributions such that $X_{0}^{\sigma}=\delta(\sigma)$ and $X_{k+1}^{\sigma}\left(\tau^{\prime}\right)=\sum_{\tau} X_{k}^{\sigma}(\tau) \cdot P\left(\tau, \tau^{\prime}\right)$. For example, the state space $\Sigma$ could be the set of all permutations of a deck of cards, and the transition function $\tau$ could describe randomly splitting the deck and interleaving the halves.

Consider the TV distance $v(k)$ between two state distributions after running $k$ steps from two states $\sigma$, $\tau$, i.e., $v(k) \triangleq \max _{\sigma, \tau} \operatorname{TV}\left(X_{k}^{\sigma}, X_{k}^{\tau}\right)$. If $v(k)$ tends to o, then there exists a unique stationary distribution $\eta$ such that $\eta(\sigma) \cdot P\left(\sigma, \sigma^{\prime}\right)=\eta\left(\sigma^{\prime}\right)$; typically, $\eta$ will be the target distribution we are trying to sample from. Furthermore, $v(k)$ provides an upper bound on the distance between the state distribution after $k$ steps to the stationary distribution $\eta$ :

$$
\max _{\sigma} \operatorname{TV}\left(X_{t}^{\sigma}, \eta\right) \leqslant v(k) .
$$

While it is usually not possible to derive $v(\mathrm{k})$ exactly, we can upper-bound $v(\mathrm{k})$ by constructing couplings of $\left(X_{t}^{\sigma}, X_{t}^{\tau}\right)$ and applying Theorems 10.7 and 10.9. In this way, we can prove bounds on the number of steps needed to get within some distance of the target distribution.

\subsubsection{Warmup: Hypercube walk}

We start off with a (rather naive) random walk for sampling $\mathrm{N}$ uniformly random bits, which serves as a toy version of the more complex random walks we will see later. Our position is a string of $\mathrm{N}$ bits (which can be regarded as a vertex of an $\mathrm{N}$-dimensional hypercube). On every iteration of the walk we uniformly sample from $\{0, \ldots, N\}$. Note that there are $N+1$ possible draws, but only $N$ coordinates: if we 
sample 0 , then we do not move, otherwise we reverse the sampled coordinate $i$ in the current position. We will show that starting from any two positions, the process mixes rapidly, i.e. starting from any position we will quickly reach the uniform distribution over positions.

Let $e(i)=(0, \ldots, 1, \ldots, 0) \in\{0,1\}^{\mathrm{N}}$ be the position where all coordinates are set to zero except for coordinate $i$, which is set to one. We also write $\oplus$ for xor applied coordinate-wise. We can model $\mathrm{K}$ steps of the random walk with the following simple PWHILE program:

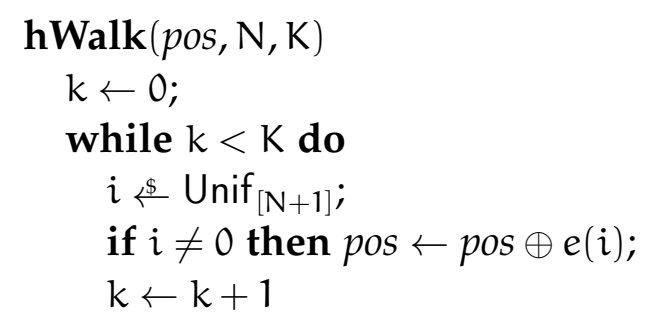

Consider two program runs, started at $\operatorname{pos}\langle 1\rangle$ and $\operatorname{pos}\langle 2\rangle$ respectively. Let $\mathrm{d}_{\mathrm{H}}$ be normalized Hamming distance between the two positions:

$$
\mathrm{d}_{\mathrm{H}} \triangleq \frac{1}{\mathrm{~N}} \sum_{i=1}^{\mathrm{N}}[\operatorname{pos}\langle 1\rangle[i] \neq \operatorname{pos}\langle 2\rangle[\mathrm{i}]] .
$$

That is, $\mathrm{d}_{\mathrm{H}}$ equals the fraction of coordinates where $\operatorname{pos}\langle 1\rangle$ and $\operatorname{pos}\langle 2\rangle$ differ. Let $\mathrm{C}(\operatorname{pos}\langle 1\rangle, \operatorname{pos}\langle 2\rangle) \subseteq[\mathrm{N}]$ be the set of differing coordinates. We specify a coupling on Unif $_{[\mathrm{N}+1]}$ by giving a bijection on $[\mathrm{N}+1]$. There are three cases:

1. $d_{H} \geqslant 2 / N$ : Let $C(\operatorname{pos}\langle 1\rangle, \operatorname{pos}\langle 2\rangle)=\left\{i_{0}, \ldots, i_{m-1}\right\}$. Take the bijection that behaves like the identity on $[\mathrm{N}+1] \backslash \mathrm{C}(\operatorname{pos}\langle 1\rangle, \operatorname{pos}\langle 2\rangle)$ and that, for all $0 \leqslant n \leqslant m$, maps $i_{n}$ to $i_{n+1}$, where we set $i_{m}=i_{0}$.

2. $d_{H}=1 / N$ : Take the bijection exchanging the differing coordinate and 0 .

3. $d_{H}=0$ : Take the identity bijection.

The coupling captures the following intuition. When $d_{H} \geqslant 2 / N$, the distance decreases by $2 / \mathrm{N}$ if we select a differing coordinate; otherwise, it remains unchanged. Likewise when $d_{H}=1 / N$, if we select the differing coordinate or 0 , then the distance decreases by $1 / \mathrm{N}$ (to 0 ); otherwise, the distance remains unchanged.

We can analyze the program hWalk using our relational pre-expectation calculus. Let the target relational expectation be $\mathrm{d}_{\mathrm{H}}$. The main step in the reasoning is to select a relational invariant for the loop. We define:

$$
J \triangleq[k\langle 1\rangle \neq k\langle 2\rangle] \cdot \infty+[k\langle 1\rangle=k\langle 2\rangle] \cdot d_{H} \cdot\left(\frac{N-1}{N+1}\right)^{K \ominus k\langle 1\rangle} .
$$


Then, we can verify for the loop while $k<K$ do bd of program hWalk that

$$
\begin{aligned}
& {[(k\langle 1\rangle<\mathrm{K}\langle 1\rangle) \wedge(\mathrm{k}\langle 2\rangle<\mathrm{K}\langle 2\rangle)] \cdot \widetilde{\mathrm{rpe}}(b d, \mathcal{J}) } \\
+ & {[(k\langle 1\rangle \geqslant \mathrm{K}\langle 1\rangle) \wedge(k\langle 2\rangle \geqslant \mathrm{K}\langle 2\rangle)] \cdot \mathrm{d}_{\mathrm{H}} } \\
+ & {[(k\langle 1\rangle<\mathrm{K}\langle 1\rangle) \neq(\mathrm{k}\langle 2\rangle<\mathrm{K}\langle 2\rangle)] \cdot \infty \quad \leqslant \quad J, }
\end{aligned}
$$

and conclude by the loop rule (Theorem 10.14):

$$
\widetilde{r p e}\left(\text { while } \mathrm{k}<\mathrm{K} \text { do } b d, \mathrm{~d}_{\mathrm{H}}\right) \leqslant \mathcal{J} .
$$

The main step here is showing that

$$
[(k\langle 1\rangle<\mathrm{K}\langle 1\rangle) \wedge(\mathrm{k}\langle 2\rangle<\mathrm{K}\langle 2\rangle)] \cdot \widetilde{\mathrm{pe}}(b d, \mathcal{J}) \leqslant[(\mathrm{k}\langle 1\rangle<\mathrm{K}\langle 1\rangle) \wedge(\mathrm{k}\langle 2\rangle\langle\mathrm{K}\langle 2\rangle)] \cdot \mathcal{J},
$$

where we use the fact that the coupling described above makes $d_{H}$ decrease.

Pushing the invariant past the initialization instruction $k \leftarrow 0$ yields:

$$
\widetilde{r p e}\left(\mathbf{h W a l k}(\text { pos, } \mathrm{N}, \mathrm{K}), \mathrm{d}_{\mathrm{H}}\right) \leqslant \widetilde{r p e}(\mathrm{~K} \leftarrow 0, \mathrm{~J})=\left(\frac{\mathrm{N}-1}{\mathrm{~N}+1}\right)^{\mathrm{K}} .
$$

Since the distance $d_{H}$ takes distance at least $1 / \mathrm{N}$ on pairs of distinct positions, by Theorem 10.9 the TV distance between the distributions over positions satisfies

$$
\begin{aligned}
v(\mathrm{~K}, \mathrm{~N}) & =\max _{\mathrm{p}_{1}, \mathrm{p}_{2} \in\{0,1\} \mathrm{N}} \operatorname{TV}\left(\llbracket \mathbf{h W a l k} \rrbracket\left(\mathrm{p}_{1}, \mathrm{~N}, \mathrm{~K}\right), \llbracket \mathbf{h W a l k} \rrbracket\left(\mathrm{p}_{2}, \mathrm{~N}, \mathrm{~K}\right)\right) \\
& \leqslant \mathrm{N}\left(1-\frac{2}{\mathrm{~N}+1}\right)^{\mathrm{K}} .
\end{aligned}
$$

Plugging in specific values gives concrete bounds between the two output distributions. Let $\rho>1$. To achieve a bound of $\mathrm{O}(1 / \rho)$ on the right hand side, we need to take $K \geqslant(1 / 2) N \log (N \rho)$. The inequality above also gives useful asymptotic information; if we set $\rho=N$, and take $K \geqslant N \log N$, the right-hand side is asymptotically bounded by $\mathrm{O}(1 / \mathrm{N})$ for large $\mathrm{N}$. We can show that this converges to the uniform distribution over vectors. We provide more details in Section 10.7. In summary, we have shown the following:

Theorem 10.18. Let $\mathrm{K}=\mathrm{N} \log \mathrm{N}$. For any initial position pos,

$$
\left.\operatorname{TV}\left(\mathbf{h W a l k}(\text { pos, } \mathrm{N}, \mathrm{K}), \mathrm{U}(\{0,1\})^{\mathrm{N}}\right)\right) \in \mathcal{O}(1 / \mathrm{N}) \text {. }
$$

\subsubsection{Random-to-top shuffle}

For our shuffling examples, we will need some notation. We view a permutation deck as a map from positions in $p \in[N]$ to names of cards in $c \in C$; deck[p] denotes the card at position $\mathrm{p}$, while $\mathrm{deck}^{-1}(\mathrm{c})$ denotes the position corresponding to card $\mathrm{c}$. 


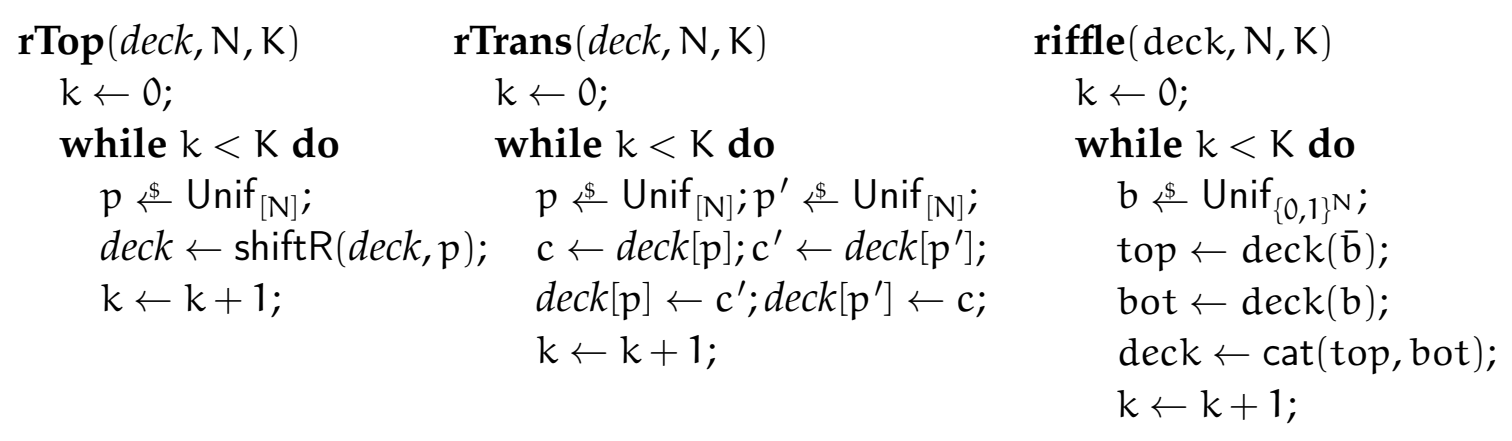

Figure 10.5: Shuffling algorithms

Summation over an empty set of indices is treated as zero, while the product over an empty set of indices is treated as one. We outline the arguments here; further details are provided in Appendix C.7.

For our first card shuffling algorithm we consider the random-to-top shuffle. In each iteration, it selects a random position in the deck and moves the card at that position to the top. ${ }^{2}$ We model this shuffle with program rTop in Figure 10.5. For a given input deck of size $\mathrm{N}$, the program repeats $\mathrm{K}$ times the process of selecting a random card and moving it to the top. The operation shift $R($ deck, $j)$ takes the block $\operatorname{deck}[0], \ldots, \operatorname{deck}[j]$ and cycles it one position to the right (thus moving deck[j] to the top), leaving the rest of the deck intact.

We are interested in bounding the distance between the stationary distributionwhich in this case is the uniform distribution-and the output distribution after $\mathrm{K}$ iterations. We will start with two decks of size $\mathrm{N}$ that are both permutations of $[\mathrm{N}]$. As in the hypercube example, we bound the pre-expectation of the normalized Hamming distance:

$$
\mathrm{d}_{\mathrm{H}} \triangleq \frac{1}{\mathrm{~N}} \sum_{i=0}^{\mathrm{N}-1}[\operatorname{deck}\langle 1\rangle[i] \neq \operatorname{deck}\langle 2\rangle[i]] .
$$

Note that $d_{H}$ takes distance at least $1 / \mathrm{N}$ on pairs of distinct permutations. If we can show that the pre-expectation of $d_{H}$ is not too big, then we can apply Theorem 10.9 to conclude that the final distributions over permutations have a close TV distance. It will be convenient to work with an auxiliary distance:

$$
\mathrm{d}_{\mathrm{M}} \triangleq(1 / \mathrm{N}) \cdot\left(\mathrm{N}-\max _{\mathrm{i}}(\forall \mathrm{j}<\mathrm{i} \cdot \operatorname{deck}\langle 1\rangle[\mathrm{j}]=\operatorname{deck}\langle 2\rangle[\mathrm{j}])\right) .
$$

The idea is that the coupling chooses identical cards on both decks and moves them to the top. This will form a block of matched cards on the top of both decks.

\footnotetext{
${ }^{2}$ This algorithm is the time-reversed version of the top-to-random shuffle, where the top card is moved to a random position. It is known that a Markov chain's convergence behavior is equivalent to that of its reversed process [7].
} 
Intuitively, $d_{M}$ measures the fraction of the deck that is not part of this top block. The target distance $d_{H}$ is upper-bounded by $d_{M}$, since $d_{M}$ counts all cards outside the first block as different. Bounds on $d_{H}$ follow from bounds on $d_{M}$. To bound the pre-expectation of $d_{M}$, we take the invariant:

$$
\mathcal{J} \triangleq[k\langle 1\rangle \neq k\langle 2\rangle] \cdot \infty+[k\langle 1\rangle=k\langle 2\rangle] \cdot d_{M} \cdot\left(\frac{N-1}{N}\right)^{K \ominus k\langle 1\rangle} .
$$

We can check that it satisfies the inequality

$$
\begin{array}{r}
{[\mathrm{k}\langle 1\rangle<\mathrm{K} \wedge \mathrm{k}\langle 2\rangle<\mathrm{K}] \cdot \widetilde{\mathrm{rpe}}(b d, \mathrm{~J})+[\mathrm{k}\langle 1\rangle \geqslant \mathrm{K} \wedge \mathrm{k}\langle 2\rangle \geqslant \mathrm{K}] \cdot \mathrm{d}_{\mathrm{H}}} \\
+[(\mathrm{k}\langle 1\rangle<\mathrm{K}) \neq(\mathrm{k}\langle 2\rangle<\mathrm{K})] \cdot \infty \leqslant \mathrm{J},
\end{array}
$$

where $b d$ is the loop body. The main case is to show the inequality for the first term when both loop guards are true: we need to bound the pre-expectation of $\mathcal{J}$ with respect to $b d$. We can bound

$$
\widetilde{r p e}(b d, \mathfrak{J}) \leqslant \mathrm{d}_{M} \cdot\left(\frac{\mathrm{N}-1}{\mathrm{~N}}\right)^{\mathrm{K} \ominus \mathrm{k}\langle 1\rangle},
$$

by applying the sampling rule (Proposition 10.13) with the coupling function $M$ that selects the same card in both decks:

$$
M\left(s_{1}, s_{2}\right)\left(p_{1}, p_{2}\right) \triangleq \begin{cases}1 / N & : \llbracket d e c k \rrbracket s_{1}\left[p_{1}\right]=\llbracket \text { deck } \rrbracket s_{2}\left[p_{2}\right] \\ 0 & : \text { otherwise. }\end{cases}
$$

The idea is that if we pick two cards in the first matched block, which happens with probability $\left(1-d_{M}\right)$, then the distance will remain the same. Otherwise, we will create at least one new matched pair in the first block and the distance will decrease by $1 / \mathrm{N}$. Hence, we can apply the loop rule (Theorem 10.14) to conclude:

$$
\widetilde{r p e}\left(\text { while } \mathrm{k}<\mathrm{K} \text { do } b d, \mathrm{~d}_{\mathrm{H}}\right) \leqslant \mathcal{J} \text {. }
$$

Computing the pre-expectation of $\mathcal{J}$ with respect to the first instruction, we have

$$
\widetilde{r p e}\left(\mathbf{r T o p}(\operatorname{deck}, \mathrm{N}, \mathrm{K}), \mathrm{d}_{\mathrm{H}}\right) \leqslant\left(\frac{\mathrm{N}-1}{\mathrm{~N}}\right)^{\mathrm{K}},
$$

noting that the distance $d_{M}$ between the initial decks is at most 1 . Since $d_{H}$ assigns pairs of distinct decks a distance at least $1 / \mathrm{N}$, Theorem 10.9 implies that the TV distance between the distributions over decks satisfies:

$$
v(\mathrm{~K}, \mathrm{~N})=\max _{\mathrm{d}_{1}, \mathrm{~d}_{2} \in[\mathrm{N}]} \operatorname{TV}\left(\llbracket \mathbf{r} T \mathbf{T o p} \rrbracket\left(\mathrm{d}_{1}, \mathrm{~N}, \mathrm{~K}\right), \llbracket \mathbf{r T o p} \rrbracket\left(\mathrm{d}_{2}, \mathrm{~N}, \mathrm{~K}\right)\right) \leqslant \mathrm{N}\left(\frac{\mathrm{N}-1}{\mathrm{~N}}\right)^{\mathrm{K}} .
$$

For example, if we choose $\mathrm{K}$ to be $\mathrm{N} \log (\mathrm{N} \rho)$, then the distance between permutation distributions is bounded by $\mathrm{O}(1 / \rho)$ for large $\mathrm{N}$ and $\rho>1$. By setting $\rho=\mathrm{N}$, we have shown the following: 
Theorem 10.19. Let $\mathrm{K}=2 \mathrm{~N} \log \mathrm{N}$, and Perm $([\mathrm{N}])$ be the set of permutations over $\mathrm{N}$. For any initial permutation of deck,

$$
\operatorname{TV}(\mathbf{r T o p}(\operatorname{deck}, \mathrm{N}, \mathrm{K}), \mathrm{U}(\operatorname{Perm}([\mathrm{N}]))) \in \mathcal{O}(1 / \mathrm{N}) .
$$

\subsubsection{Random transpositions shuffle}

Our next shuffle (rTrans in Figure 10.5) repeatedly selects two positions uniformly at random and swaps the cards, allowing for the possibility of swapping a card with itself. As before, let $d_{H}$ be the normalized Hamming distance between the two decks. We aim to bound $\overrightarrow{r p e}\left(\mathbf{r} T r a n s, d_{H}\right)$. As before, the key of the proof is finding an invariant for the loop. We take:

$$
\mathcal{J} \triangleq[k\langle 1\rangle \neq k\langle 2\rangle] \cdot \infty+[k\langle 1\rangle=k\langle 2\rangle] \cdot d_{H} \cdot\left(1-\frac{1}{N^{2}}\right)^{K \ominus k\langle 1\rangle}
$$

There are two samplings in the loop body, so we need to provide two couplings. For the first sampling $p$, we use the identity coupling. For the second sampling $\mathrm{p}^{\prime}$, we couple using the bijection induced by the two decks deck $\langle 1\rangle$ and deck $\langle 2\rangle$, i.e., the coupling matches every position $p^{\prime}\langle 1\rangle$ with the unique position $p^{\prime}\langle 2\rangle$ such that $\operatorname{deck}\left[p^{\prime}\right]\langle 1\rangle=\operatorname{deck}\left[p^{\prime}\right]\langle 2\rangle$. There are three cases: (1) if cards at $p\langle 1\rangle, p\langle 2\rangle$ are already matched, $d_{H}$ remains unchanged; (2) if positions $p^{\prime}\langle 1\rangle, p^{\prime}\langle 2\rangle$ are equal, $d_{H}$ remains unchanged; otherwise (3) $d_{H}$ decreases by 1 . This is enough to show that the invariant decreases. We can conclude:

$$
\widetilde{r p e}\left(\operatorname{rTrans}(\operatorname{deck}, \mathrm{N}, \mathrm{K}), \mathrm{d}_{\mathrm{H}}\right) \leqslant\left(1-\frac{1}{\mathrm{~N}^{2}}\right)^{\mathrm{K}}
$$

using the fact that $d_{H}$ between the inputs is at most 1. Since $d_{H}$ takes value of at least $1 / \mathrm{N}$ for pairs of distinct decks, by Theorem 10.9

$$
v(\mathrm{~K}, \mathrm{~N})=\max _{\mathrm{d}_{1}, \mathrm{~d}_{2} \in[\mathrm{N}]} \operatorname{TV}\left(\llbracket \mathbf{r} \operatorname{Trans} \rrbracket\left(\mathrm{d}_{1}, \mathrm{~N}, \mathrm{~K}\right), \llbracket \mathbf{r} \operatorname{Trans} \rrbracket\left(\mathrm{d}_{2}, \mathrm{~N}, \mathrm{~K}\right)\right) \leqslant \mathrm{N}\left(1-\frac{1}{\mathrm{~N}^{2}}\right)^{\mathrm{K}},
$$

so the distance between the deck distribution and the uniform distribution decreases as $K$ increases. If we take $K \geqslant N^{2} \log (N \rho)$, then the right-hand side is bounded asymptotically by $\mathrm{O}(1 / \rho)$ for large $\mathrm{N}$. By setting $\rho=\mathrm{N}$, we conclude:

Theorem 10.20. Let $\mathrm{K}=2 \mathrm{~N}^{2} \log \mathrm{N}$, and Perm $([\mathrm{N}])$ be the set of permutations over $\mathrm{N}$. For any initial permutation of deck,

$$
\operatorname{TV}(\mathbf{r T r a n s}(\operatorname{deck}, \mathrm{N}, \mathrm{K}), \mathrm{U}(\operatorname{Perm}([\mathrm{N}]))) \in \mathcal{O}(1 / \mathrm{N}) \text {. }
$$

Remark 10.21. Aldous' [7] bound is slightly sharper: the TV distance between output distributions is bounded by $\mathrm{O}(1 / \mathrm{N})$ asymptotically already for $\mathrm{K} \geqslant \mathrm{CN}^{2}$ for some constant C. This discrepancy appears because our proofs are carried out compositionally, while Aldous uses a global analysis. However, it is possible that a clever choice of coupling or loop invariant could let us match Aldous' bound. 


\subsubsection{Uniform riffle shuffle}

In this example we will analyze the uniform riffle shuffle, which is a more realistic model of how cards are shuffled by humans. The shuffle begins by dividing the deck in approximately two halves, and then merges the two halves in an approximately alternating manner. The reversed process, program riffle on Figure 10.5 which we analyze, takes a deck, samples a uniform random bit for each card, and then places all cards labeled with o on top of the deck without altering their relative order. After repeating this process $k$ times, for every card $i$ we have sampled a string of bits $\left(b_{i, 0}, \ldots, b_{i, k-1}\right)$, and card $i$ is on top of card $j$ if, for some $m, b_{i, k}=b_{j, k}, b_{i, k-1}=$ $b_{j, k-1}, \ldots, b_{i, m}=b_{j, m}$ and $b_{i, m-1}<b_{j, m-1}$.

The vector $\mathrm{b}$ holds $\mathrm{N}$ bits, indexed by position; $\overline{\mathrm{b}}$ negates each entry. We use shorthands for partitioning: $\operatorname{deck}(b)$ and $\operatorname{deck}(\bar{b})$ represent the sub-permutations from taking all positions where $b$ is 0 and 1 , respectively. Finally, cat concatenates two permutations.

We will take the coupling that always samples the same bit for the same card on both sides: $\mathrm{b}\left(\operatorname{deck}^{-1}(\mathrm{c})\right)\langle 1\rangle=\mathrm{b}\left(\operatorname{deck}^{-1}(\mathrm{c})\right)\langle 2\rangle$ for every $\mathrm{c} \in \mathrm{C}$. It is not hard to see that this coupling will eventually make the decks match. However, choosing an appropriate distance takes more care, since the Hamming distance may not always decrease under this coupling. We leave the details of verification to Appendix C.8. We can show the following:

Theorem 10.22. Let $\mathrm{K}=3 \log \mathrm{N}$, and Perm $([\mathrm{N}])$ be the set of permutations over $\mathrm{N}$. For any initial permutation of deck,

$$
\operatorname{TV}(\text { riffle }(\operatorname{deck}, N, K), \operatorname{Unif}\{\operatorname{Perm}([\mathrm{N}])\}) \in \mathcal{O}(1 / \mathrm{N}) \text {. }
$$

\subsection{Extensions: Proving Lower Bounds and Unifor- mity}

In this section, we describe two extensions to our random walk examples from Section 10.6: proving that the limit distribution is uniform, and proving lower bounds on the TV distance.

\subsubsection{Convergence to uniform distribution}

In Section 10.6, we showed that the Markov chains correspond to each example converge to a stationary distribution, but we did not shown that this distribution is the uniform distribution over states-if we had made an error in the implementation, the probabilistic program may converge to the wrong distribution. We can use our relational pre-expectation calculus along with Theorem 10.8 to show that the limit distribution is indeed uniform. 
We illustrate the technique for the random-to-top shuffle, but the idea is applicable to all our examples. Consider any two permutations of the deck $R_{1}, R_{2}$, and the unary expectations

$$
\mathrm{S}_{1}(\text { deck }) \triangleq\left[\text { deck }=\mathrm{R}_{1}\right] \quad \text { and } \quad \mathrm{S}_{2}(\text { deck }) \triangleq\left[\text { deck }=\mathrm{R}_{2}\right] .
$$

To show that the shuffle converges to uniform, we need to show that the expected values of $S_{1}$ and $S_{2}$ converge to the same value. Recall that Theorem 10.8 states that for any initial states $s_{1}, s_{2}$,

$$
\left|\mathbb{E}_{\llbracket \mathbf{r T o p} \rrbracket s_{1}}\left[S_{1}\right]-\mathbb{E}_{\llbracket \mathbf{r T o p} \rrbracket s_{2}}\left[S_{2}\right]\right| \leqslant\left|S_{1}-S_{2}\right|^{\#}\left(\llbracket \mathbf{r T o p} \rrbracket s_{1}, \llbracket \mathbf{r T o p} \rrbracket s_{2}\right)
$$

so it suffices to show that the right hand side converges to zero.

Computing the weakest pre-expectation of $\left|S_{1}-S_{2}\right|$ directly is difficult, so we define an alternative distance. We can see $R_{1}$ and $R_{2}$ as defining a relation (actually, a permutation $\pi$ over $[N])$ of pairs $\left(R_{1}[i], R_{2}[i]\right)$ of cards that are at the same positions. We let $\mathrm{d}$ be the distance defined by:

$$
\mathrm{d}(\operatorname{deck}\langle 1\rangle, \operatorname{deck}\langle 2\rangle) \triangleq \sum_{i=0}^{\mathrm{N}-1}[(\operatorname{deck}\langle 1\rangle[i], \operatorname{deck}\langle 2\rangle[i]) \notin \pi] .
$$

We can show that $\left|S_{1}(\operatorname{deck}\langle 1\rangle)-\mathrm{S}_{2}(\operatorname{dec} k\langle 2\rangle)\right| \leqslant \mathrm{d}(\operatorname{deck}\langle 1\rangle$, deck $\langle 2\rangle)$, since $\mathrm{d}$ takes nonnegative integer values, and whenever $d=0$, then $S_{1}$ and $S_{2}$ can only be true simultaneously. So it suffices to show that the right-hand side converges to zero. This bound can also be established by our pre-expectation calculus in much the same way as in our proof for the random-to-top shuffle, but we use a different coupling. After sampling $p\langle 1\rangle$ on the first execution we just need to pick the $p\langle 2\rangle$ on the second such that $(\operatorname{deck}\langle 1\rangle[\mathrm{p}\langle 1\rangle], \operatorname{deck}\langle 2\rangle[\mathrm{p}\langle 2\rangle]) \in \pi$. This makes $\mathrm{d}$ decrease any time a new match is formed, and once a match is formed and moved to the top, it is never undone. By starting from the same permutation $\operatorname{deck}\langle 1\rangle=\operatorname{dec}\langle 2\rangle$, this analysis shows that the rate of convergence-this time to the uniform distribution-is the same as in our previous analysis of random-to-top: $d$ converges to o at rate $(1-1 / \mathrm{N})^{\mathrm{K}}$.

\subsubsection{Proving lower bounds}

Previously, we verified upper bounds of the Total Variation distance by using the Kantorovich distance. It is also interesting to compute lower bounds on the TV distance, describing how far apart the distributions must be. We consider how to verify these bounds using the wpe calculus of McIver and Morgan [121], summarized in Figure 10.7. We will need an alternative definition of the TV distance expressed in terms of expected values rather than sets: 


$$
\begin{aligned}
& \text { wpe }(\text { skip, } \varepsilon) \triangleq \varepsilon \\
& \text { wpe }(x \leftarrow e, \mathcal{E}) \triangleq \mathcal{E}\{e / x\} \\
& \operatorname{wpe}(\mathrm{x} \leftarrow \mathrm{d}, \mathcal{E}) \triangleq \lambda s . \mathbb{E}_{\mathrm{x} \sim \mathrm{d}}[\mathcal{E}\{\mathrm{e} / \mathrm{x}\}] \\
& \text { wpe }\left(\mathrm{c} ; \mathrm{c}^{\prime}, \varepsilon\right) \triangleq \operatorname{wpe}\left(\mathrm{c}, \operatorname{wpe}\left(\mathrm{c}^{\prime}, \varepsilon\right)\right) \\
& \text { wpe (if e then } \left.c \text { else } c^{\prime}, \varepsilon\right) \triangleq[e] \cdot \text { wpe }(c, \varepsilon)+[\neg e] \cdot \text { wpe }\left(c^{\prime}, \varepsilon\right) \\
& \text { wpe (while e do } c, \varepsilon) \triangleq \operatorname{IfpX} \text {. [e] } \cdot \text { wpe }(\mathrm{c}, \mathrm{X})+[\neg e] \cdot \mathcal{E}
\end{aligned}
$$

Figure 10.6: Definition of the weakest pre-expectation operator wpe $(c, \varepsilon)$

Proposition 10.23. Let $\mu_{1}, \mu_{2} \in \mathbf{S D}(X)$. Then,

$$
\sup _{f: X \rightarrow[0,1]}\left|\mathbb{E}_{\mu_{1}}[f]-\mathbb{E}_{\mu_{2}}[f]\right|=\sup _{S \subseteq X}\left|\mu_{1}(S)-\mu_{2}(S)\right|=\operatorname{TV}\left(\mu_{1}, \mu_{2}\right) .
$$

Thus, it suffices to pick any $f: X \rightarrow[0,1]$ and compute its expected values wr.t. $\mu_{1}$ and $\mu_{2}$ to get $a$ lower bound on $\operatorname{TV}\left(\mu_{1}, \mu_{2}\right)$. This is performed with the wpe operator, which takes a program $\mathrm{c}$ and an expectation $\mathcal{F}$ : State $\rightarrow[0, \infty]$ and computes:

$$
\operatorname{wpe}(\mathfrak{c}, \mathcal{F})=\lambda s . \mathbb{E}_{x \sim \llbracket c \rrbracket s}[\mathcal{F}(\mathfrak{c})]
$$

Computing lower bounds using wpe poses some technical challenges. First, we need to find some expectation $f$ that will achieve a lower bound that is as large as possible. Second, as we are computing a difference of two expected values, we need to be able to compute exact pre-expectations-standard invariant rules for wpe only produce upper bounds on the expectation. Overcoming the first problem requires ingenuity, but the second problem can be addressed by using a technical result of Kaminski et al. [104]. We start by defining upper and lower invariants.

Definition 10.24. A family of unary expectations $\left\{\mathrm{I}_{\mathfrak{n}}\right\}_{\mathfrak{n} \in \mathbb{N}}$ is an upper $\omega$-invariant of the loop while $\mathrm{b}$ do $\mathrm{c}$ with respect to the expectation $\mathcal{F}$ if

$$
[\neg \mathrm{b}] \cdot \mathcal{F} \leqslant \mathrm{I}_{0} \quad \text { and } \quad[\mathrm{b}] \cdot \operatorname{wpe}\left(\mathrm{c}, \mathrm{I}_{\mathrm{n}}\right)+[\neg \mathrm{b}] \cdot \mathcal{F} \leqslant \mathrm{I}_{\mathrm{n}+1} .
$$

The definition of lower $\omega$-invariant is analogous, reversing the two inequalities.

These invariants can be used to compute exact weakest pre-expectations of loops.

Theorem 10.25 (Kaminski et al. [104, Theorem 5]). Let $\mathrm{I}_{\mathrm{n}}$ and $\mathrm{J}_{\mathrm{n}}$ be an upper and a lower $\omega$-invariant of while $\mathrm{b}$ do $\mathrm{c}$ with respect to $\mathcal{F}$, respectively. If the limits $\lim _{n \rightarrow \infty} \mathrm{I}_{\mathrm{n}}$ and $\lim _{n \rightarrow \infty} \mathrm{J}_{\mathrm{n}}$ exist, then

$$
\lim _{n \rightarrow \infty} J_{n} \leqslant \text { wpe }(\text { while b do } c, \mathcal{F}) \leqslant \lim _{n \rightarrow \infty} I_{n} .
$$

When the limits coincide, the weakest pre-expectation is determined exactly. 
We illustrate our technique on the example from Section 10.6.2. Let $w_{H}$ be the expression denoting the normalized Hamming weight of a vector, i.e. $w_{H} \triangleq$ $(1 / N) \sum_{i=0}^{N} p o s[i]$. We can use the expected value of $w_{H}$ to compute a lower bound for the TV distance between two runs of hWalk. We will compute this expected value by using the usual (unary) pre-expectation calculus. To compute exact weakest preconditions, we need to find upper and lower invariants. We consider the following $\omega$-invariant $\mathcal{J}_{\mathfrak{n}} \triangleq[K-n \leqslant k]\left(\mathcal{B}_{k}+\mathcal{A}_{k} \cdot \mathcal{w}_{H}\right)$, where

$$
\mathcal{A}_{\mathrm{k}} \triangleq\left(\frac{\mathrm{N}-1}{\mathrm{~N}+1}\right)^{\mathrm{K} \ominus \mathrm{k}} \quad \text { and } \quad \mathcal{B}_{\mathrm{k}} \triangleq \frac{1}{\mathrm{~N}+1} \cdot \sum_{\mathrm{i}=0}^{\mathrm{K}-\mathrm{k}-1}\left(\frac{\mathrm{N}-1}{\mathrm{~N}+1}\right)^{\mathrm{i}} .
$$

We can check that $\mathcal{J}_{n}$ is both an upper and a lower $\omega$-invariant, therefore the weakest pre-expectation for the loop is exactly $\lim _{n \rightarrow \infty} \mathcal{I}_{\mathfrak{n}}=\mathcal{B}_{k}+\mathcal{A}_{k} \cdot \mathcal{w}_{H}$. After computing its pre-expectation with respect to the first assignment, we get:

$$
\text { wpe }\left(\mathbf{h W a l k}, w_{\mathrm{H}}\right)=\mathcal{B}_{0}+\mathcal{A}_{0} \cdot w_{\mathrm{H}}
$$

Now, let $W(p) \triangleq(1 / N) \sum_{i=0}^{N} p[i]$, and let $s_{1}, s_{2}$ be any two initial states such that $s_{1}(N)=s_{2}(N)$ and $s_{1}(K)=s_{2}(K)$. By Proposition 10.23, we can lower bound the TV distance as follows:

$$
\begin{array}{r}
\mathrm{TV}\left(\llbracket \mathbf{h W a l k} \rrbracket\left(s_{1}(\text { pos }), \mathrm{N}, \mathrm{K}\right), \llbracket \mathbf{h W a l k} \rrbracket\left(s_{2}(\text { pos }), \mathrm{N}, \mathrm{K}\right)\right) \\
\geqslant \mid \text { wpe }\left(\mathbf{h W a l k}, w_{\mathrm{H}}\right)\left(\mathrm{s}_{1}\right)-\text { wpe }\left(\mathbf{h W a l k}, \boldsymbol{w}_{\mathrm{H}}\right)\left(\mathrm{s}_{2}\right) \mid \\
=\left(\frac{\mathrm{N}-1}{\mathrm{~N}+1}\right) \cdot \mid \mathrm{W}\left(\mathrm{s}_{1}(\text { pos })\right)-\mathrm{W}\left(\mathrm{s}_{2}(\text { pos })\right) \mid .
\end{array}
$$

By selecting the initial positions appropriately-essentially, picking worst-case inputs-we can derive useful lower bounds on the Total Variation distance between output distributions. For instance, taking $s_{1}$ (pos) to be the all-zeros vector and $\mathrm{s}_{2}(p o s)$ to be the all-ones vector gives:

$$
\operatorname{TV}\left(\llbracket \mathbf{h W a l k} \rrbracket\left(s_{1}(\text { pos }), \mathrm{N}, \mathrm{K}\right), \llbracket \mathbf{h W a l k} \rrbracket\left(s_{2}(\text { pos }), \mathrm{N}, \mathrm{K}\right)\right) \geqslant\left(\frac{\mathrm{N}-1}{\mathrm{~N}+1}\right)^{\mathrm{K}} .
$$

Using standard algebraic bounds, the right-hand side (and the TV distance between the two output distributions) is at least $\rho>0$ when $K<(\mathrm{N} / 2) \log \rho$.

Verifying precise lower bounds is highly challenging-for many simple examples of randomized processes, exact lower bounds are not known. Nonetheless, efforts in this direction could provide useful, complementary information when analyzing probabilistic programs.

\subsection{Extensions: Rules for Asynchronous Reasoning}

Our relational pre-expectation operator $\widetilde{r p e}(c, \varepsilon)$ can often derive useful upper bounds on the Kantorovich distance $r p e(c, \varepsilon)$, but it gives a trivial bound of infinity 
when the program c can take different branches on the two inputs. In this section, we develop techniques to give more useful bounds in the asynchronous case.

\subsubsection{Asynchronous rules for bounding the Kantorovich distance}

Our asynchronous bounds will use one-sided relational operators wpe $\langle 1\rangle(c, \varepsilon)$ (resp. wpe $\langle 2\rangle(c, \varepsilon))$ that transform relational expectations by considering that the variables labeled with $\langle 2\rangle$ (resp. labeled with $\langle 1\rangle$ ) remain unchanged and then computing the unary weakest pre-expectation wpe $(c, \mathcal{E})$ as defined in Figure 10.7 (note in particular that wpe $\langle 1\rangle(x \leftarrow e, \varepsilon)$ replaces only the variable labeled with $\langle 1\rangle)$. We use the following soundness lemma for the left version of the operator, the one for the right version being analogous.

$$
\begin{aligned}
& \text { wpe }\langle 1\rangle(\text { skip, } \varepsilon) \triangleq \varepsilon \\
& \text { wpe }\langle 1\rangle(x \leftarrow e, \varepsilon) \triangleq \varepsilon\{e\langle 1\rangle / x\langle 1\rangle\} \\
& \text { wpe }\langle 1\rangle(x \leqslant \mathrm{~d}, \mathcal{E}) \triangleq \lambda s . \mathbb{E}_{v \sim \mathrm{d}}[\mathcal{E}\{v / x\langle 1\rangle\}] \\
& \text { wpe }\langle 1\rangle\left(\mathrm{c} ; \mathrm{c}^{\prime}, \varepsilon\right) \triangleq \operatorname{wpe}\langle 1\rangle\left(\mathrm{c}, \text { wpe }\langle 1\rangle\left(\mathrm{c}^{\prime}, \varepsilon\right)\right) \\
& \text { wpe }\langle 1\rangle\left(\text { if } e \text { then } c \text { else } c^{\prime}, \varepsilon\right) \triangleq[e\langle 1\rangle] \cdot \text { wpe }\langle 1\rangle(c, \varepsilon)+[\neg e\langle 1\rangle] \cdot \text { wpe }\langle 1\rangle\left(c^{\prime}, \varepsilon\right) \\
& \text { wpe }\langle 1\rangle(\text { while } e \text { do } c, \varepsilon) \triangleq \operatorname{IfpX} \cdot[e\langle 1\rangle] \cdot \text { wpe }\langle 1\rangle(c, X)+[\neg e\langle 1\rangle] \cdot \mathcal{E}
\end{aligned}
$$

Figure 10.7: Definition of the one-sided operator wpe $\langle 1\rangle(c, \varepsilon)$

Lemma 10.26. Let $\mathrm{c}$ be a $\mathrm{PWHILE}$ program that is almost surely terminating, i.e., wpe $(\mathrm{c}, 1)=$ 1. Then, for all $s_{1}, s_{2}, \mathbb{E}_{s_{1}^{\prime} \sim \llbracket \llbracket s_{1}}\left[\mathcal{E}\left(s_{1}^{\prime}, s_{2}\right)\right] \leqslant$ wpe $\langle 1\rangle(c, \mathcal{E})\left(s_{1}, s_{2}\right)$.

Now we can present our asynchronous rules for conditionals and loops:

Theorem 10.27. Let $\mathrm{c}$ be a program that is almost surely terminating. Then:

- For conditionals with empty else branch, we can show:

$$
\begin{aligned}
r p e(\text { if } e \text { then } c, \mathcal{E}) & \leqslant[e\langle 1\rangle \wedge e\langle 2\rangle] \cdot \widetilde{r p e}(c, \mathcal{E})+[e\langle 1\rangle \wedge \neg e\langle 2\rangle] \cdot w p e\langle 1\rangle(c, \mathcal{E}) \\
& +[\neg e\langle 1\rangle \wedge e\langle 2\rangle] \cdot w p e\langle 2\rangle(c, \mathcal{E})+[\neg e\langle 1\rangle \wedge \neg e\langle 2\rangle] \cdot \mathcal{E}
\end{aligned}
$$

- Let while $\mathrm{e}$ do $\mathrm{c}$ be an almost surely terminating loop, $\rho_{\mathrm{i}}(\mathrm{s})$ be the probability that the loop does not terminate after executing the body at most $i$ times starting from state s, and:

$$
M_{\mathfrak{i}}\left(\mathcal{E}, s_{1}, s_{2}\right)=\max \left\{\mathcal{E}\left(\mathfrak{t}_{1}, \mathfrak{t}_{2}\right) \mid t_{1} \in \operatorname{supp}\left(\llbracket \mathfrak{c}_{\mathfrak{i}} \rrbracket s_{1}\right), t_{2} \in \operatorname{supp}\left(\llbracket \mathfrak{c}_{\mathfrak{i}} \rrbracket s_{2}\right)\right\}
$$


where $c_{i}$ is the first $i$ iterations of the loop. If $\rho_{i}$ and $M_{i}$ satisfy:

$$
\lim _{i \rightarrow \infty}\left(\rho_{i}\left(s_{1}\right)+\rho_{i}\left(s_{2}\right)\right) \cdot M_{i}\left(\varepsilon, s_{1}, s_{2}\right)=0
$$

for any two states $\left(\mathrm{s}_{1}, \mathrm{~s}_{2}\right)$, and if $\mathcal{J}$ is an invariant satisfying

$$
\begin{aligned}
& {[e\langle 1\rangle \wedge e\langle 2\rangle] \cdot \widetilde{r p e}(c, \mathcal{J})+[e\langle 1\rangle \wedge \neg e\langle 2\rangle] \cdot \operatorname{wpe}\langle 1\rangle(c, \mathcal{J})} \\
& +[\neg e\langle 1\rangle \wedge e\langle 2\rangle] \cdot \operatorname{wpe}\langle 2\rangle(c, \mathcal{J})+[\neg e\langle 1\rangle \wedge \neg e\langle 2\rangle] \cdot \mathcal{E} \leqslant \mathcal{J},
\end{aligned}
$$

then rpe (while e do $c, \varepsilon) \leqslant \mathcal{J}$.

Proof Sketch. The soundness of the conditional rule follows a similar argument as soundness for the definition of $\widetilde{r p e}$ for conditionals, using Lemma 10.26 for the asynchronous cases. The soundness of the loop rule is more intricate, but it follows the same strategy as in Theorem 10.14: we define a loop characteristic function based on the conditional rule (now asynchronous), show that the least fixed-point lies above rpe-this step relies on the boundedness side-condition - and finally show that the invariant rule implies that $\mathcal{J}$ is a pre-fixed-point, so it must be above the fixed point.

We detail the full proof in Appendix C.9.

\subsubsection{Example: Bounding the distance between binomial distri- butions}

Consider the following program, which simulates a binomial distribution:

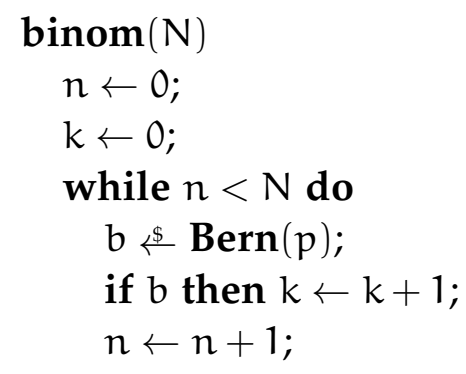

We treat $p \in[0,1]$ as a fixed constant. We will compare the distribution on the output $k$ starting from two inputs. Since the loops will run for different numbers of iterations if $\mathrm{N}\langle 1\rangle \neq \mathrm{N}\langle 2\rangle$, we will employ our asynchronous rule. We take the following invariant:

$$
\mathcal{J} \triangleq|k\langle 1\rangle-k\langle 2\rangle+p \cdot(\mathrm{N}\langle 1\rangle \ominus \mathrm{n}\langle 1\rangle)-\mathrm{p} \cdot(\mathrm{N}\langle 2\rangle \ominus \mathrm{n}\langle 2\rangle)|,
$$

We will show the following invariant bound:

$$
\begin{aligned}
& {[(n<)\langle 1\rangle \wedge(n<N)\langle 2\rangle] \cdot \widetilde{r p e}(c, J)+[(n<N)\langle 1\rangle \wedge(n \geqslant N)\langle 2\rangle] \cdot \text { wpe }\langle 1\rangle(c, J)} \\
& +[(n \geqslant N)\langle 1\rangle \wedge(n<N)\langle 2\rangle] \cdot \text { wpe }\langle 2\rangle(c, J)+[(n \geqslant N)\langle 1\rangle \wedge(n \geqslant N)\langle 2\rangle] \cdot \mathcal{E} \leqslant \mathcal{J} .
\end{aligned}
$$


In the synchronous case, we can establish the invariant by applying SAMP with the identity coupling; the inner conditional can also be analyzed synchronously. In the asynchronous case, computing the unary weakest pre-expectation establishes the invariant. Thus, the asynchronous loop rule (Theorem 10.27) gives:

$$
\operatorname{rpe}(w,|k\langle 1\rangle-k\langle 2\rangle|) \leqslant \mathcal{J}
$$

where $w$ is the loop. Applying the assignment rule, we conclude:

$$
\operatorname{rpe}(\operatorname{binom}(\mathrm{N}),|\mathrm{k}\langle 1\rangle-\mathrm{k}\langle 2\rangle|) \leqslant p \cdot|\mathrm{N}\langle 1\rangle-\mathrm{N}\langle 2\rangle| .
$$

By Theorem 10.8, this bound implies that the expected values of the output $k$ differ by at most $\mathrm{p} \cdot|\mathrm{N}\langle 1\rangle-\mathrm{N}\langle 2\rangle|$ across the two runs.

\subsection{Related work}

Proving expected sensitivity of probabilistic programs. We have shown that the quantitative logic EPRHL [42] can be embedded into the framework of this chapter (cf. Section 10.3.4), so we focus on other work. Wang et al. [158] propose an alternative method based on martingales for proving the expected sensitivity of probabilistic programs. Their technique focuses on computing the expected sensitivity when the (expected) number of iterations for a loop may be different across two related executions (i.e., loops may be asynchronous); this is similar to our asynchronous rules from Section 10.8. However, Wang et al. [158] also frame their target property in a slightly weaker way, showing that programs are Lipschitz continuous for some finite Lipschitz constant. In contrast, our method establishes bounds on this constant, which is an important aspect in many applications (e.g., it determines the rate of convergence for Markov chains). We are also able to handle the broader class of expected sensitivity properties arising from Kantorovich metrics, subsuming the notion considered by Wang et al. [158] where the output distance is the absolute difference between two expected values.

Continuity in programs and process calculi. Formal reasoning about the continuity of deterministic programs has received some attention. Chaudhuri et al. [59, 6o] were the first to give a sound, compositional framework for verifying that a program is continuous. Reed and Pierce [135] gave a type system that can verify Lipschitz continuity of functional programs (see also $[78,18,19,160]$ ). Recently, Huang et al. [96] proposed the tool PSense which can perform sensitivity analysis of probabilistic programs. Their technique relies on symbolic computation using the symbolic verifier PSI and Mathematica, and supports, e.g., the Total Variation distance and the expectation distance. PSense cannot reason, however, about general Kantorovich distances, or unbounded loops. 
Finally, in the process-algebra setting, compositional reasoning about metrics has received some attention. Gebler et al. [81] used uniform continuity to reason about the distance between recursive processes in a compositional way, while Gebler and Tini [8o] recently defined specification formats that can check uniform continuity syntactically. A more general framework for reasoning about metrics has been given by Bacci et al. [20], who presented an algebraic axiomatization of Markov processes in quantitative equational logic. Their framework supports reasoning about various metrics, including the Kantorovich metric.

\subsection{Conclusion}

We defined a pre-expectation calculus to compute upper bounds for Kantorovich metrics, and applied it to prove convergence of reinforcement learning and card shuffling algorithms, algorithmic stability of SGD, and uniformity of limit distributions. Our calculus provides theoretical foundations for reasoning about quantitative relational properties of probabilistic programs.

There are several natural directions for future work. One possible extension is to lift the requirement that programs terminate with equal probability on pairs of executions, possibly by leveraging alternative notions of the Kantorovich metric that accommodate distributions of different weight [126]. Other directions include developing a relational version of quantitative separation logic [43], and use it for proving relational properties of probabilistic heap-manipulating programs.

We also explored methods for proving lower bounds of convergence speed. In general, we are not aware of many works that prove lower bounds using program logics, with some notable exceptions [9o]. Developing more tools and techniques for reasoning about these fascinating properties is an interesting avenue for future work. 


\section{Part III}

Conclusion 


\section{CONCLUSIONS \& FUTURE WORK}

\subsection{Conclusions}

This dissertation has extended the state-of-the-art of relational verification, particularly in the higher-order setting, in several directions:

- We have developed RHOL, a relational logic for pure higher-order programs that supports both two-sided and one-sided reasoning, and that is no longer constrained by requiring that the programs we reason about have equal types or structural similarity of the programs. We have shown that RHOL does not lose any expressivity with respect to HOL by proving an equivalence theorem.

- We have demonstrated the versatility of the framework by extending it to the continuous probabilistic setting and showing how to prove lifting-based properties. We have instantiated these techniques to the probabilistic setting, and use them to develop a union-bound-based unary logic and a couplingbased relational logic.

- We have extended these logics to a stateful setting, and developed specialized rules to reason about adversarial properties, which have a natural specification in higher-order settings.

- We have also introduced a relational, lifting-based logic to reason about probabilistic processes in a step-indexed model, the topos of trees. We have use this logic to verify properties of Markov chains.

- We have explored reasoning about probabilistic programs by extending the base logic and using axioms, as opposed to liftings, and developed unary and relational logics in this setting. 
- Finally, we have provided a new approach to reason about expected sensitivity, and in doing so, we have shown that predicate transformers can also be used in the relational setting.

\subsection{Future work}

We now present some interesting directions for following the work of this dissertation.

\section{Quantitative logics}

The logics presented in the first part of the thesis were standard logics with Boolean assertions. However, the expectations from Chapter 10 are quantitative: they are maps from a set of states to the positive reals. These assertions have a categorical interpretation. We first choose a poset $(\Omega, \subseteq)$ of truth values, possibly with more structure (e.g., a complete lattice). Then, one can define a category where objects are quantitative predicates, i.e., maps with codomain $\Omega$, and morphisms are non-expansive functions between the base sets. Under some assumptions, these categories form LR fibrations, so they can be used to interpret unary and relational logics. One such instance is the category $\operatorname{Pred}_{[0, \infty]}(\mathbf{Q B S})$, that has as objects pairs $(X, F:|X| \rightarrow[0, \infty])$ of a QBS and a $[0, \infty]$-valued predicate over its carrier set, and as morphisms non-expansive Set-morphisms between the carrier sets. The expected value operator induces a lifting of the $\mathcal{P}$ monad from QBS to $\operatorname{Pred}_{[0, \infty]}(\mathbf{Q B S})$, mapping a predicate $\phi: X \rightarrow[0, \infty]$ to the function $\tilde{\phi}: \mathcal{P}(X) \rightarrow[0, \infty]$ that receives a distribution over $X$ and returns the expected value of $\phi$. This can be then used to interpret a higher-order version of expectation-based logics. Since our rules for UNIT and MLET are sound regardless of the choice of lifting, one only needs to design sound proof rules for the sampling operators.

The next step is finding a lifting that corresponds to the Kantorovich distance as presented in Chapter 10. This is more challenging since the since the techniques that can be used to find liftings, such as the TT-lifting [163] are not as well-behaved in the relational case. This kind of logic could be used to study sensitivity of higher-order programs in the presence of adversaries.

\section{Logics for generic effects}

This thesis focuses on properties of probabilistic programs, but the techniques we have presented can be extended to other effects, as we have sketched in Section 4.9. One of the strong points of our presentation is that the base logic does not need to change and the syntax of assertions does not change, and the effect is only taken into account at the semantical level, when interpreting the specifications under a lifting for the effect. For unary logics, the semantical model just requires a LR fibration 
$\mathbf{P} \rightarrow \mathbf{C}$, a monad $\mathcal{M}$ over $\mathbf{C}$ and a lifting of this monad to $\mathbf{P}$. For instance, we can model non-determinism or partiality using an appropriate monad. We can also apply the state monad transformer to these monads, as in Section 6.5, and reason about the resulting computation following similar ideas. For relational logics we can pull-back the fibration $\mathbf{P} \rightarrow \mathbf{C}$ to a fibration $\mathbf{R} \rightarrow \mathbf{C} \times \mathbf{C}$ along the product functor $\mathbf{C} \times \mathbf{C} \rightarrow \mathbf{C}$, as in our construction of the $\mathbf{B R e l}(\mathbf{Q B S})$ monad, and use a (relational) lifting of the $\mathcal{M} \times \mathcal{M}$ monad.

Investigating which effects can be given this treatment is an interesting direction for future research.

\section{Unbounded iteration in probabilistic languages}

Unbounded iteration is in general a problem for higher-order program logics, it has been pointed out that naively allowing to type programs that do not terminate can result in an unsound logic [155].

Additionally, in the case of higher-order probabilistic programs, there are also other semantical issues associated with unbounded iteration. Traditionally, un-

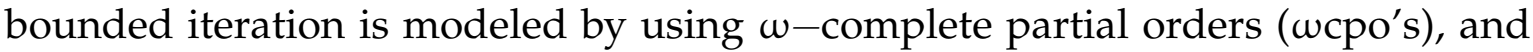
there is extensive work about extending these models to the probabilistic setting (e.g. [101]) but there are some known issues about mixing $\omega$-cpo's and probabilities [102]. Recent work on quasi-Borel $\omega$-cpo's [154] extends these ideas and provides a model for a higher-order probabilistic language with recursive terms and types. Extending our logics to this setting is a promising direction for future work.

Moreover, unbounded iteration also requires to carefully track dependencies of the gradings. Since program composition adds the gradings, a naive extension of the system with general recursion would grade the refinements of recursive functions with infinity, which provides logical information. This dependency tracking would be useful even without recursion, since it would allow to prove more precise gradings. It would be interesting to explore if we can adapt the dependency tracking of systems such as [68] to our probabilistic setting.

\section{Automation and Implementation}

This work focuses mainly on the syntax and semantics of several program logics, but we do not provide an implementation of the systems we have presented. Full automation of the proofs seems completely out of scope, however an interactive proof system in the style of Coq [151] or Agda [124], that allows the user to apply one or two-sided rules to prove a goal, is within closer reach and an interesting line for continuing our work.

Proof assistants for other relational logics have been developed, and enjoy some degree of automation. For the particular case of our lifting-based logics, the assertion language itself does not change, and the inference rules are very similar to Hoare 
logic. This similarity can potentially be exploited in the implementation of the logics by reusing systems such as EasyCrypt [28]. 


\section{CHAPTER}

TWELVE

\section{BIBLIOGRAPHY}

[1] Martín Abadi, Luca Cardelli, and Pierre-Louis Curien. Formal parametric polymorphism. In POPL 1993 Charleston, South Carolina, USA, January 1993, 1993. doi: 10.1145/158511.158622. URL http://doi.acm.org/10.1145/ 158511.158622 .

[2] Martín Abadi, Anindya Banerjee, Nevin Heintze, and Jon G. Riecke. A core calculus of dependency. In POPL 1999, San Antonio, TX, USA, January 20-22, 1999, 1999. doi: 10.1145/292540.292555. URL http://doi .acm.org/10.1145/ 292540.292555.

[3] Peter Aczel and Nicola Gambino. Collection principles in dependent type theory. In Paul Callaghan, Zhaohui Luo, James McKinna, and Robert Pollack, editors, TYPES 2000, Durham, UK, December 8-12, 2000, Selected Papers, volume 2277 of LNCS. Springer, 2000. doi: 10.1007/3-540-45842-5_1. URL http: //dx.doi.org/10.1007/3-540-45842-5_1.

[4] Peter Aczel and Nicola Gambino. The generalised type-theoretic interpretation of constructive set theory. J. Symb. Log., 71(1):67-103, 2006. doi: 10.2178/jsl/ 1140641163. URL http://dx.doi.org/10.2178/jsl/1140641163.

[5] Robin Adams and Zhaohui Luo. Classical predicative logic-enriched type theories. Ann. Pure Appl. Logic, 161(11):1315-1345, 2010. doi: 10.1016/j.apal. 2010.04.005. URL http://dx.doi.org/10.1016/j.apal.2010.04.005.

[6] Alejandro Aguirre and Shin ya Katsumata. Weakest preconditions in fibrations. Electronic Notes in Theoretical Computer Science, 352:5 - 27, 2020. ISSN 1571-0661. doi: https://doi.org/10.1016/j.entcs.2020.09.002. URL http: //www.sciencedirect.com/science/article/pii/S1571066120300487. The 36th Mathematical Foundations of Programming Semantics Conference, 2020. 
[7] David Aldous. Random walks on finite groups and rapidly mixing Markov chains. In Séminaire de Probabilités XVII 1981/82, volume 986 of Lecture Notes in Mathematics, pages 243-297. Springer-Verlag, 1983. URL https://eudml.org/ doc/113445.

[8] Philip Amortila, Doina Precup, Prakash Panangaden, and Marc G. Bellemare. A distributional analysis of sampling-based reinforcement learning algorithms. In Silvia Chiappa and Roberto Calandra, editors, The 23 rd International Conference on Artificial Intelligence and Statistics, AISTATS 2020, 26-28 August 2020, Online [Palermo, Sicily, Italy], volume 108 of Proceedings of Machine Learning Research, pages 4357-4366. PMLR, 2020. URL http://proceedings.mlr.press/v108/amortila20a.html.

[9] Torben Amtoft and Anindya Banerjee. A theory of slicing for probabilistic control flow graphs. In Bart Jacobs and Christof Löding, editors, Foundations of Software Science and Computation Structures, pages 180-196, Berlin, Heidelberg, 2016. Springer Berlin Heidelberg. ISBN 978-3-662-49630-5.

[10] Kazuyuki Asada, Ryosuke Sato, and Naoki Kobayashi. Verifying relational properties of functional programs by first-order refinement. Science of Computer Programming, 2016.

[11] Robert B. Ash and Catherine A. Doleans-Dade. Probability and Measure Theory. Academic Press, 2000.

[12] Robert Atkey. Parameterised notions of computation. J. Funct. Program., 19 (3-4):335-376, 2009. doi: 10.1017/So95679680900728X. URL https://doi.org/ 10.1017/S095679680900728X.

[13] Philippe Audebaud and Christine Paulin-Mohring. Proofs of randomized algorithms in Coq. Sci. Comput. Program., 74(8):568-589, 2009. doi: https: //doi.org/10.1016/j.scico.2007.09.002.

[14] Peter Auer, Nicolo Cesa-Bianchi, Yoav Freund, and Robert E Schapire. The nonstochastic multiarmed bandit problem. SIAM journal on computing, 32(1): 48-77, 2002.

[15] Robert J. Aumann. Borel structures for function spaces. Illinois J. Math., 5(4): 614-630, 12 1961. URL http://projecteuclid.org/euclid.ijm/1255631584.

[16] Jeremy Avigad, Johannes Hölzl, and Luke Serafin. A formally verified proof of the Central Limit Theorem. CoRR, abs/1405.7012, 2014. URL http://arxiv . org/abs/1405.7012. 
[17] Yossi Azar, Andrei Z Broder, Anna R Karlin, Nathan Linial, and Steven Phillips. Biased random walks. In Proceedings of the twenty-fourth annual ACM symposium on Theory of computing, pages 1-9, 1992.

[18] Arthur Azevedo de Amorim, Marco Gaboardi, Emilio Jesús Gallego Arias, and Justin Hsu. Really natural linear indexed type-checking. In Symposium on Implementation and Application of Functional Programming Languages (IFL), Boston, Massachusetts, pages 5:1-5:12. ACM Press, 2014. URL http://arxiv . org/abs/1503.04522.

[19] Arthur Azevedo de Amorim, Marco Gaboardi, Justin Hsu, Shin-ya Katsumata, and Ikram Cherigui. A semantic account of metric preservation. In Giuseppe Castagna and Andrew D. Gordon, editors, Proceedings of the 44th ACM SIGPLAN Symposium on Principles of Programming Languages, POPL 2017, Paris, France, January 18-20, 2017, pages 545-556. ACM, 2017.

[20] Giorgio Bacci, Radu Mardare, Prakash Panangaden, and Gordon D. Plotkin. An algebraic theory of markov processes. In Anuj Dawar and Erich Grädel, editors, Proceedings of the 33rd Annual ACM/IEEE Symposium on Logic in Computer Science, LICS 2018, Oxford, UK, July 09-12, 2018, pages 679-688. ACM, 2018. doi: 10.1145/3209108.3209177. URL https://doi .org/10.1145/3209108.3209177.

[21] Gilles Barthe, Pedro R. D'Argenio, and Tamara Rezk. Secure information flow by self-composition. In 17th IEEE Computer Security Foundations Workshop, (CSFW-17 2004), 28-30 June 2004, Pacific Grove, CA, USA, pages 100-114, 2004. doi: 10.1109/CSFW.2004.17. URL http://doi . ieeecomputersociety.org/10. 1109/CSFW. 2004.17.

[22] Gilles Barthe, Maria João Frade, Eduardo Giménez, Luís Pinto, and Tarmo Uustalu. Type-based termination of recursive definitions. Math. Struct. Comput. Sci., 14(1):97-141, 2004. doi: 10.1017/So960129503004122. URL https://doi. org/10.1017/S0960129503004122.

[23] Gilles Barthe, Benjamin Grégoire, and Santiago Zanella Béguelin. Formal certification of code-based cryptographic proofs. In Proceedings of the 36 th ACM SIGPLAN-SIGACT Symposium on Principles of Programming Languages, POPL 2009, Savannah, GA, USA, January 21-23, 2009, pages 90-101, 2009. doi: 10.1145/ 1480881.1480894. URL http://doi.acm.org/10.1145/1480881.1480894.

[24] Gilles Barthe, Benjamin Grégoire, and Santiago Zanella Béguelin. Formal certification of code-based cryptographic proofs. In POPL 2009, Savannah, GA, USA, January 21-23, 2009, 2009. doi: 10.1145/1480881.1480894. URL http://doi.acm.org/10.1145/1480881.1480894. 
[25] Gilles Barthe, Juan Manuel Crespo, and César Kunz. Relational verification using product programs. In FM 2011: Formal Methods - 17th International Symposium on Formal Methods, Limerick, Ireland, June 20-24, 2011. Proceedings, pages 200-214, 2011. doi: 10.1007/978-3-642-21437-0_17. URL http://dx. doi . org/10.1007/978-3-642-21437-0_17.

[26] Gilles Barthe, Boris Köpf, Federico Olmedo, and Santiago Zanella Béguelin. Probabilistic relational reasoning for differential privacy. In POPL 2012, Philadelphia, Pennsylvania, USA, January 22-28, 2012, 2012. doi: 10.1145/2103656. 210367o. URL http://doi.acm.org/10.1145/2103656.2103670.

[27] Gilles Barthe, Boris Köpf, Federico Olmedo, and Santiago Zanella Béguelin. Probabilistic relational reasoning for differential privacy. In John Field and Michael Hicks, editors, Proceedings of the 39th ACM SIGPLAN-SIGACT Symposium on Principles of Programming Languages, POPL 2012, Philadelphia, Pennsylvania, USA, January 22-28, 2012, pages 97-110. ACM, 2012.

[28] Gilles Barthe, François Dupressoir, Benjamin Grégoire, César Kunz, Benedikt Schmidt, and Pierre-Yves Strub. Easycrypt: A tutorial. In Foundations of Security Analysis and Design VII (FOSAD), volume 8604 of Lecture Notes in Computer Science, pages 146-166. Springer-Verlag, 2013. Tutorial Lectures.

[29] Gilles Barthe, Cédric Fournet, Benjamin Grégoire, Pierre-Yves Strub, Nikhil Swamy, and Santiago Zanella Béguelin. Probabilistic relational verification for cryptographic implementations. In Suresh Jagannathan and Peter Sewell, editors, POPL 2014, 2014.

[30] Gilles Barthe, Thomas Espitau, Benjamin Grégoire, Justin Hsu, Léo Stefanesco, and Pierre-Yves Strub. Relational reasoning via probabilistic coupling. In International Conference on Logic for Programming, Artificial Intelligence and Reasoning (LPAR), Suva, Fiji, volume 9450 of Lecture Notes in Computer Science, pages 387-401. Springer-Verlag, 2015. URL http://arxiv.org/abs/1509.03476.

[31] Gilles Barthe, Thomas Espitau, Benjamin Grégoire, Justin Hsu, Léo Stefanesco, and Pierre-Yves Strub. Relational reasoning via probabilistic coupling. In LPAR-20 2015, Suva, Fiji, November 24-28, 2015, Proceedings, 2015.

[32] Gilles Barthe, Marco Gaboardi, Emilio Jesús Gallego Arias, Justin Hsu, Aaron Roth, and Pierre-Yves Strub. Higher-order approximate relational refinement types for mechanism design and differential privacy. In ACM SIGPLANSIGACT Symposium on Principles of Programming Languages (POPL), Mumbai, India, pages 55-68, 2015.

[33] Gilles Barthe, Marco Gaboardi, Emilio Jesús Gallego Arias, Justin Hsu, Aaron Roth, and Pierre-Yves Strub. Higher-order approximate relational refinement 
types for mechanism design and differential privacy. In POPL 2015, Mumbai, India, January 15-17, 2015, 2015.

[34] Gilles Barthe, Gian Pietro Farina, Marco Gaboardi, Emilio Jesús Gallego Arias, Andy Gordon, Justin Hsu, and Pierre-Yves Strub. Differentially private bayesian programming. In CCS 2016, Vienna, Austria, October 24-28, 2016. ACM, 2016. doi: 10.1145/2976749.2978371. URL http://doi.acm.org/10. $1145 / 2976749.2978371$.

[35] Gilles Barthe, Gian Pietro Farina, Marco Gaboardi, Emilio Jesús Gallego Arias, Andy Gordon, Justin Hsu, and Pierre-Yves Strub. Differentially private bayesian programming. In ACM SIGSAC Conference on Computer and Communications Security, Vienna, Austria, October 24-28, 2016, pages 68-79, 2016. doi: 10.1145/2976749.2978371. URL http://doi . acm.org/10.1145/2976749. 2978371.

[36] Gilles Barthe, Noémie Fong, Marco Gaboardi, Benjamin Grégoire, Justin Hsu, and Pierre-Yves Strub. Advanced probabilistic couplings for differential privacy. In ACM SIGSAC Conference on Computer and Communications Security (CCS), Vienna, Austria, pages 55-67, 2016. URL https://arxiv.org/abs/1606. 07143.

[37] Gilles Barthe, Noémie Fong, Marco Gaboardi, Benjamin Grégoire, Justin Hsu, and Pierre-Yves Strub. Advanced probabilistic couplings for differential privacy. In Edgar R. Weippl, Stefan Katzenbeisser, Christopher Kruegel, Andrew C. Myers, and Shai Halevi, editors, Proceedings of the 2016 ACM SIGSAC Conference on Computer and Communications Security, Vienna, Austria, October 24-28, 2016, pages 55-67. ACM, 2016.

[38] Gilles Barthe, Marco Gaboardi, Benjamin Grégoire, Justin Hsu, and Pierre-Yves Strub. A program logic for union bounds. In Ioannis Chatzigiannakis, Michael Mitzenmacher, Yuval Rabani, and Davide Sangiorgi, editors, 43rd International Colloquium on Automata, Languages, and Programming, ICALP 2016, July 11-15, 2016, Rome, Italy, volume 55 of LIPIcs, pages 107:1-107:15. Schloss Dagstuhl Leibniz-Zentrum fuer Informatik, 2016. doi: 10.4230/LIPIcs.ICALP.2016.107. URL https://doi .org/10.4230/LIPIcs. ICALP. 2016.107.

[39] Gilles Barthe, Thomas Espitau, Benjamin Grégoire, Justin Hsu, and PierreYves Strub. Proving uniformity and independence by self-composition and coupling. CoRR, abs/1701.06477, 2017. URL http://arxiv.org/abs/1701. 06477.

[40] Gilles Barthe, Benjamin Grégoire, Justin Hsu, and Pierre-Yves Strub. Coupling proofs are probabilistic product programs. In POPL 2017, Paris, France, January 18-20, 2017, 2017. URL http://dl.acm.org/citation.cfm?id=3009896. 
[41] Gilles Barthe, Thomas Espitau, Marco Gaboardi, Benjamin Grégoire, Justin $\mathrm{Hsu}$, and Pierre-Yves Strub. An assertion-based program logic for probabilistic programs. In Amal Ahmed, editor, Programming Languages and Systems, pages 117-144, Cham, 2018. Springer International Publishing. ISBN 978-3-319-898841 .

[42] Gilles Barthe, Thomas Espitau, Benjamin Grégoire, Justin Hsu, and PierreYves Strub. Proving expected sensitivity of probabilistic programs. Proc. ACM Program. Lang., 2(POPL):57:1-57:29, 2018. doi: 10.1145/3158145. URL https://doi.org/10.1145/3158145.

[43] Kevin Batz, Benjamin Lucien Kaminski, Joost-Pieter Katoen, Christoph Matheja, and Thomas Noll. Quantitative separation logic: A logic for reasoning about probabilistic pointer programs. PACMPL, 3(POPL):34:1-34:29, 2019.

[44] João Filipe Belo. Dependently sorted logic. In Marino Miculan, Ivan Scagnetto, and Furio Honsell, editors, Types for Proofs and Programs, International Conference, TYPES 2007, Cividale del Friuli, Italy, May 2-5, 2007, Revised Selected Papers, volume 4941 of Lecture Notes in Computer Science, pages 33-50. Springer, 2007. doi: 10.1007/978-3-540-68103-8_3. URL http://dx.doi.org/10.1007/978-3-540-68103-8_3.

[45] Nick Benton. Simple relational correctness proofs for static analyses and program transformations. In Neil D. Jones and Xavier Leroy, editors, POPL 2004, 2004 .

[46] Nick Benton, Martin Hofmann, and Vivek Nigam. Abstract effects and proofrelevant logical relations. In Suresh Jagannathan and Peter Sewell, editors, The 41st Annual ACM SIGPLAN-SIGACT Symposium on Principles of Programming Languages, POPL '14, San Diego, CA, USA, January 20-21, 2014, pages 619-632. ACM, 2014. doi: 10.1145/2535838.2535869. URL https://doi.org/10.1145/ 2535838.2535869 .

[47] Lennart Beringer and Martin Hofmann. Secure information flow and program logics. In 20th IEEE Computer Security Foundations Symposium, CSF 2007, 6-8 July 2007, Venice, Italy, pages 233-248. IEEE Computer Society, 2007. doi: 10.1109/CSF.2007.30. URL http://dx.doi.org/10.1109/CSF .2007.30.

[48] P. Billingsley. Probability and Measure. Wiley Series in Probability and Statistics. Wiley, 2012. ISBN 9781118341919.

[49] Lars Birkedal, Rasmus Ejlers Møgelberg, Jan Schwinghammer, and Kristian Støvring. First steps in synthetic guarded domain theory: step-indexing in the topos of trees. Logical Methods in Computer Science, 8(4), 2012. 
[50] Ales Bizjak and Lars Birkedal. Step-indexed logical relations for probability. In Andrew M. Pitts, editor, FoSSaCS 2015, London, UK, April 11-18, 2015. Proceedings, volume 9034 of Lecture Notes in Computer Science. Springer, 2015. ISBN 978-3-662-46677-3.

[51] Aleš Bizjak, Hans Bugge Grathwohl, Ranald Clouston, Rasmus Ejlers Møgelberg, and Lars Birkedal. Guarded dependent type theory with coinductive types. In FOSSACS 2016, Eindhoven, The Netherlands, April 2-8, 2016, Proceedings, 2016.

[52] Lionel Blatter, Nikolai Kosmatov, Pascale Le Gall, and Virgile Prevosto. Deductive verification with relational properties. In Proc. of the 23 th International Conference on Tools and Algorithms for the Construction and Analysis of Systems (TACAS 2017), Uppsala, Sweden, 2017. To Appear.

[53] Dan Bogdanov, Margus Niitsoo, Tomas Toft, and Jan Willemson. Highperformance secure multi-party computation for data mining applications. Int. J. Inf. Sec., 11(6):403-418, 2012. doi: 10.1007/s10207-012-0177-2. URL https://doi.org/10.1007/s10207-012-0177-2.

[54] Johannes Borgström, Andrew D. Gordon, Michael Greenberg, James Margetson, and Jurgen Van Gael. Measure transformer semantics for bayesian machine learning. In Programming Languages and Systems - 20th European Symposium on Programming, ESOP 2011, Held as Part of the Joint European Conferences on Theory and Practice of Software, ETAPS 2011, Saarbrücken, Germany, March 26April 3, 2011. Proceedings, pages 77-96, 2011. doi: 10.1007/978-3-642-19718-5 -5. URL https://doi.org/10.1007/978-3-642-19718-5_5.

[55] Johannes Borgström, Ugo Dal Lago, Andrew D. Gordon, and Marcin Szymczak. A lambda-calculus foundation for universal probabilistic programming. In Jacques Garrigue, Gabriele Keller, and Eijiro Sumii, editors, ICFP 2016, Nara, Japan, September 18-22, 2016. ACM, 2016. ISBN 978-1-4503-4219-3.

[56] Olivier Bousquet and André Elisseeff. Stability and generalization. Journal of Machine Learning Research, 2:499-526, 2002. URL http://www.jmlr.org/ papers/v2/bousquet02a.html.

[57] Ran Canetti. Universally composable security: A new paradigm for cryptographic protocols. In Foundations of Computer Science, 2001. Proceedings. IEEE, 2001.

[58] R. Chadha, L. Cruz-Filipe, P. Mateus, and A. Sernadas. Reasoning about probabilistic sequential programs. Theoretical Computer Science, 379(1-2):142$165,2007$. 
[59] Swarat Chaudhuri, Sumit Gulwani, and Roberto Lublinerman. Continuity analysis of programs. In ACM SIGPLAN-SIGACT Symposium on Principles of Programming Languages (POPL), Madrid, Spain, pages 57-70, 2010.

[6o] Swarat Chaudhuri, Sumit Gulwani, and Roberto Lublinerman. Continuity and robustness of programs. Commun. ACM, 55(8):107-115, 2012. doi: 10. 1145/2240236.2240262. URL https://doi .org/10.1145/2240236.2240262.

[61] Ezgi Çiçek, Gilles Barthe, Marco Gaboardi, Deepak Garg, and Jan Hoffmann. Relational cost analysis. In Giuseppe Castagna and Andrew D. Gordon, editors, POPL 2017, Paris, France, January 18-20, 2017. ACM, 2017. URL http://dl . acm.org/citation. cfm?id=3009858.

[62] Michael R. Clarkson and Fred B. Schneider. Hyperproperties. In Proceedings of $\mathrm{CSF}^{\prime} 08$, pages $51-65,2008$.

[63] Ranald Clouston, Aleš Bizjak, Hans Bugge Grathwohl, and Lars Birkedal. The guarded lambda-calculus: Programming and reasoning with guarded recursion for coinductive types. Logical Methods in Computer Science, 12(3), 2016. doi: 10.2168/LMCS-12(3:7)2016. URL https://doi.org/10.2168/LMCS-12(3: 7) 2016 .

[64] Aaron R. Coble. Anonymity, information, and machine-assisted proof. Technical Report UCAM-CL-TR-785, University of Cambridge, Computer Laboratory, 2010.

[65] Ronald Cramer, Ivan Bjerre Damgard, and Jesper Buus Nielsen. Secure Multiparty Computation and Secret Sharing. Cambridge University Press, New York, NY, USA, 1st edition, 2015. ISBN 1107043050, 9781107043053.

[66] Raphä̈lle Crubillé and Ugo Dal Lago. On probabilistic applicative bisimulation and call-by-value $\lambda$-calculi. In Zhong Shao, editor, ESOP 2014, Grenoble, France, April 5-13, 2014, Proceedings. Springer, 2014. ISBN 978-3-642-54832-1.

[67] Ryan Culpepper and Andrew Cobb. Contextual equivalence for probabilistic programs with continuous random variables and scoring. In Hongseok Yang, editor, ESOP 2017, Uppsala, Sweden, April 22-29, 2017, Proceedings, 2017. ISBN 978-3-662-54433-4.

[68] Ugo Dal Lago and Marco Gaboardi. Linear Dependent Types and Relative Completeness. Logical Methods in Computer Science, 8(4), October 2012. ISSN 18605974. doi: 10.2168/LMCS-8(4:11)2012. URL https://lmcs . episciences. org/974.

[69] J. den Hartog. Probabilistic extensions of semantical models. PhD thesis, Vrije Universiteit Amsterdam, 2002. 
[70] Dominique Devriese, Lars Birkedal, and Frank Piessens. Reasoning about object capabilities with logical relations and effect parametricity. In IEEE European Symposium on Security and Privacy (EuroSEP), pages 147-162, 2016.

[71] Edsger W. Dijkstra. Guarded commands, nondeterminacy and formal derivation of programs. Communications of the ACM, 18(8):453-457, August 1975. ISSN ooo10782. doi: 10.1145/360933.360975. URL http://portal. acm.org/ citation. cfm?doid=360933.360975.

[72] Derek Dreyer, Georg Neis, Andreas Rossberg, and Lars Birkedal. A relational modal logic for higher-order stateful adts. In POPL 2010, Madrid, Spain, January 17-23, 2010, 2010. doi: 10.1145/1706299.1706323. URL http://doi . acm.org/ $10.1145 / 1706299.1706323$.

[73] Derek Dreyer, Amal Ahmed, and Lars Birkedal. Logical step-indexed logical relations. Logical Methods in Computer Science, 7(2), 2011. doi: 10.2168/LMCS-7(2: 16)2011. URL http://dx.doi.org/10.2168/LMCS-7(2:16) 2011.

[74] Joshua Dunfield and Frank Pfenning. Tridirectional typechecking. In Neil D. Jones and Xavier Leroy, editors, POPL 2004, Venice, Italy, January 14-16, 2004. ACM, 2004. doi: 10.1145/964001.964025. URL http://doi .acm.org/10.1145/ 964001.964025 .

[75] Cynthia Dwork and Aaron Roth. The algorithmic foundations of differential privacy. Foundations and Trends in Theoretical Computer Science, 9(3-4):211-407, 2014. URL http://dx.doi.org/10.1561/0400000042.

[76] Timothy S. Freeman and Frank Pfenning. Refinement types for ML. In David S. Wise, editor, Proceedings of the ACM SIGPLAN'91 Conference on Programming Language Design and Implementation (PLDI), Toronto, Ontario, Canada, June 2628, 1991, pages 268-277. ACM, 1991. doi: 10.1145/113445.113468. URL http://doi.acm.org/10.1145/113445.113468.

[77] Soichiro Fujii, Shin-ya Katsumata, and Paul-André Melliès. Towards a formal theory of graded monads. In Bart Jacobs and Christof Löding, editors, Foundations of Software Science and Computation Structures - 19th International Conference, FOSSACS 2016, Held as Part of the European Joint Conferences on Theory and Practice of Software, ETAPS 2016, Eindhoven, The Netherlands, April 2-8, 2016, Proceedings, volume 9634 of Lecture Notes in Computer Science, pages 513-530. Springer, 2016. doi: 10.1007/978-3-662-49630-5\_30. URL https://doi.org/10.1007/978-3-662-49630-5_30.

[78] Marco Gaboardi, Andreas Haeberlen, Justin Hsu, Arjun Narayan, and Benjamin C. Pierce. Linear dependent types for differential privacy. In Roberto Giacobazzi and Radhia Cousot, editors, POPL 2013, Rome, Italy 
- January 23 - 25, 2013. ACM, 2013. doi: 10.1145/2429069.2429113. URL http://doi.acm.org/10.1145/2429069.2429113.

[79] Deepak Garg, Jason Franklin, Dilsun Kirli Kaynar, and Anupam Datta. Compositional system security with interface-confined adversaries. Electr. Notes Theor. Comput. Sci., 265:49-71, 2010.

[8o] Daniel Gebler and Simone Tini. SOS specifications for uniformly continuous operators. J. Comput. Syst. Sci., 92:113-151, 2018. doi: 10.1016/j.jcss.2017.09.011. URL https://doi.org/10.1016/j.jcss.2017.09.011.

[81] Daniel Gebler, Kim G. Larsen, and Simone Tini. Compositional bisimulation metric reasoning with probabilistic process calculi. Logical Methods in Computer Science, 12(4), 2016. doi: 10.2168/LMCS-12(4:12)2016. URL https://doi.org/ 10.2168/LMCS-12(4:12) 2016.

[82] Neil Ghani, Fredrik Nordvall Forsberg, and Alex Simpson. Comprehensive parametric polymorphism: Categorical models and type theory. In Bart Jacobs and Christof Löding, editors, FOSSACS 2016, Eindhoven, The Netherlands, April 2-8, 2016, Proceedings, volume 9634 of Lecture Notes in Computer Science. Springer, 2016. doi: 10.1007/978-3-662-49630-5_1. URL http://dx.doi.org/ $10.1007 / 978-3-662-49630-5 \_1$.

[83] Neil Ghani, Fredrik Nordvall Forsberg, and Alex Simpson. Comprehensive parametric polymorphism: Categorical models and type theory. In FOSSACS 2016, Eindhoven, The Netherlands, April 2-8, 2016, Proceedings, 2016. doi: 10.1007/978-3-662-49630-5_1. URL http://dx.doi.org/10.1007/ 978-3-662-49630-5_1.

[84] Michèle Giry. A categorical approach to probability theory. In B. Banaschewski, editor, Categorical Aspects of Topology and Analysis, volume 915 of Lecture Notes in Mathematics, pages 68-85. Springer Berlin Heidelberg, 1982. ISBN 978-3540-11211-2. doi: 10.1007/BFboo92872. URL http://dx.doi.org/10.1007/ BFb0092872.

[85] Joseph A. Goguen and José Meseguer. Security policies and security models. In IEEE Symposium on Security and Privacy, pages 11-20, 1982.

[86] Noah D. Goodman, Vikash K. Mansinghka, Daniel M. Roy, Keith Bonawitz, and Joshua B. Tenenbaum. Church: a language for generative models. In UAI 2008, Proceedings of the 24th Conference in Uncertainty in Artificial Intelligence, Helsinki, Finland, July 9-12, 2008, pages 220-229, 2008. URL https://dslpitt.org/uai/displayArticleDetails.jsp?mmnu=1\& smnu=2\&article_id=1346\&proceeding_id=24. 
[87] Friedrich Gretz, Joost-Pieter Katoen, and Annabelle McIver. Operational versus weakest pre-expectation semantics for the probabilistic guarded command language. Perform. Evaluation, 73:110-132, 2014. doi: 10.1016/j.peva.2013.11. o04. URL https://doi.org/10.1016/j.peva.2013.11.004.

[88] Niklas Grimm, Kenji Maillard, Cédric Fournet, Catalin Hritcu, Matteo Maffei, Jonathan Protzenko, Tahina Ramananandro, Aseem Rastogi, Nikhil Swamy, and Santiago Zanella Béguelin. A monadic framework for relational verification: applied to information security, program equivalence, and optimizations. In June Andronick and Amy P. Felty, editors, Proceedings of the $7^{\text {th }} A C M$ SIGPLAN International Conference on Certified Programs and Proofs, CPP 2018, Los Angeles, CA, USA, January 8-9, 2018, pages 130-145. ACM, 2018. doi: 10.1145/316709o. URL https://doi .org/10.1145/3167090.

[89] Moritz Hardt, Ben Recht, and Yoram Singer. Train faster, generalize better: Stability of stochastic gradient descent. In International Conference on Machine Learning (ICML), New York, NY, volume 48 of Journal of Machine Learning Research, pages 1225-1234. JMLR.org, 2016. URL http://jmlr.org/ proceedings/papers/v48/hardt16.html.

[9o] Marcel Hark, Benjamin Lucien Kaminski, Jürgen Giesl, and Joost-Pieter Katoen. Aiming low is harder: induction for lower bounds in probabilistic program verification. Proc. ACM Program. Lang., 4(POPL):37:1-37:28, 2020.

[91] John Hatcliff and Olivier Danvy. A computational formalization for partial evaluation. Mathematical Structures in Computer Science, 7:507-541, 1997.

[92] Nevin Heintze and Jon G. Riecke. The slam calculus: Programming with secrecy and integrity. In POPL 1998, San Diego, CA, USA, January 19-21, 1998, 1998. doi: 10.1145/268946.268976. URL http://doi .acm.org/10.1145/ 268946.268976.

[93] Chris Heunen, Ohad Kammar, Sam Staton, and Hongseok Yang. A convenient category for higher-order probability theory. In 32nd Annual ACM/IEEE Symposium on Logic in Computer Science, LICS 2017, Reykjavik, Iceland, June 20-23, 2017, pages 1-12. IEEE Computer Society, 2017. doi: 10.1109/LICS.2017. 8005137. URL https://doi.org/10.1109/LICS . 2017.8005137.

[94] Johannes Hölzl. Markov chains and Markov decision processes in Isabelle/HOL. 2016. URL http://home.in.tum.de/ noelzl/mdptheory/ hoelzl2016markov-draft.pdf.

[95] Johannes Hölzl and Armin Heller. Three chapters of measure theory in Isabelle/HOL. In Marko C. J. D. van Eekelen, Herman Geuvers, Julien Schmaltz, and Freek Wiedijk, editors, Interactive Theorem Proving, ITP 2011, 
volume 6898 of Lecture Notes in Computer Science, pages 135-151. Springer, 2011.

[96] Zixin Huang, Zhenbang Wang, and Sasa Misailovic. Psense: Automatic sensitivity analysis for probabilistic programs. In Shuvendu K. Lahiri and Chao Wang, editors, Automated Technology for Verification and Analysis - 16th International Symposium, ATVA 2018, Los Angeles, CA, USA, October 7-10, 2018, Proceedings, volume 11138 of LNCS, pages 387-403. Springer, 2018. doi: 10.1007/978-3-030-0109o-4\_23. URL https://doi.org/10.1007/ 978-3-030-01090-4_23.

[97] Joe Hurd. Formal verification of probabilistic algorithms. Technical Report UCAM-CL-TR-566, University of Cambridge, Computer Laboratory, 2003.

[98] R. Impagliazzo and S. Rudich. Limits on the provable consequences of oneway permutations. In 21st Annual ACM Symposium on Theory of Computing, 1989, pages 44-61, New York, 1989. ACM.

[99] B. Jacobs. Categorical Logic and Type Theory. Number 141 in Studies in Logic and the Foundations of Mathematics. North Holland, Amsterdam, 1999.

[10o] Limin Jia, Shayak Sen, Deepak Garg, and Anupam Datta. A logic of programs with interface-confined code. In IEEE 28th Computer Security Foundations Symposium (CSF), pages 512-525, 2015.

[101] C. Jones and Gordon D. Plotkin. A probabilistic powerdomain of evaluations. In LICS '89, Pacific Grove, California, USA, June 5-8, 1989. IEEE Computer Society, 1989. ISBN o-8186-1954-6. URL http://ieeexplore.ieee.org/xpl/ mostRecent Issue $\cdot$ jsp? punumber $=249$.

[102] Achim Jung and Regina Tix. The troublesome probabilistic powerdomain. Electr. Notes Theor. Comput. Sci., 13:70-91, 1998. doi: 10.1016/S1571-0661(05) 80216-6. URL https://doi .org/10.1016/S1571-0661(05)80216-6.

[103] Ralf Jung, David Swasey, Filip Sieczkowski, Kasper Svendsen, Aaron Turon, Lars Birkedal, and Derek Dreyer. Iris: Monoids and invariants as an orthogonal basis for concurrent reasoning. In Sriram K. Rajamani and David Walker, editors, Proceedings of the 42nd Annual ACM SIGPLAN-SIGACT Symposium on Principles of Programming Languages, POPL 2015, Mumbai, India, January 15-17, 2015, pages 637-650. ACM, 2015. doi: 10.1145/2676726.2676980. URL https://doi.org/10.1145/2676726.2676980.

[104] Benjamin Lucien Kaminski, Joost-Pieter Katoen, Christoph Matheja, and Federico Olmedo. Weakest precondition reasoning for expected run-times of probabilistic programs. In European Symposium on Programming (ESOP), 
Eindhoven, The Netherlands, volume 9632 of Lecture Notes in Computer Science, pages 364-389. Springer-Verlag, 2016. doi: 10.1007/978-3-662-49498-1 \_15. URL http://dx.doi.org/10.1007/978-3-662-49498-1_15.

[105] Shin-ya Katsumata. Parametric effect monads and semantics of effect systems. In Proceedings of the 41st ACM SIGPLAN-SIGACT Symposium on Principles of Programming Languages, POPL '14, page 633-645, New York, NY, USA, 2014. Association for Computing Machinery. ISBN 9781450325448. doi: 10.1145/ 2535838.2535846. URL https://doi.org/10.1145/2535838.2535846.

[106] Georgios Kellaris, Stavros Papadopoulos, Xiaokui Xiao, and Dimitris Papadias. Differentially private event sequences over infinite streams. PVLDB, 7(12):11551166, 2014. URL http://www.vldb.org/pvldb/vol7/p1155-kellaris.pdf.

[107] Naoki Kobayashi, Étienne Lozes, and Florian Bruse. On the relationship between higher-order recursion schemes and higher-order fixpoint logic. In Proceedings of the 44th ACM SIGPLAN Symposium on Principles of Programming Languages, POPL 2017, Paris, France, January 18-20, 2017, pages 246-259, 2017. URL http://dl .acm.org/citation.cfm?id=3009854.

[108] Naoki Kobayashi, Takeshi Tsukada, and Keiichi Watanabe. Higher-order program verification via HFL model checking. In Programming Languages and Systems - 27th European Symposium on Programming, ESOP 2018, Held as Part of the European Joint Conferences on Theory and Practice of Software, ETAPS 2018, Thessaloniki, Greece, April 14-20, 2018, Proceedings, pages 711738, 2018. doi: 10.1007/978-3-319-89884-1\_25. URL https://doi.org/10. 1007/978-3-319-89884-1_25.

[109] Dexter Kozen. A probabilistic PDL. Journal of Computer and System Sciences, 30 (2):162-178, 1985 .

[110] Robbert Krebbers, Amin Timany, and Lars Birkedal. Interactive proofs in higher-order concurrent separation logic. In Giuseppe Castagna and Andrew D. Gordon, editors, POPL 2017, Paris, France, January 18-20, 2017. ACM, 2017. ISBN 978-1-4503-4660-3.

[111] Morten Krogh-Jespersen, Kasper Svendsen, and Lars Birkedal. A relational model of types-and-effects in higher-order concurrent separation logic. In POPL 2017, Paris, France, January 18-20, 2017, pages 218-231, 2017. URL http: //dl.acm.org/citation. cfm?id=3009877.

[112] Mathias Lécuyer, Vaggelis Atlidakis, Roxana Geambasu, Daniel Hsu, and Suman Jana. Certified robustness to adversarial examples with differential privacy. In 2019 IEEE Symposium on Security and Privacy, SP 2019, San Francisco, 
CA, USA, May 19-23, 2019, pages 656-672. IEEE, 2019. doi: 10.1109/SP.2019. ooo44. URL https://doi.org/10.1109/SP.2019.00044.

[113] Saunders MacLane. Categories for the Working Mathematician. Springer-Verlag, New York, 1971. Graduate Texts in Mathematics, Vol. 5.

[114] Kenji Maillard, Danel Ahman, Robert Atkey, Guido Martínez, Catalin Hritcu, Exequiel Rivas, and Éric Tanter. Dijkstra monads for all. Proc. ACM Program. Lang., 3(ICFP):104:1-104:29, 2019. doi: 10.1145/3341708. URL https://doi. org/10.1145/3341708.

[115] Kenji Maillard, Catalin Hritcu, Exequiel Rivas, and Antoine Van Muylder. The next 700 relational program logics. Proc. ACM Program. Lang., 4(POPL): 4:1-4:33, 2020.

[116] Conor McBride and Ross Paterson. Applicative programming with effects. J. Funct. Programming, 18(1):1-13, 2008.

[117] Annabelle McIver and Carroll Morgan. Abstraction, Refinement and Proof for Probabilistic Systems. Monographs in Computer Science. Springer, 2005.

[118] Paul-André Melliès and Noam Zeilberger. Functors are type refinement systems. In Sriram K. Rajamani and David Walker, editors, POPL 2015, Mumbai, India, January 15-17, 2015. ACM, 2015. doi: 10.1145/2676726.2676970. URL http://doi.acm.org/10.1145/2676726.2676970.

[119] John Miller and Moritz Hardt. When recurrent models don't need to be recurrent. CoRR, abs/1805.10369, 2018. URL http://arxiv.org/abs/1805. 10369.

[120] Eugenio Moggi. Computational lambda-calculus and monads. In Proceedings of the Fourth Annual Symposium on Logic in Computer Science (LICS '89), Pacific Grove, California, USA, June 5-8, 1989, pages 14-23. IEEE Computer Society, 1989. doi: 10.1109/LICS.1989.39155. URL https://doi.org/10.1109/LICS. 1989.39155.

[121] Carroll Morgan, Annabelle McIver, and Karen Seidel. Probabilistic predicate transformers. ACM Transactions on Programming Languages and Systems, 18(3): 325-353, 1996.

[122] Aleksandar Nanevski, Anindya Banerjee, and Deepak Garg. Dependent type theory for verification of information flow and access control policies. ACM Trans. Program. Lang. Syst., 35(2):6:1-6:41, 2013. doi: 10.1145/2491522.2491523. URL http://doi.acm.org/10.1145/2491522.2491523. 
[123] Praveen Narayanan, Jacques Carette, Wren Romano, Chung-chieh Shan, and Robert Zinkov. Probabilistic inference by program transformation in hakaru (system description). In Functional and Logic Programming - 13th International Symposium, FLOPS 2016, Kochi, Japan, March 4-6, 2016, Proceedings, pages 62-79, 2016. doi: 10.1007/978-3-319-29604-3\_5. URL https://doi.org/10.1007/ 978-3-319-29604-3_5.

[124] Ulf Norell. Dependently typed programming in agda. In Proceedings of the 4th International Workshop on Types in Language Design and Implementation, TLDI '09, page 1-2, New York, NY, USA, 2009. Association for Computing Machinery. ISBN 9781605584201. doi: 10.1145/1481861.1481862. URL https: //doi.org/10.1145/1481861.1481862.

[125] Frank Pfenning. Church and Curry: Combining intrinsic and extrinsic typing. In C.Benzmüller, C.Brown, J.Siekmann, and R.Statman, editors, Reasoning in Simple Type Theory: Festschrift in Honor of Peter B. Andrews on His 7oth Birthday, Studies in Logic 17, pages 303-338. College Publications, 2008.

[126] Benedetto Piccoli and Francesco Rossi. On properties of the generalized wasserstein distance. Archive for Rational Mechanics and Analysis, 222(3):13391365, Dec 2016. ISSN 1432-0673. doi: 10.1007/s00205-016-1026-7. URL https://doi.org/10.1007/s00205-016-1026-7.

[127] Andrew Pitts and Ian Stark. Operational reasoning for functions with local state. In Andrew Gordon and Andrew Pitts, editors, Higher Order Operational Techniques in Semantics, pages 227-273. Publications of the Newton Institute, Cambridge University Press, 1998. URL http://www.inf .ed.ac.uk/ ${ }^{\sim}$ stark/ operfl.html.

[128] Gordon Plotkin. Lambda-definability and logical relations, 1973.

[129] Gordon Plotkin. Lcf considered as a programming language. Theoretical Computer Science, 5(3):223 - 255, 1977. ISSN 0304-3975. doi: http://dx.doi. org/10.1016/0304-3975(77)90044-5. URL http://www.sciencedirect.com/ science/article/pii/0304397577900445.

[130] Gordon D. Plotkin and Martín Abadi. A logic for parametric polymorphism. In International Conference on Typed Lambda Calculi and Applications, TLCA '93, Utrecht, The Netherlands, March 16-18, 1993, Proceedings, pages 361-375, 1993. doi: 10.1007/BFbo037118. URL http://dx.doi.org/10.1007/BFb0037118.

[131] George Pólya. Über eine Aufgabe der Wahrscheinlichkeitsrechnung betreffend die Irrfahrt im Straßennetz. Mathematische Annalen, 84:149-160, 1921. URL http://eudml.org/doc/158886. 
[132] François Pottier and Vincent Simonet. Information flow inference for ML. In POPL 2002, Portland, OR, USA, January 16-18, 2002, 2002. doi: 10.1145/503272. 503302. URL http://doi .acm.org/10.1145/503272.503302.

[133] Lyle Harold Ramshaw. Formalizing the Analysis of Algorithms. PhD thesis, Computer Science, 1979.

[134] Robert Rand and Steve Zdancewic. VPHL: A Verified Partial-Correctness Logic for Probabilistic Programs. In Mathematical Foundations of Program Semantics (MFPS XXXI), 2015.

[135] Jason Reed and Benjamin C. Pierce. Distance makes the types grow stronger: a calculus for differential privacy. In ICFP 2010, Baltimore, Maryland, USA, September 27-29, 2010, pages 157-168, 2010.

[136] Stefan Richter. Formalizing integration theory with an application to probabilistic algorithms. In Konrad Slind, Annette Bunker, and Ganesh Gopalakrishnan, editors, Theorem Proving in Higher Order Logics, 17th International Conference, (TPHOL) 2004, volume 3223 of Lecture Notes in Computer Science, pages 271-286. Springer, 2004.

[137] Tetsuya Sato. Approximate relational hoare logic for continuous random samplings. In Lars Birkedal, editor, The Thirty-second Conference on the Mathematical Foundations of Programming Semantics, MFPS 2016, Carnegie Mellon University, Pittsburgh, PA, USA, May 23-26, 2016, volume 325 of Electronic Notes in Theoretical Computer Science, pages 277-298. Elsevier, 2016. doi: 10.1016/j. entcs.2016.09.043. URL https://doi.org/10.1016/j.entcs. 2016.09.043.

[138] Tetsuya Sato, Alejandro Aguirre, Gilles Barthe, Marco Gaboardi, Deepak Garg, and Justin Hsu. Formal verification of higher-order probabilistic programs: reasoning about approximation, convergence, bayesian inference, and optimization. PACMPL, 3(POPL):38:1-38:30, 2019. URL https: //dl. acm.org/citation. cfm?id=3290351.

[139] Robert E. Schapire. The strength of weak learnability. Mach. Learn., 5:197-227, 1990. doi: 10.1007/BFoo116037. URL https://doi.org/10.1007/BF00116037.

[140] Robert E Schapire. Drifting games. Machine Learning, 43(3):265-291, 2001.

[141] Adam Ścibior, Ohad Kammar, Matthijs Vákár, Sam Staton, Hongseok Yang, Yufei Cai, Klaus Ostermann, Sean K. Moss, Chris Heunen, and Zoubin Ghahramani. Denotational validation of higher-order bayesian inference. Proc. ACM Program. Lang., 2(POPL):60:1-60:29, December 2017. ISSN 2475-1421. doi: 10.1145/3158148. URL http://doi.acm.org/10.1145/3158148. 
[142] Chung-chieh Shan and Norman Ramsey. Exact bayesian inference by symbolic disintegration. In Proceedings of the 44th ACM SIGPLAN Symposium on Principles of Programming Languages, POPL 2017, Paris, France, January 18-20, 2017, pages 130-144, 2017.

[143] Marcelo Sousa and Isil Dillig. Cartesian hoare logic for verifying k-safety properties. In Proceedings of the 37th ACM SIGPLAN Conference on Programming Language Design and Implementation, PLDI 2016, Santa Barbara, CA, USA, June 13-17, 2016, pages 57-69, 2016. doi: 10.1145/2908080.2908092. URL http: //doi.acm.org/10.1145/2908080.2908092.

[144] R. Statman. Logical relations and the typed $\lambda$-calculus. Information and Control, 65(2-3):85-97, May 1985. URL http://dx.doi.org/10.1016/s0019-9958(85) $80001-2$.

[145] Sam Staton, Hongseok Yang, Frank Wood, Chris Heunen, and Ohad Kammar. Semantics for probabilistic programming: higher-order functions, continuous distributions, and soft constraints. In LICS '16, New York, NY, USA, July 5-8, 2016. ACM, 2016. ISBN 978-1-4503-4391-6.

[146] Gordon Stewart, Anindya Banerjee, and Aleksandar Nanevski. Dependent types for enforcement of information flow and erasure policies in heterogeneous data structures. In 15th International Symposium on Principles and Practice of Declarative Programming, PPDP '13, Madrid, Spain, September 16-18, 2013, pages 145-156, 2013. doi: 10.1145/2505879.2505895. URL http://doi .acm.org/10.1145/2505879.2505895.

[147] Volker Strassen. The existence of probability measures with given marginals. The Annals of Mathematical Statistics, pages 423-439, 1965. URL http: //projecteuclid.org/euclid.aoms/1177700153.

[148] Richard S. Sutton. Learning to predict by the methods of temporal differences. Mach. Learn., 3:9-44, 1988. doi: 10.1007/BFoo115009. URL https://doi.org/ $10.1007 / \mathrm{BF} 00115009$.

[149] Nikhil Swamy, Catalin Hritcu, Chantal Keller, Aseem Rastogi, Antoine Delignat-Lavaud, Simon Forest, Karthikeyan Bhargavan, Cédric Fournet, Pierre-Yves Strub, Markulf Kohlweiss, Jean Karim Zinzindohoue, and Santiago Zanella Béguelin. Dependent types and multi-monadic effects in $f^{*}$. In Rastislav Bodík and Rupak Majumdar, editors, POPL 2016, St. Petersburg, FL, USA, January 20 - 22, 2016. ACM, 2016. doi: 10.1145/2837614.2837655. URL http://doi .acm.org/10.1145/2837614.2837655. 
[150] William W. Tait. Intensional interpretations of functionals of finite type I. J. Symb. Log., 32(2):198-212, 1967. doi: 10.2307/2271658. URL http: //dx.doi.org/10.2307/2271658.

[151] The Coq Development Team. The coq proof assistant, version 8.8.o, April 2018. URL https://doi.org/10.5281/zenodo. 1219885.

[152] Aaron Turon, Derek Dreyer, and Lars Birkedal. Unifying refinement and hoarestyle reasoning in a logic for higher-order concurrency. In Greg Morrisett and Tarmo Uustalu, editors, ICFP 2013, Boston, MA, USA - September 25 - 27, 2013. ACM, 2013. ISBN 978-1-4503-2326-o. URL http://dl.acm.org/citation. cfm?id=2500365.

[153] Hiroshi Unno, Sho Torii, and Hiroki Sakamoto. Automating induction for solving horn clauses. In Computer Aided Verification - 29th International Conference, CAV 2017, Heidelberg, Germany, July 24-28, 2017, Proceedings, Part II, pages 571-591, 2017. doi: 10.1007/978-3-319-63390-9\_30. URL https://doi.org/10.1007/978-3-319-63390-9_30.

[154] Matthijs Vákár, Ohad Kammar, and Sam Staton. A domain theory for statistical probabilistic programming. PACMPL, 3(POPL):36:1-36:29, 2019. URL https: //dl. acm. org/citation. cfm?id=3290349.

[155] Niki Vazou, Eric L. Seidel, Ranjit Jhala, Dimitrios Vytiniotis, and Simon L. Peyton Jones. Refinement types for haskell. In Johan Jeuring and Manuel M. T. Chakravarty, editors, Proceedings of the 19th ACM SIGPLAN international conference on Functional programming, Gothenburg, Sweden, September 1-3, 2014, pages 269-282. ACM, 2014. doi: 10.1145/2628136.2628161. URL http://doi. acm. org/10.1145/2628136. 2628161 .

[156] Cédric Villani. Optimal transport: old and new. Springer-Verlag, 2008.

[157] Dennis Volpano, Geoffrey Smith, and Cynthia Irvine. A sound type system for secure flow analysis. Journal of Computer Security, 4(3):1-21, 1996.

[158] Peixin Wang, Hongfei Fu, Krishnendu Chatterjee, Yuxin Deng, and Ming $\mathrm{Xu}$. Proving expected sensitivity of probabilistic programs with randomized variable-dependent termination time. Proc. ACM Program. Lang., 4(POPL): 25:1-25:30, 2020.

[159] David Williams. Probability with Martingales. Cambridge University Press, 1991. doi: $10.1017 / \mathrm{CBO} 9780511813658$. 
[16o] Daniel Winograd-Cort, Andreas Haeberlen, Aaron Roth, and Benjamin C. Pierce. A framework for adaptive differential privacy. In ACM SIGPLAN International Conference on Functional Programming (ICFP), Oxford, England, pages 10:1-10:29, 2017. URL https://dl . acm.org/citation. cfm?id=3110254.

[161] Frank D. Wood, Jan-Willem van de Meent, and Vikash Mansinghka. A new approach to probabilistic programming inference. In Proceedings of the Seventeenth International Conference on Artificial Intelligence and Statistics, AISTATS 2014, Reykjavik, Iceland, April 22-25, 2014, pages 1024-1032, 2014. URL http://jmlr.org/proceedings/papers/v33/wood14.html.

[162] Hongwei Xi and Frank Pfenning. Dependent types in practical programming. In Andrew W. Appel and Alex Aiken, editors, POPL 1999, San Antonio, TX, USA, January 20-22, 1999. ACM, 1999. doi: 10.1145/292540.292560. URL http://doi.acm.org/10.1145/292540.292560.

[163] Shin ya Katsumata. Relating computational effects by $\mathrm{T} T$-lifting. Information and Computation, 222:228 - 246, 2013. ISSN 0890-5401. doi: https: //doi.org/10.1016/j.ic.2012.10.014. URL http://www.sciencedirect.com/ science/article/pii/S0890540112001551. 38th International Colloquium on Automata, Languages and Programming (ICALP 2011).

[164] Hongseok Yang. Relational separation logic. Theoretical Computer Science, 375 $(1-3): 308-334,2007$.

[165] Noam Zeilberger. Principles of type refinement. Notes for OPLSS 2016 school, 2016. URL http://noamz.org/oplss16/refinements-notes.pdf. 
algebra, 71

category, 69

Cartesian closed, 71

of categories (Cat), 69

of sets (Set), 69

coproduct, 70

currying, 71

discrete probability distribution, 131

evaluation, 71

event, 131

expected value, 63

fiber, 71

fibration, 71

complete Heyting, 72

for logical relations (LR), 72

functor, 69

contravariant, 69

reindexing, 71

graded lifting, 75

graded monad, 74

Kleisli lifting, 74

marginal, 131 measurable space, 62

measure, 62

finite measure, 62

over a QBS, 77

probability measure, 62

sigma-finite measure, 62

monad, 73

morphism, 69

natural transformation, 69

object, 69

exponential, 71

initial, 70

terminal, 70

Probabilistic coupling, 132

product, 70

pullback, 70

quasi-Borel space, 76

random variable, 63

sigma algebra, 62

Borel sigma algebra, 63

state monad transformer, 100

union bound, 63 


\section{APPENDIX OF CHAPTER 3}

\section{A.1 Example: Factorial}

This example shows that the two following implementations of factorial, with and without accumulator, are equivalent:

$$
\begin{aligned}
& \text { fact }_{1} \triangleq \text { letrec } f_{1} n_{1}=\text { case } n_{1} \text { of } 0 \mapsto 1 ; S \mapsto \lambda x_{1} . S x_{1} *\left(f_{1} x_{1}\right) \\
& \text { fact }_{2} \triangleq \text { letrec } f_{2} n_{2}=\lambda \text { acc.case } n_{2} \text { of } 0 \mapsto \text { acc; } S \mapsto \lambda x_{2} . f_{2} x_{2}\left(S x_{2} * \text { acc }\right)
\end{aligned}
$$

Our goal is to prove that:

$$
\begin{aligned}
& \emptyset \mid \emptyset \vdash \text { fact }_{1}: \text { nat } \rightarrow \text { nat } \sim \text { fact }_{2}: \text { nat } \rightarrow \text { nat } \rightarrow \text { nat }\{ \\
& \left.\forall \mathrm{n}_{1} \mathrm{n}_{2} \cdot \mathrm{n}_{1}=\mathrm{n}_{2} \Rightarrow \forall \operatorname{acc} .\left(\mathbf{r}_{1} n_{1}\right) * \text { acc }=\mathbf{r}_{2} \mathrm{n}_{2} \text { acc }\right\}
\end{aligned}
$$

Since both programs do the same number of iterations, we can do synchronous reasoning for the recursion at the head of the programs. However, the bodies of the functions have different types since fact 2 receives an extra argument, the accumulator. Therefore, we will need a one-sided application of ABS-R, before we can go back to reasoning synchronously. We will then apply the CASE rule, knowing that both terms reduce to the same branch, since $n_{1}=n_{2}$. On the zero branch, we will need to prove the trivial equality $1 *$ acc $=$ acc. On the successor branch, we will need to prove that $S x *($ fact $x) *$ acc $=$ fact $_{2} x_{2}\left(S x_{2} * a c c\right)$, knowing by induction hypothesis that such a property holds for every $m$ less that $x$.

Now we will expand on the details. We start the proof applying the LETREC rule, which has 2 premises:

1. Both functions are well-defined

2. $n_{1}=n_{2}, \forall y_{1} y_{2} \cdot\left(y_{1}, y_{2}\right)<\left(n_{1}, n_{2}\right) \Rightarrow y_{1}=y_{2} \Rightarrow \forall \operatorname{acc} .\left(f_{1} y_{1}\right) * \operatorname{acc}=f_{2} y_{2}$ acc $\vdash$ case $n_{1}$ of $0 \mapsto 1 ; S \mapsto \lambda x_{1} . S x_{1} *\left(f_{1} x_{1}\right) \sim \lambda$ acc.case $n_{2}$ of $0 \mapsto$ acc; $S \mapsto$ $\lambda x_{2} . f_{2} x_{2}\left(S x_{2} *\right.$ acc $)\left\{n_{1}=n_{2} \Rightarrow \forall\right.$ acc. $\mathbf{r}_{1} * \operatorname{acc}=\mathbf{r}_{2}$ acc $\}$ 
We assume that the first premise is provable.

To prove the second premise, we start by applying ABS-R, which leaves the following proof obligation:

$$
\begin{gathered}
\mathrm{n}_{1}=\mathrm{n}_{2}, \forall \mathrm{y}_{1} \mathrm{y}_{2} \cdot\left(\mathrm{y}_{1}, \mathrm{y}_{2}\right)<\left(\mathrm{n}_{1}, \mathrm{n}_{2}\right) \Rightarrow \mathrm{y}_{1}=\mathrm{y}_{2} \Rightarrow \forall \operatorname{acc} .\left(\mathrm{f}_{1} \mathrm{y}_{1}\right) * \operatorname{acc}=\mathrm{f}_{2} \mathrm{y}_{2} \text { acc, } \mathrm{n}_{1}=\mathrm{n}_{2} \vdash \\
\text { case } \mathrm{n}_{1} \text { of } 0 \mapsto 1 ; S \mapsto \lambda x_{1} . S x_{1} *\left(\mathrm{f}_{1} x_{1}\right) \sim \text { case } \mathrm{n}_{2} \text { of } 0 \mapsto \operatorname{acc} ; S \mapsto \lambda x_{2} . \mathrm{f}_{2} x_{2}\left(S x_{2} * \operatorname{acc}\right)\{ \\
\left.\mathbf{r}_{1} * \operatorname{acc}=\mathbf{r}_{2}\right\}
\end{gathered}
$$

Now we can apply CASE, and we have 3 premises, where $\Psi$ denotes the axioms of the previous judgment:

- $\Psi \vdash \mathrm{n}_{1} \sim \mathrm{n}_{2}\left\{\mathbf{r}_{1}=0 \Leftrightarrow \mathbf{r}_{2}=0\right\}$

- $\Psi, \mathrm{n}_{1}=0, \mathrm{n}_{2}=0 \vdash 1 \sim \operatorname{acc}\left\{\mathbf{r}_{1} * \operatorname{acc}=\mathbf{r}_{2}\right\}$

- $\Psi \vdash \lambda x_{1} . S x_{1} *\left(f_{1} x_{1}\right) \sim \lambda x_{2} . f_{2} x_{2}\left(S x_{2} * a c c\right)\left\{\forall x_{1} x_{2} \cdot n_{1}=S x_{1} \Rightarrow n_{2}=S x_{2} \Rightarrow\right.$ $\left.\left(\mathbf{r}_{1} x_{1}\right) * \operatorname{acc}=\mathbf{r}_{2} x_{2}\right\}$

Premise 1 is a direct consequence of $n_{1}=n_{2}$. Premise 2 is a trivial arithmetic identity. To prove premise 3, we first apply the ABS rule:

$$
\Psi, \mathrm{n}_{1}=S x_{1}, \mathrm{n}_{2}=S x_{2} \vdash S x_{1} *\left(f_{1} x_{1}\right) \sim f_{2} x_{2}\left(S x_{2} * a c c\right)\left\{\mathbf{r}_{1} * a c c=\mathbf{r}_{2}\right\}
$$

and then by Theorem 3.6 we can finish the proof in HOL by deriving.

$$
\Psi, \mathrm{n}_{1}=\mathrm{S} \mathrm{x}_{1}, \mathrm{n}_{2}=\mathrm{S} \mathrm{x}_{2} \vdash \mathrm{S} \mathrm{x}_{1} *\left(\mathrm{f}_{1} \mathrm{x}_{1}\right) * \mathrm{acc}=\mathrm{f}_{2} \mathrm{x}_{2}\left(\mathrm{~S} \mathrm{x}_{2} * \mathrm{acc}\right)
$$

From the premises we can first prove that $\left(x_{1}, x_{2}\right)<\left(n_{1}, n_{2}\right)$ so by the inductive hypothesis from the LETREC rule, and the $\Rightarrow_{E}$ rule, we get

$$
\forall \operatorname{acc} .\left(f_{1} x_{1}\right) * \operatorname{acc}=f_{2} x_{2} \text { acc, }
$$

which we then instantiate with $S x_{1} *$ acc to get

$$
\left(f_{1} x_{1}\right) * S x_{1} * a c c=f_{2} x_{2}\left(S x_{1} * a c c\right) .
$$

On the other hand, from the hypotheses we also have $x_{1}=x_{2}$, so by CONV we finally prove

$$
\left(f_{1} x_{1}\right) * S x_{1} * a c c=f_{2} x_{2}\left(S x_{2} * a c c\right)
$$

\section{A.2 Example: List reversal}

A related example for lists is the equivalence of reversal with and without accumulator. The structure of the proof is the same as in the factorial example, but we 
will briefly show it to illustrate how the LISTCASE rule is used. The functions are written:

$$
\begin{aligned}
& \operatorname{rev}_{1} \triangleq \text { letrec } f_{1} l_{1}=\text { case } l_{1} \text { of } \square \mapsto \square ;_{-}:{ }_{-} \mapsto \lambda h_{1} \cdot \lambda t_{1} \cdot\left(f_{1} t_{1}\right)++\left[x_{1}\right] \\
& \operatorname{rev}_{2} \triangleq \text { letrec } f_{2} l_{2}=\lambda \text { acc.case } l_{2} \text { of } \square \mapsto a c c ;_{-}::_{-} \mapsto \lambda h_{2} \cdot \lambda t_{2} \cdot f_{2} t_{2}\left(h_{2}:: \text { acc }\right)
\end{aligned}
$$

We want to prove they are related by the following judgment:

$$
\begin{gathered}
\emptyset \mid \emptyset \vdash \operatorname{rev}_{1}: \text { list }_{\tau} \rightarrow \text { list }_{\tau} \sim \operatorname{rev}_{2}: \text { list }_{\tau} \rightarrow \text { list }_{\tau} \\
\left\{\forall l_{1}, l_{2} . l_{1}=l_{2} \Rightarrow \forall \text { acc. }\left(\mathbf{r}_{1} l_{1}\right)++\operatorname{acc}=\mathbf{r}_{2} l_{2} \text { acc }\right\}
\end{gathered}
$$

By the LETREC rule, we have to prove 2 premises:

1. Both functions are well-defined.

2. $\mathrm{l}_{1}=\mathrm{l}_{2}, \forall \mathrm{m}_{1} \mathrm{~m}_{2} \cdot\left(\left|\mathrm{m}_{1}\right|,\left|\mathrm{m}_{2}\right|\right)<\left(\left|\mathrm{l}_{1}\right|,\left|\mathrm{l}_{2}\right|\right) \Rightarrow \mathrm{m}_{1}=\mathrm{m}_{2} \Rightarrow \forall \operatorname{acc} .\left(\mathrm{f}_{1} \mathrm{~m}_{1}\right)++\operatorname{acc}=$ $f_{2} m_{2}$ acc $\vdash$ case $l_{1}$ of $\square \mapsto \square ; ;_{-}: \because \mapsto \lambda h_{1} . \lambda t_{1} .\left(f_{1} t_{1}\right)++\left[x_{1}\right] \sim \lambda$ acc.case $l_{2}$ of $\square \mapsto$ acc; $:_{-} \mapsto \lambda h_{2} \cdot \lambda t_{2} \cdot f_{2} t_{2}\left(h_{2}::\right.$ acc $)\left\{\forall\right.$ acc. $\mathbf{r}_{1}++\operatorname{acc}=\mathbf{r}_{2}$ acc $\}$

For the second premise, similarly as in factorial, we apply ABS-R. We have the following premise, where $\Psi$ denotes the axioms in the previous judgment:

$$
\begin{gathered}
\Psi \vdash \text { case } l_{1} \text { of } \square \mapsto \square ;_{-}:{ }_{-} \mapsto \lambda h_{1} \cdot \lambda t_{1} \cdot\left(f_{1} t_{1}\right)++\left[x_{1}\right] \sim \\
\text { case } t_{2} \text { of } \square \mapsto a c c ;_{-}: \because h_{-} \cdot \lambda t_{2} \cdot f_{2} t_{2}\left(h_{2}:: a c c\right)\{ \\
\left.\mathbf{r}_{1}++\operatorname{acc}=\mathbf{r}_{2}\right\}
\end{gathered}
$$

and then LISTCASE, which has three premises:

- $\Psi \vdash l_{1} \sim l_{2}\left\{\mathbf{r}_{1}=\square \Leftrightarrow \mathbf{r}_{2}=\square\right\}$

- $\Psi, l_{1}=\square, l_{2}=\square \vdash \square \sim \operatorname{acc}\left\{\mathbf{r}_{1}++\operatorname{acc}=\mathbf{r}_{2}\right\}$

- $\Psi \vdash \lambda h_{1} \cdot \lambda t_{1} \cdot\left(f_{1} t_{1}\right)++\left[x_{1}\right] \sim \lambda h_{2} \cdot \lambda t_{2} \cdot f_{2} t_{2}\left(h_{2}:: a c c\right)\{$

$$
\left.\forall h_{1} t_{1} h_{2} t_{2} \cdot l_{1}=h_{1}:: t_{1} \Rightarrow l_{2}=h_{2}:: t_{2} \Rightarrow r_{1}++a c c=r_{2}\right\}
$$

We complete the proof in a similar way as in the factorial example.

\section{A.3 Example: Proof of Theorem 3.23}

We will use without proof two unary lemmas:

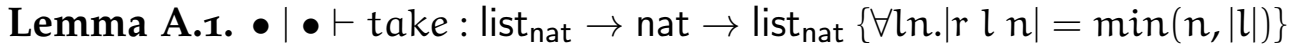

Lemma A.2. $\bullet \mid \bullet \vdash$ map $:$ list $_{\text {nat }} \rightarrow($ nat $\rightarrow$ nat $) \rightarrow$ list $_{\text {nat }}\{\forall \mathrm{lf} .|\mathrm{r} l \mathrm{f}|=|\mathrm{l}|\}$ 
Now we proceed with the proof of the theorem

We want to prove

$$
\begin{gathered}
\mathrm{l}_{1}=\mathrm{l}_{2}, \mathrm{n}_{1}=\mathrm{n}_{2}, \mathrm{~g}_{1}=\mathrm{g}_{2} \vdash \operatorname{map}\left(\text { take } \mathrm{l}_{1} \mathrm{n}_{1}\right) \mathrm{g}_{1} \sim \operatorname{take}\left(\operatorname{map} \mathrm{l}_{2} \mathrm{~g}_{2}\right) \mathrm{n}_{2}\{ \\
\left.\mathbf{r}_{1} \sqsubseteq \mathbf{r}_{2} \wedge\left|\mathbf{r}_{1}\right|=\min \left(\mathrm{n}_{1},\left|\mathrm{l}_{1}\right|\right) \wedge\left|\mathbf{r}_{2}\right|=\min \left(\mathrm{n}_{2},\left|\mathrm{l}_{2}\right|\right)\right\}
\end{gathered}
$$

where $\mathbf{r}_{1} \sqsubseteq \mathbf{r}_{2}$ is the prefix ordering and is defined as an inductive predicate:

$$
\forall \mathrm{l} . \square \sqsubseteq \mathrm{l} \quad \forall \mathrm{hl}_{1} \mathrm{l}_{2} \cdot \mathrm{l}_{1} \sqsubseteq \mathrm{l}_{2} \Rightarrow \mathrm{h}:: \mathrm{l}_{1} \sqsubseteq \mathrm{h}:: \mathrm{l}_{2}
$$

By the helping lemmas and Lemma B.5, it suffices to prove just the first conjunct:

$$
l_{1}=l_{2}, n_{1}=n_{2}, g_{1}=g_{2} \vdash \operatorname{map}\left(\text { take } l_{1} n_{1}\right) g_{1} \sim \text { take }\left(\operatorname{map} l_{2} g_{2}\right) n_{2}\left\{\mathbf{r}_{1} \sqsubseteq \mathbf{r}_{2}\right\}
$$

The derivation begins by applying the APP-R rule. We get the following judgment on $n_{2}$ :

$$
l_{1}=l_{2}, n_{1}=n_{2}, g_{1}=g_{2} \vdash n_{2}|r \geqslant| \text { take } l_{1} n_{1} \mid
$$

and a main premise:

$$
\begin{gathered}
l_{1}=l_{2}, n_{1}=n_{2}, g_{1}=g_{2} \vdash \operatorname{map}\left(\text { take } l_{1} n_{1}\right) g_{1} \sim \text { take }\left(\operatorname{map} l_{2} g_{2}\right)\{ \\
\left.\forall x_{2} \cdot x_{2} \geqslant \mid \text { take } l_{1} n_{1} \mid \Rightarrow \mathbf{r}_{1} \sqsubseteq\left(\mathbf{r}_{2} x_{2}\right)\right\}
\end{gathered}
$$

Notice that we have chosen the premise $x_{2} \geqslant \mid$ take $l_{1} n_{1} \mid$ because we are trying to prove $\mathbf{r}_{1} \sqsubseteq\left(\begin{array}{ll}\mathbf{r}_{2} & x_{2}\end{array}\right)$, which is only true if we take a larger prefix on the right than on the left. The judgment ( 1 ) is easily proven from the fact that $\mid$ take $l_{1} n_{1} \mid=$ $\min \left(n_{1},\left|l_{1}\right|\right) \leqslant n_{1}=n_{2}$, which we get from the lemmas. To prove (2) we first apply APP-L with a trivial condition $g_{1}=g_{2}$ on $g_{1}$. Then we apply APP and we have two premises:

(A) $\Psi \vdash$ take $l_{1} \mathrm{n}_{1} \sim \operatorname{map~}_{2} \mathrm{~g}_{2}\left\{\mathbf{r}_{1} \sqsubseteq \mathrm{g}_{2} \mathbf{r}_{2}\right\}$

(B) $\Psi \vdash$ map $\sim$ take \{

$\left.\forall \mathrm{m}_{1} \mathrm{~m}_{2} \cdot \mathrm{m}_{1} \sqsubseteq \mathrm{g}_{2} \mathrm{~m}_{2} \Rightarrow\left(\forall \mathrm{g}_{1} \cdot \mathrm{g}_{1}=\mathrm{g}_{2} \Rightarrow \forall \mathrm{x}_{2} \cdot \mathrm{x}_{2} \geqslant\left|\mathrm{~m}_{1}\right| \Rightarrow\left(\mathbf{r}_{1} \mathrm{~m}_{1} \mathrm{~g}_{1}\right) \sqsubseteq\left(\mathbf{r}_{2} \mathrm{~m}_{2} \mathrm{x}_{2}\right)\right)\right\}$

where $\sqsubseteq_{g}$ is defined as an inductive predicate parametrized by $\mathrm{g}$ :

$$
\forall \mathrm{l} . \square \sqsubseteq g \mathrm{l} \quad \forall \mathrm{hl}_{1} \mathrm{l}_{2} \cdot \mathrm{l}_{1} \sqsubseteq_{\mathrm{g}} \mathrm{l}_{2} \Rightarrow \mathrm{h}:: \mathrm{l}_{1} \sqsubseteq_{\mathrm{g}}(\mathrm{gh}):: \mathrm{l}_{2}
$$

We first show how to prove (A). We start by applying APP with a trivial condition for the arguments to get:

$$
\Psi \vdash \text { take } l_{1} \sim \operatorname{map}_{2}\left\{\forall x_{1} g_{2} \cdot\left(\mathbf{r}_{1} x_{1}\right) \sqsubseteq g_{2}\left(r_{2} g_{2}\right)\right\}
$$

We then apply APP, which has two premises, one of them equating $l_{1}$ and $l_{2}$. The other one is:

$$
\Psi \vdash \text { take } \sim \operatorname{map}\left\{\forall m_{1} m_{2} \cdot m_{1}=m_{2} \Rightarrow \forall x_{1} g_{2} \cdot\left(r_{1} m_{1} x_{1}\right) \sqsubseteq g_{2}\left(r_{2} m_{2} g_{2}\right)\right\}
$$


To complete this branch of the proof, we apply LETREC. We need to prove the following premise:

$$
\begin{gathered}
\Psi, m_{1}=m_{2}, \forall k_{1} k_{2} \cdot\left(k_{1}, k_{2}\right)<\left(m_{1}, m_{2}\right) \Rightarrow k_{1}=k_{2} \Rightarrow \forall x_{1} g_{2} \cdot\left(f_{1} k_{1} x_{1}\right) \sqsubseteq g_{2}\left(f_{2} k_{2} g_{2}\right) \vdash \\
\lambda n_{1} \cdot e_{1} \sim \lambda g_{2} \cdot e_{2}\left\{\forall x_{1} g_{2} \cdot\left(r_{1} x_{1}\right) \sqsubseteq g_{2}\left(r_{2} g_{2}\right)\right\}
\end{gathered}
$$

Where $e_{1}, e_{2}$ abbreviate the bodies of the functions:

$$
\begin{aligned}
& e_{1} \triangleq \text { case } m_{1} \text { of } \square \mapsto \square \\
& i_{-}::_{-} \mapsto \lambda h_{1} t_{1} \text {.case } x_{1} \text { of } 0 \mapsto \quad \text { ] } \\
& ; S \mapsto \lambda y_{1} \cdot h_{1}:: f_{1} t_{1} y_{1} \\
& e_{2} \triangleq \text { case } m_{2} \text { of } \square \mapsto \square \\
& i_{-}::_{-} \mapsto \lambda h_{2} t_{2} \cdot\left(g_{2} h_{2}\right)::\left(f_{2} t_{2} g_{2}\right)
\end{aligned}
$$

If we apply $A B S$ we get a premise:

$$
\begin{gathered}
\Psi, m_{1}=m_{2}, \forall k_{1} k_{2} \cdot\left(k_{1}, k_{2}\right)<\left(m_{1}, m_{2}\right) \Rightarrow k_{1}=k_{2} \Rightarrow \\
\forall x_{1} g_{2} \cdot\left(f_{1} k_{1} x_{1}\right) \sqsubseteq_{2}\left(f_{2} k_{2} g_{2}\right) \vdash e_{1} \sim e_{2}\left\{\mathbf{r}_{1} \sqsubseteq_{f} \mathbf{r}_{2}\right\}
\end{gathered}
$$

And now we can apply a synchronous CASE rule, since we have a premise $m_{1}=m_{2}$. This yields 3 proof obligations, where $\Psi^{\prime}$ is the set of axioms in the previous judgment:

(A.1) $\Psi^{\prime} \vdash \mathrm{m}_{1} \sim \mathrm{m}_{2}\left\{\mathbf{r}_{1}=\square \Leftrightarrow \mathbf{r}_{2}=\square\right\}$

(A.2) $\Psi^{\prime} \vdash \square \sim \square\left\{\mathbf{r}_{1} \sqsubseteq_{\mathrm{f}} \mathbf{r}_{2}\right\}$

(A.3) $\Psi^{\prime} \vdash \lambda h_{1} t_{1}$.case $x_{1}$ of $0 \mapsto \square ; S \mapsto \lambda y_{1} \cdot h_{1}:: f_{1} t_{1} y_{1} \sim$

$\lambda h_{2} t_{2} \cdot\left(g_{2} h_{2}\right)::\left(f_{2} t_{2} g_{2}\right)\left\{\forall h_{1} t_{1} h_{2} t_{2} \cdot m_{1}=h_{1}:: t_{1} \Rightarrow m_{2}=h_{2}:: t_{2} \Rightarrow\left(r_{1} h_{1} t_{1}\right) \sqsubseteq g_{2}\right.$ $\left.\left(\mathbf{r}_{2} \mathrm{~h}_{2} \mathrm{t}_{2}\right)\right\}$

Premises (A.1) and (A.2) are trivial. To prove (A.3) we first apply ABS twice:

$$
\begin{gathered}
\Psi^{\prime}, m_{1}=h_{1}:: t_{1}, m_{2}=h_{2}:: t_{2} \vdash \\
\text { case } n_{1} \text { of } 0 \mapsto \square ; S \mapsto \lambda y_{1} \cdot h_{1}:: f_{1} t_{1} y_{1} \sim\left(g_{2} h_{2}\right)::\left(f_{2} t_{2} g_{2}\right)\left\{r_{1} \sqsubseteq g_{2} \mathbf{r}_{2}\right\}
\end{gathered}
$$

Next, we apply CASE-L, which has the following two premises:

(A.3.i) $\Psi^{\prime}, \mathrm{m}_{1}=\mathrm{h}_{1}:: \mathrm{t}_{1}, \mathrm{~m}_{2}=\mathrm{h}_{2}:: \mathrm{t}_{2}, \mathrm{n}_{1}=0 \vdash \square \sim\left(\mathrm{g}_{2} \mathrm{~h}_{2}\right)::\left(\mathrm{f}_{2} \mathrm{t}_{2} \mathrm{~g}_{2}\right)\left\{\mathbf{r}_{1} \sqsubseteq \mathrm{g}_{2} \mathbf{r}_{2}\right\}$

(A.3.ii) $\Psi^{\prime}, m_{1}=h_{1}:: t_{1}, m_{2}=h_{2}:: t_{2} \vdash \lambda y_{1} \cdot h_{1}:: f_{1} t_{1} y_{1} \sim\left(g_{2} h_{2}\right)::\left(f_{2} t_{2} g_{2}\right)\left\{\forall y_{1} \cdot n_{1}=\right.$ $\left.S y_{1} \Rightarrow\left(\begin{array}{lll}\mathbf{r}_{1} & \mathrm{y}_{1}\end{array}\right) \sqsubseteq \mathrm{g}_{2} \mathbf{r}_{2}\right\}$ 
Premise (A.3.i) can be directly derived in HOL from the definition of $\Xi_{g_{2}}$. To prove (A.3.ii) we need to make use of our inductive hypothesis:

$$
\forall k_{1} k_{2} \cdot\left(k_{1}, k_{2}\right)<\left(m_{1}, m_{2}\right) \Rightarrow k_{1}=k_{2} \Rightarrow \forall x_{1} g_{2} \cdot\left(f_{1} k_{1} x_{1}\right) \sqsubseteq g_{2}\left(f_{2} k_{2} g_{2}\right)
$$

In particular, from the premises $m_{1}=h_{1}:: t_{1}$ and $m_{2}=h_{2}:: t_{2}$ we can deduce $\left(t_{1}, t_{2}\right)<\left(m_{1}, m_{2}\right)$. Additionally, from the premise $m_{1}=m_{2}$ we prove $t_{1}=t_{2}$. Therefore, from the inductive hypothesis we derive $\forall x_{1} g_{2} \cdot\left(f_{1} t_{1} x_{1}\right) \sqsubseteq g_{2}\left(f_{2} t_{2} g_{2}\right)$, and by definition of $\sqsubseteq_{2}$, and the fact that $h_{1}=h_{2}$, for every $y$ we can prove $h_{1}::\left(f_{1} t_{1} y\right) \sqsubseteq g_{2}\left(g_{2} h_{2}\right):: f_{2} t_{2}$. By Theorem 3.6, we can prove (A.3.ii).

We will now show how to prove (B) :

$$
\begin{gathered}
\Psi \vdash \operatorname{map} \sim \text { take }\left\{\forall \mathrm{m}_{1} \mathrm{~m}_{2} \cdot \mathrm{m}_{1} \sqsubseteq \mathrm{g}_{2} \mathrm{~m}_{2} \Rightarrow\right. \\
\left.\left(\forall \mathrm{g}_{1} \cdot \mathrm{g}_{1}=\mathrm{g}_{2} \Rightarrow \forall x_{2} \cdot x_{2} \geqslant\left|\mathrm{~m}_{1}\right| \Rightarrow\left(\mathbf{r}_{1} \mathrm{~m}_{1} \mathrm{~g}_{1}\right) \sqsubseteq\left(\mathbf{r}_{2} \mathrm{~m}_{2} x_{2}\right)\right)\right\}
\end{gathered}
$$

On this branch we will also use LETREC. We have to prove a premise:

$$
\Psi, \Phi \vdash \lambda g_{1} \cdot e_{2} \sim \lambda x_{2} \cdot e_{1}\left\{\forall g_{1} \cdot g_{1}=g_{2} \Rightarrow \forall x_{2} \cdot x_{2} \geqslant\left|m_{1}\right| \Rightarrow\left(\mathbf{r}_{1} g_{1}\right) \sqsubseteq\left(\mathbf{r}_{2} x_{2}\right)\right\}
$$

where

$$
\Phi \triangleq\left\{\begin{array}{c}
m_{1} \sqsubseteq g_{2} m_{2} \\
\forall k_{1} k_{2} \cdot\left(k_{1}, k_{2}\right)<\left(m_{1}, m_{2}\right) \Rightarrow k_{1} \sqsubseteq g_{2} k_{2} \Rightarrow \\
\left(\forall g_{1} \cdot g_{1}=g_{2} \Rightarrow \forall x_{2} \cdot x_{2} \geqslant\left|k_{1}\right| \Rightarrow\left(r_{1} k_{1} g_{1}\right) \sqsubseteq\left(r_{2} k_{2} x_{2}\right)\right)
\end{array}\right\}
$$

We start by applying ABS. Our goal is to prove:

$$
\begin{aligned}
& \Psi, \Phi, x_{2} \geqslant\left|m_{1}\right|, g_{1}=g_{2} \vdash \\
& \text { case } m_{1} \text { of }[\mapsto] \\
& \text { case } \mathrm{m}_{2} \text { of }[\mapsto] \\
& i_{-}:_{-} \mapsto \lambda h_{1} t_{1} \cdot\left(g_{1} h_{1}\right)::\left(f_{1} t_{1} g_{1}\right) \sim ;_{-}:_{-} \mapsto \lambda h_{2} t_{2} \text {.case } x_{2} \text { of } 0 \mapsto \square\left\{\mathbf{r}_{1} \sqsubseteq \mathbf{r}_{2}\right\} \\
& ; S \mapsto \lambda y_{2} \cdot h_{2}:: f_{2} t_{2} y_{2}
\end{aligned}
$$

Notice that we have $\alpha$-renamed the variables to have the appropriate subscript. Now we want to apply a CASE rule, but the lists over which we are matching are not necessarily of the same length. Therefore, we use the asynchronous LISTCASE-A rule. We have to prove four premises:

(B.1) $\Psi, \Phi, x_{2} \geqslant\left|m_{1}\right|, g_{1}=g_{2}, m_{1}=\square, m_{2}=\square \vdash \square \sim \square\left\{\mathbf{r}_{1} \sqsubseteq \mathbf{r}_{2}\right\}$

(B.2) $\Psi, \Phi, x_{2} \geqslant\left|m_{1}\right|, g_{1}=g_{2}, m_{1}=[\vdash \square \sim$

$\lambda h_{2} t_{2} \cdot \operatorname{case} x_{2}$ of $0 \mapsto \square ; S \mapsto \lambda y_{2} \cdot h_{2}:: f_{2} t_{2} y_{2}\left\{\forall h_{2} t_{2} \cdot m_{2}=h_{2}:: t_{2} \Rightarrow r_{1} \sqsubseteq\left(r_{2} h_{2} t_{2}\right)\right\}$

(B.3) $\Psi, \Phi, x_{2} \geqslant\left|m_{1}\right|, g_{1}=g_{2}, m_{2}=\square \vdash \lambda h_{1} t_{1} \cdot\left(g_{1} h_{1}\right)::\left(f_{1} t_{1} g_{1}\right) \sim\left[\left\{h_{1} t_{1} \cdot m_{1}=h_{1}::\right.\right.$ $\left.\mathrm{t}_{1} \Rightarrow\left(\mathbf{r}_{1} \mathrm{~h}_{1} \mathrm{t}_{1}\right) \sqsubseteq \mathbf{r}_{2}\right\}$ 
(B.4) $\Psi, \Phi, x_{2} \geqslant\left|m_{1}\right|, g_{1}=g_{2} \vdash \lambda h_{1} t_{1} \cdot\left(g_{1} h_{1}\right)::\left(f_{1} t_{1} g_{1}\right) \sim$

$\lambda h_{2} t_{2}$.case $x_{2}$ of $0 \mapsto \square ; S \mapsto \lambda y_{2} \cdot h_{2}:: f_{2} t_{2} y_{2}\{$

$\left.\forall h_{1} t_{1} h_{2} t_{2} \cdot m_{1}=h_{1}:: t_{1} \Rightarrow m_{2}=h_{1}:: t_{1} \Rightarrow\left(r_{1} h_{1} t_{1}\right) \sqsubseteq\left(r_{2} h_{2} t_{2}\right)\right\}$

Premises (B.1) and (B.2) are trivially derived from the definition of the $\sqsubseteq$ predicate. To prove premise (B.3) we see that we have premises $m_{1} \sqsubseteq g_{2} m_{2}, m_{2}=[$, and $m_{1}=h_{1}:: t_{2}$, from which we can derive a contradiction.

It remains to prove (B.4). To do so, we apply ABS twice and then NATCASE-R, which has two premises:

(B.4.i) $\Psi, \Phi, x_{2} \geqslant\left|m_{1}\right|, g_{1}=g_{2}, m_{1}=h_{1}:: t_{1}, m_{2}=h_{1}:: t_{1}, x_{2}=0 \vdash\left(g_{1} h_{1}\right)::\left(f_{1} t_{1} g_{1}\right) \sim$ [ $\left\{\mathbf{r}_{1} \sqsubseteq \mathbf{r}_{2}\right\}$

(B.4.ii) $\Psi, \Phi, x_{2} \geqslant\left|m_{1}\right|, g_{1}=g_{2}, m_{1}=h_{1}:: t_{1}, m_{2}=h_{1}:: t_{1} \vdash\left(g_{1} h_{1}\right)::\left(f_{1} t_{1} g_{1}\right) \sim \lambda y_{2} . h_{2}::$ $f_{2} t_{2} y_{2}\{$

$\left.\forall \mathrm{y}_{2} \cdot \mathrm{x}_{2}=S \mathrm{y}_{2} \Rightarrow \mathbf{r}_{1} \sqsubseteq\left(\mathbf{r}_{2} \mathrm{y}_{2}\right)\right\}$

To prove (B.4.i) we derive a contradiction between the premises. From $x_{2}=0$ and the premise $x_{2} \geqslant\left|m_{1}\right|$ we derive $m_{1}=\square$ and, together with $m_{1}=h_{1}:: t_{1}$ we arrive at a contradiction by applying NC.

To prove (B.4.ii) we need to use the induction hypothesis. From $m_{1}=h_{1}$ :: $t_{1}, m_{2}=h_{1}:: t_{1}$ we can prove that $\left|t_{1}\right|<\left|m_{1}\right|$ and $\left|t_{2}\right|<\left|m_{2}\right|$, so we can do a CUT with the i.h. and derive:

$$
\mathrm{t}_{1} \sqsubseteq \mathrm{g}_{2} \mathrm{t}_{2} \Rightarrow\left(\forall \mathrm{g}_{1} \cdot \mathrm{g}_{1}=\mathrm{g}_{2} \Rightarrow \forall \mathrm{x}_{2} \cdot \mathrm{x}_{2} \geqslant\left|\mathrm{t}_{1}\right| \Rightarrow\left(\mathrm{f}_{1} \mathrm{t}_{1} \mathrm{~g}_{1}\right) \sqsubseteq\left(\mathrm{f}_{2} \mathrm{t}_{2} \mathrm{x}_{2}\right)\right)
$$

By assumption, $\mathrm{m}_{1} \sqsubseteq \mathrm{g}_{2} \mathrm{~m}_{2}$, so $\mathrm{t}_{1} \sqsubseteq \mathrm{g}_{2} \mathrm{t}_{2}$. Moreover, also by assumption $\mathrm{g}_{1}=\mathrm{g}_{2}$, and $S y_{2}=x_{2} \geqslant\left|m_{1}\right|=S\left|t_{1}\right|$, so $y_{2} \geqslant\left|t_{1}\right|$. So if we instantiate the i.h. with $g_{1}$ and $y_{2}$, and apply CUT again, we can prove:

$$
\left(\begin{array}{lll}
f_{1} & t_{1} & g_{1}
\end{array}\right) \sqsubseteq\left(\begin{array}{lll}
f_{2} & t_{2} & y_{2}
\end{array}\right)
$$

On the other hand, since $h_{1}:: t_{1} \sqsubseteq g_{2} h_{2}:: t_{2}$, then (by elimination of $g_{g_{2}}$ ) we can derive $g_{1} h_{1}=h_{2}$ and by definition of $\sqsubseteq,\left(g_{1} h_{1}\right)::\left(f_{1} t_{1} g_{1}\right) \sqsubseteq h_{2}::\left(f_{2} t_{2} y_{2}\right)$. So we can apply Theorem 3.6 and prove (B.4.ii). This ends the proof.

\section{A.4 Example: Proof of Theorem 3.24}

We start by proving the key property of restmin, i.e.,

Lemma A.3. Let restmin ${ }_{1}$ and restmin ${ }_{2}$ denote two $\alpha$-renamings of restmin where every bound variable gets a subindex 1 or 2 respectively. Then,

$\vdash \operatorname{restmin}_{1} \sim \operatorname{restmin}_{2}\left|\forall l_{1} l_{2} \cdot d\left(l_{1}, l_{2}\right) \leqslant \delta \Rightarrow \forall h_{1} h_{2} \cdot\right| h_{1}-h_{2} \mid \leqslant \delta \Rightarrow D\left(r_{1} l_{1} h_{1}, r_{2} l_{2} h_{2}\right) \leqslant \delta$ 
Proof. The proof is a simple synchronous derivation. We start by applying LETREC, which gives us the inductive hypothesis:

$$
\begin{gathered}
\forall m_{1} m_{2} \cdot\left(\left|m_{1}\right|,\left|m_{2}\right|\right) \leqslant\left(\left|l_{1}\right|,\left|l_{2}\right|\right) \Rightarrow d\left(l_{1}, l_{2}\right) \leqslant \delta \Rightarrow \forall h_{1} h_{2} \cdot\left|h_{1}-h_{2}\right| \leqslant \delta \Rightarrow \\
D\left(r_{1} l_{1} h_{1}, r_{2} l_{2} h_{2}\right) \leqslant \delta
\end{gathered}
$$

and we apply ABS immediately after. Then we do a synchronous case analysis with the CASE rule. In the empty list case, we simply instantiate the premise that $\left|a_{1}-a_{2}\right| \leqslant \delta$. Since $d(\square, \square)=0 \leqslant \delta$, we can conclude that $D\left(\left(\square, a_{1}\right),\left(\left[, a_{2}\right)\right) \leqslant \delta\right.$.

In the $h:: t$ case, we start by applying the LET rule three times. On the first two we need to use the lemma about max and min to prove that $\left|M_{1}-M_{2}\right| \leqslant \delta$ and $\left|m_{1}-m_{2}\right| \leqslant \delta$ and introduce them in the logical context.

On the third one, to introduce the hypothesis on (rest, min), we instantiate the inductive hypothesis for restmin. Here we need to prove three facts: (1) $\left(t_{1}, t_{2}\right) \leqslant\left(l_{1}, l_{2}\right),(2)\left|m_{1}-m_{2}\right| \leqslant \delta$ and $d\left(t_{1}, t_{2}\right) \leqslant \delta$. Numbers ( 1$)$ and (3) follow from the fact that $l_{1}=h_{1}:: t_{1}$ and $l_{2}=h_{2}:: t_{2}$. Number (2) follows from the let binding of $m_{1}$ and $m_{2}$. This introduces in the logical context the premises $d\left(\right.$ rest $_{1}$, rest $\left._{2}\right) \leqslant \delta$ and $\left|\min _{1}-\min _{2}\right| \leqslant \delta$.

Finally, we need to show that $d\left(M_{1}::\right.$ rest $_{1}, M_{1}::$ rest $\left._{2}\right) \leqslant \delta$. and that $\mid \min _{1}-$ $\min _{2} \mid \leqslant \delta$. The latter follows directly from the logical context, and the former follows from the inductive definition of the distance.

Now we need to prove for ssort' that:

$$
\begin{aligned}
& \vdash \text { ssort }{ }_{1} \sim \text { ssort }_{2}^{\prime} \\
& \left\{\forall l_{1} l_{2} \cdot d\left(l_{1}, l_{2}\right) \leqslant \delta \Rightarrow \forall n_{1} n_{2} \cdot\left|l_{1}\right|=n_{1} \wedge\left|l_{2}\right|=n_{2} \leqslant \delta \Rightarrow d\left(r_{1} l_{1} n_{1}, r_{2} l_{2} n_{2}\right) \leqslant \delta\right\}
\end{aligned}
$$

The derivation is entirely synchronous and routinary. The only interesting point is instantiating the lemma above for restmin. This concludes the proof of the theorem.

\section{A.5 Proof of Theorem 3.26}

We need two straightforward lemmas in UHOL. The lemmas state that sorting preserves the length and minimum element of a list.

Lemma A.4. Let $\tau \triangleq$ list $_{\text {nat }} \rightarrow$ list $_{\text {nat. Then, }}(1) \bullet \mid \bullet \vdash$ insert : nat $\rightarrow \tau\left\{\forall x l .\left|\pi_{1}\left(\begin{array}{lll}\mathbf{r} & \times l & l\end{array}\right)\right|=\right.$ $1+|l|\}$, and (2) $\bullet \mid \bullet \vdash$ isort : $\tau\left\{\forall x .\left|\pi_{1}(\mathbf{r} x)\right|=|x|\right\}$.

Lemma A.5. Let $\tau \triangleq$ list $_{\text {nat }} \rightarrow$ list $_{\text {nat. }}$ Then, $(1) \bullet \mid \bullet \vdash$ insert : nat $\rightarrow \tau\left\{\forall x l . \operatorname{lmin}\left(\pi_{1}(\mathbf{r} \times l)\right)=\right.$ $\min (x, \operatorname{Imin}(l))\}$, and (2) $\bullet \mid \bullet \vdash$ isort : $\tau\left\{\forall x \cdot \operatorname{Imin}\left(\pi_{1}(\mathbf{r} x)\right)=\operatorname{Imin}(x)\right\}$.

Proof of Theorem 3.26. We prove the theorem using LETREC. We actually show the following stronger theorem, which yields a stronger induction hypothesis in the proof.

$$
\begin{gathered}
\bullet \mid \bullet \vdash \text { isort : } \tau \sim \text { isort : } \tau\left\{\forall x_{1} x_{2} \cdot\left(\operatorname{sorted}\left(x_{1}\right) \wedge\left|x_{1}\right|=\left|x_{2}\right|\right) \Rightarrow\right. \\
\left(\pi_{2}\left(\mathbf{r}_{1} x_{1}\right) \leqslant \pi_{2}\left(\mathbf{r}_{2} x_{2}\right)\right) \wedge \underline{\left.\left(\mathbf{r}_{1} x_{1}=\text { isort } x_{1}\right) \wedge\left(\mathbf{r}_{2} x_{2}=\text { isort } x_{2}\right)\right\}}
\end{gathered}
$$


Let $\iota$ denote the inductive hypothesis:

$$
\begin{aligned}
\iota \triangleq \forall m_{1} m_{2} \cdot\left(\left|m_{1}\right|,\left|m_{2}\right|\right)<\left(\left|x_{1}\right|,\left|x_{2}\right|\right) & \Rightarrow\left(\operatorname{sorted}\left(m_{1}\right) \wedge\left|m_{1}\right|=\left|m_{2}\right|\right) \\
& \Rightarrow \pi_{2}\left(\text { isort }_{1} m_{1}\right) \leqslant \pi_{2}\left(\text { isort }_{2} m_{2}\right) \wedge \\
& \left(\text { isort }_{1} m_{1}=\text { isort } m_{1}\right) \wedge\left(\text { isort }_{2} m_{2}=\text { isort } m_{2}\right)
\end{aligned}
$$

and e denote the body of the function isort:

$$
\begin{aligned}
e \triangleq \text { case } l \text { of } \square \mapsto([, 0) & \\
-\because{ }_{-} \mapsto \lambda \text { ht. let } s=\text { isort } t & \text { let } s^{\prime}=\text { insert } h\left(\pi_{1} s\right) \text { in } \\
& \left(\pi_{1} s^{\prime},\left(\pi_{2} s\right)+\left(\pi_{2} s^{\prime}\right)\right)
\end{aligned}
$$

By LETREC, it suffices to prove the following (we omit simple types for easier reading; they play no essential role in the proof).

$$
\begin{gathered}
\text { isort }_{1}, \text { isort }_{2}, x_{1}, x_{2}\left|\operatorname{sorted}\left(x_{1}\right),\right| x_{1}|=| x_{2} \mid, \mathfrak{} \vdash \\
e\left[\text { isort }_{1} / \text { isort }\right]\left[x_{1} / l\right] \sim e\left[\text { isort }_{2} / \text { isort }\right]\left[x_{2} / l\right]\left\{\left(\begin{array}{c}
\pi_{2} \mathbf{r}_{1} \leqslant \pi_{2} \mathbf{r}_{2} \\
\wedge \mathbf{r}_{1}=\text { isort } x_{1} \\
\wedge \mathbf{r}_{2}=\text { isort } x_{2}
\end{array}\right)\right\}
\end{gathered}
$$

Following the structure of e, we next apply the rule LISTCASE. This yields the following two main proof obligations, corresponding to the two case branches (the third proof obligation, $x_{1}=\left[\Leftrightarrow x_{2}=[\right.$ follows immediately from the assumption $\left.\left|x_{1}\right|=\left|x_{2}\right|\right)$.

$$
\begin{gathered}
\text { isort }_{1}, \text { isort }_{2}, x_{1}, x_{2}\left|\operatorname{sorted}\left(x_{1}\right),\right| x_{1}|=| x_{2} \mid, \iota, x_{1}=x_{2}=\square \vdash([, 0) \sim(\square, 0)\{ \\
\left.\left(\pi_{2} \mathbf{r}_{1} \leqslant \pi_{2} \mathbf{r}_{2}\right) \wedge\left(\mathbf{r}_{1}=\text { isort } x_{1}\right) \wedge\left(\mathbf{r}_{2}=\text { isort } x_{2}\right)\right\}
\end{gathered}
$$

$$
\begin{aligned}
& \text { isort }_{1}, \text { isort }_{2}, x_{1}, x_{2}, h_{1}, t_{1}, h_{2}, t_{2}\left|\operatorname{sorted}\left(x_{1}\right),\right| x_{1}|=| x_{2} \mid, l, x_{1}=h_{1}:: t_{1}, x_{2}=h_{2}:: t_{2} \vdash \\
& \text { let } s=\text { isort }_{1} t_{1} \quad \text { let } s=\text { isort }_{2} t_{2} \quad \mid \pi_{2} \mathbf{r}_{1} \leqslant \pi_{2} \mathbf{r}_{2} \\
& \text { let } s^{\prime}=\text { insert } h_{1}\left(\pi_{1} s\right) \text { in } \sim \text { let } s^{\prime}=\text { insert } h_{2}\left(\pi_{1} s\right) \text { in } \wedge \mathbf{r}_{1}=\text { isort } x_{1} \\
& \left(\pi_{1} s^{\prime},\left(\pi_{2} s\right)+\left(\pi_{2} s^{\prime}\right)\right) \quad\left(\pi_{1} s^{\prime},\left(\pi_{2} s\right)+\left(\pi_{2} s^{\prime}\right)\right) \quad \wedge \mathbf{r}_{2}=\text { isort } x_{2}
\end{aligned}
$$

(A.1) is immediate: By Theorem 3.6, it suffices to show that $\left(\pi_{2}(\square, 0) \leqslant \pi_{2}(\square, 0)\right) \wedge$ $\left((\square, 0)=\right.$ isort $\left.x_{1}\right) \wedge\left((\square, 0)=\right.$ isort $\left.x_{2}\right)$. Since $x_{1}=x_{2}=\square$ by assumption here, this is equivalent to $\left(\pi_{2}(\square, 0) \leqslant \pi_{2}(\square, 0)\right) \wedge((\square, 0)=$ isort $\square) \wedge((\square, 0)=$ isort $\square)$, which is trivial by direct computation.

To prove (A.2), we expand the outermost occurrences of let in both to function applications using the definition let $x=e_{1}$ in $e_{2} \triangleq\left(\lambda x . e_{2}\right) e_{1}$. Applying the rules $\mathrm{APP}$ and ABS, it suffices to prove the following for any $\phi$ of our choice. 


$$
\begin{gathered}
\text { isort }_{1}, \text { isort }_{2}, x_{1}, x_{2}, h_{1}, t_{1}, h_{2}, t_{2}\left|\operatorname{sorted}\left(x_{1}\right),\right| x_{1}|=| x_{2} \mid, l, x_{1}=h_{1}:: t_{1}, x_{2}=h_{2}:: t_{2} \vdash \\
\text { isort }_{1} t_{1} \sim \text { isort }_{2} t_{2} \mid \phi
\end{gathered}
$$

$$
\begin{aligned}
& \text { isort }_{1}, \text { isort }_{2}, x_{1}, x_{2}, h_{1}, t_{1}, h_{2}, t_{2}, s_{1}, s_{2} \mid \\
& \operatorname{sorted}\left(x_{1}\right),\left|x_{1}\right|=\left|x_{2}\right|, \iota, x_{1}=h_{1}:: t_{1}, x_{2}=h_{2}:: t_{2} \underline{\phi\left[s_{1} / r_{1}\right]\left[s_{2} / r_{2}\right]} \vdash
\end{aligned}
$$

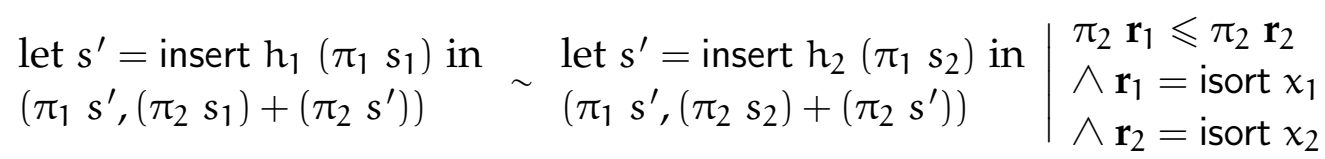

We choose $\phi$ as follows:

$\phi \triangleq \pi_{2} \mathbf{r}_{1} \leqslant \pi_{2} \mathbf{r}_{2} \wedge \mathbf{r}_{1}=\operatorname{isort}\left(t_{1}\right) \wedge \mathbf{r}_{2}=\operatorname{isort}\left(t_{2}\right) \wedge\left|\pi_{1} \mathbf{r}_{1}\right|=\left|\pi_{1} \mathbf{r}_{2}\right| \wedge \operatorname{lmin}\left(t_{1}\right)=\operatorname{lmin}\left(\pi_{1} \mathbf{r}_{1}\right)$

Proof of (A.3): By Theorem 3.6, it suffices to prove the following five statements in HOL under the context of (A.3). These statements correspond to the five conjuncts of $\phi$.

$$
\begin{aligned}
& \pi_{2}\left(\text { isort }_{1} t_{1}\right) \leqslant \pi_{2}\left(\text { isort }_{2} t_{2}\right) \\
& \text { isort }_{1} t_{1}=\text { isort } t_{1} \\
& \text { isort }_{1} t_{2}=\text { isort } t_{2} \\
& \mid \pi_{1}\left(\text { isort }_{1} t_{1}\right)|=| \pi_{1}\left(\text { isort }_{2} t_{2}\right) \mid \\
& \operatorname{Imin}\left(t_{1}\right)=\operatorname{Imin}\left(\pi_{1}\left(\text { isort }_{1} t_{1}\right)\right)
\end{aligned}
$$

(A.5)-(A.7) follow from the induction hypothesis $\iota$ instantiated with $m_{1}:=$ $t_{1}, m_{2}:=t_{2}$. Note that because $x_{1}=h_{1}:: t_{1}$ and $x_{2}=h_{2}:: t_{2}$, we can prove (in HOL) that $\left(\left|t_{1}\right|,\left|t_{2}\right|\right)<\left(\left|x_{1}\right|,\left|x_{2}\right|\right)$. Since, $\left|x_{1}\right|=\left|x_{2}\right|, x_{1}=h_{1}:: t_{1}$ and $x_{2}=h_{2}:: t_{2}$, we can also prove that $\left|t_{1}\right|=\left|t_{2}\right|$. Finally, from the axiomatic definition of sorted and the assumption $\operatorname{sorted}\left(x_{1}\right)$ it follows that sorted $\left(t_{1}\right)$. These together allow us to instantiate the i.h. $\iota$ and immediately derive (A.5)-(A.7).

To prove (A.8), we use (A.6) and (A.7), which reduces (A.8) to $\mid \pi_{1}$ (isort $\left.t_{1}\right) \mid=$ $\mid \pi_{1}$ (isort $\left.t_{2}\right) \mid$. To prove this, we apply Theorem 3 to Lemma A.4, yielding $\forall x . \mid \pi_{1}($ isort $x) \mid=$ $|x|$. Hence, we can further reduce our goal to proving $\left|t_{1}\right|=\left|t_{2}\right|$, which we already did above.

To prove (A.9), we use (A.6), which reduces (A.9) to $\operatorname{lmin}\left(t_{1}\right)=\operatorname{lmin}\left(\pi_{1}\right.$ (isort $\left.t_{1}\right)$ ). This follows immediately from Theorem 3 applied to Lemma A.5. 
This proves (A.3).

Proof of (A.4): We expand the definition of let and apply the rules APP and ABS to reduce (A.4) to proving the following for any $\phi^{\prime}$.

$$
\begin{aligned}
& \text { isort }_{1}, \text { isort }_{2}, x_{1}, x_{2}, h_{1}, t_{1}, h_{2}, t_{2}, s_{1}, s_{2} \\
& \operatorname{sorted}\left(x_{1}\right),\left|x_{1}\right|=\left|x_{2}\right|, l, x_{1}=h_{1}:: t_{1}, x_{2}=h_{2}:: t_{2}, \phi\left[s_{1} / r_{1}\right]\left[s_{2} / r_{2}\right] \vdash \\
& \text { insert } h_{1}\left(\pi_{1} s_{1}\right) \sim \text { insert } h_{2}\left(\pi_{1} s_{2}\right) \mid \phi^{\prime} \\
& \text { isort }_{1}, \text { isort }_{2}, x_{1}, x_{2}, h_{1}, t_{1}, h_{2}, t_{2}, s_{1}, s_{2}, s_{1}^{\prime}, s_{2}^{\prime} \mid \\
& \operatorname{sorted}\left(x_{1}\right),\left|x_{1}\right|=\left|x_{2}\right|, \iota, x_{1}=h_{1}:: t_{1}, x_{2}=h_{2}:: t_{2} \phi\left[s_{1} / \mathbf{r}_{1}\right]\left[s_{2} / \mathbf{r}_{2}\right], \phi^{\prime}\left[s_{1}^{\prime} / \mathbf{r}_{1}\right]\left[s_{2}^{\prime} / \mathbf{r}_{2}\right] \vdash
\end{aligned}
$$

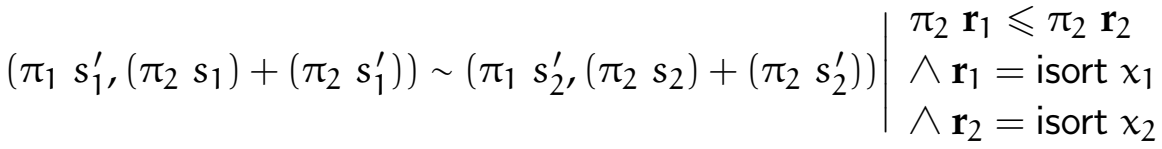

We pick the following $\phi^{\prime}$ :

$$
\phi^{\prime} \triangleq \pi_{2} \mathbf{r}_{1} \leqslant \pi_{2} \mathbf{r}_{2} \wedge \mathbf{r}_{1}=\text { insert } h_{1}\left(\pi_{1} s_{1}\right) \wedge \mathbf{r}_{2}=\text { insert } h_{2}\left(\begin{array}{ll}
\pi_{1} & s_{2}
\end{array}\right)
$$

Proof of (A.10): We start by applying Theorem 3.6. This yields three subgoals in HOL, corresponding to the three conjuncts in $\phi^{\prime}$ :

$$
\begin{gathered}
\pi_{2}\left(\text { insert } h_{1}\left(\begin{array}{ll}
\pi_{1} & s_{1}
\end{array}\right)\right) \leqslant \pi_{2}\left(\text { insert } h_{2}\left(\begin{array}{ll}
\pi_{1} & s_{2}
\end{array}\right)\right) \\
\text { insert } h_{1}\left(\begin{array}{lll}
\pi_{1} & s_{1}
\end{array}\right)=\text { insert } h_{1}\left(\begin{array}{ll}
\pi_{1} & s_{1}
\end{array}\right) \\
\text { insert } h_{2}\left(\begin{array}{lll}
\pi_{1} & s_{2}
\end{array}\right)=\text { insert } h_{2}\left(\begin{array}{lll}
\pi_{1} & s_{2}
\end{array}\right)
\end{gathered}
$$

(A.13) and (A.14) are trivial, so we only have to prove (A.12). Since $s_{1}=$ isort $t_{1}$ and $s_{2}=$ isort $t_{2}$ are conjuncts in the assumption $\phi\left[s_{1} / \mathbf{r}_{1}\right]\left[s_{2} / \mathbf{r}_{2}\right],($ A.12) is equivalent to:

$$
\pi_{2}\left(\text { insert } h_{1}\left(\pi_{1}\left(\text { isort } t_{1}\right)\right)\right) \leqslant \pi_{2} \text { (insert } h_{2}\left(\pi_{1}\left(\text { isort } t_{2}\right)\right) \text { ) }
$$

To prove this, we split cases on the shapes of $\pi_{1}$ (isort $t_{1}$ ) and $\pi_{1}$ (isort $t_{2}$ ). From the conjuncts in $\phi\left[s_{1} / \mathbf{r}_{1}\right]\left[s_{2} / \mathbf{r}_{2}\right]$, it follows immediately that $\mid \pi_{1}\left(\right.$ isort $\left.t_{1}\right)|=| \pi_{1}\left(\right.$ isort $\left.t_{2}\right) \mid$. Hence, only two cases apply:

Case: $\pi_{1}\left(\right.$ isort $\left.t_{1}\right)=\pi_{1}\left(\right.$ isort $\left.t_{2}\right)=[$. In this case, by direct computation,

$$
\pi_{2}\left(\text { insert } h_{1}\left(\pi_{1}\left(\text { isort } t_{1}\right)\right)\right)=\pi_{2}\left(\text { insert } h_{1}[)=\pi_{2}\left(\left[h_{1}\right], 0\right)=0\right. \text {. }
$$

Similarly, and $\pi_{2}$ (insert $h_{2}\left(\pi_{1}\left(\right.\right.$ isort $\left.\left.\left.t_{2}\right)\right)\right)=0$. So, the result follows trivially. 
Case: $\pi_{1}$ (isort $\left.t_{1}\right)=h_{1}^{\prime}:: t_{1}^{\prime}$ and $\pi_{1}$ (isort $\left.t_{2}\right)=h_{2}^{\prime}:: t_{2}^{\prime}$. We first argue that $h_{1} \leqslant h_{1}^{\prime}$. Note that from the second and fifth conjuncts in $\phi\left[s_{1} / \mathbf{r}_{1}\right]\left[s_{2} / \mathbf{r}_{2}\right]$, it follows that $\operatorname{Imin}\left(t_{1}\right)=\operatorname{Imin}\left(\pi_{1}\left(\right.\right.$ isort $\left.\left.t_{1}\right)\right)$. Since $\pi_{1}$ (isort $\left.t_{1}\right)=h_{1}^{\prime}:: t_{1}^{\prime}$, we further get $\operatorname{Imin}\left(t_{1}\right)=$ $\operatorname{Imin}\left(\pi_{1}\left(\right.\right.$ isort $\left.\left.t_{1}\right)\right)=\operatorname{Imin}\left(h_{1}^{\prime}:: t_{1}^{\prime}\right)=\min \left(h_{1}^{\prime}, \operatorname{Imin}\left(t_{1}^{\prime}\right)\right) \leqslant h_{1}^{\prime}$. Finally, from the axiomatic definition of sorted $\left(x_{1}\right)$ and $x_{1}=h_{1}:: t_{1}$, we derive $h_{1} \leqslant \operatorname{lmin}\left(t_{1}\right)$. Combining, we get $h_{1} \leqslant \operatorname{lmin}\left(t_{1}\right) \leqslant h_{1}^{\prime}$.

Next, $\pi_{2}$ (insert $h_{1}\left(\pi_{1}\left(\right.\right.$ isort $\left.\left.\left.t_{1}\right)\right)\right)=\pi_{2}\left(\right.$ insert $\left.h_{1}\left(h_{1}^{\prime}:: t_{1}^{\prime}\right)\right)$. Expanding the definition of insert and using $h_{1} \leqslant h_{1}^{\prime}$, we immediately get $\pi_{2}$ (insert $h_{1}\left(\pi_{1}\left(\right.\right.$ isort $\left.\left.\left.t_{1}\right)\right)\right)=$ $\pi_{2}\left(\right.$ insert $\left.h_{1}\left(h_{1}^{\prime}:: t_{1}^{\prime}\right)\right)=\pi_{2}\left(h_{1}:: h_{1}^{\prime}:: t_{1}^{\prime}, 1\right)=1$. On the other hand, it is fairly easy to prove (by case analyzing the result of $\left.h_{2} \leqslant h_{2}^{\prime}\right)$ that $\pi_{2}$ (insert $h_{2}\left(\pi_{1}\left(\right.\right.$ isort $\left.\left.\left.t_{2}\right)\right)\right)=$ $\pi_{2}\left(\right.$ insert $\left.h_{2}\left(h_{2}^{\prime}:: t_{2}^{\prime}\right)\right) \geqslant 1$. Hence,

$$
\pi_{2}\left(\text { insert } h_{1}\left(\pi_{1}\left(\text { isort } t_{1}\right)\right)\right)=1 \leqslant \pi_{2}\left(\text { insert } h_{2}\left(\pi_{1}\left(\text { isort } t_{2}\right)\right)\right) \text {. }
$$

This proves (A.15) and, hence, (A.12) and (A.10).

Proof of (A.11): By Theorem 3.6, it suffices to show the following in HOL, under the assumptions of (A.11):

$$
\begin{gathered}
\pi_{2}\left(\pi_{1} s_{1}^{\prime},\left(\pi_{2} s_{1}\right)+\left(\begin{array}{ll}
\pi_{2} & s_{1}^{\prime}
\end{array}\right)\right) \leqslant \pi_{2}\left(\pi_{1} s_{2}^{\prime},\left(\begin{array}{ll}
\pi_{2} & s_{2}
\end{array}\right)+\left(\begin{array}{ll}
\pi_{2} & s_{2}^{\prime}
\end{array}\right)\right) \\
\left(\pi_{1} s_{1}^{\prime},\left(\pi_{2} s_{1}\right)+\left(\pi_{2} s_{1}^{\prime}\right)\right)=\text { isort } x_{1} \\
\left(\pi_{1} s_{2}^{\prime},\left(\pi_{2} s_{2}\right)+\left(\pi_{2} s_{2}^{\prime}\right)\right)=\text { isort } x_{2}
\end{gathered}
$$

By computation, (A.16) is equivalent to $\left(\pi_{2} s_{1}\right)+\left(\begin{array}{ll}\pi_{2} & s_{1}^{\prime}\end{array}\right) \leqslant\left(\begin{array}{ll}\pi_{2} & s_{2}\end{array}\right)+\left(\begin{array}{ll}\pi_{2} & s_{2}^{\prime}\end{array}\right)$. Using the definition of $\phi$, it is easy to see that $\pi_{2} s_{1} \leqslant \pi_{2} s_{2}$ is a conjunct in the assumption $\phi\left[s_{1} / \mathbf{r}_{1}\right]\left[s_{2} / \mathbf{r}_{2}\right]$. Similarly, using the definition of $\phi^{\prime}, \pi_{2} s_{1}^{\prime} \leqslant \pi_{2} s_{2}^{\prime}$ is a conjunct in the assumption $\phi^{\prime}\left[s_{1}^{\prime} / \mathbf{r}_{1}\right]\left[s_{2}^{\prime} / \mathbf{r}_{2}\right]$. (A.16) follows immediately from these.

To prove (A.17), note that since $x_{1}=h_{1}:: t_{1}$, expanding the definition of isort, we get

$$
\text { isort } x_{1}=\left(\pi_{1}\left(\text { insert } h_{1}\left(\pi_{1}\left(\text { isort } t_{1}\right)\right)\right), \pi_{2}\left(\text { isort } t_{1}\right)+\pi_{2}\left(\text { insert } h_{1}\left(\pi_{1}\left(\text { isort } t_{1}\right)\right)\right)\right)
$$

Matching with the left side of (A.17), it suffices to show that $s_{1}^{\prime}=$ insert $h_{1}\left(\pi_{1}\right.$ (isort $\left.t_{1}\right)$ ) and $s_{1}=$ isort $t_{1}$. These are immediate: $s_{1}=$ isort $t_{1}$ is a conjunct in the assumption $\phi\left[s_{1} / \mathbf{r}_{1}\right]\left[s_{2} / \mathbf{r}_{2}\right]$, while $s_{1}^{\prime}=$ insert $h_{1}\left(\pi_{1}\left(\right.\right.$ isort $\left.\left.t_{1}\right)\right)$ follows trivially from this and the conjunct $s_{1}^{\prime}=$ insert $h_{1}\left(\pi_{1} s_{1}\right)$ in $\phi^{\prime}\left[s_{1}^{\prime} / \mathbf{r}_{1}\right]\left[s_{2}^{\prime} / \mathbf{r}_{2}\right]$. This proves (A.17).

The proof of (A.18) is similar to that of (A.17).

This proves (A.11) and, hence, (A.4). 


\section{APPENDIX OF CHAPTER 8}

\section{B.o.1 Equational theory of the calculus}

The denotational semantics validates the following equational theory in addition to the standard equational theory of the simply typed lambda calculus with sums and natural numbers.

Rules for fixed points, always modality and streams

$$
\begin{aligned}
& \text { fix f.t } \equiv t[\triangleright(\text { fix f. } t) / f] \\
& \operatorname{prev}(\triangleright t) \equiv t \\
& \text { letb } x \leftarrow(\text { box } u) \text { in } t \equiv t[u / x] \\
& \text { letc } x \leftarrow u \text { in } t \equiv t[u / x] \\
& \begin{aligned}
\text { hd }(x:: x s) & \equiv x \\
\operatorname{tl}(x:: x s) & \equiv x s \\
\text { hd } t:: t l t & \equiv t
\end{aligned}
\end{aligned}
$$

Rules for delayed substitutions

$$
\begin{aligned}
& \triangleright \xi[x \leftarrow t] . u \equiv \triangleright \xi . u \quad \text { if } x \text { not in } u \\
& \triangleright \xi[x \leftarrow t, y \leftarrow s] \xi^{\prime} \cdot u \equiv \triangleright \xi[y \leftarrow s, x \leftarrow t] \xi^{\prime} \cdot u \\
& \triangleright \xi[x \leftarrow \triangleright \xi . t] . u \equiv \triangleright \xi .(u[t / x]) \\
& \triangleright[x \leftarrow t] . x \equiv t
\end{aligned}
$$

Monad laws for distributions

$$
\begin{aligned}
\text { mlet } x=\operatorname{unit}(t) \text { in } u & \equiv u[t / x] \\
\text { mlet } x=t \text { in unit }(x) & \equiv t \\
\text { mlet } x_{2}=\left(\text { mlet } x_{1}=t_{1} \text { in } t_{2}\right) \text { in } u & \equiv \text { mlet } x_{1}=t_{1} \text { in }\left(\text { mlet } x_{2}=t_{2} \text { in } u\right)
\end{aligned}
$$

In particular, notice that fix does not reduce as usual, but instead the whole term is delayed before the substitution is performed. 


\section{B.I Additional background}

One consequence of Strassen's theorem is that couplings are closed under convex combinations.

Lemma B.I (Convex combinations of couplings). Let $\left(\mu_{i}\right)_{i \in I}$ and $\left(v_{i}\right)_{i \in I}$ bet two families of distributions on $\mathrm{C}_{1}$ and $\mathrm{C}_{2}$ respectively, and let $\left(\mathrm{p}_{\mathrm{i}}\right)_{\mathrm{i} \in \mathrm{I}} \in[0,1]$ such that $\sum_{i \in \mathrm{I}} p_{i}=1$. If

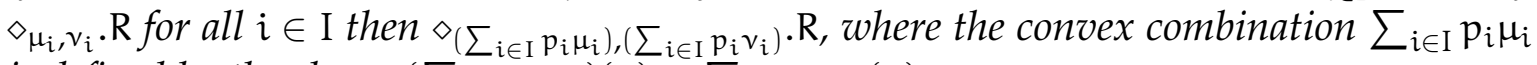
is defined by the clause $\left(\sum_{i \in \mathrm{I}} p_{i} \mu_{i}\right)(x)=\sum_{i \in \mathrm{I}} p_{i} \mu_{i}(x)$.

One obtains an asymmetric version of the lemma by observing that if $\mu_{i}=\mu$ for every $i \in I$, then $\left(\sum_{i \in I} p_{i} \mu_{i}\right)=\mu$.

One can also show that couplings are closed under relation composition.

Lemma B.2 (Couplings for relation composition). Let $\mu_{1} \in D\left(C_{1}\right), \mu_{2} \in D\left(C_{2}\right), \mu_{3} \in$ $\mathrm{D}\left(\mathrm{C}_{3}\right)$. Moreover, let $\mathrm{R} \subseteq \mathrm{C}_{1} \times \mathrm{C}_{2}$ and $\mathrm{S} \subseteq \mathrm{C}_{2} \times \mathrm{C}_{3}$. If $\diamond_{\mu_{1}, \mu_{2}} . \mathrm{R}$ and $\diamond_{\mu_{2}, \mu_{3}} . \mathrm{R}$ then $\diamond_{\mu_{1}, \mu_{3}} . \mathrm{R}$.

\section{B.2 Unary fragment}

In this section we introduce a unary system to prove properties about a single term of the guarded lambda calculus. We will start by adding some definitions Guarded HOL for the unary diamond monad, following by the derivation rules for both the non-probabilistic and the probabilistic system, plus the metatheory and an example.

\section{B.2.1 Unary fragment of GHOL}

The unary semantics of the diamond monad are:

$$
\llbracket \diamond_{[x \leftarrow \mathrm{t}]} \phi \rrbracket_{i} \triangleq\left\{(\delta, \gamma) \mid \operatorname{Pr}_{\nu \leftarrow\left(\llbracket t \rrbracket_{i}(\delta, \gamma)\right)}\left[(\delta,(\gamma, v)) \in \llbracket \phi \rrbracket_{i}\right]=1\right\}
$$

The rules are on Figure B.I

$$
\begin{gathered}
\frac{\Delta|\Sigma| \Gamma\left|\Psi \vdash \diamond_{[x \leftarrow \mathrm{t}]} \phi \quad \Delta\right| \Sigma|\Gamma, x: C| \Psi, \phi \vdash \psi}{\Delta|\Sigma| \Gamma \mid \Psi \vdash \diamond_{[x \leftarrow \mathrm{t}]} \psi} \text { MONO1 } \\
\frac{\Delta|\Sigma| \Gamma \mid \Psi \vdash \phi[\mathrm{t} / \mathrm{x}]}{\Delta|\Sigma| \Gamma \mid \Psi \vdash \diamond_{[\mathrm{x} \leftarrow \text { unit }(\mathrm{t})]} \phi} \text { UNIT1 } \\
\frac{\Delta|\Sigma| \Gamma\left|\Psi \vdash \diamond_{[\mathrm{x} \leftarrow \mathrm{t}]} \phi \quad \Delta\right| \Sigma|\Gamma, \mathrm{x}: \mathrm{C}| \Psi, \phi \vdash \diamond_{\left[\mathrm{y} \leftarrow \mathrm{t}^{\prime}\right]} \psi}{\Delta|\Sigma| \Gamma \mid \Psi \vdash \diamond_{\left[\mathrm{y} \leftarrow \text { mlet } \mathrm{x}=\mathrm{t} \text { in } \mathrm{t}^{\prime}\right]} \psi} \text { MLET1 }
\end{gathered}
$$

Figure B.1: Rules for the unary diamond modality 


\section{B.2.2 Guarded UHOL}

We start by defining the Guarded UHOL system, which allows us to prove logical properties of a term of the Guarded Lambda Calculus. More concretely, judgments have the form:

$$
\Delta|\Sigma| \Gamma \mid \Psi \vdash t: \sigma\{\phi\}
$$

where $t$ is a term well-typed in the dual context $\Delta \mid \Gamma$ and $\phi$ is a logical formula well-typed in the context $\Delta \mid \Gamma, \mathbf{r}: \sigma$ and that can refer to $t$ via the special variable $\mathbf{r}$. The logical contexts $\Sigma$ and $\Psi$ consist respectively of refinements over the contexts $\Delta$ and $\Gamma$.

\section{B.2.3 Derivation rules}

The rule NextU corresponds to the introduction of the later modality. A refinement $\Phi_{i}$ is proven on every term in the substitution, and using those as a premise, a refinement $\Phi$ is proven on $\mathrm{t}$. In the notation $\triangleright[\mathbf{r} \leftarrow \mathbf{r}] . \Phi$ the first $\mathbf{r}$ is the variable bound by the delayed substitution inside $\Phi$ while the second $\mathbf{r}$ is the distinguished variable in the refinement that refers to the term that is being typed. In other words, $\mathrm{t}$ satisfies $\triangleright[\mathbf{r} \leftarrow \mathbf{r}]$. $\Phi$ if $\triangleright[\mathbf{r} \leftarrow \mathbf{t}]$. $\Phi$. The rule PrevU corresponds to the elimination of the later modality. If we can prove $\triangleright \phi$ in a constant context, then we can also prove $\phi$. The rule BoxU applies the constant modality on a formula that can be proven on a constant context. The rule LetBoxU removes the constant modality from a formula $\Phi$ by using it as a constant premise to prove another formula $\Phi^{\prime}$. The rule LetConstU shifts constant terms between contexts. The rule FixU introduces a fixpoint and proves a refinement on it by Loeb induction. The rule ConsU proves a property on a stream from a refinement on its head and its tail. The rule ConsHatU is the analogue of Cons $\mathrm{U}$ to build constant streams. In particular, the $\hat{:}$ operator can be defined as $\lambda x$. $\lambda$ s.letb $(y, t) \leftarrow(x, s)$ in box $(y:: \Delta t)$. Conversely the rules HeadU and TailU respectively prove a property on the head and the tail of a stream from a property on the full stream.

The intended meaning for a judgment $\Delta|\Sigma| \Gamma \mid \Psi \vdash t: \tau\{\phi\}$ is: "For every valuations $\delta, \gamma$ of $\Delta$ and $\Gamma$,

$$
\llbracket \Delta|\Gamma \vdash \square \Sigma \rrbracket(\delta, \gamma) \wedge \llbracket \Delta| \Gamma \vdash \Psi \rrbracket(\delta, \gamma) \Rightarrow \llbracket \Delta \mid \Gamma, \mathbf{r}: \tau \vdash \Sigma \rrbracket(\delta,\langle\gamma, \llbracket \Delta \mid \Gamma \vdash t \rrbracket(\delta, \gamma)\rangle) ”
$$

\section{B.2.4 Metatheory}

Guarded UHOL is equivalent to Guarded HOL:

Theorem B.3 (Equivalence with Guarded HOL). For every contexts $\Delta$, $\Gamma$, type $\sigma$, term $\mathrm{t}$, sets of assertions $\Sigma, \Psi$ and assertion $\phi$, the following are equivalent:

- $\Delta|\Sigma| \Gamma \mid \Psi \vdash \mathrm{t}: \sigma\{\phi\}$ 
$\Delta|\Sigma| \Gamma, x_{1}: A_{1}, \ldots, x_{n}: A_{n} \mid \Psi, \Phi_{1}\left[x_{1} / \mathbf{r}\right], \ldots, \Phi_{n}\left[x_{n} / \mathbf{r}\right] \vdash t: A\{\Phi\}$

$\frac{\Delta|\Sigma| \Gamma\left|\Psi \vdash \mathrm{t}_{1}: \triangleright A_{1}\left\{\triangleright[\mathbf{r} \leftarrow \mathbf{r}] . \Phi_{1}\right\} \quad \ldots \quad \Delta\right| \Sigma|\Gamma| \Psi \vdash \mathrm{t}_{n}: \triangleright A_{n}\left\{\triangleright[\mathbf{r} \leftarrow \mathbf{r}] . \Phi_{n}\right\}}{\Delta|\Sigma| \Gamma \mid \Psi \vdash \triangleright\left[\mathrm{x}_{1} \leftarrow \mathrm{t}_{1}, \ldots, \mathrm{x}_{\mathrm{n}} \leftarrow \mathrm{t}_{\mathrm{n}}\right] . \mathrm{t}: \triangleright A\left\{\triangleright\left[\mathrm{x}_{1}, \ldots, \mathrm{x}_{\mathrm{n}}, \mathbf{r} \leftarrow \mathrm{t}_{1}, \ldots, \mathrm{t}_{\mathrm{n}}, \mathbf{r}\right] . \Phi\right\}} \operatorname{Next} U$

$$
\frac{\Delta|\Sigma| \cdot \mid \cdot \vdash \mathrm{t}: \triangleright A\{\triangleright[\mathbf{r} \leftarrow \mathbf{r}] . \Phi\}}{\Delta|\Sigma| \Gamma \mid \Psi \vdash \operatorname{prev} \mathrm{t}: \mathrm{A}\{\Phi\}} \operatorname{PrevU} \frac{\Delta|\Sigma| \cdot \mid \cdot \vdash \mathrm{t}: \mathrm{A}\{\Phi\}}{\Delta|\Sigma| \Gamma \mid \Psi \vdash \operatorname{box} \mathrm{t}: \square \mathrm{A}\{\square \Phi[\text { letb } x \leftarrow \mathbf{r} \text { in } x / \mathbf{r}]\}} \text { BoxU }
$$

$\frac{\Delta|\Sigma| \Gamma \mid \Psi \vdash \mathfrak{u}: \square \mathrm{B}\{\square \Phi[\text { letb } \mathrm{x} \leftarrow \mathbf{r} \text { in } \mathrm{x} / \mathbf{r}]\} \quad \Delta, \mathrm{x}: \mathrm{B}|\Sigma, \Phi[\mathrm{x} / \mathbf{r}]| \Gamma \mid \Psi \vdash \mathrm{t}: \mathrm{A}\left\{\Phi^{\prime}\right\}}{\Delta|\Sigma| \Gamma \mid \Psi \vdash \text { letb } \mathrm{x} \leftarrow \mathrm{u} \text { in } \mathrm{t}: \mathrm{A}\left\{\Phi^{\prime}\right\}}$ LetBoxU

$$
\begin{gathered}
\Delta|\Sigma| \Gamma \mid \Psi \vdash u: B\{\Phi\} \\
\frac{\Delta, x: B|\Sigma, \Phi[x / \mathbf{r}]| \Gamma \mid \Psi \vdash t: A\left\{\Phi^{\prime}\right\} \quad \text { B, } \Phi \text { constant } \quad F V(\Phi) \cap F V(\Gamma)=\emptyset}{\Delta|\Sigma| \Gamma \mid \Psi \vdash \text { letc } x \leftarrow u \text { in } \mathrm{t}: \mathrm{A}\left\{\Phi^{\prime}\right\}} \text { LetConstU }
\end{gathered}
$$

$$
\frac{\Delta|\Sigma| \Gamma, f: \triangleright A \mid \triangleright[\mathbf{r} \leftarrow f] . \Phi \vdash t: A\{\Phi\}}{\Delta|\Sigma| \Gamma \mid \Psi \vdash \text { fixf.t: } A\{\Phi\}} \text { FixU }
$$

$$
\Delta|\Sigma| \Gamma \mid \Psi \vdash x: A\left\{\Phi_{h}\right\}
$$

$$
\frac{\Delta|\Sigma| \Gamma\left|\Psi \vdash x \mathrm{~s}: \triangleright \operatorname{Str}_{\mathrm{A}}\left\{\Phi_{\mathrm{t}}\right\} \quad \Gamma\right| \Psi \vdash \forall x, \mathrm{xs} . \Phi_{\mathrm{h}}[\mathrm{x} / \mathbf{r}] \Rightarrow \Phi_{\mathrm{t}}[\mathrm{xs} / \mathbf{r}] \Rightarrow \Phi[\mathrm{x}:: \mathrm{xs} / \mathbf{r}]}{\Delta|\Sigma| \Gamma \mid \Psi \vdash x:: \mathrm{xs}: \operatorname{Str}_{\mathrm{A}}\{\Phi\}} \text { ConsU }
$$

$\Delta|\Sigma| \Gamma\left|\Psi \vdash x: A\left\{\Phi_{h}\right\} \quad \Delta\right| \Sigma|\Gamma| \Psi \vdash x s: \square \operatorname{Str}_{\mathrm{A}}\left\{\square \Phi_{\mathrm{t}}\right\}$

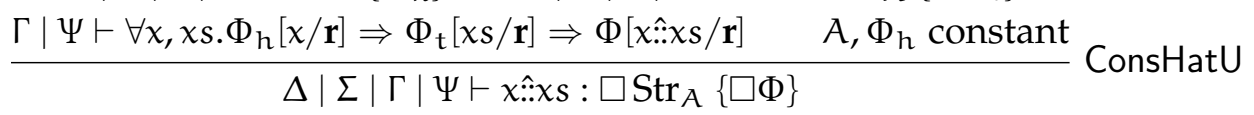

$\frac{\Delta|\Sigma| \Gamma \mid \Psi \vdash \mathrm{t}: \operatorname{Str}_{\mathrm{A}}\{\Phi[\mathrm{hd} \mathbf{r} / \mathbf{r}]\}}{\Delta|\Sigma| \Gamma \mid \Psi \vdash \mathrm{hd} \mathrm{t}: \mathrm{A}\{\Phi\}} \operatorname{HeadU} \frac{\Delta|\Sigma| \Gamma \mid \Psi \vdash \mathrm{t}: \operatorname{Str}_{\mathrm{A}}\{\Phi[\mathrm{tl} \mathbf{r} / \mathbf{r}]\}}{\Delta|\Sigma| \Gamma \mid \Psi \vdash \mathrm{tl} \mathrm{t}: \triangleright \operatorname{Str}_{\mathrm{A}}\{\Phi\}} \operatorname{TailU}$

Figure B.2: Guarded Unary Higher-Order Logic rules

- $\Delta|\Sigma| \Gamma \mid \Psi \vdash \phi[\mathrm{t} / \mathbf{r}]$

The previous result allows us to lift the soundness result from Guarded HOL to Guarded UHOL.

Corollary B.4 (Soundness and consistency). If $\Delta|\Sigma| \Gamma \mid \Psi \vdash \mathrm{t}: \sigma\{\phi\}$, then for every valuations $\delta \models \Delta, \gamma \models \Gamma$ :

$$
\llbracket \Delta \vdash \Sigma \rrbracket(\delta) \wedge \llbracket \Delta|\Gamma \vdash \Psi \rrbracket(\delta, \gamma) \Rightarrow \llbracket \Delta| \Gamma, \mathbf{r}: \sigma \vdash \phi \rrbracket(\delta, \gamma[\mathbf{r} \leftarrow \llbracket \Delta \mid \Gamma \vdash \mathrm{t} \rrbracket(\delta, \gamma)])
$$

In particular, there is no proof of $\Delta|\emptyset| \Gamma \mid \emptyset \vdash \mathrm{t}: \sigma\{\perp\}$ in Guarded UHOL. 


\section{B.2.5 Probabilistic extension}

We comment on the rules, starting from the rules of the unary logic. There are three new rules for the probabilistic case, and they all establish that an expression $u$ of type $D(D)$ satisfies the assertion $\diamond_{[y \leftarrow r]} \phi$, i.e. for every element $v$ in the support of (the interpretation of) $u$, the interpretation of $\phi$ with the valuation $[y \mapsto v]$ is true. This intuition is captured by the rule SUPP, which can be used in particular in case $u$ is a primitive distribution. The rule UNIT-U considers the case where $u$ is of the form unit $(t)$; in this case, it is clearly sufficient to know that $\phi[t / y]$ is valid. The rule MLET-U simply captures the fact that the support of mlet $x=t$ in $t^{\prime}$ is the disjoint union of the support of $t^{\prime}$ under all the assignments of $x$ to values in the support of t.

Guarded UHOL

$$
\begin{aligned}
& \frac{\Delta|\Sigma| \Gamma \mid \Psi \vdash \mathrm{t}: \mathrm{C}\{\Phi[\mathbf{r} / \mathrm{y}]\}}{\Delta|\Sigma| \Gamma \mid \Psi \vdash \operatorname{unit}(\mathrm{t}): \mathrm{D}(\mathrm{C})\left\{\diamond_{[\mathrm{y} \leftarrow \mathbf{r}]} \Phi\right\}} \text { UNIT }-\mathrm{U} \\
& \frac{\Delta|\Sigma| \Gamma\left|\Psi \vdash \mathrm{t}: \mathrm{D}(\mathrm{C})\left\{\diamond_{[\mathrm{x} \leftarrow \mathrm{r}]} \Phi\right\} \quad \Delta\right| \Sigma|\Gamma, \mathrm{x}: \mathrm{C}| \Psi, \phi \vdash \mathrm{t}^{\prime}: \mathrm{D}(\mathrm{D})\left\{\diamond_{[\mathrm{y} \leftarrow \mathbf{r}]} \psi\right\}}{\Delta|\Sigma| \Gamma \mid \Psi \vdash \operatorname{mlet} x=\mathrm{t} \operatorname{in~}^{\prime}: \mathrm{D}(\mathrm{D})\left\{\diamond_{[\mathrm{y} \leftarrow \mathbf{r}]} \psi\right\}} \text { MLET }-\mathrm{U} \\
& \frac{\Delta|\Sigma| \Gamma \mid \Psi \vdash \operatorname{Pr}_{z \sim u}[\phi[z / y]]=1}{\Delta|\Sigma| \Gamma \mid \Psi \vdash u: D(D)\left\{\diamond_{[y \leftarrow r]} \phi\right\}} \operatorname{SUPP}-U
\end{aligned}
$$

Figure B.3: Proof rules for probabilistic constructs - unary case

Finally, we prove an embedding lemma for Guarded UHOL. The proof can be carried by induction on the structure of derivations, or using the equivalence between Guarded UHOL and Guarded HOL (Theorem 3.3).

Lemma B.5 (Embedding lemma). Assume that:

- $\Delta|\Sigma| \Gamma \mid \Psi \vdash \mathrm{t}_{1}: \sigma_{1}\{\phi\}$

- $\Delta|\Sigma| \Gamma \mid \Psi \vdash t_{2}: \sigma_{2}\left\{\phi^{\prime}\right\}$

Then $\Delta|\Sigma| \Gamma \mid \Psi \vdash \mathrm{t}_{1}: \sigma_{1} \sim \mathrm{t}_{2}: \sigma_{2}\left\{\phi\left[\mathbf{r}_{1} / \mathbf{r}\right] \wedge \phi^{\prime}\left[\mathbf{r}_{2} / \mathbf{r}\right]\right\}$.

\section{B.2.6 Unary example: Every two}

We define the every 2 function, which receives a stream and returns another stream consisting of the elements at even positions in the input stream. Note that this function, while productive, cannot be built with the type $S \operatorname{tr} \rightarrow S t r$, since we need to take twice the tail of the argument, which would have type $\triangleright \triangleright S t r$, and then a Str cannot be built. Instead, we need to use the constant modality as follows: 


$$
\begin{aligned}
& \text { every } 2: \square \operatorname{Str} \rightarrow \operatorname{Str} \\
& \text { every } 2 \triangleq \text { fix every } 2 . \lambda s . \hat{h d}(\hat{t} l s)::(\operatorname{every} 2 \circledast \operatorname{next}(\hat{t} l(\hat{t l} s)))
\end{aligned}
$$

Where the hid and $\hat{t l}$ functions are not the native ones, but rather they are defined as:

$$
\begin{array}{ll}
\hat{\text { hd }:} \square \mathrm{Str} \rightarrow \text { nat } & \hat{\mathrm{tl}}: \square \mathrm{Str} \rightarrow \square \mathrm{Str} \\
\hat{\mathrm{hd}} \triangleq \lambda \text { s.letb } x \leftarrow \mathrm{s} \text { in hd } x & \hat{\mathrm{tl}} \triangleq \lambda \text { s.letb } x \leftarrow s \text { in box (prev (tl } x) \text { ) }
\end{array}
$$

The property we want to prove is:

$$
\cdot|\cdot| \text { ones }: \square \operatorname{Str} \mid \Psi \vdash \text { every } 2: \square \operatorname{Str} \rightarrow \operatorname{Str}\{\forall \text { s.s }=\text { ones } \Rightarrow \mathbf{r} s=(\text { letb } x \leftarrow s \text { in } x)\}
$$

where ones is the constant stream containing only the number 1 defined as:

$$
\text { ones } \triangleq \text { box (fix f. } 1:: \mathrm{f})
$$

For which we can prove the following properties:

$$
\Psi \triangleq \hat{h d} \text { ones }=1, \hat{t l} \text { ones }=\text { ones }
$$

In the rest of the proof we omit the empty contexts $\Delta$ and $\Sigma$. We start by applying the Fix rule, which has the premise:

$$
\begin{gathered}
\text { ones }: \square \text { Str, every } 2: \triangleright(\square \operatorname{Str} \rightarrow \operatorname{Str}) \mid \\
\Psi, \triangleright[\mathbf{r} \leftarrow \text { every } 2] . \forall \text { s.s }=\text { ones } \Rightarrow \mathbf{r} s=\text { letb } x \leftarrow s \text { in } x \vdash \\
\lambda s .(\cdots): \square \operatorname{Str} \rightarrow \operatorname{Str}\{\forall \text { s.s }=\text { ones } \Rightarrow(\mathbf{r} s)=\text { letb } x \leftarrow s \text { in } x\}
\end{gathered}
$$

We apply the Abs rule inmediately after:

$$
\begin{gathered}
\text { ones }: \square \text { Str, every } 2: \triangleright(\square \text { Str } \rightarrow \text { Str }), s: \square \text { Str } \mid \\
\Psi, \triangleright[\mathbf{r} \leftarrow \text { every } 2] . \forall \text { s.s }=\text { ones } \Rightarrow \mathbf{r} s=\text { letb } x \leftarrow s \text { in } x, s=\text { ones } \vdash \\
\hat{h d}(\hat{t l} s)::(\text { every } 2 \circledast \triangleright(\hat{t l}(\hat{t} l s))): S \operatorname{tr}\{\mathbf{r}=\text { letb } x \leftarrow s \text { in } x\}
\end{gathered}
$$

By SUB and the equivalence letb $x \leftarrow s$ in $x \equiv$ letb $x \leftarrow$ ones in $x$, we can change the conclusion of the judgment. Now we use the Cons rule, which has three premises:

1. ones : $\square$ Str, every $2: \triangleright(\square$ Str $\rightarrow$ Str $), s: \square$ Str $\Psi, \triangleright[\mathbf{r} \leftarrow$ every 2$] . \forall s . s=$ ones $\Rightarrow \mathbf{r} s=$ letb $x \leftarrow s$ in $x, s=$ ones $\vdash \hat{h d}(\hat{t} \hat{l} s):$ nat $\{\mathbf{r}=1\}$

2. ones: $\square$ Str, every $2: \triangleright(\square$ Str $\rightarrow$ Str $), s: \square \operatorname{Str}$

$\Psi, \triangleright[\mathbf{r} \leftarrow$ every 2$] . \forall s . s=$ ones $\Rightarrow \mathbf{r} s=$ letb $x \leftarrow s$ in $x, s=$ ones $\vdash$ $($ every $2 \circledast \triangleright(\hat{t} \hat{l}(\hat{t l} s))): \operatorname{Str}\{\triangleright[\mathbf{r} \leftarrow \mathbf{r}] . \mathbf{r}=$ letb $x \leftarrow$ ones in $x\}$ 
3. ones: $\square$ Str, every2: $\triangleright(\square$ Str $\rightarrow$ Str $), s: \square$ Str

$\Psi, \triangleright[\mathbf{r} \leftarrow$ every 2$] . \forall$ s.s $=$ ones $\Rightarrow \mathbf{r} s=$ letb $x \leftarrow s$ in $x, s=$ ones $\vdash$

$\forall y, y s . y=1 \Rightarrow \triangleright[z s \leftarrow y s] .(z s=$ letb $x \leftarrow$ ones in $x) \Rightarrow y::$ ys $=($ letb $x \leftarrow$ ones in $x$ )

Premise ( 1 ) is a consequence of the properties of ones. To prove premise (3) we reduce the letbox with the box inside ones, and do some reasoning using the definition of the fixpoint. To prove the premise (2) we first desugar the term we are typing:

$$
\text { every } 2 \circledast \triangleright(\hat{t} l(\hat{t l} s))) \triangleq \triangleright[g \leftarrow \operatorname{every} 2, t \leftarrow \triangleright(\hat{t} l(\hat{t l} s))] . g t
$$

and then we apply Next which has the following premises:

- ones : $\square$ Str, every2 : $\triangleright(\square$ Str $\rightarrow$ Str $), s: \square$ Str $\mid$

$\Psi, \triangleright[\mathbf{r} \leftarrow$ every 2$] . \forall$ s.s $=$ ones $\Rightarrow \mathbf{r} s=$ letb $x \leftarrow s$ in $x, s=$ ones $\vdash$

every2 $: \triangleright(\square$ Str $\rightarrow$ Str $)\{\triangleright[\mathbf{r} \leftarrow \mathbf{r}] .(\forall s=$ ones $\Rightarrow \mathbf{r} s=$ letb $x \leftarrow s$ in $x)\}$

- ones : $\square$ Str, every2 $: \triangleright(\square$ Str $\rightarrow$ Str $), s: \square$ Str

$\Psi, \triangleright[\mathbf{r} \leftarrow$ every 2$] .(\forall s . s=$ ones $\Rightarrow \mathbf{r} s=$ letb $x \leftarrow s$ in $x), s=$ ones $\vdash$

$\triangleright(\hat{\mathrm{tl}}(\hat{\mathrm{tl}} \mathrm{s})): \triangleright \square \operatorname{Str}\{\triangleright[\mathbf{r} \leftarrow \mathbf{r}] .(\mathbf{r}=$ ones $)\}$

- ones : $\square$ Str, every2 : $\triangleright(\square$ Str $\rightarrow$ Str $), \mathrm{s}: \square$ Str, g: $\square$ Str $\rightarrow$ Str, t : $\square$ Str

$\Psi, \triangleright[\mathbf{r} \leftarrow$ every 2$] .(\forall$ s.s $=$ ones $\Rightarrow \mathbf{r} s=$ letb $x \leftarrow s$ in $x), s=$ ones,

$\forall$ s.s $=$ ones $\Rightarrow \mathrm{gs}=$ letb $x \leftarrow \mathrm{s}$ in $\mathrm{x}, \mathrm{t}=$ ones $\vdash \mathrm{g} \mathrm{t}: \operatorname{Str}\{\mathbf{r}=$ (letb $\mathrm{x} \leftarrow$

ones in $x)\}$

The first premise is just an application of the Var rule. The second premise can be proven as a consequence of the properties of ones. Finally, the third premise can be proven with some simple logical reasoning in HOL. This concludes the proof. 


\section{APPENDIX OF CHAPTER 10}

\section{C.1 Background: Real Analysis}

The following are standard convergence results in real analysis, see for instance [159]. In all of them we consider a sequence of relational expectations $\mathcal{E}_{n}$ : State $\times$ State $\rightarrow$ $\mathbb{R}_{\geqslant 0}^{\infty}$ and a distribution $\mu: \operatorname{SD}($ State $\times$ State $)$.

Lemma C.1 (Fatou's Lemma). Let $\mathcal{E}_{\mathrm{n}}$ be a monotone increasing sequence of relational expectations. Then,

$$
\mathbb{E}_{\mu}\left[\lim _{n \rightarrow \infty} \mathcal{E}_{n}\right] \leqslant \lim _{n \rightarrow \infty} \mathbb{E}_{\mu}\left[\mathcal{E}_{n}\right] .
$$

Fatou's Lemma also holds when $\mathcal{E}_{n}$ is not a monotone sequence (replacing the limit by a limit inferior), but the monotone version suffices for our purposes.

Now we present a result that will be useful in showing convergence of couplings. A similar result can be found in the monograph Villani [156, Theorem 5.19].

Theorem C.2 (Convergence of couplings). Let $v_{i}$ and $\rho_{i}$ denote two sequences of subdistributions with countable support over $\mathrm{X}$, converging pointwise to $v$ and $\rho$ respectively. Let $\mu_{i} \in \Gamma\left(\nu_{i}, \rho_{i}\right)$ be a sequence of couplings of $v_{i}$ and $\rho_{i}$. Then there exists a subsequence $\mu_{i}^{\prime}$ of $\mu_{i}$ that converges to a coupling $\mu \in \Gamma(\nu, \rho)$.

Proof. The proof proceeds in two steps. First we show that there exists a convergent subsequence of couplings. By the Bolzano-Weierstrass theorem, $[0,1]$ is sequentially compact, i.e., every sequence in $[0,1]$ has a subsequence that converges in $[0,1]$. Moreover, countable products preserve sequential compactness. Since every $v_{i}$ and $\rho_{i}$ have countable support, so does every $\mu_{i}$, so we can consider the sequence $\left\{\mu_{i}\right\}_{i \in \mathbb{N}}$ as a sequence over $[0,1]^{\mathcal{S}}$ where $\mathcal{S}=\cup_{i} \operatorname{supp}\left(\mu_{i}\right)$. Since this is a sequentially compact space, we can extract a subsequence $\left\{\mu_{i}^{\prime}\right\}_{i \in \mathbb{N}}$ that converges pointwise to some distribution, call it $\mu \in \mathbf{S D}(X \times X)$; let $\left\{v_{i}^{\prime}\right\}_{i \in \mathbb{N}}$ and $\left\{\rho_{i}^{\prime}\right\}_{i \in \mathbb{N}}$ be the corresponding 
subsequences of $\left\{v_{i}\right\}_{i \in \mathbb{N}}$ and $\left\{\rho_{i}\right\}_{i \in \mathbb{N}}$ such that $\mu_{i}^{\prime} \in \Gamma\left(v_{i}^{\prime}, \rho_{i}^{\prime}\right)$. Since they are subsequences of convergent sequences, these were convergent, $\left\{v_{i}^{\prime}\right\}_{i \in \mathbb{N}}$ converges to $v$ and $\left\{\rho_{i}^{\prime}\right\}_{i \in \mathbb{N}}$ converges to $\rho$.

The main task is showing that $\mu$ is indeed a coupling of $\nu$ and $\rho$, i.e., $\mu \in \Gamma(\nu, \rho)$. We consider the first marginal condition. Let $\epsilon>0$ be any positive number. Since $\rho$ is a distribution over a countable set, there exists a finite set $S(\epsilon)$ such that $\sum_{x_{2} \notin S(\epsilon)} \rho\left(x_{2}\right)<\epsilon$. We first show that:

$$
\lim _{i \rightarrow \infty} \sum_{x_{2} \notin S(\epsilon / 2)} \rho_{i}\left(x_{2}\right)=0 .
$$

Since $\left\{\rho_{i}^{\prime}\right\}_{i}$ converges pointwise to $\rho$, it also converges in $L_{1}$. So, there exists a finite number $N(\epsilon)$ such that for all $i>N(\epsilon)$, we have:

$$
\sum_{x_{2} \in X}\left|\rho_{i}^{\prime}\left(x_{2}\right)-\rho\left(x_{2}\right)\right|<\epsilon .
$$

Thus for all $i>N(\epsilon / 2)$, we have:

$$
\begin{aligned}
\sum_{x_{2} \notin S(\epsilon / 2)} \rho_{i}^{\prime}\left(x_{2}\right) & =\sum_{x_{2} \notin S(\epsilon / 2)}\left(\rho_{i}^{\prime}\left(x_{2}\right)-\rho\left(x_{2}\right)\right)+\sum_{x_{2} \notin S(\epsilon / 2)} \rho\left(x_{2}\right) \\
& \leqslant \sum_{x_{2} \in X}\left|\rho_{i}^{\prime}\left(x_{2}\right)-\rho\left(x_{2}\right)\right|+\epsilon / 2 \\
& \leqslant \epsilon .
\end{aligned}
$$

Since $\epsilon$ was arbitrary, this establishes Eq. (C.I). Now, let $x_{1} \in X$ be any element. We 
can compute:

$$
\begin{aligned}
& \left|v\left(x_{1}\right)-\left(\pi_{1}(\mu)\right)\left(x_{1}\right)\right|=\left|v\left(x_{1}\right)-\sum_{x_{2} \in X} \lim _{i \rightarrow \infty} \mu_{i}\left(x_{1}, x_{2}\right)\right| \\
& \leqslant\left|v\left(x_{1}\right)-\sum_{x_{2} \in S(\epsilon)} \lim _{i \rightarrow \infty} \mu_{i}\left(x_{1}, x_{2}\right)\right|+\sum_{x_{2} \notin S(\epsilon)} \lim _{i \rightarrow \infty} \mu_{i}\left(x_{1}, x_{2}\right) \\
& \text { (triangle ineq.) } \\
& =\left|v\left(x_{1}\right)-\lim _{i \rightarrow \infty} \sum_{x_{2} \in S(\epsilon)} \mu_{i}\left(x_{1}, x_{2}\right)\right|+\sum_{x_{2} \notin S(\epsilon)} \lim _{i \rightarrow \infty} \mu_{i}\left(x_{1}, x_{2}\right) \\
& \leqslant\left|v\left(x_{1}\right)-\lim _{i \rightarrow \infty} \sum_{x_{2} \in S(\epsilon)} \mu_{i}\left(x_{1}, x_{2}\right)\right|+\sum_{x_{2} \notin S(\epsilon)} \lim _{i \rightarrow \infty} \rho_{i}\left(x_{2}\right) \quad\left(\pi_{2}\left(\mu_{i}\right)=\rho_{i}\right) \\
& \left.\leqslant\left|v\left(x_{1}\right)-\lim _{i \rightarrow \infty} \sum_{x_{2} \in S(\epsilon)} \mu_{i}\left(x_{1}, x_{2}\right)\right|+\sum_{x_{2} \notin S(\epsilon)} \rho\left(x_{2}\right) \quad \text { (limit } \rho\right) \\
& \left.\leqslant\left|v\left(x_{1}\right)-\lim _{i \rightarrow \infty} \sum_{x_{2} \in S(\epsilon)} \mu_{i}\left(x_{1}, x_{2}\right)\right|+\epsilon \quad \text { (def. } S(\epsilon)\right) \\
& =\left|v\left(x_{1}\right)-\lim _{i \rightarrow \infty} v_{i}\left(x_{1}\right)+\lim _{i \rightarrow \infty} \sum_{x_{2} \notin S(\epsilon)} \mu_{i}\left(x_{1}, x_{2}\right)\right|+\epsilon \quad\left(\pi_{1}\left(\mu_{i}\right)=v_{i}\right) \\
& =\left|\lim _{i \rightarrow \infty} \sum_{x_{2} \notin S(\epsilon)} \mu_{i}\left(x_{1}, x_{2}\right)\right|+\epsilon \\
& \leqslant \lim _{i \rightarrow \infty} \sum_{x_{2} \notin S(\epsilon)} \rho_{i}\left(x_{2}\right)+\epsilon=\epsilon . \quad \text { (by Eq. (C.1 ) }
\end{aligned}
$$

Since $\epsilon>0$ and $x_{1} \in X$ are arbitrary, this shows the first marginal condition $\nu=\pi_{1}(\mu)$. The second marginal condition follows similarly, and so $\mu \in \Gamma(\nu, \rho)$ as desired.

\section{C.2 Section 2: Omitted Proofs}

Theorem 10.7. For the direction " $\leqslant$ ", it suffices to show that $\left|\mu_{1}(S)-\mu_{2}(S)\right| \leqslant \mathbb{E}_{\mu}[\mathcal{E}]$ for every $\mu \in \Gamma\left(\mu_{1}, \mu_{2}\right)$ and every $S \subseteq X$. By the property of marginals and monotonicity of probabilities, we have:

$$
\begin{aligned}
\left|\mu_{1}(S)-\mu_{2}(S)\right| & =|\mu(S \times X)-\mu(X \times S)|=|\mu(S \times(X \backslash S))-\mu((X \backslash S) \times S)| \\
& \leqslant \max (\mu(S \times(X \backslash S)), \mu((X \backslash S) \times S)) \leqslant \mu\left(\left\{\left(x_{1}, x_{2}\right) \mid x_{1} \neq x_{2}\right\}\right) \\
& =\mathbb{E}_{\mu}[\mathcal{E}] .
\end{aligned}
$$

For the other direction, we construct a so-called optimal coupling. For every $x \in X$, let $\mu_{0}(x)=\min \left(\mu_{1}(x), \mu_{2}(x)\right)$. The optimal coupling for $\left(\mu_{1}, \mu_{2}\right)$ is defined by:

$$
\mu\left(x_{1}, x_{2}\right)= \begin{cases}\mu_{0}\left(x_{1}\right) & : x_{1}=x_{2} \\ \frac{\left(\mu_{1}\left(x_{1}\right)-\mu_{0}\left(x_{1}\right)\right) \cdot\left(\mu_{2}\left(x_{2}\right)-\mu_{0}\left(x_{2}\right)\right)}{\operatorname{TV}\left(\mu_{1}, \mu_{2}\right)} & : x_{1} \neq x_{2},\end{cases}
$$


where $0 / 0:=0$. One can check that $\mu$ is a coupling for $\left(\mu_{1}, \mu_{2}\right)$ and that $\mu\left(\left[x_{1} \neq x_{2}\right]\right)=$ $\operatorname{TV}\left(\mu_{1}, \mu_{2}\right)$.

Theorem 10.8. It suffices to show $\left|\mathbb{E}_{\mu_{1}}\left[f_{1}\right]-\mathbb{E}_{\mu_{2}}\left[f_{2}\right]\right| \leqslant \mathbb{E}_{\mu}[\mathcal{E}]$ for every $\mu \in \Gamma\left(\mu_{1}, \mu_{2}\right)$, which follows from the marginal properties of couplings, linearity of expectation, and the fact that $\left|\mathbb{E}_{\mu}[\mathrm{f}]\right| \leqslant \mathbb{E}_{\mu}[|\mathrm{f}|]$ :

$$
\begin{aligned}
& \left|\mathbb{E}_{\mu_{1}}\left[f_{1}\right]-\mathbb{E}_{\mu_{2}}\left[f_{2}\right]\right|=\left|\mathbb{E}_{\mu}\left[\lambda\left(x_{1}, x_{2}\right) . f_{1}\left(x_{1}\right)\right]-\mathbb{E}_{\mu}\left[\lambda\left(x_{1}, x_{2}\right) . f_{2}\left(x_{2}\right)\right]\right| \\
= & \left|\mathbb{E}_{\mu}\left[\lambda\left(x_{1}, x_{2}\right) . f_{1}\left(x_{1}\right)-f_{2}\left(x_{2}\right)\right]\right| \leqslant \mathbb{E}_{\mu}\left[\lambda\left(x_{1}, x_{2}\right) .\left|f_{1}\left(x_{1}\right)-f_{2}\left(x_{2}\right)\right|\right]=\mathbb{E}_{\mu}[\mathcal{E}] .
\end{aligned}
$$

Theorem 10.9. The proof follows from Theorem 10.7 and from the observations that $(\rho \cdot \mathcal{E})^{\#}=\rho \cdot \mathcal{E}^{\#}$ and that $\mathcal{\varepsilon} \leqslant \mathcal{E}^{\prime}$ implies $\mathcal{E}^{\#} \leqslant \mathcal{E}^{\prime \#}$, taking the pointwise order in both cases.

\section{C.3 Soundness and Continuity: Omitted Proofs}

The syntactic relational pre-expectation transformer is a monotonic operator.

Lemma C.3 (Monotonicity of $\widetilde{r p e}(\mathrm{c},-))$. Let $\mathcal{E}$ be a relational expectation and let $\mathrm{c}$ be a program. Then $\widetilde{p p e}(\mathrm{c},-)$ and $\Phi_{\varepsilon, \mathrm{c}}(-)$ are monotonic, i.e. for any two relational expectations $\mathcal{E}_{1}, \mathcal{E}_{2}$ such that $\mathcal{E}_{1} \leqslant \mathcal{E}_{2}$, we have rpe $\left(\mathrm{c}, \mathcal{E}_{1}\right) \leqslant \widetilde{r p e}\left(\mathrm{c}, \mathcal{E}_{2}\right)$ and $\Phi_{\mathcal{E}, \mathrm{c}}\left(\mathcal{E}_{1}\right) \leqslant \Phi_{\mathcal{\varepsilon}, \mathrm{c}}\left(\mathcal{E}_{2}\right)$.

Proof. The latter result is a corollary from the former. By definition,

$$
\Phi_{\mathcal{E}, c, e}\left(\mathcal{E}_{1}\right)=[e\langle 1\rangle \wedge e\langle 2\rangle] \cdot \widetilde{r p e}\left(c, \mathcal{E}_{1}\right)+[\neg e\langle 1\rangle \wedge \neg e\langle 2\rangle] \cdot \mathcal{E}+[e\langle 1\rangle \neq e\langle 2\rangle] \cdot \infty
$$

and

$$
\Phi_{\varepsilon, c, e}\left(\varepsilon_{2}\right)=[e\langle 1\rangle \wedge e\langle 2\rangle] \cdot \widetilde{r p e}\left(c, \varepsilon_{2}\right)+[\neg e\langle 1\rangle \wedge \neg e\langle 2\rangle] \cdot \mathcal{E}+[e\langle 1\rangle \neq e\langle 2\rangle] \cdot \infty .
$$

So given $\widetilde{r p e}\left(c, \varepsilon_{1}\right) \leqslant \widetilde{r p e}\left(c, \varepsilon_{2}\right)$ we can conclude $\Phi_{\mathcal{E}, c, e}\left(\varepsilon_{1}\right) \leqslant \Phi_{\varepsilon, c, e}\left(\varepsilon_{2}\right)$.

The former result is proven by induction on $c$ :

- skip. Then

$$
\widetilde{r p e}\left(\text { skip, } \varepsilon_{1}\right)=\varepsilon_{1} \leqslant \varepsilon_{2}=\widetilde{r p e}\left(\text { skip, } \varepsilon_{2}\right)
$$

- $x \leftarrow e$. Then

$$
\widetilde{r p e}\left(x \leftarrow e, \mathcal{E}_{1}\right)=\mathcal{E}_{1}\{e\langle 1\rangle, e\langle 2\rangle / x\langle 1\rangle, x\langle 2\rangle\}
$$

and

$$
\widetilde{r p e}\left(x \leftarrow e, \varepsilon_{2}\right)=\varepsilon_{2}\{e\langle 1\rangle, e\langle 2\rangle / x\langle 1\rangle, x\langle 2\rangle\}
$$

Consider a pair of states $s_{1}, s_{2}$ then:

$$
\begin{aligned}
\mathcal{E}_{1}\{e\langle 1\rangle, e\langle 2\rangle / x\langle 1\rangle, x\langle 2\rangle\}\left(s_{1}, s_{2}\right) & =\mathcal{E}_{1}\left(s_{1}\left\{s_{1}(e) / x\right\}\right)\left(s_{2}\left\{s_{2}(e) / x\right\}\right) \\
& \leqslant \varepsilon_{2}\left(s_{1}\left\{s_{1}(e) / x\right\}\right)\left(s_{2}\left\{s_{2}(e) / x\right\}\right) \\
& =\mathcal{E}_{2}\{e\langle 1\rangle, e\langle 2\rangle / x\langle 1\rangle, x\langle 2\rangle\}\left(s_{1}, s_{2}\right)
\end{aligned}
$$


- $x \leqslant d$. Then,

$$
\widetilde{\operatorname{rpe}}\left(\mathrm{x} \leftarrow \mathrm{d}, \mathcal{E}_{1}\right)=\inf _{\mu \in \Gamma\left(\mu_{1}, \mu_{2}\right)} \mathbb{E}_{\mu}\left[\mathcal{E}_{1}\right]
$$

and

$$
\widetilde{r p e}\left(x \leqslant \mathrm{~d}, \mathcal{E}_{2}\right)=\inf _{\mu \in \Gamma\left(\mu_{1}, \mu_{2}\right)} \mathbb{E}_{\mu}\left[\mathcal{E}_{2}\right]
$$

Let $\mu \in \Gamma\left(\mu_{1}, \mu_{2}\right)$ be an arbitrary coupling. By monotonicity of the expectation, then $\mathbb{E}_{\mu}\left[\mathcal{E}_{1}\right] \leqslant \mathbb{E}_{\mu}\left[\mathcal{E}_{2}\right]$, and therefore the infimum for $\mathcal{E}_{1}$ is less or equal than the one for $\varepsilon_{2}$.

- $c ; c^{\prime}$. By the induction hypothesis,

$$
\widetilde{r p e}\left(\mathrm{c} ; \mathrm{c}^{\prime}, \varepsilon_{1}\right)=\widetilde{r p e}\left(\mathrm{c}, \widetilde{r p e}\left(\mathrm{c}^{\prime}, \varepsilon_{1}\right)\right) \leqslant \widetilde{r p e}\left(\mathrm{c}, \widetilde{r p e}\left(\mathrm{c}^{\prime}, \varepsilon_{2}\right)\right)=\widetilde{r p e}\left(\mathrm{c} ; \mathrm{c}^{\prime}, \varepsilon_{2}\right)
$$

Note that the inequality needs two applications of the I.H., one to show that $\widetilde{r p e}\left(c^{\prime}, \varepsilon_{1}\right) \leqslant \widetilde{r p e}\left(c^{\prime}, \varepsilon_{2}\right)$ and another one to show $\widetilde{r p e}\left(c, \widetilde{r p e}\left(c^{\prime}, \varepsilon_{1}\right)\right) \leqslant \widetilde{r p e}\left(c, \widetilde{r p e}\left(c^{\prime}, \varepsilon_{2}\right)\right)$.

- if $e$ then $c$ else $c^{\prime}$. By the induction hypothesis (applied at $c$ and $c^{\prime}$ ),

$$
\begin{aligned}
& \widetilde{r p e}\left(\text { if } e \text { then } c \text { else } c^{\prime}, \varepsilon_{1}\right)= \\
& =[e\langle 1\rangle \wedge e\langle 2\rangle] \cdot \widetilde{r p e}\left(c, \varepsilon_{1}\right)+[\neg e\langle 1\rangle \wedge \neg e\langle 2\rangle] \cdot \widetilde{r p e}\left(c^{\prime}, \varepsilon_{1}\right)+[e\langle 1\rangle \neq e\langle 2\rangle] \cdot \infty \\
& \leqslant[e\langle 1\rangle \wedge e\langle 2\rangle] \cdot \widetilde{r p e}\left(c, \varepsilon_{2}\right)+[\neg e\langle 1\rangle \wedge \neg e\langle 2\rangle] \cdot \widetilde{r p e}\left(c^{\prime}, \varepsilon_{2}\right)+[e\langle 1\rangle \neq e\langle 2\rangle] \cdot \infty \\
& =\widetilde{r p e}\left(\text { if } e \text { then } c \text { else } c^{\prime}, \varepsilon_{2}\right)
\end{aligned}
$$

- while $e$ do $c$. Then,

$$
\begin{aligned}
& \widetilde{r p e}\left(\text { while } e \text { do } c, \varepsilon_{1}\right) \\
& \quad=\text { IfpX. }[e\langle 1\rangle \wedge e\langle 2\rangle] \cdot \widetilde{r p e}(\mathrm{c}, X)+[\neg e\langle 1\rangle \wedge \neg e\langle 2\rangle] \cdot \varepsilon_{1}+[e\langle 1\rangle \neq e\langle 2\rangle] \cdot \infty \\
& \widetilde{r p e}\left(\text { while } e \text { do } c, \varepsilon_{2}\right) \\
& \quad=\operatorname{IfpX.}[e\langle 1\rangle \wedge e\langle 2\rangle] \cdot \widetilde{r p e}(\mathrm{c}, X)+[\neg e\langle 1\rangle \wedge \neg e\langle 2\rangle] \cdot \varepsilon_{2}+[e\langle 1\rangle \neq e\langle 2\rangle] \cdot \infty
\end{aligned}
$$

Existence of the least fixed points is guaranteed by monotonicity of the functionals, which follows from the inductive hypothesis applied to $c$. Suppose $X_{2}$ is the least fixpoint of the second expression. We will show that it is a pre-fixpoint of the first expression.

$$
\begin{aligned}
& {[e\langle 1\rangle \wedge e\langle 2\rangle] \cdot \widetilde{r p e}\left(c, X_{2}\right)+[\neg e\langle 1\rangle \wedge \neg e\langle 2\rangle] \cdot \mathcal{E}_{1}+[e\langle 1\rangle \neq e\langle 2\rangle] \cdot \infty} \\
& \leqslant[e\langle 1\rangle \wedge e\langle 2\rangle] \cdot \widetilde{r p e}\left(c, X_{2}\right)+[\neg e\langle 1\rangle \wedge \neg e\langle 2\rangle] \cdot \varepsilon_{2}+[e\langle 1\rangle \neq e\langle 2\rangle] \cdot \infty \\
& =X_{2}
\end{aligned}
$$

By Knaster-Tarski, the least fixed point of a monotonically increasing operator is the greatest lower bound of the set of pre-fixpoints. From this we conclude $\widetilde{r p e}\left(\right.$ while e do $\left.c, \varepsilon_{1}\right) \leqslant X_{2}$. 
We need a lemma about the existence of a coupling realizing the minimum Kantorovich distance.

Lemma C.4. Let $\mu_{1}, \mu_{2} \in \mathbf{S D}$ (State) be two subdistributions of finite support with the same weight, and let $\mathcal{E}$ : State $\times$ State $\rightarrow \mathbb{R}_{\geqslant 0}^{\infty}$ be a relational expectation. There exists a coupling $\mu \in \Gamma\left(\mu_{1}, \mu_{2}\right)$ realizing the minimum Kantorovich distance:

$$
\mathbb{E}_{\mu}[\mathcal{E}]=\inf _{\mu \in \Gamma\left(\mu_{1}, \mu_{2}\right)} \mathbb{E}_{\mu}[\mathcal{E}]=\mathcal{E}^{\#}\left(\mu_{1}, \mu_{2}\right) .
$$

This is an extremely simple case of standard existence results in the theory of optimal transport (see, e.g., Theorem 4.1 in Villani's monograph [156]). We include a proof to keep the exposition self-contained.

Proof. Let $\mathrm{d}^{*}=\inf _{\mu \in \Gamma\left(\mu_{1}, \mu_{2}\right)} \mathbb{E}_{\mu}[\mathcal{E}]$ be the infimum distance. If $\mathrm{d}^{*}=\infty$ then the product coupling realizes the distance. Otherwise, suppose that the infimum $\mathrm{d}^{*}$ is finite. By the definition of infimum, there exists a sequence of couplings $\mu^{(1)}, \mu^{(2)}, \cdots \in \Gamma\left(\mu_{1}, \mu_{2}\right)$ such that

$$
\lim _{k \rightarrow \infty} \mathbb{E}_{\mu^{(k)}}[\mathcal{E}]=\mathrm{d}^{*} .
$$

Without loss of generality, we may assume that for each $k$ the distance $\mathbb{E}_{\mu^{(k)}}[\mathcal{E}]$ is finite as well. Let $S=\cup_{k} \operatorname{supp}\left(\mu^{(k)}\right)$ be the union of the supports of all $\mu^{(k)}$. Since $\mu_{1}, \mu_{2}$ have countable support, $S$ is countable. Since all the expected distances are finite, in fact all pairs of states $\left(s_{1}, s_{2}\right) \in S$ have $\mathcal{E}\left(s_{1}, s_{2}\right)<\infty$. By Theorem C.2 we can find a subsequence of $\mu^{(\mathrm{k})}$ that is converging pointwise; define:

$$
\mu\left(s_{1}, s_{2}\right)=\lim _{k \rightarrow \infty} \mu^{(k)}\left(s_{1}, s_{2}\right)
$$

for every $s_{1}, s_{2} \in$ State, where the limit is taken over the subsequence (so it exists). Then $\mu$ is indeed a coupling in $\Gamma\left(\mu_{1}, \mu_{2}\right)$. To show that $\mu$ realizes the infimum distance, we derive:

$$
\begin{aligned}
\mathbb{E}_{\mu}[\mathcal{E}] & =\sum_{\left(s_{1}, s_{2}\right) \in S} \mathcal{E}\left(s_{1}, s_{2}\right) \cdot \mu\left(s_{1}, s_{2}\right) \\
& =\sum_{\left(s_{1}, s_{2}\right) \in S} \mathcal{E}\left(s_{1}, s_{2}\right) \cdot \lim _{k \rightarrow \infty} \mu^{(k)}\left(s_{1}, s_{2}\right) \\
& \leqslant \sum_{\left(s_{1}, s_{2}\right) \in S} \lim _{k \rightarrow \infty} \mathcal{E}\left(s_{1}, s_{2}\right) \cdot \mu^{(k)}\left(s_{1}, s_{2}\right) \\
& \leqslant \lim _{k \rightarrow \infty} \sum_{\left(s_{1}, s_{2}\right) \in S} \mathcal{E}\left(s_{1}, s_{2}\right) \cdot \mu^{(k)}\left(s_{1}, s_{2}\right) \\
& =\lim _{k \rightarrow \infty} \mathbb{E}_{\mu^{(k)}}[\mathcal{E}] \\
& =d^{*} .
\end{aligned}
$$

The first inequality is because $\mathcal{E}$ may take value infinity; the second inequality is by Fatou's lemma. 
Continuity proceeds in two steps. We first need a lemma about continuity of the Kantorovich distance. While it seems challenging to establish this lemma for distributions with infinite support, we establish it for distributions with finite support.

Lemma C.5. Let $\mu_{1}, \mu_{2} \in \mathbf{S D}$ (State) be two distributions with finite support, and let $\mathcal{E}_{\mathrm{n}}$ : State $\times$ State $\rightarrow \mathbb{R}_{\geqslant 0}^{\infty}$ be a monotonically increasing chain of relational expectations converging pointwise to $\mathcal{E}$ : State $\times$ State $\rightarrow \mathbb{R}_{\geqslant 0}^{\infty}$. Then:

$$
\inf _{\mu \in \Gamma\left(\mu_{1}, \mu_{2}\right)} \mathbb{E}_{\mu}[\mathcal{E}]=\inf _{\mu \in \Gamma\left(\mu_{1}, \mu_{2}\right)} \mathbb{E}_{\mu}\left[\lim _{n \rightarrow \infty} \mathcal{E}_{n}\right]=\lim _{n \rightarrow \infty} \inf _{\mu \in \Gamma\left(\mu_{1}, \mu_{2}\right)} \mathbb{E}_{\mu}\left[\mathcal{E}_{n}\right] .
$$

Proof. If $\mu_{1}, \mu_{2}$ have different weights, then both infimums are infinity and we are done. It is not hard to show that

$$
\lim _{n \rightarrow \infty} \inf _{\mu \in \Gamma\left(\mu_{1}, \mu_{2}\right)} \mathbb{E}_{\mu}\left[\mathcal{E}_{n}\right] \leqslant \inf _{\mu \in \Gamma\left(\mu_{1}, \mu_{2}\right)} \mathbb{E}_{\mu}[\mathcal{E}],
$$

since $\varepsilon_{n} \leqslant \mathcal{E}$ and the coupling realizing the infimum (which exists by Lemma C.4) is a valid coupling in each of the limit terms.

Showing the other direction is more involved. Define the finite relations

$$
\begin{aligned}
R_{<\infty} & =\left\{\left(s_{1}, s_{2}\right) \mid \mathcal{E}\left(s_{1}, s_{2}\right)<\infty\right\} \cap\left(\operatorname{supp}\left(\mu_{1}\right) \times \operatorname{supp}\left(\mu_{2}\right)\right) \\
R_{\infty} & =\left(\operatorname{supp}\left(\mu_{1}\right) \times \operatorname{supp}\left(\mu_{2}\right)\right) \backslash R_{<\infty} .
\end{aligned}
$$

We first consider the case where

$$
\inf _{\mu \in \Gamma\left(\mu_{1}, \mu_{2}\right)} \mathbb{E}_{\mu}[\mathcal{E}]=\infty
$$

This means that every coupling must put weight on $R_{<\infty}$. To see this fact, note that the following infimum is realized by some coupling $\mu^{*}$ :

$$
\inf _{\mu \in \Gamma\left(\mu_{1}, \mu_{2}\right)} \mathbb{E}_{\mu}\left[R_{\infty}\right]
$$

If $\mu^{*}\left(R_{\infty}\right)=0$, then $\mu^{*}$ does not place any mass on points where $\varepsilon$ is infinity. Since $\mu^{*}$ has finite support, this means that $\mathbb{E}_{\mu^{*}}[\mathcal{E}]$ would be finite, a contradiction. So, we have:

$$
\inf _{\mu \in \Gamma\left(\mu_{1}, \mu_{2}\right)} \mathbb{E}_{\mu}\left[R_{\infty}\right]=\inf _{\mu \in \Gamma\left(\mu_{1}, \mu_{2}\right)} \mathbb{E}_{\mu}\left[R_{\infty}\right] \geqslant \rho>0 .
$$

for some constant $\rho$. Now, let $M$ be any real number greater than $\rho$, and take $N$ large enough so that for every $\left(s_{1}, s_{2}\right) \in R_{\infty}$, we have $\varepsilon_{n}\left(s_{1}, s_{2}\right)>M / \rho$ for all $n>N$. Such an $N$ must exist since $R_{\infty}$ is finite, and $\mathcal{E}_{n}\left(s_{1}, s_{2}\right)$ is tending to infinity for $\left(s_{1}, s_{2}\right) \in R_{\infty}$. We now have

$$
\inf _{\mu \in \Gamma\left(\mu_{1}, \mu_{2}\right)} \mathbb{E}_{\mu}\left[\mathcal{E}_{n}\right] \geqslant \inf _{\mu \in \Gamma\left(\mu_{1}, \mu_{2}\right)} \mathbb{E}_{\mu}\left[\left[R_{\infty}\right] \cdot \mathcal{E}_{n}\right] \geqslant(M / \rho) \cdot \rho \geqslant M
$$


for all $n>N$. Since this is true for $M$ arbitrarily large, we must have

$$
\lim _{n \rightarrow \infty} \inf _{\mu \in \Gamma\left(\mu_{1}, \mu_{2}\right)} \mathbb{E}_{\mu}\left[\mathcal{E}_{n}\right]=\infty \geqslant \inf _{\mu \in \Gamma\left(\mu_{1}, \mu_{2}\right)} \mathbb{E}_{\mu}[\mathcal{E}]
$$

as claimed.

Otherwise, suppose the infimum equals $w^{*}<\infty$. Let $M=\sup _{\left(s_{1}, s_{2}\right) \in R_{<\infty}} \mathcal{E}\left(s_{1}, s_{2}\right)$ be the largest finite value assigned by $\mathcal{E}$. Since $\varepsilon_{n}\left(s_{1}, s_{2}\right)$ tends to infinity for all $\left(s_{1}, s_{2}\right) \in R_{\infty}$ and $R_{\infty}$ is finite, we may take a subsequence $\mathcal{E}_{n}^{\prime}$ such that $\mathcal{E}_{n}^{\prime}\left(s_{1}, s_{2}\right) \geqslant n$ for all $\left(s_{1}, s_{2}\right) \in R_{\infty}$. Let $v_{i}^{\prime}$ be a coupling realizing the infimum

$$
\inf _{\mu \in \Gamma\left(\mu_{1}, \mu_{2}\right)} \mathbb{E}_{\mu}\left[\mathcal{E}_{i}^{\prime}\right]
$$

Since this infimum is less than $w^{*}$, we have $v_{i}^{\prime}\left(s_{1}, s_{2}\right)<w^{*} / n$ for every $\left(s_{1}, s_{2}\right) \in R_{\infty}$. Since each $v_{i}^{\prime}$ has finite support and takes values in $[0,1]$, by the Bolzano-Weierstrass theorem there exists a subsequence $v_{i}^{\prime \prime}$ converging pointwise to $v^{*}$; we write $\varepsilon_{i}^{\prime \prime}$ for the corresponding expectations. Note that $v_{i}^{\prime \prime} \in \Gamma\left(\mu_{1}, \mu_{2}\right)$ is a coupling, and $v_{i}^{\prime \prime}\left(R_{\infty}\right)=0$.

Now let $\epsilon>0$. Let $N$ be such that for all $n>N$ and $\left(s_{1}, s_{2}\right) \in R_{<\infty}$, we have $\left|v_{n}^{\prime \prime}\left(s_{1}, s_{2}\right)-v^{*}\left(s_{1}, s_{2}\right)\right|<\epsilon / M$; such an $N$ exists since the distributions have finite support. Then since $\mathcal{E}_{n}^{\prime \prime}\left(s_{1}, s_{2}\right) \leqslant \mathcal{E}\left(s_{1}, s_{2}\right) \leqslant M$ for all $\left(s_{1}, s_{2} \in R_{<\infty}\right.$, and $v^{*}\left(s_{1}, s_{2}\right)=0$ for all $\left(s_{1}, s_{2}\right) \in R_{\infty}$, we have

$$
\mathbb{E}_{v^{*}}\left[\mathcal{E}_{n}^{\prime \prime}\right]<\inf _{\mu \in \Gamma\left(\mu_{1}, \mu_{2}\right)} \mathbb{E}_{\mu}\left[\mathcal{E}_{n}^{\prime \prime}\right]+\epsilon
$$

for all $n>N$. The monotone convergence theorem implies:

$$
\mathbb{E}_{\gamma^{*}}[\mathcal{E}]=\lim _{n \rightarrow \infty} \mathbb{E}_{\gamma^{*}}\left[\mathcal{E}_{n}^{\prime \prime}\right] \leqslant \lim _{n \rightarrow \infty} \inf _{\mu \in \Gamma\left(\mu_{1}, \mu_{2}\right)} \mathbb{E}_{\mu}\left[\mathcal{E}_{n}^{\prime \prime}\right]+\epsilon .
$$

On the other hand, we have the bound

$$
\inf _{\mu \in \Gamma\left(\mu_{1}, \mu_{2}\right)} \mathbb{E}_{\mu}[\mathcal{E}] \leqslant \mathbb{E}_{\gamma^{*}}[\mathcal{E}] .
$$

Since both bounds hold for all $\epsilon$, we can conclude:

$$
\inf _{\mu \in \Gamma\left(\mu_{1}, \mu_{2}\right)} \mathbb{E}_{\mu}[\mathcal{E}] \leqslant \lim _{n \rightarrow \infty} \inf _{\mu \in \Gamma\left(\mu_{1}, \mu_{2}\right)} \mathbb{E}_{\mu}\left[\mathcal{E}_{n}^{\prime \prime}\right]=\lim _{n \rightarrow \infty} \inf _{\mu \in \Gamma\left(\mu_{1}, \mu_{2}\right)} \mathbb{E}_{\mu}\left[\mathcal{E}_{n}\right] .
$$

Now, we can prove continuity of relational pre-expectations, provided that programs sample from distributions with finite support. Note that such programs can still produce distributions with infinite support, for instance by sampling in a loop.

Theorem (Continuity). Let c be a program where all primitive distributions have finite support, and let $\mathcal{E}_{n}$ : State $\times$ State $\rightarrow \mathbb{R}_{\geqslant 0}^{\infty}$ be a monotonically increasing chain of relational expectations converging pointwise to $\mathcal{E}$ : State $\times$ State $\rightarrow \mathbb{R}_{\geqslant 0}^{\infty}$. Then,

$$
\widetilde{\operatorname{rpe}}(\mathrm{c}, \mathcal{E})=\sup _{\mathrm{n} \in \mathbb{N}} \widetilde{\operatorname{rpe}}\left(\mathrm{c}, \mathcal{E}_{\mathrm{n}}\right) \text {. }
$$


Proof of Theorem 10.12. By induction on the structure of the program.

- skip. Then,

$$
\widetilde{r p e}(\mathbf{s k i p}, \mathcal{E})=\mathcal{E}=\sup _{\mathbf{n} \in \mathbb{N}} \mathcal{E}_{\mathfrak{n}}=\sup _{\mathbf{n} \in \mathbb{N}} \widetilde{\operatorname{rpe}}\left(\mathbf{s k i p}, \varepsilon_{\mathfrak{n}}\right)
$$

- $x \leftarrow e$. Then,

$$
\begin{aligned}
\widetilde{r p e}(x \leftarrow e, \mathcal{E}) & =\mathcal{E}\{e\langle 1\rangle, e\langle 2\rangle / x\langle 1\rangle, x\langle 2\rangle\} \\
& =\sup _{n \in \mathbb{N}} \varepsilon_{n}\{e\langle 1\rangle, e\langle 2\rangle / x\langle 1\rangle, x\langle 2\rangle\} \quad \text { (subst. continuous) } \\
& =\sup _{n \in \mathbb{N}} \widetilde{r p e}\left(x \leftarrow e, \varepsilon_{n}\right)
\end{aligned}
$$

- $x \leqslant$ d. Let $s_{1}, s_{2}$ be any two states. By assumption, $\llbracket d \rrbracket s_{1}$ and $\llbracket d \rrbracket s_{2}$ have finite support, so $\mu_{1}=\llbracket x \leqslant d \rrbracket s_{1}$ and $\mu_{2}=\llbracket x \leqslant d \rrbracket s_{2}$ also have finite support. By Lemma C.5 applied to $\mu_{1}, \mu_{2}$, we have

$$
\begin{aligned}
\widetilde{r p e}(x \leqslant d, \mathcal{E})\left(s_{1}, s_{2}\right) & =\inf _{\mu \in \Gamma\left(\mu_{1}, \mu_{2}\right)} \mathbb{E}_{\mu}[\mathcal{E}] \\
& =\lim _{n \in \mathbb{N}} \inf _{\mu \in \Gamma\left(\mu_{1}, \mu_{2}\right)} \mathbb{E}_{\mu}\left[\mathcal{E}_{n}\right] \\
& =\lim _{n \in \mathbb{N}} \widetilde{r p e}\left(x \notin d, \varepsilon_{n}\right)\left(s_{1}, s_{2}\right) .
\end{aligned}
$$

By monotonicity, the sup and the lim coincide.

- $c ; c^{\prime}$. Then,

$$
\begin{aligned}
& \widetilde{r p e}\left(\mathrm{c} ; \mathrm{c}^{\prime}, \varepsilon\right)=\widetilde{r p e}\left(\mathrm{c}, \widetilde{r p e}\left(\mathrm{c}^{\prime}, \varepsilon\right)\right) \\
& =\widetilde{\operatorname{rpe}}\left(\mathrm{c}, \sup _{\mathrm{n} \in \mathbb{N}} \widetilde{\mathrm{rpe}}\left(\mathrm{c}^{\prime}, \varepsilon_{\mathrm{n}}\right)\right) \\
& =\sup _{n \in \mathbb{N}} \widetilde{r p e}\left(\mathrm{c}, \widetilde{r p e}\left(\mathrm{c}^{\prime}, \varepsilon_{\mathrm{n}}\right)\right) \\
& =\sup _{n \in \mathbb{N}} \widetilde{r p e}\left(c ; c^{\prime}, \varepsilon_{n}\right)
\end{aligned}
$$

- if $e$ then $c$ else $c^{\prime}$. Then,

$$
\begin{aligned}
& \widetilde{r p e}\left(\text { if } e \text { then } c \text { else } c^{\prime}, \varepsilon\right) \\
& =[e\langle 1\rangle \wedge e\langle 2\rangle] \cdot \widetilde{p p e}(\mathrm{c}, \mathcal{E})+[\neg e\langle 1\rangle \wedge \neg e\langle 2\rangle] \cdot \widetilde{p p e}\left(c^{\prime}, \mathcal{E}\right)+[e\langle 1\rangle \neq e\langle 2\rangle] \cdot \infty \\
& =[e\langle 1\rangle \wedge e\langle 2\rangle] \cdot \sup _{n \in \mathbb{N}} \widetilde{\operatorname{rpe}}\left(\mathbf{c}, \varepsilon_{\mathfrak{n}}\right)+[\neg e\langle 1\rangle \wedge \neg e\langle 2\rangle] \cdot \sup _{n \in \mathbb{N}} \widetilde{\operatorname{rpe}}\left(\mathbf{c}^{\prime}, \varepsilon_{\mathfrak{n}}\right)+[e\langle 1\rangle \neq e\langle 2\rangle] \cdot \infty \\
& =\sup _{n \in \mathbb{N}}\left([e\langle 1\rangle \wedge e\langle 2\rangle] \cdot \widetilde{r p e}\left(c, \varepsilon_{n}\right)+[\neg e\langle 1\rangle \wedge \neg e\langle 2\rangle] \cdot \widetilde{r p e}\left(c^{\prime}, \varepsilon_{n}\right)+[e\langle 1\rangle \neq e\langle 2\rangle] \cdot \infty\right) \\
& =\sup _{n \in \mathbb{N}} \widetilde{r p e}\left(\text { if } e \text { then } c \text { else } c^{\prime}, \varepsilon_{\mathfrak{n}}\right)
\end{aligned}
$$


- while e do c. Then,

$$
\begin{aligned}
& \widetilde{r p e}(\text { while } e \text { do } c, \mathcal{E})=\operatorname{IfpX} . \Phi_{c, \mathcal{E}}(X), \quad \text { where } \\
& \Phi_{c, \mathcal{E}}(\mathrm{X}) \triangleq[e\langle 1\rangle \wedge e\langle 2\rangle] \cdot \widetilde{r p e}(\mathrm{c}, \mathrm{X})+[\neg e\langle 1\rangle \wedge \neg e\langle 2\rangle] \cdot \mathcal{E}+[e\langle 1\rangle \neq e\langle 2\rangle] \cdot \infty
\end{aligned}
$$

We claim that:

$$
\begin{aligned}
\widetilde{r p e}(\text { while e do } c, \mathcal{E}) & =\operatorname{IfpX} . \Phi_{c, \sup _{n} \in \mathbb{N}} \varepsilon_{n}(X) \\
& =\operatorname{IfpX} \sup _{n \in \mathbb{N}} \Phi_{c, \varepsilon_{n}}(X) \\
& =\sup _{n \in \mathbb{N}} \operatorname{Ifp} X . \Phi_{c, \varepsilon_{n}}(X) \\
& =\sup _{n \in \mathbb{N}} \widetilde{r p e}\left(\text { while e do } c, \varepsilon_{n}\right)
\end{aligned}
$$

(definition)

Equality (1) follows from:

$$
\begin{aligned}
& \text { IfpX. } \Phi_{c, \sup _{n} \in \mathbb{N}} \varepsilon_{n}(X)= \\
& =\mid \mathrm{fpX} .[e\langle 1\rangle \wedge e\langle 2\rangle] \cdot \widetilde{\operatorname{rpe}}(\mathrm{c}, \mathrm{X})+[\neg e\langle 1\rangle \wedge \neg e\langle 2\rangle] \cdot \sup _{\mathrm{n} \in \mathbb{N}} \varepsilon_{\mathrm{n}}+[e\langle 1\rangle \neq e\langle 2\rangle] \cdot \infty \\
& =\mid \mathrm{IfpX} . \sup _{\mathrm{n} \in \mathbb{N}}\left([e\langle 1\rangle \wedge e\langle 2\rangle] \cdot \widetilde{\mathrm{rp} e}(\mathrm{c}, \mathrm{X})+[\neg e\langle 1\rangle \wedge \neg e\langle 2\rangle] \cdot \mathcal{E}_{n}+[e\langle 1\rangle \neq e\langle 2\rangle] \cdot \infty\right)
\end{aligned}
$$

To show (2) we note that-by the Knaster-Tarski fixpoint theorem and the fact that $\Phi_{c, \varepsilon_{n}}(X)$ is monotonic-Ifp is itself a supremum (over the ordinals), namely

$$
\text { IfpX. } \sup _{n \in \mathbb{N}} \Phi_{c, \varepsilon_{n}}(X)=\sup _{m} \sup _{n \in \mathbb{N}} \Phi_{c, \varepsilon_{n}}^{m}(0) .
$$

Hence, the two suprema can be swapped.

We are now ready to show soundness (Theorem 10.11).

Theorem (Soundness). Let c be a program, and suppose that $\mathcal{E}$ : State $\times$ State $\rightarrow \mathbb{R}_{\geqslant 0}^{\infty}$ is a relational expectation. Then

$$
\operatorname{rpe}(\mathrm{c}, \mathcal{\varepsilon}) \leqslant \widetilde{r p e}(\mathrm{c}, \mathcal{E}) .
$$

Equivalently, if $\widetilde{r p e}(c, \mathcal{E})\left(s_{1}, s_{2}\right)$ is finite for input states $s_{1}, s_{2} \in$ State then there exists a coupling $\mu_{s_{1}, s_{2}} \in \Gamma\left(\llbracket c \rrbracket s_{1}, \llbracket c \rrbracket s_{2}\right)$ such that

$$
\mathbb{E}_{\mu_{s_{1}, s_{2}}}[\varepsilon] \leqslant \widetilde{r p e}(c, \varepsilon)\left(s_{1}, s_{2}\right) .
$$

Proof. Given $v \in X$, we write $\delta(v)$ for the point distribution centered at $v$, and given $\mu \in \mathbf{S D}(X)$ and $f: X \rightarrow \operatorname{SD}(X)$, we write $\mathbb{E}_{\mu}[f]$ for the distribution bind. Throughout, let $\left(s_{1}, s_{2}\right) \in$ State $\times$ State be two initial states. We prove the second, equivalent formulation by induction on the structure of $c$. Suppose that $\widetilde{r p e}(c, \mathcal{E})\left(s_{1}, s_{2}\right)$ is finite. 
- skip. Take the coupling $\delta\left(s_{1}, s_{2}\right)$. Then

$$
\mathbb{E}_{\delta\left(s_{1}, s_{2}\right)}[\mathcal{E}]=\mathcal{E}\left(s_{1}, s_{2}\right)=\widetilde{\operatorname{rpe}}(\text { skip, } \mathcal{E})\left(s_{1}, s_{2}\right) \text {. }
$$

- $x \leftarrow e$. Analogous to skip, but taking the coupling $\delta\left(s_{1}^{\prime}, s_{2}^{\prime}\right)$, where $s_{i}^{\prime}=s_{i}[x \mapsto$ $\left.\llbracket e \rrbracket s_{i}\right]$.

- $x \notin d$. Let $F$ : State $\rightarrow \mathbf{S D}($ State $)$ be defined as $F=\llbracket x \notin d \rrbracket . F\left(s_{1}\right)$ and $F\left(s_{2}\right)$ must have equal weights for $\widetilde{r p e}(x \notin d, \mathcal{E})\left(s_{1}, s_{2}\right)$ to be finite and evidently $F\left(s_{1}\right)$ and $F\left(s_{2}\right)$ both have countable support, so Lemma C.4 implies that there exists a coupling $\mu \in \Gamma\left(\llbracket x \leqslant d \rrbracket s_{1}, \llbracket x \leqslant d \rrbracket s_{2}\right)$ such that

$$
\mathbb{E}_{\mu}[\mathcal{E}]=\mathcal{E}^{\#}\left(\llbracket x \notin \mathrm{d} \rrbracket s_{1}, \llbracket x \leftarrow \mathrm{d} \rrbracket s_{2}\right)=\widetilde{r p e}(x \notin \mathrm{d}, \mathcal{E}) \text {. }
$$

- $c ; c^{\prime}$. By induction, there exists a coupling $\mu_{s_{1}, s_{2}} \in \Gamma\left(\llbracket c \rrbracket s s_{1}, \llbracket c \rrbracket s_{2}\right)$ such that

$$
\mathbb{E}_{\mu_{s_{1}, s_{2}}}\left[\widetilde{r p e}\left(c^{\prime}, \mathcal{E}\right)\right] \leqslant \widetilde{r p e}\left(c ; c^{\prime}, \mathcal{E}\right)\left(s_{1}, s_{2}\right)<\infty .
$$

Thus, $\widetilde{r p e}\left(c^{\prime}, \varepsilon\right)\left(s_{1}^{\prime}, s_{2}^{\prime}\right)$ must be finite for all pairs $\left(s_{1}^{\prime}, s_{2}^{\prime}\right) \in \operatorname{supp}\left(\mu_{s_{1}, s_{2}}\right) \triangleq$ $S$. Again by induction, for all $\left(s_{1}^{\prime}, s_{2}^{\prime}\right) \in S$ there exists a coupling $\mu_{s_{1}^{\prime}, s_{2}^{\prime}}^{\prime} \in$ $\Gamma\left(\llbracket c^{\prime} \rrbracket s_{1}^{\prime}, \llbracket c^{\prime} \rrbracket s_{2}^{\prime}\right)$ such that

$$
\mathbb{E}_{\mu_{s_{1}^{\prime}, s_{2}^{\prime}}^{\prime}}[\mathcal{E}] \leqslant \widetilde{r p e}\left(\mathrm{c}^{\prime}, \mathcal{E}\right)\left(s_{1}^{\prime}, \mathrm{s}_{2}^{\prime}\right)<\infty
$$

Define the following joint distribution:

$$
\mu_{s_{1}, s_{2}}^{*}\left(x_{1}, x_{2}\right)=\mathbb{E}_{\left(y_{1}, y_{2}\right) \sim \mu_{s_{1}, s_{2}}}\left[\mu_{y_{1}, y_{2}}^{\prime}\left(x_{1}, x_{2}\right)\right] .
$$

By a routine calculation, it is not hard to show that $\mu^{*}$ is indeed a coupling in $\Gamma\left(\llbracket c ; c^{\prime} \rrbracket s_{1}, \llbracket c ; c^{\prime} \rrbracket s_{2}\right)$. Let's for instance compute the first marginal (the second 
marginal is analogous):

$$
\begin{aligned}
& \pi_{1}\left(\mu_{s_{1}, s_{2}}^{*}\right)\left(x_{1}\right)=\sum_{x_{2} \in \text { State }} \mu_{s_{1}, s_{2}}^{*}\left(x_{1}, x_{2}\right) \\
& =\sum_{x_{2} \in \text { State }} \mathbb{E}_{\left(y_{1}, y_{2}\right) \sim \mu_{s_{1}, s_{2}}}\left[\mu_{y_{1}, y_{2}}^{\prime}\left(x_{1}, x_{2}\right)\right] \\
& =\mathbb{E}_{\left(y_{1}, y_{2}\right) \sim \mu_{s_{1}, s_{2}}}\left[\sum_{x_{2} \in \text { State }} \mu_{y_{1}, y_{2}}^{\prime}\left(x_{1}, x_{2}\right)\right] \\
& =\mathbb{E}_{\left(y_{1}, y_{2}\right) \sim \mu_{s_{1}, s_{2}}}\left[\left(\llbracket c^{\prime} \rrbracket y_{1}\right)\left(x_{1}\right)\right] \\
& =\sum_{y_{1} \in \text { State }} \sum_{y_{2} \in \text { State }}\left(\llbracket c^{\prime} \rrbracket y_{1}\right)\left(x_{1}\right) \cdot \mu_{s_{1}, s_{2}}\left(y_{1}, y_{2}\right) \\
& =\left(\sum_{y_{1} \in \text { State }}\left(\llbracket \mathfrak{c}^{\prime} \rrbracket y_{1}\right)\left(x_{1}\right)\right) \cdot\left(\sum_{y_{2} \in \text { State }} \mu_{s_{1}, s_{2}}\left(y_{1}, y_{2}\right)\right) \\
& =\sum_{y_{1} \in \text { State }}\left(\llbracket c^{\prime} \rrbracket y_{1}\right)\left(x_{1}\right) \cdot\left(\llbracket c \rrbracket s s_{1}\right)\left(y_{1}\right) \\
& =\left(\llbracket c ; c^{\prime} \rrbracket s_{1}\right)\left(x_{1}\right)
\end{aligned}
$$

Combining inequalities and applying monotonicity of expectations yields

$$
\mathbb{E}_{\mu_{s_{1}, s_{2}}^{*}}[\mathcal{E}]=\mathbb{E}_{\mu_{s_{1}, s_{2}}}\left[\mathbb{E}_{\mu_{-,-}^{\prime}}[\varepsilon]\right] \leqslant \mathbb{E}_{\mu_{s_{1}, s_{2}}}\left[\widetilde{r p e}\left(\mathrm{c}^{\prime}, \mathcal{E}\right)\right] \leqslant \widetilde{r p e}\left(\mathrm{c} ; \mathrm{c}^{\prime}, \mathcal{E}\right)\left(s_{1}, s_{2}\right) .
$$

- if $e$ then $c$ else $c^{\prime}$. Note that $e$ cannot be different between $s_{1}$ and $s_{2}$, otherwise

$$
\widetilde{r p e}\left(\text { if } e \text { then } c \text { else } c^{\prime}, \varepsilon\right)\left(s_{1}, s_{2}\right)
$$

is infinite. Thus, there are two possible cases: either $e$ is true in both $s_{1}, s_{2}$, or $e$ is false in both $s_{1}, s_{2}$. In the first case, we have:

$$
\llbracket \text { if } e \text { then } c \text { else } c^{\prime} \rrbracket s_{i}=\llbracket c \rrbracket s_{i} .
$$

By induction, there exists a coupling $\mu_{\mathfrak{t}}\left(s_{1}, s_{2}\right) \in \Gamma\left(\llbracket c \rrbracket s_{1}, \llbracket c \rrbracket s_{2}\right)$ such that

$$
\mathbb{E}_{\mu_{\mathfrak{t}}\left(s_{1}, s_{2}\right)}[\mathcal{E}] \leqslant \widetilde{r p e}(c, \mathcal{E})\left(s_{1}, s_{2}\right)=\widetilde{r p e}\left(\text { if } e \text { then } c \text { else } c^{\prime}, \mathcal{E}\right)\left(s_{1}, s_{2}\right)
$$

since the right-hand side is finite by assumption. Similarly, if $e$ is false in both $s_{1}$ and $s_{2}$, by induction there exists a coupling $\mu_{f}\left(s_{1}, s_{2}\right) \in \Gamma\left(\llbracket \mathfrak{c}^{\prime} \rrbracket s_{1}, \llbracket c^{\prime} \rrbracket s_{2}\right)$ such that

$$
\mathbb{E}_{\mu_{\mathrm{f}}\left(s_{1}, s_{2}\right)}[\varepsilon] \leqslant \widetilde{r p e}\left(c^{\prime}, \mathcal{E}\right)\left(s_{1}, s_{2}\right)=\widetilde{r p e}\left(\text { if } e \text { then } c \text { else } c^{\prime}, \mathcal{E}\right)\left(s_{1}, s_{2}\right)
$$

since the right-hand side is finite by assumption. Thus, we can define the desired coupling by case analysis:

$$
\mu\left(s_{1}, s_{2}\right) \triangleq \begin{cases}\mu_{\mathfrak{t}}\left(s_{1}, s_{2}\right) & : \llbracket e \rrbracket s_{1}=\llbracket e \rrbracket s_{2}=t t \\ \mu_{f}\left(s_{1}, s_{2}\right) & : \llbracket e \rrbracket s_{1}=\llbracket e \rrbracket s_{2}=f f \\ & : \llbracket e \rrbracket s_{1} \neq \llbracket e \rrbracket s_{2} \quad \text { (impossible) }\end{cases}
$$


- while e do c. By induction on $c$, for any pair of states $s_{1}^{\prime}, s_{2}^{\prime}$ and any expectation $\mathcal{E}_{\mathrm{c}}$ such that $\widetilde{r p e}\left(\mathrm{c}, \mathcal{E}_{\mathrm{c}}\right)\left(\mathrm{s}_{1}^{\prime}, \mathrm{s}_{2}^{\prime}\right)$ is finite, there exists a coupling $v_{s_{1}, s_{2}} \in$ $\Gamma\left(\llbracket c \rrbracket s_{1}, \llbracket c \rrbracket s_{2}\right)$ such that

$$
\mathbb{E}_{{v_{s_{1}, s_{2}}}}\left[\mathcal{E}_{\mathrm{c}}\right] \leqslant \mathcal{E}_{\mathrm{c}}\left(s_{1}^{\prime}, s_{2}^{\prime}\right) \text {. }
$$

Now, let's consider the loop. We define the following loop approximants:

$$
\begin{aligned}
c_{0} & \triangleq \text { while } t t \text { do skip } \\
c_{i+1} & \triangleq \text { if } e \text { then } c ; c_{i} \text { else skip }
\end{aligned}
$$

Each approximant executes at most $i$ iterations of the loop; the zero-th approximant returns the zero distribution and does not execute any iterations of the loop body. By definition, the relational pre-expectation of $\mathcal{E}$ with respect to $c_{0}$ is:

$$
\widetilde{r p e}\left(c_{0}, \mathcal{E}\right)=\operatorname{Ifp} X . \Phi_{\mathcal{E}, \text { skip }}(X),
$$

where the characteristic functional of a loop while e do $c$ is defined as:

$$
\Phi_{\mathcal{E}, \mathrm{c}}(X) \triangleq[e\langle 1\rangle \wedge e\langle 2\rangle] \cdot \widetilde{\operatorname{rp} e}(\mathrm{c}, \mathrm{X})+[\neg e\langle 1\rangle \wedge \neg e\langle 2\rangle] \cdot \mathcal{E}+[e\langle 1\rangle \neq e\langle 2\rangle] \cdot
$$

It is easy to show that the constant zero relational expectation is a fixed point for the loop $c_{0}$, and evidently it must be the least fixed point. So, $\widetilde{r p e}\left(c_{0}, \varepsilon\right)=0$. Let

$$
\begin{aligned}
\mathcal{E}_{0} & \triangleq \widetilde{\operatorname{rpe}}\left(c_{0}, \varepsilon\right)=0 \\
\mathcal{E}_{i+1} & \triangleq \widetilde{\operatorname{rpe}}\left(c_{i+1}, \mathcal{E}\right) \\
& =[e\langle 1\rangle \wedge e\langle 2\rangle] \cdot \widetilde{\operatorname{rpe}}\left(c, \varepsilon_{i}\right)+[\neg e\langle 1\rangle \wedge \neg e\langle 2\rangle] \cdot \mathcal{E}+\cdot[e\langle 1\rangle \neq e\langle 2\rangle] \cdot \infty
\end{aligned}
$$

By induction and definition of relational pre-expectation, $\varepsilon_{i}=\Phi_{\varepsilon, c}^{i}(0)$. Furthermore, by monotonicity (Lemma C.3) $\Phi_{\varepsilon, c}^{i}(0)$ is a monotone increasing sequence in $i$.

We now need two small lemmas.

Lemma C.6. For every $\mathrm{j} \in \mathbb{N}$, program $\mathrm{c}$, and relational expectation $\mathcal{E}$, we have:

$$
\Phi_{\mathcal{E}, \mathrm{c}}^{\mathrm{j}}(0) \leqslant \operatorname{IfpX} . \Phi_{\mathcal{E}, \mathrm{c}}(\mathrm{X})=\widetilde{r p e}(\text { while e do } c, \mathcal{E}) .
$$

Proof. By induction on $j$. The base case $j=0$ is clear, and the inductive step follows by monotonicity (Lemma C.3):

$$
\Phi_{\varepsilon, \mathrm{c}}^{\mathrm{j}+1}(0)=\Phi_{\varepsilon, \mathrm{c}}\left(\Phi_{\mathcal{\varepsilon}, \mathrm{c}}^{\mathrm{i}}(0)\right) \leqslant \Phi_{\varepsilon, \mathrm{c}}\left(\operatorname{Ifp} X . \Phi_{\mathcal{E}, \mathrm{c}}(X)\right)=\operatorname{Ifp} X . \Phi_{\varepsilon, \mathrm{c}}(X) .
$$

Now, let $\left(s_{1}, s_{2}\right)$ be two given input states such that $\widetilde{r p e}($ while e do $c, \mathcal{E})\left(s_{1}, s_{2}\right)<$ $\infty$. As an immediate consequence, $\Phi_{\mathcal{E}, c}^{i}(0)\left(s_{1}, s_{2}\right)$ must be finite for all $i$, so $\mathcal{E}_{\mathfrak{i}}\left(s_{1}, s_{2}\right)$ are all finite. 
Lemma C.7. For all $\mathbf{j} \in \mathbb{N}$ and $\left(s_{1}^{\prime}, s_{2}^{\prime}\right) \in$ State $\times$ State, if $\varepsilon_{j}\left(s_{1}^{\prime}, s_{2}^{\prime}\right)<\infty$ then there exists a coupling $\mu_{j, s_{1}^{\prime}, s_{2}^{\prime}} \in \Gamma\left(\llbracket c_{j} \rrbracket s_{1}^{\prime}, \llbracket c_{j} \rrbracket s_{2}^{\prime}\right)$ such that

$$
\mathbb{E}_{\mu_{j, s_{1}^{\prime}, s_{2}^{\prime}}}[\mathcal{E}] \leqslant \mathcal{E}_{\mathfrak{j}}\left(s_{1}^{\prime}, s_{2}^{\prime}\right)<\infty \text {. }
$$

Proof. By induction on $j$. The base case $j=0$ is clear, taking the null coupling that assigns probability zero to every pair of states. For the inductive step, we have

$$
\varepsilon_{j+1}=[e\langle 1\rangle \wedge e\langle 2\rangle] \cdot \widetilde{\operatorname{rpe}}\left(c, \varepsilon_{j}\right)+[\neg e\langle 1\rangle \wedge \neg e\langle 2\rangle] \cdot \varepsilon+[e\langle 1\rangle \neq e\langle 2\rangle] \cdot \infty .
$$

Note that e must be equal in $s_{1}^{\prime}$ and $s_{2}^{\prime}$, since $\varepsilon_{j+1}\left(s_{1}^{\prime}, s_{2}^{\prime}\right)$ is finite. There are two cases. If $e$ is false in $s_{1}^{\prime}$ and $s_{2}^{\prime}$, then $\llbracket c_{j+1} \rrbracket s_{1}^{\prime}=\delta\left(s_{1}^{\prime}\right)$ and $\llbracket c_{j+1} \rrbracket s_{2}^{\prime}=\delta\left(s_{2}^{\prime}\right)$. We can define the coupling $\mu_{s_{1}^{\prime}, s_{2}^{\prime}}=\delta\left(s_{1}^{\prime}, s_{2}^{\prime}\right) \in \Gamma\left(\llbracket c_{j+1} \rrbracket s_{1}^{\prime}, \llbracket c_{j+1} \rrbracket s_{2}^{\prime}\right)$ and we are done, since

$$
\mathbb{E}_{\mu_{s_{1}^{\prime}, s_{2}^{\prime}}}[\mathcal{E}]=\mathcal{E}\left(s_{1}^{\prime}, s_{2}^{\prime}\right)=\mathcal{E}_{j+1}\left(s_{1}^{\prime}, s_{2}^{\prime}\right) \text {. }
$$

Otherwise, suppose that $e$ is true in $s_{1}^{\prime}$ and $s_{2}^{\prime}$. Since $\varepsilon_{j+1}\left(s_{1}^{\prime}, s_{2}^{\prime}\right)<\infty$, we must have $\widetilde{r p e}\left(\mathrm{c}, \mathcal{E}_{\mathrm{j}}\right)\left(\mathrm{s}_{1}^{\prime}, \mathrm{s}_{2}^{\prime}\right)<\infty$ as well. Hence by the (outer) induction on the structure of the program, there exists a coupling $v_{s_{1}^{\prime}, s_{2}^{\prime}} \in \Gamma\left(\llbracket c \rrbracket s_{1}^{\prime}, \llbracket c \rrbracket s_{2}^{\prime}\right)$ such that

$$
\mathbb{E}_{v_{s_{1}^{\prime}, s_{2}^{\prime}}}\left[\mathcal{E}_{j}\right] \leqslant \mathcal{E}_{j}\left(s_{1}^{\prime}, s_{2}^{\prime}\right)<\infty .
$$

As a result, for all states $\left(t_{1}, t_{2}\right) \in \operatorname{supp}\left(v_{s_{1}^{\prime}, s_{2}^{\prime}}\right)$, we must have $\varepsilon_{j}\left(t_{1}, t_{2}\right)$ finite as well. By the (inner) induction hypothesis on $j$, there is a coupling $\mu_{j, t_{1}, t_{2}} \in$ $\Gamma\left(\llbracket c_{j} \rrbracket t_{1}, \llbracket c_{j} \rrbracket t_{2}\right)$ such that

$$
\mathbb{E}_{\mu_{j, t_{1}, t_{2}}}[\mathcal{E}] \leqslant \mathcal{E}_{j}\left(t_{1}, t_{2}\right)<\infty .
$$

Now, we can define the coupling for the $(j+1)$-th approximants:

$$
\mu_{j+1, s_{1}^{\prime}, s_{2}^{\prime}} \triangleq \mathbb{E}_{v_{s_{1}^{\prime}, s_{2}^{\prime}}}\left[\mu_{j,-,-}\right]
$$

We first check the distance condition. By definition, we have:

$$
\begin{aligned}
\mathbb{E}_{\mu_{j+1, s_{1}^{\prime}, s_{2}}}[\mathcal{E}] & =\mathbb{E}_{\left(\mathrm{t}_{1}, \mathrm{t}_{2}\right) \sim v_{s_{1}^{\prime}, s_{2}^{\prime}}}\left[\mathbb{E}_{\mu_{j, t_{1}, \mathrm{t}_{2}}}[\mathcal{E}]\right] \\
& \leqslant \mathbb{E}_{\left(\mathrm{t}_{1}, \mathrm{t}_{2}\right) \sim v_{s_{1}^{\prime}, s_{2}^{\prime}}}\left[\mathcal{E}_{j}\left(\mathrm{t}_{1}, \mathrm{t}_{2}\right)\right] \\
& \leqslant \mathcal{E}_{j}\left(s_{1}^{\prime}, s_{2}^{\prime}\right) \\
& \leqslant \mathcal{E}_{j+1}\left(s_{1}^{\prime}, s_{2}^{\prime}\right)
\end{aligned}
$$

The marginal condition is not hard to show, using the marginal properties of $v_{s_{1}^{\prime}, s_{2}^{\prime}}$ and $\mu_{j, t_{1}, t_{2}}$ combined with the definition of approximants: since $e$ is true in $s_{1}^{\prime}$ and $s_{2}^{\prime}$, we have $\llbracket c_{j+1} \rrbracket s_{1}^{\prime}=\llbracket c ; c_{j} \rrbracket s_{1}^{\prime}$ and $\llbracket c_{j+1} \rrbracket s_{2}^{\prime}=\llbracket c ; c_{j} \rrbracket s_{2}^{\prime}$. The proof follows the case for sequential composition. 
Since $\varepsilon_{\mathfrak{i}}\left(s_{1}, s_{2}\right)<\infty$ by assumption, we may apply Lemma C.7 with input states $s_{1}, s_{2}$ and expectations $\mathcal{E}_{i}$ to produce a sequence of couplings $\mu_{i, s_{1}, s_{2}} \in$ $\Gamma\left(\llbracket c_{i} \rrbracket s_{1}, \llbracket c_{i} \rrbracket s_{2}\right)$ such that

$$
\mathbb{E}_{\mu_{i, s_{1}, s_{2}}}[\mathcal{E}] \leqslant \mathcal{E}_{i}\left(s_{1}, s_{2}\right)=\widetilde{\operatorname{rpe}}\left(c_{i}, \mathcal{E}\right)=\Phi_{\mathcal{E}, \mathrm{c}}^{i}(0)<\infty .
$$

By Theorem C.2, from the sequence $\mu_{i, s_{1}, s_{2}}$ we can extract a subsequence $\mu_{i, s_{1}, s_{2}}^{\prime}$ (and a corresponding subsequence $c_{i}^{\prime}$ of $c_{i}$ ) that converges monotonically to a coupling satisfying

$$
\tilde{\mu}_{s_{1}, s_{2}} \in \Gamma\left(\lim _{i \rightarrow \infty} \llbracket c_{i}^{\prime} \rrbracket s_{1}, \lim _{i \rightarrow \infty} \llbracket c_{i}^{\prime} \rrbracket s_{2}\right)=\Gamma\left(\llbracket \text { while e do } c \rrbracket s_{1}, \llbracket \text { while e do } c \rrbracket s_{2}\right),
$$

by the definition of semantics for loops. All that remains to show is:

$$
\mathbb{E}_{\left(s_{1}^{\prime}, s_{2}^{\prime}\right) \sim \tilde{\mu}_{s_{1}, s_{2}}}\left[\mathcal{E}\left(s_{1}^{\prime}, s_{2}^{\prime}\right)\right] \leqslant \widetilde{r p e}(\text { while e do } c, \mathcal{E})\left(s_{1}, s_{2}\right) \text {. }
$$

We can compute:

$$
\begin{aligned}
& \mathbb{E}_{\left(s_{1}^{\prime}, s_{2}^{\prime}\right) \sim \tilde{\mu}_{s_{1}, s_{2}}}\left[\mathcal{E}\left(s_{1}^{\prime}, s_{2}^{\prime}\right)\right]=\sum_{\left(s_{1}^{\prime}, s_{2}^{\prime}\right) \in \text { State } \times \text { State }} \mathcal{E}\left(s_{1}^{\prime}, s_{2}^{\prime}\right) \cdot \lim _{i \rightarrow \infty} \mu_{i, s_{1}, s_{2}}^{\prime}\left(s_{1}^{\prime}, s_{2}^{\prime}\right) \\
& \leqslant \sum_{\left(s_{1}^{\prime}, s_{2}^{\prime}\right) \in \text { State } \times \text { State }} \lim _{i \rightarrow \infty} \mathcal{E}\left(s_{1}^{\prime}, s_{2}^{\prime}\right) \cdot \mu_{i, s_{1}, s_{2}}^{\prime}\left(s_{1}^{\prime}, s_{2}^{\prime}\right) \\
& \text { ( } \mathcal{E} \text { may be } \infty \text { ) } \\
& \leqslant \lim _{i \rightarrow \infty} \sum_{\left(s_{1}^{\prime}, s_{2}^{\prime}\right) \in \text { State } \times \text { State }} \mathcal{E}\left(s_{1}^{\prime}, s_{2}^{\prime}\right) \cdot \mu_{i, s_{1}, s_{2}}^{\prime}\left(s_{1}^{\prime}, s_{2}^{\prime}\right) \\
& \text { (by Fatou's lemma) } \\
& \leqslant \lim _{i \rightarrow \infty}\left(\widetilde{r p e}\left(c_{i}^{\prime}, \mathcal{E}\right)\left(s_{1}, s_{2}\right)\right) \\
& =\left(\lim _{i \rightarrow \infty} \widetilde{\operatorname{rpe}}\left(c_{i}^{\prime}, \mathcal{E}\right)\right)\left(s_{1}, s_{2}\right) \\
& \text { (by construction) } \\
& =\lim _{i \rightarrow \infty}\left(\Phi_{\varepsilon, c}^{i}(0)\right)\left(s_{1}, s_{2}\right) \\
& \leqslant\left(\operatorname{lfp} X . \Phi_{\varepsilon, c}(X)\right)\left(s_{1}, s_{2}\right) \\
& =\widetilde{r p e}(\text { while e do } c, \mathcal{E})\left(s_{1}, s_{2}\right) \text {. }
\end{aligned}
$$

\section{C.4 Embedding Relational Hoare Logics}

Proof of Theorem 10.15. We adopt the convention that $f(\infty) \triangleq \infty$, even if $f$ is constant. The proof is by induction on the derivation.

Case Assn: By definition, we have:

$$
\begin{aligned}
& \widetilde{\operatorname{rpe}}(x \leftarrow e, \mathcal{E}+[\neg \mathrm{Q}] \cdot \infty)= \\
& =\operatorname{id}(\mathcal{E}\{e\langle 1\rangle, e\langle 2\rangle / x\langle 1\rangle, x\langle 2\rangle\})+[\neg \mathrm{Q}\{e\langle 1\rangle, e\langle 2\rangle / x\langle 1\rangle, x\langle 2\rangle\}] \cdot \infty
\end{aligned}
$$


AssN $\overline{\vdash\left\{\mathrm{Q}\left[e_{1}\langle 1\rangle, e_{2}\langle 2\rangle / x_{1}\langle 1\rangle, x_{2}\langle 2\rangle\right] ; \mathcal{E}\left[e_{1}\langle 1\rangle, e_{2}\langle 2\rangle / x_{1}\langle 1\rangle, x_{2}\langle 2\rangle\right]\right\} x_{1} \leftarrow e_{1} \sim \text { id } x_{2} \leftarrow e_{2}\{\mathrm{Q} ; \mathcal{E}\}}$

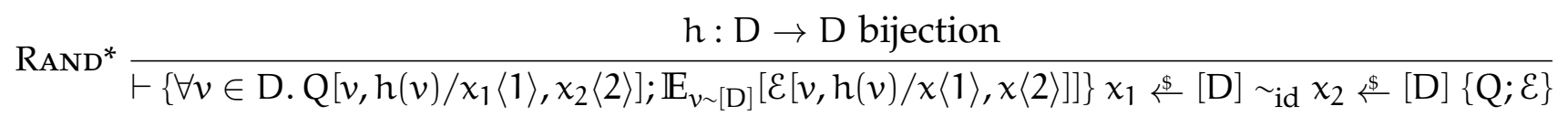

$$
\operatorname{SEQ} \frac{\vdash\{P ; \varepsilon\} c_{1} \sim_{f} c_{2}\left\{Q ; \mathcal{E}^{\prime}\right\} \quad \vdash\left\{Q ; \mathcal{E}^{\prime}\right\} c_{1}^{\prime} \sim_{f^{\prime}} c_{2}^{\prime}\left\{R ; \mathcal{E}^{\prime \prime}\right\}}{\vdash\{P ; \varepsilon\} c_{1} ; c_{1}^{\prime} \sim_{f^{\prime} \circ f} c_{2} ; c_{2}^{\prime}\left\{R ; \mathcal{E}^{\prime \prime}\right\}}
$$$$
\vdash\left\{\mathrm{P} \wedge \mathrm{e}_{1}\langle 1\rangle ; \varepsilon\right\} \mathrm{c}_{1} \sim_{\mathrm{f}} \mathrm{c}_{2}\left\{\mathrm{Q} ; \mathcal{E}^{\prime}\right\}
$$

CoND $\frac{\vdash\left\{\mathrm{P} \wedge \neg e_{1}\langle 1\rangle ; \mathcal{E}\right\} \mathrm{c}_{1}^{\prime} \sim_{f} \mathrm{c}_{2}^{\prime}\left\{\mathrm{Q} ; \mathcal{E}^{\prime}\right\} \quad \models \mathrm{P} \rightarrow \mathrm{e}_{1}\langle 1\rangle=\mathrm{e}_{2}\langle 2\rangle}{\vdash\{\mathrm{P} ; \mathcal{E}\} \text { if } \mathrm{e}_{1} \text { then } \mathrm{c}_{1} \text { else } \mathrm{c}_{1}^{\prime} \sim_{\mathrm{f}} \text { if } \mathrm{e}_{2} \text { then } \mathrm{c}_{2} \text { else } \mathrm{c}_{2}^{\prime}\left\{\mathrm{Q} ; \mathcal{E}^{\prime}\right\}}$

$$
\vdash\left\{\mathrm{P} \wedge v\langle 1\rangle=\mathrm{k} ; \varepsilon_{\mathrm{k}}\right\} \mathrm{c}_{1} \sim_{\mathrm{f}_{\mathrm{k}}} \mathrm{c}_{2}\left\{\mathrm{P} \wedge v\langle 1\rangle=\mathrm{k}-1 ; \varepsilon_{\mathrm{k}-1}\right\}
$$

While $\frac{\models \mathrm{P} \rightarrow e_{1}\langle 1\rangle=e_{2}\langle 1\rangle \wedge\left(v\langle 1\rangle \leqslant 0 \leftrightarrow \neg e_{1}\langle 1\rangle\right)}{\vdash\left\{\mathrm{P} \wedge v\langle 1\rangle=\mathrm{n} ; \varepsilon_{\mathrm{n}}\right\} \text { while } e_{1} \text { do } c_{1} \sim_{f_{1} \circ \ldots \circ f_{n}} \text { while } e_{2} \text { do } c_{2}\left\{\mathrm{P} \wedge v\langle 1\rangle=0 ; \varepsilon_{0}\right\}}$

CONSEQ $\frac{\vdash\{\mathrm{P} ; \mathcal{E}\} \mathrm{c}_{1} \sim_{f} \mathrm{c}_{2}\left\{\mathrm{Q} ; \mathcal{E}^{\prime}\right\} \quad \models \mathrm{P}^{\prime} \rightarrow\left(\mathrm{P} \wedge \mathrm{f}(\mathcal{E}) \leqslant \mathrm{f}^{\prime}\left(\mathcal{E}^{\prime \prime}\right)\right) \wedge \mathrm{Q} \rightarrow \mathrm{Q}^{\prime} \wedge\left(\mathcal{E}^{\prime \prime \prime} \leqslant \mathcal{E}^{\prime}\right)}{\vdash\left\{\mathrm{P}^{\prime} ; \mathcal{E}^{\prime \prime}\right\} \mathrm{c}_{1} \sim_{\mathrm{f}^{\prime}} \mathrm{c}_{2}\left\{\mathrm{Q}^{\prime} ; \mathcal{E}^{\prime \prime \prime}\right\}}$

$$
\operatorname{CASE} \frac{\vdash\{\mathrm{P} \wedge \mathrm{R} ; \mathcal{E}\} \mathrm{c}_{1} \sim_{f} \mathrm{c}_{2}\left\{\mathrm{Q} ; \mathcal{E}^{\prime}\right\} \quad \vdash\{\mathrm{P} \wedge \neg \mathrm{R} ; \mathcal{E}\} \mathrm{c}_{1} \sim_{\mathrm{f}} \mathrm{c}_{2}\left\{\mathrm{Q} ; \mathcal{E}^{\prime}\right\}}{\vdash\{\mathrm{P} ; \mathcal{E}\} \mathrm{c}_{1} \sim_{\mathrm{f}} \mathrm{c}_{2}\left\{\mathrm{Q} ; \mathcal{E}^{\prime}\right\}}
$$

FRAME-D $\frac{\vdash\{P ; \mathcal{E}\} \mathrm{c}_{1} \sim_{f} \mathrm{c}_{2}\left\{\mathrm{Q} ; \mathcal{E}^{\prime}\right\} \quad \mathrm{f}(z)=\mathrm{k} \cdot z \text { where } k \geqslant 1 \quad \mathrm{FV}\left(\mathcal{E}^{\prime \prime}\right) \cap \mathrm{MV}\left(\mathrm{c}_{1}, \mathrm{c}_{2}\right)=\emptyset}{\vdash\left\{\mathrm{P} ; \mathcal{E}+\mathcal{E}^{\prime \prime}\right\} \mathrm{c}_{1} \sim_{\mathrm{f}} \mathrm{c}_{2}\left\{\mathrm{Q} ; \mathcal{E}^{\prime}+\mathcal{E}^{\prime \prime}\right\}}$

Figure C.1: EPRHL proof pules 
Case Rand*: By the proof rule for sampling (Proposition 10.13) taking the coupling function given by the bijection coupling $M\left(s_{1}, s_{2}\right)=M_{h}$, we have:

$$
\begin{aligned}
& \widetilde{\operatorname{rpe}}(x \notin[\mathrm{D}], \mathcal{E}+[\neg \mathrm{Q}] \cdot \infty) \leqslant \\
& \leqslant \mathbb{E}_{v \sim \llbracket[\mathrm{D}] \rrbracket}[\mathcal{E}\{v, \mathrm{~h}(v) / x\langle 1\rangle, x\langle 2\rangle\}+[\neg \mathrm{Q}\{v, \mathrm{~h}(v) / x\langle 1\rangle, x\langle 2\rangle\}] \cdot \infty] \\
& \leqslant \mathbb{E}_{v \sim[[D] \rrbracket}[\mathcal{E}\{v, \mathrm{~h}(v) / x\langle 1\rangle, x\langle 2\rangle\}]+[\forall v \in \mathrm{D} . \neg \mathrm{Q}\{v, \mathrm{~h}(v) / x\langle 1\rangle, x\langle 2\rangle\}] \cdot \infty
\end{aligned}
$$

where the second inequality is because each element $v \in \mathrm{D}$ has positive probability under $\llbracket[D] \rrbracket$, so if $\neg Q\{v, h(v) / x\langle 1\rangle, x\langle 2\rangle\}$ for some $v \in D$ then both sides are infinite.

Case Seq: By induction, we have:

$$
\begin{aligned}
& \widetilde{\operatorname{rpe}}\left(\mathrm{c}^{\prime}, \mathcal{E}^{\prime \prime}+[\neg \mathrm{R}] \cdot \infty\right) \leqslant \mathrm{f}^{\prime}\left(\mathcal{E}^{\prime}\right)+[\neg \mathrm{Q}] \cdot \infty \\
& \widetilde{\operatorname{rpe}}\left(\mathrm{c}, \mathcal{E}^{\prime}+[\neg \mathrm{Q}] \cdot \infty\right) \leqslant \mathrm{f}(\mathcal{E})+[\neg \mathrm{P}] \cdot \infty
\end{aligned}
$$

Then, we can conclude:

$$
\begin{aligned}
\widetilde{\operatorname{rpe}}\left(\mathrm{c} ; \mathrm{c}^{\prime}, \mathcal{E}^{\prime \prime}+[\neg \mathrm{R}] \cdot \infty\right) & =\widetilde{\operatorname{rpe}}\left(\mathrm{c}, \widetilde{\operatorname{rpe}}\left(\mathrm{c}^{\prime}, \mathcal{E}^{\prime \prime}+[\neg \mathrm{R}] \cdot \infty\right)\right) \\
& =\widetilde{\operatorname{rpe}}\left(\mathrm{c}, \mathrm{f}^{\prime}\left(\mathcal{E}^{\prime}\right)+[\neg \mathrm{Q}] \cdot \infty\right) \\
& \leqslant \mathrm{f}^{\prime}\left(\widetilde{\operatorname{rpe}}\left(\mathrm{c}, \mathcal{E}^{\prime}+[\neg \mathrm{Q}] \cdot \infty\right)\right) \\
& \leqslant \mathrm{f}^{\prime}(\mathrm{f}(\mathcal{E})+[\neg \mathrm{P}] \cdot \infty) \\
& =\mathrm{f}^{\prime} \circ \mathrm{f}(\mathcal{E})+[\neg \mathrm{P}] \cdot \infty
\end{aligned}
$$

Case Cond: By induction, we have:

$$
\begin{aligned}
& \widetilde{\operatorname{rpe}}\left(\mathrm{c}, \mathcal{E}^{\prime}+[\neg \mathrm{Q}] \cdot \infty\right) \leqslant \mathrm{f}\left(\mathcal{E}^{\prime}\right)+[\neg(\mathrm{P} \wedge \mathrm{e}\langle 1\rangle)] \cdot \infty \\
& \widetilde{\operatorname{rpe}}\left(\mathrm{c}^{\prime}, \mathcal{E}^{\prime}+[\neg \mathrm{Q}] \cdot \infty\right) \leqslant \mathrm{f}\left(\mathcal{E}^{\prime}\right)+[\neg(\mathrm{P} \wedge \neg \mathrm{e}\langle 1\rangle)] \cdot \infty
\end{aligned}
$$

Then, we have:

$$
\begin{aligned}
& \widetilde{\text { rpe }}\left(\text { if e then } c \text { else } c^{\prime}, \mathcal{E}^{\prime}+[\neg \mathrm{Q}] \cdot \infty\right) \\
& =[e\langle 1\rangle \wedge e\langle 2\rangle] \cdot \widetilde{r p e}\left(\mathrm{c}, \mathcal{E}^{\prime}+[\neg \mathrm{Q}] \cdot \infty\right)+[\neg e\langle 1\rangle \wedge \neg e\langle 2\rangle] \cdot \widetilde{r p e}\left(\mathrm{c}^{\prime}, \mathcal{E}^{\prime}+[\neg \mathrm{Q}] \cdot \infty\right) \\
& +[\neg(e\langle 1\rangle=e\langle 2\rangle)] \cdot \infty \\
& \leqslant[e\langle 1\rangle \wedge e\langle 2\rangle] \cdot\left(f\left(\mathcal{E}^{\prime}\right)+[\neg(\mathrm{P} \wedge e\langle 1\rangle)] \cdot \infty\right) \\
& +[\neg e\langle 1\rangle \wedge \neg e\langle 2\rangle] \cdot\left(f\left(\mathcal{E}^{\prime}\right)+[\neg(\mathrm{P} \wedge \neg e\langle 1\rangle)] \cdot \infty\right)+[\neg(e\langle 1\rangle=e\langle 2\rangle)] \cdot \infty \\
& \leqslant[e\langle 1\rangle \wedge e\langle 2\rangle] \cdot\left(f\left(\mathcal{E}^{\prime}\right)+[\neg(\mathrm{P} \wedge e\langle 1\rangle)] \cdot \infty\right) \\
& +[\neg e\langle 1\rangle \wedge \neg e\langle 2\rangle] \cdot\left(\mathrm{f}\left(\mathcal{E}^{\prime}\right)+[\neg(\mathrm{P} \wedge \neg e\langle 1\rangle)] \cdot \infty\right)+[\neg \mathrm{P}] \cdot \infty \\
& (\models \mathrm{P} \rightarrow \mathrm{e}\langle 1\rangle=\mathrm{e}\langle 2\rangle) \\
& =([e\langle 1\rangle \wedge e\langle 2\rangle]+[\neg e\langle 1\rangle \wedge \neg e\langle 2\rangle]) \cdot f\left(\mathcal{E}^{\prime}\right)+[\neg P] \cdot \infty \\
& \text { (boolean alg.) } \\
& =\mathrm{f}\left(\mathcal{E}^{\prime}\right)+[\neg \mathrm{P}] \cdot \infty
\end{aligned}
$$


Case While: Let $n$ be any natural number. For any natural number $0<m \leqslant n$, we write $f^{(m)}=f_{1} \circ \cdots \circ f_{m}$ and we define $f^{(0)}=i d$. We will show:

$\widetilde{r p e}\left(\right.$ while e do $\left.c, \varepsilon_{0}+[\neg(\mathrm{P} \wedge v\langle 1\rangle=0)] \cdot \infty\right) \leqslant \mathrm{f}^{(\mathrm{n})}\left(\mathcal{E}_{\mathrm{n}}\right)+[\neg(\mathrm{P} \wedge v\langle 1\rangle=\mathrm{n})] \cdot \infty$

We take the following loop invariant:

$$
\mathcal{J}_{\mathrm{n}} \triangleq \sum_{j=0}^{\mathrm{n}}\left([\mathrm{P} \wedge v\langle 1\rangle=j] \cdot \mathrm{f}^{(\mathrm{j})}\left(\mathcal{E}_{\mathrm{j}}\right)\right)+[\neg(\mathrm{P} \wedge v\langle 1\rangle \leqslant \mathrm{n})] \cdot \infty
$$

We claim that:

$$
\begin{aligned}
& {[e\langle 1\rangle \wedge e\langle 2\rangle] \cdot \widetilde{r p e}\left(c, \mathcal{J}_{\mathfrak{n}}\right)+[\neg e\langle 1\rangle \wedge \neg e\langle 2\rangle] \cdot\left(\mathcal{E}_{0}+[\neg(\mathrm{P} \wedge v\langle 1\rangle=0)] \cdot \infty\right)} \\
& +[e\langle 1\rangle \neq e\langle 2\rangle] \cdot \infty \leqslant \mathcal{J}_{\mathfrak{n}} .
\end{aligned}
$$

Both sides are infinite if $e\langle 1\rangle \neq e\langle 2\rangle$, or $\neg(\mathrm{P} \wedge v\langle 1\rangle \leqslant n)$, or $\neg e\langle 1\rangle \wedge \neg e\langle 2\rangle \wedge v \neq 0$. So, it suffices to prove:

$$
\begin{aligned}
& {[P \wedge e\langle 1\rangle \wedge e\langle 2\rangle \wedge v\langle 1\rangle \leqslant n] \cdot \widetilde{r p e}\left(\mathrm{c}, \mathcal{J}_{\mathfrak{n}}\right)+[\mathrm{P} \wedge \neg e\langle 1\rangle \wedge \neg e\langle 2\rangle] \cdot \mathcal{E}_{0}} \\
& \leqslant[\mathrm{P} \wedge v\langle 1\rangle \leqslant \mathrm{n}] \cdot \mathcal{J}_{\mathfrak{n}}=\sum_{j=0}^{n}[\mathrm{P} \wedge v\langle 1\rangle=j] \cdot \mathrm{f}^{(j)}\left(\mathcal{E}_{j}\right) .
\end{aligned}
$$

If $\mathrm{P} \wedge \neg e\langle 1\rangle \wedge \neg e\langle 2\rangle$ holds, then $v\langle 1\rangle=0$ holds by assumption. So by definition of $\mathcal{J}_{n}$ :

$$
[\mathrm{P} \wedge \neg e\langle 1\rangle \wedge \neg e\langle 2\rangle] \cdot \mathcal{E}_{0}=[\mathrm{P} \wedge v\langle 1\rangle=0] \cdot \mathrm{f}^{(0)}\left(\mathcal{E}_{0}\right) \leqslant \mathcal{J}_{n} .
$$

If $\mathrm{P} \wedge \mathrm{e}\langle 1\rangle \wedge e\langle 2\rangle \wedge v\langle 1\rangle \leqslant \mathrm{n}$ holds, then suppose that $v\langle 1\rangle=\mathrm{k}$ with $0<\mathrm{k} \leqslant \mathrm{n}$. We have:

$$
\begin{aligned}
& {[P \wedge e\langle 1\rangle \wedge e\langle 2\rangle \wedge v\langle 1\rangle=k] \cdot \widetilde{r p e}\left(\mathrm{c}, \mathrm{J}_{\mathfrak{n}}\right)} \\
& \leqslant[\mathrm{P} \wedge \mathrm{e}\langle 1\rangle \wedge \mathrm{e}\langle 2\rangle \wedge v\langle 1\rangle=\mathrm{k}] \cdot \widetilde{\mathrm{rpe}}\left(\mathrm{c}, \sum_{j=0}^{\mathrm{n}}[\mathrm{P} \wedge v\langle 1\rangle=\mathrm{j}] \cdot \mathrm{f}^{(\mathrm{j})}\left(\mathcal{E}_{\mathrm{j}}\right)\right. \\
& +[\neg(\mathrm{P} \wedge v\langle 1\rangle=\mathrm{k}-1)] \cdot \infty) \\
& =[\mathrm{P} \wedge e\langle 1\rangle \wedge e\langle 2\rangle \wedge v\langle 1\rangle=\mathrm{k}] \cdot \widetilde{r p e}\left(\mathrm{c},[\mathrm{P} \wedge v\langle 1\rangle=\mathrm{k}-1] \cdot \mathrm{f}^{(\mathrm{k}-1)}\left(\mathcal{E}_{\mathrm{k}-1}\right)\right. \\
& +[\neg(\mathrm{P} \wedge v\langle 1\rangle=\mathrm{k}-1)] \cdot \infty) \\
& \leqslant[P \wedge e\langle 1\rangle \wedge e\langle 2\rangle \wedge v\langle 1\rangle=k] \cdot f^{(k-1)}\left(\widetilde { r p e } \left(c, \varepsilon_{k-1}\right.\right. \\
& +[\neg(\mathrm{P} \wedge v\langle 1\rangle=\mathrm{k}-1)] \cdot \infty)) \\
& \leqslant[P \wedge e\langle 1\rangle \wedge e\langle 2\rangle \wedge v\langle 1\rangle=k] \cdot f^{(k-1)}\left(f_{k}\left(\mathcal{E}_{k}\right)+[\neg(P \wedge v\langle 1\rangle=k)] \cdot \infty\right) \\
& \text { (induction) } \\
& =[P \wedge e\langle 1\rangle \wedge e\langle 2\rangle \wedge v\langle 1\rangle=k] \cdot f^{(k)}\left(\mathcal{E}_{k}\right) \\
& \leqslant[P \wedge e\langle 1\rangle \wedge e\langle 2\rangle \wedge v\langle 1\rangle=k] \cdot J_{n}
\end{aligned}
$$


Above, we have used the induction hypothesis:

$$
\widetilde{r p e}\left(\mathrm{c}, \mathcal{E}_{\mathrm{k}-1}+[\neg(\mathrm{P} \wedge v\langle 1\rangle=\mathrm{k}-1)] \cdot \infty\right) \leqslant \mathrm{f}_{\mathrm{k}}\left(\mathcal{E}_{\mathrm{k}}\right)+[\neg(\mathrm{P} \wedge v\langle 1\rangle=\mathrm{k})] \cdot \infty .
$$

By the loop rule, we can conclude:

$$
\begin{gathered}
\widetilde{r p e}(\text { while e do } c, \mathcal{E}+[\neg(P \wedge v\langle 1\rangle=0)] \cdot \infty) \\
\quad \leqslant \mathcal{J}_{n} \leqslant f^{(n)}\left(\mathcal{E}_{n}\right)+[\neg(P \wedge v\langle 1\rangle=n)] \cdot \infty .
\end{gathered}
$$

Case Conseq: By induction, we have:

$$
\begin{aligned}
& \widetilde{\operatorname{rpe}}\left(\mathrm{c}, \mathcal{E}^{\prime \prime \prime}+\left[\neg \mathrm{Q}^{\prime}\right] \cdot \infty\right)=\widetilde{\operatorname{rpe}}\left(\mathrm{c},[\mathrm{Q}] \cdot \mathcal{E}^{\prime \prime \prime}+\left[\neg \mathrm{Q}^{\prime}\right] \cdot \infty\right) \\
& \leqslant \widetilde{r p e}\left(c, \mathcal{E}^{\prime}+\left[\neg \mathrm{Q}^{\prime}\right] \cdot \infty\right) \\
& \leqslant \mathrm{f}(\mathcal{E})+[\neg \mathrm{P}] \cdot \infty \\
& \leqslant \mathrm{f}(\mathcal{E})+\left[\neg \mathrm{P}^{\prime}\right] \cdot \infty \\
& =\left[\mathrm{P}^{\prime}\right] \cdot f(\varepsilon)+\left[\neg \mathrm{P}^{\prime}\right] \cdot \infty \\
& \leqslant\left[\mathrm{P}^{\prime}\right] \cdot \mathrm{f}^{\prime}\left(\mathcal{E}^{\prime \prime}\right)+\left[\neg \mathrm{P}^{\prime}\right] \cdot \infty \\
& \leqslant f^{\prime}\left(\mathcal{E}^{\prime \prime}\right)+\left[\neg P^{\prime}\right] \cdot \infty
\end{aligned}
$$

Case Case: By induction, we have:

$$
\begin{aligned}
& \widetilde{\operatorname{rpe}}\left(\mathrm{c}, \mathcal{E}^{\prime}+[\neg \mathrm{Q}] \cdot \infty\right) \leqslant \mathrm{f}\left(\mathcal{E}^{\prime}\right)+[\neg(\mathrm{P} \wedge \mathrm{R})] \cdot \infty \\
& \widetilde{\operatorname{rpe}}\left(\mathrm{c}, \mathcal{E}^{\prime}+[\neg \mathrm{Q}] \cdot \infty\right) \leqslant \mathrm{f}\left(\mathcal{E}^{\prime}\right)+[\neg(\mathrm{P} \wedge \neg \mathrm{R})] \cdot \infty
\end{aligned}
$$

Thus, we have:

$$
\begin{array}{rrr}
\widetilde{\operatorname{rpe}}\left(\mathrm{c}, \mathcal{E}^{\prime}+[\neg \mathrm{Q}] \cdot \infty\right) & \leqslant \mathrm{f}\left(\mathcal{E}^{\prime}\right)+\min ([\neg(\mathrm{P} \wedge \mathrm{R})],[\neg(\mathrm{P} \wedge \neg \mathrm{R})]) \cdot \infty \quad \text { (induction) } \\
& \leqslant \mathrm{f}\left(\mathcal{E}^{\prime}\right)+[\neg \mathrm{P}] \cdot \infty & \text { (boolean alg.) }
\end{array}
$$

Case Frame-D: We have:

$$
\begin{aligned}
\widetilde{\operatorname{rpe}}\left(\mathrm{c}, \mathcal{E}^{\prime}+\mathcal{E}^{\prime \prime}+[\neg \mathrm{Q}] \cdot \infty\right) & \leqslant \widetilde{\operatorname{rpe}}\left(\mathrm{c}, \mathcal{E}^{\prime}+[\neg \mathrm{Q}] \cdot \infty\right)+\mathcal{E}^{\prime \prime} \\
& \leqslant \mathrm{f}(\mathcal{E})+[\neg \mathrm{P}] \cdot \infty+\mathcal{E}^{\prime \prime} \\
& \leqslant \mathrm{f}\left(\mathcal{E}+\mathcal{E}^{\prime \prime}\right)+[\neg \mathrm{P}] \cdot \infty
\end{aligned}
$$

\section{C.5 Convergence of TD(o): Omitted details}

We start by analyzing the inner loop $w_{\text {in }}$. We first show that

$$
\widetilde{\operatorname{rpe}}\left(w_{\text {in }},\|\mathrm{W}\langle 1\rangle-\mathrm{W}\langle 2\rangle\|_{\infty}\right) \leqslant \mathcal{J}_{\text {in }}
$$


for the invariant $\mathcal{J}_{\text {in }}$ :

$$
\begin{aligned}
& \mathcal{J}_{\text {in }} \triangleq[i\langle 1\rangle \neq i\langle 2\rangle] \cdot \infty \\
& \quad+[i\langle 1\rangle=i\langle 2\rangle] \cdot \max _{l<|S|}\left([l<i\langle 1\rangle] \cdot|W\langle 1\rangle[l]-W\langle 2\rangle[l]|+[i\langle 1\rangle \leqslant l] \cdot k \cdot\|V\langle 1\rangle-V\langle 2\rangle\|_{\infty}\right) .
\end{aligned}
$$

Let $c_{\text {in }}$ be the body, and $c_{\text {samp }}$ be the three sampling statements. Applying INv, it suffices to show:

$$
\begin{aligned}
& {[i\langle 1\rangle<|\mathcal{S}| \wedge i\langle 2\rangle<|\mathcal{S}|] \cdot \widetilde{\text { rpe }}\left(c_{\text {in }}, \mathcal{J}_{\text {in }}\right)+[i\langle 1\rangle \geqslant|\mathcal{S}| \wedge i\langle 2\rangle \geqslant|\mathcal{S}|] \cdot\|W\langle 1\rangle-W\langle 2\rangle\|_{\infty}} \\
& +[i\langle 1\rangle \neq i\langle 2\rangle] \cdot \infty \leqslant \mathcal{J}_{\text {in }}
\end{aligned}
$$

We describe how to bound the key part of the invariant, $\widetilde{\operatorname{rpe}}\left(\mathrm{c}_{\mathrm{in}}, \mathcal{J}_{\mathrm{in}}\right)$ in the first term; the other cases are simpler. Computing the pre-expectation for the last two instructions gives us

$$
\widetilde{\operatorname{rpe}}\left(c_{\text {in }}, \mathcal{J}_{\text {in }}\right) \leqslant \widetilde{r p e}\left(c_{\text {samp }},[i\langle 1\rangle=i\langle 2\rangle] \cdot \mathcal{J}\right),
$$

where $\mathcal{I}$ is the following relational expectation:

$$
\max _{l<|S|}\left(\begin{array}{l}
{[l<i\langle 1\rangle] \cdot|W\langle 1\rangle[l]-W\langle 2\rangle[l]|} \\
+[l=i\langle 1\rangle] \cdot|(1-\alpha) \cdot(V[i]\langle 1\rangle-V[i]\langle 2\rangle)+\alpha \cdot(r\langle 1\rangle-r\langle 2\rangle+\gamma \cdot(V[j]\langle 1\rangle-V[j]\langle 2\rangle))| \\
+[i\langle 1\rangle+1 \leqslant l] \cdot k \cdot\|V\langle 1\rangle-V\langle 2\rangle\|_{\infty}
\end{array}\right) .
$$

By taking an appropriate coupling, we will show that $\widetilde{r p e}\left(c_{s a m p}, \mathcal{J}\right)$ is at most $\mathcal{J}_{\text {in }}$. For sampling $j$, we take the coupling function where if $a\langle 1\rangle=a\langle 2\rangle$, then we take the identity coupling ensuring $j\langle 1\rangle=j\langle 2\rangle$, otherwise we take the product coupling. We take the same coupling for sampling $r$. Finally for sampling $a$, we take the identity coupling ensuring $a\langle 1\rangle=a\langle 2\rangle$. When $i\langle 1\rangle=i\langle 2\rangle, j\langle 1\rangle=j\langle 2\rangle$, and $r\langle 1\rangle=r\langle 2\rangle$. By applying rule SAMP, we can upper-bound $\widetilde{r p e}\left(c_{i n}, J_{i n}\right)$ by $[i\langle 1\rangle=i\langle 2\rangle=i]$ times:

$$
\rho\left(v_{a}, v_{r}, v_{j}, i\right) \cdot \max _{l<|S|}\left(\begin{array}{l}
{[l<i] \cdot|\mathrm{W}\langle 1\rangle[\mathrm{l}]-\mathrm{W}\langle 2\rangle[\mathrm{l}]|} \\
+[\mathrm{l}=\mathrm{i}] \cdot\left|(1-\alpha) \cdot(\mathrm{V}[\mathrm{i}]\langle 1\rangle-\mathrm{V}[\mathrm{i}]\langle 2\rangle)+\alpha \cdot\left(\gamma \cdot\left(\mathrm{V}\langle 1\rangle\left[v_{j}\right]-\mathrm{V}\langle 2\rangle\left[v_{j}\right]\right)\right)\right| \\
+[i+1 \leqslant l] \cdot k \cdot\|\mathrm{V}\langle 1\rangle-\mathrm{V}\langle 2\rangle\|_{\infty}
\end{array}\right) .
$$

taking a sum over triples $\left(v_{a}, v_{r}, v_{j}\right) \in \mathcal{A} \times \mathcal{R} \times \mathcal{S}$ and $\rho\left(v_{a}, v_{r}, v_{j}, i\right)$ is the probability of drawing $v_{a}, v_{r}, v_{j}$ in current state $i$; note that for any fixed $i<|\mathcal{S}|$, the coefficients sum to 1 .

Now for any $l<|\mathcal{S}|$ and any input states, at most one of the three summands in the max is non-zero. We can bound the first and last summands:

$$
\begin{array}{rr}
{[l<i] \cdot|W\langle 1\rangle[l]-W\langle 2\rangle[l]| \leqslant \mathcal{J}_{\text {in }}} & (\text { since } l<i) \\
{[i+1 \leqslant l] \cdot k \cdot\|V\langle 1\rangle-V\langle 2\rangle\|_{\infty} \leqslant \mathcal{J}_{\text {in }}} & (\text { since } i \leqslant l)
\end{array}
$$




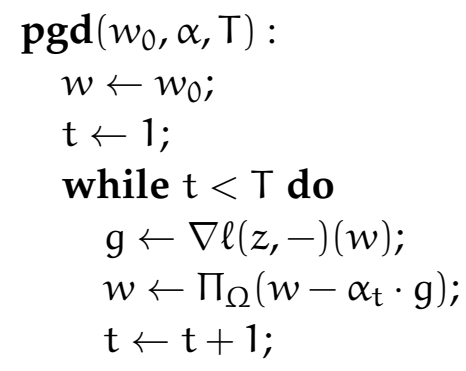

Figure C.2: Projected Gradient Descent (PGD)

For the second summand, we have:

$$
\begin{aligned}
& {[l=i] \cdot\left|(1-\alpha) \cdot(\mathrm{V}\langle 1\rangle[i]-\mathrm{V}\langle 2\rangle[i])+\alpha \gamma \cdot\left(\mathrm{V}\langle 1\rangle\left[v_{j}\right]-\mathrm{V}\langle 2\rangle\left[v_{j}\right]\right)\right|} \\
& \leqslant[l=i] \cdot\left|(1-\alpha) \cdot\|\mathrm{V}\langle 1\rangle-\mathrm{V}\langle 2\rangle\|_{\infty}+\alpha \gamma \cdot\|\mathrm{V}\langle 1\rangle-\mathrm{V}\langle 2\rangle\|_{\infty}\right| \\
& \left.\leqslant[l=i] \cdot k \cdot\|\mathrm{V}\langle 1\rangle-\mathrm{V}\langle 2\rangle\|_{\infty} \quad \quad \text { (since } i \leqslant l\right) \\
& \leqslant \mathcal{J}_{\text {in }}, \quad
\end{aligned}
$$

Putting everything together, we have:

$$
\begin{aligned}
\widetilde{r p e}\left(c_{\text {in }}, J_{\text {in }}\right) & \leqslant[i\langle 1\rangle=i\langle 2\rangle=i] \cdot \sum_{v_{a}, v_{r}, v_{j}} \text { Eq. (C.2)) } \\
& \leqslant[i\langle 1\rangle=i\langle 2\rangle=i] \cdot \sum_{v_{a}, v_{r}, v_{j}} \rho\left(v_{a}, v_{r}, v_{j}, i\right) \cdot J_{i n} \\
& =J_{\text {in }},
\end{aligned}
$$

establishing the inner invariant.

\section{C.6 Verifying robustness of Projected Gradient Descent (PGD)}

This example is inspired by an analysis by Miller and Hardt [119]. Let $\Omega \subseteq \mathbb{R}^{\mathrm{d}}$ be a compact and convex set of feasible parameters, and let $\Pi_{\Omega}: \mathbb{R}^{\mathrm{d}} \rightarrow \Omega$ be the Euclidean projection sending a point from $\mathbb{R}^{\mathrm{d}}$ to the closest point in $\Omega$ under the Euclidean distance. Given a loss function $\ell$, initial parameters $w_{0}$, and a sequence of step sizes $\left\{\alpha_{t}\right\}_{t}$, the program pgd in Figure C.2 runs projected gradient descent for $T$ iterations.

Consider running this algorithm with two different loss functions $\ell\langle 1\rangle$ and $\ell\langle 2\rangle$, satisfying the following conditions:

1. Gradients are close. For any parameter $w \in \mathbb{R}^{\mathrm{d}}$,

$$
\|\nabla \ell\langle 1\rangle(z,-)(w)-\nabla \ell\langle 2\rangle(z,-)(w)\| \leqslant \gamma .
$$


2. Gradient of loss function is Lipschitz. For any two parameters $w, w^{\prime} \in \mathbb{R}^{\mathrm{d}}$,

$$
\left\|\nabla \ell\langle 1\rangle(z,-)(w)-\nabla \ell\langle 1\rangle(z,-)\left(w^{\prime}\right)\right\| \leqslant \beta\left\|w-w^{\prime}\right\| .
$$

Taking the step sizes $\alpha_{t} \leqslant \alpha / t$, we can bound the distance between final weights $\|w\langle 1\rangle-w\langle 2\rangle\|$ from running projected gradient descent on the loss functions $\ell\langle 1\rangle$ and $\ell\langle 2\rangle$ by showing the following bound on the relational pre-expectation, which matches the analysis of Miller and Hardt [119]:

$$
\widetilde{r p e}\left(\operatorname{pgd}\left(w_{0}, \alpha, T\right),\|w\langle 1\rangle-w\langle 2\rangle\|\right) \leqslant \alpha \gamma T^{\alpha \beta+1}
$$

Intuitively, this property means that small changes to the loss function in PGD do not lead to large changes in the learned parameters.

To start the proof, we take the following loop invariant:

$$
\begin{aligned}
\mathcal{J} & \triangleq[\mathrm{t}\langle 1\rangle \neq \mathrm{t}\langle 2\rangle] \cdot \infty \\
& +[\mathrm{t}\langle 1\rangle=\mathrm{t}\langle 2\rangle] \cdot\|w\langle 1\rangle-w\langle 2\rangle\| \prod_{j=t}^{\mathrm{T}}\left(1+\alpha_{j} \beta\right) \\
& +[\mathrm{t}\langle 1\rangle=\mathrm{t}\langle 2\rangle] \cdot \sum_{s=\mathrm{t}\langle 1\rangle}^{\mathrm{T}} \alpha_{s} \gamma \prod_{j=s+1}^{\mathrm{T}} \exp \left(1+\alpha_{j} \beta\right)
\end{aligned}
$$

To apply the loop rule, we need to check

$$
\begin{aligned}
& {[(\mathrm{t}<\mathrm{T})\langle 1\rangle \wedge(\mathrm{t}<\mathrm{T})\langle 2\rangle] \widetilde{\operatorname{rpe}}(\mathrm{c}, \mathcal{J})+[(\mathrm{t} \geqslant \mathrm{T})\langle 1\rangle \wedge(\mathrm{t} \geqslant \mathrm{T})\langle 2\rangle] \mathcal{E}} \\
& +[(\mathrm{t}<\mathrm{T})\langle 1\rangle \neq(\mathrm{t}<\mathrm{T})\langle 2\rangle] \cdot \infty \leqslant \mathcal{J} .
\end{aligned}
$$

The main case is when both loop guards are true and when both loop counters are equal. Taking the relational pre-expectation for the loop body in this case, we have:

$$
\begin{aligned}
& \widetilde{r p e}(\mathrm{c}, \mathrm{J})= \\
& \left.=\left\|\Pi_{\Omega}\left(w-\alpha_{t} \cdot \nabla \ell(z,-)(w)\right)\langle 1\rangle-\Pi_{\Omega}\left(w-\alpha_{t} \cdot \nabla \ell(z,-)(w)\right)\langle 2\rangle\right\| \prod_{j=t}^{T}(1\rangle+1+\alpha_{j} \beta\right) \\
& +\sum_{s=t\langle 1\rangle+1}^{T} \alpha_{s} \gamma \prod_{j=s+1}^{T}\left(1+\alpha_{j} \beta\right) \\
& \leqslant\left(\|w\langle 1\rangle-w\langle 2\rangle\|+\alpha_{t}\|\nabla \ell(z,-)(w)\langle 1\rangle-\nabla \ell(z,-)(w)\langle 2\rangle\|\right) \prod_{j=t\langle 1\rangle+1}^{T}\left(1+\alpha_{j} \beta\right) \\
& +\sum_{s=t\langle 1\rangle+1}^{T} \alpha_{s} \gamma \prod_{j=s+1}^{T}\left(1+\alpha_{j} \beta\right) \\
& \left.\leqslant\left(\|w\langle 1\rangle-w\langle 2\rangle\|+\alpha_{t} \beta\|w\langle 1\rangle-w\langle 2\rangle\|+\alpha_{t} \gamma\right) \prod_{j=t}^{T}(1)+\alpha_{j} \beta\right)+\sum_{s=t}^{T} \alpha_{s} \gamma \prod_{j=s+1}^{T}\left(1+\alpha_{j} \beta\right) \\
& =\mathfrak{J}
\end{aligned}
$$


Pushing the invariant past the initial assignment instructions and taking the same step sizes $\alpha_{t} \leqslant \alpha / t$ as Miller and Hardt [119], we conclude:

$$
\begin{aligned}
& \widetilde{r p e}\left(\operatorname{pgd}\left(w_{0}, \alpha, \mathrm{T}\right),\|w\langle 1\rangle-w\langle 2\rangle\|\right) \leqslant \sum_{s=1}^{\mathrm{T}} \alpha_{s} \gamma \prod_{j=s+1}^{\mathrm{T}}\left(1+\alpha_{j} \beta\right) \\
& \leqslant \sum_{s=1}^{T} \alpha_{s} \gamma \prod_{j=s+1}^{T} \exp \left(\alpha_{j} \beta\right) \\
& \leqslant \sum_{s=1}^{T} \frac{\alpha \gamma}{s} \prod_{j=s+1}^{T} \exp \left(\frac{\alpha \beta}{j}\right) \\
& =\sum_{s=1}^{T} \frac{\alpha \gamma}{s} \exp \left(\alpha \beta \sum_{j=s+1}^{T} \frac{1}{j}\right) \\
& \leqslant \sum_{s=1}^{\mathrm{T}} \frac{\alpha \gamma}{\mathrm{s}} \exp (\alpha \beta \log (\mathrm{T} / \mathrm{s})) \\
& \leqslant \alpha \gamma T^{\alpha \beta} \sum_{s=1}^{T} \frac{1}{s^{\alpha \beta+1}} \\
& \leqslant \alpha \gamma \top^{\alpha \beta+1} \text {. }
\end{aligned}
$$

\section{C.7 Random-to-top: Omitted details}

Axioms We assume a few axioms about the shiftR operation. Let $a_{1}, a_{2}$ be two decks and $J$ such that $\forall i .(0 \leqslant i \leqslant J) \Rightarrow a_{1}[i]=a_{2}[i]$. Then,

- If $j \leqslant J$ and $a_{i}^{\prime}=\operatorname{shiftR}\left(a_{i}, j\right)$, then $\forall i .(0 \leqslant i \leqslant J) \Rightarrow a_{1}^{\prime}[i]=a_{2}^{\prime}[i]$

- If $j_{1}, j_{2}>J, a_{i}^{\prime}=\operatorname{shiftR}\left(a_{i}, j_{i}\right)$, and $a_{1}\left[j_{1}\right]=a_{2}\left[j_{2}\right]$, then $\forall i .(0 \leqslant i \leqslant J+1) \Rightarrow a_{1}^{\prime}[i]=$ $a_{2}^{\prime}[i]$

Additionally, if $a$ is a permutation of $[N]$, then, for all $i<N$, so is $\operatorname{shiftR}(a, i)$.

Establishing the invariant Let $\mathrm{C} \triangleq(\mathrm{N}-1) / \mathrm{N}$. Recall the loop invariant:

$$
\mathcal{J} \triangleq[k\langle 1\rangle \neq k\langle 2\rangle] \cdot \infty+[k\langle 1\rangle=k\langle 2\rangle] \cdot d_{M} \cdot C^{\max (0, k-k\langle 1\rangle)}
$$

We check that it satisfies the loop rule:

$$
\begin{aligned}
& {[\mathrm{k}\langle 1\rangle<\mathrm{K} \wedge \mathrm{k}\langle 2\rangle<\mathrm{K}] \cdot \widetilde{\operatorname{rpe}}(\mathrm{c}, \mathrm{J})+[\mathrm{k}\langle 1\rangle \geqslant \mathrm{K} \wedge \mathrm{k}\langle 2\rangle \geqslant \mathrm{K}] \cdot \mathrm{F}} \\
& +[(\mathrm{k}\langle 1\rangle<\mathrm{K}) \neq(\mathrm{k}\langle 2\rangle<\mathrm{K})] \cdot \infty \leqslant \mathrm{J},
\end{aligned}
$$


If $[k\langle 1\rangle \neq k\langle 2\rangle] \infty$ then the right-hand side of the inequality is $\infty$, and it is satisfied. Otherwise, if $[k\langle 1\rangle \geqslant k \wedge k\langle 2\rangle \geqslant k]$ then we need to check that, indeed,

$$
\mathrm{F} \leqslant \mathrm{d}_{M} \cdot \mathrm{C}^{\max (0, \mathrm{~K}-\mathrm{k}\langle 1\rangle)}=\mathrm{d}_{\mathrm{M}}
$$

Finally, if $[k\langle 1\rangle<k \wedge k\langle 2\rangle<k]$, we compute the pre-expectation of the loop body with respect to J. Let $J^{\prime}$ be the pre-expectation of the loop body without the sampling, i.e.,

$$
\begin{aligned}
J^{\prime} & \triangleq[k\langle 1\rangle+1 \neq k\langle 2\rangle+1] \cdot \infty \\
& +[k\langle 1\rangle+1=k\langle 2\rangle+1] \cdot(1 / N) \cdot\left(N-\max _{i}\left(\forall j<i \cdot a^{\prime}\langle 1\rangle[j]=a^{\prime}\langle 2\rangle[j]\right) \cdot C^{\max (0, k-k\langle 1\rangle-1)}\right)
\end{aligned}
$$

where $a^{\prime}\langle 1\rangle=\operatorname{shiftR}(a\langle 1\rangle, y\langle 1\rangle)$ and $a^{\prime}\langle 2\rangle=\operatorname{shiftR}(a\langle 2\rangle, y\langle 2\rangle)$. In the following, let $l^{\prime}$ denote $\max _{i}\left(\forall j<i a^{\prime}\langle 1\rangle[j]=a^{\prime}\langle 2\rangle[j]\right)$. We pick a coupling induced by a bijection $\pi$ such that, for all $z, a\langle 1\rangle[z\langle 1\rangle]=\mathrm{a}\langle 2\rangle[\pi(z\langle 1\rangle)]$. The pre-expectation induced by this assignment is:

$$
\begin{aligned}
\mathcal{J}^{\prime \prime} & \triangleq[k\langle 1\rangle+1 \neq k\langle 2\rangle+1] \cdot \infty \\
& +[k\langle 1\rangle+1=k\langle 2\rangle+1] \cdot(1 / N) \cdot\left(N-\max _{i}\left(\forall j<i \cdot a^{\prime \prime}\langle 1\rangle[j]=a^{\prime \prime}\langle 2\rangle[j]\right) \cdot C^{\max (0, k-k\langle 1\rangle-1)}\right)
\end{aligned}
$$

where $a^{\prime \prime}\langle 1\rangle=\operatorname{shiftR}(a\langle 1\rangle, y)$ and $a^{\prime}\langle 2\rangle=\operatorname{shiftR}(a\langle 2\rangle, \pi(y))$.

Now we have to compute the expected value of $J^{\prime \prime}$ when we sample y uniformly from Unif ${ }_{[N]}$. There are two cases. If $y<l^{\prime}$, then $\pi(y)=y$, and $a\langle 1\rangle[y]=a\langle 2\rangle[y]$, and

$$
\max _{i}\left(\forall j<i . a^{\prime \prime}\langle 1\rangle[j]=a^{\prime \prime}\langle 2\rangle[j]\right)=\max _{i}(\forall j<i . a\langle 1\rangle[j]=a\langle 2\rangle[j])
$$

where we use the first axiom of shiftR. The probability of this happening is precisely $l^{\prime} / N=1-d_{M}$. In the other case, by the second axiom of shiftR

$$
\max _{i}\left(\forall j<i . a^{\prime \prime}\langle 1\rangle[j]=a^{\prime \prime}\langle 2\rangle[j]\right)+1 \leqslant \max _{i}(\forall j<i . a\langle 1\rangle[j]=a\langle 2\rangle[j])
$$

This case happens with probability $d_{M}$. The inequality arises from the fact that we may have matches below $l^{\prime}$. From the expression above we derive:

$$
\begin{aligned}
& (1 / N)\left(N-\max _{i}\left(\forall j<i . a^{\prime \prime}\langle 1\rangle[j]=a^{\prime \prime}\langle 2\rangle[j]\right)\right) \\
& \leqslant(1 / N)\left(N-\max _{i}(\forall j<i . a\langle 1\rangle[j]=a\langle 2\rangle[j])-1\right) \\
& =d_{M}-1 / N
\end{aligned}
$$

Using this inequality, we can bound the pre-expectation of the loop invariant (simplifying under the assumptions $[k\langle 1\rangle \geqslant k \wedge k\langle 2\rangle \geqslant k]$ and $[k\langle 1\rangle=k\langle 2\rangle])$ :

$$
\begin{aligned}
\mathbb{E}_{y_{\mathcal{S}}^{\mathbb{S} \text { Unif }_{[N]}}}\left[\mathcal{J}^{\prime \prime}\right] & \leqslant\left(1-d_{M}\right) \cdot d_{M} \cdot C^{K-k\langle 1\rangle-1}+d_{M} \cdot\left(d_{M}-1 / N\right) \cdot C^{K-k\langle 1\rangle-1} \\
& =C^{K-\langle 1\rangle-1} \cdot d_{M} \cdot\left(\left(1-d_{M}\right)+\left(d_{M}-1 / N\right)\right) \\
& =C^{K-\langle 1\rangle-1} \cdot d_{M} \cdot C \\
& =C^{K-\langle 1\rangle} \cdot d_{M}=\mathcal{J}
\end{aligned}
$$


This finishes the proof of the premise of the loop rule. Note that we did not explicitly compute the pre-expectation of the loop invariant, we just found an upper bound which is enough to apply the loop rule.

\section{C.8 Uniform riffle: Omitted details}

Axioms We use some axioms about permutations, filtering, and concatenation.

- Let perm $\left(a_{1}, a_{2}\right)$ be the predicate that $a_{1}$ and $a_{2}$ are permutations of $C$. Then if we split a deck into two pieces and concatenate them, the result is a permutation of the original. Formally, for any bit-vector $b$ we have:

$$
\operatorname{perm}(a, \operatorname{cat}(a(\bar{b}), a(b)))
$$

- Let $a_{1}, a_{2}$ be permutations, $b_{1}, b_{2}$ be bitstrings, and $a_{1}^{\prime}, a_{2}^{\prime}$ be

$$
a_{i}^{\prime}=\operatorname{cat}\left(a_{i}\left(\bar{b}_{i}\right), a_{i}\left(b_{i}\right)\right) .
$$

Then if $b_{1}, b_{2}$ match cards in $a_{1}, a_{2}$, i.e., $b_{1} \circ a_{1}^{-1}=b_{2} \circ a_{2}^{-1}$, then we can bound the size of blocks in the block decomposition of $a_{1}^{\prime}, a_{2}^{\prime}$ as:

$\forall c \in[C] .\left|B D\left(a_{1}^{\prime}, a_{2}^{\prime}\right)(c)\right| \leqslant \bar{b}\left(a_{1}^{-1}(c)\right)\left(\bar{b}\left(B D\left(a_{1}, a_{2}\right)(c)\right)\right)+b\left(a_{1}^{-1}(c)\right)\left(b\left(B D\left(a_{1}, a_{2}\right)(c)\right)\right)$

where we write $b(P)$ and $\bar{b}(P)$ to mean the total number of ones in $b$ and $\bar{b}$ at the positions $P$.

- Summing the previous bound over all cards gives:

$$
\sum_{c \in C}\left|B D\left(a_{1}^{\prime}, a_{2}^{\prime}\right)(c)\right| \leqslant \sum_{[c] \in B D\left(a_{1}, a_{2}\right)} \bar{b}\left(B D\left(a_{1}, a_{2}\right)(c)\right)^{2}+b\left(B D\left(a_{1}, a_{2}\right)(c)\right)^{2}
$$

where the right-hand side sums over the equivalence classes of cards / positions induced by the block decomposition.

Defining the distance Defining the distance between decks requires some care. Consider the following distance based on positions:

$$
\mathrm{d}_{\mathrm{P}}\left(\operatorname{deck}_{1}, \operatorname{deck}_{2}\right) \triangleq\left(1 / \mathrm{N}^{2}\right) \sum_{\mathrm{c} \in \mathrm{C}}\left|\operatorname{deck}_{1}^{-1}(\mathrm{c})-\operatorname{deck}_{2}^{-1}(\mathrm{c})\right|
$$

This distance measures the total difference between the positions of each card in deck $_{1}$ and its counterpart in deck $_{2}$, normalized to be in $[0,1]$; and $d_{\mathrm{P}}=0$ holds only when $d e c k_{1}=d e c k_{2}$. However, it is not easy to directly show that this distance is monotonically decreasing in expectation-indeed, some terms in the sum may 
actually increase. Instead, we define an upper bound $d_{c}$ on $\left|\operatorname{dec}_{1}^{-1}(\mathrm{c})-\operatorname{dec}_{2}^{-1}(\mathrm{c})\right|$ for every card. The sum $d_{M} \triangleq 1 / N^{2} \sum_{c \in C} d_{c}$ will be an upper bound of $d_{P}$, and $d_{M}$ decreases monotonically to zero.

We will define $d_{c}$ in terms of a few concepts from the theory of permutations. Given two decks deck , deck $_{2}$ and a permutation $\pi$ on positions taking deck to deck $_{2}$, there is a unique cyclical decomposition of $\pi$, i.e., we can partition the positions into $\mathrm{P}_{1}, \ldots, \mathrm{P}_{k}$ such that $\pi$ moves positions in $\mathrm{P}_{i}$ as a single cycle. We define a block decomposition of $\pi$ to be a partition of the positions $B_{1}, \ldots, B_{j}$ such that each block is contiguous, and $\pi$ acts as a permutation on each $B_{i}$. A block decomposition is minimal if no block can be further decomposed; it is not hard to show that a minimal block decomposition must be unique. When deck $_{1}$, deck $k_{2}$ are permutations, we write $\mathrm{BD}\left(\right.$ deck $_{1}$, deck $\left._{2}\right)$ for the block decomposition induced by two decks deck ${ }_{1}$ and deck 2 . Finally, to define the distance, for every card $c \in C$ we let:

$$
\mathrm{d}_{\mathrm{c}} \triangleq\left|\mathrm{BD}\left(\operatorname{deck}_{1}, \operatorname{deck}_{2}\right)(\mathrm{c})\right|-1
$$

where $\mid \mathrm{BD}\left(\right.$ deck $\left._{1}, \operatorname{deck}_{2}\right)(\mathrm{c}) \mid$ is the size of the block containing card $\mathrm{c}$ in deck 1 and deck $_{2}$; both positions must be in the same block. The size of each block is at least 1 , and if the distance $d_{c}$ is zero then $c$ must be at the same position in deck $k_{1}$ and deck . It is not hard to show that the size of the c's block is at least the difference in c's position across deck $_{1}$ and deck $_{2}$ :

$$
\left|\operatorname{deck}_{1}^{-1}(\mathrm{c})-\operatorname{deck}_{2}^{-1}(\mathrm{c})\right| \leqslant \mathrm{d}_{\mathrm{c}}
$$

so $d_{c}=0$ implies that $\mathrm{c}$ is at the same position in deck ${ }_{1}$ and $a_{2}$. (However, the reverse implication may not hold.) As a result, we can upper bound our target distance

$$
\mathrm{d}_{\mathrm{P}} \leqslant \frac{1}{\mathrm{~N}^{2}} \sum_{\mathrm{c} \in \mathrm{C}} \mathrm{d}_{\mathrm{c}}=\mathrm{d}_{\mathrm{M}}
$$

Now, we turn to the loop. Let $\Phi$ be the binary invariant

$$
\Phi \triangleq \operatorname{perm}(\operatorname{deck}\langle 1\rangle, \operatorname{deck}\langle 2\rangle) \wedge k\langle 1\rangle=k\langle 2\rangle \wedge\left(\mathrm{b} \circ \operatorname{deck}^{-1}\right)\langle 1\rangle=\left(\mathrm{b} \circ \operatorname{deck}^{-1}\right)\langle 2\rangle
$$

and take the following invariant expectation:

$$
\mathcal{J}=[\neg \Phi] \cdot \infty+[\Phi] \cdot \mathrm{d}_{M} \cdot(1 / 2)^{(\mathrm{K}-\mathrm{k}\langle 1\rangle)_{+}}
$$

We want to verify that:

$$
\begin{aligned}
& {[(\mathrm{k}<\mathrm{K})\langle 1\rangle \wedge(\mathrm{k}<\mathrm{K})\langle 2\rangle] \cdot \widetilde{r p e}(b d, \mathcal{J}) } \\
+ & {[(\mathrm{k} \geqslant \mathrm{K})\langle 1\rangle \wedge(\mathrm{k} \geqslant \mathrm{K})\langle 2\rangle] \cdot \mathrm{d}_{\mathrm{P}} } \\
+ & {[(\mathrm{k}<\mathrm{K})\langle 1\rangle \neq(\mathrm{k}<\mathrm{K})\langle 2\rangle] \cdot \infty \quad \leqslant \quad \mathcal{J}, }
\end{aligned}
$$

where $b d$ is the loop body. The cases $[(k \geqslant K)\langle 1\rangle \wedge(k \geqslant K)\langle 2\rangle]$ and $[(k<K)\langle 1\rangle \neq$ $(\mathrm{k}<\mathrm{K})\langle 2\rangle]$ are almost immediate. The main case is when $[(\mathrm{k}<\mathrm{K})\langle 1\rangle \wedge(\mathrm{k}<\mathrm{K})\langle 2\rangle]$. 
Focusing on the case where $\Phi$ holds (otherwise there is nothing to show), this boils down to:

$$
\mathbb{E}_{\mathrm{b}}\left[\mathrm{d}_{M}(\operatorname{cat}(\operatorname{deck}(\overline{\mathrm{b}}), \operatorname{deck}(\mathrm{b}))\langle 1\rangle, \operatorname{cat}(\operatorname{deck}(\overline{\mathrm{b}}), \operatorname{deck}(\mathrm{b}))\langle 2\rangle)\right] \leqslant \frac{1}{2} \mathrm{~d}_{M},
$$

i.e., each iteration of the loop halves the invariant, where the expected value is taken over $b\langle 1\rangle \sim\{0,1\}^{N}$ and $b\langle 2\rangle$ is coupled so that $\left(b \circ\right.$ deck $\left.^{-1}\right)\langle 1\rangle=\left(\mathrm{b} \circ\right.$ deck $\left.^{-1}\right)\langle 2\rangle$. Above, we write $d_{M}\left(x_{1}, x_{2}\right)$ as shorthand for $d_{M}\left[x_{1}, x_{2} / \operatorname{deck}\langle 1\rangle\right.$, deck $\left.\langle 2\rangle\right]$.

The inequality follows from the permutation axioms, and from the mean and variance of the binomial distribution-for deck , deck $_{2}$ fixed, $\overline{\mathrm{b}}\left(\mathrm{BD}\left(\right.\right.$ deck $_{1}$, deck $\left.\left._{2}\right)\right)$ and $\mathrm{b}\left(\mathrm{BD}\left(\right.\right.$ deck $_{1}$, deck $\left.\left._{2}\right)\right)$ each follow the binomial distribution with $\mid \mathrm{BD}\left(\right.$ deck $_{1}$, deck $\left._{2}\right)(\mathrm{c}) \mid$ trials and parameter $1 / 2$. This completes the proof for the body of the loop. Finally, we push the invariant past the initialization of the procedure, and we have the bound:

$$
\widetilde{\text { rpe }}\left(\operatorname{riffle}(\text { deck, } N, K), d_{P}\right) \leqslant[\neg \Phi]+[\Phi] \cdot d_{M} \cdot(1 / 2)^{K} \leqslant[\neg \Phi]+[\Phi] \cdot(1 / 2)^{K} .
$$

since the initial distance $d_{M}$ is at most 1 . Given that $d_{P}$ assigns different decks a distance of at least $1 / \mathrm{N}^{2}$, Theorem 10.9 implies that the TV distance between the deck distributions is at most

$$
v(K, N)=\max _{d_{1}, d_{2} \in[N]} \operatorname{TV}\left(\llbracket \text { riffle } \rrbracket\left(d_{1}, N, K\right), \llbracket \text { riffle } \rrbracket\left(d_{2}, N, K\right)\right) \leqslant N^{2}\left(\frac{1}{2}\right)^{K},
$$

so the distributions converge to one another and to the uniform distribution exponentially quick. If we take $K \geqslant \log _{2}\left(N^{2} \rho\right), v(K)$ is asymptotically bounded by $O(1 / \rho)$ for large $N$. When setting $\rho=N$, we establish the following guarantee.

Theorem C.8. Let $\mathrm{K}=3 \log \mathrm{N}$, and Perm $([\mathrm{N}])$ be the set of permutations over $\mathrm{N}$. For any initial permutation of deck,

$$
\operatorname{TV}(\operatorname{riffle}(\operatorname{deck}, \mathrm{N}, \mathrm{K}), \operatorname{Unif}\{\operatorname{Perm}([\mathrm{N}])\}) \in \mathcal{O}(1 / \mathrm{N})
$$

Establishing the invariant Recall that we need to show:

$$
\mathbb{E}_{\mathrm{b}}\left[\mathrm{d}_{M}(\operatorname{cat}(\operatorname{deck}(\overline{\mathrm{b}}), \operatorname{deck}(\mathrm{b}))\langle 1\rangle, \operatorname{cat}(\operatorname{deck}(\overline{\mathrm{b}}), \operatorname{deck}(\mathrm{b}))\langle 2\rangle)\right] \leqslant \frac{1}{2} \mathrm{~d}_{M}(\operatorname{deck}\langle 1\rangle, \operatorname{deck}\langle 2\rangle),
$$

i.e., each iteration of the loop halves the invariant, where the expected value is taken over $b\langle 1\rangle \sim\{0,1\}^{N}$ and $b\langle 2\rangle$ is coupled so that $\left(b \circ \operatorname{deck}^{-1}\right)\langle 1\rangle=\left(b \circ\right.$ deck $\left.^{-1}\right)\langle 2\rangle$. Writing $a_{1}, a_{2}=\operatorname{deck}\langle 1\rangle,\langle 2\rangle$, and $a_{1}^{\prime}, a_{2}^{\prime}=\operatorname{cat}(\operatorname{deck}(\bar{b}), \operatorname{deck}(b))\langle 1\rangle,\langle 2\rangle$, and $b_{1}, b_{2}=$ 
$\mathrm{b}\langle 1\rangle,\langle 2\rangle$, the permutation axioms give:

$$
\begin{aligned}
& \mathbb{E}_{b}\left[d_{M}\left(a_{1}^{\prime}, a_{2}^{\prime}\right)\right]= \\
& =\frac{1}{N^{2}} \sum_{c \in C} \mathbb{E}_{b}\left[\left|B D\left(a_{1}^{\prime}, a_{2}^{\prime}\right)(c)\right|-1\right] \\
& \leqslant \frac{1}{N^{2}} \sum_{[c] \in B D\left(a_{1}, a_{2}\right)} \mathbb{E}_{b}\left[\bar{b}\left(B D\left(a_{1}, a_{2}\right)(c)\right)^{2}\right]+\mathbb{E}_{b}\left[b\left(B D\left(a_{1}, a_{2}\right)(c)\right)^{2}\right]-\left|B D\left(a_{1}, a_{2}\right)(c)\right| \\
& =\frac{1}{2 N^{2}} \sum_{[c] \in B D\left(a_{1}, a_{2}\right)}\left|B D\left(a_{1}, a_{2}\right)(c)\right|^{2}-\left|B D\left(a_{1}, a_{2}\right)(c)\right| \\
& =\frac{1}{2 N^{2}} \sum_{c \in C}\left(\left|B D\left(a_{1}, a_{2}\right)(c)\right|-1\right) \\
& =\frac{1}{2} d_{M}\left(a_{1}, a_{2}\right) .
\end{aligned}
$$

\section{C.9 Asynchronous rules: Omitted details}

We prove soundness of the asynchronous rules.

Proof of Theorem 10.27. We start with the rule for conditionals. Let $c$ be a program that is almost surely terminating, let $\varepsilon$ be a relational pre-expectation, and let $s_{1}, s_{2} \in$ State be two states. If $s_{1}(e)=s_{2}(e)$, then the bound follows from soundness of synchronous case (Theorem 10.11):

$$
\begin{aligned}
\operatorname{rpe}(\text { if } e \text { then } c, \mathcal{E})\left(s_{1}, s_{2}\right) & \leqslant \widetilde{r p e}(\text { if } e \text { then } c, \mathcal{E})\left(s_{1}, s_{2}\right) \\
& =([e\langle 1\rangle \wedge e\langle 2\rangle] \cdot \widetilde{r p e}(c, \mathcal{E})+[\neg e\langle 1\rangle \wedge \neg e\langle 2\rangle] \cdot \mathcal{E})\left(s_{1}, s_{2}\right) .
\end{aligned}
$$

Otherwise if $e$ is true in $s_{1}$ and false in $s_{2}$, then:

$$
\operatorname{rpe}(\text { if e then } c, \mathcal{E})\left(s_{1}, s_{2}\right)=\inf _{\mu \in \Gamma\left(\llbracket c \rrbracket s_{1}, \delta\left(s_{2}\right)\right)} \mathbb{E}_{\mu}[\mathcal{E}] \leqslant \operatorname{wpe}\langle 1\rangle(c, \mathcal{E})\left(s_{1}, s_{2}\right)
$$

by Lemma 10.26. The case where $e$ is false in $s_{1}$ and true in $s_{2}$ is almost identical.

Next, we consider the asynchronous rule for loops. Let while e do c be almost surely terminating. We define a sequence of loop approximants:

$$
\begin{aligned}
c_{0} & \triangleq \text { skip } \\
c_{i+1} & \triangleq(\text { if } e \text { then } c) ; c_{i}
\end{aligned}
$$

When the loop is almost surely terminating, we have the following equivalence:

$$
\llbracket \text { while e do } c \rrbracket s=\lim _{i \rightarrow \infty}\left(\llbracket c_{i} \rrbracket s\right)
$$

for any input state s, and the limit of distributions exists. 
Our overall argument proceeds much like the proof for the synchronous case. We first show that the least-fixed point of a characteristic function of the loop is an upper bound on pre-expectation. Then, we argue that the asynchronous loop rule shows that $\mathcal{J}$ is a fixed point with respect to the characteristic function, so it must also be an upper bound. We work with the following characteristic function:

$$
\begin{aligned}
\Psi_{\mathcal{E}, \mathrm{c}}\left(\mathcal{E}^{\prime}\right) \triangleq[e\langle 1\rangle \wedge e\langle 2\rangle] \cdot \widetilde{\operatorname{rpe}}\left(\mathrm{c}, \mathcal{E}^{\prime}\right)+[\neg e\langle 1\rangle \wedge \neg e\langle 2\rangle] \cdot \mathcal{E} \\
+[e\langle 1\rangle \wedge \neg e\langle 2\rangle] \cdot w p e\langle 1\rangle\left(\mathrm{c}, \mathcal{E}^{\prime}\right)+[\neg e\langle 1\rangle \wedge e\langle 2\rangle] \cdot w p e\langle 2\rangle\left(\mathrm{c}, \mathcal{E}^{\prime}\right) .
\end{aligned}
$$

By Lemma C.3 and monotonicity of the weakest pre-expectation operator, the operator $\Psi_{\varepsilon, \mathrm{c}}$ is monotone. Thus, the least fixed-point exists:

$$
\mathcal{L}_{\mathcal{E}, \mathrm{c}}=\operatorname{Ifp} X . \Psi_{\mathcal{E}, \mathrm{c}}(X) .
$$

We can inductively define:

$$
\begin{aligned}
\mathcal{E}_{0} & \triangleq[\neg e\langle 1\rangle \wedge \neg\langle 2\rangle] \cdot \mathcal{E} \\
\mathcal{E}_{i+1} & \triangleq[e\langle 1\rangle \wedge e\langle 2\rangle] \cdot \widetilde{\operatorname{rpe}}\left(c, \mathcal{E}_{i}\right)+[\neg e\langle 1\rangle \wedge \neg e\langle 2\rangle] \cdot \mathcal{E} \\
& +[e\langle 1\rangle \wedge \neg e\langle 2\rangle] \cdot \operatorname{wpe}\langle 1\rangle\left(c, \mathcal{E}_{i}\right)+[\neg e\langle 1\rangle \wedge e\langle 2\rangle] \cdot \operatorname{wpe}\langle 2\rangle\left(c, \mathcal{E}_{i}\right) .
\end{aligned}
$$

By definition $\varepsilon_{i}=\Psi_{\varepsilon, c}^{i}\left(\varepsilon_{0}\right)$, and by monotonicity $\varepsilon_{i}$ is a monotone increasing sequence. Furthermore, for any expectation $\mathcal{E}^{\prime}$ we have $\varepsilon_{0} \leqslant \Psi_{\mathcal{E}, \mathrm{c}}\left(\mathcal{E}^{\prime}\right)$, hence $\mathcal{E}_{0} \leqslant \mathcal{L}_{\mathcal{E}, \mathrm{c}}$. By monotonicity of $\Psi_{\varepsilon, \mathrm{c}}$, we have $\varepsilon_{i} \leqslant \mathcal{L}_{\mathcal{\varepsilon}, \mathrm{c}}$ for every $i$.

We now prove an analogue of Lemma C.7.

Lemma C.9. For all $j \in \mathbb{N}$ and $\left(s_{1}^{\prime}, s_{2}^{\prime}\right) \in$ State $\times$ State, there exists $\mu_{j, s_{1}^{\prime}, s_{2}^{\prime}} \in \Gamma\left(\llbracket c_{j} \rrbracket s_{1}^{\prime}, \llbracket c_{j} \rrbracket s_{2}^{\prime}\right)$ such that

$$
\mathbb{E}_{\mu_{j, s_{1}^{\prime}, s_{2}^{\prime}}}[\mathcal{E}] \leqslant \mathcal{E}_{j}\left(s_{1}^{\prime}, s_{2}^{\prime}\right)+\left(\rho_{j}\left(s_{1}^{\prime}\right)+\rho_{j}\left(s_{2}^{\prime}\right)\right) \cdot M_{j}\left(\mathcal{E}, s_{1}^{\prime}, s_{2}^{\prime}\right)
$$

where $\rho_{j}(s)$ is the probability of e being true in $\llbracket c_{j} \rrbracket s$, and:

$$
M_{j}\left(\mathcal{E}, s_{1}^{\prime}, s_{2}^{\prime}\right)=\max \left\{\mathcal{E}\left(t_{1}, t_{2}\right) \mid t_{1} \in \operatorname{supp}\left(\llbracket c_{j} \rrbracket s_{1}^{\prime}\right), t_{2} \in \operatorname{supp}\left(\llbracket c_{j} \rrbracket s_{2}^{\prime}\right)\right\} .
$$

Proof. By induction on $j$. The base case $j=0$ is clear, taking the coupling $\delta\left(s_{1}^{\prime}, s_{2}^{\prime}\right)$. For the inductive step, we have

$$
\begin{aligned}
\mathcal{E}_{j+1} \triangleq[e\langle 1\rangle \wedge e\langle 2\rangle] \cdot \widetilde{\operatorname{rpe}}\left(\mathrm{c}, \mathcal{E}_{j}\right)+[\neg e\langle 1\rangle \wedge \neg e\langle 2\rangle] \cdot \mathcal{E} \\
+[e\langle 1\rangle \wedge \neg e\langle 2\rangle] \cdot \operatorname{wpe}\langle 1\rangle\left(\mathrm{c}, \varepsilon_{j}\right)+[\neg e\langle 1\rangle \wedge e\langle 2\rangle] \cdot \operatorname{wpe}\langle 2\rangle\left(\mathrm{c}, \mathcal{E}_{j}\right) .
\end{aligned}
$$

There are four cases.

Case: $s_{1}^{\prime}(e)=f f$ and $s_{2}^{\prime}(e)=f f$. In this case, $\llbracket c_{j+1} \rrbracket s_{1}^{\prime}=\delta\left(s_{1}^{\prime}\right)$ and $\llbracket c_{j+1} \rrbracket s_{2}^{\prime}=\delta\left(s_{2}^{\prime}\right)$. We can define the coupling $\mu_{s_{1}^{\prime}, s_{2}^{\prime}}=\delta\left(s_{1}^{\prime}, s_{2}^{\prime}\right) \in \Gamma\left(\llbracket c_{j+1} \rrbracket s_{1}^{\prime}, \llbracket c_{j+1} \rrbracket s_{2}^{\prime}\right)$ and we are done, since

$$
\mathbb{E}_{\mu_{s_{1}^{\prime}, s_{2}^{\prime}}}[\mathcal{E}]=\mathcal{E}\left(s_{1}^{\prime}, s_{2}^{\prime}\right)=\mathcal{E}_{j+1}\left(s_{1}^{\prime}, s_{2}^{\prime}\right)
$$


Case: $s_{1}^{\prime}(e)=t t$ and $s_{2}^{\prime}(e)=t t$. In this case, $\llbracket c_{j+1} \rrbracket s_{1}^{\prime}=\llbracket c ; c_{j} \rrbracket s_{1}^{\prime}$ and $\llbracket c_{j+1} \rrbracket s_{2}^{\prime}=\llbracket c ; c_{j} \rrbracket s_{2}^{\prime}$. If $\varepsilon_{j+1}\left(s_{1}^{\prime}, s_{2}^{\prime}\right)$ is infinite we are done, so suppose that it is finite. By Theorem 10.11, there exists a coupling $v_{s_{1}^{\prime}, s_{2}^{\prime}} \in \Gamma\left(\llbracket c \rrbracket s s_{1}^{\prime}, \llbracket c \rrbracket s s_{2}^{\prime}\right)$ such that

$$
\mathbb{E}_{v_{s_{1}^{\prime}, s_{2}^{\prime}}}\left[\mathcal{E}_{j}\right] \leqslant \mathcal{E}_{j}\left(s_{1}^{\prime}, s_{2}^{\prime}\right) \text {. }
$$

By the induction hypothesis, there is a coupling $\mu_{j, t_{1}, t_{2}} \in \Gamma\left(\llbracket c_{j} \rrbracket t_{1}, \llbracket c_{j} \rrbracket t_{2}\right)$ such that

$$
\mathbb{E}_{\mu_{j, t_{1}, t_{2}}}[\mathcal{E}] \leqslant \mathcal{E}_{j}\left(t_{1}, t_{2}\right)+\left(\rho_{j}\left(t_{1}\right)+\rho_{j}\left(t_{2}\right)\right) \cdot M_{j}\left(\mathcal{E}, t_{1}, t_{2}\right) .
$$

Now, we can define the coupling for the $(j+1)$-th approximants:

$$
\mu_{j+1, s_{1}^{\prime}, s_{2}^{\prime}} \triangleq \mathbb{E}_{v_{s_{1}^{\prime}, s_{2}^{\prime}}}\left[\mu_{j,-,-}\right]
$$

We first check the distance condition. By definition, we have:

$$
\begin{aligned}
\mathbb{E}_{\mu_{j+1, s_{1}, s_{2}^{\prime}}}[\mathcal{E}] & =\mathbb{E}_{\left(t_{1}, t_{2}\right) \sim v_{s_{1}^{\prime}, s_{2}^{\prime}}}\left[\mathbb{E}_{\mu_{j, t_{1}, t_{2}}}[\mathcal{E}]\right] \\
& \leqslant \mathbb{E}_{\left(t_{1}, t_{2}\right) \sim v_{s_{1}^{\prime}, s_{2}^{\prime}}}\left[\mathcal{E}_{j}\left(t_{1}, t_{2}\right)+\left(\rho_{j}\left(t_{1}\right)+\rho_{j}\left(t_{2}\right)\right) \cdot M_{j}\left(\mathcal{E}, t_{1}, t_{2}\right)\right] \\
& \leqslant \mathcal{E}_{j}\left(s_{1}^{\prime}, s_{2}^{\prime}\right)+\left(\rho_{j+1}\left(s_{1}^{\prime}\right)+\rho_{j+1}\left(s_{2}^{\prime}\right)\right) \cdot M_{j+1}\left(\mathcal{E}, s_{1}^{\prime}, s_{2}^{\prime}\right) \\
& \leqslant \mathcal{E}_{j+1}\left(s_{1}^{\prime}, s_{2}^{\prime}\right)+\left(\rho_{j+1}\left(s_{1}^{\prime}\right)+\rho_{j+1}\left(s_{2}^{\prime}\right)\right) \cdot M_{j+1}\left(\mathcal{E}, s_{1}^{\prime}, s_{2}^{\prime}\right) .
\end{aligned}
$$

The marginal condition is not hard to show, using the marginal properties of $v_{s_{1}^{\prime}, s_{2}^{\prime}}$ and $\mu_{j, t_{1}, t_{2}}$ combined with the definition of approximants: since $e$ is true in $s_{1}^{\prime}$ and $s_{2}^{\prime}$, we have $\llbracket c_{j+1} \rrbracket s_{1}^{\prime}=\llbracket c ; c_{j} \rrbracket s_{1}^{\prime}$ and $\llbracket c_{j+1} \rrbracket s_{2}^{\prime}=\llbracket c ; c_{j} \rrbracket s_{2}^{\prime}$. The proof follows the case for sequential composition.

Case: $s_{1}^{\prime}(e)=t t$ and $s_{2}^{\prime}(e)=f f$. In this case, $\llbracket c_{j+1} \rrbracket s_{1}^{\prime}=\llbracket c ; c_{j} \rrbracket s_{1}^{\prime}$ and $\llbracket c_{j+1} \rrbracket s_{2}^{\prime}=\llbracket$ skip $\rrbracket s_{2}^{\prime}$. By Lemma 10.26, there exists a coupling $v_{s_{1}^{\prime}, s_{2}^{\prime}} \in \Gamma\left(\llbracket c \rrbracket s_{1}^{\prime}, \llbracket\right.$ skip $\left.\rrbracket s_{2}^{\prime}\right)$ such that

$$
\mathbb{E}_{v_{s_{1}^{\prime}, s_{2}^{\prime}}}\left[\mathcal{E}_{j}\right] \leqslant \text { wpe }\langle 1\rangle(c, \mathcal{E})\left(s_{1}^{\prime}, s_{2}^{\prime}\right)=\mathcal{E}_{j}\left(s_{1}^{\prime}, s_{2}^{\prime}\right)
$$

By the induction hypothesis, there is a coupling $\mu_{j, t_{1}, t_{2}} \in \Gamma\left(\llbracket c_{j} \rrbracket t_{1}, \llbracket c_{j} \rrbracket t_{2}\right)$ such that

$$
\mathbb{E}_{\mu_{j, t_{1}, t_{2}}}[\mathcal{E}] \leqslant \mathcal{E}_{j}\left(t_{1}, t_{2}\right)+\left(\rho_{j}\left(t_{1}\right)+\rho_{j}\left(t_{2}\right)\right) \cdot M_{j}\left(\mathcal{E}, t_{1}, t_{2}\right) .
$$

Now, we can define the coupling for the $(j+1)$-th approximants:

$$
\mu_{j+1, s_{1}^{\prime}, s_{2}^{\prime}} \triangleq \mathbb{E}_{v_{s_{1}^{\prime}, s_{2}^{\prime}}}\left[\mu_{j,-,-}\right]
$$

The distance and marginal conditions follow as in the previous case.

Case: $s_{1}^{\prime}(e)=f f$ and $s_{2}^{\prime}(e)=t$. In this case, $\llbracket c_{j+1} \rrbracket s_{1}^{\prime}=\llbracket$ skip $\rrbracket s_{1}^{\prime}$ and $\llbracket c_{j+1} \rrbracket s_{2}^{\prime}=\llbracket c ; c_{j} \rrbracket s_{2}^{\prime}$. By Lemma 10.26, there exists a coupling $v_{s_{1}^{\prime}, s_{2}^{\prime}} \in \Gamma\left(\llbracket\right.$ skip $\left.\rrbracket s_{1}^{\prime}, \llbracket c \rrbracket s_{2}^{\prime}\right)$ such that

$$
\mathbb{E}_{v_{s_{1}^{\prime}, s_{2}^{\prime}}}\left[\mathcal{E}_{j}\right] \leqslant w p e\langle 2\rangle(\mathrm{c}, \mathcal{E})\left(s_{1}^{\prime}, s_{2}^{\prime}\right)=\mathcal{E}_{j}\left(s_{1}^{\prime}, s_{2}^{\prime}\right) \text {. }
$$


By the induction hypothesis, there is a coupling $\mu_{j, t_{1}, t_{2}} \in \Gamma\left(\llbracket c_{j} \rrbracket t_{1}, \llbracket c_{j} \rrbracket t_{2}\right)$ such that

$$
\mathbb{E}_{\mu_{j, t_{1}, t_{2}}}[\mathcal{E}] \leqslant \mathcal{E}_{j}\left(t_{1}, t_{2}\right)+\left(\rho_{j}\left(t_{1}\right)+\rho_{j}\left(t_{2}\right)\right) \cdot M_{j}\left(\mathcal{E}, t_{1}, t_{2}\right) .
$$

Now, we can define the coupling for the $(j+1)$-th approximants:

$$
\mu_{j+1, s_{1}^{\prime}, s_{2}^{\prime}} \triangleq \mathbb{E}_{v_{s_{1}^{\prime}, s_{2}^{\prime}}}\left[\mu_{j,-,-}\right]
$$

The distance and marginal conditions follow as in the previous case.

Thus, we may apply Lemma C.9 with input states $s_{1}, s_{2}$ and expectations $\mathcal{E}_{i}$ to produce a sequence of couplings $\mu_{i, s_{1}, s_{2}} \in \Gamma\left(\llbracket c_{i} \rrbracket s_{1}, \llbracket c_{i} \rrbracket s_{2}\right)$ such that

$$
\begin{aligned}
\mathbb{E}_{\mu_{i, s_{1}, s_{2}}}[\mathcal{E}] & \leqslant \mathcal{E}_{i}\left(s_{1}, s_{2}\right)+\left(\rho_{i}\left(s_{1}\right)+\rho_{i}\left(s_{2}\right)\right) \cdot M_{i}\left(\mathcal{E}, s_{1}, s_{2}\right) \\
& =\Psi_{\mathcal{E}, \mathrm{c}}^{i}\left(\mathcal{E}_{0}\right)\left(s_{1}, s_{2}\right)+\left(\rho_{i}\left(s_{1}\right)+\rho_{i}\left(s_{2}\right)\right) \cdot M_{i}\left(\mathcal{E}, s_{1}, s_{2}\right) .
\end{aligned}
$$

By Theorem C.2, we can extract a subsequence $\mu_{i, s_{1}, s_{2}}^{\prime}$ (with a corresponding subsequence $c_{i}^{\prime}$ of $c_{i}$ ) from the sequence $\mu_{i, s_{1}, s_{2}}$ that converges monotonically to a coupling satisfying

$$
\tilde{\mu}_{s_{1}, s_{2}} \in \Gamma\left(\lim _{i \rightarrow \infty} \llbracket c_{i}^{\prime} \rrbracket s_{1}, \lim _{i \rightarrow \infty} \llbracket c_{i}^{\prime} \rrbracket s_{2}\right)=\Gamma\left(\llbracket \text { while e do } c \rrbracket s_{1}, \llbracket \text { while e do } c \rrbracket s_{2}\right),
$$

where the equality holds because the loop is almost surely terminating. All that remains to show is:

$$
\mathbb{E}_{\left(s_{1}^{\prime}, s_{2}^{\prime}\right) \sim \tilde{\mu}_{s_{1}, s_{2}}}\left[\mathcal{E}\left(s_{1}^{\prime}, s_{2}^{\prime}\right)\right] \leqslant \widetilde{r p e}(\text { while e do } c, \mathcal{E})\left(s_{1}, s_{2}\right) .
$$

We can compute:

$$
\begin{aligned}
\mathbb{E}_{\left(s_{1}^{\prime}, s_{2}^{\prime}\right) \sim \tilde{\mu}_{s_{1}, s_{2}}\left[\mathcal{E}\left(s_{1}^{\prime}, s_{2}^{\prime}\right)\right]}=\sum_{\left(s_{1}^{\prime}, s_{2}^{\prime}\right) \in \text { State } \times \text { State }} \mathcal{E}\left(s_{1}^{\prime}, s_{2}^{\prime}\right) \cdot \lim _{i \rightarrow \infty} \mu_{i, s_{1}, s_{2}}^{\prime}\left(s_{1}^{\prime}, s_{2}^{\prime}\right) \\
\leqslant \sum_{\left(s_{1}^{\prime}, s_{2}^{\prime}\right) \in \text { State } \times \text { State }} \lim _{i \rightarrow \infty} \mathcal{E}\left(s_{1}^{\prime}, s_{2}^{\prime}\right) \cdot \mu_{i, s_{1}, s_{2}}^{\prime}\left(s_{1}^{\prime}, s_{2}^{\prime}\right) \quad(\mathcal{E} \text { may be } \infty) \\
\leqslant \lim _{i \rightarrow \infty} \sum_{\left(s_{1}^{\prime}, s_{2}^{\prime}\right) \in \text { State } \times \text { State }} \mathcal{E}\left(s_{1}^{\prime}, s_{2}^{\prime}\right) \cdot \mu_{i, s_{1}, s_{2}}^{\prime}\left(s_{1}^{\prime}, s_{2}^{\prime}\right)
\end{aligned}
$$

Finally, the premise of the asynchronous loop rule implies that $\Psi_{\mathcal{E}, \mathrm{c}}(\mathcal{J}) \leqslant \mathcal{J}$, i.e., $\mathcal{J}$ is a pre-fixed-point of $\Psi_{\varepsilon, c}$. Since $\mathcal{L}_{\mathcal{E}, \mathrm{c}}$ is the least fixed point, we have:

$\operatorname{rpe}($ while e do $c, \mathcal{E})\left(s_{1}, s_{2}\right) \leqslant \mathbb{E}_{\left(s_{1}^{\prime}, s_{2}^{\prime}\right) \sim \tilde{\mu}_{s_{1}, s_{2}}}\left[\mathcal{E}\left(s_{1}^{\prime}, s_{2}^{\prime}\right)\right] \leqslant \mathcal{L}_{\mathcal{E}, c}\left(s_{1}, s_{2}\right) \leqslant \mathcal{J}\left(s_{1}, s_{2}\right)$. 